\title{
IntechOpen
}

\section{Cystic Fibrosis in the Light of New Research}

Edited by Dennis Wat 



\section{CYSTIC FIBROSIS IN THE LIGHT OF NEW RESEARCH}

Edited by Dennis Wat 


\section{Contributors}

Michael Sebastian Daniel Kormann, Magali Taulan, Teresinha Leal, Emer P. Reeves, Michelle White, Fatma Gargoum, Niall Browne, Killian Hurley, Noel G. McElvaney, Martin Walshaw, Gareth Huw Jones, Paula Dyce, Hassett, Cormac McCarthy, Alessandro Franciosis, Orla O'Carroll, Patrick Sinn, Ashley Cooney, Caroline Raynal, Anne Bergougnoux, Mireille Claustres, Dennis Wat, Rosaria Casciaro, Federico Cresta, Rosa Patricia Arias Llorente, David González-Jiménez, Juan José Diaz-Martín, Carlos GarcÃa Bousoño, Alda Marques, Terry W. Chin, Monica Borgatti

\section{(c) The Editor(s) and the Author(s) 2015}

The moral rights of the and the author(s) have been asserted.

All rights to the book as a whole are reserved by INTECH. The book as a whole (compilation) cannot be reproduced, distributed or used for commercial or non-commercial purposes without INTECH's written permission.

Enquiries concerning the use of the book should be directed to INTECH rights and permissions department (permissions@intechopen.com).

Violations are liable to prosecution under the governing Copyright Law.

\section{(c)) BY}

Individual chapters of this publication are distributed under the terms of the Creative Commons Attribution 3.0 Unported License which permits commercial use, distribution and reproduction of the individual chapters, provided the original author(s) and source publication are appropriately acknowledged. If so indicated, certain images may not be included under the Creative Commons license. In such cases users will need to obtain permission from the license holder to reproduce the material. More details and guidelines concerning content reuse and adaptation can be foundat http://www.intechopen.com/copyright-policy.html.

\section{Notice}

Statements and opinions expressed in the chapters are these of the individual contributors and not necessarily those of the editors or publisher. No responsibility is accepted for the accuracy of information contained in the published chapters. The publisher assumes no responsibility for any damage or injury to persons or property arising out of the use of any materials, instructions, methods or ideas contained in the book.

First published in Croatia, 2015 by INTECH d.o.o.

eBook (PDF) Published by IN TECH d.o.o.

Place and year of publication of eBook (PDF): Rijeka, 2019.

IntechOpen is the global imprint of IN TECH d.o.o.

Printed in Croatia

Legal deposit, Croatia: National and University Library in Zagreb

Additional hard and PDF copies can be obtained from orders@intechopen.com

Cystic Fibrosis in the Light of New Research

Edited by Dennis Wat

p. $\mathrm{cm}$.

ISBN 978-953-51-2152-7

eBook (PDF) ISBN 978-953-51-7248-2 


\section{We are IntechOpen, \\ the world's leading publisher of Open Access books}

Built by scientists, for scientists

\section{$3,800+$}

Open access books available

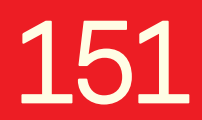

Countries delivered to

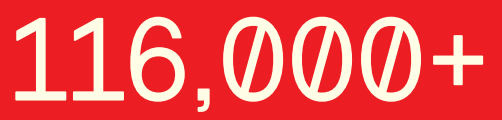

International authors and editors
$120 \mathrm{M}+$

Downloads

Our authors are among the

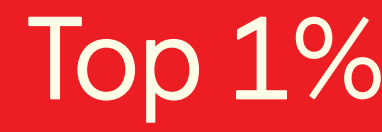

most cited scientists

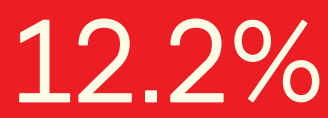

Contributors from top 500 universities

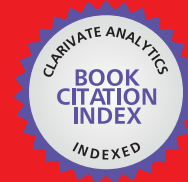

WEB OF SCIENCE ${ }^{\mathrm{TM}}$

Selection of our books indexed in the Book Citation Index in Web of Science ${ }^{\mathrm{TM}}$ Core Collection (BKCI)

Interested in publishing with us?

Contact book.department@intechopen.com

Numbers displayed above are based on latest data collected.

For more information visit www.intechopen.com

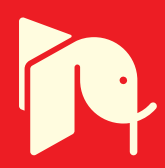





\section{Meet the editor}

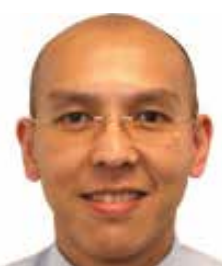

Dennis Watt obtained his degree in Medicine (MB BCh) in Cardiff, UK, in 1998 and was awarded Doctor of Medicine (MD) for his research on the impact of respiratory viruses in Cystic Fibrosis from the University of Cardiff. He trained in pulmonary medicine in Cambridge. During this period, he had the opportunity to work in the Cystic Fibrosis Unit at Papworth, UK. In 2009, he was appointed as Consultant Respiratory Physician in the Cambridge Centre for Lung Infection at Papworth Hospital, dedicated to looking after patients with Cystic Fibrosis and non-CF bronchiectasis. In 2012, Dennis moved to Liverpool for family reasons and joined the Knowsley Community COPD Service. Under his stewardship, this service has since won the Health Service Journal 'Respiratory Integrated Care Award' in 2013. Apart from his vast experience practising CT and COPD, he also has experience in asthma, non-CF bronchiectasis, sleep medicine and oxygen therapy for the management of chronic lung diseases.

Dennis has published widely in respiratory medicine, including peer-reviewed journals, abstracts and book chapters. He has also been a reviewer for a number of high-impact peer-reviewed journals. 



\section{Contents}

Preface XIII

Section 1 Basic Science for the Clinician 1

Chapter 1 Factors Affecting Prognosis and Prediction of Outcome in Cystic Fibrosis Lung Disease 3

Cormac McCarthy, Orla O'Carroll, Alessandro N. Franciosi and Noel

G. McElvaney

Chapter 2 Outcome Measures for Respiratory Physiotherapy in Cystic Fibrosis - Challenges and Advances 37

Alda Marques, Joana Cruz, Cristina Jácome and Ana Oliveira

Chapter 3 Oxidative Stress in Cystic Fibrosis $\mathbf{7 3}$

D. González Jiménez, J.J. Díaz Martín, R.P. Arias Llorente and C. Bousoño García

Section 2 Clinical Aspect of Cystic Fibrosis 85

Chapter 4 Cystic Fibrosis Related Diabetes 87

Paula Dyce, Gareth Huw Jones and Martin J Walshaw

Chapter 5 Cystic Fibrosis and Fertility 111

Rosaria Casciaro, Federico Cresta, Federica Favilli and Laura

Minicucci

Chapter 6 Medications to Enhance CFTR Activity 131

Terry W. Chin 
Section 3 Microbiology of Cystic Fibrosis 141

Chapter 7 Respiratory Virus in Cystic Fibrosis - A Review of the Literature 143

Dennis Wat

Chapter 8 An Overview of Infections in Cystic Fibrosis Airways and the Role of Environmental Conditions on Pseudomonas aeruginosa Biofilm Formation and Viability 171

Cameron T. McDaniel, Warunya Panmanee and Daniel J. Hassett

Section 4 CFTR - Mutations and Modifiers 199

Chapter 9 New Molecular Diagnosis Approaches - From the Identification of Mutations to their Characterization 201 Anne Bergougnoux, Magali Taulan-Cadars, Mireille Claustres and Caroline Raynal

Chapter 10 Emerging Roles of microRNAs in Cystic Fibrosis - From Pathogenesis to Development of New Therapies 233 Sabrina Noel and Teresinha Leal

Chapter 11 New Research on the Importance of Cystic Fibrosis Transmembrane Conductance Regulator Function for Optimal Neutrophil Activity 249

Michelle M. White, Fatma Gargoum, Niall Browne, Killian Hurley, Noel G. McElvaney and Emer P. Reeves

Chapter 12 Role of Non-coding RNAs in Cystic Fibrosis $\mathbf{2 7 9}$ Jessica Varilh, Jennifer Bonini and Magali Taulan-Cadars

Section 5 Cystic Fibrosis: Genetic Correction 307

Chapter 13 Therapy for Cystic Fibrosis Caused by Nonsense Mutations 309

Roberto Gambari, Giulia Breveglieri, Francesca Salvatori, Alessia Finotti and Monica Borgatti

Chapter 14 Integrating Viral and Nonviral Vectors for Cystic Fibrosis Gene Therapy in the Airways 327

Ashley L Cooney, Paul B McCray, Jr and Patrick L. Sinn 
Chapter 15 In vivo Gene Correction of Cystic Fibrosis 357

Michael Sebastian Daniel Kormann 



\section{Preface}

Cystic Fibrosis $(\mathrm{CF})$ is the most common autosomal recessive inherited disease. The incidence of CF in the UK is around 1 in 2,500 live births with 1 in 25 carrying a CF gene mutation. $\mathrm{CF}$ is a multisystem disorder and is characterised by chronic suppurative lung disease and by exocrine pancreatic insufficiency, which affects gastrointestinal function and causes restricted growth and maturation. CF also causes obstructive azoospermia and thus male infertility. It is an autosomal recessive disorder and is accounted for by mutations in the Cystic Fibrosis Transmembrane Conductance Regulator (CFTR) gene. The main function of CFTR in many tissues is to regulate and participate in the transport of chloride ions across epithelial cell membranes. Thus far, more than 1,900 mutations have been described in this gene, but the most common mutation worldwide is caused by the deletion of phenylalanine at position 508 (Delta F508) of the CFTR.

The existing therapeutic modalities for CF lung disease are predominantly reliant on the mechanical clearance of airway secretions, treatment of infection with novel antibiotics and intense nutritional support and care delivered by dedicated specialist CF centres. All these have contributed to the improved life expectancy and quality of life in recent decades. However, antibiotic treatments are at times fraught with difficulties of bacterial resistance and cumulative toxicity, and they are also cumbersome and time consuming for patients and families. Eventual respiratory failure is the main cause of mortality in CF. Hence, there is a pressing need for the development of newer treatment approaches.

This book has attracted leading experts in this field, covering a variety of topics regarding this fascinating condition ranging from microbiology, immunologic aspects, therapeutic options and the exciting genetic management of this condition.

It is well recognised that Pseudomonas aeruginosa is the major infective organism in adult CF lungs; there is a dedicated chapter that defines some of the niches which enable this organism to thrive in the CF lung and the available therapeutic options.

Another chapter presents an overview on the impact of respiratory viruses in $\mathrm{CF}$, providing an insight on the interaction between viruses and bacteria as well as the preventative and therapeutic measures that are currently available for the management of viral lung infection in CF.

There is also a chapter that outlines how the prognosis of this life-limiting illness has changed in the last decade and some co-morbidities in CF that may influence its long-term outcomes. It also outlines some useful clinical measurements which may enable clinicians to make predictions on CF prognosis. 
CF-related diabetes is an emerging area. This book recounts the difficulties in securing this diagnosis and the advantages of continuous glucose monitoring rather than the traditional oral glucose tolerance test as a diagnostic tool for this condition. It also explains how some lifestyle changes may potentially improve glycaemic control.

As CF prognosis improves, this poses a question about fertility in patients with CF. This subject also poses challenges to clinicians when addressing fertility issues with CF patients. This book provides some advanced treatment options for infertility in CF and the subsequent impact of pregnancy on lung function progression, diabetes control and nutritional status.

Oxidative stress has been implicated as a causative factor in the etiology of progressive lung damage in CF. Supplementation with antioxidant micronutrients might therefore be helpful in rendering progression of CF lung disease. A section is this book is dedicated to looking at the main pathways of oxidative stress in $\mathrm{CF}$ and the role of new antioxidant treatments.

Despite advances in our understanding of the molecular and cellular bases of $\mathrm{CF}$, there persists an enigma as to why recruited neutrophils fail to eradicate bacterial infections in the lung. This books provides an overview of neutrophil functions in CF as well as the alterations of cellular activities that lead to the pathogenesis of this disease.

Genetic treatment is a hot topic in CF. This book encompasses a collection of chapters that provide an overview of the therapies that are designed to enhance CFTR protein activity. The book also presents some theoretical possibilities and primary data in preclinical models using genome editing and technologies that support in vivo gene correction of CFTR. All these aspects provide hope to enhance the overall prognosis of this condition. This is a book aimed for clinicians, scientists and researchers with specialist interest in CF. I hope that they will gain a greater insight into this fascinating condition, as I have done. Through the interesting ideas expressed by the authors, I hope this will generate future research ideologies and innovations which may have significant impacts on this life-limiting illness.

I would like to use this opportunity to thank all the authors who have contributed to this book; without their effort, this book would not have materialised. I would also like to thank INTECH for appointing me as the Editor and providing me with guidance throughout the development of this book.

Finally, I would like to thank my wife, Pauline, and my three lovely children, Lucinda, Harrison and Edison, for sacrificing their precious family time for the preparation of this book.

Dr Dennis Wat

Consultant Chest Physician Liverpool Heart and Chest Hospital, United Kingdom 
Section 1

Basic Science for the Clinician 

Chapter 1

\title{
Factors Affecting Prognosis and Prediction of Outcome in Cystic Fibrosis Lung Disease
}

\author{
Cormac McCarthy, Orla O'Carroll, \\ Alessandro N. Franciosi and Noel G. McElvaney \\ Additional information is available at the end of the chapter
}

http://dx.doi.org/10.5772/60899

\begin{abstract}
Cystic fibrosis (CF) is a multisystem disorder with a significantly shortened life expectancy with the major cause of mortality related to lung disease. Inflammation is seen in the CF airways from a very early age and contributes significantly to symptoms and disease progression. As the condition worsens over time, lung function declines, usually measured by Forced Expiratory Volume in 1 second (FEV1)\% predicted, and extra-pulmonary complications often manifest. While the life expectancy in CF is still short, the median age of death and predicted survival age are continually increasing. Therapeutic interventions for CF have improved significantly in the last 20 years and now there are targeted therapies towards specific elements in CF that may impact upon exacerbation frequency, symptoms, and eventually mortality due to lung disease.
\end{abstract}

As life expectancy in CF increases, the need for predicting prognosis becomes more and more important. Numerous factors affect prognosis in $\mathrm{CF}$ and can be used to ultimately predict outcomes. These factors can be constant or dynamic variables ranging from genetic mutation and gender to clinical measurements including pulmonary function and weight. Further variables that affect prognosis in CF include Diabetes Mellitus, sex, and pancreatic insufficiency. Furthermore, genotype is becoming more and more important as novel targeted therapies are developed that may affect survival and improve prognosis.

While prognosis in CF has traditionally been associated with FEV1\% predicted, decrements in lung function that are associated with recurrent pulmonary exacerbations are increasingly important, and these are increasingly common as CF lung 
disease progresses. What drives these pulmonary exacerbations is bacterial colonisation, particularly Pseudomonas aeruginosa, with early eradication shown to improve prognosis. Nutrition and weight are also very important in CF and low body mass index has been shown to predict poor outcomes. There are several clinical prediction tools in CF, both radiological and clinical and many are too complex to be used routinely in patient care. However, newer tools aimed at predicting outcomes based on readily available objective measure are now available, including the CF-ABLE score.

In this chapter we outline, firstly, how prognosis in CF has changed over the last decade as a result of changes in treatment, better diagnostics, and improved care. Secondly, we describe the effects that genotype, pancreatic status, gender, and diabetic status have upon outcome. Thirdly, this chapter highlights the usefulness and importance of clinical measurements, including lung function, radiology, bacteriology, and blood and sputum biomarkers of disease and inflammation in predicting outcomes and how changes in these parameters influence prognosis. Finally, we summarise the prediction tools that have been utilised in CF to predict survival and how these may be utilised in clinical practice.

In conclusion, the most sensitive way of predicating prognosis currently remains a multifaceted approach, including several markers of disease and the use of all factors and a composite clinical prediction tool is suggested to stratify patient risk.

Keywords: Prognosis, prediction, survival, outcome, cystic fibrosis

\section{Introduction}

Cystic fibrosis (CF) is a multisystem inflammatory condition that is associated with a significantly shortened life span, primarily as a result of the pulmonary manifestations of the disease [1]. For many years pulmonary function measurements have been utilised as the primary surrogate of disease severity, with forced expiratory volume in 1 second (FEV1) used to assess clinical status of both patients and to predict mortality [2,3]. However, in the last two decades there has been a significant improvement in survival in CF and this subsequently has consequences on how to treat patients and predict prognosis in this complex condition. With longer life expectancy it is essential to better predict outcome and prognosticate in $\mathrm{CF}$, thus the use of survival or death as an outcome measure has become almost negligible in clinical trials or indeed in studies to predict prognosis. Hence, the development of surrogate markers or disease severity is increasingly important in CF; these range from physiological measurements of lung function, biomarkers, radiological measures, and composite scoring systems and are becoming essential in CF care and development of new drugs. With groundbreaking therapeutic breakthroughs in CF over the last decade, particularly in the modulation of CFTR function [4], the use of surrogate outcomes has become more apparent. This has led to development of new 
imaging modalities such as flurodeoxyglucose positron emission tomography (FDG-PET) imaging [5-7] and hyperpolarised helium magnetic resonance imaging (He3-MRI) [8], as well as increased use of multiple breath washout (MBW) and lung clearance index (LCI) to assess disease severity [9]. Moreover, there has been huge progress in the research into and development of biomarkers of inflammation in CF [10-12], both systemic and pulmonary inflammation that correlates with clinical condition and can predict outcome, further highlighting the deeper understating of the pathophysiological changes in CF and the translational research ongoing in this area. Furthermore, the use of new composite scoring systems, taking into account many aspects of this multisystem condition have been developed to aid with the classification of disease severity and predict outcome over a defined period [13].

In this chapter we will outline, firstly, how prognosis in CF has changed over the last decade as a result of changes in treatment, better diagnostics, and improved care. Secondly, the chapter will describe the effects genotype, such as pancreatic status, gender, and diabetic status, have upon outcome. Thirdly, this chapter will highlight the usefulness and importance of clinical measurements, including lung function, radiology, bacteriology, and blood and sputum biomarkers of disease and inflammation in predicting outcomes and how changes in these parameters influence prognosis. Finally, the chapter will summarise the prediction tools that have been created in CF, both clinical and research tools, which utilise measurements of disease and radiological evidence of bronchiectasis to predict survival and how these may be utilised in clinical practice.

\section{Prognosis in cystic fibrosis}

Life expectancy in patients with cystic fibrosis is in a constant state of change. Whilst there are undoubtedly significant further gains to be made, the improvement in predicted survival in cystic fibrosis sufferers has been a relative success story since its original description as a clinical entity in 1938. Median life expectancy, the time period in which half of a given population will die, has increased from a few months in the 1940s to as high as 41 years old in the current era [14] at present in many countries. The predicted median survival of people born with CF today continues to rise.

Numerous factors have contributed to the changing statistics in CF prognosis. Earlier and more sensitive detection methods, centralised specialist multidisciplinary care and evidence-based research have provided patients, their families, and clinicians with an environment that facilitates the long-term management of this complex multisystem disorder. Identification of increasing numbers of CF genotypes (many of which are characterised by phenotypically milder variants) has also contributed to increasing the CF population and this consequently affects the overall statistics on outcome and prognosis.

What is relevant to a patient diagnosed in infancy with a severe form of CF may not be relevant to a patient diagnosed in middle adulthood. Equally, statistics on survival from one country may not relate accurately to another, not because of difference in treatment alone, but because of differences in the predominating demographics in the two cohorts and methodologies used 
in assessing outcomes. As such, a "one size fits all" approach to prognosticating is not appropriate. In this chapter, we review the evidence regarding prognosis based on key clinical and demographic parameters, and the biomarkers and prediction tools that may be used to predict outcome.

\section{Influence of genotype on prognosis in cystic fibrosis}

$\mathrm{CF}$ is the most common lethal genetic disease of Caucasian populations and is caused by genetic mutations of the cystic fibrosis transmembrane regulator (CFTR) gene. The CFTR gene encodes an ATP- and CAMP-dependent chloride channel expressed on the apical membrane of epithelial and certain non-epithelial cells throughout the body. To date, greater than 1,000 CFTR mutations have been discovered. In the lung, it also regulates the activity of the ENaC channel (an apical sodium transport channel) defects in which mediate the majority of pathogenic processes in the main target organ in CF sufferers.

Commonly, the multitude of various CFTR mutations are classified into 6 distinct groups based on their ultimate effects on a range of cellular mechanisms such as transcription, processing within the cell, localization of the channel, and quantity of correctly functioning protein. Class I includes mutations with complete lack of production of protein while Class VI involves unstable functional protein being produced that is then degraded at the cell surface (Table 1).

\begin{tabular}{llll}
\hline Class & Effect & Mutation Type & Example \\
\hline I & No functional protein produced & $\begin{array}{l}\text { Premature stop codons: } \\
\text { nonsense; splicing; deletions }\end{array}$ & W128X; R553X; G542X \\
\hline II & Defective processing and maturation & $\begin{array}{l}\text { Missense; small deletions or } \\
\text { insertions }\end{array}$ & F508del; N1303K \\
\hline III & Defects in regulation of channel opening & $\begin{array}{l}\text { Missense; small deletions or } \\
\text { insertions }\end{array}$ & G551D; G551S; G1349D \\
\hline IV & Defective Chloride transport & $\begin{array}{l}\text { Missense; small deletions or } \\
\text { insertions }\end{array}$ & R117H; R334W, R347P \\
\hline V & Reduction of wild-type mRNA & Partial splicing & A455E \\
\hline VI & Increased turnover of unstable protein at cell & Missense; nonsense & 120del23; N287Y; 4279insA \\
& surface & & \\
\hline
\end{tabular}

Table 1. List of CFTR Mutations.

The most common CFTR mutation worldwide is delF508 that accounts for upwards of $70 \%$ of cases of $\mathrm{CF}$ and has long been associated with more severe disease and less favourable clinical outcomes [15-17]. Conversely, several mutations have been found to be associated with more favourable outcomes and milder clinical phenotypes. In a study based on the US CF Registry in 2003, it was found that significantly different mortality rates were observed when CFTR 
genotype was classified according to the effects on quantitative protein production. The authors proposed that groups with severely reduced levels of CFTR (I-III) had a more severe clinical phenotype and higher mortality than groups with some residual CFTR function (IVVI) [18]. A follow up study found that 'high risk' patients (I-III) had a two-fold greater risk of death when compared to 'low risk' (IV-VI) patients [19] and this risk was not fully explained by lung function, pancreatic insufficiency, Pseudomonas aeruginosa (PA) colonisation, or nutritional factors. Hence, CFTR genotype may be useful as an initial measure of prognosis in early CF diagnosis when it is often the only available information about the disease. Knowledge of the genetic processes involved in CF is leading the way for new targeted therapies that ideally will improve mortality. One such therapy is ivacaftor, a potentiator drug that enhances gating at the cell surface in patients with the class III G551D mutation. This novel medication has been associated with sustained increases in FEV1 of up to $10 \%$, reduced frequency of pulmonary exacerbation, weight gain, and subjective improvements in quality of life (CFQ-R) scores [4, 20,21]. However, there is wide phenotypic variance observed frequently among patients with identical genotypes. This is observed in the clinical realms of lung function, pancreatic and diabetic status, nutritional status, and response to medications and this leads to reluctance to link genotype too closely with phenotype in terms of predicting clinical outcomes and prognosis.

\section{Impact of gender on prognosis}

It has long been recognized that there is a significant gender difference in terms of both morbidity and mortality in cystic fibrosis. Many studies, throughout early and current cystic fibrosis research, have focused on the poorer outcomes observed in female patients when compared with their male counterparts. This dichotomy endures across the areas of microbial colonization, lung function, frequency of exacerbations, and overall survival. However, an explanation for this vast difference remains to be found.

A notable early study conducted in Canada investigated the effects of numerous variables on mortality and found that on average, males had overall increased survival rates of greater than 5 years when compared with female cohorts from the same time period (1970-1989) [22]. These results were echoed across international cohorts for similar time frames and were postulated to relate to the lower bone mineral density observed in female populations. Over time, as survival in cystic fibrosis increased, this gender difference has persisted with Rosenfeld et al. in 1997 confirming a gender difference in CF survival (25.3 vs. 28.4 females vs. males) in a cohort of over 20,000 patients [23], and Harness-Brumley et al. in more recent 2014 study observed this difference in greater than 32,000 North American patients (36.0 years vs. 38.7 years, females vs. males) [24]. Despite accounting for variables known to influence CF-related mortality, it was demonstrated that female gender is an independent significant risk factor for death. Furthermore, women were found to be colonized at an earlier age with various pathogens and that their clinical course was much worse when colonized with common CF pathogens [24]. 
Though the relationship between gender and adverse outcomes has been described in a multitude of studies over a vast period of time, a full explanation of this dichotomy remains elusive. Much current research now centres on the role of estrogen in female CF patients. The primary female sex hormone is $17 \beta$-estradiol $\left(\mathrm{E}_{2}\right)$ and circulates in the body bound to sex hormone binding globulin, interacting with target tissues through a range of estrogen receptors (ERs) expressed on the cell surface. $E_{2}$ levels naturally vary over the course of the normal menstrual cycle and $E_{2}$ further dehydrates the already compromised airway surface liquid seen in $\mathrm{CF}$ and peak levels of $\mathrm{E}_{2}$ lead to an increased risk of infection and subsequent exacerbation [25]. Furthermore, high levels of $\mathrm{E}_{2}$ have been shown to promote TLR hyporesponsiveness to a range of bacteria driven by an inhibition of interleukin-8 (IL-8) release [26]. In a study targeted to investigate the effects of $\mathrm{E}_{2}$ on Pseudomonas Aeruginosa (PA). it was found that high levels of $\mathrm{E}_{2}$ promotes mucoid conversion, alginate synthesis, and genetic mutations in mucins lending increased infectivity and virulence to PA in those exposed to $E_{2}$ [15]. An interesting addendum to this study was the observation that the individuals studied had lower rates of exacerbation and required a lower number of antibiotic courses if they were using the combined oral contraceptive pill [15]. As exacerbation rate is commonly quoted as a predictor for mortality, there is scope here to assess the role of targeted estrogen therapies on mortality and the gender dichotomy in $\mathrm{CF}$.

\section{Relationship between exocrine disease and prognosis in cystic fibrosis}

Pancreatic insufficiency is an extremely common complication of CF and affects over $85 \%$ of patients at some point during the course of their disease [27]. Loss of pancreatic exocrine function leads to malabsorption of fat, protein, and micronutrients, which in turn causes failure to thrive, steatorrhoea symptoms, and fat-soluble vitamin deficiencies. This arises as a consequence of obstruction of proximal intralobular ducts in the pancreas due to inspissated mucus plugs and tends to arise early in the disease. Chronic pancreatitis is another less common manifestation generally associated with milder (IV-VI) CF genotypes [28, 29], as residual pancreatic acinar tissue is a prerequisite for pancreatitis to develop. Pancreatitis affects over $10 \%$ of CF patients and tends to occur in a chronic relapsing and remitting fashion. It is thought to be due to a combination of obstructive tubulopathy and acidification of the acinar lumen due to reduced ductal bicarbonate secretion. Those with symptomatic pancreatitis can often become pancreatic insufficient as their disease progresses. A recent review of the European Cystic Fibrosis registry demonstrated that pancreatic insufficiency was associated with a statistically significant decreases in FEV1\%, with pancreatic insufficient patients twice as likely as sufficient patients to experience severe lung disease, defined as $\mathrm{FEV}<40 \%$ predicted [30]. This indicates that lack of pancreatic exocrine function is associated with worsening prognosis.

\section{Cystic fibrosis-related diabetes is associated with poor prognosis}

Cystic fibrosis-related diabetes (CFRD) is a common comorbidity with an estimated prevalence of $20 \%$ in adolescents and $40 \%-50 \%$ in adults [31]. As with other disease-related comorbidities, 
incidence of CFRD continues to increase as life expectancy improves. It encompasses a spectrum of disease from impaired glucose tolerance to CFRD with fasting hyperglycaemia. Impaired chloride channel function leads to thick pancreatic secretions that cause obstructive damage to the exocrine pancreas and subsequent architectural disturbance in islet cells with loss of endocrine function. Insulin deficiency is compounded by insulin resistance that occurs during pulmonary exacerbations as a consequence of increased levels of growth hormone, cortisol, catecholamines, and inflammatory cytokines [32]. Not all patients with CF go on to develop CFRD, and it has been demonstrated that multiple extrinsic factors contribute to disease pathogenesis such as malabsorption, immunosuppressant therapy following lung transplantation, use of glucocorticosteroids, and the presence of liver disease.

CFRD has a significant impact upon clinical parameters of disease and thus impacts upon prognosis. Insulin deficiency and hyperglycaemia negatively affects pulmonary function with the rate of decline in FEV1\% over a 4 year period found to be related to the severity of insulin deficiency [33]. Moreover, insulin replacement therapy can improve both nutritional status and pulmonary function in patients with CFRD [34]. Hyperglycaemia also impacts clinically, with moderately elevated blood glucose levels leading to increased airway glucose concentrations, which in turn promotes the growth of various respiratory pathogens and can increase exacerbation rate [35]. CFRD also impacts, predictably, on nutritional status with both insulin deficiency and hyperglycaemia exerting effects. The classical complications of diabetes mellitus also contribute to morbidity in patients with CFRD. Macrovascular complications have not been documented, despite the increasing life expectancy of these patients. However, microvascular complications are common and include mild neuropathy as the most common manifestation with prevalence rates similar to those for non-CF diabetics [36]. Furthermore in one study, retinopathy occurred in $16 \%$ of CFRD patients and microalbuminaemia in $14 \%$ in a cohort of patients who had diabetes for more than 10 years [36].

In terms of direct mortality, CFRD has been shown to have a negative prognostic effect, with increased mortality seen in association with poorer nutritional status and greater severity of lung disease $[37,38]$. In addition, CFRD mortality has been shown to be increased in female cohorts when compared to males [39]. However, over time there have been sustained improvements in CFRD-associated mortality with both female and male mortality decreasing in the period between 1992 and 2003 [31], this effect is presumably a reflection of increased awareness of the importance of CFRD and improvements in diagnosis and treatment strategies. CFRD is a common and complex co-morbidity in CF which negatively impacts on clinical outcomes and mortality. There is evidence to show that early treatment of CFRD can promote improved nutritional status, pulmonary function, and ultimately improve outcome.

\section{Cystic fibrosis-related liver disease and prognosis}

As the life expectancy of patients with CF continues to improve, cystic fibrosis-related liver disease (CFLD) is becoming increasingly more prevalent with an incidence estimated of up to $30 \%$, according to multiple studies. Liver disease firstly manifests in its most simple form as 
biochemical derangements in liver enzymes. Structural disease develops next and manifests as fatty infiltration on ultrasound scan or increased bile content in liver parenchyma. Decompensated liver disease occurs last, which can involve portal hypertension, ascites, variceal disease, and impaired coagulation. These disease processes occur as a consequence of altered viscous bile due to abnormal CFTR-regulated ion transport across cholangiocytes. Consequently, biliary flow is reduced and there is obstruction of intrahepatic bile ducts. This causes damage to hepatocytes and cholangiocytes through inflammatory processes, bile duct proliferation, and portal tract fibrosis. The pathogenesis of fatty liver disease in CF is less well understood and has been loosely attributed to fatty acid deficiency, malnutrition, and insulin resistance [40].

Attempts have been made to link the development of CFLD with the presence of certain underlying CFTR mutations, the assumption being that CFLD would be seen with increasing frequency in patients with the classically severe or high-risk phenotypes as described above. However, this relationship has not been demonstrated to date. Non-CFTR modifier genes have been proposed to increase susceptibility to development of CFLD and it is thought that identifying these genetic modifiers may allow early identification of patients at risk. To date only polymorphisms in the SERPINA1 allele, which codes for an alpha-1 antitrypsin, has been demonstrated to be significantly related to CFLD and portal hypertension [41].

Liver disease is widely cited as the third most common cause of death in $\mathrm{CF}$ patients after respiratory failure and transplant complications, with a mortality rate estimated at $2.5 \%$ [30] and a higher mortality risk is observed in those with liver disease than in those without [40]. However, there continues to be massive variability in the severity of CFLD without adequate explanation as to why it affects only certain patients, thus it is a complex prognostic index.

\section{Clinical measurements of disease severity and how they predict prognosis}

Whilst recognising the importance of tailoring care of patients with CF to the individual genotypes and associated co-morbidities is essential, many of these factors are established and in many ways less dynamically modifiable than others in clinical practice.

The prognostic value of more dynamic markers of clinical condition offer more practical longterm targets around which to focus treatment and goals in the individual patient. On a day to day basis, these parameters guide practice and stratify patients in terms of expected outcomes.

\section{Pulmonary function and how it predicts prognosis}

Spirometric measurement of lung function, specifically FEV1, has been the pre-eminent surrogate marker of disease staging in $\mathrm{CF}$ for a long time. As it is reproducible, readily available, and cheap, it provides longitudinal measurement of airflow obstruction over years. 
Seminal work by Kerem et al. in 1992 suggested that as an independent factor, FEV1 reliably predicts the relative risk of mortality, with a baseline FEV1 of less than $30 \%$ predicted giving a $50 \%$ two-year mortality [3,42]. Following on from this work, further studies suggested that rate of decline of FEV1 could more accurately identify the most at risk patients [2, 43, 44]. Interestingly there is evidence that high baseline FEV1 is a risk factor for greater FEV1 decline and that this phenomenon may be explained by less aggressive prescribing patterns in patients with preserved lung function [45].

Few would argue that FEV1 is invaluable in clinical practice; however, it does fall short in identifying early lung disease where spirometry is often normal. This is especially the case in paediatric CF centres, where lung disease is often in its early stages or where technical limitations in performing reliable expiratory manoeuvres are more common. Recent interest has arisen in lung disease assessment using the Lung Clearance Index (LCI), a measurement derived from the multiple-breath inert gas washout. Whilst this technique is time consuming and less readily available, there is growing evidence that it may be more sensitive than FEV1 in diagnosing early lung disease $[46,47]$ and that abnormal LCI in the setting of normal FEV1 may predict future FEV1 decline [9]. Moreover, LCI correlates with high resolution computerised tomography (HRCT) findings [48] and predicts exacerbations and time to first exacerbation [49]. As such LCI may prove to be an appealing tool for more sensitively detecting improvements in lung health when assessing novel therapeutic options [50] and become a useful prognostic index.

\section{Microbial colonisation in cystic fibrosis and its effect upon prognosis}

Bacterial colonisation of the airways is the catalyst for cyclical infectious exacerbations of $\mathrm{CF}$, leading to acute, subacute, and chronic inflammation. The identification of organism and subsequent in-vitro sensitivity testing helps in guiding treatment, antibiotic choices, and even infection control measures. The individual impact of specific organisms on prognosis and survival has been a focus of interest in clinical research for some time.

\section{Pseudomonas aeruginosa}

The most prominent pathogen with a specific affinity for the CF lung is Pseudomonas Aeruginosa (PA). Though its prevalence in CF populations varies with age [51], it is the most common airway pathogen in adult CF patients. PA colonisation predicts lower FEV1 at the time of culture $[52,53]$ and greater rate of decline in pulmonary function over time [54-56] when compared with Methicillin Sensitive Staphylococcus Aureus colonised (MSSA) and noncolonised patients. Moreover, evidence suggests that patients in whom PA colonisation is eradicated in a systematic manner have a significant reduction in treatment burden and days spent in hospital. This, in turn, can result in reduced expense on treatment of exacerbations [57]. 


\section{Staphylococcus aureus}

Amongst the earliest pathogens to become clinically prominent in the life of patients with $\mathrm{CF}$ is Staphylococcus Aureus (SA) [58]. Its prevalence globally is $44 \%-56 \%$ (2008) in its methicillin sensitive form (MSSA) and $8 \%-23 \%$ for methicillin resistant strains (MRSA). The difficulty in accurately establishing the relevance of SA as a pathogen in CF may lie in the complexity involved in characterising the specific virulence of the strains involved. MSSA, Small colony variant MSSA (SCV-MSSA) and MRSA (both community acquired-MRSA and healthcare associated MRSA) all differ in resistance patterns and apparent virulence. A study by Hoffman et al. in 2006 [59] suggested that co-colonisation with MSSA and PA favoured the formation of SCV-MSSA. Subsequent work by Besier et al. [60] associated colonisation with SCV-MSSA with worse lung function, more PA co-infection and more antibiotic resistance than those with MSSA. This is especially telling when colonisation with PA and SA is not uncommon. During a 1990s US-based clinical trial [61] an analysis of baseline sputum found 43\% of participants to culture both PA and SA. Current evidence suggest that patients culturing MRSA have worse lung function and require more antibiotics than those with MSSA [62] and that PA/MRSA cocolonisation is associated with more pronounced decline in lung function than PA/MSSA cocolonisation [63]. Controversy still exists surrounding the long established practice of prescribing prophylactic antimicrobials with activity against MSSA. Although studies have suggested reduced cough and less culture positivity in the setting of prophylaxis, no clear benefits in terms of outcomes have been observed. Furthermore, several studies have pointed to increased and earlier incidence of PA culture in patients undergoing MSSA suppression therapy [64]. Whilst guidelines published by international bodies vary on their recommendation regarding chronic anti-staphylococcal use, a recent Cochrane review found no convincing evidence for treatment [65] and reiterated concerns around the increase in PA colonisation.

\section{Burkholderia cepacia complex}

Long mistaken for a Pseudomonas, Burkholderia species were identified more accurately in the 1980s and 1990s as diagnostic techniques improved. Now recognised as perhaps the most virulent pathogens in $\mathrm{CF}$, they present a major treatment challenge. The Burkholderia Cepacia Complex (BCC) is comprised of at least 17 distinct species of gram-negative bacteria. Termed Genomovars, these species vary in prevalence and apparent virulence. The most common BCC strains identified are B.Cenocepacia and B.Multivorans. Evidence from 2002 revealed a four-fold mortality risk in patients colonised with epidemic B.Cenocepacia, associated with a more rapid rate of lung function decline versus others (FEV1 -1.9\% vs. $-0.3 \%$ per annum) [66]. Further data from 2004 showed B.Cenocepacia was associated with a statistically significant higher rate of lung function decline $(-140 \mathrm{ml} /$ year vs. $-32 \mathrm{ml} /$ year $(\mathrm{p}=0.01))$ and reduction in BMI when compared to PA and B.Multivorans [67]. Whilst evidence is mounting that specific BCC genomovars have a hierarchy of virulence with B.Cenocepacia associated with the highest morbidity, it is worth noting that progressive disease can be caused by a multitude of strains. "Cepacia Syndrome", a constellation of sepsis, fevers, and leucocytosis and fulminant decline 
most often resulting in death, has been described with genomovars other than B.Cenocepacia [68]. The true determinant of virulence may best be assessed by identification of specific epidemic strains within distinct genomovars. Moreover, strains associated with poor outcomes in the native lungs of CF sufferers may not cause the worst outcomes in post-transplant patients. A 2008 study assessing mortality in CF patients on transplant waiting list and posttransplant revealed a higher waiting-list five year mortality in B.Multivorans and non-epidemic strain B.Cenocepacia carriers than those with epidemic strains of B.Cenocepacia. Furthermore, 5year post-transplant mortality was highest in the non-epidemic B.Cenocepacia cohort and carriers of Burkholderia Gladioli, an organism not included in the BCC group, and generally considered less relevant as a CF pathogen [69]. Clearly a deeper understanding of the relevance and prognostic implications of specific BCC colonisation is needed. Despite the significant risk posed by Burkholderia species in the CF population, evidence for eradication protocols and recommended antibiotic regimens is lacking, which is concerning as it possibly has the most significant effect upon prognosis of all bacterial colonisers [70].

\section{Non-tuberculous Mycobacteria (NTM)}

A 2013 study by Bryant et al. [71] demonstrating patient to patient transmission of Mycobacterium Abscessus (subspecies Massiliense) in a CF centre re-focused attention on the clinical management and implications of NTM colonisation. Prevalence (6\%-14\%) of NTM organisms is variable and changing with time [72] and patient phenotype appears to dictate colonisation with younger, more malnourished patients with more severe genotypes culturing more Mycobacterium Abscessus Complex (MBASC) organisms than Mycobacterium Avium Complex (MAC) $[73,74]$. Recurrent NTM culture positive status has been shown to be associated with progression of HRCT changes [75], however, despite these findings, statistical evidence indicating worse outcomes in pulmonary function or mortality is lacking. With the employment of prophylactic macrolide treatment for reduction of exacerbations, significant concerns were raised regarding the possibility of developing macrolide resistance in colonising NTM species; hence, NTM culture positivity is a contraindication to macrolide prophylaxis. Longterm macrolide use may, however, have a protective effect in reducing incidence of NTM culture positivity as highlighted by Coolen et al. in 2015 [76]. This finding could have a significant impact on prognosis and treatment options, as NTM colonisation is still considered a contraindication to transplant in some centres despite growing evidence that outcomes posttransplant in this cohort are acceptable [77].

\section{Other microbial organisms in cystic fibrosis}

Aspergillus Fumigatus (AspF) is a common airway fungus found in the CF lung. Previous treatment was aimed largely at patients meeting criteria for diagnosis of Allergic Bronchopulmonary Aspergillosis (ABPA). More recent evidence, however, suggests that AspF carriage correlates with more severe changes on HRCT [78] and can predict greater rate of FEV1 decline 
in patients who are a) colonised but not sensitised, b) sensitised but do not meet ABPA diagnostic criteria, and c) those with a diagnosis of ABPA [79]. Suitable studies assessing the value of AspF eradication in patients with CF not meeting ABPA criteria are lacking.

Stenotrophomonas Maltophilia (SM) and its relevance has been a point of debate for some time. Previously regarded as a bystander in the CF microbiome there is growing belief that it represents a true pathogen with potentially significant impact of mortality [80]. Antibiotic options for the treatment of SM are limited and dedicated studies assessing treatment effectiveness are lacking [81].

Candida Species are highly prevalent in CF airway culture [82]. Difficulty arises in differentiating oropharyngeal contamination from bronchial colonisation without bronchoscopic sampling. Data from 2010 suggests that colonisation with Candida Albicans is associated with increased exacerbation rate and significant decline in FEV1 [83]. There is a paucity of data relating to the benefits of treating Candida Species routinely in CF.

\section{Impact of respiratory failure on prognosis in cystic fibrosis}

Despite being a predictable outcome of progressive lung disease, the management of respiratory failure in CF, both hypoxic (Type 1 Respiratory Failure) and hypercapnic (Type 2 Respiratory Failure), is lacking in terms of an evidence base [84]. Prescribing habits for oxygen (nocturnal, ambulatory, or resting) borrow largely from guidelines for other conditions. Hypoxia as a determinant of prognosis therefore has not been assessed, although there are suggestions that patients at higher mortality risk have an association with worsening six minute walk test results [85]. However, baseline hypercapnia has been shown to be an independent risk factor for death, even in patients with an FEV1 greater than $30 \%$ predicted [86]. There is a paucity of reliable data regarding the benefits of maintenance non-invasive bilevel positive airway pressure ventilation (BIPAP) in CF. One randomised placebo controlled trial from 2008 [87] did show significant benefits with nocturnal BIPAP in terms of symptoms (quality of life questionnaires scores, dyspnoea indices, and chest symptoms), improvements in modified shuttle walk test distances, and improved nocturnal (but not diurnal) arterial hypercapnia, but no significant improvements were shown in lung function. Data does suggest, however, that in the setting of acute respiratory failure requiring admission to intensive care units, non-invasive ventilation (NIV) was associated with significantly improved outcomes when compared to intubation and mechanical ventilation in patients with CF [88-90]. Hence, the importance of respiratory failure in predicting prognosis needs further study in CF.

\section{Nutritional status and weight in determining outcome in cystic fibrosis}

The impact of malnutrition in CF is of great interest; exocrine and endocrine dysfunction, coupled with high basal metabolic requirements in the setting of chronic disease, exposes 
patients to a high risk of malnourishment. Low BMI in CF is known to increase in incidence with age and be closely implicated in worsening lung function [91,92] and predicts worse outcome over a 4-year period [93]. Furthermore, patients awaiting lung transplantation who require nutritional intervention have been shown to have a higher risk of death [86] and improving the nutritional status of malnourished patients may ultimately improve lung function, even in cohorts with relatively advanced lung disease [94-96].

\section{Bone mineral density in cystic fibrosis}

Low bone mineral density (BMD) and subsequently osteopenia and osteoporosis are common in CF. Thus far, no convincing association has been shown between lung function and BMD and predictors of low BMD include BMI, weight, and age [97, 98]. Despite much research focus in this area, recent evidence raises the possibility that, unlike lung function and life expectancy, there has not been a significant improvement inter-generationally in BMD [99]. The lack of clinical data proving concrete improvements in outcomes with bisphosphonate therapy, other than BMD change itself, has left a clinical conundrum regarding the management of low BMD in CF [100]. Further studies are needed to guide clinical decisions which are currently dictated by experience and judgement. However, there is no prognostic value in BMD scores in CF patients.

\section{Biomarkers of disease severity and how they predict prognosis}

\subsection{Sputum and bronchoalveolar lavage fluid biomarkers}

The need for useful and clinically relevant biomarkers is vital, and their use as predictors of prognosis as well as surrogates of clinical response to treatment is increasingly important. Numerous different sputum biomarkers have been investigated and used to correlate with clinical condition, including correlating with exacerbation frequency, pulmonary function, microbiological colonisation and overall prognosis. The use of sputum biomarkers is ideal in CF as patients produce significant amounts of sputum, hence samples are easy to obtain and samples are routinely collected to assess bacterial colonisation as part of best practice care.

Several studies have demonstrated how neutrophil elastase (NE) activity is significantly increased in the CF lung and a recent study by Sly et al. revealed how NE activity is an early biomarker for the development of bronchiectasis in CF [101]. The levels of NE in CF bronchoalveolar lavage fluid (BALF) correlate inversely with FEV1 [10] and furthermore the higher the level of NE detectable the more rapid the decline in lung function [12], neutrophil counts also correlate well with these measures. These results support the use of NE levels and activity as a useful biomarker available from BALF or sputum, and many other potential biomarkers are benchmarked against this measure as well as clinical correlation. Several inflammatory cytokines which are known to be elevated in the CF lung have potential to be useful biomarkers also, particularly interleukin-8 (IL-8) and tumour necrosis alpha (TNF- $\alpha$ ). IL-8 is a neutrophil 
chemo-attractant and elevated levels are seen in CF BALF [102] and levels measured in sputum correlate inversely with FEV1 [10]. IL-8 and TNF- $\alpha$ levels are elevated at the time of an acute pulmonary exacerbation, and have been shown to significantly decrease in response to antibiotic treatment in an inverse pattern compared to lung function measurements [103]. Furthermore, the levels of IL-8 found in the lungs of CF patients correlates with microbial colonisation, with higher levels identifiable in patients colonised with Pseudomonas Aeruginosa (PA) and Staphylococcus Aureus (SA) compared to non-colonised CF patients and healthy controls [104]. Further support for the usefulness of these cytokines as markers is how they correlate with symptoms of deterioration as reported by patients [105], hence IL- 8 and TNF$\alpha$ are markers of degree of lung damage and bacterial colonisation. There are limitations to the use of sputum and BALF biomarkers such as these, as they do not always correlate with all clinical measurements of disease; in one study IL-8 did not correlate [106] with modified Bhalla scores [107], or other reversible changes on high resolution computed tomography (HRCT) that did correlate with lung function measurements.

Glycosaminoglycans (GAGs) are involved in the modulation of IL-8 activity in BALF and increased expression of GAGs is related to the sustained inflammation in the CF lung, hence the levels of GAGs may be a potential biomarker of disease progression in CF as their expression is related to the neutrophil chemotaxis in the lung [108]. Other cytokines including IL-10 and IL-4 are also significantly elevated in CF BALF compared to healthy controls [109]. However, fewer studies have been done on these cytokines.

Several other sputum and BALF biomarkers have been studied and some have more potential future utility than others. Some of the early sputum biomarkers investigated were nitrites, with sputum $\mathrm{NO}_{2} / \mathrm{NO}_{3}$ shown to be significantly higher in CF patients with acute exacerbations [110] and correlated with neutrophil counts and inversely with FEV1 [111]. However, the levels did not return to normal despite intensive antibiotic treatment, hence negating their potential use as a marker of response to treatment [110]. Biomarkers that correlate to PA colonisation also include club cell secretory protein (CCSP), which is inversely related to the NE concentration in $\mathrm{CF}$ sputum and patients with pseudomonas aeruginosa have significantly lower CCSP than non-colonised individuals [112]. Furthermore, levels of leukotriene B4 (LTB4) correlate with PA colonisation in CF compared to other organisms [104].

To date, the most accurate sputum and BALF biomarker in CF remains NE activity and levels, hence several other biomarkers have been compared to this to demonstrate their usefulness. Other proteases such as matrix metalloproteinases 2 (MMP-2) and MMP-9 correlate well with increased levels noted in BALF which possessed increased NE activity [109], while cathepsins do not correlate with PA colonisation or exacerbations [113]. Biomarkers specific to pulmonary exacerbations are ideal as defining the response to treatment is very difficult in $\mathrm{CF}_{\text {,; }}$ these include mucins MUC5AB and MUC5AC which are degraded at the time of exacerbation and demonstrate increased sialylation compared to controls [114]. Granulocyte-macrophage colony-stimulating factor (GM-CSF) levels at the time of pulmonary exacerbation predicts a larger acute decline in FEV1[115], which is highly predictive of poor prognosis [13], hence this may be a useful marker of future disease progression. Finally, calprotectin, a neutrophil derived protein, may also be a useful biomarker, where levels both in sputum and serum have 
been demonstrated to decrease significantly following treatment of an acute exacerbation and predicted time to next exacerbation [116], this is extremely useful as both exacerbations and lung function decline are the most sensitive markers of disease progression in CF.

\subsection{Serum biomarkers as markers of systemic inflammation}

Similar to sputum biomarkers, the use of serum biomarkers is highly appealing to monitor disease progression and also predict outcomes. Several studies have investigated in $\mathrm{CF}$ an array of potentially useful peptides and cytokines measurable in serum that correlate with exacerbation severity, length and recurrence as well as lung function and microbiological colonisation. While there are many studies of biomarkers of inflammation in $\mathrm{CF}$ and it further strengthens our understanding of the disease, the use of biomarkers has not become routine clinical practice, hence the need for accurate measurements of both pulmonary and systemic inflammation, which makes serum levels a key factor in this process as they may also be useful in measuring the response to treatment and predicting prognosis especially as new therapeutic options emerge.

As discussed previously, markers of neutrophil activity and elastase levels and activity in the lung correlate excellently with clinical condition, hence the use of neutrophil markers from serum would be ideal as biomarkers. One such biomarker is calprotectin, a neutrophil derived protein that is released during neutrophil activation, which has been shown to correlate with the resolution of exacerbations [116], as well as correlating with radiological scores and pulmonary symptoms [117]. CD16b is a receptor expressed by human neutrophils which binds immunoglobulins and is involved in the inflammatory response in CF. Levels of alpha-1 antitrypsin (AAT) complexed to CD16b (AAT:CD16b complex) have been shown to be significantly higher in $\mathrm{CF}$ patients than healthy controls, and correlate to other pro-inflammatory cytokines including IL-8 and TNF- $\alpha$ [11]. Plasma concentrations of AAT:CD16b complex at the time of acute pulmonary exacerbation responded to antibiotic treatment and demonstrated a significant correlation to FEV1 improvement. These results suggest this may be a potentially useful biomarker of acute exacerbations and hence a useful prognostic tool.

The use of cytokines, similarly to sputum, is often viewed as an ideal surrogate of disease state, and many have been studied. Immunoglobulin G (IgG) and IL-6 have been shown previously to correlate with mortality, however, when adjusted for confounding factors, in particular FEV1, this correlation did not remain independent [118]. There is significant cross correlation between inflammatory markers such as TNF- $\alpha$, IL- 8 and cathepsins as well as IL-6 and IgG. However, none have shown any significant correlation with mortality. As survival becomes longer in this disease, the need increases for biomarkers that correlate together with other indices of disease such as pulmonary function, bacterial colonisation, exacerbation frequency, and radiological changes.

Leucocyte ribonucleic acid (RNA) measurements may be one such biomarker for potential future use. In identifying the presence of airway infection, whole blood levels of leucocyte RNA is an accurate measure and is more sensitive than FEV1 or c-reactive protein (CRP), and together with FEV1 is highly sensitive [119]. Other novel biomarkers include anti-neutrophil cytoplasmic antibodies specific for bactericidal/permeability-increasing protein (BPI-ANCA). 
Bactericidal/permeability-increasing protein (BPI) is one of the most potent endogenously produced antibacterials secreted by neutrophils, and autoantibodies against this protein are present in up to $90 \%$ of CF patients [120]. Levels of BPI-ANCA correlate strongly with markers of lung disease and it may indicate a pathogenic role in CF [121] and a recent study has shown that the presence of BPI-ANCA correlated significantly with a poor outcome, being death or requiring a lung transplantation. Furthermore, in patients colonised with PA the outcome was significantly worse if associated with this biomarker [122].

While no single biomarker of disease severity exists from either sputum or serum, there are several potential biomarkers that need further study and more robust results to support their routine use in clinical practice.

One biomarker that has recently re-emerged as a useful index of disease severity is the measurement of CFTR function. This may be measured by nasal potential difference (NPD) and sweat chloride concentration. It is important as a biomarker as it has been shown that CFTR and sweat chloride concentration at diagnosis predicts long term prognosis [123]. NPD is now reproducible in a more robust manor than previously, as it was difficult to accurately to so in the past [124]. The use of this biomarker has been further established in the recent clinical trials of CFTR modulators and correctors such as ivacaftor, with a change in NPD and sweat chloride seen as marker of improvement and correlating with improvements in lung function and BMI, whether this will be a long-term prognostic indictor remains to be seen [4].

\section{Radiological methods to predict prognosis}

Due to the complexity of CF lung disease, multiple modalities are needed to fully assess the extent of disease severity, including radiological imaging. Several imaging techniques have been employed and studied in CF; with plain radiograph, CT, and MRI all having varying abilities to predict prognosis, as well as correlate with clinical condition, and a number of scoring systems exist to qualify the extent of pulmonary involvement. High-resolution CT imaging of the lungs is currently the most sensitive method of assessing the structural changes in CF. HRCT permits airway thickness measurements and the extent of bronchiectactic changes, and these changes closely correlate with FEV1 and often adult patients with CF show more acute changes in HRCT abnormalities than decline in spirometric results [125]. The progression of bronchiectasis also correlates with CFTR genotype and levels of neutrophilic inflammation [126], while also significantly predicting exercise capacity in CF patients [127].

Newer methods to image the lungs in CF include hyperpolarised helium magnetic resonance imaging (He3-MRI) [8] and flurodeoxyglucose positron emission tomography (FDG-PET) imaging [5-7]. Both modalities correlate closely with abnormalities seen on HRCT and improve with antibiotic treatment, making them both sensitive useful tools in the acute setting. He3MRI may identify early ventilatory changes in paediatric disease before identifiable changes on HRCT [128] and may better correlate with spirometry in assessing response after exacerbation treatment [8]. These modalities are not currently in widespread use due to both cost and availability, however, He3-MRI may become more routine in the future as life expectancy 
increases and the risk associated with increased cumulative exposure to ionising radiation associated with CT and FDG-PET [129].

A number of different radiological scoring systems have been developed to assess disease burden in CF. Older scoring systems were based on chest radiograph changes, and these included the Brasfield [130], National Institutes of Health (NIH) chest radiograph [131], and the Royal Children's Hospital ( $\mathrm{RCH}$ ) chest radiograph score [132]. While these were somewhat useful, they correlated poorly with spirometry and with clinical condition [133]. With the continual improvement in CT imaging modalities, HRCT has become the gold standard for assessing structural abnormalities, and several robust validated scoring systems exist for CT abnormalities. These include the Brody II score [134] and the CF-CT score [135], while one of the older original scoring tools, the Bhalla score, for bronchiectasis is still widely used in modified versions [107]. The Bhalla scoring system is an objective measure incorporating all aspects of CF structural abnormalities and has been validated and correlates with spirometry[136], clinical condition [127, 137], and has some correlation with health related quality of life [138], however, there is little correlation between these scores and other biomarkers of inflammation [106].

Radiological scoring systems are useful in assessing the extent of structural changes in $\mathrm{CF}$, and correlate with certain aspects of the disease, however, they alone are not sufficient to predict prognosis and are likely more beneficial when used as part of composite clinical prediction tools.

\section{Usefulness of clinical prediction tools in cystic fibrosis}

As previously outlined in this chapter, life expectancy continues to improve in patients with CF due to a combination of improvements in treatment, level of care, and diagnostic tools, as well as multidisciplinary input and the development of disease-specific centres. With continually improving clinical outcomes, the need arises to better predict the prognosis of CF at an individual and group level. This need was first identified in 1958 with the development of the Shwachman-Kulczycki (SK) score to assess the severity of CF [139]. This score formed part of a study which monitored 105 patients for 5 years, the first of its kind to assess the long term progress of young CF patients. It was seen that there was increased frequency of CF patients surviving to adolescence and young adulthood and demonstrated a need to assess disease severity in order to predict the likely course of the disease.

There are many criticisms of the SK score as it is largely a subjective measure that depends on the clinical estimation of the examiner. Another drawback is that it does not include evaluations of pulmonary function. It was also devised with reference to a paediatric population in a time when life expectancy was drastically decreased. However, there have been a range of studies since its conception that have validated this score against a host of parameters increasingly used to measure prognosis. For example, Brasfield et al. observed a significant correlation between the SK score and chest radiography in an evaluation of over 640 chest radiogrpahs of $118 \mathrm{CF}$ patients [130]. A more recent evaluation of the usefulness of this score 
to assess disease severity was undertaken by Stollar et al. in 2011 where [140] significant correlations were demonstrated between FEV1, chest radiograph, HRCT, 6-minute walk test (6MWT), and SK score. It was felt that the SK score adequately reflects radiographic and functional impairments in patients with greater impairment of lung function, however, the score was less useful in patients with preserved lung function (FEV1 $>70 \%$ ). Regardless of its shortcomings, the SK score is regarded as a milestone in the history of CF and continues to serve as a somewhat tool in the determination of disease severity.

This score formed the basis for the development of a multitude of clinical prediction tools, the majority of which involve clinical parameters, radiological parameters, or a combination of both. One such early radiological scoring system is the Brasfield score [130], which is based on the chest radiograph findings of patients with CF and encompasses a maximum score of 25, with points scored according to the severity of air trapping, linear markings, nodular cystic lesions, large lesions, and an impression of the overall general severity of the radiograph. However, it similarly is a subjective scoring system and there can be inter-rater differences. The Brasfield score does correlate with pulmonary function tests and the SK score and is reproducible. A disadvantage of the Brasfield score is that it was developed to include radiographs assessed by a team of radiologists and clinicians, while the Northern score [141] is an advance on the Brasfield score which allows for a single examiner to determine the radiographic severity based on assessing chest radiographs divided into lung quadrants. The maximum score is 20, with higher scores reflecting poorer outcomes due to increased severity of disease. This score was validated against the Brasfield score and an earlier score, ChrispinNorman [142], and was found to be equal in terms of consistency, reproducibility, and accuracy in reflecting overall clinical status as measured by the SK score.

The development of widespread use of computed tomography in clinical practice has led to the inclusion of HRCT in prognostic tools for CF. Nathanson et al. proposed the first such score which involved dividing the lung CT into 12 distinct zones in order to classify the severity of bronchiectasis on a 5-point scale in each zone [143]. This, once again, was validated against the SK and Brasfield scoring systems along with the results of pulmonary function tests carried out on the subjects. As discussed, Bhalla et al. [107] further developed the idea of CT scoring systems in order to aid selection for lung transplantation and put forward a score based on the severity of 9 different radiological parameters including peribronchial thickening, extent of bronchiectasis, and extent of mucus impaction. This score is very reproducible and correlates strongly with pulmonary function, hence is the gold standard for HRCT evaluation.

Clinically-based prognostic tools play a central role in the management of CF patients and serve to direct treatments, investigations, and multidisciplinary input. The National Institute of Health scale, developed in 1973, proposed a comprehensive scoring system which included multiple parameters including baseline demographics, various measures of lung function, and presence of common complications encountered in the disease [131]. A 100-point scale derived from these measurements correlated with severity of disease, the higher scores being associated with poorer outcomes. It is a complex and cumbersome scale that reduces the likelihood for its application across the broader clinical setting. However, it is a reliable and reproducible 
score which encompasses many aspects of the disease and this has led to its continued use in CF-related research.

With the advancement of CF survival and life expectancy a need to develop simple and reliable prognostic tools for use in the clinical setting became apparent. In 1997, Hayllar et al. developed a predictive index based on research from over 400 patients and studied and correlated the index result with a mortality curve [144]. In this way, a simple calculation could be made to estimate 6-month and 1-year survival rates for a given score. The score was based on height, presence of hepatomegaly, white blood cell count, FEV1, and forced vital capacity (FVC). This, and scores like it, revolutionised the approach to prognosis in previously unchartered territory and survival estimates became central to prognostic tools. Liou et al. advanced on the NIH and Hayllar scores to present a 5-year prognostic tool, the longest range survival model in the field at its time of development [145]. It is based on a composite score of 8 clinical factors, as well as measurement of FEV1 and is a comprehensive, reproducible, reliable prognostic tool. However, it remains a complicated score to perform clinically and so focus has shifted in recent times to the development of simplified prediction tools that can be used within the time constraints of the current clinical setting. The CF-ABLE score was devised with this in mind and it involves the measurement of 4 of the major clinical parameters encountered in day to day practice [93]. Age, BMI, FEV1, and frequency of pulmonary exacerbations have been evaluated on a 7-point scale for correlation with prognosis and it has been found that patients with higher scores have a $26 \%$ chance of a poor outcome (death or transplantation) within 4 years of measurement. This score has been validated on a national registry and highlights the applicability of simple prediction tools that can be employed in the clinical environment.

Clinical and radiological prediction tools have emerged as a vital resource in the treatment of CF. They allow us to stratify patients by disease severity and to a certain extent allow us to predict likely adverse outcomes in patients with poor prognostic indices. A multitude of prognostic tools have been developed since the original SK score as CF research has continued to expand, each reflecting the changing face of $\mathrm{CF}$ treatment and diagnostic tools. Clinical practice favours the use of simple reliable scoring systems which take into account easily measurable parameters, such as the Hayllar score and the more up to date CF-ABLE score. Future research will need to involve the development of further prognostic tools to reflect the changes occurring in the management of CF (Table 2).

\begin{tabular}{llllll}
\hline Name of Score & $\begin{array}{l}\text { Year } \\
\text { Published }\end{array}$ & $\begin{array}{l}\text { Number of } \\
\text { patients in cohort }\end{array}$ & Parameters Used & Strengths & Limitations \\
\hline Shwachman- & 1958 & 105 CF patients & - General activity & - Uncomplicated & - Subjective \\
Kulzcizy & & followed for 5 & - Physical exam & assessment & - No inclusion of \\
[139] & years & - Nutrition & - Reproducible & measurement of \\
& & - Chest radiograph & - High correlation & pulmonary \\
& & & with pulmonary & function
\end{tabular}




\begin{tabular}{|c|c|c|c|c|c|}
\hline Name of Score & $\begin{array}{l}\text { Year } \\
\text { Published }\end{array}$ & $\begin{array}{l}\text { Number of } \\
\text { patients in cohort }\end{array}$ & Parameters Used & Strengths & Limitations \\
\hline & & & & $\begin{array}{l}\text {-High correlation } \\
\text { with Brasfield score }\end{array}$ & $\begin{array}{l}\text { - Developed in } \\
\text { paediatric } \\
\text { population }\end{array}$ \\
\hline NIH Score [131] & 1973 & $\begin{array}{l}73 \text { patients aged } 3 \\
\text { to } 30 \text { years } \\
\text { followed for a } \\
\text { period between } 3 \\
\text { and } 6 \text { years }\end{array}$ & $\begin{array}{l}100 \text { point scale of severity of } \\
\text { lung and general parameters } \\
\text { as well as common } \\
\text { complications: } \\
\text { - Chest Radiograph } \\
\text { - Pulmonary function } \\
\text { - Pulmonary exacerbations } \\
\text { - Pneumothorax } \\
\text { - Haemoptysis } \\
\text { - Pulmonary surgery } \\
\text { - Cor Pulmonale } \\
\text { - Lung auscultation } \\
\text { - Cough and expectoration } \\
\text { - Weight } \\
\text { - Activity } \\
\text { - General attitude } \\
\text { - ABG } \\
\text { - GI complications } \\
\text { - Infertility } \\
\text { - Salt depletion } \\
\text { - Osteoarthropathy }\end{array}$ & $\begin{array}{l}\text { - Comprehensive } \\
\text { s- Demonstrated to } \\
\text { be useful in } \\
\text { prognosis and } \\
\text { assessment of } \\
\text { disease evolution }\end{array}$ & $\begin{array}{l}\text { - Complex scoring } \\
\text { system } \\
\text { - Overestimates } \\
\text { rare clinical } \\
\text { elements } \\
\text { - Does not include } \\
\text { patients younger } \\
\text { than } 5 \text { years of age } \\
\text { - High variability } \\
\text { in pulmonary } \\
\text { function evaluation }\end{array}$ \\
\hline $\begin{array}{l}\text { Brasfield } \\
{[130]}\end{array}$ & 1979 & $\begin{array}{l}643 \text { chest } \\
\text { radiographs in } 118 \\
\text { CF patients }\end{array}$ & $\begin{array}{l}\text { Composite score of presence } \\
\text { and severity of each of the } \\
\text { following: } \\
\text { - Air trapping } \\
\text { - Linear markings } \\
\text { - Nodular cystic lesions } \\
\text { - Large lesions } \\
\text { - Overall impression of } \\
\text { severity }\end{array}$ & $\begin{array}{l}\text { - Reproducible } \\
\text { - Strong correlation } \\
\text { with clinical severity } \\
\text { - Uncomplicated } \\
\text { assessment }\end{array}$ & $\begin{array}{l}\text { - Somewhat } \\
\text { subjective in } \\
\text { ymeasurement of } \\
\text { overall severity } \\
\text { - Inflexible } \\
\text { - Designed for } \\
\text { team of } \\
\text { radiologists }\end{array}$ \\
\hline $\begin{array}{l}\text { Nathanson } \\
\text { [143] }\end{array}$ & 1991 & $\begin{array}{l}28 \mathrm{HRCT} \text { of } \mathrm{CF} \\
\text { patients }\end{array}$ & $\begin{array}{l}\text { Severity of bronchiectasis } \\
\text { and mucous impaction on a } \\
5 \text { point scale as measured in } \\
12 \text { distinct lung zones }\end{array}$ & $\begin{array}{l}\text { - Good correlation } \\
\text { with existing } \\
\text { radiographic scores } \\
\text { and clinical } \\
\text { outcomes }\end{array}$ & $\begin{array}{l}\text { - No studies in } \\
\text { reproducibility }\end{array}$ \\
\hline
\end{tabular}




\begin{tabular}{|c|c|c|c|c|c|}
\hline Name of Score & $\begin{array}{l}\text { Year } \\
\text { Published }\end{array}$ & $\begin{array}{l}\text { Number of } \\
\text { patients in cohort }\end{array}$ & Parameters Used & Strengths & Limitations \\
\hline Bhalla [107] & 1991 & $\begin{array}{l}\text { HRCT scans of } 14 \\
\text { patients studies by } \\
3 \text { radiologists }\end{array}$ & $\begin{array}{l}\text { 3-point severity score of the } \\
\text { following parameters: } \\
\text { - Bronchiectasis severity } \\
\text { - Peribronchial thickening } \\
\text { - Bronchiectasis extent } \\
\text { - Extent of mucoid } \\
\text { impaction } \\
\text { - Abscesses } \\
\text { - General impression } \\
\text { - Number of blisters } \\
\text { - Emphysema extent } \\
\text { - Collapse/consolidation }\end{array}$ & $\begin{array}{l}\text { - Excellent } \\
\text { agreement between } \\
\text { examiners } \\
\text { - Reproducible } \\
\text { - High correlation } \\
\text { with pulmonary } \\
\text { function tests }\end{array}$ & $\begin{array}{l}\text { - Extensive number } \\
\text { of parameters } \\
\text { - Complex scoring } \\
\text { system }\end{array}$ \\
\hline $\begin{array}{l}\text { Northern Score } \\
\text { [141] }\end{array}$ & 1994 & $\begin{array}{l}45 \text { chest } \\
\text { radiographs read } \\
\text { by } 10 \text { clinical } \\
\text { physicians }\end{array}$ & $\begin{array}{l}\text { Severity of radiographic } \\
\text { alterations in each lung } \\
\text { quadrant: } \\
\text { - 0: normal } \\
\text { - 1: minimally increased } \\
\text { linear signs or nodular cystic } \\
\text { lesions } \\
\text { - 2: moderately diffuse } \\
\text { nodular cystic lesions, more } \\
\text { pronounced linear signs } \\
\text { - 3: severe; profuse cystic } \\
\text { lesions, extensive collapse/ } \\
\text { consolidation } \\
\text { - 4: very severe; small areas } \\
\text { of visible lung with dense } \\
\text { infiltrates throughout }\end{array}$ & $\begin{array}{l}\text { - Allows for single- } \\
\text { examiner approach } \\
\text { - Better agreement } \\
\text { seen among } \\
\text { examiners } \\
\text { c- Reproducible } \\
\text { - Uncomplicated }\end{array}$ & $\begin{array}{l}\text { - May overly } \\
\text { simplify picture } \\
\text { and extent of lung } \\
\text { disease } \\
\text { - No strong } \\
\text { correlation with } \\
\text { clinical measures }\end{array}$ \\
\hline Hayllar [144] & 1997 & $\begin{array}{l}\text { Retrospective data } \\
\text { analysed on } 403 \\
\text { patients over } 18 \\
\text { year period }\end{array}$ & $\begin{array}{l}\text { Predictive index based on } \\
\text { measurement of: } \\
\text { - Height } \\
\text { - Hepatomegaly } \\
\text { - FVC (\%) } \\
\text { - FEV1 (\%) } \\
\text { - White blood cell count } \\
\text { Mortality curve developed } \\
\text { to relate predictive index to } \\
\text { survival probability }\end{array}$ & $\begin{array}{l}\text { - Directly relates } \\
\text { clinical measures to } \\
\text { an estimate of } \\
\text { survival } \\
\text { - Externally } \\
\text { validated } \\
\text { - Reproducible }\end{array}$ & $\begin{array}{l}\text { - Excludes some } \\
\text { known predictors } \\
\text { of mortality } \\
\text { - Developed based } \\
\text { on data in 1970s } \\
\text { and 1980s; does not } \\
\text { reflect improved } \\
\text { diagnostic } \\
\text { techniques }\end{array}$ \\
\hline
\end{tabular}




\begin{tabular}{|c|c|c|c|c|c|}
\hline Name of Score & $\begin{array}{l}\text { Year } \\
\text { Published }\end{array}$ & $\begin{array}{l}\text { Number of } \\
\text { patients in cohort }\end{array}$ & Parameters Used & Strengths & Limitations \\
\hline $\begin{array}{l}\text { Liou } \\
\text { Predictive 5- } \\
\text { year } \\
\text { survivorship } \\
\text { model of Cystic } \\
\text { Fibrosis } \\
\text { [145] }\end{array}$ & 2001 & $\begin{array}{l}\text { Retrospective data } \\
\text { collected } 5820 \\
\text { patients in US } \\
\text { registry between } \\
1986 \text { and } 1993\end{array}$ & $\begin{array}{l}\text { Composite score of the } \\
\text { presence or value of } \\
\text { following parameters to } \\
\text { predict 5-year survival: } \\
\text { - Age } \\
\text { - Gender (M=0, F=1) } \\
\text { - FEV1 (\%) } \\
\text { - Weight-for-age z score } \\
\text { - Pancreatic insufficiency } \\
\text { - Diabetes mellitus } \\
\text { - Staphylococcus aureus } \\
\text { - Burkholderia cepacia } \\
\text { - No. of acute exacerbations } \\
\text { (0-5) }\end{array}$ & $\begin{array}{l}\text { - Comprehensive } \\
\text { - Widely applicable } \\
\text { - Validated } \\
\text { - User-friendly }\end{array}$ & $\begin{array}{l}\text { - Does not account } \\
\text { for radiological } \\
\text { findings } \\
\text { - Prediction rule is } \\
\text { derived from the } \\
\text { same cohort as the } \\
\text { validation group }\end{array}$ \\
\hline $\begin{array}{l}\text { CF-ABLE Score } \\
\text { [93] }\end{array}$ & 2013 & $\begin{array}{l}49 \text { CF patients } \\
\text { followed over a } 7- \\
\text { year period. } \\
\text { Validated in } 370 \\
\text { patients collected } \\
\text { from national } \\
\text { registry over } 5 \\
\text { years }\end{array}$ & $\begin{array}{l}\text { 7-point scale based on } \\
\text { evaluation of: } \\
\text { - Age }<24=1 \text { point } \\
\text { - BMI }<20.1 \mathrm{~kg} / \mathrm{m}^{2}=1 \\
\text { - FEV1 }(\%)<52 \%=3.5 \\
\text { - Number of exacerbations } \\
\text { in past } 3 \text { months }>1=3.5 \\
\text { points } \\
\text { Score of }>5 \text { indicates } 26 \% \\
\text { chance of poor outcome in } \\
\text { the next } 4 \text { years }\end{array}$ & $\begin{array}{l}\text { - Common clinical } \\
\text { measurements used } \\
\text { - Easy to caluclate } \\
\text { - Validated }\end{array}$ & $\begin{array}{l}\text { - Difficult to define } \\
\text { pulmonary } \\
\text { exacerbation } \\
\text { - Adult only score }\end{array}$ \\
\hline
\end{tabular}

Table 2. Summary of Clinical Prediction Tools in Cystic Fibrosis.

\section{Conclusion}

Over the last two decades, prognosis has improved significantly in CF and this has led to the need to better predict outcomes. Subsequently, many studies have looked at all aspects of the disease and identified modifiable risks, as well as identified biomarkers to follow disease progression. This increased knowledge of the condition has allowed improved treatment and more phenotypically specific therapy to be developed. Many different clinical features and markers predict outcome and this is a complex area, and a multifaceted approach to risk stratifying patients is needed. However, what must be not overlooked as the survival increases in $\mathrm{CF}$ are the extrapulmonary manifestations and the significant psychosocial issues associated with chronic illness. It is important to note that up to $22 \%$ of CF patients demonstrate symptoms of depression, $10 \%$ report anxiety symptoms, and up to $5 \%$ report suicidal thoughts [146]. The 
incidence of depression and anxiety is $2-3$ times higher than age matched community control individuals [147]. This is highly important and there is an association between depression and lower FEV1 [146]. Also, patients with well-preserved spirometry who have depression demonstrate more significant decline in lung function than those with no depressive symptoms [148]. Depression is associated with negative medication beliefs leading to lower medication adherence, which ultimately may lead to poorer prognosis, hence it is important to evaluate patients holistically beyond the medical complications of CF when assessing prognosis [149]. Other important factors that are easily overlooked are socio-economic issues that impact upon survival, including lower household income and socio-economic status [150-152], larger family size with more than one person with CF [153], and exposure to cigarette smoke [154] all predicting a poor outcome. These risk factors are sometimes more difficult to quantify and hence are often overlooked. They are also difficult to measure as part of composite prediction tools, but they must be taken into account when assessing the prognostic indices of each individual with CF.

As survival continues to improve, the use of composite scoring systems and multi-disciplinary approach to improving care is essential in CF. Many clinical prediction tools exist for $\mathrm{CF}$ and some are more useful in clinical practice than others, however, these are underutilised in general and should be employed in more clinical trials, as well as routine care.

In summary, the prognosis of CF has improved significantly over the last two decades and may improve further as newer medications are developed. The need to accurately predict prognosis is essential as the decision for lung transplantation or to aggressively treat certain aspects of disease may be more tailored and appropriate per individual, this improving survival further. The most sensitive way of predicating prognosis currently remains a multifaceted approach, including several markers of disease and the use of all factors and a composite clinical prediction tool is suggested to stratify patient risk.

\section{Author details}

Cormac McCarthy", Orla O’Carroll, Alessandro N. Franciosi and Noel G. McElvaney

*Address all correspondence to: cmccarthy@rcsi.ie

Respiratory Research Division, Department of Medicine, Royal College of Surgeons in Ireland, Education and Research Centre, Beaumont Hospital, Dublin, Ireland

\section{References}

[1] Ramsey, B.W., Management of pulmonary disease in patients with cystic fibrosis. N Engl J Med, 1996. 335(3): p. 179-88. 
[2] Rosenbluth, D.B., et al., Lung function decline in cystic fibrosis patients and timing for lung transplantation referral. Chest, 2004. 126(2): p. 412-9.

[3] Kerem, E., et al., Prediction of mortality in patients with cystic fibrosis. N Engl J Med, 1992. 326(18): p. 1187-91.

[4] Ramsey, B.W., et al., A CFTR potentiator in patients with cystic fibrosis and the G551D mutation. N Engl J Med, 2011. 365(18): p. 1663-72.

[5] Klein, M., et al., 18F-fluorodeoxyglucose-PET/CT imaging of lungs in patients with cystic fibrosis. Chest, 2009. 136(5): p. 1220-8.

[6] Amin, R., et al., Cystic fibrosis: detecting changes in airway inflammation with FDG PET/CT. Radiology, 2012. 264(3): p. 868-75.

[7] Chen, D.L., J.J. Atkinson, and T.W. Ferkol, FDG PET imaging in cystic fibrosis. Semin Nucl Med, 2013. 43(6): p. 412-9.

[8] McMahon, C.J., et al., Hyperpolarized 3helium magnetic resonance ventilation imaging of the lung in cystic fibrosis: comparison with high resolution CT and spirometry. Eur Radiol, 2006. 16(11): p. 2483-90.

[9] Aurora, P., et al., Lung clearance index at 4 years predicts subsequent lung function in children with cystic fibrosis. Am J Respir Crit Care Med, 2011. 183(6): p. 752-8.

[10] Mayer-Hamblett, N., et al., Association between pulmonary function and sputum biomarkers in cystic fibrosis. Am J Respir Crit Care Med, 2007. 175(8): p. 822-8.

[11] Reeves, E.P., et al., A novel neutrophil derived inflammatory biomarker of pulmonary exacerbation in cystic fibrosis. J Cyst Fibros, 2012. 11(2): p. 100-7.

[12] Sagel, S.D., et al., Sputum biomarkers of inflammation and lung function decline in children with cystic fibrosis. Am J Respir Crit Care Med, 2012. 186(9): p. 857-65.

[13] McCarthy, C., et al., The CF-ABLE Score: A Novel Clinical Prediction Rule for Prognosis in Cystic Fibrosis. Chest, 2012.

[14] Cystic Fibrosis Foundation Patient Registry Annual Data Report 2013. 2013; Available from: http://www.cff.org/UploadedFiles/research/ClinicalResearch/PatientRegistryReport/2013_CFF_Patient_Registry_Annual_Data_Report.pdf.

[15] Kerem, E., et al., The relation between genotype and phenotype in cystic fibrosis--analysis of the most common mutation (delta F508). N Engl J Med, 1990. 323(22): p. 1517-22.

[16] Johansen, H.K., et al., Severity of cystic fibrosis in patients homozygous and heterozygous for delta F508 mutation. Lancet, 1991. 337(8742): p. 631-4.

[17] Santis, G., et al., Independent genetic determinants of pancreatic and pulmonary status in cystic fibrosis. Lancet, 1990. 336(8723): p. 1081-4. 
[18] McKone, E.F., et al., Effect of genotype on phenotype and mortality in cystic fibrosis: a retrospective cohort study. Lancet, 2003. 361(9370): p. 1671-6.

[19] McKone, E.F., C.H. Goss, and M.L. Aitken, CFTR genotype as a predictor of prognosis in cystic fibrosis. Chest, 2006. 130(5): p. 1441-7.

[20] Davies, J.C., et al., Efficacy and safety of ivacaftor in patients aged 6 to 11 years with cystic fibrosis with a G551D mutation. Am J Respir Crit Care Med, 2013. 187(11): p. 1219-25.

[21] McKone, E.F., et al., Long-term safety and efficacy of ivacaftor in patients with cystic fibrosis who have the Gly551Asp-CFTR mutation: a phase 3, open-label extension study (PERSIST). Lancet Respir Med, 2014. 2(11): p. 902-10.

[22] Corey, M. and V. Farewell, Determinants of mortality from cystic fibrosis in Canada, 1970-1989. Am J Epidemiol, 1996. 143(10): p. 1007-17.

[23] Rosenfeld, M., et al., Gender gap in cystic fibrosis mortality. Am J Epidemiol, 1997. 145(9): p. 794-803.

[24] Harness-Brumley, C.L., et al., Gender differences in outcomes of patients with cystic fibrosis. J Womens Health (Larchmt), 2014. 23(12): p. 1012-20.

[25] Coakley, R.D., et al., 17beta-Estradiol inhibits Ca2+-dependent homeostasis of airway surface liquid volume in human cystic fibrosis airway epithelia. J Clin Invest, 2008. 118(12): p. 4025-35.

[26] Chotirmall, S.H., et al., 17Beta-estradiol inhibits IL-8 in cystic fibrosis by up-regulating secretory leucoprotease inhibitor. Am J Respir Crit Care Med, 2010. 182(1): p. 62-72.

[27] Nousia-Arvanitakis, S., Cystic fibrosis and the pancreas: recent scientific advances. J Clin Gastroenterol, 1999. 29(2): p. 138-42.

[28] Ahmed, N., et al., Molecular consequences of cystic fibrosis transmembrane regulator (CFTR) gene mutations in the exocrine pancreas. Gut, 2003. 52(8): p. 1159-64.

[29] Ooi, C.Y., et al., Type of CFTR mutation determines risk of pancreatitis in patients with cystic fibrosis. Gastroenterology, 2011. 140(1): p. 153-61.

[30] Kerem, E., et al., Factors associated with FEV1 decline in cystic fibrosis: analysis of the ECFS patient registry. Eur Respir J, 2014. 43(1): p. 125-33.

[31] Moran, A., et al., Cystic fibrosis-related diabetes: current trends in prevalence, incidence, and mortality. Diabetes Care, 2009. 32(9): p. 1626-31.

[32] Hardin, D.S., et al., Insulin resistance is associated with decreased clinical status in cystic fibrosis. J Pediatr, 1997. 130(6): p. 948-56.

[33] Milla, C.E., W.J. Warwick, and A. Moran, Trends in pulmonary function in patients with cystic fibrosis correlate with the degree of glucose intolerance at baseline. Am J Respir Crit Care Med, 2000. 162(3 Pt 1): p. 891-5. 
[34] Nousia-Arvanitakis, S., A. Galli-Tsinopoulou, and M. Karamouzis, Insulin improves clinical status of patients with cystic-fibrosis-related diabetes mellitus. Acta Paediatr, 2001. 90(5): p. 515-9.

[35] Galutira, D., P.W. Allderdice, and W.S. Davidson, HaeIII RFLP for salivary proline-rich protein gene probe (pPRPII2.2RP). Nucleic Acids Res, 1991. 19(23): p. 6667.

[36] Schwarzenberg, S.J., et al., Microvascular complications in cystic fibrosis-related diabetes. Diabetes Care, 2007. 30(5): p. 1056-61.

[37] Koch, C., et al., Presence of cystic fibrosis-related diabetes mellitus is tightly linked to poor lung function in patients with cystic fibrosis: data from the European Epidemiologic Registry of Cystic Fibrosis. Pediatr Pulmonol, 2001. 32(5): p. 343-50.

[38] Apparailly, F., et al., Adeno-associated virus pseudotype 5 vector improves gene transfer in arthritic joints. Hum Gene Ther, 2005. 16(4): p. 426-34.

[39] Milla, C.E., J. Billings, and A. Moran, Diabetes is associated with dramatically decreased survival in female but not male subjects with cystic fibrosis. Diabetes Care, 2005. 28(9): p. 2141-4.

[40] Moyer, K. and W. Balistreri, Hepatobiliary disease in patients with cystic fibrosis. Curr Opin Gastroenterol, 2009. 25(3): p. 272-8.

[41] Bartlett, J.R., et al., Genetic modifiers of liver disease in cystic fibrosis. JAMA, 2009. 302(10): p. 1076-83.

[42] Grasemann, H., H.G. Wiesemann, and F. Ratjen, [The importance of lung function as a predictor of 2-year mortality in mucoviscidosis]. Pneumologie, 1995. 49(8): p. 466-9.

[43] Milla, C.E. and W.J. Warwick, Risk of death in cystic fibrosis patients with severely compromised lung function. Chest, 1998. 113(5): p. 1230-4.

[44] Konstan, M.W., et al., Design and powering of cystic fibrosis clinical trials using rate of FEV(1) decline as an efficacy endpoint. J Cyst Fibros, 2010. 9(5): p. 332-8.

[45] Morgan, W.J., et al., Probability of treatment following acute decline in lung function in children with cystic fibrosis is related to baseline pulmonary function. J Pediatr, 2013. 163(4): p. 1152-7 e2.

[46] Lum, S., et al., Early detection of cystic fibrosis lung disease: multiple-breath washout versus raised volume tests. Thorax, 2007. 62(4): p. 341-7.

[47] Gustafsson, P.M., P. Aurora, and A. Lindblad, Evaluation of ventilation maldistribution as an early indicator of lung disease in children with cystic fibrosis. Eur Respir J, 2003. 22(6): p. 972-9.

[48] Gustafsson, P.M., et al., Multiple-breath inert gas washout and spirometry versus structural lung disease in cystic fibrosis. Thorax, 2008. 63(2): p. 129-34. 
[49] Vermeulen, F., et al., Lung clearance index predicts pulmonary exacerbations in young patients with cystic fibrosis. Thorax, 2014. 69(1): p. 39-45.

[50] Davies, J., et al., Assessment of clinical response to ivacaftor with lung clearance index in cystic fibrosis patients with a G551D-CFTR mutation and preserved spirometry: a randomised controlled trial. Lancet Respir Med, 2013. 1(8): p. 630-8.

[51] Linnane, B., et al., The findings of a clinical surveillance bronchoalveolar lavage programme in pre-school patients with cystic fibrosis. Pediatr Pulmonol, 2014.

[52] Kerem, E., et al., Pulmonary function and clinical course in patients with cystic fibrosis after pulmonary colonization with Pseudomonas aeruginosa. J Pediatr, 1990. 116(5): p. 714-9.

[53] Rosenfeld, M., et al., Baseline characteristics and factors associated with nutritional and pulmonary status at enrollment in the cystic fibrosis EPIC observational cohort. Pediatr Pulmonol, 2010. 45(9): p. 934-44.

[54] Pamukcu, A., A. Bush, and R. Buchdahl, Effects of pseudomonas aeruginosa colonization on lung function and anthropometric variables in children with cystic fibrosis. Pediatr Pulmonol, 1995. 19(1): p. 10-5.

[55] Konstan, M.W., et al., Risk factors for rate of decline in FEV1 in adults with cystic fibrosis. J Cyst Fibros, 2012. 11(5): p. 405-11.

[56] Pentz, A., et al., The impact of chronic pseudomonal infection on pulmonary function testing in individuals with cystic fibrosis in Pretoria, South Africa. S Afr Med J, 2014. 104(3): p. 191-4.

[57] Lillquist, Y.P., E. Cho, and A.G. Davidson, Economic effects of an eradication protocol for first appearance of Pseudomonas aeruginosa in cystic fibrosis patients: 1995 vs. 2009. J Cyst Fibros, 2011. 10(3): p. 175-80.

[58] Nixon, G.M., et al., Early airway infection, inflammation, and lung function in cystic fibrosis. Arch Dis Child, 2002. 87(4): p. 306-11.

[59] Hoffman, L.R., et al., Selection for Staphylococcus aureus small-colony variants due to growth in the presence of Pseudomonas aeruginosa. Proc Natl Acad Sci U S A, 2006. 103(52): p. 19890-5.

[60] Besier, S., et al., Prevalence and clinical significance of Staphylococcus aureus small-colony variants in cystic fibrosis lung disease. J Clin Microbiol, 2007. 45(1): p. 168-72.

[61] Burns, J.L., et al., Microbiology of sputum from patients at cystic fibrosis centers in the United States. Clin Infect Dis, 1998. 27(1): p. 158-63.

[62] Ren, C.L., et al., Presence of methicillin resistant Staphylococcus aureus in respiratory cultures from cystic fibrosis patients is associated with lower lung function. Pediatr Pulmonol, 2007. 42(6): p. 513-8. 
[63] Hubert, D., et al., Association between Staphylococcus aureus alone or combined with Pseudomonas aeruginosa and the clinical condition of patients with cystic fibrosis. J Cyst Fibros, 2013. 12(5): p. 497-503.

[64] Ratjen, F., et al., Effect of continuous antistaphylococcal therapy on the rate of $P$. aeruginosa acquisition in patients with cystic fibrosis. Pediatr Pulmonol, 2001. 31(1): p. 13-6.

[65] Smyth, A.R. and S. Walters, Prophylactic anti-staphylococcal antibiotics for cystic fibrosis. Cochrane Database Syst Rev, 2014. 11: p. CD001912.

[66] Ledson, M.J., et al., Outcome of Burkholderia cepacia colonisation in an adult cystic fibrosis centre. Thorax, 2002. 57(2): p. 142-5.

[67] Courtney, J.M., et al., Clinical outcome of Burkholderia cepacia complex infection in cystic fibrosis adults. J Cyst Fibros, 2004. 3(2): p. 93-8.

[68] Jones, A.M., et al., Burkholderia cenocepacia and Burkholderia multivorans: influence on survival in cystic fibrosis. Thorax, 2004. 59(11): p. 948-51.

[69] Murray, S., et al., Impact of burkholderia infection on lung transplantation in cystic fibrosis. Am J Respir Crit Care Med, 2008. 178(4): p. 363-71.

[70] Regan, K.H. and J. Bhatt, Eradication therapy for Burkholderia cepacia complex in people with cystic fibrosis. Cochrane Database Syst Rev, 2014. 10: p. CD009876.

[71] Bryant, J.M., et al., Whole-genome sequencing to identify transmission of Mycobacterium abscessus between patients with cystic fibrosis: a retrospective cohort study. Lancet, 2013. 381(9877): p. 1551-60.

[72] Qvist, T., et al., Shifting paradigms of nontuberculous mycobacteria in cystic fibrosis. Respir Res, 2014. 15: p. 41.

[73] Catherinot, E., et al., Mycobacterium avium and Mycobacterium abscessus complex target distinct cystic fibrosis patient subpopulations. J Cyst Fibros, 2013. 12(1): p. 74-80.

[74] Qvist, T., et al., Epidemiology of nontuberculous mycobacteria among patients with cystic fibrosis in Scandinavia. J Cyst Fibros, 2015. 14(1): p. 46-52.

[75] Olivier, K.N., et al., Nontuberculous mycobacteria. II: nested-cohort study of impact on cystic fibrosis lung disease. Am J Respir Crit Care Med, 2003. 167(6): p. 835-40.

[76] Coolen, N., et al., Reduced risk of nontuberculous mycobacteria in cystic fibrosis adults receiving long-term azithromycin. J Cyst Fibros, 2015.

[77] Lobo, L.J., et al., Lung transplant outcomes in cystic fibrosis patients with pre-operative Mycobacterium abscessus respiratory infections. Clin Transplant, 2013. 27(4): p. 523-9.

[78] McMahon, M.A., et al., Radiological abnormalities associated with Aspergillus colonization in a cystic fibrosis population. Eur J Radiol, 2012. 81(3): p. e197-202. 
[79] Fillaux, J., et al., Assessment of Aspergillus sensitization or persistent carriage as a factor in lung function impairment in cystic fibrosis patients. Scand J Infect Dis, 2012. 44(11): p. 842-7.

[80] Waters, V., et al., Chronic Stenotrophomonas maltophilia infection and mortality or lung transplantation in cystic fibrosis patients. J Cyst Fibros, 2013. 12(5): p. 482-6.

[81] Amin, R. and V. Waters, Antibiotic treatment for Stenotrophomonas maltophilia in people with cystic fibrosis. Cochrane Database Syst Rev, 2014. 4: p. CD009249.

[82] Gungor, O., et al., Frequency of fungi in respiratory samples from Turkish cystic fibrosis patients. Mycoses, 2013. 56(2): p. 123-9.

[83] Chotirmall, S.H., et al., Sputum Candida albicans presages FEV(1) decline and hospitaltreated exacerbations in cystic fibrosis. Chest, 2010. 138(5): p. 1186-95.

[84] Elphick, H.E. and G. Mallory, Oxygen therapy for cystic fibrosis. Cochrane Database Syst Rev, 2013. 7: p. CD003884.

[85] Martin, C., et al., Prognostic value of six minute walk test in cystic fibrosis adults. Respir Med, 2013. 107(12): p. 1881-7.

[86] Belkin, R.A., et al., Risk factors for death of patients with cystic fibrosis awaiting lung transplantation. Am J Respir Crit Care Med, 2006. 173(6): p. 659-66.

[87] Young, A.C., et al., Randomised placebo controlled trial of non-invasive ventilation for hypercapnia in cystic fibrosis. Thorax, 2008. 63(1): p. 72-7.

[88] Texereau, J., et al., Determinants of mortality for adults with cystic fibrosis admitted in Intensive Care Unit: a multicenter study. Respir Res, 2006. 7: p. 14.

[89] Efrati, O., et al., Outcome of patients with cystic fibrosis admitted to the intensive care unit: is invasive mechanical ventilation a risk factor for death in patients waiting lung transplantation? Heart Lung, 2010. 39(2): p. 153-9.

[90] Berlinski, A., et al., Invasive mechanical ventilation for acute respiratory failure in children with cystic fibrosis: outcome analysis and case-control study. Pediatr Pulmonol, 2002. 34(4): p. 297-303.

[91] Steinkamp, G. and B. Wiedemann, Relationship between nutritional status and lung function in cystic fibrosis: cross sectional and longitudinal analyses from the German CF quality assurance (CFQA) project. Thorax, 2002. 57(7): p. 596-601.

[92] Gozdzik, J., et al., Relationship between nutritional status and pulmonary function in adult cystic fibrosis patients. J Physiol Pharmacol, 2008. 59 Suppl 6: p. 253-60.

[93] McCarthy, C., et al., The CF-ABLE score: a novel clinical prediction rule for prognosis in patients with cystic fibrosis. Chest, 2013. 143(5): p. 1358-64.

[94] Steinkamp, G., et al., [Stabilization of lung function in cystic fibrosis during long-term tube feeding via a percutaneous endoscopic gastrostomy]. Pneumologie, 1990. 44(10): p. 1151-3. 
[95] Steinkamp, G. and H. von der Hardt, Improvement of nutritional status and lung function after long-term nocturnal gastrostomy feedings in cystic fibrosis. J Pediatr, 1994. 124(2): p. 244-9.

[96] Stephenson, A.L., et al., Longitudinal trends in nutritional status and the relation between lung function and BMI in cystic fibrosis: a population-based cohort study. Am J Clin Nutr, 2013. 97(4): p. 872-7.

[97] Donovan, D.S., Jr., et al., Bone mass and vitamin $D$ deficiency in adults with advanced cystic fibrosis lung disease. Am J Respir Crit Care Med, 1998. 157(6 Pt 1): p. 1892-9.

[98] Tschopp, O., et al., Osteoporosis before lung transplantation: association with low body mass index, but not with underlying disease. Am J Transplant, 2002. 2(2): p. 167-72.

[99] Putman, M.S., et al., Trends in bone mineral density in young adults with cystic fibrosis over a 15year period. J Cyst Fibros, 2015.

[100] Conwell, L.S. and A.B. Chang, Bisphosphonates for osteoporosis in people with cystic fibrosis. Cochrane Database Syst Rev, 2014. 3: p. CD002010.

[101] Sly, P.D., et al., Risk factors for bronchiectasis in children with cystic fibrosis. N Engl J Med, 2013. 368(21): p. 1963-70.

[102] McElvaney, O.J., et al., The effect of the decoy molecule PA401 on CXCL8 levels in bronchoalveolar lavage fluid of patients with cystic fibrosis. Mol Immunol, 2015. 63(2): p. 550-8.

[103] Colombo, C., et al., Cytokine levels in sputum of cystic fibrosis patients before and after antibiotic therapy. Pediatr Pulmonol, 2005. 40(1): p. 15-21.

[104] Bodini, A., et al., Biomarkers of neutrophilic inflammation in exhaled air of cystic fibrosis children with bacterial airway infections. Pediatr Pulmonol, 2005. 40(6): p. 494-9.

[105] Karpati, F., F.L. Hjelte, and B. Wretlind, TNF-alpha and IL-8 in consecutive sputum samples from cystic fibrosis patients during antibiotic treatment. Scand J Infect Dis, 2000. 32(1): p. 75-9.

[106] Dakin, C.J., et al., Relationship between sputum inflammatory markers, lung function, and lung pathology on high-resolution computed tomography in children with cystic fibrosis. Pediatr Pulmonol, 2002. 33(6): p. 475-82.

[107] Bhalla, M., et al., Cystic fibrosis: scoring system with thin-section CT. Radiology, 1991. 179(3): p. 783-8.

[108] Reeves, E.P., et al., The involvement of glycosaminoglycans in airway disease associated with cystic fibrosis. ScientificWorldJournal, 2011. 11: p. 959-71.

[109] Bergin, D.A., et al., Airway inflammatory markers in individuals with cystic fibrosis and non-cystic fibrosis bronchiectasis. J Inflamm Res, 2013. 6: p. 1-11.

[110] Linnane, S.J., et al., Total sputum nitrate plus nitrite is raised during acute pulmonary infection in cystic fibrosis. Am J Respir Crit Care Med, 1998. 158(1): p. 207-12. 
[111] Anil, N., et al., Induced sputum nitrites correlate with FEV1 in children with cystic fibrosis. Acta Paediatr, 2010. 99(5): p. 711-4.

[112] Laguna, T.A., et al., Sputum club cell protein concentration is associated with pulmonary exacerbation in cystic fibrosis. J Cyst Fibros, 2014.

[113] Naudin, C., et al., Human cysteine cathepsins are not reliable markers of infection by Pseudomonas aeruginosa in cystic fibrosis. PLoS One, 2011. 6(9): p. e25577.

[114] Schulz, B.L., et al., Glycosylation of sputum mucins is altered in cystic fibrosis patients. Glycobiology, 2007. 17(7): p. 698-712.

[115] Liou, T.G., et al., Sputum biomarkers and the prediction of clinical outcomes in patients with cystic fibrosis. PLoS One, 2012. 7(8): p. e42748.

[116] Gray, R.D., et al., Sputum and serum calprotectin are useful biomarkers during CF exacerbation. J Cyst Fibros, 2010. 9(3): p. 193-8.

[117] Golden, B.E., et al., Calprotectin as a marker of inflammation in cystic fibrosis. Arch Dis Child, 1996. 74(2): p. 136-9.

[118] Moffitt, K.L., et al., Inflammatory and immunological biomarkers are not related to survival in adults with Cystic Fibrosis. J Cyst Fibros, 2014. 13(1): p. 63-8.

[119] Nick, J.A., et al., Blood mRNA biomarkers for detection of treatment response in acute pulmonary exacerbations of cystic fibrosis. Thorax, 2013. 68(10): p. 929-37.

[120] Schultz, H., et al., BPI-ANCA of pediatric cystic fibrosis patients can impair BPI-mediated killing of E. coli DH5alpha in vitro. Pediatr Pulmonol, 2004. 37(2): p. 158-64.

[121] Dorlochter, L., et al., Anti-neutrophil cytoplasmatic antibodies and lung disease in cystic fibrosis. J Cyst Fibros, 2004. 3(3): p. 179-83.

[122] Lindberg, U., et al., BPI-ANCA and long-term prognosis among 46 adult CF patients: a prospective 10-year follow-up study. Clin Dev Immunol, 2012. 2012: p. 370107.

[123] McKone, E.F., et al., Association of sweat chloride concentration at time of diagnosis and CFTR genotype with mortality and cystic fibrosis phenotype. J Cyst Fibros, 2015.

[124] Rowe, S.M., et al., Optimizing nasal potential difference analysis for CFTR modulator development: assessment of ivacaftor in CF subjects with the G551D-CFTR mutation. PLoS One, 2013. 8(7): p. e66955.

[125] Judge, E.P., et al., Pulmonary abnormalities on high-resolution CT demonstrate more rapid decline than FEV1 in adults with cystic fibrosis. Chest, 2006. 130(5): p. 1424-32.

[126] Mott, L.S., et al., Progression of early structural lung disease in young children with cystic fibrosis assessed using CT. Thorax, 2012. 67(6): p. 509-16.

[127] Dodd, J.D., et al., Thin-section CT in patients with cystic fibrosis: correlation with peak exercise capacity and body mass index. Radiology, 2006. 240(1): p. 236-45. 
[128] Bannier, E., et al., Hyperpolarized 3He MR for sensitive imaging of ventilation function and treatment efficiency in young cystic fibrosis patients with normal lung function. Radiology, 2010. 255(1): p. 225-32.

[129] O'Connell, O.J., et al., Radiologic imaging in cystic fibrosis: cumulative effective dose and changing trends over 2 decades. Chest, 2012. 141(6): p. 1575-83.

[130] Brasfield, D., et al., The chest roentgenogram in cystic fibrosis: a new scoring system. Pediatrics, 1979. 63(1): p. 24-9.

[131] Taussig, L.M., et al., A new prognostic score and clinical evaluation system for cystic fibrosis. J Pediatr, 1973. 82(3): p. 380-90.

[132] Holzer, F.J., A. Olinsky, and P.D. Phelan, Variability of airways hyper-reactivity and allergy in cystic fibrosis. Arch Dis Child, 1981. 56(6): p. 455-9.

[133] Sawyer, S.M., et al., Critical evaluation of three chest radiograph scores in cystic fibrosis. Thorax, 1994. 49(9): p. 863-6.

[134] Brody, A.S., et al., High-resolution computed tomography in young patients with cystic fibrosis: distribution of abnormalities and correlation with pulmonary function tests. J Pediatr, 2004. 145(1): p. 32-8.

[135] Wainwright, C.E., et al., Effect of bronchoalveolar lavage-directed therapy on Pseudomonas aeruginosa infection and structural lung injury in children with cystic fibrosis: a randomized trial. JAMA, 2011. 306(2): p. 163-71.

[136] Pereira, F.F., et al., Correlation between Bhalla score and spirometry in children and adolescents with cystic fibrosis. Rev Assoc Med Bras, 2014. 60(3): p. 216-21.

[137] Shah, R.M., et al., High-resolution CT in the acute exacerbation of cystic fibrosis: evaluation of acute findings, reversibility of those findings, and clinical correlation. AJR Am J Roentgenol, 1997. 169(2): p. 375-80.

[138] Eshed, I., et al., Bronchiectasis: correlation of high-resolution CT findings with health-related quality of life. Clin Radiol, 2007. 62(2): p. 152-9.

[139] Shwachman, H. and L.L. Kulczycki, Long-term study of one hundred five patients with cystic fibrosis; studies made over a five- to fourteen-year period. AMA J Dis Child, 1958. 96(1): p. 6-15.

[140] Stollar, F., et al., Shwachman-Kulczycki score still useful to monitor cystic fibrosis severity. Clinics (Sao Paulo), 2011. 66(6): p. 979-83.

[141] Conway, S.P., et al., The chest radiograph in cystic fibrosis: a new scoring system compared with the Chrispin-Norman and Brasfield scores. Thorax, 1994. 49(9): p. 860-2.

[142] Chrispin, A.R. and A.P. Norman, The systematic evaluation of the chest radiograph in cystic fibrosis. Pediatr Radiol, 1974. 2(2): p. 101-5. 
[143] Nathanson, I., et al., Ultrafast computerized tomography of the chest in cystic fibrosis: a new scoring system. Pediatr Pulmonol, 1991. 11(1): p. 81-6.

[144] Hayllar, K.M., et al., A prognostic model for the prediction of survival in cystic fibrosis. Thorax, 1997. 52(4): p. 313-7.

[145] Liou, T.G., et al., Predictive 5-year survivorship model of cystic fibrosis. Am J Epidemiol, 2001. 153(4): p. 345-52.

[146] Quon, B.S., et al., Prevalence of Symptoms of Depression and Anxiety in Adults With Cystic Fibrosis Based on the PHQ-9 and GAD-7 Screening Questionnaires. Psychosomatics, 2014.

[147] Quittner, A.L., et al., Prevalence of depression and anxiety in patients with cystic fibrosis and parent caregivers: results of The International Depression Epidemiological Study across nine countries. Thorax, 2014. 69(12): p. 1090-7.

[148] Fidika, A., M. Herle, and L. Goldbeck, Symptoms of depression impact the course of lung function in adolescents and adults with cystic fibrosis. BMC Pulm Med, 2014. 14: p. 205.

[149] Hilliard, M.E., et al., Medication Beliefs Mediate Between Depressive Symptoms and Medication Adherence in Cystic Fibrosis. Health Psychol, 2014.

[150] Schechter, M.S., et al., Association of socioeconomic status with the use of chronic therapies and healthcare utilization in children with cystic fibrosis. J Pediatr, 2009. 155(5): p. 634-9 e1-4.

[151] O'Connor, G.T., et al., Median household income and mortality rate in cystic fibrosis. Pediatrics, 2003. 111(4 Pt 1): p. e333-9.

[152] O'Connor, G.T., et al., Case-mix adjustment for evaluation of mortality in cystic fibrosis. Pediatr Pulmonol, 2002. 33(2): p. 99-105.

[153] Lavie, M., et al., Several siblings with Cystic Fibrosis as a risk factor for poor outcome. Respir Med, 2015. 109(1): p. 74-8.

[154] Collaco, J.M., et al., Interactions between secondhand smoke and genes that affect cystic fibrosis lung disease. JAMA, 2008. 299(4): p. 417-24. 

Chapter 2

\title{
Outcome Measures for Respiratory Physiotherapy in Cystic Fibrosis - Challenges and Advances
}

\author{
Alda Marques, Joana Cruz, Cristina Jácome and \\ Ana Oliveira \\ Additional information is available at the end of the chapter \\ http://dx.doi.org/10.5772/60674
}

\begin{abstract}
Respiratory physiotherapy is part of the routine management of patients with cystic fibrosis. It normally consists of airway clearance techniques and exercise training. The evidence of such interventions has been questioned. Nevertheless, the lack of evidence should not be interpreted as lack of benefit. Instead, attention to methodological issues, such as the selection of the outcome measures, is needed, as they may hamper the establishment of the effectiveness of respiratory physiotherapy techniques. Hence, this chapter presents and discusses the strengths and weaknesses of conventional and emerging outcome measures possibly to be used (i) in clinical practice before, during and after each session of respiratory physiotherapy to monitor its effectiveness; (ii) before and after the respiratory physiotherapy treatment (i.e., normally characterised by weeks of intervention) and (iii) in applied research in respiratory physiotherapy used in the management for cystic fibrosis. A comprehensive overview of the available outcome measures is provided, with particular emphasis on their strengths and limitations that should be recognised when interpreting the results.
\end{abstract}

Keywords: Respiratory physiotherapy, outcome measures, cystic fibrosis

\section{Introduction}

Respiratory physiotherapy is a non-pharmacological treatment commonly provided to patients with cystic fibrosis (CF) [1]. According to international guidelines, respiratory 
physiotherapy is a key element of care for patients with CF, as it aims at both rehabilitation and prevention [2]. Specifically, respiratory physiotherapy is used to deal with the progressive loss of pulmonary function accompanied by symptoms of cough, excessive sputum production, dyspnoea, exercise intolerance, reduced functionality and impaired quality of life. To respond to these multiple problems and needs, respiratory physiotherapy involves a range of strategies and techniques, such as airway clearance, exercise training and breathlessness management, which have an overall aim of reducing the progression of the disease [3, 4].

However, there is a lack of evidence to suggest the superiority of one technique over the other [5] and to determine which strategies promote the adherence of this population to regular physical activity [6]. Nevertheless, the lack of evidence does not mean lack of benefit. Instead, methodological issues, such as the selection of the outcome measures, may hamper the establishment of the effectiveness of the respiratory physiotherapy techniques.

Respiratory physiotherapists use several outcome measures to monitor and evaluate their interventions. Most of the clinically available outcome measures are not specific for the physiotherapy intervention employed and may be affected by other factors. This means that there are no gold standard outcome measures specifically related to respiratory physiotherapy interventions. Thus, in all areas of respiratory physiotherapy, one of the barriers to generate evidence has been the lack of accurate, reliable, sensitive and valid outcome measures. To overcome this problematic issue, new measures have been emerging.

This chapter starts by providing an overview of the problem. It then presents and discusses the strengths and weaknesses of the commonly used clinical outcome measures and other measures that have been gaining interest in the assessment and monitoring of respiratory physiotherapy interventions in CF. The chapter ends with a brief conclusion. A comprehensive overview of the available outcome measures is sought to be provided, with particular emphasis on their strengths and limitations that should be recognised when interpreting the results.

\section{Outcome measures for respiratory physiotherapy}

Respiratory physiotherapy in CF involves a wide range of interventions and among them airway clearance techniques and exercise training are recognised as the most important. The primary aim of airway clearance techniques is to relieve the airway obstruction by promoting the normal mucociliary clearance mechanism of the lungs and facilitating expectoration, thus reducing the risk of infection and inflammation. A variety of airway clearance techniques have been developed. Some involve airway oscillation, some are independently performed and others require electricity or physical assistance [5]. Exercise training is advocated as an important package of care delivered to patients with CF [5], since exercise intolerance has been associated with reduced survival [45]. Observed benefits of exercise training include slow pulmonary function decline [46], reduced dyspnoea and improved exercise capacity, muscle strength and health-related quality of life (HRQoL) [47].

Although adhering to airway clearance techniques [7] and exercise training is generally regarded as beneficial for patients with $\mathrm{CF}$, there is no consensus about the superiority of one 
technique over the other [5]. Methodological issues, such as the underpowered samples or the selection of the outcome measures, may explain this lack of evidence and thus hamper the establishment of the effectiveness of these interventions. A clear example of this issue has been the use of forced expiratory volume in the first second $\left(\mathrm{FEV}_{1}\right)$ as a gold standard to assess the impact of the mentioned interventions for many years, which is currently considered as not a sensitive measure to be used in respiratory physiotherapy [5].

Most of the clinically available outcome measures are not specifically related to the physiotherapy intervention employed and may be affected by other factors. This means that there is no gold standard outcome measure that is specifically related to respiratory physiotherapy interventions. Moreover, there are many doubts about the accuracy, reliability, sensitivity and validity of the current measures. Given this problematic situation, several researchers have been investigating the potential of other objective, simple and non-invasive measures to be used as outcome measures in respiratory physiotherapy.

The outcome measures most commonly used by respiratory physiotherapists to monitor their interventions and evaluate their practice are: $\mathrm{FEV}_{1}$, respiratory sounds, sputum weight, measures of oxygenation, chest radiography, dyspnoea, exercise capacity and HRQoL. Computerised respiratory sounds, lung ultrasound, fat-free mass, inspiratory muscle strength and endurance, physical activity and burden of treatment are some examples of these emerging outcome measures to assess and monitor respiratory physiotherapy interventions in CF. Each one of these outcome measures, their strengths and weaknesses are presented in detail below according to their novelty in the field (i.e., conventional and emerging).

\subsection{Conventional outcome measures}

\subsubsection{Forced expiratory volume in the first second}

The most common pulmonary function test performed to assess respiratory physiotherapy interventions is the forced spirometry, i.e., the volume and/or flow of air that can be inhaled and exhaled as a function of time. The procedure consists in three distinct phases: (1) maximal inspiration followed by an expiration at functional residual capacity; (2) a "blast" of exhalation; and (3) continued complete exhalation until the end of test [8]. First, the patient should exhale until he or she reaches the functional residual capacity and then be instructed to inhale rapidly and completely. In this phase, the mouthpiece should be placed in the patient's mouth and indications should be given for the patient to blow as much and as fast as possible and to keep blowing until totally emptying the lungs. Spirometry has been described as a cost-effective, simple, reliable, valid and easy-to-interpret bedside measure [8]. The most used pulmonary function parameter is the forced expiratory volume in the first second $\left(\mathrm{FEV}_{1}\right)$, followed by the forced vital capacity (FVC) and the ratio between $\mathrm{FEV}_{1} / \mathrm{FVC}$. Measurements are taken considering patient's gender, age, height, weight and race and are then compared with predicted values.

Spirometry, namely $\mathrm{FEV}_{1}$, has been used to assess the effectiveness of respiratory physiotherapy interventions. However, contradictory findings have emerged. Pfleger et al. (1992) found significant improvements in $\mathrm{FEV}_{1}$ after autogenic drainage and high-pressure PEP-mask (n=14, 
Cohen's $d_{z} 0.09$ and 0.22) [9]. Jarad, Powell and Smith (2010) reported a statistically significant reduction in FEV1 following hydro-acoustic therapy ( $n=19$, Cohen's dz=0.12) or flutter $(n=19$, Cohen's dz=0.06) [10]. Nevertheless, these changes returned to baseline on the second study day [10].

In two recent reviews where conventional physiotherapy [11] and the active cycle of breathing techniques (ACBT) [12] were compared with other airway clearance techniques, no significant differences were observed between the techniques in terms of pulmonary function measured with spirometry [11]. Nevertheless, when oscillating devices for airway clearance were used, significant results were observed in pulmonary function, the $\mathrm{FEV}_{1}$ being the primary outcome measure more frequently reported [13].

Findings in the literature about the effectiveness of respiratory physiotherapy interventions in $\mathrm{CF}$ remain controversial when $\mathrm{FEV}_{1}$ is considered as the outcome measure. This is in part due to the fact that accuracy and sensitivity of spirometry depends on many factors that are difficult to control and not related to the intervention itself. Some examples of these factors are the transducer characteristics, presence or absence of an in-line filter, presence or absence of a display, patient's mood and motivation to cooperate, relationship between the patient and the technician, among others. Therefore, spirometry might be unsuitable or its reliability may be affected in a number of situations, for example if the equipment or settings change, if patients are unwilling or unable to collaborate (e.g. children, people with dementia), or if pain or discomfort is present. Hence, this measure should be routinely used to characterise the pulmonary function of patients with $\mathrm{CF}$, but not to assess the effectiveness of respiratory physiotherapy interventions.

\subsubsection{Respiratory sounds}

Lung auscultation, performed with conventional stethoscopes, is one of the oldest and most used techniques to diagnose and monitor respiratory diseases [14, 15]. It consists in acquiring acoustic signals from the lung structures during spontaneous or controlled volume or flow breathing, and classifying the respiratory sounds as normal or abnormal (e.g., adventitious respiratory sounds, such as crackles and wheezes) [15]. Auscultation is recognised as an efficient and safe method for the early detection of respiratory diseases as it is non-invasive, practical, low cost and easy to apply in all clinical settings and patients, irrespective of patients' age and severity of the disease [14-16].

The efficiency of this method depends on the hearing ability of the health professionals [17], their capacity to memorise different sound patterns [18] and on the quality of the acoustic properties of the stethoscope being used [17]. Considering these limitations and subjectivity, reliability studies have been performed. In CF there are no reliability studies using conventional stethoscopes. However, in other respiratory diseases, poor to fair correlations between different raters have been reported, either in taped recorded sounds (kappa $=0.26$ and coefficient of reliability of less than $60 \%)[19,20]$ and real-time auscultation $(-0.02<$ kappa $<0.77)$ [21]. Using digital stethoscopes, one study conducted in adult patients with CF assessed the interrater agreement between real-time manual annotation of respiratory sounds and automatic detection of respiratory sounds through a computerised system [22]. Poor to moderate 
correlations were found $(-0.20<\mathrm{kappa}<0.60)$ [22]. Similar results were found in children with respiratory diseases $(-0.08<\mathrm{kappa}<0.86)$ [23-25]. Direct comparisons between conventional and digital stethoscopes showed that, although digital stethoscopes have a better sound quality [26], the performance of health professionals to detect respiratory sounds is not enhanced by its use [27].

It is also important to note that, in all the studies, the recognition of crackles was always less accurate than the recognition of wheezes. As respiratory sounds in CF are mainly characterised by the presence of crackles [28] and conventional auscultation has provided poor reliability results, particularly for detection of crackles, it can be concluded that respiratory sounds are not a reliable outcome measure for CF diagnosis and monitoring. Also due to its poor reliability, conventional auscultation has not been used as an outcome measure for respiratory physiotherapy interventions in recent research [7]. Nevertheless, due to its simplicity and wide availability in all clinical settings, it is still recurrently used in clinical practice by physiotherapists to monitor patients with CF and to define therapeutic approaches [3]. Despite these limitations, the advantages of using lung auscultation should not be overlooked, and currently significant research efforts are being conducted to create equipment capable of overcoming the subjectivity associated with conventional auscultation while preserving its main advantages (i.e., portability, patients' minimal cooperation and cost-effectiveness) [16].

\subsubsection{Sputum weight}

Mucus is transported from the bronchial airways towards the exterior by mucociliary clearance, spontaneous cough and through a range of airway clearance techniques, such as directed huffs and coughs. Subsequently, secretions are either expectorated or swallowed. During respiratory physiotherapy sessions, patients are encouraged to expectorate to a cup. Sputum volume or weight can then be used as an outcome measure for respiratory physiotherapy.

While sputum volume has shown to be difficult to determine with precision, sputum weight (either dry or wet) has shown to be more accurate [29]. In a recent Cochrane review on the effectiveness of respiratory physiotherapy interventions in respiratory diseases, five of the eight studies used sputum weight as an outcome measure, out of which four were conducted in patients with CF [7]. Mortensen et al. (1991) found that patients expectorated 8-8.6g of sputum weight after airway clearance techniques and $0 \mathrm{~g}$ during a control day (range $0-2.1 \mathrm{~g}$ ) [30]. In Pfeger et al. (1992), the mean weight of expectorated mucus with spontaneous cough was approximately $17 \mathrm{~g}$ whereas with airway clearance techniques ranged from 34 to $45 \mathrm{~g}(\mathrm{n}=14)$ [9]. Although no mean and standard deviation was provided, Rossman et al. (1982) also found a higher volume of expectorated secretions during the different forms of chest physiotherapy compared to a control session $(n=6)$ [31]. However, Jarad et al. (2010) found no significant differences in wet or dry sputum weight between a positive expiratory pressure (PEP) device (flutter) or a placebo [10].

Another study conducted by Osman et al. (2010) compared high-frequency chest wall oscillation with conventional airway clearance techniques in 29 patients with CF using wet sputum weight. Expectorated sputum in a single session was significantly different between the techniques ( $p<0.001$; Cohen's $\left.d_{z}=0.72\right)$ [32]. 
The problem with sputum weight remains that the lack of expectoration during respiratory physiotherapy techniques does not mean that airway clearance techniques are not effective. It is very common to expectorate after respiratory physiotherapy sessions or to swallow secretions even during the session, which means that sputum weight expectorated during a session may underestimate the effect of airway clearance techniques. On the other hand, expectoration may be contaminated with saliva [30]. Sputum weight can, therefore, be both over- and under-estimated and it is not surprising that contradictory findings have been found in the literature.

Although simple to collect and measure, sputum collected during and following the treatments is not considered a reliable measure of alveolar recruitment, airway clearance or even sensitive to small changes. In fact, this outcome measure has been frequently questioned [29, 30, 33-35] and is no longer considered as a valid outcome measure for studies in airway clearance techniques [36].

\subsubsection{Measures of oxygenation}

Blood gas measurements are used to evaluate a person's lung function and acid-base balance, i.e., it measures the amount of oxygen and carbon dioxide that is in the blood and determines its levels of acidity $(\mathrm{pH})$. The test results provide information about the partial pressure of oxygen $\left(\mathrm{PaO}_{2}\right)$, partial pressure of carbon dioxide $\left(\mathrm{PaCO}_{2}\right)$ and hydrogen ion activity $(\mathrm{pH})$ in arterial blood, as well as indices of bicarbonate concentration, base excess and oxygen saturation. The analysis is performed on the blood collected from a person's artery and is therefore, an invasive and relatively complex procedure. Although it is possible to measure oxygenation more accurately, given the nature of the procedure, it is not practical to be performed on a routine basis to monitor respiratory physiotherapy treatments. Hence, transcutaneous pulse oximetry (reported as the percentage of saturation of arterial haemoglobin by oxygen $\left[\mathrm{SaO}_{2}\right]$ ) has become the most common method in clinical practice and research for measuring oxygenation [37].

Peripheral oxygen saturation is commonly used as it is simple to perform via pulse oximeters and finger probes. Pulse oximeters monitor the saturation of haemoglobin with oxygen (i.e., oxyhaemoglobin). This is possible because blood changes its colour as haemoglobin absorbs various amounts of light depending on its saturation with oxygen. Oxyhaemoglobin absorbs greater amounts of infrared light and does not absorb much red light, but as the haemoglobin oxygen saturation drops, more and more red light is absorbed and the blood becomes darker. Hence, pulse oximeters emit two wavelengths of light, red at $660 \mathrm{~nm}$ and near-infrared at 940 $\mathrm{nm}$ from the finger probe [38]. The pulse oximeter directly senses the absorption of light and translates it through complex signal processing to a function of the arterial oxygen saturation. A microprocessor integrates the data and, through an elaborate calibration algorithm based on human volunteer data, the oxygen saturation can be estimated. This measure allows constant monitoring of heart rate as well, being ideal to monitor patient's safety during interventions.

Nonetheless, this measure can provide unreliable readings due to several factors such as haemoglobin level, arterial blood flow to the vascular bed, oximetry sensor location and 
temperature, fluorescent or direct sunlight, jaundice, discoloration of the nail bed, nail polish, bruising under the nail, motion artefacts, intravascular dyes and skin pigmentation. All these are discussed in detail elsewhere [38].

In two Cochrane reviews about respiratory physiotherapy techniques, insufficient evidence was found regarding peripheral oxygen saturation as an outcome measure $[7,12]$. Osman et al. (2010) compared high-frequency chest wall oscillation with conventional airway clearance techniques in 29 patients with CF. It was found that, compared to the baseline, non-significant changes in peripheral oxygen saturation were observed during or after any of the treatments applied (Cohen's $d_{z}$ from 0 to 0.28 ) [32]. However, in a recent study, the addition of noninvasive ventilation to chest physiotherapy resulted in a significant reduction in the proportion of treatment time with peripheral oxygen saturation below $90 \%(\mathrm{p}<0.001)$ [39].

Controversial evidence exists on the potential of peripheral oxygen saturation as an adequate measure to assess the effectiveness of respiratory physiotherapy. Hence, this measure seems ideal to monitor patients' safety but it may not present the required specificity and/or sensitivity to assess changes caused by respiratory physiotherapy interventions.

\subsubsection{Dyspnoea}

Dyspnoea is defined as "a subjective experience of breathing discomfort that consists of qualitatively distinct sensations that vary in intensity" [40]. In a study conducted with 123 patients with $\mathrm{CF}$, dyspnoea was reported to be present in $74 \%$ of the patients [41] and therefore, it is an important problem to consider in this population. As a subjective experience, adequate assessment depends on patient self-report. Depending on the circumstances in which breathing discomfort occurs and the history of similar sensations, dyspnoea may be perceived as a threat associated with anxiety, fear or depression, and it may be viewed as a sign of disease. This symptom has multidimensional aspects involving physiological, psychological, social and environmental factors that result in a behavioural response. The assessment of multidimensional aspects of dyspnoea has assumed significance in recent years [40].

Dyspnoea has been assessed using scales and questionnaires, however, important differences exists between these instruments, particularly in what they measure (e.g., one instrument may ask what breathing feels like, whereas another may ask how distressing it is or how it impacts on patient's performance or quality of life), in the rating task (i.e., what patients are instructed to rate), and in whether measurements are performed on real time or involve the recall of a specific episode. These differences make comparisons across studies difficult.

In one study exploring the effectiveness of non-invasive ventilation during chest physiotherapy [39], it was found that dyspnoea scores, assessed with the Borg scale, increased in slightly lesser extent following chest physiotherapy assisted with non-invasive ventilation than chest physiotherapy alone $\left(2.26 \pm 1.96\right.$ vs. $2.69 \pm 1.82, p=0.02$, Cohen's $\left.d_{z}=0.23\right)$. Marques (2008), however, found that dyspnoea assessed with the modified Borg scale was not significantly different after one session of airway clearance techniques (Cohen's $d_{z}=0.06$ ) [42]. Enright et al. (2004) investigated the effect of an 8-week inspiratory muscle training $(n=19)$ and also did not 
find significant differences is dyspnoea, using the dyspnoea domain of the Chronic Respiratory Disease Questionnaire (Cohen's $d_{z} 0.07$ to 0.37 ) [43].

A combination of unidimensional and multidimensional tools will probably be the best approach for clinical assessment. Measurement of dyspnoea has to be seen in context, taking into consideration the patient's history, physical examination and diagnostic tests [44]. As dyspnoea is a symptom perceived by patients, it has the potential to contribute for early management of the disease, for adjusting the respiratory physiotherapy interventions and, thus, for improving outcomes in patients.

\subsubsection{Chest radiography}

Several imaging techniques are available to diagnose and monitor a respiratory condition. Within the respiratory field, chest radiography is the most commonly used. Although relatively simple to perform, and in itself being relevant and reliable, the measure presents several important limitations to be used as an outcome measure for respiratory physiotherapy. The interpretation of chest radiography imaging is somewhat complex and presents high levels of inter-observer subjectivity [45], the reports detailing either the presence or the absence of any abnormalities are not commonly available immediately after the exam, its portability is limited to places where health and safety radiation protection standards can be ensured, as considerable doses of radiation are involved and is difficult to perform in non-collaborative populations such as children or people with dementia. These factors prevent the use of this measure to monitor respiratory physiotherapy patients with the required frequency.

In two long-term studies ( $\mathrm{n}=36$ and $\mathrm{n}=32$ ), conducted for approximately one year comparing different respiratory physiotherapy airways clearance techniques in patients with $\mathrm{CF}$, chest radiography was used as an outcome measure. However, no significant differences were shown between interventions [46, 47]. In the most recent Cochrane reviews [11, 12] about physiotherapy in $\mathrm{CF}$, chest radiography is no longer reported as an outcome measure in the included studies.

It therefore, seems that chest radiography is ideal for contributing to diagnosis and providing a measure of improvement or deterioration over time but might not be the most appropriate to be used as an outcome measure for respiratory physiotherapy.

\subsubsection{Exercise capacity}

The assessment of exercise capacity, expressed as the maximal workload achievable or the peak oxygen consumption on a progressive maximal test, has been used to measure patients' functional capacity and limitation, facilitates a safe exercise prescription and identifies changes in patients' performance as a result of an intervention $[48,49]$. There are two types of clinically applicable tests to assess exercise capacity in patients with CF: laboratory-based tests and field tests $[50,51]$.

Laboratory-based tests, such as the cardiopulmonary exercise testing (CPET) [48, 49], are the gold standard for evaluating the causes of exercise intolerance in patients with respiratory 
diseases, as they provide a comprehensive assessment of the physiological responses to exercise involving the respiratory, cardiovascular and musculoskeletal systems [52]. CPET consists of a progressive exercise performed at increasing levels of intensity either on a treadmill or cycle ergometer, with simultaneous monitoring of breath-by-breath measures of airflow (e.g., $\mathrm{VO}_{2}, \mathrm{VCO}_{2}$ ) along with heart rate, peripheral oxygen saturation and exerciserelated symptom responses (e.g. dyspnoea, leg discomfort) [49]. There are a number of protocols that can be used to perform a CPET in patients with $\mathrm{CF}$, but the most commonly reported is the Godfrey protocol [53]. The CPET has been shown to be reproducible in young and adult patients with CF [54,55]. In patients with CF $(n=23)$ enrolled in a strength and endurance training for 6 months, significant differences were observed in $\mathrm{VO}_{2}$ peak between 3 and 6 months (Cohen's $d_{z}=2.01$ ) and between 18 and 24 months (Cohen's $d_{z}=2.22$ ) [56]. Despite its sensitivity to change, the application of CPET is still limited in clinical settings [57]. This may be attributable to the need for expensive equipment and technical expertise. For this reason, field tests are more commonly used by physiotherapists to assess changes in exercise capacity [58].

Field tests are simple clinical exercise tests that do not require expensive equipment [59] and, thus, are suitable to be used at different settings of respiratory physiotherapy practice, such as hospitals, private practices, at home or in community environments. In patients with CF, the field tests most frequently used are the 6-minute walk test (6MWT), the 3-min step test (3MST) and the modified shuttle test (MST) [51,58]. The 6MWT measures the maximal distance that a patient can walk in 6 minutes over a marked course (usually a corridor) following a standardised protocol [60]. The 6MWT has been recommended for patients with CF [60] and is valid and reliable for assessing exercise capacity in children [61,62] and adults [63] with CF. The product of the distance walked during a $6 \mathrm{MWT}$ and body mass have been reported to correlate with aerobic capacity $\left(\mathrm{VO}_{2} \mathrm{max}\right)$ in children with $\mathrm{CF}$ [64], thus supporting the use of this test in clinical settings. Furthermore, the 6MWT was found to be valuable for identifying patients who might experience oxygen desaturation and physical impairment in daily activities [65]. For this reason, the 6MWT has been considered more reflective of activities of daily living than other walk tests [59]. Numerous reference equations of the 6MWT are available for children [66-69] and adults [70].

The 3MST is a simple test that requires the patient to step at a rate of 30 steps per min on and off a step with $15 \mathrm{~cm}$ (6 inch) of height, during 3 minutes. This test was developed for children with CF by Balfour-Lynn et al. (1998) [71] based on the original Master two-step exercise test (1929) [72]. One major advantage of the 3MST is that it does not depend on patient's motivation, since the cadence of steps is fixed and determined by a metronome. One limitation concerns to the fact that, as step height and rate are kept constant, the workload varies between patients depending on their height and lower limb length [51, 58]. The 3MST has been found to be repeatable [71] and sensitive to changes in pulmonary function and peripheral oxygen saturation in children with CF following a course of intravenous antibiotics for acute respiratory exacerbations [73]. This is an important finding since even physiotherapists without access to a formal exercise laboratory may evaluate the response to an intervention using a simple field test. When compared to the 6MWT, the 3MST elicited a significantly greater change in 
heart rate and breathlessness in children with CF, along with a comparable [71] or higher [74] fall in oxygen saturation, suggesting that this test is a more vigorous exercise challenge. Nevertheless, Narang et al. (2003) showed that, in children with mild disease, important information such as the exercise-related desaturation that occurs at higher exercise levels may be missed with this test [75].

While the 6MWT and 3MST may be submaximal tests for patients with mild-to-moderate CF, the MST allows maximal exercise capacity to be reached [58]. The MST [76] consists of a validated adaptation of the Incremental Shuttle Walk Test [77], specifically developed for adults with CF. This adaptation allowed individuals to walk and run at increasing speeds over 15 levels with a maximum speed of $10.2 \mathrm{~km} / \mathrm{h}$ [76]. Bradley et al. $(1999,2000)$ studied the validity, reliability and sensitivity of the MST in adults with CF [76, 78]. When compared to a laboratory-based treadmill test, they showed that $90 \%$ of the variation of directly measured $\mathrm{VO}_{2}$ peak was explained by the variation in MST performance [76]. Moreover, the MST was effective in evoking a symptom-limited exercise response (i.e., peak heart rate and peak rating of perceived dyspnoea) similar to what is found in treadmill tests [76]. Thus, it may be a valid alternative when laboratory-based exercise testing cannot be performed. The validity of the MST has also been assessed in children with $\mathrm{CF}$ (correlation of the MST distance with $\mathrm{VO}_{2}$ peak, $\mathrm{r}=0.663 \mathrm{p}<0.01$ ) [79]. In adults with CF, the MST was found to be reliable (correlation between trials for distance completed and symptoms reported, $\mathrm{r}=0.99, \mathrm{p}<0.01$ ) and repeatable (coefficients of repeatability: distance completed, four shuttles; peak heart rate, 6 beats/min; peak oxygen saturation, 4\%) [78]. The sensitivity of the test was assessed by measuring the change in MST performance after 2 weeks of antibiotic therapy in patients admitted to hospital with acute respiratory exacerbations. An effect size of 1.18 was achieved, suggesting that this is a highly sensitive measure [78]. This test has also been used to assess changes in exercise capacity after 2 months of exercise training and significant differences were found (median number of shuttles: from 100 (range 21-150) to 105 (44-150)) [80]. Despite the good measurement properties, it was recently argued that the 15-level MST developed by Bradley et al. (1999) [76] still remained sub-maximal for some patients with CF and, thus, levels were extended to 25 in order to create a truly maximal test [81].

There is no "best" exercise test. The selection of the test will depend upon the aspect of exercise capacity of interest, availability of resources (i.e., time to perform the test, staff and equipment) and patient's characteristics (e.g., age, disease stage) [50,51]. For example, if the aetiology of a patient's reduced exercise capacity is of interest, a laboratory-based test would be more appropriate, while a field test could be used in large population studies. In addition, a young child ( $<7$ years old) may not be able to cooperate sufficiently for a formal laboratory exercise testing. Physiotherapists have the important role of selecting the best exercise test for a specific individual according to the specific question being asked and the specificities of each test.

\subsubsection{Health-related quality of life}

Health-related quality of life (HRQoL) has been extensively studied in patients with CF, especially in the last decades due to the improvement in patients' life expectancy [82]. Several instruments have been used to assess HRQoL in CF, either generic [83-85] or disease-specific 
[85-87]. One disadvantage of generic questionnaires is that they lack the sensitivity needed to assess areas of functioning that are critically important for patients with CF [88]. Diseasespecific questionnaires were developed in an attempt to better understand CF specific issues in clinical practice and research.

The most commonly used disease-specific questionnaires include the Cystic Fibrosis Questionnaire (CFQ) and the Cystic Fibrosis Quality of Life questionnaire. The CFQ was developed for assessing HRQoL of patients with $\mathrm{CF}$ and encompasses general domains of HRQoL (physical functioning, role functioning, vitality, health perceptions, emotional functioning and social functioning), as well as domains specific to CF (body image, eating disturbances, treatment burden, and respiratory and digestive symptoms) [89]. Three versions of the instrument have been developed: one for adolescents aged 14 years or older and adults (CFQTeen/Adult), and two for assessing children 6-13 years old, one to be completed by the child (CFQ-Child) and the other by parents (CFQ-Parent) [89, 90]. Each version takes around 15 minutes to fill in. The different versions of the CFQ questionnaire have shown good psychometric properties (validity [88-90], reliability [89, 90], internal consistency [88-90] and responsiveness [90]). Hebestreit et al. (2010) found that patients with $C F(n=23)$ enrolling in strength and endurance training for 6 months improved their subjective health perception (CFQ domain) significantly between months 3 and 6 (Cohen's $d_{z}=0.92$ ) [56]. Schmidt et al. (2011) evaluated a 12-week individually tailored unsupervised aerobic exercise programme in 14 patients with $\mathrm{CF}$ and also found significant differences in emotional functioning and treatment burden domains of the CFQ (Cohen's $d_{z}$ of 0.29 and 1.03) [91]. Thus, this questionnaire may be used in clinical practice to assess HRQoL of patients with $\mathrm{CF}$, document the progression of disease and explore the effects of respiratory physiotherapy interventions.

The Cystic Fibrosis Quality of Life questionnaire was specifically developed for adolescents (14 years old or older) and adults, presenting good validity (concurrent and discriminate), internal consistency, test-retest reliability results and responsiveness [92]. One advantage of this instrument is the inclusion of domains concerning wider impacts of the disease on patient's lives (e.g., interpersonal relationships, career issues and future concerns), which are not found in other CF questionnaires. Though, it is not adaptable to children.

In sum, disease-specific instruments present good psychometric properties and seem appropriate for several different applications in patients with $\mathrm{CF}$, such as to identify problems and intervene on an individual basis, compare different CF groups, or detect changes in patients' HRQoL as a result of disease progression or interventions.

\subsection{Emerging outcome measures}

\subsubsection{Computerised respiratory sounds}

Computerised auscultation consists of recording patient's respiratory sounds with a digital stethoscope and automatically analysing and classifying them based on specific signal characteristics [93]. Computerised auscultation allows to objectively detect, characterise and identify both normal and adventitious (i.e., crackles and wheezes) respiratory sounds within the breathing cycle [93]. 
To assess computerised respiratory sounds, the patient is positioned in the sitting or supine position (for long-term assessments) and instructed to breath from his/her mouth. Successive or simultaneous recordings are taken from the trachea plus six chest locations (i.e., right and left: anterior, lateral and posterior positions), using a single or a multichannel equipment (Figures 1 and 2) [94].

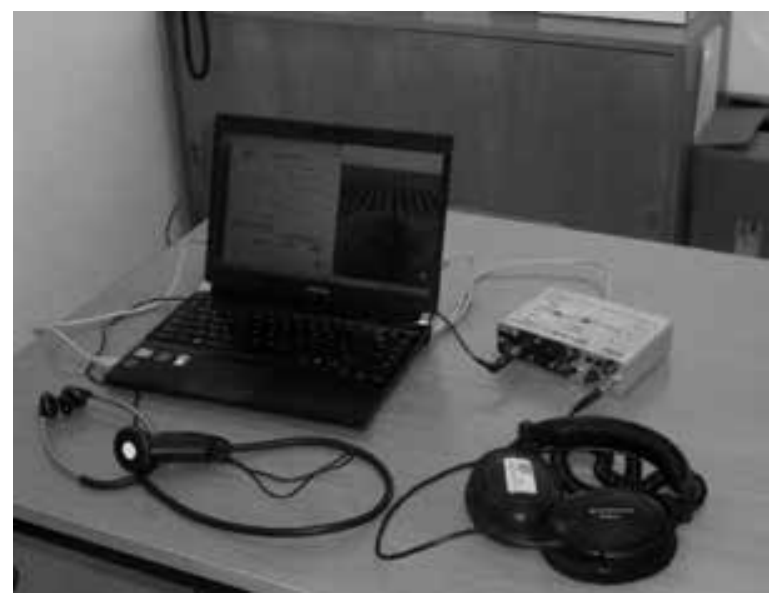

Figure 1. Single-channel equipment for computerised respiratory sound analysis.

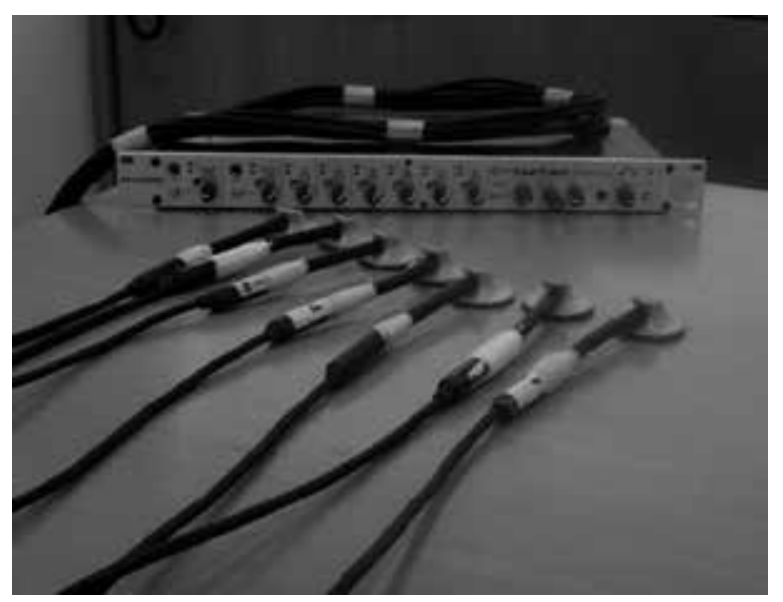

Figure 2. Multi-channel equipment for computerised respiratory sound analysis.

Seven to ten respiratory cycles at tidal breathing or at a flow of 1 to $1.5 \mathrm{~L}$ should be recorded to ensure the stability of sound and quality of the analysis [94]. Then, the sound is filtered using a combination of low-pass and high-pass filters in cascade to reduce sound artefacts (e.g., muscle, heart sounds and movement frequencies) and specialised algorithms for respiratory sound detection and analysis are implemented [94]. 
A wide range of analysis methods exists and new ones are continuously being proposed. However, the ones with more evidence are time-frequency analysis, fast Fourier and wavelet transformations, neural networks, periodogram and auto- regressive models [93, 95-97]. Specifically for patients with CF, a time-frequency wheeze detector has already been validated, demonstrating high levels of sensitivity (77.2\%) and specificity (98.4\%) [98]. Nevertheless, algorithms for the detection of crackles in patients with CF were not found and, thus, further studies are needed.

The reliability of computerised respiratory sounds has been mainly studied in adults with CF [99] and children with asthma, pneumonia [100] and bronchiolitis [23]. In adults with CF, one study assessed the test-retest reliability of two main parameters of crackles (i.e., the initial defection width and the two cycle duration). The intra-subject reliability of crackle parameters was found to be 'good' to 'excellent' $(0.76<$ intraclass correlation coefficients $<0.94)$ with no systematic bias. The smallest real difference found for the initial defection width was between 0.30 and $0.66 \mathrm{~ms}$, and for the two-cycle duration between 1.57 and $2.42 \mathrm{~ms}$. The reliability of wheezes has only been assessed in children with bronchiolitis, presenting moderate to good agreement $(0.77<\mathrm{kappa}<0.79)$ [99]. The reliability of computerised respiratory sounds to assess wheezing in adults and its overall reliability in children with CF is still unexplored.

Due to its simplicity and increasing reliability, computerised respiratory sounds have been used as outcome measures for pharmacological and respiratory physiotherapy interventions in children and adult patients with several respiratory diseases, including CF [93]. Marques et al. (2008) investigated the effect of one single session of respiratory physiotherapy using the ACBT in 17 adult patients with CF [42]. The initial defection width and two cycle duration of crackles were analysed. Considering the mean of all participants, no significant differences were found in the analysed parameters. However, when the individual data of each participant was considered, significant changes were observed in the initial defection width of 9 patients $(53 \%)$ and in the two-cycle duration of 10 participants (59\%).

Considering the detection of wheezes and their analysis, one study assessing the sensitivity of computerised respiratory sounds to defect bronchial hyperactivity in 23 children with CF, following an induced methacholine challenge, found a sensitivity of $50 \%$ and a specificity of $100 \%$ [101]. Studies in patients with lower respiratory tract infection, a common form of exacerbation in CF [102], have shown that computerised respiratory sounds are effective in detecting changes following pharmacological and respiratory physiotherapy interventions [93]. Small to large effects were found in the number of crackles (Cohen's $d_{z}: 0.14$ to 1.65), peak frequency (Cohen's $\mathrm{d}_{\mathrm{z}}$ : 0.11 to 0.47 ), two-cycle duration (Cohen's $\mathrm{d}_{\mathrm{z}}: 0.83$ to 0.85 ) and initial/ largest deflection width (Cohen's $\mathrm{d}_{\mathrm{z}}$ : 0.38 to 1.25$)$. Better results have been found in the detection and characterisation of wheezes, with medium to large effect sizes for the number and occupation rate of wheezes (Cohen's $\mathrm{d}_{\mathrm{z}}$ : 0.34 to 4.30 ) [93].

One of the disadvantages of computerised respiratory sounds concerns the complexity and costs associated with the equipment and subsequent sound analysis. Nevertheless, an emerging body of health and engineer researchers have been gathering efforts to produce more simple and efficient hardware/software that can be used in clinical practice [101, 103]. 
Despite the scarce evidence of computerised respiratory sounds in $\mathrm{CF}$, it seems that this measure might offer potential to assess the short- and long-term effects of respiratory physiotherapy interventions in these patients. However, more research is still required to determine the parameters of computerised respiratory sounds (i.e., number, frequency, duration) that are more sensitive to change after an intervention and to establish the reference values that will allow physiotherapists to interpret with confidence the results obtained from the computerised auscultation.

\subsubsection{Lung ultrasound}

Lung ultrasound (LU) is a simple, non-invasive and radiation-free methodology [104] mainly used in critical care, emergency medicine, trauma surgery and pulmonary medicine for diagnostic purposes [105]. However, due to its practical and secure character, which enables its use as often as required, LU has become an attractive alternative imaging technique for monitoring patients on whom thoracic computed tomography (CT) cannot be performed on a routine basis or where chest $X$-ray presents serious limitations in terms of sensitivity and specificity [106]. In fact, the international evidence-based guidelines for LU recommend this technique to monitor aeration changes and the effects of therapy in a number of acute respiratory diseases, including acute pulmonary edema, acute respiratory distress syndrome, acute lung injury, community-acquired pneumonia, ventilator-associated pneumonia and recovery from lavage of alveolar proteinosis (level A) [105].

Usually, LU is performed using a 3-5 $\mathrm{MHz}$ convex transducer to visualise deeper lung structures [107, 108]. A high-frequency 5-12 MHz linear probe is most effective in visualising the chest wall, pleura and the lung peripheral parenchyma [108]. A complete examination of the chest requires longitudinal, transversal and oblique-array probes to be placed along the rib spaces, proceeding from top to bottom in the ventral-dorsal direction, along of 12 regions of interest (parasternal, medial clavicular, anterior axillary, medial, and posterior right and left chest walls) [106, 108]. Anterior examination should be performed with the patient in the supine or semi-lateral position [106] while posterior examination should take place with the patient seated [107].

LU has shown to be reliable in the diagnosis of several acute respiratory conditions, such as pneumothorax (sensitivity of $65-100 \%$; specificity of $78-100 \%$ ) [108-110], including the diagnosis of this condition in patients with CF (specificity of 100\%) [109], interstitial syndrome (sensitivity and specificity of $94 \%$ ), lung consolidation (sensitivity of 90 to $95 \%$; specificity of $95 \%$ ), pleural effusion (sensitivity of 90 to $100 \%$; specificity of $100 \%$ ) [110] and atelectasis (sensitivity of $88 \%$; specificity of $89 \%$ ) [104]. One study assessed the inter-subject reliability of $\mathrm{LU}$ in the detection of atelectasis and reported a very good agreement (kappa=0.90; 95\%CI 0.75 to 1) [104]. When compared with other imaging equipment (e.g. chest X-ray and MRI), this measure showed similar or even better reliability results [104, 107, 110].

No data has been found regarding the use of LU to monitor respiratory physiotherapy interventions in stable or exacerbated patients with CF. Nevertheless, considering its high accuracy in detecting signals commonly presented in CF exacerbations, such as atelectasis and consolidation [111], and its increasing impact in the management of acute respiratory condi- 
tions [105], it is reasonable to conclude that LU may be a promising measure to monitor the effectiveness of respiratory physiotherapy in patients with acute exacerbations of CF. Considering stable $\mathrm{CF}$, one study used LU to characterise diaphragm thickness as a way to infer about its muscle mass. The authors reported an excellent inter- and intra-subject agreement $(90 \%$ and $91 \%$, respectively) and showed that LU was capable of detecting differences between patients with low and high rates of fat-free mass [112]. From these findings, it can be hypothesised that LU may also play a role in the assessment of the effectiveness of inspiratory muscle training and general exercise training programmes in increasing the diaphragm thickness of patients with stable $\mathrm{CF}$, and thus, its muscle mass. Nevertheless, studies assessing the LU validity, reliability and responsiveness to change are needed before it can be recommended as an outcome measure for respiratory physiotherapy interventions.

At this point, there is no evidence to recommend $\mathrm{LU}$ as an outcome measure for respiratory physiotherapy in patients with CF. However, the advantages presented by LU over other imaging measures and its good performance as a diagnostic tool, should motivate further investigation on the validity and reliability of this measure to assess respiratory physiotherapy interventions in this population.

\subsubsection{Fat-free mass}

Fat free mass (FFM) is the component of body mass that represents muscle mass and protein stores [113] and it is a critical determinant of maximal exercise capacity [114]. It is known that, in patients with $\mathrm{CF}$, lower FFM is also associated with lower $\mathrm{FEV}_{1}$ percentage predicted and more frequent respiratory exacerbations $[115,116]$. Maintaining appropriate levels of FFM is therefore crucial for maintaining the overall functional capacity in patients with CF [114].

The gold standard to assess FFM are the 4-C models, which divide body weight into fat, water and remaining fat-free dry tissue, with the last item further divided into proteins and minerals. The 4-C models require measurements of body weight, body water, body volume and bone mineral [117] and thus several specialised equipment, such as dual-energy $X$-ray, measures of air displacement plethysmography (BOD POD) and $\mathrm{D}_{2} \mathrm{O}$ analysis, are needed. The partial measures are then pulled together in a predictive equation [118]. Although all devices involved are valid and reliable (intraclass correlation coefficient $>0.99$ ), the improved accuracy of the 4$\mathrm{C}$ models may be offset by the potential propagation of errors due to the inherent measurement error of each device used to assess each variable [118]. Also, it appears not to be suitable for widespread clinical use due to the need for specialised equipment, experienced personnel and large amount of time. Thus, its main value lies in the quality of its evidence in supporting treatment approaches, rather than in routine practical application [117, 119].

For application of respiratory physiotherapy clinical practice, 2-C models, which require only the use of one device, are the most recommended and have shown large correlations with the 4 -C models. These are bioelectrical impedance $\left(r>0.79, R^{2}=0.70\right), C T$ scans $\left(r>0.83, R^{2}=0.96\right), M R I$ $(r>0.91)$, and specially dual-energy $X$-ray $(r>0.91)$ [120, 121]. Indirect strategies involving calculations based on anthropometric measurements, such as skinfold thickness analysis, are controversial. Measuring FFM by measurement of skinfolds implies that no index of this component of weight is directly measured, and thus it is based on the assumption that 
measuring fatness reflects lean body mass as well. Therefore, its use has not been recommended [117]. Nevertheless, studies that show medium correlations between measurements of skinfold thickness and $4-C$ models $\left(R^{2}=0.62\right)$ exist [120] and support the usability of this measure to monitor FFM irrespective of the clinical severity of CF [122].

FFM has been used as an outcome measure in respiratory physiotherapy interventions for children and adults with CF, mainly for the assessment of different types of exercise training. In the study of Selvadurai et al. (2002), 66 children with exacerbated CF significantly increased their FFM after five sessions of exercise training (aerobic or resistance training) or chest physiotherapy, independently of the protocol applied (Cohen's $d_{z}: 1.65$ to 5.22) [123]. Sosa et al. (2012) did not find improvements in the percentage of FFM of children with stable CF following an 8-week exercise training protocol (78.1 to 79.4\%; Cohen's $d_{z} 0.47$ ) [119], however, significant differences were reached when a component of inspiratory muscle training was added (81.6 to 82.6\%; Cohen's d $\mathrm{d}_{\mathrm{z}}$ 0.85) [124]. Gruber et al. (2014) compared the effect of a 6week interval exercise training vs. standard exercise training in 43 adults with CF [125]. Significant improvements in FFM were only observed in the standard exercise training group (41.7 to $43.3 \mathrm{~kg}$; Cohen's $d_{z}$ 0.29). These four studies, with its medium and large effect sizes, demonstrated that FFM is an adequate outcome measure to assess the effectiveness of respiratory physiotherapy or just exercise training protocols in patients with CF.

Despite the limited evidence available, FFM assessed with 2-C or 4-C models appears to be a valid, reliable and usable measure to assess respiratory physiotherapy interventions in $\mathrm{CF}$.

\subsubsection{Inspiratory muscle strength}

One of the functions of the muscles is to develop strength. In the specific case of the inspiratory muscles, strength is usually estimated as pressure [126]. Several techniques have been described to measure inspiratory muscle strength, however, the maximum static inspiratory pressure at the mouth (Pimax) is one of the most widely used [126].

The assessment of Pimax requires patients to make a maximum inspiratory (Mueller manoeuvre) effort at or near the residual volume, maintained for at least 1-1.5 seconds. These tests are volitional and require patient's full cooperation. For these reasons, this technique is usually performed by an experienced health professional to assure adequate instruction and encouragement. The manoeuver is repeated until the variation between measures is less than $20 \%$. Pimax is usually expressed in absolute values $\left(\mathrm{cmH}_{2} \mathrm{O}\right)$ or as a percentage of the predicted values.

Pimax is a simple, low-cost and well tolerated technique. In addition, through the hand-held and portable electronic pressure transducers, the technique is easily used by physiotherapists in a wide range of clinical settings (e.g., primary care, hospital wards, intensive care units). Another advantage of using this technique is the availability of reference values for healthy children, adults and the elderly, enabling the interpretation of results [127, 128].

The reliability of this measure is well established in people with non-CF bronchiectasis [129] and healthy people [130]. In patients with CF, only one study was found assessing the testretest reliability of Pimax [131]. Pimax had an excellent coefficient of reliability (89\%) and 
intraclass correlation coefficient $(0.88)$ in patients with $C F(n=20)$, in line with the results obtained in healthy individuals (coefficient of reliability $91 \%$; intraclass correlation coefficient 0.87) [131]. One of the disadvantages of this study is the fact that it was conducted only with adult patients with CF (22.7 \pm 3.4 years old). The reliability of Pimax in children with CF is still unexplored.

Due to its simplicity and adequate reliability, Pimax has been used as an outcome measure of inspiratory muscle training programmes. In the study by de Jong et al. (2001), seven patients with CF improved their Pimax from 105 to $123 \mathrm{cmH}_{2} \mathrm{O}$ after 6 weeks of inspiratory muscle training (Cohen's $d_{z}$ 0.82) [132]. Enright et al. (2004) investigated the effect of an 8-week inspiratory muscle training with a high $(n=9)$ and low $(n=10)$ training intensity. Large effects were found using the Pimax, with improvements from $114-134 \mathrm{cmH}_{2} \mathrm{O}$ to $155-159 \mathrm{cmH}_{2} \mathrm{O}$ (Cohen's $d_{z} 1.01$ and 1.34) [43]. Amelina et al. (2006), after a 6-week inspiratory muscle training, found that patients with CF $(n=10)$ had a mean improvement from $77 \%$ to $91 \%$ of the predicted Pimax ( $p=0.023$; Cohen's $d_{z}$ 0.73) [133]. These three studies, with its medium and large effect sizes, demonstrated that Pimax is an adequate outcome measure to assess the effectiveness of inspiratory muscle training.

Pimax has also been used as an outcome measure to determine the effectiveness of noninvasive ventilation during chest physiotherapy [39]. It was found that Pimax was maintained following ACBT assisted with non-invasive ventilation, resulting in a significant difference compared with ACBT alone (mean difference from standard treatment $9.04 \mathrm{cmH}_{2} \mathrm{O}, 95 \% \mathrm{CI} 4.25$ to $13.83, \mathrm{p}=0.006$ ) [39].

Despite the limited evidence available, it seems that physiotherapists can confidently rely on Pimax to assess the effectiveness of respiratory physiotherapy interventions in patients with CF.

\subsubsection{Inspiratory muscle endurance}

Inspiratory muscles, in addition to developing strength, have to be able to sustain muscular tasks over time - also known as endurance [126]. Inspiratory muscle endurance is a highly complex ability, which provides insight about the resistance to fatigue of inspiratory muscles and about the function of the inspiratory pump.

Dyspnoea, one of the primary complaints of patients with $\mathrm{CF}$, has been related to inspiratory muscle fatigue. In adults with CF $(n=18)$, inspiratory muscle endurance was found to be strongly correlated with exercise dyspnoea $(\mathrm{r}=-0.72)$ and explained $48 \%$ of the variability of this symptom [134]. In patients with advanced CF, the assessment and monitoring of inspiratory muscle endurance may have a greater importance since, in these patients, activities of daily living may be limited by their ability to sustain ventilation. Therefore, the measure of inspiratory muscle endurance seems to be useful to evaluate the determinants of dyspnoea and fatigue in patients with CF.

A number of distinct techniques have been employed to measure endurance of the inspiratory muscles [126]. In CF, two main techniques have been employed: (i) ventilatory endurance tasks $[43,112,131]$ and (ii) endurance to external loads $[132,134]$. 
In ventilatory endurance tasks, inspiratory muscle endurance has been measured through the sustained maximum inspiratory pressure (SMIP). This sustained pressure is determined using an electronic manometer, with a fixed leak via a $2 \mathrm{~mm}$ diameter during the inspiratory manoeuver, and a specific computer software. The leak sets a maximum flow during the inspiratory effort and allows continuous measurement of pressure over a full range of lung volumes, until no further pressure can be generated. During this technique, patients are asked to take a maximal and sustained inspiratory effort from residual volume to total lung capacity. SMIP is measured as the area under the pressure-time curve and is generally expressed in absolute values (pressure-time units) [43, 112, 131].

The coefficient of reliability for measurements of SMIP in CF was previously established as $90 \%$ [131]. Albinni et al. (2004) reported that inspiratory muscle endurance, measured by the SMIP, improved significantly in patients with CF $(n=16)$ after 12 weeks of inspiratory muscle training ( $p=0.0002$ ) [135]. Enright et al. (2004) investigated the effect of an 8-week inspiratory muscle training with a training intensity of either $80 \%$ of maximal inspiratory effort $(n=9)$ or $20 \%$ of maximal inspiratory effort $(n=10)$ [43]. The SMIP improved significantly in the two groups (from 654-782 to 808-923 pressure-time units; Cohen's $d_{z} 0.46$ and 0.51) [43].

In endurance to external loads, inspiratory muscle endurance has been determined with incremental loading tests using threshold devices. During these tests, patients have to generate sufficient inspiratory pressure to open the valve and allow inspiratory flow. The test starts with an inspiratory load of $20-30 \%$ of Pimax for $2 \mathrm{~min}$. The load is then increased every $2 \mathrm{~min}$ in increments of $10 \%$ of Pimax. The maximal load is defined by the maximal inspiratory pressure sustained for 1 or $2 \mathrm{~min}$ (Plim), which can be expressed in absolute values and as a percentage of the Pimax $[132,134]$.

The reliability of the Plim in CF has not yet been explored. In patients with COPD and in healthy people, however, it has been demonstrated that the reproducibility of the inspiratory pressure of the threshold was excellent, with small coefficients of variation in both groups $(<1 \%)[136]$. Future studies should assess the reliability and test-retest reliability of the Plim in the CF population.

Only one small study was found using Plim as an outcome measure in CF population. In this study, patients with CF ( $n=7)$ were submitted to a 6-week inspiratory muscle training. Plim, expressed as a percentage of the Pimax, increased significantly from $49 \%$ to $66 \%$ (Cohen's $\mathrm{d}_{z}$ 1.29) [132]. Using Plim, inspiratory muscle training was also found to have large effect sizes in patients with COPD ( $n=16$; Cohen's $d_{z}$ 0.83) [137] and with chronic heart failure $(n=16$; Cohen's $d_{z} 1.09$ ) [138]. Further research is needed to assess the sensitivity and responsiveness of Plim to inspiratory muscle training in patients with CF.

Both SMIP and Plim appear to be adequate outcome measures to assess the effectiveness of inspiratory muscle training in the inspiratory endurance of patients with CF. Nevertheless, this evidence emerges from few studies with small sample sizes. More research is needed to determine the responsiveness of these two outcome measures to inspiratory muscle training, as well as to other respiratory interventions, such as respiratory retraining. 


\subsubsection{Physical activity}

Given the clinical implications of regular physical activity in patients' pulmonary function [139], exercise tolerance [140] and airway clearance [141], its assessment and monitoring in patients with CF has also become a topic of great interest in research and clinical practice. An assessment of physical activity (and inactivity) in the free living environment can be performed using subjective and objective methods [142, 143], although no gold standard is still available.

Subjective methods rely on the individuals' self-report through physical activity questionnaires. These instruments are simple, inexpensive and easy to employ for routine assessment of patients' physical activity levels [142]. Examples of questionnaires used in CF include the Habitual Activity Estimation Scale [144] and the Seven Day Physical Activity Recall [145]. Although the Habitual Activity Estimation Scale has presented good test-retest reliability results (intraclass correlation coefficients of 0.72 ) in patients with CF [146], a previous systematic review concluded that these two instruments were not able to generate valid activity data or provide a valid assessment of aerobic fitness at the individual level [147]. Thus, the use of these questionnaires for individual assessment and counselling may provide imprecise data. For these purposes, objective measures are recommended.

Objective measures to assess daily physical activity include heart rate monitoring devices and motion sensors, such as pedometers, accelerometers and multisensor devices $[143,148]$. These measures have been used in studies involving patients with CF [149-151] and show promising results in evaluating the impact of respiratory physiotherapy interventions [56, 123]. Given the small size of the devices, low participant burden and relatively low cost, objective measures are appropriate for use in research and clinical practice [148]. Heart rate monitoring devices enable the assessment of patients' level of exertion since heart rate increases in a linear fashion with oxygen consumption, especially in moderate to strenuous intensity activities [152]. As such, these devices have been mostly used as a feedback tool to ensure patients' compliance with the intensity recommendations when exercising at home [56, 153]. Regarding motion sensors, there are a large number of options available that makes the choice of the best device challenging. Pedometers are designed to measure the number of steps taken by an individual by detecting vertical movement at the hip or waist. They may be desirable in simpler studies due to their lower cost and limited data [143]. Furthermore, since pedometers provide immediate feedback to the user [143], they may be valuable in self-monitoring interventions delivered to patients with CF. Despite their potential applications, no data is still available regarding the validity and reliability of pedometers in CF [154]. Still, pedometers have provided good reliability results in healthy and chronic respiratory diseases except when walking at slower speeds [155].

Although more expensive than pedometers, accelerometers provide a more detailed analysis of daily physical activity by capturing the frequency, duration and intensity of physical activity through the collection of body accelerations during movements [143]. They have also the advantage of storing the data for several weeks [143]. Multisensor devices combine accelerometry data with physiologic information collected from other sensors, such as heart rate and skin temperature, and also have a memory function. The use of accelerometers and multisensor devices in CF has increased dramatically in recent years [140,146, 156-158] and this will likely 
continue with the technology advances. The clinimetric properties of these devices in CF are described in a recent systematic review [154]. The authors found that only one accelerometer (ActiGraph model 7164) and one multisensor device (BodyMedia SenseWear armband) were tested. The ActiGraph presented good convergent validity [157, 159], test-retest reliability (intraclass correlation coefficient of 0.63 ) and feasibility [146, 157] in adolescent and adult patients with CF. Discriminate validity [160] and responsiveness [123] were only evaluated in children with CF. Selvadurai et al (2002), exploring the effects of exercise training in children with $\mathrm{CF}$ admitted to hospital $(\mathrm{n}=66)$, found a significant improvement in activity levels after five sessions of endurance training $\left(8.64 \%, p<0.001\right.$, Cohen's $\left.d_{z} 0.82\right)$ or resistance training $\left(3.81 \%, p<0.001\right.$, Cohen's $\left.d_{z} 0.85\right)$ measured by accelerometry [123]. Therefore, accelerometers may be a useful tool to assess changes in physical activity levels of patients with $\mathrm{CF}$ in response of respiratory physiotherapy interventions. Regarding the SenseWear armband device, its validity (discriminate, convergent and concurrent) [140,161] was only determined for adult patients with $\mathrm{CF}$ and no data on reliability and responsiveness exist. Further research is needed to determine which motion sensors provide the best clinimetric properties in $\mathrm{CF}$ in order to improve physiotherapy assessment. Moreover, as children have typically higher physical activity levels than adults [162], validation studies should be conducted in children and adults with CF. Finally, it would be useful to develop specific guidelines for the use (e.g., number of monitoring days, duration) of these motion sensors in $\mathrm{CF}$, in order to standardise the collection of activity data and optimise their interpretation.

\subsubsection{Burden of treatment}

The concept of burden of treatment has been receiving increasing attention in patients with CF. Burden of treatment is described as the increased demand experienced from performing self-care activities required to undertake treatment regimens and monitor health outcomes [163]. Recent evidence demonstrated, however, that from a patient's perspective, treatment burden is beyond the workload arising from treatment regimens, being experienced in three disruption domains: biological (physical side effects), biographical (sense of self) and relational (impact on valued relationships) [164].

A large observational cohort study explored treatment complexity in patients with $\mathrm{CF}(\mathrm{n}=7252)$ over a 3-year period [165]. It may be hypothesised that treatment regimens would be more complex only among patients with more severe disease. Indeed, in this cohort, the highest treatment complexity was presented by patients with more severe disease. Nevertheless, over the 3-year period, the complexity of treatment regimens increased in all age and disease severity groups. This study showed that the recommended management of CF resulted in high burden of treatment for patients.

In the specific case of respiratory physiotherapy, vigorous airway clearance and exercise regimens are recommended for patients with CF [166], which may result in increased burden of treatment. Burden of treatment, in turn, is associated with non-adherence and poor health outcomes [158, 163, 167-169]. This is particularly important for physiotherapists since the level of adherence to exercise and physiotherapy is generally reported to be poor (40-55\%) [170, $171]$, in contrast with moderate to high adherence to nebulised medications, pancreatic enzymes and antibiotics (65-95\%) [170, 172]. 
These levels of adherence suggest that patients with CF make decisions based on the complexity of recommended therapies they can complete, while fulfilling their responsibilities and commitments to family and work. As the number of adjunct therapies increase in $\mathrm{CF}$, there may be a point at which perceived treatment burden outweighs the benefits of new or adjunct therapies, adversely affecting patient's adherence. Physiotherapists therefore, need to be sensitive recognising, understanding and supporting the reduction of burden of treatment, in order to balance the potential benefits and burdens of physiotherapy interventions and maximise adherence $[158,173]$.

The recognition of this concept led to the development of specific instruments for assessing burden of treatment. Burden of treatment has been incorporated into the CFQ [89]. Specifically, the burden of treatment domain is comprised of three questions: 'to what extent do your treatments make your daily life more difficult?', 'how much time do you currently spend each day on your treatments?' and 'how difficult is it for you to do your treatments each day?'. The score ranges from 0 to 100, with lower scores representing higher burden of treatment.

The burden of treatment domain of the CFQ has been used in recent studies evaluating the effectiveness of novel interventions [91, 174]. In a trial with patients with CF ( $n=12)$, assessing the effect of a nebulised hypertonic saline therapy, a significant improvement was found in respiratory symptoms together with an increased in perceived burden of treatment (Cohen's $d_{z}$ 3.05) [174]. Schmidt et al. (2011) evaluated a 12-week individually tailored unsupervised aerobic exercise programme in patients with $\mathrm{CF}(\mathrm{n}=14)$ [91]. Patients were instructed to exercise at least 30 minutes, three times a week with a heart rate target above $70 \%$ of their maximum. This study showed that an exercise programme could significantly increase $\mathrm{VO}_{2}$ max and, at same time, significantly decrease the perceived burden of treatment (Cohen's $d_{z}$ 1.03) [91]. These two studies, despite being distinct, show that the burden of treatment domain of the CFQ is sensitive to change over time and demonstrate large effect sizes. Physiotherapists can, therefore, confidently rely on the burden of treatment domain of the CFQ to assess their interventions.

One of the disadvantages of the burden of treatment domain is the fact that it mainly addresses the complexity and time consuming routine of self-care. However, recent research has demonstrated that burden of treatment goes beyond these aspects and is experienced as biological, biographical and relational disruptions [164]. Therefore, when designing future instruments and methods for assessing burden of treatment, these three disruption domains should also be taken into account. This highlights the relevance of assessing burden of treatment as an outcome measure of respiratory physiotherapy in CF. The evaluation of burden of treatment will also inform the development of new and minimally disruptive interventions.

\section{Conclusions}

This chapter presented and discussed the strength and weaknesses of the outcome measures currently applied or emerging in CF respiratory physiotherapy interventions. It provided a comprehensive overview of the most commonly used, and also addressed the less used and 
some even emerging, outcome measures, which show potential to overcome some of the barriers to build an evidence base for respiratory physiotherapy practice in patients with $\mathrm{CF}$.

The chapter presented outcome measures possibly to be applied (i) in clinical practice before, during and after each session of respiratory physiotherapy to monitor its effectiveness; (ii) before and after the respiratory physiotherapy treatment (i.e., normally characterised by weeks of intervention) and (iii) clinically, but which main interest is fundamental and/or applied research in $\mathrm{CF}$ respiratory physiotherapy.

In a time where the relationship between "best care" versus "burden of treatment" is discussed, two factors seem crucial for respiratory physiotherapy: (1) to take into consideration family and patient's preferences when providing treatments and (2) build a sound evidence base. For the latter, a shift of the commonly used outcome measures, namely $\mathrm{FEV}_{1}$, is essential, and a strong consideration to start applying new outcome measures is recommended.

\section{Author details}

Alda Marques ${ }^{1,2^{*}}$, Joana Cruz $^{1,2}$, Cristina Jácome ${ }^{1,2}$ and Ana Oliveira ${ }^{1,2}$

*Address all correspondence to: amarques@ua.pt

1 Lab 3R - Respiratory Rehabilitation Research Laboratory, School of Health Sciences, University of Aveiro (ESSUA), Aveiro, Portugal

2 Center for Health Technology and Services Research (CINTESIS), School of Health Sciences, University of Aveiro, Aveiro, Portugal

\section{References}

[1] McIlwaine MP, Lee Son NM, Richmond ML. Physiotherapy and cystic fibrosis: what is the evidence base? Curr Opin Pulm Med. 2014;20(6):613-7.

[2] Holland AE, Button BM. Physiotherapy for cystic fibrosis in Australia: knowledge and acceptance of the Consensus Statement recommendations. Respirology. 2013;18(4):652-6.

[3] Bradley JM, Moran FM, Stuart Elborn J. Evidence for physical therapies (airway clearance and physical training) in cystic fibrosis: an overview of five Cochrane systematic reviews. Respir Med. 2006;100(2):191-201.

[4] Bott J, Blumenthal S, Buxton M, Ellum S, Falconer C, Garrod R, et al. Guidelines for the physiotherapy management of the adult, medical, spontaneously breathing patient. Thorax. 2009;64(Suppl 1):i1-i52. 
[5] Main E. Airway clearance research in CF: the 'perfect storm' of strong preference and effortful participation in long-term, non-blinded studies. Thorax. 2013;68(8):701-2.

[6] Cox NS, Alison JA, Holland AE. Interventions for promoting physical activity in people with cystic fibrosis. Cochrane Database Syst Rev. 2013;12:CD009448.

[7] Warnock L, Gates A, van der Schans CP. Chest physiotherapy compared to no chest physiotherapy for cystic fibrosis. Cochrane Database Syst Rev. 2013;9:CD001401.

[8] Miller MR, Hankinson J, Brusasco V, Burgos F, Casaburi R, Coates A, et al. Standardisation of spirometry. Eur Respir J. 2005;26(2):319-38.

[9] Pfleger A, Theissl B, Oberwaldner B, Zach MS. Self-administered chest physiotherapy in cystic fibrosis: a comparative study of high-pressure PEP and autogenic drainage. Lung. 1992;170(6):323-30.

[10] Jarad NA, Powell T, Smith E. Evaluation of a novel sputum clearance technique - hydro-acoustic therapy (HAT) in adult patients with cystic fibrosis: a feasibility study. Chron Respir Dis. 2010;7(4):217-27.

[11] Main E, Prasad A, Schans C. Conventional chest physiotherapy compared to other airway clearance techniques for cystic fibrosis. Cochrane Database Syst Rev. 2005(1):Cd002011.

[12] McKoy NA, Saldanha IJ, Odelola OA, Robinson KA. Active cycle of breathing technique for cystic fibrosis. Cochrane Database Syst Rev. 2012;12:Cd007862.

[13] Morrison L, Agnew J. Oscillating devices for airway clearance in people with cystic fibrosis. Cochrane Database Syst Rev. 2009(1):Cd006842.

[14] Xavier GN, Duarte ACM, Melo-Silva CA, dos Santos CEVG, Amado VM. Accuracy of pulmonary auscultation to detect abnormal respiratory mechanics: A cross-sectional diagnostic study. Med Hypotheses. 2014;83(6):733-4.

[15] Abbas A, Fahim A. An automated computerized auscultation and diagnostic system for pulmonary diseases. J Med Syst. 2010;34(6):1149-55.

[16] Bohadana A, Izbicki G, Kraman SS. Fundamentals of lung auscultation. N Engl J Med. 2014;370(8):744-51.

[17] Sovijärvi ARA, Vanderschoot J, Earis JE. Standardization of computerized respiratory sound analysis. Eur Respir Rev. 2000;10(77):974-87.

[18] Marques A, Bruton A, Barney A. Clinically useful outcome measures for physiotherapy airway clearance techniques: a review. Physi Ther Rev. 2006;11(4):299-307.

[19] Brooks D, Wilson L, Kelsey C. Accuracy and reliability of 'specialized' physical therapists in auscultating tape-recorded lung sounds. Physiother Can. 1993;45(1):21-4. 
[20] Allingame S, Williams T, Jenkins S, Tucker B. Accuracy and reliability of physiotherapists in the interpretation of tape-recorded lung sounds. Aust J Physiother. 1995;41(3):179-84.

[21] Brooks D, Thomas J. Interrater reliability of auscultation of breath sounds among physical therapists. Phys Ther. 1995;75(12):1082-8.

[22] Marques A, Oliveira A, Jácome C, Dinis J, Pinho C. 236 Agreement between real-time auscultation and computerised respiratory analyses. J Cyst Fibros.12:S108.

[23] Elphick HE, Lancaster GA, Solis A, Majumdar A, Gupta R, Smyth RL. Validity and reliability of acoustic analysis of respiratory sounds in infants. Arch Dis Child. 2004;89(11):1059-63.

[24] Morrow B, Angus L, Greenhough D, Hansen A, McGregor G, Olivier O, et al. The reliability of identifying bronchial breathing by auscultation. International Journal of Therapy and Rehabilitation. 2010;17(2):69-75.

[25] Puder LC, Fischer HS, Wilitzki S, Usemann J, Godfrey S, Schmalisch G. Validation of computerized wheeze detection in young infants during the first months of life. BMC Pediatr. 2014;14:257.

[26] Tourtier JP, Libert N, Clapson P, Tazarourte K, Borne M, Grasser L, et al. Auscultation in flight: comparison of conventional and electronic stethoscopes. Air Med J. 2011;30(3):158-60.

[27] Hoffmann C, Falzone E, Verret C, Pasquier P, Leclerc T, Donat N, et al. Brief report: pulmonary auscultation in the operating room: a prospective randomized blinded trial comparing electronic and conventional stethoscopes. Anesth Analg. 2013;117(3): 646-8.

[28] Zemanick ET, Harris JK, Conway S, Konstan MW, Marshall B, Quittner AL, et al. Measuring and improving respiratory outcomes in cystic fibrosis lung disease: opportunities and challenges to therapy. J Cyst Fibros. 2010;9(1):1-16.

[29] Falk M, Kelstrup M, Andersen JB, Kinoshita T, Falk P, Stovring S, et al. Improving the ketchup bottle method with positive expiratory pressure, PEP, in cystic fibrosis. Eur J Respir Dis. 1984;65(6):423-32.

[30] Mortensen J, Falk M, Groth S, Jensen C. The effects of postural drainage and positive expiratory pressure physiotherapy on tracheobronchial clearance in cystic fibrosis. Chest. 1991;100(5):1350-7.

[31] Rossman CM, Waldes R, Sampson D, Newhouse MT. Effect of chest physiotherapy on the removal of mucus in patients with cystic fibrosis. Am Rev Respir Dis. 1982;126(1):131-5. 
[32] Osman LP, Roughton M, Hodson ME, Pryor JA. Short-term comparative study of high frequency chest wall oscillation and European airway clearance techniques in patients with cystic fibrosis. Thorax. 2010;65(3):196-200.

[33] Braggion C, Cappelletti LM, Cornacchia M, Zanolla L, Mastella G. Short-term effects of three chest physiotherapy regimens in patients hospitalized for pulmonary exacerbations of cystic fibrosis: a cross-over randomized study. Pediatr Pulmonol. 1995;19(1):16-22.

[34] Kluft J, Beker L, Castagnino M, Gaiser J, Chaney H, Fink RJ. A comparison of bronchial drainage treatments in cystic fibrosis. Pediatr Pulmonol. 1996;22(4):271-4.

[35] Oermann CM, Sockrider MM, Giles D, Sontag MK, Accurso FJ, Castile RG. Comparison of high-frequency chest wall oscillation and oscillating positive expiratory pressure in the home management of cystic fibrosis: a pilot study. Pediatr Pulmonol. 2001;32(5):372-7.

[36] Pryor JA, Tannenbaum E, Scott SF, Burgess J, Cramer D, Gyi K, et al. Beyond postural drainage and percussion: Airway clearance in people with cystic fibrosis. J Cyst Fibros. 2010;9(3):187-92.

[37] Elphick HE, Mallory G. Oxygen therapy for cystic fibrosis. Cochrane Database Syst Rev. 2013;7:CD003884.

[38] Chan ED, Chan MM, Chan MM. Pulse oximetry: understanding its basic principles facilitates appreciation of its limitations. Respir Med. 2013;107(6):789-99.

[39] Holland AE, Denehy L, Ntoumenopoulos G, Naughton MT, Wilson JW. Non-invasive ventilation assists chest physiotherapy in adults with acute exacerbations of cystic fibrosis. Thorax. 2003;58(10):880-4.

[40] Parshall MB, Schwartzstein RM, Adams L, Banzett RB, Manning HL, Bourbeau J, et al. An official American Thoracic Society statement: update on the mechanisms, assessment, and management of dyspnea. Am J Respir Crit Care Med. 2012;185(4):43552.

[41] Stenekes SJ, Hughes A, Gregoire MC, Frager G, Robinson WM, McGrath PJ. Frequency and self-management of pain, dyspnea, and cough in cystic fibrosis. J Pain Symptom Manage. 2009;38(6):837-48.

[42] Marques A. The use of computer aided lung sound analysis to characterise adventitious lung sounds: a potential outcome measure for respiratory therapy. Southampton: University of Southampton; 2008.

[43] Enright S, Chatham K, Ionescu AA, Unnithan VB, Shale DJ. Inspiratory muscle training improves lung function and exercise capacity in adults with cystic fibrosis. Chest. 2004;126(2):405-11. 
[44] Bausewein C, Booth S, Higginson IJ. Measurement of dyspnoea in the clinical rather than the research setting. Curr Opin Support Palliat Care. 2008;2(2):95-9.

[45] Neuman MI, Lee EY, Bixby S, Diperna S, Hellinger J, Markowitz R, et al. Variability in the interpretation of chest radiographs for the diagnosis of pneumonia in children. J Hosp Med. 2012;7(4):294-8.

[46] Mcllwaine PM, Wong LT, Peacock D, Davidson AG. Long-term comparative trial of conventional postural drainage and percussion versus positive expiratory pressure physiotherapy in the treatment of cystic fibrosis. J Pediatr. 1997;131(4):570-4.

[47] Mcllwaine PM, Wong LT, Peacock D, Davidson AG. Long-term comparative trial of positive expiratory pressure versus oscillating positive expiratory pressure (flutter) physiotherapy in the treatment of cystic fibrosis. J Pediatr. 2001;138(6):845-50.

[48] Clinical exercise testing with reference to lung diseases: indications, standardization and interpretation strategies. ERS Task Force on Standardization of Clinical Exercise Testing. European Respiratory Society. Eur Respir J. 1997;10(11):2662-89.

[49] ATS/ACCP Statement on cardiopulmonary exercise testing. Am J Respir Crit Care Med. 2003;167(2):211-77.

[50] Orenstein DM. Exercise testing in cystic fibrosis. Pediatr Pulmonol. 1998;25(4):223-5.

[51] Rogers D, Prasad SA, Doull I. Exercise testing in children with cystic fibrosis. J R Soc Med. 2003;96(Suppl 43):23-9.

[52] Palange P, Ward SA, Carlsen K-H, Casaburi R, Gallagher CG, Gosselink R, et al. Recommendations on the use of exercise testing in clinical practice. Eur Respir J. 2007;29(1):185-209.

[53] Godfrey S, Mearns M. Pulmonary function and response to exercise in cystic fibrosis. Arch Dis Child. 1971;46(246):144-51.

[54] McKone EF, Barry SC, FitzGerald MX, Gallagher CG. Reproducibility of maximal exercise ergometer testing in patients with cystic fibrosis. Chest. 1999;116(2):363-8.

[55] Saynor ZL, Barker AR, Oades PJ, Williams CA. Reproducibility of maximal cardiopulmonary exercise testing for young cystic fibrosis patients. J Cyst Fibros. 2013;12(6):644-50.

[56] Hebestreit H, Kieser S, Junge S, Ballmann M, Hebestreit A, Schindler C, et al. Longterm effects of a partially supervised conditioning programme in cystic fibrosis. Eur Respir J. 2010;35(3):578-83.

[57] Stevens D, Oades PJ, Armstrong N, Williams CA. A survey of exercise testing and training in UK cystic fibrosis clinics. J Cyst Fibros. 2010;9(5):302-6.

[58] Urquhart D. Exercise testing in cystic fibrosis: why (and how)? J R Soc Med. 2011;104(suppl 1):S6-S14. 
[59] Solway S, Brooks D, Lacasse Y, Thomas S. A qualitative systematic overview of the measurement properties of functional walk tests used in the cardiorespiratory domain. Chest. 2001;119(1):256-70.

[60] ATS statement: guidelines for the six-minute walk test. Am J Respir Crit Care Med. 2002;166(1):111-7.

[61] Gulmans VAM, van Veldhoven NHMJ, de Meer K, Helders PJM. The six-minute walking test in children with cystic fibrosis: reliability and validity. Pediatr Pulmonol. 1996;22(2):85-9.

[62] Cunha MT, Rozov T, de Oliveira RC, Jardim JR. Six-minute walk test in children and adolescents with cystic fibrosis. Pediatr Pulmonol. 2006;41(7):618-22.

[63] Gruet M, Brisswalter J, Mely L, Vallier J-M. Use of the peak heart rate reached during six-minute walk test to predict individualized training intensity in patients with cystic fibrosis: validity and reliability. Arch Phys Med Rehabil. 2010;91(4):602-7.

[64] Lesser DJ, Fleming MM, Maher CA, Kim SB, Woo MS, Keens TG. Does the 6-min walk test correlate with the exercise stress test in children? Pediatr Pulmonol. 2010;45(2):135-40.

[65] Ziegler B, Rovedder PM, Lukrafka JL, Oliveira CL, Menna-Barreto SS, Dalcin Pde T. Submaximal exercise capacity in adolescent and adult patients with cystic fibrosis. J Bras Pneumol. 2007;33(3):263-9.

[66] Priesnitz CV, Rodrigues GH, da Silva Stumpf C, Viapiana G, Cabral CP, Stein RT, et al. Reference values for the 6-min walk test in healthy children aged 6-12 years. Pediatr Pulmonol. 2009;44(12):1174-9.

[67] Klepper SE, Muir N. Reference values on the 6-minute walk test for children living in the United States. Pediatr Phys Ther. 2011;23(1):32-40.

[68] Kanburoglu MK, Ozdemir FM, Ozkan S, Tunaoglu FS. Reference values of the 6-minute walk test in healthy turkish children and adolescents between 11 and 18 years of age. Respir Care. 2014;59(9):1369-75.

[69] Ulrich S, Hildenbrand F, Treder U, Fischler M, Keusch S, Speich R, et al. Reference values for the 6-minute walk test in healthy children and adolescents in Switzerland. BMC Pulm Med. 2013;13(1):49.

[70] Dourado VZ. Equações de referência para o teste de caminhada de seis minutos em indivíduos saudáveis. Arq Bras Cardiol. 2011;96:e128-e38.

[71] Balfour-Lynn IM, Prasad SA, Laverty A, Whitehead BF, Dinwiddie R. A step in the right direction: assessing exercise tolerance in cystic fibrosis. Pediatr Pulmonol. 1998;25(4):278-84. 
[72] Master AM, Oppenheimer ET. A simple exercise tolerance test for circulatory efficiency with standard tables for normal individuals. Am J Med Sci. 1929;177(2):22343.

[73] Pike SE, Prasad SA, Balfour-Lynn IM. Effect of intravenous antibiotics on exercise tolerance (3-min step test) in cystic fibrosis*. Pediatr Pulmonol. 2001;32(1):38-43.

[74] Aurora P, Prasad SA, Balfour-Lynn IM, Slade G, Whitehead B, Dinwiddie R. Exercise tolerance in children with cystic fibrosis undergoing lung transplantation assessment. Eur Respir J. 2001;18(2):293-7.

[75] Narang I, Pike S, Rosenthal M, Balfour-Lynn IM, Bush A. Three-minute step test to assess exercise capacity in children with cystic fibrosis with mild lung disease. Pediatr Pulmonol. 2003;35(2):108-13.

[76] Bradley J, Howard J, Wallace E, Elborn S. Validity of a modified shuttle test in adult cystic fibrosis. Thorax. 1999;54(5):437-9.

[77] Singh SJ, Morgan MD, Scott S, Walters D, Hardman AE. Development of a shuttle walking test of disability in patients with chronic airways obstruction. Thorax. 1992;47(12):1019-24.

[78] Bradley J, Howard J, Wallace E, Elborn S. Reliability, repeatability, and sensitivity of the modified shuttle test in adult cystic fibrosis. Chest. 2000;117(6):1666-71.

[79] Rogers D, Smith P, John N, Oliver W, IJM D. Validity of a modified shuttle walk test as a measure of exercise tolerance in paediatric CF patients. J Cys Fibros. 2002;1(Suppl 11):139.

[80] Paranjape SM, Barnes LA, Carson KA, von Berg K, Loosen H, Mogayzel Jr PJ. Exercise improves lung function and habitual activity in children with cystic fibrosis. J Cyst Fibros. 2012;11(1):18-23.

[81] Elkins MR, Dentice RL, Bye PT. Validation of the MST-25: an extension of the modified shuttle test (MST). J Cyst Fibros. 2009;8, Supplement 2(0):S70.

[82] Royce FH, Carl JC. Health-related quality of life in cystic fibrosis. Curr Opin Pediatr. 2011;23(5):535-40.

[83] Britto MT, Kotagal UR, Hornung RW, Atherton HD, Tsevat J, Wilmott RW. Impact of recent pulmonary exacerbations on quality of life in patients with cystic fibrosis. Chest. 2002;121(1):64-72.

[84] Congleton J, Hodson ME, Duncan-Skingle F. Quality of life in adults with cystic fibrosis. Thorax. 1996;51(9):936-40.

[85] Thomas C, Mitchell P, O'Rourke P, Wainwright C. Quality-of-life in children and adolescents with cystic fibrosis managed in both regional outreach and cystic fibrosis center settings in Queensland. J Pediatr. 2006;148(4):508-16. 
[86] Gee L, Abbott J, Conway SP, Etherington C, Webb AK. Quality of life in cystic fibrosis: the impact of gender, general health perceptions and disease severity. J Cyst Fibros. 2003;2(4):206-13.

[87] Abbott J, Hart A, Morton A, Gee L, Conway S. Health-related quality of life in adults with cystic fibrosis: the role of coping. J Psychosom Res. 2008;64(2):149-57.

[88] Modi AC, Quittner AL. Validation of a disease-specific measure of health-related quality of life for children with cystic fibrosis. J Pediatr Psychol. 2003;28(8):535-46.

[89] Quittner AL, Buu A, Messer MA, Modi AC, Watrous M. Development and validation of the cystic fibrosis questionnaire in the United States: A health-related quality-oflife measure for cystic fibrosis. Chest. 2005;128(4):2347-54.

[90] Henry B, Aussage P, Grosskopf C, Goehrs JM. Development of the Cystic Fibrosis Questionnaire (CFQ) for assessing quality of life in pediatric and adult patients. Qual Life Res. 2003;12(1):63-76.

[91] Schmidt AM, Jacobsen U, Bregnballe V, Olesen HV, Ingemann-Hansen T, Thastum $\mathrm{M}$, et al. Exercise and quality of life in patients with cystic fibrosis: a 12-week intervention study. Physiother Theory Pract. 2011;27(8):548-56.

[92] Gee L, Abbott J, Conway SP, Etherington C, Webb AK. Development of a disease specific health related quality of life measure for adults and adolescents with cystic fibrosis. Thorax. 2000;55(11):946-54.

[93] Marques AS, Oliveira AL, Jacome CI. Computerized adventitious respiratory sounds as outcome measures for respiratory therapy: a systematic review. Respir Care. 2014;59(5):765-76.

[94] Rossi M, Sovijarvi ARA, Piirila P, Vannuccini L, Dalmasso FVJ. Environmental and subject conditions and breathing manoeuvres for respiratory sound recordings. Eur Respir Rev. 2000;10(77):611-5.

[95] Oliveira A, Marques A. Respiratory sounds in healthy people: a systematic review. Respir Med. 2014;108(4):550-70.

[96] Gurung A, Scrafford CG, Tielsch JM, Levine OS, Check W. Computerized lung sound analysis as diagnostic aid for the detection of abnormal lung sounds: a systematic review and meta-analysis. Respir Med. 2011;105(9):1396-403.

[97] Reichert S, Gass R, Brandt C, Andres E. Analysis of respiratory sounds: state of the art. Clinical medicine Circulatory, respiratory and pulmonary medicine. 2008;2:4558.

[98] Oliveira D, Pinho C, Marques A, Dinis J. Validation of a time-frequency wheeze detector in cystic fibrosis: a pilot study. Eur Respir J. 2011;38(55):237s. 
[99] Marques A, Bruton A, Barney A. The reliability of lung crackle characteristics in cystic fibrosis and bronchiectasis patients in a clinical setting. Physiol Meas. 2009;30(9): 903-12.

[100] Prodhan P, Dela Rosa RS, Shubina M, Haver KE, Matthews BD, Buck S, et al. Wheeze detection in the pediatric intensive care unit: comparison among physician, nurses, respiratory therapists, and a computerized respiratory sound monitor. Respir Care. 2008;53(10):1304-9.

[101] Sanchez I, Powell RE, Pasterkamp H. Wheezing and airflow obstruction during methacholine challenge in children with cystic fibrosis and in normal children. Am Rev Respir Dis. 1993;147(3):705-9.

[102] van Ewijk BE, van der Zalm MM, Wolfs TFW, van der Ent CK. Viral respiratory infections in cystic fibrosis. J Cyst Fibros. 2005;4, Supplement 2(0):31-6.

[103] Pinho C, Oliveira A, Oliveira D, Dinis J, Marques A. Lungsounds@UA Interface and Multimedia Database. Int J E-Health Med Commun. 2014;5(1):81-95.

[104] Acosta CM, Maidana GA, Jacovitti D, Belaunzaran A, Cereceda S, Rae E, et al. Accuracy of transthoracic lung ultrasound for diagnosing anesthesia-induced atelectasis in children. Anesthesiology. 2014;120(6):1370-9.

[105] Volpicelli G, Elbarbary M, Blaivas M, Lichtenstein DA, Mathis G, Kirkpatrick AW, et al. International evidence-based recommendations for point-of-care lung ultrasound. Intensive Care Med. 2012;38(4):577-91.

[106] Xirouchaki N, Kondili E, Prinianakis G, Malliotakis P, Georgopoulos D. Impact of lung ultrasound on clinical decision making in critically ill patients. Intensive Care Med. 2014;40(1):57-65.

[107] Reissig A, Copetti R, Mathis G, Mempel C, Schuler A, Zechner P, et al. Lung ultrasound in the diagnosis and follow-up of community-acquired pneumonia: a prospective, multicenter, diagnostic accuracy study. Chest. 2012;142(4):965-72.

[108] Gardelli G, Feletti F, Nanni A, Mughetti M, Piraccini A, Zompatori M. Chest ultrasonography in the ICU. Respir Care. 2012;57(5):773-81.

[109] Slater A, Goodwin M, Anderson KE, Gleeson FV. COPD can mimic the appearance of pneumothorax on thoracic ultrasound. Chest. 2006;129(3):545-50.

[110] Xirouchaki N, Magkanas E, Vaporidi K, Kondili E, Plataki M, Patrianakos A, et al. Lung ultrasound in critically ill patients: comparison with bedside chest radiography. Intensive Care Med. 2011;37(9):1488-93.

[111] Ng MY, Flight W, Smith E. Pulmonary complications of cystic fibrosis. Clin Radiol. 2014;69(3):e153-e62. 
[112] Enright S, Chatham K, Ionescu AA, Unnithan VB, Shale DJ. The influence of body composition on respiratory muscle, lung function and diaphragm thickness in adults with cystic fibrosis. J Cyst Fibros. 2007;6(6):384-90.

[113] Matel JL, Milla CE. Nutrition in cystic fibrosis. Semin Respir Crit Care Med. 2009;30(5):579-86.

[114] Klijn PH, van der Net J, Kimpen JL, Helders PJ, van der Ent CK. Longitudinal determinants of peak aerobic performance in children with cystic fibrosis. Chest. 2003;124(6):2215-9.

[115] Engelen MP, Schroder R, Van der Hoorn K, Deutz NE, Com G. Use of body mass index percentile to identify fat-free mass depletion in children with cystic fibrosis. Clin Nutr. 2012;31(6):927-33.

[116] Bolton CE, Ionescu AA, Evans WD, Pettit RJ, Shale DJ. Altered tissue distribution in adults with cystic fibrosis. Thorax. 2003;58(10):885-9.

[117] Wells JC, Fewtrell MS. Measuring body composition. Arch Dis Child. 2006;91(7):6127.

[118] Moon JR, Stout JR, Smith-Ryan AE, Kendall KL, Fukuda DH, Cramer JT, et al. Tracking fat-free mass changes in elderly men and women using single-frequency bioimpedance and dual-energy X-ray absorptiometry: a four-compartment model comparison. Eur J Clin Nutr. 2013;67 Suppl 1:S40-6.

[119] Santana Sosa E, Groeneveld IF, Gonzalez-Saiz L, Lopez-Mojares LM, Villa-Asensi JR, Barrio Gonzalez MI, et al. Intrahospital weight and aerobic training in children with cystic fibrosis: a randomized controlled trial. Med Sci Sports Exerc. 2012;44(1):2-11.

[120] Mijnarends DM, Meijers JM, Halfens RJ, ter Borg S, Luiking YC, Verlaan S, et al. Validity and reliability of tools to measure muscle mass, strength, and physical performance in community-dwelling older people: a systematic review. Journal of the American Medical Directors Association. 2013;14(3):170-8.

[121] Atherton RR, Williams JE, Wells JC, Fewtrell MS. Use of fat mass and fat free mass standard deviation scores obtained using simple measurement methods in healthy children and patients: comparison with the reference 4-component model. PLoS ONE. 2013;8(5):e62139.

[122] de Meer K, Gulmans VA, Westerterp KR, Houwen RH, Berger R. Skinfold measurements in children with cystic fibrosis: monitoring fat-free mass and exercise effects. Eur J Pediatr. 1999;158(10):800-6.

[123] Selvadurai HC, Blimkie CJ, Meyers N, Mellis CM, Cooper PJ, Van Asperen PP. Randomized controlled study of in-hospital exercise training programs in children with cystic fibrosis. Pediatr Pulmonol. 2002;33(3):194-200.

[124] Santana-Sosa E, Gonzalez-Saiz L, Groeneveld IF, Villa-Asensi JR, Barrio Gómez de Aguero MI, Fleck SJ, et al. Benefits of combining inspiratory muscle with 'whole 
muscle' training in children with cystic fibrosis: a randomised controlled trial. $\mathrm{Br} \mathrm{J}$ Sports Med. 2013. Epub: 2013/05/16.

[125] Gruber W, Orenstein DM, Braumann KM, Beneke R. Interval exercise training in cystic fibrosis - effects on exercise capacity in severely affected adults. J Cyst Fibros. 2014;13(1):86-91.

[126] ATS/ERS Statement on Respiratory Muscle Testing. Am J Respir Crit Care Med. 2002;166(4):518-624.

[127] Enright PL, Kronmal RA, Manolio TA, Schenker MB, Hyatt RE. Respiratory muscle strength in the elderly. correlates and reference values. Cardiovascular Health Study Research Group. Am J Respir Crit Care Med. 1994;149(2 Pt 1):430-8.

[128] Wilson SH, Cooke NT, Edwards RH, Spiro SG. Predicted normal values for maximal respiratory pressures in caucasian adults and children. Thorax. 1984;39(7):535-8.

[129] Moran F, Piper A, Elborn JS, Bradley JM. Respiratory muscle pressures in non-CF bronchiectasis: repeatability and reliability. Chron Respir Dis. 2010;7(3):165-71.

[130] McConnell AK, Copestake AJ. Maximum static respiratory pressures in healthy elderly men and women: issues of reproducibility and interpretation. Respiration. 1999;66(3):251-8.

[131] Enright S, Unnithan VB, Davies D. Reproducibility of measurements of inspiratory work capacity in cystic fibrosis patients. Respir Physiol Neurobiol. 2006;150(1):35-43.

[132] de Jong W, van Aalderen WM, Kraan J, Koeter GH, van der Schans CP. Inspiratory muscle training in patients with cystic fibrosis. Respir Med. 2001;95(1):31-6.

[133] Amelina E, Cherniak A, Chikina S, Krasovsky S, Appaeva A. Inspiratory muscle training (IMT) in cystic fibrosis adults. Eur Respir J. 2006:716s.

[134] Leroy S, Perez T, Neviere R, Aguilaniu B, Wallaert B. Determinants of dyspnea and alveolar hypoventilation during exercise in cystic fibrosis: impact of inspiratory muscle endurance. J Cyst Fibros. 2011;10(3):159-65.

[135] Albinni S, Rath R, Renner S, Eichler I. Additional inspiratory muscle training intensifies the beneficial effects of cycle ergonometer training in patients with cystic fibrosis. J Cyst Fibros. 2004;Suppl1:S63.

[136] Gosselink R, Wagenaar RC, Decramer M. Reliability of a commercially available threshold loading device in healthy subjects and in patients with chronic obstructive pulmonary disease. Thorax. 1996;51(6):601-5.

[137] Hill K, Jenkins SC, Philippe DL, Cecins N, Shepherd KL, Green DJ, et al. High-intensity inspiratory muscle training in COPD. Eur Respir J. 2006;27(6):1119-28. 
[138] Dall'Ago P, Chiappa GR, Guths H, Stein R, Ribeiro JP. Inspiratory muscle training in patients with heart failure and inspiratory muscle weakness: a randomized trial. J Am Coll Cardiol. 2006;47(4):757-63.

[139] Schneiderman JE, Wilkes DL, Atenafu EG, Nguyen T, Wells GD, Alarie N, et al. Longitudinal relationship between physical activity and lung health in patients with cystic fibrosis. Eur Respir J. 2014;43(3):817-23.

[140] Troosters T, Langer D, Vrijsen B, Segers J, Wouters K, Janssens W, et al. Skeletal muscle weakness, exercise tolerance and physical activity in adults with cystic fibrosis. Eur Respir J. 2009;33(1):99-106.

[141] Baldwin DR, Hill AL, Peckham DG, Knox AJ. Effect of addition of exercise to chest physiotherapy on sputum expectoration and lung function in adults with cystic fibrosis. Respir Med. 1994;88(1):49-53.

[142] Andre D, Wolf DL. Recent advances in free-living physical activity monitoring: a review. J Diabetes Sci Tech (Online). 2007;1(5):760-7.

[143] Strath SJ, Kaminsky LA, Ainsworth BE, Ekelund U, Freedson PS, Gary RA, et al. Guide to the Assessment of Physical Activity: Clinical and Research Applications: A Scientific Statement from the American Heart Association. Circulation. 2013. Epub 2013/10/14.

[144] Hay J, Cairney J. Development of the habitual activity estimation scale for clinical research: A systematic approach. Pediatric Exercise Science. 2006;18(2):193-202.

[145] Sallis JF, Haskell WL, Wood PD, Fortmann SP, Rogers T, Blair SN, et al. Physical activity assessment methodology in the Five-City Project. Am J Epidemiol. 1985;121(1): 91-106.

[146] Wells GD, Wilkes DL, Schneiderman-Walker J, Elmi M, Tullis E, Lands LC, et al. Reliability and validity of the habitual activity estimation scale (HAES) in patients with cystic fibrosis. Pediatr Pulmonol. 2008;43(4):345-53.

[147] Hulzebos E, Dadema T, Takken T. Measurement of physical activity in patients with cystic fibrosis: a systematic review. Expert Rev Respir Med. 2013;7(6):647-53.

[148] Trost SG, O'Neil M. Clinical use of objective measures of physical activity. Br J Sports Med. 2013. Epub 2013/12/05

[149] Aznar S, Gallardo C, Fiuza-Luces C, Santana-Sosa E, López-Mojares LM, Santalla A, et al. Levels of moderate-vigorous physical activity are low in Spanish children with cystic fibrosis: A comparison with healthy controls. J Cyst Fibros. 2014;13(3):335-40.

[150] Quon BS, Patrick DL, Edwards TC, Aitken ML, Gibson RL, Genatossio A, et al. Feasibility of using pedometers to measure daily step counts in cystic fibrosis and an assessment of its responsiveness to changes in health state. J Cyst Fibros. 2012;11(3): 216-22. 
[151] Ward N, White D, Rowe H, Stiller K, Sullivan T. Physical activity levels of patients with cystic fibrosis hospitalised with an acute respiratory exacerbation. Respir Med. 2013;107(7):1014-20.

[152] American College of Sports Medicine. ACSM's resource manual for guidelines for exercise testing and prescription. 9th ed. Philadelphia: Lippincott Williams \& Wilkins; 2014.

[153] Johnson MR, Ferkol TW, Shepherd RW. Energy cost of activity and exercise in children and adolescents with cystic fibrosis. J Cyst Fibros. 2006;5(1):53-8.

[154] Bradley JM, Kent L, Elborn JS, O'Neill B. Motion sensors for monitoring physical activity in cystic fibrosis: what is the next step? Physical Therapy Reviews. 2010;15(3): 197-203.

[155] Turner LJ, Houchen L, Williams J, Singh SJ. Reliability of pedometers to measure step counts in patients with chronic respiratory disease. J Cardiopulm Rehabil Prev. 2012;32(5):284-91.

[156] Dias A, Gorzelniak L, Jörres RA, Fischer R, Hartvigsen G, Horsch A. Assessing physical activity in the daily life of cystic fibrosis patients. Perv Mobile Comput. 2012;8(6): 837-44.

[157] Hebestreit H, Kieser S, Rüdiger S, Schenk T, Junge S, Hebestreit A, et al. Physical activity is independently related to aerobic capacity in cystic fibrosis. Eur Respir J. 2006;28(4):734-9.

[158] Savi D, Quattrucci S, Internullo M, De Biase RV, Calverley PMA, Palange P. Measuring habitual physical activity in adults with cystic fibrosis. Respir Med. 2013;107(12): 1888-94.

[159] Janz KF, Cassady SL, Barr RN, Kelly JM. Monitoring exercise in children and adolescents with cystic fibrosis: validation of the CSA accelerometer. Cardiopulm Phys Ther J. 1995;6(2):3-8.

[160] Selvadurai HC, Blimkie CJ, Cooper PJ, Mellis CM, Van Asperen PP. Gender differences in habitual activity in children with cystic fibrosis. Arch Dis Child. 2004;89(10): 928-33.

[161] Dwyer TJ, Alison JA, McKeough ZJ, Elkins MR, Bye PTP. Evaluation of the SenseWear activity monitor during exercise in cystic fibrosis and in health. Respir Med. 2009;103(10):1511-7.

[162] Troiano RP, Berrigan D, Dodd K, Mâsse L, Tilert T, McDowell M. Physical Activity in the United States Measured by Accelerometer. Med Sci Sports Exerc. 2008;40(1):1818. 
[163] May C, Eton D, Boehmer K, Gallacher K, Hunt K, MacDonald S, et al. Rethinking the patient: using burden of treatment theory to understand the changing dynamics of illness. BMC Health Serv Res. 2014;14(1):281.

[164] Demain S, Goncalves AC, Areia C, Marques A, Marcos AJ, Oliveira R, et al. Living with and managing treatment burden in long-term conditions: A systematic review of qualitative research. PLoS Med. 2015; in press.

[165] Sawicki GS, Ren CL, Konstan MW, Millar SJ, Pasta DJ, Quittner AL. Treatment complexity in cystic fibrosis: trends over time and associations with site-specific outcomes. J Cyst Fibros. 2013;12(5):461-7.

[166] Flume PA, Robinson KA, O'Sullivan BP, Finder JD, Vender RL, Willey-Courand DB, et al. Cystic fibrosis pulmonary guidelines: airway clearance therapies. Respir Care. 2009;54(4):522-37.

[167] Gallacher K, May CR, Montori VM, Mair FS. Understanding patients' experiences of treatment burden in chronic heart failure using normalization process theory. Ann Fam Med. 2011;9(3):235-43.

[168] Karamanidou C, Weinman J, Horne R. A qualitative study of treatment burden among haemodialysis recipients. J Health Psychol. 2014;19(4):556-69.

[169] Eton DT, Ramalho de Oliveira D, Egginton JS, Ridgeway JL, Odell L, May CR, et al. Building a measurement framework of burden of treatment in complex patients with chronic conditions: a qualitative study. Patient Rel Outcome Meas. 2012;3:39-49.

[170] Conway SP, Pond MN, Hamnett T, Watson A. Compliance with treatment in adult patients with cystic fibrosis. Thorax. 1996;51(1):29-33.

[171] Abbott J, Dodd M, Bilton D, Webb AK. Treatment compliance in adults with cystic fibrosis. Thorax. 1994;49(2):115-20.

[172] Kettler L, Sawyer S, Winefield H, Greville H. Determinants of adherence in adults with cystic fibrosis. Thorax. 2002;57(5):459-64.

[173] Jordan S, Philpin S, Warring J, Cheung WY, Williams J. Percutaneous endoscopic gastrostomies: the burden of treatment from a patient perspective. J Adv Nurs. 2006;56(3):270--81.

[174] Donaldson SH, Bennett WD, Zeman KL, Knowles MR, Tarran R, Boucher RC. Mucus Clearance and Lung Function in Cystic Fibrosis with Hypertonic Saline. N Engl J Med. 2006;354(3):241-50. 

Chapter 3

\title{
Oxidative Stress in Cystic Fibrosis
}

\author{
D. González Jiménez, J.J. Díaz Martín, \\ R.P. Arias Llorente and C. Bousoño García \\ Additional information is available at the end of the chapter \\ http://dx.doi.org/10.5772/60661
}

\begin{abstract}
Airway infection leads to progressive damage of the lungs in patients with cystic fibrosis (CF). Oxidative stress has been implicated as a causative factor in the aetiology of that process. Supplementation with antioxidant micronutrients (vitamin E, vitamin C, B-carotene and selenium), docosahexaenoic acid or glutathione might therefore be helpful in maintaining an adequate redox balance. Current literature suggests a relationship between oxidative status and lung function. In this chapter we will summarize the main pathways of oxidative stress, focusing on results of new antioxidant treatments.
\end{abstract}

Keywords: Oxidative stress, fat-soluble vitamins, $\beta$-carotene, glutathione, essential fatty acid, antioxidants

\section{Introduction}

\subsection{Oxidative stress pathways in cystic fibrosis}

Colonization of the respiratory tract by bacterial pathogens in the mucus of CF patients leads to a sustained inflammatory response characterized by massive influx of polymorphonuclear neutrophils and the activation of macrophages, eosinophils, monocytes and lymphocytes. An integral part of this inflammation is the production and release of free radicals such as superoxide (O2-) and hydroxide (OH-), which can induce oxidative stress. In fact, elevated levels of proinflammatory cytokines, especially interleukins (IL-1 $\beta$, IL-6, IL-8), tumour necrosis factor- $\alpha$ and potent neutrophil chemoattractants found in bronchoalveolar lavage, are 
involved in the production of pro-oxidants leading to apoptosis and tissular damage. Conversely, the production of immunosuppressive cytokine IL-10 with anti-inflammatory properties is reduced or even suppressed [1].

Thus, the severe and recurrent respiratory inflammation ultimately leads to excessive activated neutrophils and macrophages, which contribute to the generation of free radicals. Furthermore, defects in the Cystic Fibrosis Transmembrane Regulator (CFTR) can directly affect transport and glutathione homeostasis, while maldigestion and malabsorption related to exocrine pancreatic insufficiency impair the absorption of fat-soluble vitamins and antioxidants. It has been suggested that the chloride channel CFTR also regulates glutathione, disturbing the balance between pro- and anti-oxidants and promoting oxidative stress, which may play an important role in Cystic Fibrosis Related Diabetes, a serious complication associated with a dramatic increase in morbidity and mortality [2].

Although the cause of CF is well established, the pathogenesis of this progressive multisystemic disease is not yet fully understood. In fact, the broad spectrum of phenotypes and severity in CF patients that carry the same combination of mutations suggests additional environmental or genetic factors.

The CFTR dysfunction in the pancreas causes exocrine pancreatic insufficiency in almost $90 \%$ of patients with CF. This leads to fat malabsorption, which explains the difficulty to gain or at least maintain weight, and the high incidence of fat-soluble-vitamins and antioxidant (vitamins A, E, and D and carotenoids) deficiency, and also essential-fatty-acids deficiency. Obviously, the reduced availability of dietary antioxidants may further increase oxidative stress in CF patients, which apparently plays an important role in multiorgan pathophysiology of CF.

Consequently, the products of lipid peroxidation, which are markers of oxidative stress, have been detected in exhaled breath condensate, as well as in blood and urine of CF patients. Thus products of lipid peroxidation are unstable molecules that can reach distant sites to exert various effects, including activation of the fibroblast cells in the presence of inflammation, which further increases oxidative stress [3].

\section{Management of oxidative stress in cystic fibrosis}

Nutrition plays an essential role in the survival and quality of life of CF patients. CF patients have high caloric requirements due to an increased resting energy expenditure (REE), bacterial infection, and malabsorption. REE is higher in CF patients with a more severe phenotype. Lung function and nutritional status are closely correlated, and the severe weight loss can lead to a decrease in lean body mass, with consequences for respiratory muscles. There is a significant correlation between growth retardation and the severity of pulmonary involvement [4]. Recently, Yen et al. found that greater weight at age four years is associated with greater height, better pulmonary function, fewer complications of $\mathrm{CF}$, and better survival through the age of 18 years. Furthermore, greater weight for age in the peripubertal period is associated, on 
average, with improved tempo and timing of pubertal height growth [5]. Long-term nutritional management is as integral a part of modern care as pulmonary therapy, and is intimately linked to pulmonary outcomes.

Dietetic management is based on the replacement of pancreatic enzymes and fat-soluble vitamins (A, D, E, K), together with a high-protein diet and high calorie intake (120-125\% of the normal recommended daily allowance). The recommended Body Mass Index (BMI) for adult CF patients is $\geq 22 \mathrm{~kg} / \mathrm{m} 2$ for females and $\geq 23 \mathrm{~kg} / \mathrm{m} 2$ for males. Overnight enteral nutrition via nasogastric tube or gastrostomy provides supplementary nutritional support when BMI is suboptimal [6].

\section{a. Docosahexaenoic acid (DHA)}

Essential fatty acid (EFA) imbalance has been identified in CF patients and is characterized by a decrease in docosahexaenoic acid (DHA) and linoleic acid and an increase in arachidonic acid (AA) [7]. These characteristics were mainly attributed to intestinal malabsorption due to exocrine pancreatic insufficiency. In the last few years, new mechanisms have been proposed, such as intensification of the b-oxidation of polyunsaturated fatty acids (PUFAs), inadequate dietary EFA consumption, the possibility of an intrinsically defective EFA metabolism in CF epithelial cells, an increase in the production of proinflammatory eicosanoids, a rise in the peroxidation of PUFAs, and finally an impairment of desaturases or hepatic lipase activity [8].

It has been observed that long-term intake of the daily mixtures of fatty acids (eicosapentaenoic, docosahexaenoic, linoleic and $\gamma$-linolenic acid) at a low dose has a positive effect on lung function and inflammation in adult CF patients. The total number of exacerbations after a year of supplementation was reduced, while the lean-body-mass and lung-function parameters measured by spirometry were increased. In addition, supplementation led to improved parameters of oxidation, inflammation (IgG and $\operatorname{IgM}$ ) and other clinical parameters [9]. 8isoprostane, a free-radical product of lipid peroxidation which is a consequence of oxidative stress, appears to be a prognostic factor in deterioration of lung function in a short time in patients infected with Burkholderia cenocepacia. The measured concentration of 8-isoprostane in exhaled breath condensate of 24 patients did not show any significant relationship with the clinical parameters during a one- and three-year study [10].

Forty-three CF patients were enrolled in a randomized double-blind placebo-controlled study with three fatty-acid blends containing mainly n-3 or n-6 FA, or saturated fatty acid acting as placebo [11]. After three months, in the omega-3 fatty-acid-supplemented group, a significant decrease in the inflammatory markers, erythrocyte sedimentation rate and IL-8 was reported. Another, longer-term study (17 participants) demonstrated a significant increase in essentialfatty-acid content in neutrophil membranes and a significant decrease in the leukotriene B4 to leukotriene B5 ratio in participants taking omega-3 supplements compared to the placebo [12].

\section{b. Fat-soluble vitamins}

Supplementation of CF patients with vitamin $E$ and $\beta$-carotene has been effective in preventing oxidative lung damage [13], as seen by a decrease in lipid peroxidation products. It is believed that these antioxidants have an important role in maintaining or restoring essential-fatty-acid 
status by protecting polyunsaturated fatty acid from oxidative degradation, as their supplementation augments levels of plasma polyunsaturated fatty acid.

Carotenoids

Levels of plasma carotenoids such as $\beta$-carotene, $\beta$-cryptoxanthin, and total lycopene are significantly lowered in CF patients and this has been associated with higher susceptibility to lipid peroxidation. Rust et al. [14] examined the effect of long-term oral $\beta$-carotene supplementation in patients with CF. Patients of the CF supplementation group received $1 \mathrm{mg} \beta$ carotene/kg body weight/day (maximally $50 \mathrm{mg} \beta$-carotene/day). During high-dose treatment, a significant decrease in the MDA level and a correction of total antioxidative capacity was observed.

Renner et al. reported distinct clinical benefits from high-dose $(1 \mathrm{mg} / \mathrm{kg}$ body weight/ day, maximum $50 \mathrm{mg} /$ day) supplements. Their patients required significantly fewer antibiotics during the phase of high-dose $\beta$-carotene supplementation and showed a decrease in pulmonary exacerbations [15]. Lepage et al. reported that the two-month supplementation of CF patients with $4.42 \mathrm{mg} \beta$-carotene, three times per day, led to the normalization of increased MDA level and increased plasma $\beta$-carotene from $0.08 \pm 0.03$ to $3.99 \pm 0.92 \mu \mathrm{M}$ [16].

At the same time, toxicity issues have been raised for supplementation with water- miscible vitamin A formulations in CF patients, which may increase serum retinol and possible risk of CF-associated liver and bone complications [17]. However, $\beta$-carotene supplementation seems to be safe since it does not affect serum concentrations of other carotenoides and retinol [14]. Recent studies have analysed the use and safety of a new CF polyvitamin (AquADEKs ${ }^{\circledR}$ ), which comprises almost $90 \%$ vitamin A in retinol form. Patients on AquADEKs ${ }^{\circledR}$ maintained a high level of serum $\beta$-carotene, but serum retinol was not above the normal levels. $\beta$-carotene levels were associated with lung function and better nutritional status [18,19], while lipid peroxidation markers were not affected [20].

Vitamin E ( $\alpha$-tocopherol)

$\alpha$-tocopherol acts as a membrane antioxidant closely associated with polyunsaturated fatty acids. Vitamin E's antioxidative properties might be helpful in reducing the negative effects of free radicals. Current recommended supplementation of vitamin E in CF patients only includes $\alpha$-tocopherol. Supplementation with high levels of $\alpha$-tocopherol alone may result in further imbalances in CF patients: such supplementation has been shown to deplete $\gamma$ tocopherol in the blood and tissues [21]. Papas et al. [16] evaluated vitamin E supplementation with mixed tocopherols. The increase in the blood of levels of $\gamma$-tocopherol may be particularly important for $\mathrm{CF}$ patients due to its function as a scavenger of reactive nitrogen species and its synergistic effects with $\alpha$-tocopherol [22]. Cystic fibrosis is characterized by neutrophildominated airway inflammation. Activated neutrophils release oxidants, proteases, and cytokines, further sustaining and increasing the inflammatory response and causing direct injury to the lungs. Improved antioxidant capacity with $\gamma$-tocopherol, especially if present in the lungs, could potentially decrease oxidant-mediated damage and limit the cytokinemediated neutrophil recruitment. It has also been reported that reduced serum levels of vitamin $\mathrm{E}$ are associated with an increased rate of pulmonary exacerbations in CF [23]. 


\section{c. Other vitamins and micronutrients}

\section{Vitamin C}

Vitamin $C$ is present in the respiratory lining fluid of human lungs, and local deficit occurs during oxidative stress. Experimental findings confirm that vitamin $\mathrm{C}$ induced the openings of CFTR Cl- channels without a detectable increase in intracellular cyclic AMP levels. Vitamin $\mathrm{C}$ instilled into the nasal epithelium of human subjects effectively activates $\mathrm{Cl}$ - transport, too.

The pool of vitamin $C$ in the respiratory tract represents a potential nutraceutical and pharmaceutical target for the complementary treatment of oxidative stress in patients with CF [24]. In a study by Winklhofer-Roob et al. [25] on 122 children and young adults with CF, ascorbicacid concentrations decreased with age, and low vitamin $C$ levels were associated with the highest indexes of inflammation, so the authors concluded that ascorbic acid could interact with an inflammation-amplifying circle of activation of alveolar neutrophils and macrophages.

Water-soluble vitamins seem to be well absorbed by patients with $\mathrm{CF}$, but there is documented evidence of poor dietary intake. A supplement of at least 50-100 mg vitamin C/day should be prescribed for patients with an unbalanced diet, or if there is evidence of deficiency.

\section{Selenium}

Dietary intake of selenium is inversely related to inflammatory markers such as sialic acid and triacylglycerol [26]. Moreover, a possible role of selenium in the modulation of serum complement 3 , which may be an early marker of metabolic syndrome manifestations, has also been documented.

Wood et al. carried out an eight-week, double-blind, randomized intervention trial, providing two groups of patients with low- and high-dose vitamin supplements (500 $\mu \mathrm{g}$ vitamin $\mathrm{A}$ and $10 \mathrm{mg}$ vitamin $\mathrm{E}$ vs. $500 \mu \mathrm{g}$ vitamin A, $25 \mathrm{mg} \beta$-carotene, $200 \mathrm{mg}$ vitamin $\mathrm{E}, 300 \mathrm{mg}$ vitamin C and $90 \mu \mathrm{g}$ selenium). They demonstrated significant improvement of clinical indicators after treatment. Increased serum $\beta$-carotene, selenium, and fatty-acid concentrations were linked to improved lung function [27].

Consensus regarding supplementation of antioxidants in CF to include selenium is yet to be established. Two studies have attempted to demonstrate successfully how the administration of $2.8 \mu \mathrm{g} / \mathrm{kg} /$ day and $90 \mu \mathrm{g} /$ day of selenium can decrease oxidative stress in CF [28].

\section{d. Glutathione and n-acetylcysteine}

The discovery that $\mathrm{CF}$ is associated with significantly diminished efflux of reduced glutathione (GSH) from most cells in the body [29] offers a new perspective on the pathophysiology of this disease. GSH plays several important roles; among the most important are the following: 1) primary water-soluble antioxidant; 2) mucolytic capable of cleaving disulphide bonds; and 3) regulator of immune-system function [30].

The relationship between redox ratio (GSH:GSSG) and total glutathione (GSH+GSSG) and the initiation of inflammation is well established [29,30]. GSH is also an important component of the epithelial lining fluid of the intestines, helping to keep intestinal mucus thin, serving to 
defend the intestinal system against reactive oxygen species, and keeping inflammation in check under normal circumstances [31].

In a recent placebo-controlled, randomized, double-blinded, clinical trial in 44 paediatric $\mathrm{CF}$ patients aged between 18 months and 10 years [32], treatment with oral glutathione (65 $\mathrm{mg} / \mathrm{kg} /$ day) increased weight and BMI z score and improved measures of gut inflammation (faecal calprotectin) over the course of six months, without adverse side effects. The authors therefore concluded that Oral GSH might primarily be beneficial in those children with more severe inflammation of the gut, and suggested that early intervention with oral glutathione in young CF children with growth failure could forestall decline in pulmonary function in later years.

Several studies have investigated the potential therapeutic role of inhaled GSH in patients with CF. Three short-term clinical trials, including a placebo-controlled one, have shown the tolerability and efficacy of inhaled GSH on pulmonary function in these subjects [33-34]. A recent 12-month randomized single-blind placebo-controlled trial demonstrated the efficacy of inhaled GSH (600 mg twice daily) on lung function in CF adults [35]. Three months of therapy with inhaled GSH resulted in a statistically significant improvement in percentagepredicted FEV1, measured as a pre-post difference from baseline values, when compared to the placebo, which persisted at six and nine but not at 12 months. A reduced compliance with therapy in adult patients could explain the decrease in FEV1 values registered in the last visit. The best improvements in functional parameters were registered in the subgroup of patients with moderate lung disease (FEV1 below $81 \%$ ). These results are in concordance with those reported by Griese et al. [36], who showed a significant increase of FEV1 absolute values (but not when expressed as percentage-predicted) from the baseline after three months of GSH therapy.

$\mathrm{N}$-acetylcysteine (NAC), a well-known cysteine donor for the synthesis of glutathione, has been used in different diseases to treat GSH deficiency [37]. High-dose oral NAC has been shown to increase neutrophil GSH levels, decrease airway neutrophil recruitment and reduce neutrophilic release of airway elastase in CF patients [38]. Skov et al. demonstrated that highdose oral NAC (1200 mg x 2/day for 30 days) in CF patients with chronic P. Aeruginosa infection decreased the level of oxidized vitamin C [39].

Indications of a positive effect of NAC treatment on the lung function of a subgroup of $\mathrm{CF}$ patients have previously been published [40]. Recently, a placebo-controlled randomized clinical trial (70 CF patients) was conducted in the USA to study the effect of oral NAC on lung inflammation (ClinicalTrials.gov Identifier: NCT00809094). Oral NAC was administered in a dose of $1800 \mathrm{mg}$ /day divided into two dosages over a period of 24 weeks and the effects on the sputum levels of human neutrophil elastase (HNE) were assessed as a primary end-point. While no statistical significant difference was found between the two groups with regard to the primary end-point, an improvement in the predicted FEV1\% was observed in the NACtreated group.

A recent Cochrane review on the use of thiol derivatives, such as NAC, did not find sufficient evidence to recommend the use of these compounds in the management of CF lung disease, but concluded that further studies were warranted [41]. 
$\gamma$-Glutamylcysteine ethyl ester (GCEE) is another potentially interesting GSH pro-drug, which has proved to have some efficacy in the amelioration of oxidative stress, e.g., in experimental myocardial infarction [42] and central-nervous-system conditions (see, e.g., [43]). However, GCEE has not been investigated in CF yet.

New CFTR modulation therapies are being designed to correct the function of the defective protein (CFTR) made by the CF gene, allowing chloride and sodium to move properly in and out of cells lining the lungs and other organs. N6022 is a new injectable compound that modulates the function of the defective CFTR protein and decreases inflammation in the lung. N6022 is the first of a new class of compounds that increase levels of an important signalling molecule in the body called S-nitrosoglutathione, or GSNO. These novel compounds have been shown in preliminary results (Phase $1 \mathrm{~b}$ trial) to increase the amount of CFTR that reaches the cell membrane and to stabilize CFTR so that its function can be improved.

\section{Conclusion}

In conclusion, there appears to be conflicting evidence regarding the clinical effectiveness of antioxidant supplementation in CF patients. Based on the available evidence, glutathione (administered either orally or by inhalation) and high doses of $\beta$-carotene appear to improve lung function in some cases and decrease oxidative stress. Further studies, especially in very young patients, examining clinically relevant outcomes, dose levels and other promising therapies like CFTR modulation, are necessary before a firm conclusion can be made regarding the effects on oxidative stress in these patients.

\section{Author details}

D. González Jiménez ${ }^{1}$, J.J. Díaz Martín ${ }^{1}$, R.P. Arias Llorente ${ }^{2 *}$ and C. Bousoño García ${ }^{1}$

*Address all correspondence to: soyrosapatricia@hotmail.com

1 Pediatric Gastroenterology and Nutrition Unit, Hospital Universitario Central de Asturias, Oviedo, Spain

2 Service of Neonatology, Hospital Universitario Central de Asturias, Oviedo, Spain

\section{References}

[1] Back EI, Frindt C, Nohr D, Frank J, Ziebach R, Stern M, Ranke M, Biesalski HK. Antioxidant deficiency in cystic fibrosis: when is the right time to take action?. Am J Clin Nutr. 2004; 80: 374-384. 
[2] Gao L, Kim KJ, Yankaskas JR, Forman HJ. Abnormal glutathione transport in cystic fibrosis airway epithelia. AJP - Lung Physiol. 1999; 277: 113-118.

[3] Ntimbane T, Comte B, Mailhot G, Berthiaume Y, Poitout V, Prentki M, Rabasa-Lhoret R, Levy E. Cystic fibrosis-related diabetes: from CFTR dysfunction to oxidative stress. Clin Biochem Rev. 2009; 30: 153-177.

[4] Haack A, Carvalho Garbi Novaes MR. Multidisciplinary care in cystic fibrosis: a clinical-nutrition review. Nutr Hosp. 2012; 27: 362-371.

[5] Yen EH, Quinton H, Borowitz D. Better nutritional status in early childhood is associated with improved clinical outcomes and survival in patients with cystic fibrosis. J Pediatr. 2013; 162: 530-5, e531.

[6] Garattini E, Bilton D, Cremona G, Hodson M. Adult cystic fibrosis care in the $21^{\text {st }}$ century. Monaldi Arch Chest Dis. 2011; 75: 178-184.

[7] Njoroge SW, Laposata M, Katrangi W, Seegmiller AC. DHA and EPA reverse cystic fibrosis-related FA abnormalities by suppressing FA desaturase expression and activity. J Lipid Res. 2012; 53: 257-265.

[8] Mimoun M, Coste TC, Lebacq J, Lebecque P, Wallemacq P, Leal T, Armand M. Increased tissue arachidonic acid and reduced linoleic acid in a mouse model of cystic fibrosis are reversed by supplemental glycerophospholipids enriched in docosahexaenoic acid. J Nutr. 2009; 139: 2358-2364.

[9] Olveira G, Olveira C, Acosta E, Espíldora F, Garrido-Sánchez L, García-Escobar E, Rojo-Martínez G, Gonzalo M, Soriguer F. Fatty acid supplements improve respiratory, inflammatory and nutritional parameters in adults with cystic fibrosis. Arch Bronconeumol. 2010; 46: 70-77.

[10] Fila L, Grandcourtová A, Chládek J, Musil J. Oxidative stress in cystic fibrosis patients with Burkholderia cenocepacia airway colonization: relation of 8-isoprostane concentration in exhaled breath condensate to lung function decline. Folia Microbiol (Praha). 2014; 59: 217-222.

[11] Keen C, Olin AC, Eriksson S, Ekman A, Lindblad A, Basu S, Beermann C, Strandvik B. Supplementation with fatty acids influences the airway nitric oxide and inflammatory markers in patients with cystic fibrosis. J Pediatr Gastroenterol Nutr. 2010; 50: 537-544.

[12] Panchaud A, Sauty A, Kernen Y, Decosterd LA, Buclin T, Boulat O, Hug C, Pilet M, Roulet $\mathrm{M}$. Biological effects of a dietary omega-3 polyunsaturated fatty acids supplementation in cystic fibrosis patients: a randomized, crossover placebo-controlled trial. Clin Nutr. 2006; 25: 418-427.

[13] Rivas-Crespo MF, González Jiménez D, Acuña Quirós MD, Sojo Aguirre A, Heredia González S, Díaz Martín JJ, Garagorri Otero JM, Lázaro Almarza A, Bousoño-García 
C. High serum retinol and lung function in young patients with cystic fibrosis. J Pediatr Gastroenterol Nutr. 2013; 56: 657-662.

[14] Rust P, Eichler I, Renner S, Elmadfa I. Long-term oral beta-carotene supplementation in patients with cystic fibrosis effects on antioxidative status and pulmonary function. Ann Nutr Metab. 2000; 44: 30-37.

[15] Renner S, Rath R, Rust P, Lehr S, Frischer T, Elmadfa I, Eichler I. Effects of beta-carotene supplementation for six months on clinical and laboratory parameters in patients with cystic fibrosis. Thorax. 2001; 56: 48-52.

[16] Lepage G, Champagne J, Ronco N, Lamarre A, Osberg I, Sokol RJ, Roy CC. Supplementation with carotenoids corrects increased lipid peroxidation in children with cystic fibrosis. Am J Clin Nutr. 1996; 64: 87-93.

[17] Graham-Maar RC, Schall JI, Stettler N, Zemel BS, Stallings VA. Elevated vitamin A intake and serum retinol in preadolescent children with cystic fibrosis. Am J Clin Nutr. 2006; 84: 174-182.

[18] Papas KA, Sontag MK, Pardee C, Sokol RJ, Sagel SD, Accurso FJ, Wagener JS. A pilot study on the safety and efficacy of a novel antioxidant rich formulation in patients with cystic fibrosis. J Cyst Fibros. 2008; 7: 60-67.

[19] Sadowska-Woda I, Rachel M, Pazdan J, Bieszczad-Bedrejczuk E, Pawliszak K. Nutritional supplement attenuates selected oxidative stress markers in pediatric patients with cystic fibrosis. Nutr Res. 2011; 31: 509-518.

[20] Sagel SD, Sontag, MK Anthony MM, Emmett, P Papas KA. Effect of an antioxidantrich multivitamin supplement in cystic fibrosis. J Cyst Fibros. 2011; 10: 31-36.

[21] Huang SH, Schall JI, Zemel BS, Stallings VA. Vitamin E status in children with cystic fibrosis and pancreatic insufficiency. J Pediatr. 2006; 148: 556-559.

[22] Christen S, Woodall AA, Shigenaga MK, Southwell-Keely PT, Duncan MW, Ames BN. Gamma-tocopherol traps mutagenic electrophiles such as $\mathrm{NO}(\mathrm{X})$ and complements alpha-tocopherol: physiological implications. Proc Natl Acad Sci USA. 1997; 94: 3217-3222.

[23] Hakim F, Kerem E, Rivlin J, Bentur L, Stankiewicz H, Bdolach-Abram T, Wilschanski M. Vitamins A and E and pulmonary exacerbations in patients with cystic fibrosis. J Pediatr Gastroenterol Nutr. 2007; 45: 347-53.

[24] Fischer H, Schwarzer C, Illek B. Vitamin C controls the cystic fibrosis transmembrane conductance regulator chloride channel. Proc Natl Acad Sci. 2004; 101: 3691-3696.

[25] Winklhofer-Roob BM, Ellemunter H, Fruhwirth M, Schlegel-Haueter SE, Khoschsorur G, van't Hof MA, Shmerling DA. Plasma vitamin C concentrations in patients with cystic fibrosis: evidence of associations with lung inflammation. Am J Clin Nutr. 1997; 65: 1858-1866. 
[26] Zulet MA, Puchau B, Hermsdorff HH, Navarro C, Martinez JA. Dietary selenium intake is negatively associated with serum sialic acid and metabolic syndrome features in healthy young adults. Nutr Res. 2009; 29: 41-48e.

[27] Wood LG, Fitzgerald DA, Lee AK, Garg ML. Improved antioxidant and fatty acid status of patients with cystic fibrosis after antioxidant supplementation is linked to improved lung function. Am J Clin Nutr. 2003; 77: 150-159.

[28] Shamseer L, Adams D, Brown N, Johnson JA, Vohra S. Antioxidant micronutrients for lung disease in cystic fibrosis. Cochrane Database Syst Rev. 8 December 2010; 12: CD007020.

[29] Kogan I, Ramjeesingh M, Li C, Kidd JF, Wang Y, Leslie EM, Cole SP, Bear CE. CFTR directly mediates nucleotide-regulated glutathione flux. EMBO J. 2003; 22: 1981-1989.

[30] Velsor LW, van Heeckeren A, Day BJ. Antioxidant imbalance in the lungs of cystic fibrosis transmembrane conductance regulator protein mutant mice. Am J Physiol Lung Cell Mol Physiol. 2001; 281: L31-8.

[31] Hoensch H, Morgenstern I, Petereit G, Siepmann M, Peters WH, Roelofs HM, Kirch $\mathrm{W}$. Influence of clinical factors, diet, and drugs on the human upper gastrointestinal glutathione system. Gut. 2002; 50: 235-240.

[32] Visca A, Bishop CT, Hilton S, Hudson VM. Oral Reduced L-Glutathione Improves Growth in Pediatric Cystic Fibrosis Patients: A Randomized Clinical Trial. J Pediatr Gastroenterol Nutr. January 28 2015. [Epub ahead of print]

[33] Griese M, Ramakers J, Krasselt A, Starosta V, Van Koningsbruggen S, Fischer R, Ratjen F, Müllinger B, Huber RM, Maier K, Rietschel E, Scheuch G. Improvement of alveolar glutathione and lung function but not oxidative state in cystic fibrosis. Am J Respir Crit Care Med. 2004; 169: 822-888.

[34] Bishop C, Hudson VM, Hilton SC, Wilde C. A pilot study of the effect of inhaled buffered reduced glutathione on the clinical status of patients with cystic fibrosis. Chest. 2005; 127: 308-317.

[35] Calabrese C, Tosco A, Abete P, Carnovale V, Basile C, Magliocca A, Quattrucci S, De Sanctis S, Alatri F, Mazzarella G, De Pietro L, Turino C, Melillo E, Buonpensiero P, Di Pasqua A, Raia V. Randomized, single blind, controlled trial of inhaled glutathione vs. placebo in patients with cystic fibrosis. J Cyst Fibros. 2015; 14: 203-210.

[36] Griese M, Kappler M, Eismann C, Ballmann M, Junge S, Rietschel E, van Koningsbruggen-Rietschel S, Staab D, Rolinck-Werninghaus C, Mellies U, Köhnlein T, Wagner T, König S, Teschler H, Heuer HE, Kopp M, Heyder S, Hammermann J, Küster P, Honer M, Mansmann U, Beck-Speier I, Hartl D, Fuchs C; Glutathione Study Group, Hector A. Inhalation treatment with glutathione in patients with cystic fibrosis. A randomized clinical trial. Am J Respir Crit Care Med. 2013; 188: 83-89. 
[37] Atkuri K, Mantovani JJ, Herzenberg LA. N-Acetylcysteine a safe antidote for cysteine/glutathione deficiency, Curr Opin Pharmacol. 2007; 7: 355-359.

[38] Tirouvanziam R, Conrad CK, Bottiglieri T, Herzenberg LA, Moss RB. High dose oral N-acetylcysteine, a glutathione prodrug, modulates inflammation in cystic fibrosis. Proc Natl Acad Sci. 2006; 103: 4628-4633.

[39] Skov M, Pressler T, Lykkesfeldt J, Poulsen HE, Jensen PØ, Johansen HK, Qvist T, Kræmer D, Høiby N, Ciofu O. The effect of short-term, high-dose oral N-acetylcysteine treatment on oxidative stress markers in cystic fibrosis patients with chronic $\mathrm{P}$. aeruginosa infection - A pilot study. J Cyst Fibros. 2015; 14: 211-218.

[40] Stafanger G, Koch C. N-acetylcysteine in cystic fibrosis and Pseudomonas aeruginosa infection: clinical score, spirometry and ciliary motility. Eur Respir J. 1989; 2: 234-237.

[41] Tam J, Nash EF, Ratjen F, Tullis E, Stephenson A. Nebulized and oral thiol derivatives for pulmonary disease in cystic fibrosis. Cochrane Database Syst Rev. 2013; 7: CD007168.

[42] Hoshida S, Kuzuya T, Yamashita N, Nishida M, Kitahara S, Hori M, Kamada T, Tada M. Gamma-glutamylcysteine ethyl ester for myocardial protection in dogs during ischemia and reperfusion. J Am Coll Cardiol. 1994; 24: 1391-1397.

[43] Lok J, Leung W, Zhao S, Pallast S, van Leyen K, Guo S, Wang X, Yalcin A, Lo EH. Gamma-glutamylcysteine ethyl ester protects cerebral endothelial cells during injury and decreases blood-brain barrier permeability after experimental brain trauma. J Neurochem. 2011; 118: 248-255. 



\section{Section 2}

Clinical Aspect of Cystic Fibrosis 



\title{
Chapter 4
}

\section{Cystic Fibrosis Related Diabetes}

\author{
Paula Dyce, Gareth Huw Jones and \\ Martin J Walshaw \\ Additional information is available at the end of the chapter \\ http://dx.doi.org/10.5772/61129
}

\begin{abstract}
Cystic fibrosis related diabetes (CFRD) is a common co-morbidity of cystic fibrosis. It is associated with diminishing lung function, poorer nutritional state, and a worse prognosis and its early diagnosis and treatment is important. In addition, the adverse pulmonary effects of CFRD may be present for up to 10 years prior to diagnosis.

Prevalence increases with age from 1 to $2 \%$ below 10 years of age, $20 \%$ of adolescents and $40-50 \%$ by adulthood, and up to $90 \%$ of adults with CF will have some degree of glucose intolerance by 40 years of age.

Although its pathophysiology is not well understood, the primary cause is thought to be the fatty infiltration of the pancreas leading to fibrosis and destruction of the pancreatic cells resulting in beta cell destruction and progressive insulin deficiency.

Oral glucose tolerance testing (OGTT) remains the method of choice for a diagnosis of type I, type II and gestational diabetes; it is less discriminating in CFRD where early glucose trends are important, and continuous glucose monitoring may prove a more useful diagnostic and management tool.

Diabetic complications occur in CFRD and annual screening for these is necessary. As life expectancy rises, diabetic complications may become more prevalent.

This chapter discusses the diagnosis and treatment of CFRD and touches on developments in research which will enhance the future management of this increasingly common and important manifestation of the CF condition.
\end{abstract}

Keywords: Cystic fibrosis related diabetes, Screening, Oral glucose tolerance testing, Continuous glucose monitoring, Cystic Fibrosis Related Diabetes 


\section{Introduction}

Cystic Fibrosis Related Diabetes (CFRD) is a form of abnormal glucose handling (dysglycaemia) that is quite different to other forms of diabetes. It is uncommon in childhood, rarely occurring in those under 10 years of age, but its incidence rises inexorably as patients survive longer, affecting up to $50 \%$ of $\mathrm{CF}$ adults $[1,2]$.

The development of CFRD is associated with a significantly higher mortality rate $[2,3,4]$, and has a deleterious impact on pulmonary function [5] with the degree of glucose intolerance being directly related to the decline in FEV1 [6]. Those with CFRD suffer more frequent infective exacerbations and have a higher burden of colonisation with pathogens such as Pseudomonas aeruginosa and Burkholderia cepacia as well as a poorer nutritional status - all of which are known to be associated with poorer outcomes [7].

However, dysglycaemia is a progressive phenomenon in many CF patients, and the associated increased morbidity occurs many years prior to a formal diagnosis of CFRD $[8,9,10]$. This has fuelled the debate as to how the diagnosis should be made - the current "gold standard" diagnostic test for diabetes mellitus (the oral glucose tolerance test, OGTT) [11] may not be appropriate in the CF population, since the optimal time to introduce treatment (currently limited exclusively to insulin-based therapies) may be at an earlier stage of dysglycaemia.

We will explore these issues as well as consider future developments in this emerging area of CF care.

\section{Epidemiology, prevalence and incidence}

Historically, CFRD was typically only seen in paediatric practice as a pre-terminal complication in those with advanced respiratory failure. It remains relatively rare in children under the age of 10 (2), affects less than 5\% of individuals during their early teen years, but the incidence rises exponentially with age, occurring in approximately $20 \%$ of adolescents and up to $50 \%$ of individuals in their fourth decade. The average age of onset for CFRD is 18-21 years (12).

Furthermore, it is estimated that $70-90 \%$ of all adult CF patients will have some degree of glucose intolerance and therefore dysglycaemia is the commonest co-morbidity that complicates care in adult CF and its prevalence is likely to increase further as life expectancy continues to rise.

In addition to age, other factors associated with an increased incidence of CFRD are severity of CFTR mutation, poor pulmonary function, low BMI, pancreatic insufficiency, liver dysfunction, and corticosteroid use $(5,7,13,14)$.

\section{Pathophysiology}

The pathophysiology of CFRD is still poorly understood. CFTR may have a role in the cell signalling that contributes to both the timely release of and response to insulin and restoration of abnormal CFTR function. 
However, although Ivacaftor (which corrects the underlying gene product defect in patients with a gating mutation) has reportedly led to normalisation of sugar handling in a single individual [15], it seems likely that the pathways directly involving CFTR are of only minor significance to overall glucose homeostasis.

CFRD involves a complex interplay between a state of relative insulin deficiency, particularly during the first phase response, and variable insulin resistance during infective exacerbations. Some level of dysglycaemia is detectable in most pancreatic-insufficient patients and the hallmark of CFRD is the presence of post-prandial glucose excursions that may be followed by spontaneous hypoglycaemic episodes.

Below we consider mechanisms that contribute to abnormal glucose handling in CF.
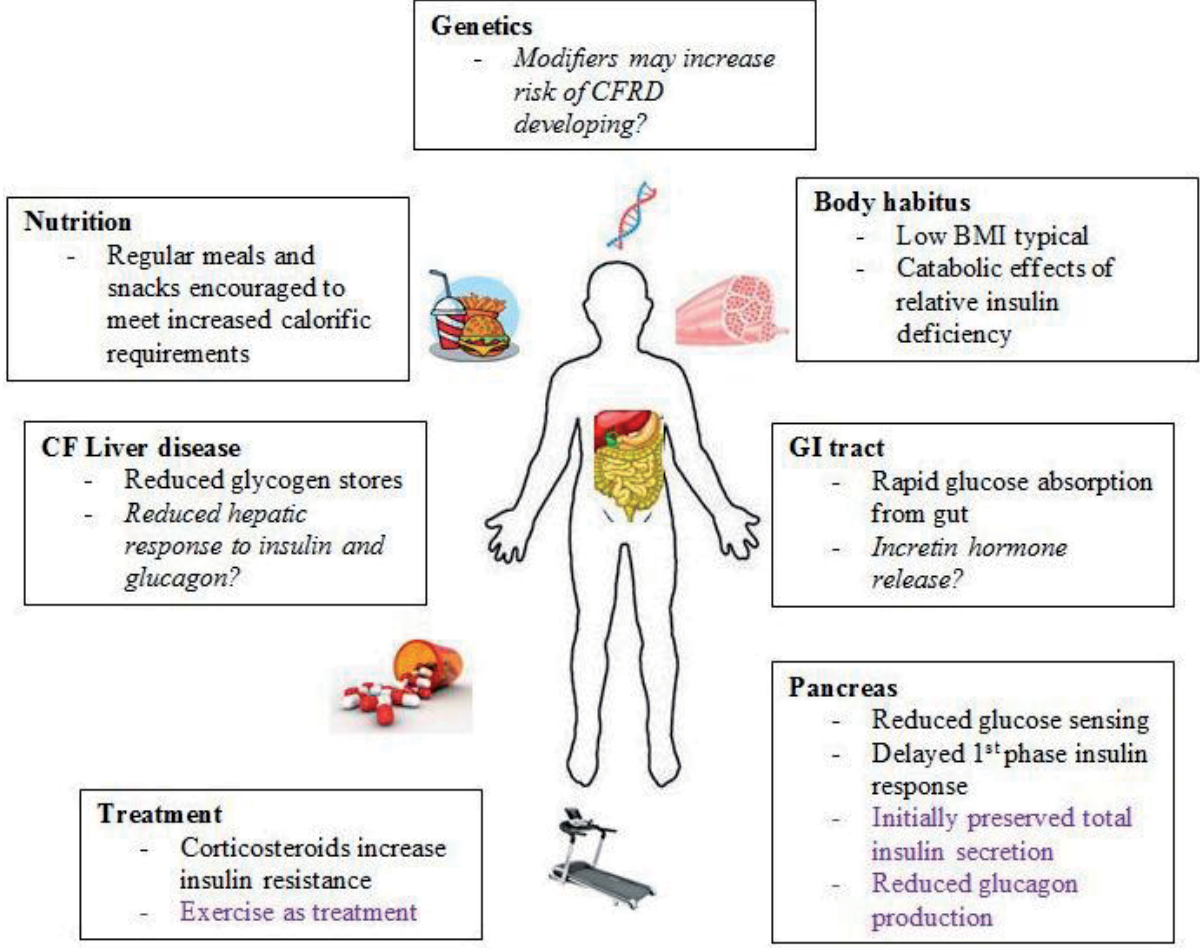

Figure 1. Factors influencing glucose handling in CF. Italicised - unclear role. Purple - factors pre-disposing to reactive hypoglycaemic episodes

\subsection{Pancreatic responses in $\mathrm{CF}$}

The predominant mechanism underpinning dysglycaemia in CF patients is sluggish clearance of proteolytic enzymes from the pancreatic ducts causing progressive $\beta$-cell destruction that directly reduces insulin levels, as well as causing ischaemic damage and amyloid deposition [16], further impairing the ability of remaining islet cells to sense and respond to glucose levels 
appropriately [17]. It seems likely that the same mechanism might also prevent incretin hormones (e.g. GLP-1 and GIP) from evoking their physiological insulinogenic effect during the post-prandial period, although this is an area still under investigation (see below).

Loss of pancreatic endocrine function is a continuum in patients with CF with impaired release of both insulin and glucagon [18]. Those with significant pancreatic insufficiency (almost 90\% of patients with CF use pancreatic enzyme supplements [5]) have deranged glucose handling even if not formally diabetic [17], typically displaying a slow and prolonged insulin response to stimulation $[18,19]$.

The initial abnormality seen in CF patients is a delayed first-phase insulin response combined with preserved total insulin secretion that occurs in response to various stimuli even in $\mathrm{CF}$ subjects with normal glucose tolerance [10, 18, 20, 21, 22, 23]. Even at this early stage, patient outcomes can be affected and the loss of pulmonary function is directly related to the degree of dysglycaemia [6]: this has implications for CFRD screening and diagnosis (see below).

The CFTR mutation also causes rapid absorption of glucose from the small intestine compared to the non-CF gut [23]. This combination of rapid glucose absorption and slow prolonged insulin response helps to explain both the typical high post-prandial glucose excursions seen in this group (Fig. 2) (an independent marker of worsening clinical status prior to a formal CFRD diagnosis [10]), as well as the symptomatic hypoglycaemia (often termed reactive or rebound) that may develop several hours later, as insulin continues to be secreted despite falling glucose levels (see Fig. 2) [24, 25]. A tendency to spontaneous hypoglycaemia may be further exacerbated by intense periods of exercise, something that forms a cornerstone of treatment in CF [11] (see below).

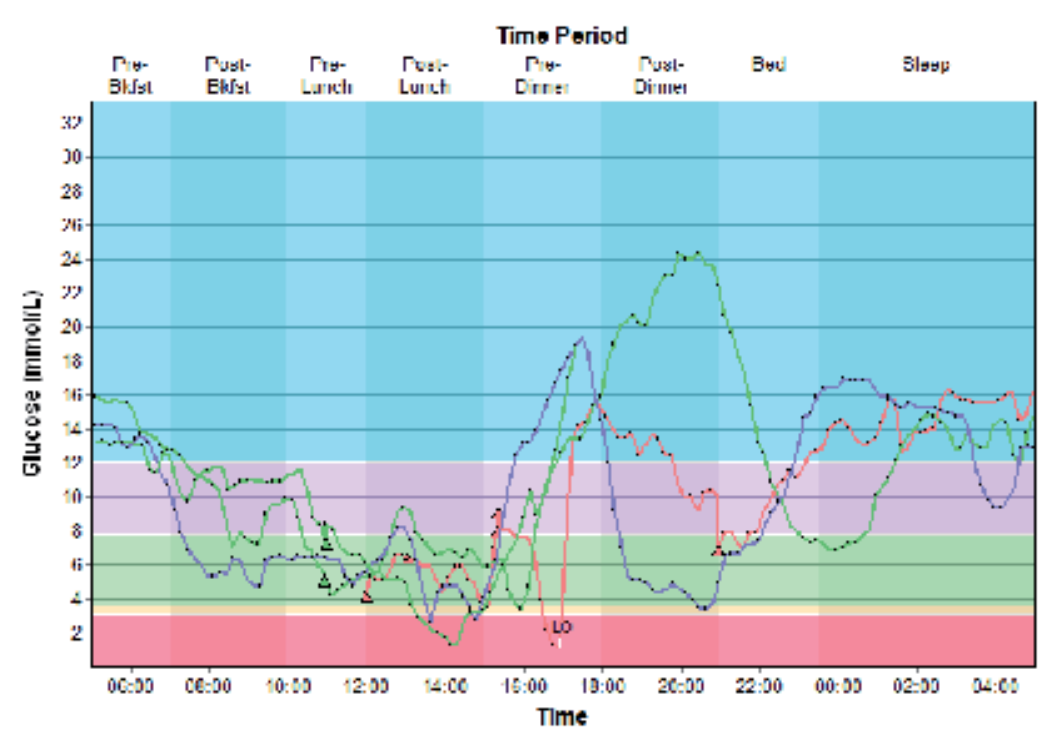

Figure 2. Continuous glucose monitoring showing a typical glucose profile seen in CFRD 
As progressive damage to the pancreas accumulates, there is an associated loss of endocrine tissue such that over time patients become relatively insulinopaenic [26]. Although pancreatic $\beta$-cell mass may be significantly reduced in patients with CFRD [27, 28], absolute insulinopaenia and ketoacidosis, typical features of Type 1 Diabetes (T1DM) [29], do not occur even in the minority of patients with sufficient pancreatic damage to cause fasting hyperglycaemia. Indeed, the presence of true ketoacidosis is so rare in this population that its occurrence should stimulate investigation for the development of concurrent T1DM [11, 30, 31]. It is possible that the loss of $\alpha$-cells as part of the indiscriminate pancreatic damage that occurs in CF results in relatively low glucagon levels, protecting against the development of ketosis as well as adding to the propensity for symptomatic hypoglycaemia [32].

\subsection{Insulin sensitivity in CF}

The role of insulin sensitivity in the development of CFRD is unclear: CFTR may have a theoretical role in cell signalling to increase glucose uptake by peripheral tissues but such responses are predominantly mediated through GLUT receptors. Insulin resistance increases during periods of acute illness [33] including pulmonary exacerbations which may frequently occur in those with an underlying suppurative lung condition. Hardin et al. [34] suggested that insulin sensitivity may be abnormal even in clinically stable CF patients, where on-going indolent infection may up-regulate inflammatory pathways, thereby inducing a degree of chronic insulin resistance.

However, despite these mechanisms, it is relatively rare for pancreatic-sufficient CF individuals, even when colonised with pathogens and suffering regular exacerbations, to develop significant dysglycaemia [35] and overall it has been shown that insulin resistance is not a major determinant of the development of diabetes in CF [36].

It is recognised that at times of high inflammatory stresses or concurrent glucocorticoid use such as during a pulmonary exacerbation - insulin resistance may contribute to or unmask clinically relevant dysglycaemia in predisposed individuals [17, 30,34] and extra glucose monitoring is therefore recommended during such periods [37] since initiation or escalation of treatment may be required [30]. As the nutritional aspects of CF care are increasingly well addressed particularly by the early institution of highly effective pancreatic enzyme supplementation, it is becoming increasingly common for patients with CF to have a raised BMI indeed almost a quarter of patients in one recent series were reported to be overweight or obese [38]. It may be that in future, insulin resistance will play a larger role in the development of dysglycaemia in this population.

\subsection{Other factors}

Not all CF individuals with pancreatic insufficiency develop CFRD, raising the possibility that other genetic factors might play a part [39].

However, with few exceptions, antibodies associated with the development of T1DM have not been found in CFRD [40-42]. Genetic variation in the CAPN10 gene, which encodes Calpain-10, may be a common risk factor for the development of both CFRD and T2DM [43], explaining 
in part why CF individuals with a familial history of T2DM are at increased risk of developing CFRD themselves [35] despite a lack of insulin resistance.

It seems likely that genetic modifiers have a significant role in determining to what extent dysglycaemia complicates the clinical course of an individual with cystic fibrosis. [35].

Glucose handling may also be disturbed in individuals with CF-related liver disease, particularly in severe cases where the hepatic uptake and storage of glucose is diminished, potentially rendering treatment for hypoglycaemia with exogenous glucagon ineffective. Chronic kidney disease, which may develop in CF because of recurrent uroliathiasis/nephrocalcinosis, repeated exposure to nephrotoxic medications or indeed as a complication of CFRD itself, may also effect glucose regulation.

Although it is unclear how important this is for overall blood sugar control, severe renal failure may lead to an accumulation of exogenous insulin therapy causing an apparent improvement in diabetic control (if judged by insulin requirements) as well as predisposing to hypoglycaemic episodes.

Incretin hormones released from the bowel are a major determinant of pancreatic responses in the post-prandial period, causing significantly more insulin to be secreted compared to the presence of glucose alone - this so-called incretin effect is lost in T2DM and a range of treatments that target components of the diffuse endocrine system have revolutionised the treatment of this condition. Whilst it has been shown that enteroendocrine cells are present in the expected amounts in the bowels of both animal models and direct intestinal biopsies from children with CF [44, 45], the incretin effect itself has not been definitively investigated in a CF setting, with various small-scale studies showing conflicting results. Whether or not the incretin system is attenuated in $\mathrm{CF}$, it is unclear whether augmenting its function pharmacologically would in any way improve the responses of a pathologically damaged pancreas.

Considering the mechanisms above, it is clear to see that although CFRD has a number of features in common with both T1DM and T2DM it is clearly significantly different to both - a comparison of CFRD with the more common forms of diabetes is shown in Table 1.

\begin{tabular}{llll}
\hline & CFRD & T1DM & T2DM \\
\hline Insulin resistance & Variable & Minimal & Significant \\
\hline Insulin deficiency & Relative & Absolute & Variable \\
\hline Typical BMI & Low & Low & High \\
\hline Complications & $\begin{array}{l}\text { Pulmonary, weight loss, } \\
\text { microvascular }\end{array}$ & Microvascular & Macrovascular \\
\hline Treatment & Insulin & Insulin & Oral agents $+/$ - insulin \\
\hline Ketosis & Very rare & Common & Rare \\
\hline
\end{tabular}

Table 1. Comparison of CFRD with other forms of diabetes 


\section{Screening and diagnosis}

Dysglycaemia is often detectable for several years prior to a formal diagnosis of CFRD, and early detection is important to prevent pulmonary function decline and weight loss developing $[6,8]$. Timely intervention with appropriate treatment may have a profound impact on a patient's health and well-being. In the next section, we will discuss the various methods of screening for CFRD.

\subsection{Diagnosis}

The World Health Organisation, Diabetes UK, American Diabetes Association and UK CF Trust all consider that the formal diagnosis of CFRD should be based on responses to a standard $75 \mathrm{~g}$ oral glucose tolerance test (see Table 2) and recommend that it is carried out annually from late childhood. Given the impact of pulmonary decline in early CFRD, tighter thresholds were discussed, but currently the general diagnostic criteria are still applied to CF.

- 2-hr 75g OGTT plasma glucose result $\geq 11.1 \mathrm{mmol} / 1$

- Fasting plasma glucose $\geq 7.0 \mathrm{mmol} / \mathrm{l}$

- HBA1C $\geq 6.5 \%$ (A1C $<6.5 \%$ does not rule out CFRD because this value is often spuriously low in CF.)

- Classical symptoms of diabetes (polyuria and polydipsia) in the presence of a casual glucose level $\geq 11.1 \mathrm{mmol} / 1$

Table 2. Diagnostic criteria for CFRD (any of the following)

These criteria were originally established by consensus opinion in 1998 [3]. The previously used sub-categorisation of the diabetic response to recognise whether or not fasting hyperglycaemia was also present (often abbreviated as CFRD FH+ and CFRD FH-, respectively), is now considered to be obsolete since the differentiation adds little to management [46].

The OGTT was originally designed to detect individuals, predominantly with insulin resistance, who were most likely to benefit from intervention aimed at preventing the microvascular sequelae of dysglycaemia. Although these do occur in CFRD, they are relatively late phenomena, where pulmonary decline (leading to increased mortality) occurs much earlier.

Furthermore, OGTT responses are highly variable in the CF population, where insulin resistance waxes and wanes and glucose absorption alters depending on a variety of factors peculiar to this cohort [17]. An opportunistic annual screen for diabetes carried out when an individual with CF is well may poorly reflect their overall glucose handling.

Screening for diabetes based on the 2-hour glucose value alone has been shown to be of less use in the CF population [47]. Furthermore, the dose of glucose used to stress the endocrine function of the pancreas during an OGTT may be inadequate in CF, where individuals have high calorific requirements and may routinely ingest carbohydrate loads much higher than the $75 \mathrm{~g}$ bolus typically used in the diagnostic test.

Given these facts, as well as the typical insulin response profile seen in patients with $\mathrm{CF}$, basing the diagnosis of, as well as screening for, CFRD by means of an OGTT, as is currently recom- 
mended [11], is clearly imperfect [48], leading to the conclusion that the under-diagnosis of clinically relevant dysglycemia is common.

Patients with CF may experience significant hyperglycaemic excursions shortly after eating, which then rapidly correct to the euglycaemic range as the delayed first-phase insulin response belatedly begins; this not only devalues diagnostic tests such as the OGTT (which uses the 2hour glucose value to diagnose diabetes) but also means that measures of average glucose control, such as glycosylated haemoglobin levels (the HbA1c test), may be misleadingly normal or even low in this group and therefore of much less clinical relevance in CFRD than in other forms of diabetes [49]. One study from Denmark found only $16 \%$ of patients had elevated $\mathrm{HbA} 1 \mathrm{c}$ values at the time CFRD was diagnosed [50]; others have found similar results [49]. The utility of $\mathrm{HbA1c}$ may be further devalued by the increased red cell turnover commonly seen in CF patients $[49,51]$. As progressive decline in pancreatic endocrine function in the CF population is related to increased morbidity years before a formal diagnosis of CFRD is made by OGTT $[8,9,10]$ and given that $\mathrm{HbA} 1 \mathrm{c}$ is at best an unreliable marker of glucose handling [49], other methods of assessing clinically important dysglycaemia, such as 1-hour OGTT result, serial glucose monitoring or Continuous Glucose Monitoring (CGM), have now been advocated as better markers of clinically relevant dysglycaemia.

Elevation of 1-hour glucose level during an OGTT has been shown to be a better predictor of declining pulmonary function than a standard 2-hour result [9] as well as correlating better with other methods of diabetic screening [53]. Currently, the UK CF Trust advocates the use of both a standard 2-hour OGTT as well as a period of serial glucose monitoring (that entails checking levels before and 2-hours after meals and at bedtime for several days) to establish if clinically relevant hyperglycaemia requiring treatment is present [49].

For effective serial glucose monitoring to take place, patients must be empowered and educated with regards to the technique including timing, hand washing, correct sampling procedures as well as meter and strip management. Misleading results have been documented [54] due to poor technique, therefore patient-recorded measurements should be considered with a degree of caution, particularly one-off abnormal readings. Additionally, there is a risk that patients, fearful of a further increase in their treatment burden associated with being diagnosed with CFRD, will report lower glucose readings, fail to document high levels or simply fabricate results altogether.

However, a CGM system circumvents many of the inherent weaknesses associated with selfreported serial monitoring whilst enabling an accurate glucose profile to be established. Worn for a period of 3-5 days typically on the arm or abdomen, a CGM system consists of a transmitter with a flexible plastic sensor which sits just below the skin allowing regular measurement of interstitial glucose. Readings are sent wirelessly to a receiver automatically every minute without any user input, although an occasional finger-prick reading is required to calibrate readings against blood glucose levels.

The use of CGM has been validated in the CF population [55] whilst others have shown that it may be superior to other modes of screening for dysglycaemia [56]. The visual output obtained from a CGM can be a useful tool in highlighting sugar control issues with patients 


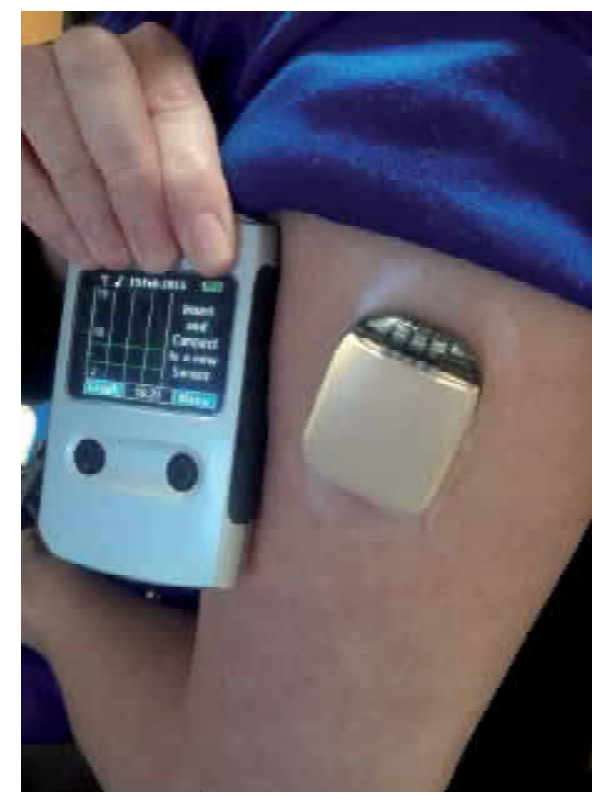

Figure 3. CGM in situ with separate base unit

and allow for highly individualised treatment regimens to be decided. A positive CGM result - that is a value of more than $7.87 .8 \mathrm{mmol} / \mathrm{l}$ for over $4.5 \%$ of the test - is associated with a worse prognosis in terms of CF-specific endpoints (i.e. pulmonary function and weight) as well as being a better predictor of future risk of development of CFRD than an OGTT [57], which has been shown to give unreliable results when used as a screening tool [58].

\section{Clinical features, signs and symptoms}

The biochemical and clinical detection and diagnosis of CFRD can be difficult, since symptoms of polyuria, polydipsia and weight loss only occur in one third of cases [8]. An unexplained decline in pulmonary function or difficulty maintaining or gaining weight should alert health care professionals to the possibility of underlying significant dysglycaemia, impaired glucose tolerance or even CFRD. Poor weight gain or weight loss can often be identified in the preceding 12 months prior to a diagnosis of CFRD [57].

\section{Consequences of dysglycaemia in $\mathrm{CF}$}

Multiple studies and reviews of large registry datasets have demonstrated a clear link between dysglycaemia and poor outcomes in pulmonary health in CF $[3,5,43]$, but the mechanism by which hyperglycaemia, often intermittent rather than sustained in this population, causes the 
observed decline in lung function is less clear. Plausible theories include direct pulmonary damage or suppression of various components of the immune system in the setting of hyperglycaemia leading to an increased risk of pulmonary infections which might also be more severe in nature [14], as has been demonstrated in other types of diabetes [44]. Insulin therapy has been shown to reduce sputum pathogen levels in CF patients [45]. It has also been hypothesised that the predominant factor in the decline in both respiratory function and nutritional status is a direct consequence of the altered protein metabolism experienced by $\mathrm{CF}$ patients who become relatively deficient in insulin therefore losing its important anabolic effects [14]. CFRD has been considered to be a risk factor for the development of distal intestinal obstruction syndrome in cystic fibrosis patients [59]; however, it is unclear how strong the association really is between these two conditions [60] or what causal mechanism might be involved.

\subsection{Microvascular complications}

Microvascular complications may develop in CFRD leading to the typical associated pathologies (retinopathy, nephropathy and neuropathy), any of which may significantly impact the on-going management of the condition, particularly the development of visual impairment, gastroparesis and renal failure.

Where glucose control has deteriorated to the point that fasting hyperglycaemia is present (a relatively rare occurrence in the CFRD population), a significant proportion will also have developed retinopathy and microalbuminaemia (16\% and $14 \%$, respectively) [61]. Almost half of all CFRD patients will display some degree of neuropathy, in most cases where the dysglycaemia has been present for over a decade [43].

Examples of autonomic dysfunction and gastroparesis have been reported [61], although the latter condition may be difficult to differentiate from the delayed gastric emptying that occurs in up to $50 \%$ of the $\mathrm{CF}$ population regardless of their glucose tolerance [43].

Overall, the prevalence of microvascular complications is less in CFRD than in other forms of diabetes, possibly due to the partially preserved endogenous insulin production and sensitivity leading to relatively short periods of hyperglycaemia.

\subsection{Macrovascular complications}

Although there is nothing to suggest dysglycaemia is not a significant risk factor for the development of macrovascular complications in individuals with $\mathrm{CF}$, as it is in other populations, at present their occurrence very infrequently complicates the management of CFRD.

Although there are case reports of both coronary artery disease and strokes occurring in diabetic CF patients $[62,63]$, there is at present no evidence of excess mortality attributable to cardiovascular or cerebrovascular disease despite these patients often having co-existing dyslipidaemia.

Currently, the consequences of significant dysglycaemia are likely to manifest earlier and more profoundly in other ways, e.g. deteriorating lung function, but as the life expectancy of the 
general CF population continues to rise there is likely to be both a concurrent increase in the incidence of CFRD and ultimately macrovascular complications.

\section{Treatment and management}

Treatment of CFRD is currently exclusively limited to insulin therapy, since by the time CFRD is formally diagnosed the predominant issue is one of failure of insulin secretion. Evidence of improved outcomes with insulin therapy is established for those who have a positive OGTT with and without fasting hyperglycaemia $[2,39,64]$. It is also recommended that insulin is initiated in those patients that exhibit diabetic responses whilst suffering an infective exacerbation [11], which presumably reflects a group with poor pancreatic reserve, meaning that they are unable to respond adequately when peripheral insulin resistance increases due to higher levels of inflammation or treatment with corticosteroids. The UK CF Trust (2004) guidelines for the management of CFRD [11] have produced recommendations for instigation of treatment, which are as follows; Treatment should be considered:

- When impaired glucose tolerance on OGTT is associated with weight loss or deteriorating clinical condition

- When there are episodes of transient hyperglycaemia

- When a diabetic glucose tolerance on OGTT, but normal glucose monitoring, is associated with weight loss or deteriorating clinical condition

Definite indications for initiating treatment are:

- CF-related diabetes, that is, diabetic OGTT and/or regular hyperglycaemia

- Pregnancy with impaired glucose tolerance or diabetes

Better awareness and more aggressive management of CFRD have led to a considerable improvement in outcomes over the last 5 years including mortality [2]. As discussed above, the concept of truly normal glucose tolerance in the majority of adult CF patients, especially if pancreatic-insufficient, is a fallacy and all such patients could be reasonably considered in a 'pre-diabetic' state; however, the role of early treatment to prevent CFRD is less clear. Small studies have previously suggested only a trend towards improvement in various outcomes when CF patients with impaired glucose tolerance (IGT) received long-acting insulins [65].

As total insulin secretion is often preserved or only marginally decreased in non-diabetic $\mathrm{CF}$ patients, the use of insulin therapy in such a group may not be as efficacious and any benefits must be weighed against the increased treatment burden (which is considerable for the vast majority of CF patients) and the risk of hypoglycaemia.

However, there is now increasing evidence to support initiation of treatment in non-diabetic groups with improvement in pulmonary and other clinical outcomes seen after insulin treatment was started [64, 66, 67], particularly those with positive CGM results [57], although 
it is currently too early to know if such benefits are sustained long-term or will impact upon mortality rates.

The treatment of CFRD can prove challenging with treatment strategies having to constantly be changed due to the variability and disease progression of CF. There are many different variables associated with $\mathrm{CF}$ that have to be taken into consideration when managing CFRD.

\subsection{Pharmacological treatments of CFRD}

Whenever treatment for dysglycaemia in CF patients is initiated, the only modality with an established evidence base is insulin therapy. Biguinide therapy potentially offers antiinflammatory activity as well as anti-diabetic effects and was shown to improve glucose control in 4 patients without significant side effects over a 10-year period [68]. However, given that insulin resistance is not the predominant abnormality in stable CF patients [36], coupled with concerns about an increased risk of hepatitis, lactic acidosis, weight loss and pancreatitis [69], metformin has not been widely used in this cohort.

A small study $(n=12)$ using acarbose in a group of CF patients with IGT receiving inpatient antibiotics for a pulmonary exacerbation showed an improvement in glucose profile compared to placebo but also a very high incidence of GI side effects [70].

Secretagogues, such as the sulphonylureas, have been shown to be of little benefit in a CF setting whilst increasing the risk of symptomatic hypoglycaemia in this group [71].

Dietary modification can play a role in decreasing very high post-prandial glucose excursions in CF patients and Balzer et al. have suggested that adopting a low glycaemic index diet could be advantageous in CFRD [72]. As such, insulin remains the only recommended treatment for CFRD [11]; however, even the use of short-acting insulin in a bolus regime, attempting to control post-prandial glucose excursions, still risks symptomatic hypoglycaemia developing as endogenous hormone levels belatedly rise.

The incretin system is particularly important in the post-prandial handling of carbohydrate; therefore, agents that enhance its effects are of considerable interest in the management of CFRD, particularly as theoretically at least they have a much lower propensity to cause hypoglycaemia. However, the widespread use of these agents has not yet been investigated in a CF-specific setting, although initial small-scale use seems to have been well tolerated [73].

\subsection{Insulin regimes for insulin therapy}

Different types of insulin are available for use to treat CFRD. Their different modes of action need to be correlated with glucose profiles and individual requirements of the patient in order to manage glucose levels effectively.

The main categories of insulin are:

- Long-acting insulin analogues - Normally administered once daily with a duration of 18-24 hours, achieving a steady state after 2-3 days (e.g. insulin glargine; detemir or degludec). 
- Intermediate insulin - Onset of action 2-3 hours with a duration of 16-35 hours (e.g. insulin isophane).

- Short-acting insulin - Onset of action 30-60 minutes and duration of up to 8 hours (e.g. soluble insulin).

- Rapid-acting insulin analogues - Onset of action 15 minutes and duration of action 2-5 hours (e.g. insulin aspart, lispro or glulisine).

- Pre- mix insulin - Onset of action 30-60 minutes duration up to 24 hours (e.g. biphasic isophane insulin).

- Pre-mix insulin analogue - Onset of action 15 minutes duration up to 24 hours (e.g. biphasic insulin aspart, lispro).

Typically, either a basal/bolus or a combination of both is used to treat CFRD [74].

Short acting rapid insulin before meals remains the insulin of choice for those without fasting hyperglycaemia.

Taking into account treatment burden, long lasting basal insulin needs to be considered and is frequently given [74].

Pre-mix insulin is used in some cases although with the variability in eating patterns this may prove detrimental.

\subsection{Multidisciplinary approach to management}

When diagnosing and managing patients with CFRD, it is of paramount importance that the multidisciplinary team have excellent knowledge relating to this complication. In ideal circumstances an endocrinologist with experience of CF will form part of the regular multidisciplinary team caring for patients, but in many centres the task of coordinating diabetic care will be delegated to a specialist nurse.

Communication is crucial to ensure patients are fully informed about the condition, particularly the differences between CFRD and other types of diabetes as patients may come with pre-conceived notions that could impact their engagement with treatment and on-going management, e.g. undertake wholly inappropriate calorie restriction.

Wherever possible, both the specialist CF dietician and clinical psychologist should be involved at the earliest opportunity to ensure the best possible expert care and management for the patient.

\section{Screening for complications in CFRD}

An important aspect of managing CFRD is effective screening for the development of complications. As such it is recommended that at least annually patients with CFRD have the following assessed: 
- Clinical history - number of admissions with reasons

- Height, weight and BMI

- Pulmonary function

- Blood pressure

- Alcohol and smoking

- Hypoglycaemia - identify cause and optimise treatment

- Exercise

- Foot examination - pedal pulses, sensory and vibration check.

- Sexual dysfunction

- Frequency of distal intestinal obstruction syndrome (DIOS)

- Full dietetic review - Meals, snacks, enzymes, supplements, feeds.

- Insulin therapy

- Injection technique.

- Insulin site check

- Home blood glucose monitoring.

- Psychosocial support

- Urine sample for Microalbuminuria

- $\mathrm{HbA} 1 \mathrm{c}$

- Urea and electrolytes, creatinine clearance (selected cases)

- Lipid profile

- Retinopathy screening referral

Additionally, annual screening is an opportunity to identify educational gaps and discuss how the patient is coping with their CFRD.

\section{The challenges of CFRD}

The distinct glucose profile typically seen in CFRD presents clear challenges not only for establishing the diagnosis in the first place but also for managing the condition in general.

\subsection{Dietary considerations}

The primary aim of nutritional management in relation to CFRD is the achievement of a normal nutritional status [3]. CFRD has different and conflicting dietary recommendations from that 
of type 1 or type 2 diabetes. CFRD patients have up to $150 \%$ the calorific requirements of other diabetic patients and require a diet containing both high fat and protein levels.

Patients with CF require regular snacks in between meals in order to meet their increased metabolic requirements and often resort to consuming foodstuff that is high in refined sugars - such as so called 'energy drinks' - in order to maintain their weight, especially as it obviates the need to medicate with enzyme supplements [75].

Ingestion of products that contain even modest quantities of highly refined sugar can not only cause glucose excursions but may also precipitate a reactive hypoglycaemic episode - a phenomenon that can be seen during $7-15 \%$ of diagnostic OGTTs [24, 76].

The use of a diet rich in carbohydrates that have a low glycaemic index (GI) is encouraged in type 2 diabetics both to aid blood glucose control and weight reduction, however little robust evidence exists of benefit from a similar approach in a CFRD population and there are concerns that low GI diets might lead to inappropriate weight loss in this group [72].

Recommendations from American researchers have suggested that carbohydrate counting may have a role in CFRD [3] however in other countries this approach is reserved for T1DM alone, with the CF dietetic community in the United Kingdom favouring regular meals and snacks containing a mixture of both complex and refined carbohydrates be taken with or just after eating other foods [75]- an approach that anecdotally can reduce both the post-prandial glucose excursions as well as subsequent reactive hypoglycaemia occurring.

Dietary assessment is therefore an important part of the management of CFRD particularly to assess and modify refined sugar intake whilst ensuring overall calorific requirements and a healthy weight are maintained.

\subsection{Exercise}

Regular exercise forms an important cornerstone of the general management of CF and is widely advocated [46] although direct evidence that it specifically improves overall blood sugar control in CFRD is lacking [77] although there is an on-going trial exploring this currently [78] CFRD patients need to be aware of the risk of precipitating hypoglycaemia during exercise and should be educated about extra monitoring during times of exertion.

\subsection{Liver disease}

CF affects all of the major organs in the body including the liver and the prevalence of CFRD is higher in patients who have liver disease [79]. As discussed previously significant liver disease may lead to a reduction in hepatic glycogen stores, exacerbating the risk of symptomatic hypoglycaemia developing and potentially reducing the response to exogenous glucagon used to treat such episodes. In addition, although it may be difficult to quantify, CFliver disease could lead to subtle impairments of hepatic insulin secretion and catabolism which may contribute to the overall dysglycaemia suffered by this group [79]. 


\subsection{Hypoglycaemia}

As mentioned elsewhere in this chapter hypoglycaemia is not uncommon in CFRD [24]. Furthermore, it has been demonstrated that hypoglycaemia awareness is also impaired as a result of frequent subclinical hypoglycaemic episodes and a diminished glucagon response [32]. Therefore, careful consideration must be given to when blood sugar monitoring is carried out as well as the timing, type and amount of insulin used for treatment.

\subsection{Corticosteroids}

Oral corticosteroids (e.g. prednisolone) are frequently used during pulmonary exacerbations in $\mathrm{CF}$, and the concomitant elevation in blood glucose may necessitate a change in diabetes management in these patients. Patients may also require increased supervision and treatment during times of infection where blood glucose levels often fluctuate rapidly. There is evidence that CF patients with normoglycaemia exhibit diabetic glucose tolerance during pulmonary exacerbations [80]. This is likely to be due to the stress of infection and inflammation that unmasks the early alterations in glucose homeostasis [81]. Additionally, when infection subsides and patients stop corticosteroids, blood glucose can dramatically drop causing hypoglycaemia and careful support and advice is required during this time.

\section{Future developments}

There are a number of areas currently at a research stage that may be of benefit in the management of CFRD.

Many centres are moving away from a reliance on the standard 2-hour OGTT to diagnose CFRD, with the use of CGM becoming more widespread to detect clinically significant dysglycemia at an earlier stage.

A number of clinical trials are exploring the use of incretin based therapy in individuals with CF $[82,83]$ and such therapies theoretically offer an ideal combination of glucose lowering effects with a smaller risk of precipitating hypoglycaemia, especially as concerns about the risk of pancreatitis associated with these type of medications have somewhat lessened recently [84].

Ultimately novel experimental treatments being developed for other forms of diabetes such as stem cell implantation may be applicable to individuals with CFRD.

\section{Author details}

Paula Dyce, Gareth Huw Jones and Martin J Walshaw*

*Address all correspondence to: mwalshaw@doctors.org.uk

Adult CF Centre, Liverpool Heart \& Chest Hospital, United Kingdom 


\section{References}

[1] Van den Berg JM, Kouwenberg JM, Heijerman HG. Demographics of glucose metabolism in cystic fibrosis. J Cyst Fibros. 2009;8(4):276-9.

[2] Moran A, Dunitz J, Nathan B, Saeed A, Holme B, Thomas W. Cystic fibrosis-related diabetes: current trends in prevalence, incidence, and mortality. Diabetes Care. 2009;32(9):1626-31

[3] Moran A, Hardin D, Rodman D, Allen HF, Beall RJ, Borowitz D, et al. Diagnosis, screening and management of cystic fibrosis related diabetes mellitus: a consensus conference report. Diabetes Res Clin Pract. 1999;45(1):61-73.

[4] Bismuth E, Laborde K, Taupin P, Velho G, Ribault V, Jennane F, et al. Glucose tolerance and insulin secretion, morbidity, and death in patients with cystic fibrosis. $\mathrm{J} \mathrm{Pe}$ diatr. 2008;152(4):540-5, 5.e1.

[5] Koch C, Rainisio M, Madessani U, Harms HK, Hodson ME, Mastella G, et al. Presence of cystic fibrosis-related diabetes mellitus is tightly linked to poor lung function in patients with cystic fibrosis: data from the European Epidemiologic Registry of Cystic Fibrosis. Pediatr Pulmonol. 2001;32(5):343-50.

[6] Milla CE, Warwick WJ, Moran A. Trends in pulmonary function in patients with cystic fibrosis correlate with the degree of glucose intolerance at baseline. Am J Respir Crit Care Med. 2000;162(3 Pt 1):891-5.

[7] Marshall BC, Butler SM, Stoddard M, Moran AM, Liou TG, Morgan WJ. Epidemiology of cystic fibrosis-related diabetes. J Pediatr. 2005;146(5):681-7.

[8] Lanng S, Thorsteinsson B, Nerup J, Koch C. Influence of the development of diabetes mellitus on clinical status in patients with cystic fibrosis. Eur J Pediatr. 1992;151(9): 684-7.

[9] Brodsky J, Dougherty S, Makani R, Rubenstein RC, Kelly A. Elevation of 1-hour plasma glucose during oral glucose tolerance testing is associated with worse pulmonary function in cystic fibrosis. Diabetes Care. 2011;34(2):292-5.

[10] Costa M, Potvin S, Hammana I, Malet A, Berthiaume Y, Jeanneret A, et al. Increased glucose excursion in cystic fibrosis and its association with a worse clinical status. $J$ Cyst Fibros. 2007;6(6):376-83.

[11] Cystic Fibrosis Trust: Management of Cystic Fibrosis Related Diabetes Clinical Care Guidelines (2004), CF Trust, Bromley.

[12] Moran A, Doherty L, Wang X, Thomas W. Abnormal glucose metabolism in cystic fibrosis. J Pediatr, 1998;133(1):10-17. 
[13] Adler AI, Shine BS, Chamnan P, Haworth CS, Bilton D. Genetic determinants and epidemiology of cystic fibrosis-related diabetes: results from a British cohort of children and adults. Diabetes Care. 2008;31:1789-94.

[14] Solomon MP, Wilson DC, Corey M, Kalnins D, Zielenski J, Tsui LC, Pencharz P, Durie $\mathrm{P}$, Sweezey NB. Glucose intolerance in children with cystic fibrosis. J Pediatr. 2003;142:128-132.

[15] Resolution of cystic fibrosis-related diabetes with Ivacaftor therapy. Am J Resp Critic Care Med. 2014;190(5):590-91.

[16] Couce M, O'Brien TD, Moran A, Roche PC, Butler PC. Diabetes mellitus in cystic fibrosis is characterized by islet amyloidosis. J Clin Endocrinol Metab. 1996;81(3):1267-72.

[17] Brennan AL, Geddes DM, Gyi KM, Baker EH. Clinical importance of cystic fibrosisrelated diabetes. J Cyst Fibros. 2004;3(4):209-22.

[18] Moran A, Diem P, Klein DJ, Levitt MD, Robertson RP. Pancreatic endocrine function in cystic fibrosis. J Pediatr. 1991;118(5):715-23.

[19] Cucinotta D, De Luca F, Arrigo T, Di Benedetto A, Sferlazzas C, Gigante A, et al. First-phase insulin response to intravenous glucose in cystic fibrosis patients with different degrees of glucose tolerance. J Pediatr Endocrinol. 1994;7(1):13-7.

[20] Lippe BM, Sperling MA, Dooley RR. Pancreatic alpha and beta cell functions in cystic fibrosis. J Pediatr. 1977;90:751-55 [PubMed].

[21] Arrigo T, Cucinotta D, Conti Nibali S, Di Cesare E, Di Benedetto A, Magazzù G, De Luca F. Longitudinal evaluation of glucose tolerance and insulin secretion in non-diabetic children and adolescents with cystic fibrosis: results of a two-year follow-up. Acta Paediatr. 1993;82:249-53 [PubMed].

[22] Lanng S, Thorsteinsson B, Røder ME, Orskov C, Holst JJ, Nerup J, Koch C. Pancreas and gut hormone responses to oral glucose and intravenous glucagon in cystic fibrosis patients with normal, impaired, and diabetic glucose tolerance. Acta Endocrinologica. 1993;128:207-214 [PubMed].

[23] Frase LL, Strickland AD, Kachel GW, Krejs GJ. Enhanced glucose absorption in the jejunum of patients with cystic fibrosis. Gastroenterology. 1985;88(2):478-84.

[24] Battezzati A, Battezzati PM, Costantini D, et al. Spontaneous hypoglycemia in patients with cystic fibrosis. Eur J Endocrinol. 2007;156:369-76.

[25] Hartling SG, Garne S, Binder C, et al. Proinsulin, insulin, and C-peptide in cystic fibrosis after an oral glucose tolerance test. Diabetes Res. 1988;7:165-169.

[26] Lombardo F, De Luca F, Rosano M, Sferlazzas C, Lucanto C, Arrigo T, et al. Natural history of glucose tolerance, beta-cell function and peripheral insulin sensitivity in cystic fibrosis patients with fasting euglycemia. Eur J Endocrinol. 2003;149(1):53-9. 
[27] Soejima K, Landing BH. Pancreatic islets in older patients with cystic fibrosis with and without diabetes mellitus: morphometric and immunocytologic studies. Pediatr Pathol. 1986;6(1):25-46.

[28] Löhr M, Goertchen P, Nizze H, Gould NS, Gould VE, Oberholzer M, et al. Cystic fibrosis associated islet changes may provide a basis for diabetes. An immunocytochemical and morphometrical study. Virchows Arch A Pathol Anat Histopathol. 1989;414(2):179-85.

[29] Swartz LM, Laffel LM. A teenage girl with cystic fibrosis-related diabetes, diabetic ketoacidosis, and cerebral edema. Pediatr Diabetes. 2008;9(4 Pt 2):426-30.

[30] Laguna TA, Nathan BM, Moran A. Managing diabetes in cystic fibrosis. Diabetes Obes Metab. 2010;12(10):858-64.

[31] Atlas AB, Finegold DN, Becker D, Trucco M, Kurland G. Diabetic ketoacidosis in cystic fibrosis. Am J Dis Child. 1992;146(12):1457-8.

[32] Drummond, R., Ross, E., Bicknell, S., Small, M. and Jones, G. Insulin therapy in patients with cystic fibrosis related diabetes mellitus: benefit, timing of initiation and hypoglycaemia. Pract Diab Int. 2011;28: 177-182. doi: 10.1002/pdi.1588.

[33] Yki-Jarvinen H, Sammalkorpi K, Koivisto VA, Nikkila EA. Severity, duration, and mechanisms of insulin resistance during acute infections. J Clin Endocrinol Metab. 1989;69(2):317-23.

[34] Hardin DS, Leblanc A, Marshall G, Seilheimer DK. Mechanisms of insulin resistance in cystic fibrosis. Am J Physiol Endocrinol Metab. 2001;281(5):E1022-8.

[35] Blackman SM, Hsu S, Vanscoy LL, Collaco M, Ritter SE, Naugton K, Cutting GR. Genetic modifiers play a substantial role in diabetes complicating cystic fibrosis. J Clin Endocrinol Metabol. 2009: 94(4):1302-1309.

[36] Mohan K, Miller H, Dyce P, Grainger R, Hughes R, Vora J, et al. Mechanisms of glucose intolerance in cystic fibrosis. Diabet Med. 2009;26(6):582-8.

[37] O'Riordan SM, Robinson PD, Donaghue KC, Moran A. Management of cystic fibrosis-related diabetes in children and adolescents. Pediatr Diabetes. 2009;10 Suppl 12:43-50.

[38] Hanna RM, Weiner DJ. Overweight and obesity in patients with cystic fibrosis: A center-based analysis. Pediatr Pulmonol. 2015;50:35-41. doi: 10.1002/ppul.23033

[39] Moran A. Cystic fibrosis-related diabetes: an approach to diagnosis and management. Pediatr Diabetes. 2000;1:41-8.

[40] Carrington M, Krueger LJ, Holsclaw DS Jr, Iannuzzi MC, Dean M, Mann D. Cystic fibrosis-related diabetes is associated with HLA DQB1 alleles encoding Asp-57- molecules. J Clin Immunol. 1994;14:353-58. 
[41] Minicucci L, Cotellessa M, Pittaluga L, Minuto N, d'Annunzio G, Azanzini MA, Lorini R. Beta-cell autoantibodies and diabetes mellitus family history in cystic fibrosis. J Pediatr Endocrinol Metab. 2005;18:755-60.

[42] Nousia-Arvanitakis S, Galli-Tsinopoulou A, Dracoulacos D, Karamouzis M, Demitriadou A. Islet autoantibodies and insulin dependent diabetes mellitus in cystic fibrosis. J Pediatr Endocrinol Metab. 2000;13:319-24.

[43] Moran A, Becker D, Casella SJ, Gottleib PA, Kirkman MS, Marshall, BS, Slovis B. The CFRD consensus conference Committee: Epidemiology, pathophysiology, and prognostic implications of cystic fibrosis-related diabetes. Diabetes Care. 2010;33(12): 2677-83.

[44] Hjelte L, Flodstrom-Tullberg M, Nilsson A, Wierup N, Sundler F. Neuroendocrine characterization of the intestine of F508del CFTR mice. J Cyst Fibros. 2011 June;10 Suppl 1: S69:A270.

[45] Buchanan KD, Kerr JI, Johnston CF, Redmond AO, Craig BG. The diffuse endocrine system in cystic fibrosis. Scand J Gastroenterol Suppl. 1983;82:155-63.

[46] Moran A, Brunzell C, Cohen RC, et al., the CFRD Guidelines Committee. Clinical Care Guidelines for Cystic Fibrosis-Related Diabetes: A position statement of the American Diabetes Association and a clinical practice guideline of the Cystic Fibrosis Foundation, endorsed by the Pediatric Endocrine Society. Diabetes Care. 2010;33(12): 2697-2708. doi:10.2337/dc10-1768.

[47] Dobson L, Sheldon CD and Hattersley AT. Conventional measures underestimate glycaemia in cystic fibrosis patients. Diabetic Med. 2004;21:691-96. doi: 10.1111/j. 1464-5491.2004.01219.x

[48] Walshaw M. Routine OGTT screening for CFRD - no thanks. J Royal Soc Med. 2009;102(Suppl 1):40-44. doi:10.1258/jrsm.2009.s19009.

[49] Holl RW, Buck C, Babka C, Wolf A, Thon A. HbA1c is not recommended as a screening test for diabetes in cystic fibrosis. Diabetes Care. 2000;23(1):126.

[50] Lanng S, Hansen A, Thorsteinsson B, Nerup J, Koch C. Glucose tolerance in patients with cystic fibrosis: five year prospective study. BMJ. 1995;311(7006):655-59. doi: 10.1136/bmj.311.7006.655.

[51] Godbout A, Hammana I, Potvin S, Mainville D, Rakel A, Berthiaume Y, Rabasa-Lhoret $\mathrm{R}$. No relationship between mean plasma glucose and glycated haemoglobin in patients with cystic fibrosis-related diabetes. Diabetes Metabol. 2008;34(6):568-73.

[52] Brennan AL, Gyi KM, Wood DM, Hodson ME, Geddes DM, Baker EH. Relationship between glycosylated haemoglobin and mean plasma glucose concentration in cystic fibrosis. J Cyst Fibros. 2006;5:27-31.pmid:16202666. 
[53] Dyce P, Jones GH, Walshaw M. Comparison of continuous glucose monitoring (CGM) and oral glucose tolerance testing (OGTT) in the detection of CFRD. Paediatr Pulmonol. 2014.1 (49):S38-574.

[54] Clarke S F, Foster JR. A history of blood glucose meters and their role in self-monitoring of diabetes mellitus. Br J Biomed Sci. 2012;69 (2): 83-93.

[55] O'Riordan SMP, Hindmarsh P, Hill NR, et al. Validation of continuous glucose monitoring in children and adolescents with cystic fibrosis: a prospective cohort study. Diabetes Care. 2009;32(6):1020-22. doi:10.2337/dc08-1925.

[56] Khammar A, Stremler N, Dubus JC, Gross G, Sarles J, Reynaud R. Value of continuous glucose monitoring in screening for diabetes in cystic fibrosis. Arch Pediatr. 2009 December;16(12):1540-6. doi: 10.1016/j.arcped.2009.09.007. Epub 2009 Oct 24. French.

[57] Hameed S, Morton JR, Jaffé A, Field PI, Belessis Y, Yoong T, et al. Early glucose abnormalities in cystic fibrosis are preceded by poor weight gain. Diabetes Care. 2010;33(2):221-6.

[58] Sterescu AE, Rhodes B, Jackson R, Dupuis A, Hanna A, Wilson DC, et al. Natural history of glucose intolerance in patients with cystic fibrosis: ten-year prospective observation program. J Pediatr. 2010;156(4):613-7.

[59] Colomboa C, Ellemunter HB, Houwenc R, Munckd A, Taylor C, Wilschanski MF. Guidelines for the diagnosis and management of distal intestinal obstruction syndrome in cystic fibrosis patients. J Cyst Fibro. 2011; 10(Suppl 2):S24-S28.

[60] Leichsenring M, Rueckel B, Meissner P, Kratzer W. The variability of carbohydrate antigen 19-9 (CA 19-9) levels in cystic fibrosis patients. J Cyst Fibro. 2014; 13(Supplement 2):S95.

[61] Schwarzenberg SJ, Thomas W, Olsen TW, Grover T, Walk D, Milla C, Moran A. Microvascular complications in cystic fibrosis-related diabetes. Diabetes Care. 2007;30:1056-61.

[62] Perrin FMR, Serino W. Ischaemic heart disease - a new issue in cystic fibrosis? J R Soc Med. 2010;103:S44-S48.

[63] Simmonds NJ, Cullinan P, Hodson ME. Growing old with cystic fibrosis - the characteristics of long-term survivors of cystic fibrosis. Respir Med. 2009 April;103(4):629-35. doi: 10.1016/j.rmed.2008.10.011. Epub 2008 Nov 20.

[64] Rolon MA, Benali K, Munck A, Navarro J, Clement A, Tubiana-Rufi N, Czernichow P, Polak M. Cystic fibrosis-related diabetes mellitus: clinical impact of prediabetes and effects of insulin therapy. Acta Paediatr. 2001;90:860-67.

[65] Minicucci L, Haupt M, Casciaro R, De Alessandri A, Bagnasco F, Lucidi V, et al. Slow-release insulin in cystic fibrosis patients with glucose intolerance: a randomized clinical trial. Pediatr Diabetes. 2012;13(2):197-202. 
[66] Dobson L, Hattersley AT, Tiley S, Elworthy S, Oades PJ, Sheldon CD. Clinical improvement in cystic fibrosis with early insulin treatment. Arch Dis Child. 2002;87(5): 430-31.

[67] Bizzarri C, Lucidi V, Ciampalini P, Bella S, Russo B, Cappa M. Clinical effects of early treatment with insulin glargine in patients with cystic fibrosis and impaired glucose tolerance. J Endocrinol Invest. 2006;29(3):RC1-4.

[68] Onady GM, Langdon LJ. Insulin versus oral agents in the management of Cystic Fibrosis Related Diabetes: a case based study. BMC Endocr Disord. 2006;6:4.

[69] Kongsuphol P, Cassidy D, Romeiras F, Schreiber R, Mehta A, Kunzelmann K. Metformin treatment of diabetes mellitus increases the risk for pancreatitis in patients bearing the CFTR-mutation S573C. Cell Physiol Biochem. 2010;25(4-5):389-96.

[70] Kentrup H, Bongers H, Spengler M, Kusenbach G, Skopnik H. Efficacy and safety of acarbose in patients with cystic fibrosis and impaired glucose tolerance. Eur J Pediatr. 1999;158(6):455-59.

[71] [Cochrane] Onady GM, Stolfi A. Insulin and oral agents for managing cystic fibrosisrelated diabetes. Cochrane Database Syst Rev. 2013;7:CD004730.

[72] Balzer BW, Graham CL, Craig ME, Selvadurai H, Donaghue KC, Brand-Miller JC, et al. Low glycaemic index dietary interventions in youth with cystic fibrosis: a systematic review and discussion of the clinical implications. Nutrients. 2012;4(4):286-96.

[73] Hellman, J. Fischier, A. Hollsing. Incretin-based treatment of diabetes related to cystic fibrosis: a case study. J Cyst Fibro. 2014;13 (2):S126.

[74] Kelly A, Moran, A. Update on cystic fibrosis related diabetes. J Cyst Fibro. 2013;(12) 318-31.

[75] Ashworth, F., et al. The management of cystic fibrosis related diabetes. Brit J Homecare 1. 1999;136-140.

[76] Radike K, Molz K, Holl RW, Poeter B, Hebestreit H, Ballmann M. Diabetes Care. 2011 April;34(4):e43. doi: 10.2337/dc10-2286. No abstract available.

[77] Dwyer TJ, Elkins MR, Bye PTP. The role of exercise in maintaining health in cystic fibrosis. Curr Opin Pulm Med. 2011;17 (6): 455-60.

[78] [ClinicalTrials.gov [Internet]. Bethesda (MD): National Library of Medicine (US).2000 Feb 29 -. Identifier NCT02127957, Effects of an Exercise Program Among CF Patients With Dysglycemia (FKEX); 2014 Apr 18 [cited 2015 Feb 13]; [about 4 screens]. Available from: https://clinicaltrials.gov/ct2/show/NCT02127957.]

[79] Holstein, A, et al. Clinical implications of hepatogenous diabetes in liver cirrhosis. J Gastroenterology Hepatol. 2002;17(6): 677-81. 
[80] Sc NN, Shoseyov D, Kerem E, Zangen DH. Patients with cystic fibrosis and normoglycemia exhibit glucose tolerance during pulmonary exacerbation. J Cyst Fibro. 2010; 9:199-204.

[81] Zeller, M., et al. Glycemia in acute coronary syndromes. Diabetes Metabol 2006;32: 2S42-2S47.

[82] ClinicalTrials.gov [Internet]. Bethesda (MD): National Library of Medicine (US). 2000 Feb 29 -. Identifier NCT00967798. Prevention of Cystic Fibrosis Diabetes. August 27, 2009 [cited February 13 2015] Available from: https:/clinicaltrials.gov/ct2/show/ NCT00967798

[83] ClinicalTrials.gov [Internet]. Bethesda (MD): National Library of Medicine (US). 2000 Feb 29 -. Identifier NCT01879228. Effect of Chronic Incretin-based Therapy in Cystic Fibrosis. June 12, 2013 [cited February 13 2015] Available from https://clinicaltrials.gov/ct2/show/NCT01879228

[84] [Pancreatic Safety of Incretin-Based Drugs - FDA and EMA Assessment. Amy G. Egan, M.D., M.P.H., Eberhard Blind, M.D., Ph.D., Kristina Dunder, M.D., Pieter A. de Graeff, M.D., B. Timothy Hummer, Ph.D., Todd Bourcier, Ph.D., and Curtis Rosebraugh, M.D., M.P.H. N Engl J Med 2014; 370:794-797]. 

Chapter 5

\title{
Cystic Fibrosis and Fertility
}

\author{
Rosaria Casciaro, Federico Cresta, \\ Federica Favilli and Laura Minicucci
}

Additional information is available at the end of the chapter

http://dx.doi.org/10.5772/60616

\begin{abstract}
In the last 20 years, the prognosis of cystic fibrosis (CF) has slightly increased and nowadays more than $50 \%$ of $\mathrm{CF}$ patients are adults. An obvious consequence of this deep change is the increasing question about fertility in both males and females.

Almost $97 \%$ of male CF patients are infertile, having significant anatomical abnormalities of the reproductive tract, in most cases a congenital bilateral absence of the vas deferens (CBAVD); even if anatomical defect plays an important role, cystic fibrosis transmembrane conductance regulator (CFTR) is directly involved in many aspects of male reproduction, with well-known consequences in spermatozoa capacitation and bicarbonate secretion.

Actually, male CF patients can become parents with assisted fertilization techniques: intracytoplasmic sperm injection (ICSI), currently the most used fertilization treatment worldwide, has dramatically improved the assisted reproduction outcomes for men with obstructive azoospermia.

Most CF women have a normal reproductive tract and may be able to conceive spontaneously, but multifactorial fertility problems can affect them also: the main cause could be the difficult transport of sperm through the female reproductive tract, secondary to thick secretions, but also lung function and nutritional status at the time of conception significantly influence their fertility.
\end{abstract}

Keywords: Cystic fibrosis, CFTR, Congenital bilateral absence of the vas deferens, Fertility, In vitro fertilization, Pregnancy 


\section{Introduction}

As the life expectancy of cystic fibrosis (CF) patients continues to increase, and more patients become adults with a chronic disease, researching the impact of this disorder on male and female infertility has become increasingly important.

Although most men with CF have significant anatomical abnormalities of the reproductive tract causing infertility, most women with CF have anatomically normal reproductive tracts and up to half of them may be able to conceive spontaneously.

Assisted reproductive technologies can help both infertile male and female patients with CF in achieving successful parenthood. In addition, for women more health characteristics including baseline pulmonary function have to be evaluated as predictors of health and pregnancy outcomes.

\section{Fertility in men with cystic fibrosis}

$\mathrm{CF}$ is a systemic illness that affects multiple organ systems, including lungs, endocrine and epithelial tissues, gastrointestinal system, pancreas, and reproductive tract. Because of the dramatic improvement made in prognosis in CF population in the last two decades, reproductive function has become one of the new red flags in the management of $\mathrm{CF}$ adult patients. Infertility in CF males has been extensively studied and found in most cases to be secondary to atrophy or malformation of the vas deferens, leading to an obstructive azoospermia (Figure 1).

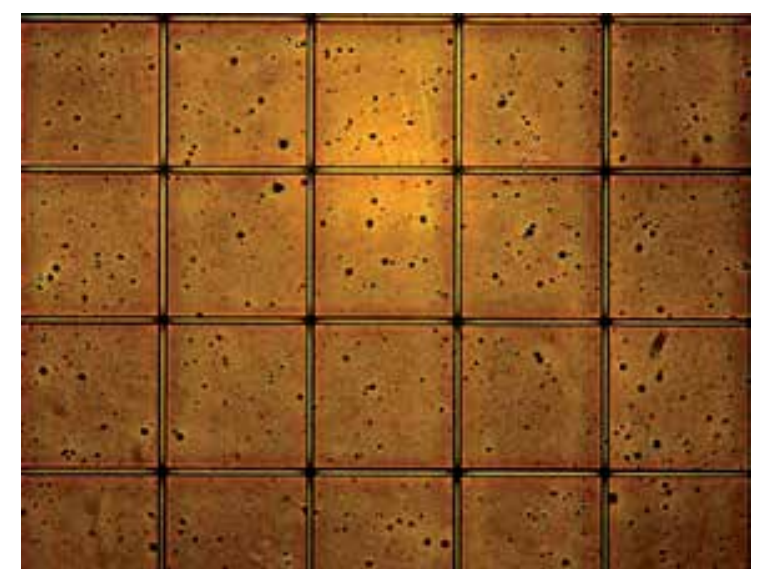

Figure 1. Spermiogram performed in our 34-year-old patient affected by CF documents azoospermia

\subsection{Pathogenesis}

Cystic fibrosis transmembrane conductance regulator (CFTR) is a gene located on chromosome 7 (7q31.2), encoding for a protein located in the apical membranes of epithelial cells; it was 
identified in 1989 and its role in the pathogenesis of CF is now well known. CF is a disease characterized by a defect in electrolyte and fluid transport in exocrine tissues; it could present several different clinical manifestations, including chronic lung disease, pancreas insufficiency, and infertility [1].

In literature, it is reported that almost $97 \%$ of male CF patients are infertile [2]: this infertility is primarily secondary to an obstructive azoospermia. The defective CFTR ion channel function causes an early obstruction of male genital tract, due to the dehydrated secretions: this mechanism drives to deep structural changes in reproductive tract, causing in most cases a congenital bilateral absence of the vas deferens (CBAVD). In early studies on CF adult patients with azoospermia, CBAVD was reported in all the population studied. At the light of these findings, Holscalaw et al. [3] speculated a unique genetic cause of CF and CBAVD. Usually, the proximal part of epididymis is present and this allows the sperm collection in CF patients to obtain spermatozoa.

Obstructive dysfunction is not the only cause of infertility in males with CF: further studies demonstrated that CFTR may also play a critical role in spermatogenesis and sperm maturation [4]; an increased CFTR mutation frequency in a population of men with reduced sperm quality is also reported [5]. Histological examination of CF testicular biopsies shows a wide range of spermatogenesis abnormalities, including a decreased count of mature spermatids and maturation arrest. These findings could be the expressions of CFTR abnormalities in seminiferous tubules or spermatozoa.

CFTR plays a role in many aspects of male reproduction, with well-known consequences in CBAVD and CF. It has not only ion channel functions but also it is a versatile signaling molecule and interacts with more than 180 other proteins. CFTR is expressed throughout the whole genital tract [6], but we do not know yet what is CFTR's role in the male accessory glands other than the epididymis.

A significant role in sperm function was also suggested by the involvement of CFTR in uterine $\mathrm{HCO}_{3}{ }^{-}$secretion and its effect on the fertilizing capacity of sperm [7]. CFTR is present in human sperm and it is involved in both sperm motility and capacitation phases. CF mice sperm has reduced sperm motility and capacitation with reduced fertility rate in vitro and in vivo [8]: these findings suggest a significant role of CFTR in sperm functions also.

\subsection{CBAVD}

CBAVD is a congenital condition in which vas deferens fails to develop properly, causing male infertility because of the total obstruction of reproductive tract. CBAVD accounts for approximately $1-2 \%$ of all infertility in males and is the result of genetic abnormalities [9]. More than half of the men with CBAVD (62-80\%) carry a CFTR mutation and this condition is considered to be one of the most common CFTR-related diseases. Anatomical abnormalities include bilateral or unilateral absence of the vas deferens and seminal vesicles anomalies.

Subjects with CBAVD usually have no clinical symptoms of CF, but the finding of subclinical CF features is not uncommon (mild chloride elevation at sweat test, chronic sinusitis, nasal polyps) and actually many experts consider CBAVD as a mild CF form [9]. 


\subsection{Other genital abnormalities}

Genital abnormalities may develop early in $\mathrm{CF}$, but in children these are less common than described in adults. In 2002, Blau et al. [10] described genital abnormalities in male children with $\mathrm{CF}$, performing pelvic and scrotal ultrasonography in $12 \mathrm{CF}$ boys aged 2-12 years. They found seminal vesicles hypoplasia, testicular microlithiasis, and abnormalities of the epididymal head, such as cysts, hypo-, or hyper-echogenicity. These findings are more frequent in pancreatic-insufficient than in pancreatic-sufficient $\mathrm{CF}$ patients. The reported experience represents a very small population of CF children, and larger longitudinal studies will be necessary to better define the onset and progression of urogenital abnormalities in CF males.

In CF adults, testes are usually symmetric and have a normal echogenicity, but mild inhomogeneity or striated appearance can be documented with ultrasounds. Focal inhomogeneities seem to be rare, as testicular nodules. Didymus cysts, epididymal cysts (also multiple) with sediments and/or calcifications are common. Usually, vas deferens is absent, but a structure attributable to spermatic cord could be revealed bilaterally or unilaterally in some cases, usually with a significant stenosis.

Several features can influence the anatomical genital phenotype in these patients (as genotype, clinical features, age), and further studies will be crucial to find risk factors and significant correlation for these abnormalities in CF.

\subsection{Fertility management in men with $\mathrm{CF}$}

Over the last 20 years, the relevant improvement in survival of CF patients and the concomitant development of new assisted conception methods have significantly increased the opportunities for these patients to become parents. It is therefore very important to start an early and effective management of fertility issues in males with CF.

The clinical management of reproductive issues in males affected by CF has to begin during puberty with periodic evaluation of testicular volume/consistency and all the other virilization signs, indicating a congruous testosterone production. Hormonal levels (as LH, FSH, and testosterone serum concentration) are usually normal in male $\mathrm{CF}$, indicating a regular spermatogenesis in most cases. It could be also useful as a deeper examination to detect the presence of vas deferens (usually palpable in the upper portion of scrotum), but the definitive diagnosis of CBAVD can be made with radiological exams.

There is not a considerable literature about the morphological study of the scrotum in adult CF patients, but trans-rectal ultrasounds could be considered a good instrument to evaluate abnormalities in shape, volume, and structure of testes, epididymis, and spermatic cord.

Also scrotal ultrasound with high-definition instruments is non-invasive and executable without any discomfort for the patient and could be useful in order to analyze the extra pelvic portion of the vas deferens (from the groin to the testicle) and all the scrotal structures.

The diagnosis of azoospermia could be simply supported and confirmed by semen analysis. In case of seminal vesicles abnormalities, semen analysis will also show an acid $\mathrm{pH}$, due to lack of fructose concentrations in sperm, and often the volume of ejaculate is low: in these 
cases, the sperm is produced by the prostate, with no contribute from vesicles. Typically, men with the absence of the vas deferens have low-volume (often less than $0.5 \mathrm{ml}$ ) and acidic semen [11].

\subsection{Assisted reproduction in men with $\mathrm{CF}$}

\subsubsection{Counseling}

CF men and their partners, who want to start assisted reproduction treatments, need an adequate genetic, medical, and psychological counseling. Genetic tests have to be performed in the patient (if not already done) and in the partner, in order to define the risk of generating an affected child. In case of incomplete genetic assessment or high risk of CF recurrence, prenatal or pre-implantation genetic diagnosis could also be recommended; it is important to consider ethic and legislative issues, because in some countries pre-implantation genetic tests are not allowed. All these procedures have relevant psychological, ethical, and sanitary costs, so alternative measures (as adoption or use of sperm donor) could be considered.

Another important issue is the prognosis of the potential father affected by CF. This delicate aspect asks the intervention of CF-clinician and psychologist, together with an experienced fertility specialist.

\subsubsection{Sperm collection}

Sperm retrieval is a procedure used to obtain sperm for fertility purposes. In general, it is necessary in case of azoospermia or if men are unable to ejaculate. In almost all cases, sperm retrieval must be utilized in combination with in vitro fertilization (IVF) and intracytoplasmic sperm injection (ICSI) for reasonable pregnancy rates to be obtained [12].

The sperm collection could be performed by aspiration from epididymis or directly from the testis. In CF the proximal part of the epididymis is usually present, so the procedure is usually quietly simple. The techniques of collection include:

- Percutaneous epididymal sperm aspiration (PESA) from the caput of the epididymis, usually simple to localize and good reservoir of sperm; aspiration is performed with fine needle after local anesthetization and is usually fast and well tolerated by the patient

- Microsurgical epididymal sperm aspiration (MESA)

- Percutaneous testicular aspiration (TESA) performed with local anesthetization (Figure 2) as for PESA

- Testicular surgical biopsy (Figure 3)

- Testicular sperm extraction (TESE), an in vivo microdissection of testicular tubules finalized to the identification of normal tubules (Figure 4), suitable for an effective sperm collection 


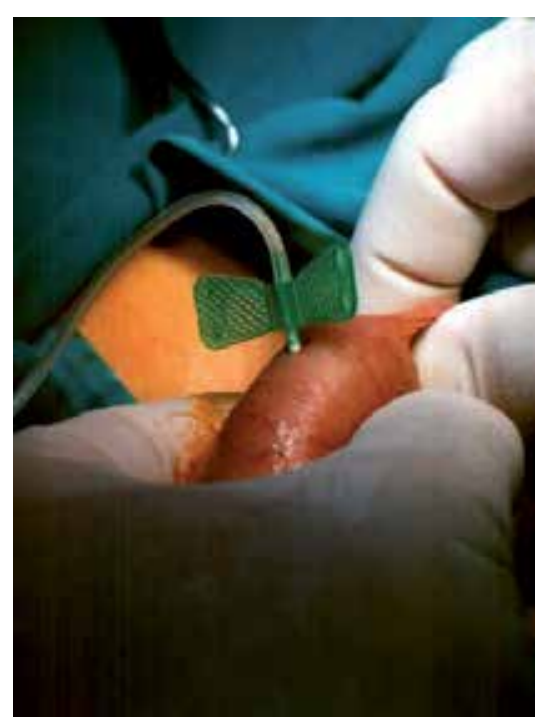

Figure 2. Percutaneous testicular aspiration (TESA) performed with local anesthetization

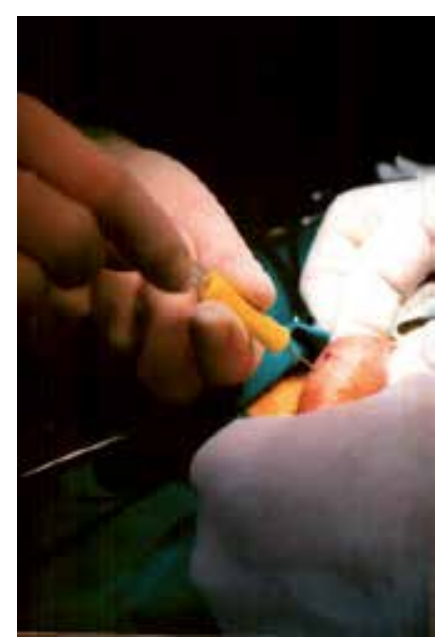

Figure 3. Percutaneous testicular biopsy

Cryopreservation of sperm is possible and represents a good chance for future use, but usually repeated epididymal aspiration is required. These procedures are usually performed on the same day of the partner's oocytes collection (Figure 5) to assure high counts of motile sperm in the time of fecundation. 


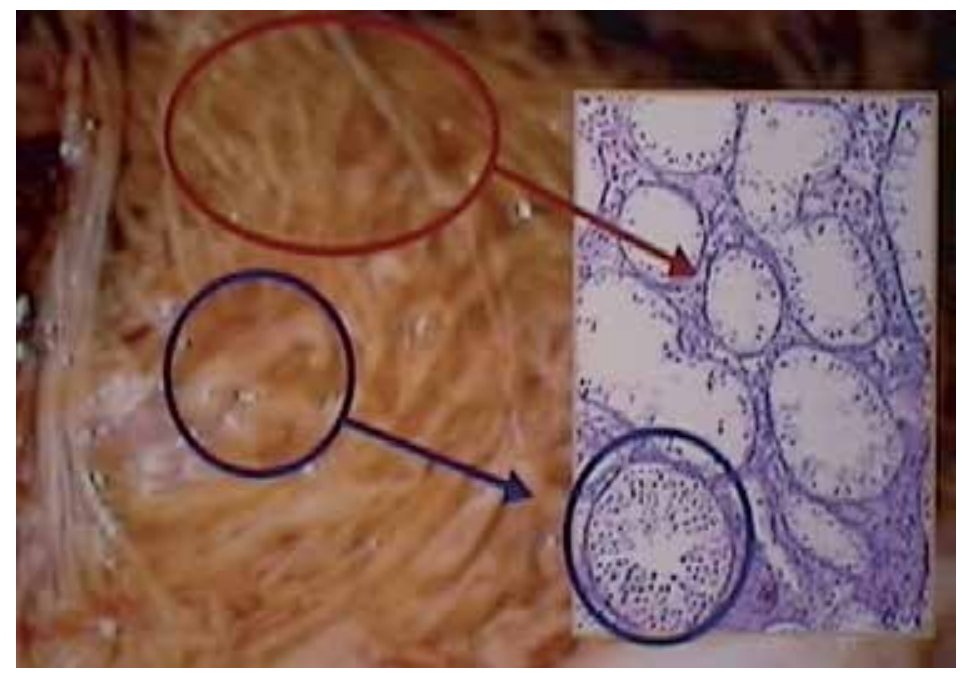

Figure 4. Microsurgical identification of tubules with spermatogenesis (TESE technique)

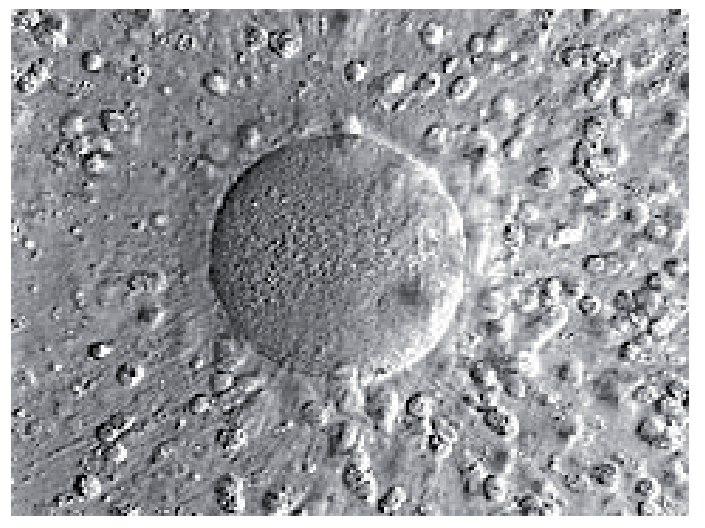

Figure 5. Oocytes culture

\subsubsection{Assisted reproduction treatments}

The first experience of in vitro fertilization with sperm obtained by epididymal aspiration was in 1985 [13]; in the next years, several experiences also in CBAVD patients have been reported [14]. In that period, the fertilization rates were low (less than $20 \%$ ), with very poor birth rates.

A dramatic increase in assisted reproduction outcomes for men with obstructive azoospermia has been represented by ICSI, introduced first in the 1990s. The first large experience with ICSI in the United States was published by Sherins et al. in 1995 [15]. This technique is generally performed following an in vitro fertilization procedure to extract one to several oocytes from a woman. The procedure is done under a microscope, using multiple micromanipulation devices, in order to allow the direct injection of a single sperm in a human oocyte. 
This technique is currently the most used fertilization treatment worldwide and it has deeply increased the chances of successful fertilizations and pregnancies; ICSI technique allows to have the same fertilization and pregnancy rates between CF males and men with no vas deferens obstructive disease (Figure 6).

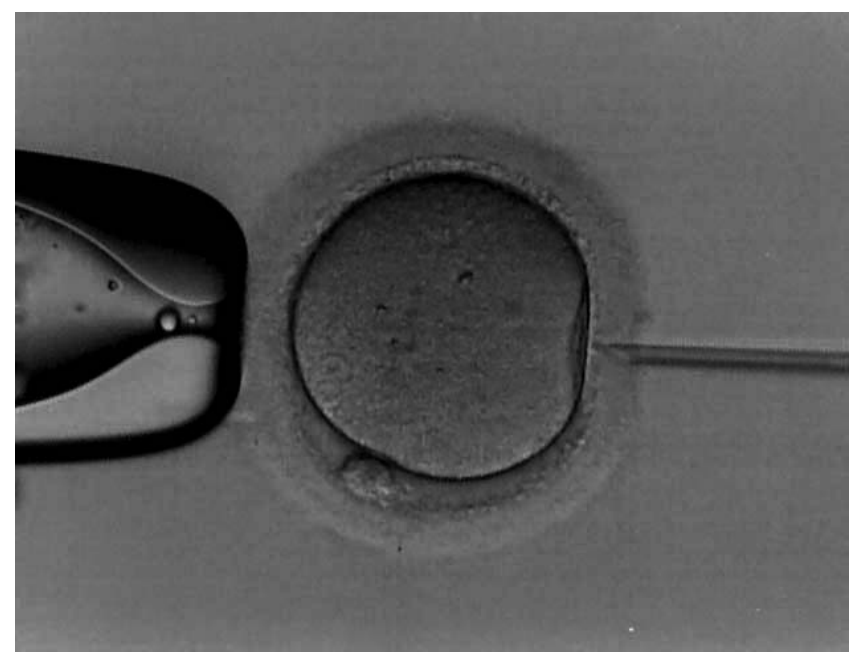

Figure 6. ICSI technique

\section{Fertility in women with cystic fibrosis}

Although most men with CF have significant anatomical abnormalities of the reproductive tract, most $\mathrm{CF}$ women have an anatomically normal reproductive tract and may be able to conceive spontaneously, but in literature it is reported that only slightly more than half of them have spontaneous pregnancies. The fertility problems in CF female are multifactorial: the main cause would be the difficult transport of sperm through the female reproductive tract, secondary to thick secretions; but certainly also the underlying medical conditions (especially lung function and nutritional status) have a major impact on fertility in these patients. The improved health and longevity of CF women naturally leads to an increased number of CF women who become or desire to become pregnant.

The first reported successful pregnancy in a CF woman was in 1960 [16], and in 196613 pregnancies in 10 different patients were reported [17]. These early reports were discouraging. However, with aggressive management of infections and significant improvement in pulmonary and nutritional interventions, pregnancies today are well tolerated in $\mathrm{CF}$, especially in women with mild to moderate disease $[18,19]$. The North American CF Registry reported in 2007 that $3-4 \%$ of CF women over 17 years old become pregnant each year [20]. 


\subsection{Pathogenesis}

Despite CF has long been associated with female infertility, the underlying causes remain unclear. Actually, we know that the majority of CF women have a normal fertility, but ovulation disturbance may occur in patients with advanced disease [21], also with amenorrhea in the most compromised subjects. Although most girls have normal menstrual cycles, there is a higher incidence of missed or irregular periods and amenorrhea. This is more likely in those with a reduced percentage of body fat and may occur in case of malnutrition or alimentary disorders.

Fertility problems may be related to lung disease severity, poor weight, and to an unsatisfying control of CF-related diabetes [22]. Historically, the predictors of poor pregnancy outcome for mother and/or fetus were a forced vital capacity (FVC) of less than $50 \%$ of the predicted value and poor nutritional status. In the past, an FVC of less than $50 \%$ of the predicted value was an absolute contraindication to pregnancy.

For several years, it has been postulated that the thickened cervical mucus present in CF women reproductive tract could lead to fertility disturbance. Normally, when the egg is released from the ovary, cervical and uterine mucus thins and allows easier passage of sperm into the uterus and fertilization. Increased thickness of this mucus in women with CF may theoretically act as a barrier to sperm penetration and could reduce fertility, but nowadays the majority of women with CF can become pregnant without any difficulty.

In 2008, Hodges et al. [23] studied the mouse model of CF, indicating that in CF mice the major cause of decreased fertility is the impaired sperm transport within female reproductive tract. In their experience, excess cervical mucus played a minor role, because instead of a physical barrier, the decreased fertilization seemed to be due to an inadequate fluid production in reproductive system, with a subsequent decrease in sperm number in the oviduct. CFTR could play not only an important role in female reproductive tract fluid control but also in sperm capacitation with its bicarbonate transport.

\subsection{Impact of pregnancy on CF}

The physiologic changes associated with pregnancy may contribute to increased morbidity and mortality risks for the mother in CF women. Volumetric increase of abdomen with the consequent upward displacement of diaphragm causes a decrease in functional residual volume, and the concomitant increase in resting minute ventilation can lead to a relevant breath disturbance. Also gas exchange has deep alterations in pregnant women, with an increased alveolar-arterial oxygen gradient, especially in supine position [24].

In a recent study [25], it is reported that adjusting for the FEV1 percent predicted, weight, height, and pulmonary exacerbation rate per year, pregnancy is not associated with an increased risk of death. Pregnancy did not appear to be harmful even in a subset of women with diabetes mellitus or with FEV1 less than $40 \%$ of predicted. Important predictors of pregnancy outcome for the fetus are the severity of maternal pulmonary impairment and nutritional status; in women with advanced lung disease, preterm delivery is very common. 
McMullen et al. [26] in 2006 characterized health outcomes in CF pregnant women, comparing them with a group of never-pregnant CF population: this large observational study showed a nonsignificant difference in terms of FEV1 decline between the two groups $(6.8 \%$ in pregnant group and $4.7 \%$ in never-pregnant, $P=0.61$ ). Respiratory exacerbations and hospitalizations were increased during pregnancy, as well as the number of outpatient visits and administered therapies.

The risk for congenital anomalies in the fetus is not increased in CF gravidas and breastfeeding is possible without complications.

\subsection{Risk factors}

The pregravid pulmonary function is clearly the greatest outcome predictor in CF women, but there are multiple clinical prognostic markers to consider. Women with poor nutritional status, pulmonary hypertension, and relevant decrease in pulmonary function during the first 3 months of gestation have to be informed about the high risk of maternal mortality and should consider therapeutic abortion [27].

\subsubsection{Lung function}

FEV1\% predicted, PO2, and PCO2 are important predictors of pregnancy outcomes. In 1995, Edenborough et al. [28] reported that a pregravid FEV1 $<60 \%$ caused greater pulmonary function decrease, a higher frequency of preterm infants, and also a higher mortality. However, successful pregnancies have been reported also in CF patients with compromised lung function $\left(\mathrm{FEV}_{1}<50 \%\right.$ of that predicted), and most patients seem to return to baseline pulmonary status after pregnancy.

The only absolute contraindication to pregnancy in CF is represented by pulmonary hypertension, which is correlated with higher rates of mortality in pregnancy. An echocardiogram performed before pregnancy can be useful to individuate underlying pulmonary hypertension and cor pulmonale, in order to advise the patient about the high mortality risk in case of pregnancy [24].

\subsubsection{Pancreatic insufficiency and diabetes mellitus}

In old literature, pancreatic insufficiency was considered as a major risk factor in pregnancy outcomes. Actually, with the modern pancreatic enzymes supplementations, it is a nonsignificant issue in CF pregnancies.

CF women planning pregnancy have to be tested for glucose intolerance before conception and the test has to be repeated at 20 weeks of gestational age. Insulin therapy is indicated in case of abnormalities in blood glucose monitoring.

\subsubsection{Nutritional status}

Clinician should advise to reach a pregravid weight before conception up to $90 \%$ of the ideal body weight. Poor nutritional status is without any doubt one of the most relevant risk factor 
for pregnancy outcomes in $\mathrm{CF}$, and a severe malnutrition (BMI $<18 \mathrm{~kg} / \mathrm{m}^{2}$ ) is a relative contraindication to pregnancy. A weight gain of 11-12 kg during pregnancy is recommended [29]; poor outcome is particularly associated with a maternal gain less than $4.5 \mathrm{Kg}$. A high caloric intake should be maintained and a poor weight gain is an indication for aggressive nutritional intervention (dietary supplementation, nasogastric, or gastrostomy feeding).

\subsubsection{Microbiological issues}

Burkholderia cepacia complex has been considered for several years a relative contraindication for pregnancy in $C F$, because this infection seemed to be related with a higher maternal mortality [30]. B. cepacia (particularly B. cenocepacia and B. multivorans) is also considered related to preterm delivery, weight loss, and rapid pulmonary function decline [31], but further studies are needed to better define its role in CF pregnancies.

\subsubsection{Pulmonary transplantation}

Limited cases of pregnancy after lung transplantation have been reported; most experience comes from renal transplantation. Compared to other solid organ transplants, lung recipients experience more frequent rejections during pregnancy and also a higher rate of graft loss postpartum [32]. Graft dysfunction is unpredictable and may occur anytime during pregnancy, leading to progressive decline and also eventual death after delivery. However, further studies are necessary to determine long-term maternal survival.

Prematurity and neonatal complications in these pregnancies are very high (56\% and 33\%, respectively), but no long-term consequences on children are reported [33].

\subsection{Management of pregnancy in CF women}

The management of pregnancy in CF requests necessarily a multidisciplinary approach. Most of the literature on pregnancy in CF is constituted by case reports and a few national centre based reviews. Unfortunately, at the moment, we have no available trials about any aspect of pregnancy management in CF. In 2008, Edenborough et al. [34] published the guidelines for the management of pregnancy in women with $\mathrm{CF}$, based on the review of the literature and experience of pediatricians, adult and transplant physicians, nurses, physiotherapists, dietitians, pharmacists and psychologists experienced in $\mathrm{CF}$, and also anesthetists and obstetricians with experience of CF pregnancy.

\subsubsection{Counselling}

Counselling consists in helping the CF patient planning pregnancy and her partner to explain the risks of their decision, such as medical implications, treatment options, and also the impact of a toddler on the everyday life of a CF woman.

Genetic features, such as the risk of recurrence of the disease, have also to be discussed with the couple. CF genotype, if not already known, have to be defined and the partner should also 
be tested before conception. Genetic counsellors should be involved in order to discuss these delicate issues with the couple.

Genetic tests have a sensitivity $<100 \%$ with detection rates from $70 \%$ to $95 \%$ of CFTR mutations. If the partner has not been tested, given a CF carrier frequency of 1:25, the risk of an affected infant is 1:50; while if the partner is a CF carrier, the risk is 1:2.

When the partner is a known carrier or if he has not been tested, clinicians should suggest to perform prenatal genetic diagnosis to the couple, with the analysis of chorionic villus sample (CVS) within the first trimester of gestation. This procedure includes technical risks that should be discussed with the couple.

Psychological counselling is also an important part of the counselling activities: CF team should provide information about sexual health and reproduction to all of their patients, particularly teenage girls. Psychologists and clinicians play an important role also in psychological counselling for CF women who want to become pregnant, even if women with advanced disease with a very strong wish to have a child may proceed whatever the advices.

\subsubsection{Medications during pregnancy}

Most drugs have not been tested on pregnant women. Relevant issues about their use in pregnancy are timing of exposure (periconception, first, second, third trimester, or perinatally), systemic availability of the drug, and its ability to cross the placenta. Side effects can consist in teratogenesis, growth retardation, death, renal insufficiency, neurological disorders, stillbirth, etc.

Many pregnancies are unplanned and drugs could have been taken at the time of conception and continued in the first weeks of pregnancy, and many women with serious illnesses required treatment to be continued. Even if nowadays there is experience to guide prescribing in pregnancy, the principle remains to avoid drug use where possible, except when the risk of the drug is outweighed by the risk of the condition being treated.

The Swedish FASS information catalog provides information on the risks of drugs to the fetus during pregnancy and to the infant during lactation. Each drug is classified to one category of safety:

1. Drugs that have been used widely during pregnancy and are assumed safe for the fetus

2. Drugs not known to cause harm to the human fetus but with insufficient experience to consider them safe. This category can be subdivided into

3. drugs that have been demonstrated to cause no harm in animal studies

4. drugs with insufficient animal data

5. drugs that have been demonstrated to harm the fetus only in animal studies

6. Drugs that could theoretically cause harm to the fetus by their pharmacological actions

7. Drugs known or believed to cause harm to the fetus 
Patient's therapy should be reviewed during the discussion of a potential pregnancy, even if most of the routine CF medications are safe and could be continued. Contraindicated drugs have to be discontinued. $\beta$-lactams are safe in pregnancy and aminoglycosides at conventional doses have not showed toxic results. Once-daily tobramicin has been tested in second and third trimesters, showing safe results. Ciprofloxacin has been widely used during pregnancy with no certain side effects, but its use is indicated only if vital for the mother.

\subsubsection{Lung function and infections}

CF women can become pregnant in all pulmonary disease stage, but the outcome for the mother and the newborn is closely related to lung function (FEV1 predicted) and clinical stability. Lung function should be optimized and chronic infections may be suppressed before pregnancy: oral flucloxacillin could be administered in case of Staphylococcus aureus colonization; for Pseudomonas aeruginosa nebulized colistin or aminoglycoside treatment should be employed.

When a pregnancy is unplanned, pregnancy is frequently connected to a worsening in lung function: if necessary, one or more courses of IV antibiotic treatment could be administered in the usual format also during pregnancy ( $\beta$-lactam + aminoglycoside).

During the first trimester, most patients will feel breathless and the frequency of hospitalizations could rise up: at each visit, physical examination, sputum cultures, weight and oxygen saturation measurement, and pulmonary function test should be performed.

\subsubsection{Physiotherapy}

If the pregnancy is planned, preconceptional period could represent a good opportunity to optimize the daily physiotherapy program. Inhalation therapies and techniques should also be reviewed and optimized; the timing of these treatments in relation to airway clearance therapy (ACT) is a relevant feature during pregnancy, especially in CF patients who produce big volumes of sputum. ACT adherence and technique have to be adapted in pregnant women. Advice on physical exercise and pelvic floor strength should be given as soon as possible.

The breathing pattern is affected by physiological and mechanical changes during pregnancy, and in the last trimester these features can lead to increase in closing volume and determine atelectasis. Physiotherapists should meet the pregnant patients weekly to optimize the physiotherapy regimen, monitor lung function and sputum production in terms of colour and quantity. Also maintaining exercise capacity should be useful during pregnancy and in last trimester.

\subsubsection{Nutrition}

Dietetic counselling is crucial in preconceptional period, because maternal nutritional state is one of the most important factors influencing outcomes for mother and infant. A low prepregnancy BMI is strictly associated with reduced birth weight. Preconceptional assessment is similar to non-CF population and should be performed by a CF specialist dietitian, who can advise not only on increasing energy density of the diet but also oral supplements and invasive 
nutritional support (enteral tube feeding) can be purposed to the patient if nutritional status is unsatisfactory. Vitamin supplementation with folic acid, vitamin A, and vitamin D are recommended.

An overall weight gain of $12.5 \mathrm{Kg}$ is considered normal and in $\mathrm{CF}$ it is recommended a weight gain of at least $11 \mathrm{Kg}$. Also gastro-oesophageal reflux, heartburn, nausea, recurrent vomiting, and constipation may occur more frequently in women with $\mathrm{CF}$ and required clinicians monitoring.

\subsubsection{Diabetes care}

A pre-pregnancy diagnosis of CF-related diabetes and gestational diabetes are associated with a poorer prognosis; in literature, it is reported that an unsatisfactory glycemic control in the first trimester is associated with an increased risk of teratogenesis. During preconceptional counselling an OGTT is recommended, if not already performed, and blood sugars have to be monitored during lung exacerbations. Usually, OGTT is repeated at 20 weeks gestation and glycaemia should be measured at every visit: if random values are high, another OGTT could be repeated at 28 weeks.

Insulin is the recommended treatment for diabetes in $\mathrm{CF}$ also during pregnancy. A high calorie intake should also be assessed in diabetic patients.

\subsubsection{Termination of pregnancy}

Guidelines about when to terminate pregnancy in a woman with advanced CF remain fluid. The indications may be psychosocial (in order to prevent serious injury to the mental health of the pregnant woman) or medical.

The only absolute contraindication to pregnancy is a pre-existing pulmonary hypertension with cor pulmonale; also chronic hypoxia could be considered a contraindication to pregnancy. There are no clear indications about the FEV1 cut-off to recommend pregnancy termination.

There are also some relative contraindications to pregnancy in CF women:

1. Poor nutritional status (BMI $<18 \mathrm{~kg} / \mathrm{m}^{2} ;<85 \%$ ideal body weight)

2. Uncontrolled CF-related diabetes

3. Burkholderia cepacia infection

4. Significant liver disease

\subsubsection{Delivery}

Most pregnancies in CF end in spontaneous vaginal delivery. Caesarean section is indicated only in case of maternal or fetal sufferance, preferably with spinal anesthesia. In CF, a high proportion $(26-46 \%)$ of pregnancies end up with a spontaneous or therapeutic preterm delivery, and the usual indication is represented by maternal conditions. Usually, failing lung function and hypoxia occur in patients with a significant low pre-pregnant lung function; 
persisting hypoxia and onset of headache are in these cases severe signs and could request oxygen support or non-invasive ventilation.

In peripartum, pain and anxiety can lead to hyperventilation and decrease in alveolar gas exchanges; hypoxia, hypercarbia, and respiratory acidosis occur rapidly in patients with compromised lung function. An adequate analgesia should be performed, because it reduces pain, fear, and fatigue; oxygen support can be useful in case of desaturations.

\section{Our experience}

In our CF Center, we have 230 patients in regular follow-up and 153 of these are $>18$ years old. In the last few years, fertility issues increased significantly in our population, surely because of the significant improvement in prognosis and the easier access to the assisted reproduction treatments.

During the last 20 years, 12 of our male patients recurred to in vitro fertilization with sperm obtained by epididymal aspiration and in 6 cases had successful reproduction (with twin pregnancy in 2 cases and in 1 case triplet pregnancy). All the newborns enjoyed good health and no recurrence of CF has been detected (all the partners were tested for CFTR mutations before the conception). In the other six cases in vitro fertilization was not successful and one of these three patients decided to use heterologous fertilization by sperm donor, with a subsequent successful pregnancy.

In the same period four of our female patients recurred to in vitro fertilization with two successful pregnancies (in one case twin pregnancy). Also in these cases the newborns enjoyed good health and no recurrence of CF has been detected (all the partners were tested for CFTR mutations before the conception).

In our population 12 of our patients (10 women and 2 men) had spontaneous conceptions with 18 successful pregnancies. Also in these cases no recurrence of the disease has been detected, even if in some cases the diagnosis of $\mathrm{CF}$ in these patients had been performed after the delivery and in most cases the partners had not been tested for CFTR mutations before the conception. Three of our female patients, after several attempts to become pregnant spontaneously, decided and obtained to adopt a child.

Actually, no literature is available about scrotal imaging in males affected by CF. We decided to realize an original study, performing scrotal ultrasound examination with high-definition technique, to better evaluate abnormalities in shape, volume, and structure of the testes, epididymis, and spermatic cord in a group of adult patients ( $>18$ years) affected by CF. Preliminary results seem to show an increased incidence of testicular and epididymal abnormalities in comparison to the general population, but in most cases these seem to be secondary to the obstruction of vas deferens [FigureS 7,8,9]. 


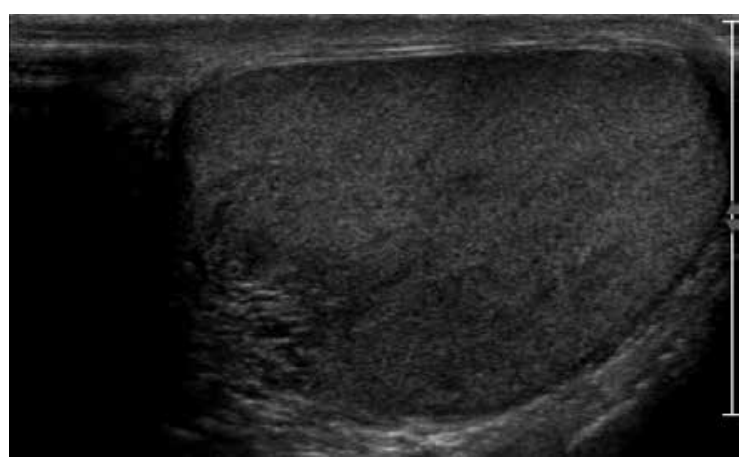

Figure 7. US Figure of rete testis ectasia with structure inhomogeneities in a CF patient

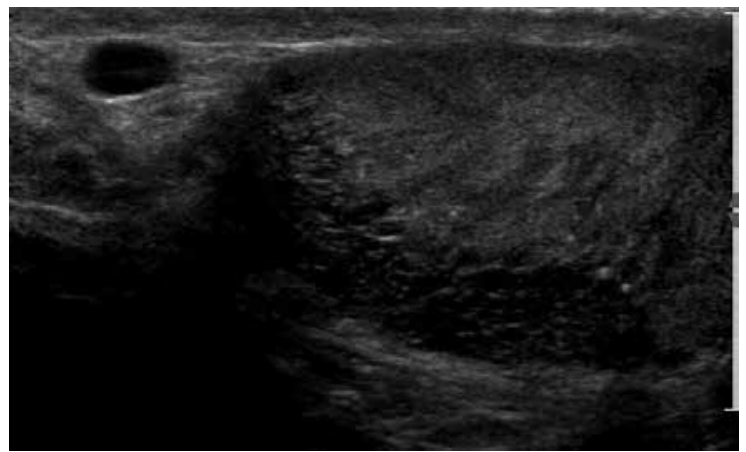

Figure 8. US Figure of rete testis ectasia with structure inhomogeneities with a little epididymal cyst in a CF patient

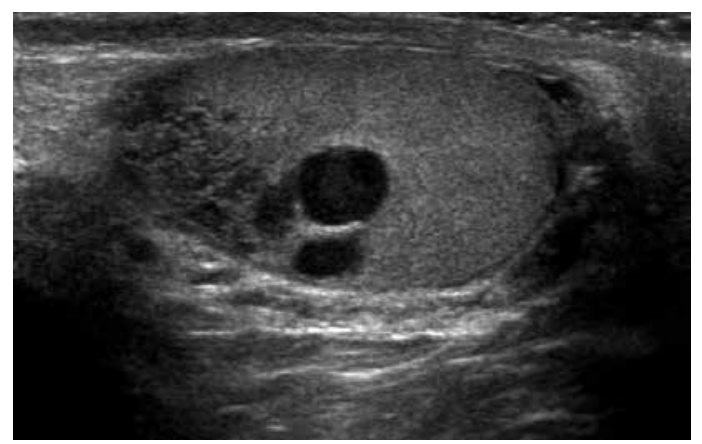

Figure 9. US Figure of intradidymal cysts (one with corpuscular content) in a CF patient 


\section{Nomenclature}

$\mathrm{CF}=$ Cystic fibrosis

$\mathrm{CFTR}=$ Cystic fibrosis transmembrane conductance regulator

$\mathrm{CBAVD}=$ Congenital bilateral absence of the vas deferens

$\mathrm{LH}=$ Luteinizing hormone

$\mathrm{FSH}=$ Follicle stimulating hormone

$\mathrm{US}=$ ultrasounds

$\mathrm{IVF}=$ in vitro fertilization

ICSI = Intracytoplasmic sperm injection

PESA = Percutaneous epididymal sperm aspiration

MESA = Microsurgical epididymal sperm aspiration

TESA $=$ Percutaneous testicular aspiration

TESE $=$ Testicular sperm extraction

$\mathrm{FVC}=$ forced vital capacity

FEV1 = Forced expiratory volume in 1 second

PO2 $=$ Partial pressure of oxygen

PCO2 $=$ Partial pressure of carbon dioxide

$\mathrm{BMI}=$ Body mass index

\section{Acknowledgements}

We want to thank Professor Lorenzo Derchi (IRCCS A.O.U San Martino - IST, Radiology Unit, Genova) for the original Figures of scrotal ultrasounds performed on our CF patients.

We want to thank Dr. Mauro Costa (Ospedale Evangelico Internazionale, Reproduction Medicine Unit, Genova) for the collaboration.

\section{Author details}

Rosaria Casciaro*, Federico Cresta, Federica Favilli and Laura Minicucci

*Address all correspondence to: rosariacasciaro@ospedale-gaslini.ge.it

Pneumology Unit - Cystic Fibrosis Center, IRCCS Giannina Gaslini, Genova, Italy 


\section{References}

[1] Quinton PM. Physiological basis of cystic fibrosis: a historical perspective. Physiol Rev 1999;79(Suppl):S3-22; PMID:9922374.

[2] Taussig LM, Lobeck CC, di Sant'Agnese PA, Ackerman DR, Kattwinkel J. Fertility in males with cystic fibrosis. N Engl J Med 1972;287:586-9; PMID:5055208.

[3] Holsclaw DS, Perlmutter AD, Jockin H, Shwachman H, Genital abnormalities in male patients with cystic fibrosis. J Urol 1971;106:568-74; PMID:4399160.

[4] Meschede D, Dworniczak B, Behre HM, Kliesch S, Claustres M, Nieschlag E, Horst J. CFTR gene mutations in men with bilateral ejaculatory-duct obstruction and anomalies of the seminal vesicles. Am J Hum Genet 1997;61:1200-2.

[5] van der Ven K, Messer L, van der Ven H, Jeyendran RS, Ober C. Cystic fibrosis mutation screening in healthy men with reduced sperm quality. Hum Reprod 1996;11:513-7; PMID:8671256.

[6] Trezise AE, Linder CC, Grieger D, Thompson EW, Meunier H, Griswold MD, Buchwald M. CFTR expression is regulated during both the cycle of the seminiferous epithelium and the oestrous cycle of rodents. Nat Genet 1993;3:157-64; PMID 7684647.

[7] Wang XF, Zhou CX, Shi QX, Yuan YY, Yu MK, Ajonuma LC, Ho LS, Lo PS, Tsang LL, Liu $Y$, et al. Involvement of CFTR in uterine bicarbonate secretion and the fertilizing capacity of sperm. Nat Cell Biol 2003; 5:902-6; PMID 14515130.

[8] Xu WM, Shi QX, Chen WY, Zhou CX, Ni Y, Rowlands DK, Yi Liu G, Zhu H, Ma ZG, Wang $X F$, et al. Cystic fibrosis transmembrane conductance regulator is vital to sperm fertilizing capacity and male fertility. Proc Natl Acad Sci USA 2007;104:981621; PMID:17519339.

[9] Durieu I, Bey-Omar F, Rollet J, et al. Diagnostic criteria for cystic fibrosis in men with congenital absence of vas deferens. Med (Baltimore) 1995;74:42-7.

[10] Blau H, Freud E, Mussaffi H, Werner M, Konen O, Rathaus V. Urogenital abnormalities in male children with cystic fibrosis. Arch Dis Child 2002 Aug;87(2):135-8.

[11] Wilschanski M, Corey M, Durie P, et al. Diversity of reproductive tract abnormalities in men with cystic fibrosis. JAMA 1996;276:607-8.

[12] Smith HC. Fertility in men with cystic fibrosis assessment, investigations and management. Ped Resp Rev 2010;11:80-3.

[13] Temple-Smith PD, Southwick GJ, Yates CA, Trounson AO, de Kretser DM. Human pregnancy by in vitro fertilization (IVF) using sperm aspirated from the epididymis. J in Vitro Fert Embryo Transf 1985;2:119-22. 
[14] Silber SJ, Balmaceda J, Borrero C, Ord T, Asch R. Pregnancy with sperm aspiration from the proximal head of epididymis: a new treatment for congenital absence of the vas deferens. Fertil Steril 1988;50:525-8.

[15] Sherins RJ, Thorsell LP, Dorfmann A, Dennison-Lagos L, Calvo LP, Krysa L, Coulam $\mathrm{CB}$, Schulman JD. Intracytoplasmic sperm injection facilitates pregnancies even in the most severe forms of male infertility. Fertil Steril 1995;Aug 64(2):369-75.

[16] Siegel B, Siegel S. Pregnancy and delivery in a patient with cystic fibrosis of the pancreas. Obstet Gynecol 1960;16:438-40.

[17] Grand RJ, Talamo RC, di San'Agnese PA, et al. Pregnancy in cystic fibrosis of the pancreas. JAMA 1966;195:993.

[18] Edenborough FP, Stableforth DE, Mackenzie WE. The outcome of 72 pregnancies in 55 women with cystic fibrosis in United Kingdom 1977-1986. BJOG 2000;254-61.

[19] Gilljam M, Antoniou M, Shin J, et al. Pregnancy in cystic fibrosis: fetal and maternal outcomes. Chest 2000;118:86-91.

[20] Cystic fibrosis foundation. Patient registry: annual data report 2007. Bethesda, Maryland.

[21] Johannesson M, Csemiczky G, Landgren BM, Hjelte L, Gotllieb C. Female patients with cystic fibrosis suffer from reproductive endocrinological disorders despite good clinical status. Hum Reprod 1998;13:2092-7.

[22] Edenborough FP. Women with cystic fibrosis and their potential for reproduction. Thorax 2001;Aug 56(8):649-55.

[23] Hodges CA, Palmert MR, Drumm ML. Infertility in females with cystic fibrosis is multifactorial: evidence from mouse models. Endocrin. 2008;Jun 149(6):2790-7. DOI: 10.1210/en.2007-1581. Epub 2008 Mar 6.

[24] Whitty JE, Cystic fibrosis in pregnancy. Clin Obstet Gynecol 2010;53(2):369-76.

[25] Goss CH, Rubenfeld GD, Otto K, et al. The effect of pregnancy on survival in women with cystic fibrosis. Chest 2003;124:1460-8.

[26] McMullen AH, Pasta DJ, Frederick PD, et al. Impact of pregnancy on women with cystic fibrosis. Chest 2006;129:3.

[27] Lau EMT, Moriarty C, Ogle R, Bye PT. Pregnancy and cystic fibrosis. Ped Resp Rev 11(2010):90-4.

[28] Edenborough FP, Stableforth DE, Webb AK. Outcome of pregnancy in women with cystic fibrosis. Thorax 1995;50:170-4.

[29] Hilman BC, Aitken ML, Constantinescu M. Pregnancy in patients with cystic fibrosis. Clin Obstet Gynecol 1996;39:70-86. 
[30] Tanser SJ, Hodson ME, Geddes DM. Case report of death during pregnancy in patients with cystic fibrosis: three out of four patients were colonized by Burkholderia cepacia. Respir Med 2000;94:1004.

[31] Bose D, Yentis SM, Fauvel NJ. Caeserean section in a parturient with respiratory failure caused by cystic fibrosis. Anaesthesia 1997;52:578-82.

[32] Gyi KM, Hodson ME, Yacoub MY. Pregnancy in cystic fibrosis lung transplant recipients: case series and review. J Cyst Fibros 2006;5:171-6.

[33] Chetty SP, Shaffer BL, Norton ME. Management of pregnancy in women with genetic disorders: Part 2: Inborn errors of metabolism, cystic fibrosis, neurofibromatosis type 1, and Turner syndrome in pregnancy. Obstet Gynecol Surv. 2011;Dec 66(12): 765-76.

[34] Edenborough FP, Borgo G, Knoop C, Lannefors L, et al. Guidelines for the management of pregnancy in women with cystic fibrosis. J Cyst Fibros 2008; 7:S2-32. 
Chapter 6

\title{
Medications to Enhance CFTR Activity
}

\author{
Terry W. Chin \\ Additional information is available at the end of the chapter \\ http://dx.doi.org/10.5772/60768
}

\begin{abstract}
Since the basic defect in cystic fibrosis (CF) involves a defective cell surface protein controlling chloride channel transport across cell membranes, medications which are developed to enhance the cystic fibrosis transmembrane conductance regulator (CFTR) protein should result in improvement in patients with CF. The presence of over 2000 genetic mutations have made these efforts difficult. However, a classification scheme of these mutations has allowed three basic approaches: to bypass missense mutations by having the cellular translation machinery read through the premature stop codon, to enhance the "gating" function of the CFTR protein on the cell surface, and to correct a defective CFTR protein "trafficking" though the cytoplasm to be inserted properly in the cell membrane. This chapter will review clinical trials using drugs which are designed to enhance CFTR protein activity.
\end{abstract}

Keywords: Cystic fibrosis, CFTR, CFTR modulators, CFTR potentiators, CFTR correctors, VC-770, ivacaftor, VX-809, lumacaftor, VX-661, PTC124, ataluren

\section{Introduction}

The identification of the genetic defect in CF allowed the classification of CFTR mutations into six types of mutations. The over 2000 mutations which has been described can be placed in one of these classes: Class I mutations, which may be the result of the genetic mutation resulting in a stop codon or a shift in the reading frame in the messenger RNA which eventually results in a downstream stop codon, do not produce a complete CFTR protein. Class ii mutations result in an altered CFTR protein which is degraded in the endoplasmic reticulum and/or Golgi systems within the cell. Some proteins may be able to make it to the cell surface and have 
variable levels of function (or Class III, IV, or VI effects). Class III mutations result in amino acid substitutions which affect how the CFTR protein is regulated on the cell surface, usually decreasing the channel opening. Class IV mutations result in amino acid substitutions which affect how the CFTR protein functions in its conduction of chloride ions. Class V mutations result in decreased production of normal functioning CFTR protein. Therefore, there is a reduced amount of normal CFTR protein on the cell surface. Finally, class VI mutations are those which affect the stability of CFTR.

Awareness of the types of genetic mutations affecting the normal functioning of the CFTR protein has resulted in searches for treatments directed at various CFTR dysfunctions. This chapter will review recent attempts to develop treatments specific for the various classes of mutations.

\section{Premature termination codons}

In approximately $10 \%$ of patients with $\mathrm{CF}$, the responsible mutation results in a nonsense mutation that terminates the CFTR protein production due to a premature stop codon in the CFTR messenger ribonucleic acid (mRNA). The resultant truncated protein cannot properly transport chloride ion across the membrane. PTC Therapeutics, Inc. had discovered a small molecule drug ataluren (PTC124 ${ }^{\circledR}$ ) which enables the mRNA containing the premature stop codon to be read through the ribosome. The molecule is a 1,2,4-oxadiazole with a molecular weight of 284 Daltons. Using cell culture as well as a mouse model of CF, investigators determined that oral administration of ataluren was effective as long plasma concentrations in the range of 2 to $10 \mathrm{mcg} / \mathrm{mL}$ will result in functional CFTR activity. [1, 2] There was no evidence of nonspecific read through of normal stop codons. It did not appear to be teratogenic in rats and rabbits. However, there did appear to be inhibition of cytochrome P450 (CYP2C9) at therapeutic concentrations of ataluren. Therefore, monitoring blood levels of medications which are primarily metabolized by this enzyme (such as warfarin or phenytoin) may be needed clinically.

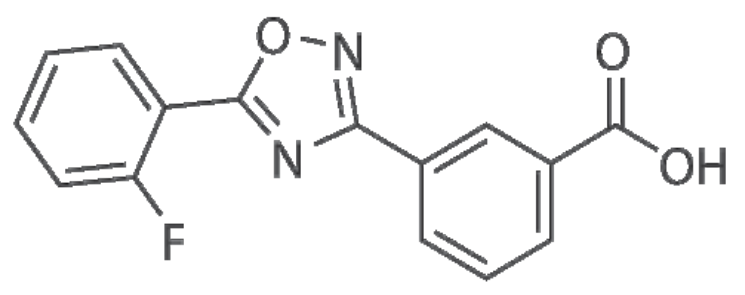

Figure 1. Structural formula of atalaren

Phase 1 studies indicate that serum ataluren levels of 2 to $10 \mathrm{mcg} / \mathrm{mL}$ can be achieved with a three times per day (TID) dosing schedule. Since administration with meals can result in prolonged levels in the blood, dosing after a meal appears to be desired. 
Phase 2a studies have been conducted in 30 children and 47 adult patients in the United States, Israel, and Europe. [3, 4, 5] Using transepithelial potential difference (TEPD), which assesses transepithelial chloride conductance, as the primary outcome measure, these studies found statistically significant improvements. Pulmonary function testing also showed positive trends with decreased sputum volume and thickness, easier elimination of sputum, decreased coughing, and increased quality of life. There were no serious adverse drug effects. Phase $2 \mathrm{a}$ extension study looked at a 3-month administration of ataluren and confirmed its effectiveness and safety. [6] Optimal responses appear to be with doses of 10-, 10-, 20-mg/kg on the TID schedule and were seen with numerous different missense mutations.

The encouraging results of these preliminary studies prompted PTC Therapeutics to conduct a Phase 3, randomized, double-blind, placebo-controlled study to look at the effects of 48 weeks of therapy on pulmonary function and clinical symptoms in CF patients with the appropriate mutation. Over 400 patients were enrolled and showed positive trends favoring ataluren over placebo with an increase in percent of predicted for forced expiratory volume in 1 second (FEV1) and decreased pulmonary exacerbation rate. [7] A subgroup of patients who were not receiving inhaled antibiotics (primarily tobramycin) showed an even greater improvement on ataluren. The study continued to show the drug was well tolerated and had a good safety profile. There were some cases of creatinine elevation which were associated with the combination of potential nephrotoxic antibiotics with ataluren. These results resulted in conducting another international, multicenter study in which patients will not be allowed to receive treatment with chronic inhaled aminoglycosides (such as tobramycin or TOBI) in early 2015. The aim is to randomize approximately 208 patients who will receive either ataluren or placebo for 48 weeks.

\section{Potentiators}

The presence of some CFTR on the cell surface in mutation classes III, IV, and VI suggests another approach may be to increase the "gating" function of these protein molecules. The most common gating mutation is G551D, a missense mutation that results in the replacement of a glycine for an aspartic acid at position 551 of the CFTR protein. The resulting CFTR protein is present at the cell surface but does not open and close properly, which is called defective channel gating. The effort to find an effective "potentiator" of the channel opening has resulted in the Federal Drug Administration (FDA) approval of the first medication which modulates the function of CFTR, ivacaftor (Kalydeco), in January 2012.

Ivacaftor (or VX-770) was developed by Vertex Pharmaceuticals, Inc., to potentiate the action of the mutated CFTR on the cell surface. It has a molecular weight of 392 and a molecular formula of $\mathrm{C}_{24} \mathrm{H}_{28} \mathrm{~N}_{2} \mathrm{O}_{3}$. Studies with recombinant cell lines with G551D-CFTR indicated that VX-770 did result in increased total chloride transport by greater than 10\%. [8] There was evidence to indicate that it acted directly on the mutated CFTR protein to keep the channel open. It apparently has similar activity on other mutant CFTR forms resulting from other CFTR gating mutations. However, since the G551D mutation affects about $5 \%$ of all CF patients and 


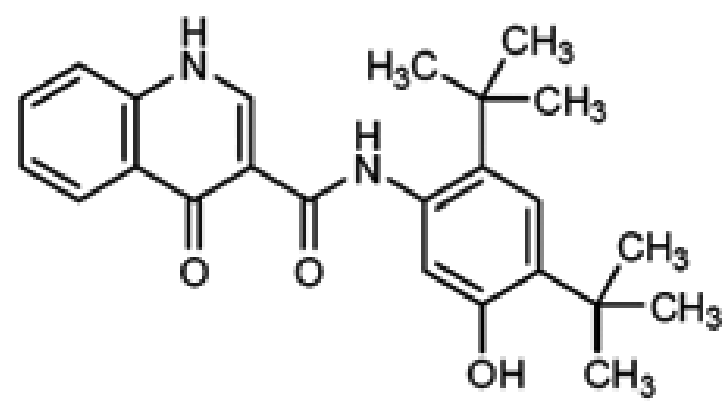

Figure 2. Structural formula of ivacaftor

is the most common gating mutation, initial studies focused on patients with this particular mutation.

Phase 1 studies indicated that ivacaftor is primarily metabolized in the liver and moderate hepatic impairment may reduce its elimination. Since in vitro studies indicated that ivacaftor is a substrate of CYP3A4/5 and therefore, strong (e.g., ketoconazole) and moderate (e.g., fluconazole) CYP3A inhibitors as well as strong CYP3A inducers (e.g., rifampin) will affect ivacaftor's metabolism. A detailed list of the numerous drug interactions is found in the package insert of ivacaftor (Kalydeco ${ }^{\circledR}$ ) (see http://www.accessdata.fda.gov/drugsatfda_docs/ label/2012/203188lbl.pdf).

An initial phase 2 study by Accurso found that the safety profile of ivacaftor in adult patients with at least one G551D mutation was excellent for a wide variety of daily doses (from $50 \mathrm{mg}$ to $500 \mathrm{mg}$ for 2 to 4 weeks). An improvement in FEV1 and drop in sweat chloride were also noted (although these were not primary end points of the study). [9] This prompted two randomized, placebo-controlled, double blind studies eventually resulting in FDA approval. Ivacaftor $150 \mathrm{mg}$ twice daily for 48 weeks was studied in 167 patients 12 years or older with at least one G551D mutation. There was a 10.6\% improvement in predicted FEV1 from baseline versus placebo. Additionally, a 55\% reduction in pulmonary exacerbation was also observed. Sweat chloride levels dropped to a mean of $47.8 \mathrm{mmol} / \mathrm{L}$ compared with $100.0 \mathrm{mmol} / \mathrm{L}$ in the placebo group. There was improvement in quality of life measurements as well as a more significant increase in weight gain (4.1 kg increase) in those receiving ivacaftor. [10] An additional pediatric study of 52 patients 6 to 12 years of age showed a 12.5\% improvement in FEV1 and a $2.8 \mathrm{~kg}$ increase in weight. A decrease in sweat chloride of $54 \mathrm{mmol} / \mathrm{L}$ was also seen. [11] In both studies, the incidence of adverse events was similar in both treated and placebo groups.

Similar results were observed in studies examining non-G551D gating mutations. [12] These 36 patients also showed significant improvements in FEV1, sweat chloride measurements, changes in BMI, and quality of life indices. Therefore, the FDA also approved ivacaftor for G178R, S549N, S549R, G551S, G970R, G1244E, S1251N, G1349D, and S1255P mutations in February 2014. Finally, in December 2014, one additional mutation, R117H, was added by the FDA to the list of approved CF mutations for ivacaftor administration after 69 patients greater 
than 6 years of age were studied showing similar efficacy and safety data. [13] However, their decrease in sweat chloride was only $24.0 \mathrm{mmol} / \mathrm{L}$ and BMI treatment differences were not significant.

Since its initial FDA approval in 2012, follow-up studies in 144 patients with the G551D mutation have confirmed the persistence of lung function improvement $(9.4 \%$ increase in adults and $10.3 \%$ in children) for up to 144 weeks on ivacaftor. [14] Additionally, treated patients continued to have reduced hospitalizations and decreased Pseudomonas aeruginosa culture rates, which may be related to increased mucociliary clearance. [15] There was a reduced rate of decline in FEV1. The absolute increase in weight was $5.1 \mathrm{~kg}$ in adults and 14.8 $\mathrm{kg}$ in children after almost 3 years, indicating that there was an increased rate of weight gain and improved BMI. [16] Not all the nutritional improvement may be attributed to improved lung function since treated patients also showed a normalizing in small bowel $\mathrm{pH}$ which may have improved pancreatic enzyme function.[14]

Although the FDA approval was for patients greater than 6 years of age, Davies et al. found ivacaftor to be well-tolerated in 34 children between 6 months and 5 years of age at doses 50 to $75 \mathrm{mg}$ twice a day. [17] They also showed a significant decrease in sweat chloride with a mean drop of $44 \mathrm{mmol} / \mathrm{L}$, and many patients showed improvement in pancreatic function with increases in stool elastase. However, $14.7 \%$ showed a higher rate of elevated liver function test results indicating the need to especially monitor these younger patients.

\section{Correctors}

Most patients with CF have the F508del mutation in which the defective CFTR protein has defective folding and processing in the endoplasmic reticulum, resulting in minimal amounts of CFTR at the cell surface. After screening a large number of molecules, Vertex Pharmaceuticals found two in which the CFTR molecule appears to be "corrected" in its "trafficking" through the cytoplasmic to the cell membrane. Importantly, the F508del-CFTR corrected by VX-809 (subsequently named lumacaftor) appears to have biochemical and functional characteristics similar to normal CFTR such as biochemical susceptibility to proteolysis, time in the plasma membrane, and single-channel openness. [18]

Although there was a statistically significant reduction in sweat chloride values, no effect was seen in CFTR function in the nasal epithelium as measured by nasal potential difference (NPD) or in lung function as measured by FEV1. Therefore, although it appeared to be safe and was well tolerated at all doses, there did not appear to be any significant effect on CFTR function in upper and lower respiratory tracts. Indeed, a phase 2 study with 140 patients did not show any difference in lung function although there was a slight reduction in sweat chloride of 2.9 $\mathrm{mmol} / \mathrm{L}$ which was barely significant $(\mathrm{p}=0.04)$.

VX-661 is another corrector with very similar structure to lumacaftor (see Figures 3 and 4). In a Phase 2 study of 128 adult patients with homozygous F508del mutation in whom four doses of VX-661 (10, 30, 100, and $150 \mathrm{mg})$ dosed once daily were given either alone or with ivacaf- 


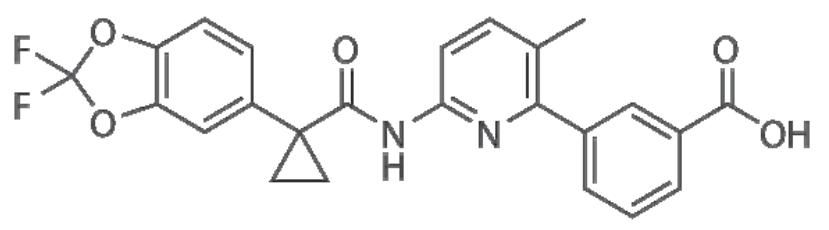

Figure 3. Structural formula of lumacaftor (VX-809)

tor150 mg twice a day for 28 days. Interim results found decreases in sweat chloride with both treatments. However, significant increases in FEV1 were found in both doses $100 \mathrm{mg}$ and 150 mg of VX-661 with ivacaftor at $9 \%$ and 7.5\% over baseline, respectively. [19]<smiles>CC(C)(CO)c1cc2cc(NC(=O)C3(c4ccc5c(c4)OC(F)(F)O5)CC3)c(F)cc2n1C[C@H](O)CO</smiles>

Figure 4. Please Add Caption

[20] There were significant improvements in the mean absolute change in percent predicted of FEV1 and BMI after 24 weeks. Because there appeared to be no difference in two doses studied, the submission to the FDA selected lumacaftor $400 \mathrm{mg}$ BID plus ivacaftor $250 \mathrm{mg}$ BID. An open label roll-over study is still in progress and expected to continue for another 24 weeks.

Patients receiving just VX-661 monotherapy in the Phase 2 study also did not show any improvement, further supporting the strategy of combining a corrector and potentiator in treating patients with F508del mutation. In 2015, Vertex is starting a series of large-scale Phase 3 studies examining the efficacy of the combined therapy with VC-661 and ivacaftor in patients with one or two copies of the F508del mutation.

\section{Conclusions}

The capability of molecules to enhance CFTR protein activity offers potential new treatment options for patients with CF. Long-term follow-up studies on ivacaftor, the first CFTR modulator to obtain FDA approval, look very promising. However, the drawback with these targeted therapies is the wide range of CFTR dysfunction seen in CF. Expanding the use of ivacaftor to nine other gating mutations only benefit about $10 \%$ of the total $\mathrm{CF}$ patient population. 
Combination therapy appears to offer the optimal opportunity for many more patients with $\mathrm{CF}$ to benefit from modulating CFTR protein. The preliminary studies on ivacaftor with lumacaftor for patients with homozygous F508del mutation look promising and may benefit another $50 \%$ of patients. Whether the combination of VC-661 and ivacaftor is more effective remains to be seen for these patients and those with one F508del and a gating mutation, which represents another $30 \%$ of patients.

Although Vertex has been the first to develop chemicals which correct trafficking of F508delCFTR and restoring its activity, other companies such as Norvartis are also actively testing other molecules, such as picolinamide-based compounds. [21] It is hoped that with increased competition from other companies, the high cost of these medications can be lessened.

\section{Author details}

Terry W. Chin

Address all correspondence to: tchin@memorialcare.org

Miller Children's Hospital, Long Beach, University of California, Irvine, USA

\section{References}

[1] Du M, Liu X, Welch EM, et al. PTC124 is an orally bioavailable compound that promotes suppression of the human CFTR-G542X nonsense allele in a CF mouse model. Proc Natl Acad Sci USA. 2008 Feb 12;105(6):2064-9.

[2] Targets genetic disorders caused by nonsense mutations. Nature. 2007 May 3;447(7140):87-91.

[3] Kerem E, Hirawat S, Armoni S, et al. Effectiveness of PTC124 treatment of cystic fibrosis caused by nonsense mutations: aprospective phase II trial. Lancet. 2008 Aug 30;372(9640):719-27. Epub 2008 Aug 20.

[4] Clancy JP, Konstan MW, Rowe SM, et al. A Phase 2 study of PTC124 in cystic fibrosis patients harboring premature stop mutations. Pediatr Pulmonol 2006;40 (Suppl29): 301 (abst \#269).

[5] Sermet-Gaudelus I, De Boeck K, Casimir G, et al. Children with nonsense-mutation mediated cystic fibrosis respond to investigational treatment with PTC124. Pediatr Pulmonol. 2008 Oct;43(Suppl S31):313 (abst \#316).

[6] Kerem E, YaakovY, Armoni S, et al. PTC124 induces time-dependent improvements in chloride conductance and clinical parameters in patients with nonsense-mutationmediated cystic fibrosis. Pediatr Pulmonol. 2008 Oct;43(Suppl S31):294 (abst \#266). 
[7] Rowe SM, Sermet-Gaudelus I, Konstan M, et al. Results of the Phase 3 Study of Ataluren in Nonsense Mutation Cystic Fibrosis (nmCF). Pediatr Pulmonol. 2012 Sep; 47(S35): 290 (abst \#193).

[8] Van Goor F, Hadida S, Groothuis PD, et al. Rescue of CF airway epithelial cell function in vitro by a CFTR potentiator, VX-770. Proc Natl Acad Sci U S A. 2009;106(44): 18825-30.

[9] Please Add Reference

[10] Please Add Reference

[11] Davies JC1, Wainwright CE, Canny GJ, et al. Efficacy and safety of ivacaftor in patients aged 6 to 11 years with cystic fibrosis with a G551D mutation. Am J RespirCrit Care Med. 2013 Jun 1;187(11):1219-25. doi: 10.1164/rccm.201301-0153OC.

[12] De Boeck K, Munck A, Walker S, et al. Efficacy and safety of ivacaftor in patients with cystic fibrosis and a non-G551D gating mutation. J Cyst Fibros. 2014 Dec;13(6): 674-80. doi: 10.1016/j.jcf.2014.09.005. Epub 2014 Sep 26.

[13] Moss RB, Flume PA, Elborn JS, et al. Ivacaftor treatment in patientswith cystic fibrosis who have an R117H-CFTR mutation, the KONDUCT study. J Cyst Fibros 2014;13(S2):S44 (abst WS23.6).

[14] McKone EF, Borowitz D2, Drevinek P3, et al. Long-term safety and efficacy of ivacaftor in patients with cystic fibrosis who have the Gly551Asp-CFTR mutation: a phase 3, open-label extension study (PERSIST). Lancet Respir Med. 2014 Nov;2(11):902-10. doi: 10.1016/S2213-2600(14)70218-8. Epub 2014 Oct 9.

[15] Rowe SM, Heltshe SL, Gonska T, et al. Clinical Mechanism of the Cystic Fibrosis Transmembrane Conductance Regulator Potentiator Ivacaftor in G551D-mediated Cystic Fibrosis. Am J RespirCrit Care Med 2014;190(2):175-184. doi: 10.1164/rccm. 201404-0703OC.

[16] Sawicki GS, McKone EF, Pasta DJ, et al.The effect of ivacaftor on weight over three years in patients with CF and a G551K-CFTR mutation. Pediatr Pulmonol 2014; 49(S38):289 (abst \#207).

[17] Davies JC, Robertson S, Green Y, et al. An open-label study of the safety, pharmacokinetics, and pharmodynamics of ivacaftor in patients aged 2 to 5 years with $\mathrm{CF}$ and a CFTR-gating mutation: the kiwi study. Pediatr Pulmonol 2014; 49(S38):286 (abst \#200).

[18] Van Goor F, Hadida S, Grootenhuis PD, et al. Correction of the F508del-CFTR protein processing defect in vitro by the investigational drug VX-809. Proc Natl Acad Sci U S A. 2011 Nov 15;108(46):18843-8. doi: 10.1073/pnas.1105787108. Epub 2011 Oct 5.

[19] Donaldson S, Pilewski J, M. Griese M, et al. VX-661, an investigational CFTR corrector, in combination with ivacaftor, a CFTR potentiator, in patients with CF and ho- 
mozygous for the F508Del-CFTR mutation: Interim analysis. J Cystic Fibrosis 2013 June;12(Suppl 1):S14 (abst WS7.3).

[20] Ramsey B, Boyle MP, Elborn J, et al. Effect of lumacaftor in combination with ivacaftor in patients with cystic fibrosis who are homozygous for F508del-CFTR: Transport study. Pediatr Pulmonol 2014;49(S38):305 (abs \# 250).

[21] Norman P. Novel picolinamide-based cystic fibrosis transmembrane regulator modulators: evaluation of WO2013038373, WO2013038376, WO2013038381, WO2013038386 and WO2013038390. Expert OpinTher Pat. 2014 Jul;24(7):829-37. doi: 10.1517/13543776.2014.876412. Epub 2014 Jan 7. 



\section{Section 3}

Microbiology of Cystic Fibrosis 



\title{
Respiratory Virus in Cystic Fibrosis - A Review of the Literature
}

\author{
Dennis Wat
}

Additional information is available at the end of the chapter

http://dx.doi.org/10.5772/60905

\begin{abstract}
Life expectancy in Cystic Fibrosis (CF) has improved dramatically in the last few decades; this is very much due to the emergence of disease-modifying treatments, optimisation of nutritional status and the inception of specialist CF units. However, progressive obstructive lung disease characterised by chronic inflammation, bacterial colonisation and recurrent infections of the lung, resulting in irreversible pulmonary damage, remains the major cause of mortality in individuals with CF. Historically, bacterial infections are the major pathogens accounting for clinical deterioration in $\mathrm{CF}$. More recently, there has been emerging evidence to support respiratory viruses being accountable for the colonisation of bacteria and progression of lung disease in $\mathrm{CF}$. This chapter sought to provide an overview on the impact of respiratory viruses in CF lung disease, the interaction between viruses and bacteria, the preventative and therapeutic measures that are currently available for the management of viral lung disease in $\mathrm{CF}$.
\end{abstract}

Keywords: Cystic fibrosis, respiratory virus, bacteria, Pseudomonas aeruginosa

\section{Introduction}

Cystic Fibrosis (CF) is the most commonly inherited potentially lethal disease amongst the Caucasian ancestry. The prevalence of CF is reported as 0.737 per 10,000 in 27 European Union countries [1]. The United States (US) Cystic Fibrosis Patient Registry reports a similar prevalence of $0.797 \mathrm{CF}$ patients per 10,000 people [2]. It is an autosomal recessive disease and is 
caused by mutations in the Cystic Fibrosis Transmembrane Conductance Regulator gene (CFTR) [3]. The most common mutation is caused by deletion of phenylalanine at position 508 (Delta F508) of the CFTR on chromosome 7, which accounts for approximately 70\% of CF cases. The primary function of CFTR in many tissues is to regulate and participate in the transport of chloride ions across epithelial cell membranes. To date, more than 1,900 mutations have been described in this gene.

CF is a multisystem disease as CFTR is expressed in different organs [4]; however, the lungs are the predominant organs that bear the brunt of the disease [5]. Recurrent pulmonary infections may start at very early stages in the lives of patients with CF. It has been hypothesised that low airway surface liquid volume and impaired mucociliary clearance are responsible for the pathogenesis of lung infections. These in turn lead to impaired bacterial clearance from respiratory epithelial cells [6]. Pulmonary infections remain the greatest cause of poor life quality, morbidity and mortality in CF that eventually lead to premature death in this condition [7].

Apart from chronic lung disease with recurrent exacerbations, exocrine pancreatic insufficiency is also a feature that leads to malabsorption and subsequently growth retardation and maturation. Endocrine pancreatic insufficiency is another feature of CF with the manifestation of diabetes. Obstructive azoospermia in male CF patients leads to male infertility.

The median survival from CF has taken great strides over the past 40 years as a consequence of the introduction of specialist centre care, nutritional optimisation, prevention and aggressive management of pulmonary exacerbations [8]. In the United Kingdom (UK) CF population in 2012, the median survival was reported as 43.5 years, compared to 38.8 years for the population in 2008 as per the UK CF Registry [9]. It has been postulated that the continuing improvement in survival of $\mathrm{CF}$ patients in successive cohorts means that the previous prediction of patients with CF living beyond a median age of 50 years is not impossible. The recent introduction of Ivacaftor to the management of CF patients with G551D CFTR mutations may further enhance the overall survival [10].

Historically, bacteria have been the predominant cause for respiratory exacerbations. The presences of some organisms including Staphylococcus aureus, Pseudomonas aeruginosa and Burkhoderia cepacia in the airways have been shown to lead to clinical deterioration [11-13] and may subsequently lead to morbidity and mortality. Pulmonary exacerbations are associated with acquisition of new organisms and increased concentration of airway flora [14]. The new acquisitions of $P$. aeruginosa in CF have been demonstrated to occur in the winter months coinciding with the peak of respiratory viral infections $[15,16]$. In the event of a pulmonary exacerbation the absence of pyrexia, raised inflammatory markers and systemic response, pathogens other than bacteria can be the potential cause. Respiratory viruses have been implicated by a number of studies in the last 30 years as potentiators for CF exacerbations [17-29]. Influenza is a substantial health threat; it is associated with approximately 36,000 deaths and 220,000 hospitalisations in the USA on an annual basis [30]. The recent emergence of novel influenza virus (H1N1) further heightened the awareness of influenza-like illness. CF Pulmonary exacerbation rates have also been shown to be significantly increased during the winter months and are highly associated with the influenza season [31]. Respiratory viruses that are 
associated with the exacerbations of $C F$ include influenza $A$ and $B$, respiratory syncytial virus $(R S V)$, parainfluenza virus (PIV) types 1 to 4, rhinovirus, metapneumovirus, coronavirus and adenovirus.

In the last 30 years, there have been a number of published studies depicting the impact of respiratory viruses in $\mathrm{CF}$. A number of studies have also demonstrated the relationship between respiratory viruses and bacteria in the pathogenesis of CF exacerbations [15, 32]. The introduction of molecular diagnostic technologies has further enhanced the awareness of respiratory viral aetiology in $\mathrm{CF}$ exacerbations as they have much higher detection rates than traditional methods. However, further understanding is required to appreciate their relationship in order to allow the development of potential novel treatment. If indeed respiratory virus does lead to secondary bacterial infection in $\mathrm{CF}$, viral vaccinations and anti-viral therapies would be important therapeutic options for CF. On the other hand, the currently commercially available vaccines and anti-virals for the prevention and treatment of respiratory viral infections are limited; they are primarily for influenza infection. The potential development of new vaccines and anti-virals is an exciting field which may offer alternate therapeutic opportunities for CF exacerbations.

This chapter will focus on the literature regarding respiratory viruses in CF and their clinical implications, the detection techniques for viruses and their differences in sensitivities, the interaction between viruses and bacteria, and the management of viral infections.

\section{Viral respiratory infections in $\mathrm{CF}$}

Early studies looking at respiratory viruses in CF relied on repeated serological testing, either alone [20] or in combination with viral cultures for viral detection [21-25]. These methods are relatively insensitive and more recent studies have utilised molecular-based methodologies [18, 26-28, 33-36]. All these studies produced different results in terms of prevalence of respiratory viruses in CF. The differences can be due to different methodologies, different sampling methods; the differences can also be accountable by different populations studied as the prognosis for CF has improved with each successive birth cohort.

Wang et al. [25] described the relationship between respiratory viral infections and deterioration in clinical status in CF almost 30 years ago. In this 2- year prospective study [25], viruses were identified through serology and nasal lavage in 49 patients with CF (mean age 13.7 years) on a quarterly basis and at the onset of exacerbations. Although the CF patients had more respiratory illnesses than sibling controls (3.7 versus 1.7/year), there were no differences in virus identification rates (1.7/year). The rate of proven virus infection was significantly correlated with the decline in lung functions, nutritional status, radiology score, and frequency and duration of hospitalisation.

More recent studies suggest no difference in the frequency of either upper respiratory tract illness (URTI) episodes [22] or proven respiratory viral infections [24] between children with $\mathrm{CF}$ and healthy controls, but children with CF have significantly more episodes of lower airway 
symptoms than controls [22, 24]. Ramsey et al. [24] prospectively compared the incidence and effect of viral infections on pulmonary function and clinical scores in 15 school-age patients with CF aged between 5 and 21 years and their healthy siblings. Over a 2-year period, samples were taken at regular two monthly intervals and during acute respiratory illnesses (ARI) for pharyngeal culture and serology for respiratory viruses. There was a total of 68 ARI episodes that occurred in the patients with CF and in 19 episodes there was an associated virus identified. A total of 49 infective agents were identified either during ARIs or at routine testing in the patients with CF; 14 were identified on viral isolation (rhinovirus on 11 occasions), whilst 35 were isolated on seroconversion (PIV on 12, RSV on 9 and M. pneumoniae on 6 occasions). At the time of an ARI, the virus isolation and seroconversion rates were $8.8 \%$ and $19.1 \%$, respectively, in children with CF compared to $15.0 \%$ and $15.0 \%$, respectively, for the healthy siblings. In contrast, the rates of virus isolation and seroconversion at routine 2 monthly visits were $5.6 \%$ and $16.2 \%$, respectively, for children with CF and $7.7 \%$ and $20.2 \%$, respectively, for the healthy siblings. There was no significant difference in the rate of viral infections between the patients with CF and their sibling controls, as measured either by culture or serology. The rate of viral infections was higher in younger children (both CF and controls); however, the rate of decline in pulmonary function and severity score was both lower in the younger children with CF and the ones with more viral infections. The authors concluded that there were no significant adverse effects with viral infections in CF.

Likewise, Hiatt [22] assessed respiratory viral infections over three winters in 22 infants less than 2 years of age with CF (30 patient seasons), and 27 age-matched controls (28 patient seasons). The average number of acute respiratory illness per winter was the same in the control and CF groups (5.0 vs. 5.0). However, only 4 of the 28 control infants had lower respiratory tract symptoms in association with the respiratory tract illness, compared with 13 out of the 30 infants with CF (Odd ratio - 4.6; 95\% confidence interval 1.3 and 16.5; p-value $<0.05) ; 7$ of the infants with CF cultured $R S V$, of whom 3 required hospitalisation. In contrast, none of the controls required hospitalisation. Pulmonary function measured by rapid chest compression technique was significantly reduced in the infants with CF after the winter months and was associated with two interactions; $R S V$ infection with lower respiratory tract infection and male sex with lower respiratory tract infection.

From previous reports, two viral agents appear to have the greatest effect on respiratory status in $C F$, namely RSV and influenza, possibly because the uses of viral culture and serology have underestimated the effects of rhinovirus. In younger children, $R S V$ is a major pathogen resulting in an increased rate of hospitalisation. Abman et al. [37] prospectively followed up 48 children with CF diagnosed through newborn screening and documented the effect of $R S V$ infection. Eighteen of the infants were admitted into hospital a total of 30 times over a mean follow-up of 28 months (range 5-59]. In 7 of these infants RSV was isolated, and their clinical course was severe with 3 requiring mechanical ventilation and 5 necessitating chronic oxygen therapy. Over the next 2 years, these infants had significantly more frequent respiratory symptoms and lower Brasfield chest radiograph [38] scores than non-RSV-infected counterparts. 
In older children and adults with CF, influenza seems to have the greatest effect. Pribble et al. [23] assessed acute pulmonary exacerbation isolates from 54 patients with CF. Over the year of the study, 80 exacerbations were identified, of which 21 episodes were associated with an identified viral agent (influenza $A-5$ episodes; influenza $B-4$ episodes; $R S V-3$ episodes) with most agents identified on serology. Compared to other respiratory viruses, infection with influenza was associated with a more significant drop in pulmonary function $\left(\mathrm{FEV}_{1}\right.$ declined by $26 \%$ compared with $6 \%$ ). There were also a higher proportion of patients with a greater than $20 \%$ drop in $\mathrm{FEV}_{1}$ within the influenza infected cohort. A retrospective study in older patients with chronic $P$. aeruginosa infection reported an acute deterioration in clinical status in association with influenza $A$ virus infection, which was confirmed by serology [39].

Over a 1-year period, Smyth et al. [27] prospectively investigated 108 patients with CF (mean age of 7.9 years) using a combination of viral immunofluorescence, culture and seroconversion to identify respiratory viruses. With the exception of rhinovirus, a semi-nested reverse transcriptase PCR technique was used. During the study, 76 subjects had 157 respiratory exacerbations (1.5 episodes/patient/year) and a viral agent was identified in 44 episodes, 25 of which were rhinovirus and an equal distribution of other viruses was identified almost always on seroconversion. Rhinovirus identification was associated with significantly more days of intravenous antibiotics, whereas, those children in whom a non-rhinovirus was identified had a significantly greater decrease in $\mathrm{FEV}_{1}$ over the year of the study. When all viruses were considered as a whole, patients had significantly greater decline in Shwachman score [40] and days of intravenous antibiotics use.

Collinson et al. [26] followed 48 children with CF over a 15-month period using viral cultures for viral detection, with the exception of picornaviruses where polymerase chain reaction (PCR) was used; 38 children completed the study and there were 147 symptomatic upper respiratory tract infections (URTIs), 2.7 episodes/child/year, with samples available for 119 episodes. Picornaviruses were identified in 51 (43\%) of these episodes, of which 21 (18\%) were rhinoviruses. In those children old enough to perform spirometry, there was significant reduction in both FVC and $\mathrm{FEV}_{1}$ in association with URTIs, with little difference in severity of reduction whether a picornavirus was identified or not. Maximal mean drop in $\mathrm{FEV}_{1}$ was $16.5 \%$, at 1-4 days after onset of symptoms, but a deficit of $10.3 \%$ persisted at 21-24 days. Those with more URTIs appeared to have greater change in total Shwachman score [40] and Chrispin-Norman score [41] over the study. Six children isolated a P.aeruginosa for the first time during the study, 5 at the time of a URTI and only 1 was asymptomatic at the time of first isolation. However, the data from this study have to be handled with care as the term 'URTI' does not necessarily imply a positive viral isolation.

Punch et al. [42] used a multiplex reverse transcriptase PCR (RT-PCR) assay combined with an enzyme-linked amplicon hybridization assay (ELAHA) for the identification of seven common respiratory viruses in the sputum of $38 \mathrm{CF}$ patients; 53 sputum samples were collected over 2 seasons and 12 (23\%) samples from 12 patients were positive for a respiratory virus (4 for influenza B, 3 for parainfluenza type 1, 3 for influenza $A$ and 2 for RSV). There were no statistical associations between virus status and demographics, clinical variables or isolation rates for $P$. aeruginosa, S. aureus or A. fumigatus. 
Olesen and colleagues [28] obtained sputum and laryngeal aspirates from children with CF over a 12-month period in outpatient clinics. They achieved a viral detection rate of $16 \%$, with rhinovirus being the most prevalent virus. $\mathrm{FEV}_{1}$ was significantly reduced during viral infection $(-12.5 \%, \mathrm{p}=0.048)$, with the exception of rhinovirus infection. The authors were not able to demonstrate a positive correlation between respiratory viruses and bacterial infections in their studied population as the type or frequency of bacterial infection during or after viral infections were not altered. They also concluded that clinical viral symptoms had a very poor predictive value (0.39) for a positive viral test.

Our group in 2004 [36] utilised 'real-time' multiplex Nucleic Acid Sequenced Based Amplification to examine the role of respiratory viruses in CF children. Over an 18-month period, a viral detection rate of $46 \%$ was achieved during reported episodes of respiratory illness. The results compared favourably with previous studies and it may be that earlier studies relied heavily on repeated serological testing, either alone [20] or in combination with viral isolation [21-25]. The viral detection rate was $18.3 \%$ from routine nasal samples. However, this was comparable to the seroconversion rate of $12.3 \%$ as reported by Wang et al. [25]. Ramsey and colleagues [24] also achieved a similar seroconversion rate of $16.2 \%$ from asymptomatic samples. These results suggest that a laboratory method with a higher sensitivity for viral detection does not increase the detection rate in asymptomatic samples, implying that false positives are not necessarily more common than less sensitive diagnostic methods. Influenza $A$ and $B$ viruses were the major viruses in causing respiratory exacerbations in $C F$ and both viruses are more commonly detected during pulmonary exacerbations; 22 of 88 [23\%) viruses found in this study were influenza viruses $(A \mathcal{E} B$ ). The result is consistent with majority of the previous studies which showed that influenza virus represented between $12 \%$ and $27 \%$ of all viruses detected. However, the findings are in contrast with other studies where rhinovirus is the major virus in $\mathrm{CF}$ exacerbations [26, 28, 43]. The influenza vaccine uptake rate during the study period was up to 70\% [36]. It is possible that the detection rate for influenza virus could have been higher had the vaccine coverage not been this high.

Asner et al. [44] performed an observational cross-sectional study of CF children from a large paediatric referral centre investigating the association between respiratory viruses and pulmonary exacerbations by taking mid-turbinate swabs, sputum and throat swab samples that were tested by a direct immunofluorescent antibody assay and a multiplex PCR panel. Forty-three patients were recruited into the study. Pulmonary function tests, quality of life and severity scores were recorded. Sputum cell counts, bacterial density and cytokines were measured. Twenty-six (60.5\%) subjects were tested positive for at least one respiratory virus by any diagnostic method applied to any sample type. Of the 26 virus positive subjects, 17 $(65.4 \%)$ were positive for one virus and the remaining $9(34.6 \%)$ were positive for two or more viruses. Coxsackielechovirus was the most commonly identified pathogen (29.4\%) amongst the 17 subjects that were positive for one virus. Virus-positive patients were younger $(p=0.047)$ and more likely to be male $(p=0.029)$. They were also more likely to present with fever $(p=0.019)$, have higher CF clinical severity $(\mathrm{p}=0.041)$ and lower quality of life scores $(\mathrm{p}=0.022)$. However, virus-positive and negative patients had similar IL-8, neutrophil percentage, elastase levels and 26 additional cytokines levels between both groups. The authors reported a higher rate of 
viral detection with mid-turbinate swabs than with sputum samples. The study was primarily conducted in the outpatient setting and subjects may have milder form of exacerbations which may in turn explain the lack of inflammatory response in virus-positive subjects. There was also a significant median age difference between the virus-positive and negative groups (6.9 vs. 13 years, $\mathrm{p}=0.047$ ), with respiratory viral infection being more common in younger children presumably related to the delay maturation of the immune system.

A CF centre in Milan (Italy) led by Esposito and colleagues [43] showed that human rhinovirus was the most frequently isolated virus from $\mathrm{CF}$ patients $<25$ years of age during respiratory exacerbations and when subjects were clinically stable over a 1-year period. Molecular techniques were utilised to isolate viruses from nasopharyngeal samples. The authors demonstrated that human rhinovirus was more common among patients with pulmonary exacerbations than among clinically stable patients. The human rhinovirus viral load was however similar in subjects with or without acute respiratory exacerbations $(p=0.46)$. There were no correlations between the associated clinical condition and viral load as well as between bacterial colonisation, colonising bacterial, and viral infections between the 2 groups. This finding is similar to de Almeida et al. [17] who did not show a difference in viral infection between the exacerbation and clinically stable groups. Therefore, this raises a possibility that isolation of rhinovirus from nasopharyngeal swabs does not always indicate that it is a cause of exacerbation as it may be explained by a coincidental upper airways infection, a carrier state, or prolonged shedding of a pathogen that caused a previous infection [45].

In 2009, a novel swine pandemic influenza A virus (H1N1) was identified. Nash et al. [46] showed that the symptoms of CF patients infected with H1N1 tend to be mild. There was no significant reduction in $\mathrm{FEV}_{1} \%$ predicted, $\mathrm{FVC} \%$ predicted and body mass index regardless of whether the patients were positive or negative for H1N1. Colombo et al. [47] performed a multi-centre survey showing that diagnostic testing did not identify clinical characteristics specifically associated with H1N1 infections. Similarly, they did not show a significant decline in lung function associated with this infection. To date, the significance of H1N1 infection in CF remains undefined.

In contrast, the data regarding respiratory viral infection in adults is sparse. An observational study conducted by Hoek et al. [48] over a 1-year period amongst adult CF patients yielded a viral isolation rate of 33\% [8/24] utilising molecular techniques and conventional methods. Etherington and colleagues [18] from an adult CF centre published a retrospective case control study looking at the prevalence of respiratory viruses during exacerbations. Viral throat swabs were taken from all patients presenting with an acute pulmonary exacerbation requiring intravenous antibiotic treatment over a 12-month period. Viral isolation was performed by PCR. There were 432 pulmonary exacerbations in 180 adults. In total, there was a total positive viral isolation in 42 exacerbations indicating a prevalence of $9.7 \%$. Rhinovirus was the commonest isolated virus and was found on 29 occasions (69\%). Influenza A/H1N1 was isolated in seven patients $(16.7 \%)$. They demonstrated a measurable impact of viral infections in CF as exacerbations associated with a positive viral PCR had a greater fall in lung function at presentation with higher levels of inflammatory markers. These patients also received more 
days of intravenous antibiotics, showed less response to treatment and had a shorter time to next pulmonary exacerbation compared to matched controls.

Flight et al. [35] followed up 100 adult CF patients prospectively for 12 months. Sputum, nose swabs and throat swabs were collected every 2 months and at the onset of pulmonary exacerbation for virus detection. PCR assays for adenovirus, influenza A\&B, human metapneumovirus, parainfluenza 1-3, respiratory syncytial virus and human rhinovirus were performed on each sample. Symptom scores, spirometry and inflammatory markers were measured at each visit. Overall, virology results were available for 626 of 649 completed study visits. Of these, $191(30.5 \%)$ were positive for a respiratory virus including 9 episodes of dual viral infection. Human rhinovirus accounted for $72.5 \%$ of viruses. Overall incidence of viral respiratory infection (VRI) was 1.66 (95\% CI 1.39 to 1.92) cases/patient-year. VRI was associated with increased risk of pulmonary exacerbation $(\mathrm{OR}=2.19 ; 95 \% \mathrm{CI} 1.56$ to 3.08 ; $\mathrm{p}<0.001)$ and prescription of antibiotics (OR=2.26; 95\% CI 1.63 to 3.13; $\mathrm{p}<0.001$ ). Virus-positive visits were associated with higher respiratory symptom scores and greater C-reactive protein levels. Virus-positive exacerbations had a lower acute fall in $\mathrm{FEV}_{1}$ than virus-negative exacerbations ( $12.7 \%$ vs. $15.6 \%$; $\mathrm{p}=0.040)$. The incidence of exacerbations, but not VRI, was associated with greater lung function decline over 12 months (-1.79\% per pulmonary exacerbation/year; $95 \%$ CI -3.4 to $-0.23 ; \mathrm{p}=0.025$ ).

Experimental data on the effects of viral infections in CF are limited. Toll-like receptors (TLRs) have recently been identified as key mediators of the innate response and they recognise pathogens through detection of conserved microbial structures that are absent from the host. Kurt-Jones et al. [49] found that RSV persisted longer in the lungs of infected TLR4-deficient mice compared to normal mice. Haynes et al. [50] also demonstrated that TLR4-deficient mice when challenged with $R S V$ exhibited impaired natural killer cell trafficking and impaired virus clearance compared to normal ones. Limited human studies have demonstrated the important role of TLRs in host response against many major groups of mammalian pathogens [51]. The relationship between TLR and respiratory virus including $R S V$ in humans will require further studies before it can be established.

Some studies have suggested a higher viral replication when there is an impairment of the innate host defence in CF. Influenza titres were significantly increased in a mouse model which were chronically infected with $P$. aeruginosa compared to control model [52]. This in turn led to an increase in susceptibility to fatal streptococcus pneumonia infection. Increased virus replication was also found after PIV infection of CF human airway epithelial cells, compared to controls [53]. One of the possible causes of increased virus replication and of virus persistence might be a reduced production of respiratory nitric oxide (NO), which is a vital part of innate antiviral defence mechanism [54]. Increased production of $\mathrm{NO}$ protects against viral infections. In CF patients, expression of the NO producing enzyme NO synthase type 2 (NOS2) is considerably reduced.

$\mathrm{Xu}$ et al. [55] showed that CF cells that were infected with influenza A had less IFN-related antiviral gene induction at $24 \mathrm{~h}$ but more significant inflammatory cytokine gene induction at $1 \mathrm{~h}$ after infection. Therefore, the lesser antiviral and greater early inflammatory response may explain the severe respiratory illness of CF patients with viral infections. Sutanto and co- 
workers [56] showed that CF airway epithelial cells had a marked increase in IL-8 production, a reduction in apoptosis and an increased viral replication compared with airway epithelial cells from healthy children following exposure to human rhinovirus. This is despite the fact that CF and healthy airway epithelial cells have similar basal and stimulated expression of IL-8 in response to pro-inflammatory stimuli. The increment of IL-8, together with a reduction of apoptotic responses by CF cells to human rhinovirus, could contribute to augmented airway inflammation in the setting of recurrent viral infections early in life.

Azithromycin has previously been shown to offer anti-rhinoviral activity in bronchial epithelial cells and, during rhinovirus infection by increasing the production of interferon-stimulated genes [57]. However, the role of anti-viral properties of Azithromycin in CF is not clearly defined. Schögler et al. [58] showed that primary bronchial epithelial cells from CF children that were pre-treated with Azithromycin had a seven-fold reduction in rhinovirus replication without inducing cell death. Azithromycin also increased RV-induced pattern recognition receptor, IFN and IFN-stimulated gene mRNA levels when measured by real-time quantitative PCR. Therefore, it is likely that Azithromycin pre-treatment reduces RV replication in CF bronchial epithelial cells, possibly through the amplification of the antiviral response mediated by the IFN pathway.

\section{Detection of respiratory viruses}

The diagnostic accuracy and sensitivity of respiratory viral detection is determined by several factors:

1. Appropriate respiratory specimen for testing - Nasal swabs, nasopharyngeal aspirates, nasal swabs and mid-turbinate sampling are reasonable sampling methods in young children who may not be able to expectorate. However, in older patients, sputum is easy to obtain, painless and quick. Bronchoalveolar lavage (BAL) is a useful intervention to obtain specimen in the distal airways but it is more invasive. However, it can provide useful information regarding the activities of respiratory viruses and bacteria in the distal airways.

2. Appropriate specimen transport method - There are recognised procedures for transporting clinical specimens for diagnostic virology testing. These procedures should be adhered to closely to enhance the chances of isolating the viral organism. For instance, nasal swabs should be transported in viral transport medium and all specimens should be refrigerated if there is a delay of more than 2 hours in reaching the laboratory.

3. Detection methods - Molecular techniques have superseded many conventional methods such as viral culture and serology as they are far more sensitive and specific; in addition, they have a more rapid turn-around, allowing diagnostic technology to have an immediate impact on clinical management. The advantages of such technology will allow the appropriate utilisation of anti-virals, many of which are virus-specific; it may play a role in complying with hospital infection control policy; finally, it can provide useful infor- 
mation to public health authorities such that public health policies can be adjusted accordingly, e.g. the outbreak of SARS and influenza H5N1 virus.

4. Utilisation of real-time multiplex amplification technique allows multiple viruses being quantified even if the copy number of the viral target is low.

More recently, Virochip has been shown to be a pan-virus microarray platform that is capable of detection of known as well as novel viruses in a single assay simultaneously [59]. Probes chosen for Virochip can identify nodes in the viral taxonomy at the family, genus and species levels. As the Virochip probes are updated regularly, the extent of probes that can be covered are ever increasing, up to 36,000. It has a diagnostic sensitivity comparable to PCR for detecting respiratory genomes at levels as low as 100 genome copies. At the present time, Virochip is very much a research tool, and several issues must be addressed before it can be used as a routine test for virus detection in the clinical setting, including cost, diagnostic accuracy, repeatability, and sensitivity/specificity for virus detection. In addition, the clinical implication of novel viruses in the human respiratory tract is not yet defined. Therefore, the accurate interpretation of Virochip in the clinical setting remains a formidable task. For example, where specimens are polymicrobial or viral material are present at low levels, clinical and epidemiological information might be required to draw clinically meaningful conclusions.

\section{Interaction between respiratory viruses and bacteria}

In a 25-year retrospective review from the Danish CF clinic, the first isolation of $P$. aeruginosa was most likely between October and March [16] coinciding with the peak of the RSV season. However, there are a number of other possible viral agents that would broadly fit the winter season, most notably influenza, rhinovirus and metapneumovirus; therefore, these findings must be interpreted with caution.

An increase in immunoglobulin A ( $\operatorname{Ig} \mathrm{A})$ antibodies to the O-antigen of P. aeruginosa is noted in $62 \%$ of viral infections [60]. This suggests a possible 'microbial synergism' between bacterial infections and infections with respiratory viruses in CF.

The first bacterial isolation of a given organism in CF has also been shown to often follow a viral infection. In the 17-month prospective study reported by Collinson et al. [26], 5 of the 6 first isolations of $P$. aeruginosa were made during the symptomatic phase of an upper respiratory tract infection or three weeks thereafter. In contrast, only one of the 6 initial infections with $P$. aeruginosa was identified during the asymptomatic period. Similarly, $H$. influenzae was recovered for the first time from 3 children within 3 weeks of an upper respiratory tract infection and the one new $S$. aureus infection was identified immediately following a viral infection.

Armstrong and colleagues have reported that $50 \%$ of CF respiratory exacerbations requiring hospitalisation are associated with isolation of a respiratory virus [21]. In their prospective study of repeated BAL in infants over a 5-year period, a respiratory virus was identified in $52 \%$ of infants hospitalised for a respiratory exacerbation, most commonly $R S V ; 11$ of the 31 
hospitalised infants (35\%) acquired P. aeruginosa in the subsequent 12-60 month follow-up, compared to 3 of 49 (6\%) non-hospitalised infants (Relative risk 5.8). This indicates that RSV infection was identified immediately following a viral infection.

Respiratory viruses can disrupt the airway epithelium and precipitate bacterial adherence. Influenza $A$ infection has been shown to cause epithelial shedding to basement membrane with submucosal oedema and neutrophil infiltrate [61], while both influenza and adenovirus have a cytopathic effect on cultured nasal epithelium leading to destruction of the cell monolayer [62]. This epithelial damage results in an increase in the permeability of the mucosal layer [63, 64] and possibly facilitating bacterial adherence. Bacteria can also utilise viral glycoproteins and other virus-induced receptors on host cell membrane as bacterial receptors in order to adhere to virus-infected cells $[65,66]$.

Kim et al. [67] found that invariant natural killer T cells induce a type of macrophage activation driving the secretion of interleukin-13 leading to the production of globlet cell metaplasia and airway hyperactivity following infection with Sendai virus. The term 'invariant' stems from the fact that all invariant natural killer $T$ cells in humans and mice use a unique $T$ cell receptor that is essential for interaction with CD1d. CD1d molecules present lipid antigens to T lymphocytes rather than peptide antigens as in the case of major histocompatibility complex (MHC) class I and II molecules. Historically, MHC class II dependent CD4 and T lymphocytes, through their response to stimulation by environmental allergens, are keys to the pathogenesis of human asthma. The findings by the authors lead to the notion of the use of anti-interleukin-13 therapy as a potential therapy in patients.

Viral infections might predispose to secondary bacterial infections by impairing mucociliary function and triggering host inflammatory receptors $[68,69]$. This phenomenon has been demonstrated both in vivo and in vitro [70, 71]. Avadhanula et al. [72] showed that different respiratory viruses use different mechanisms to enhance the adherence of bacteria to respiratory epithelial cells. In particular, RSV and PIV type 3 up-regulate intercellular adhesion molecule-1 (ICAM-1), carcinoembryonic adhesion molecule 1 (CEACAM1) and platelet activating factor receptor (PAFr) but not mucin on the surfaces of A549, BEAS-2B and NHBE but not SAE cell lines. Much of the increased bacterial adhesion following RSV infection could be blocked by antibodies directed against these receptors. A549 and BEAS-2B are transformed cell lines derived from type II alveolar and normal bronchial cells, respectively. NHBE and SAE cells are primary epithelial cells obtained from bronchi and distal bronchial tree and are likely to include a heterogeneous population of cells.

Mechanisms independent of the expression of conventional receptors for bacteria, such as binding to viral proteins, could be responsible for enhanced adhesion [73]. Immunofluorescence microscopy demonstrates that bacteria binding to RSV-infected A549 cells adhere not only to these cells expressing viral antigens but also to uninfected epithelial cells. These data suggest that the ability to augment bacterial adhesion may result from a factor served by infected cells that exert a paracrine effect on adjacent epithelium. Cytokines or other inflammatory molecules are potential good candidates for such a mediator. 
Rhinovirus has been shown to potentiate bacterial infections by inhibiting the secretion of TNF alpha and interleukin- 8 by macrophages in vitro following co-infection with gram negative bacterial products, lipopolysaccharide (LPS), and gram positive bacterial products, lipoteichoic acid (LTA) [74]. This rhinovirus-dependent impairment of the macrophage immune response was not mediated by autocrine production of the anti-inflammatory cytokines interleukin-10 and PGE2, or by down-regulation of the cell surface receptor for LTA and LPS. In addition, the authors also show that rhinovirus inhibit the phagocytosis of bacterial products by macrophages. These findings support the notion that rhinovirus exposure resulted in a reduced ability to innate and adaptive immune responses against bacterial products, hence promoting the occurrence of bacterial and viral co-infections.

The lower respiratory tract is protected by local mucociliary mechanisms that involve the integration of the ciliated epithelium, periciliary fluid and mucus. Mucus acts as a physical and chemical barrier onto which particles and organisms adhere. Cilia lining the respiratory tract propel the overlying mucus to the oropharynx where it is either swallowed or expectorated. Influenza viral infection has been shown to precipitate the loss of cilial beat, and shedding of the columnar epithelial cells generally within 48 hours of infection [75]. Pittet et al. [76] showed that a prior influenza infection of tracheal cells in vivo does not increase the initial number of pneumococci found during the first hour of infection, but it does significantly reduce mucociliary velocity, and thereby reduces pneumococcal clearance during the first 2 hours after pneumococcal infection at both 3 and 6 days after an influenza infection. The defects in pneumococcal clearance were greatest at 6 days after influenza infection. Changes to the tracheal epithelium induced by influenza virus may increase susceptibility to a secondary S. pneumoniae infection by increasing pneumococcal adherence to the tracheal epithelium and/or decreasing the clearance of $S$. pneumoniae via the mucociliary escalator of the trachea, and thus increasing the risk of secondary bacterial infection.

De Vrankrijker et al. [77] showed that mice that were co-infected with RSV and P. aeruginosa had a 2,000 times higher colony-forming units (CFU) count of $P$. aeruginosa in the lung homogenates compared to mice that were infected with $P$. aeruginosa alone. Co-infected mice also had more severe lung function changes. These results suggest that $R S V$ can facilitate the initiation of acute $P$. aeruginosa infection.

Another study also showed that $H$. influenzae and S. pneumoniae bind to both free RSV virions and epithelial cells transfected with cell-membrane-bound $G$ protein, but not to secreted $G$ protein. Pre-incubation with specific anti-G antibody significantly reduces bacterial adhesion to $G$ protein-transfected cells [78].

Stark et al. [79] showed that mice that were exposed to $R S V$ had significantly decreased $S$. pneumonia, S. aureus or $P$. aeruginosa clearance 1 to 7 days after $R S V$ exposure. Mice that were exposed to both $R S V$ and bacteria had a higher production of neutrophil-induced peroxide but less production of myeloperoxidase compared to mice that were exposed to S. pneumoniae alone. This suggests that functional changes in the recruited neutrophils may contribute to the decreased bacterial clearance. 
More recently, Chattoraj et al. [15] demonstrated that acute infection of primary CF airway epithelial cells with rhinovirus liberates planktonic bacteria from biofilm. Superinfection with rhinovirus stimulates robust chemokine responses from $C F$ airway epithelial cells that were pre-treated with mucoid $P$. aeruginosa. The authors also showed that these chemokine responses lead to a liberation of bacteria from mucoid $P$. aeruginosa biofilm and transmigration of planktonic bacteria from the apical to the basolateral surface of mucociliary-differentiated CF airway epithelial cells. Planktonic bacteria, which are more pro-inflammatory than their biofilm counterparts, stimulate increased chemokine responses in CF airway epithelial cells which, in turn, may contribute to the pathogenesis of CF exacerbations and subsequent prolonged intravenous antibiotic use and hospitalisation.

Contrary to the above reports, Chin et al. [32] performed a prospective study over a 2-year period on 35 adult CF patients. P. aeruginosa sputum density was analysed during stable, exacerbation and post-exacerbation assessments. PCR was used to detect respiratory viruses during exacerbations. The sputum density of $P$. aeruginosa in patients with or without a viral infection was compared using quantitative culture or by PCR. Twenty-two patients experienced 30 exacerbations during the study period; $50 \%$ were associated with a viral infection. There was no change in sputum density of $P$. aeruginosa from the stable to exacerbation state. Virus-associated exacerbations did not result in significant increases in $P$. aeruginosa sputum density compared to non-viral exacerbations.

Contrary to the above findings, Asner et al. [44] found the mean total bacterial density in sputum samples in virus-positive patients being two logs lower than that found in virusnegative patients $(\mathrm{p}=0.299)$. However, this could be explained by the fact that the median age of the virus-positive group was significantly lower than the virus-negative group. Viruspositive and virus-negative patients had similar IL-8, neutrophil percentage and neutrophil elastase levels.

Similarly, Kieninger et al. [80] performed a comprehensive investigation of the inflammatory response of CF airway epithelial cells on virus infection. Strong cytokine production was found in all cells studied, with the magnitude and type of inflammation differing depending on cell type and virus used. There was no exaggerated inflammatory response in $\mathrm{CF}$, either during cytokine production or at the transcriptional level. Instead, there was a trend towards lower cytokine production in CF airway epithelial cells after virus infection, which was associated with increased cell death. The lower inflammatory response in CF can also be explained by additional pathophysiological mechanisms, such as interactions between anti-viral and proinflammatory pathways, which are likely to be involved [81]. It could also be speculated that because of chronic activation of pro-inflammatory pathways, CF airway epithelial cells are not able to respond sufficiently to further stimuli, such as virus infections. This might, in turn, lead to a lack of recruitment of effector immune cells resulting in longer duration and more severe respiratory symptoms.

TLRs are key mediators of type I interferon (IFN) during viral infections by recognizing various viral components. TLR7 and TLR9 have become apparent as universally important in inducing type I IFN during infection with most viruses, particularly by plasmacytoid dendritic cells [82]. New intracellular viral pattern recognition receptors leading to type I IFN production have 
been identified. CFTR mutations have been shown to affect the epithelial induction of type I IFN expression by airway cells in response to P. aeruginosa infection [83]. This is achieved by abolishing this signalling pathway, an important component of the innate immune system that protects mucosal surfaces. Based on available evidence, chronic colonisation of $P$. aeruginosa in CF airways can be hypothesised to increase the predisposition of viral infections; however, more in-depth studies are required to elucidate this hypothesis.

Taken together, these findings suggest conflicting data regarding the inflammatory response of the CF airway epithelium on virus infection and to some extent the symbiotic relationship between viruses and bacteria. Nonetheless, respiratory viruses may lead to epithelial disruption, increase neutrophil influx, inhibition of macrophage phagocytosis, destruction of mucociliary escalator, down-regulation of cilia beat, liberation of pro-inflammatory planktonic $P$. aeruginosa from biofilm and increased neutrophil-induced peroxide release, indirectly facilitating bacterial infection of the airway.

\section{Prevention and treatment for respiratory viruses}

The diversity of viral serotypes in causing infection has made vaccine preparation very difficult. Frequent mutations of viral proteins of RNA viruses (e.g. genetic drift and shift of influenza) have further hampered the prevention of the illness.

In the UK, it has been reported that 2,150 deaths during the 2011/12 season was attributable to influenza [84], though some of the deaths may be attributed to $R S V$. Influenza vaccines are the only commercially available vaccines against common respiratory viruses. They have been used since the mid-1940s and they now have an established role in the prevention of influenza $A$ and $B$ infections. Inactivated influenza vaccine is effective even in young children including those younger than 2 years [85]. The waning of vaccine-induced immunity over time requires annual re-immunisation even if the vaccine antigens are unchanged.

Recent vaccines contain antigens of two influenza A subtypes, strains of the currently circulating H3N2 and H1N1 (Swine flu) subtypes, and one influenza B virus. The current recommendation for influenza vaccination in the UK is to offer it to those over the age of 65 , those with chronic heart, respiratory (including CF) or renal diseases and those who are diabetic or immunosuppressed.

Our group [34] recently showed that influenza vaccination provides protection against influenza acquisition in patients with $C F$, with 1 of 41 patients vaccinated having a positive nasal swab for influenza compared to 4 of the 22 non-vaccinated patients ( $p=0.046$ ). Although influenza vaccination does not appear to have any impact on respiratory exacerbation rates, it does have a role in preventing live infections. In our study, respiratory exacerbation rates in the preceding 10 months before the study between the vaccinated and non-vaccinated groups were similar, indicating that these were unlikely to be the reasons influencing the decision on immunisation. The decision may be secondary to a combination of patient/parent education, social background, awareness of vaccination and accessibility of vaccination. 
Due to the lack of randomised controlled studies looking at the efficacy of influenza vaccine in $\mathrm{CF}$, the Cochrane review recommends clinicians to make their own judgements on the benefits and risks of this therapy in this cohort of patients [86]. In addition to vaccine, neuraminidase inhibitors have been shown to have a role in preventing influenza $A$ and $B$ infections [87].

Rhinovirus has more than 100 serotypes; therefore, it will be unlikely that a unifying vaccine can be developed. VP4, one of the non-enveloped capsids, is highly conserved among all of the rhinoviruses; anti-VP4 antibodies have recently been generated and been shown to have the potential for future vaccine development [88].

The development of an $R S V$ vaccine has been hampered by the experience with formalininactivated whole $R S V$ vaccine in the 1960s, as it caused $80 \%$ of $R S V$ vaccinees to become hospitalised compared with $5 \%$ of controls, as well as two fatalities [89]. Current major research work has focused on a prophylaxis using a humanised mouse monoclonal antibody, Palizivumab. In patients with CF, monthly Palizivumab injection significantly reduced the hospitalisation rate for acute respiratory illness during the $R S V$ season compared to those who were not immunised $(\mathrm{p}<0.05)$. The former group also had fewer hospital days for acute respiratory illness [90]. However, the Cochrane database systematic reviews were not able to draw any firm conclusions on the safety and tolerability of RSV prophylaxis with Palivizumab in infants with cystic fibrosis up to 2 years of age due to a lack of randomised controlled studies [91]. Further studies are required to evaluate the safety and effectiveness of this treatment in CF patients.

There is currently no licensed PIV vaccine. The formalin-inactivated vaccine generated in the 1960s was not able to prevent PIV infection and was soon abandoned. Recently, recombinant bovine PIV type 3 and human PIV type 3 attenuated vaccines are being evaluated in animal models as vectors for the delivery of other viral antigens such as RSV-G and RSV-F proteins. This bivalent vaccine combination provides high level of resistance to challenges with PIV type 3 and $R S V$ in animal models [92].

The conventional methods of vaccination are via the intramuscular and subcutaneous routes. Mucosal immunisation has recently been introduced as it represents an attractive manner of delivering vaccines. It is fast, simple, non-invasive and can be carried out by unskilled individuals. The use of mucosal vaccination seems logical in that most of respiratory viral infections initially start at the mucosal sites and therefore induce local immunity. In the autumn/winter of 2014/15 the annual nasal spray flu vaccine (Fluenz Tetra) became available for children aged 2, 3 and 4 year as part of the UK NHS childhood vaccination programme. The nasal spray flu vaccine is also for children aged 2-18 years who are "at risk" from flu, such as children with long-term health conditions.

Amantadine has been the conventional anti-viral against influenza. However, it is strainspecific as it is only effective against influenza $A$ and has common side effects such as insomnia, poor concentration and irritability. It is now largely being replaced by neuraminidase inhibitors such as Zanamivir and Oseltamivir, which are licensed for the treatment of influenza $A$ and 
$B$, including avian flu H5N1 and swine flu H1N1. However, Amantadine still has a role in dealing with Oseltamivir-resistant $\mathrm{H} 1 \mathrm{~N} 1$ virus. In children and adults, early initiation of neuraminidase inhibitors within 48 hours of the onset of symptoms can reduce the duration of flulike symptoms by 0.5 to 2.5 days [93]. Early use of these medications can also reduce development of complications such as pneumonia [94]. The 2009 pandemic H1N1 virus remains susceptible to neuraminidase inhibitors, and Oseltamivir has been used extensively for treatment related to this viral infection. Resistance to Oseltamivir has been reported with H1N1 viral infection but this is mainly restricted to immunocompromised individuals [95]. Zanamivir has a poor oral bioavailability, and intranasal application has been shown to be effective in treating experimental influenza infection with the reduction in symptoms caused, virus shedding and development of otitis media [96]. Intravenous use of Peramivir or Zanamivir could be lifesaving in critically ill patients with influenza infection [97,98]. However, currently the Cochrane database of systematic reviews does not recommend the routine use of neuraminidase inhibitors in influenza infection in CF because of the absence of high level evidence for the effectiveness of these interventions [99].

Ribavarin, a synthetic guanosine nucleoside that has a broad spectrum of anti-viral activity, has been used for treatment of infections related to RSV, metapneumovirus, and parainfluen$z a$ and influenza viruses [100]. Potential benefits of ribavarin therapy include the inhibition of RSV-specific IgE production in nasal secretions, which has been associated with the development of hypoxaemia and wheezing [101] and it has improved pulmonary functions [102]. Controlled studies also show that the use of ribavarin is effective in reducing the clinical severity score, duration of mechanical ventilation, supplemental oxygen use and days of hospitalisation [103]. Aerosolised ribavarin has been used for the treatment of $R S V$-related bronchiolitis and pneumonia. Intravenous formulation could be used for treatment of severe pneumonia, caused by infection $R S V$, metapneumovirus, or parainfluen$z a$ virus, on the basis of experience in immunocompromised patients [104]. Bonney et al. have shown that metapneumovirus can be successfully treated with a combination of intravenous ribavarin and immunoglobulin [105].

Although rhinovirus is the major cause of colds, its vast amount of serotypes has made development of anti-virals against it problematic. A $90 \%$ of rhinovirus serotypes gain entry into epithelial cells using ICAM-1 cellular receptors, and blockade of these receptors in experimental studies has shown reduced infection severity [106], but further study is required before this treatment option becomes widely available. Macrolide antibiotics, Bafilomycin A1 and Erythromycin have been shown to inhibit ICAM-1 epithelial expression and hypotheses about their potential as anti-inflammatory agents have yet to be definitive, as clinical proof is either negative or inconclusive [107].

Recently, an anti-rhinoviral agent known as Plecoranil, which acts by inhibiting the uncoating of Picornaviruses [108], the RV 3C protease inhibitor, Ruprintrivir[109] and soluble ICAM-1, Tremacamra[106] have shown promising results in early-stage clinical trials, but each of these medications was derailed by a combination of cost, pharmacokinetics, toxicity, drug interactions, and limited efficacy [110]. 
A previous study suggests that the increased morbidity in CF patients after virus infection is not due to an exaggerated inflammatory response of the airway epithelium but rather linked to increased cell death. Thus, they provide a rationale for implementing therapies aimed at controlling viruses and their replication rather than primarily targeting inflammation. In this respect, a promising candidate is the macrolide-antibiotic azithromycin, which is increasingly used in CF patients as a beneficial immunomodulatory agent [111] and has recently been shown to possess anti-viral properties [57].

\section{Conclusion}

As we become increasingly knowledgeable about the impact of respiratory virus infections in the context of CF exacerbations, screening for respiratory viruses should be part of the routine investigations for any CF patients that present with exacerbation symptoms. Using the appropriate sampling method in conjunction with sensitive and specific diagnostic technology will enable us to make appropriate clinical decisions surrounding the use of anti-virals and antibiotics.

Gaining further understanding in the pathogenesis of virus-induced respiratory exacerbations in CF may allow the development of new therapeutic techniques. If viral infection does predispose to bacterial infection, then influencing the interaction between viruses and bacteria could be a next pathway to diminish respiratory morbidity in patients with $\mathrm{CF}$. The development of novel therapies will be exciting and this may improve their quality of life and prolong the lifespan of patients with CF.

However, there are still a number of research dilemmas that remain unanswered:

1. What are the standardised definitions of CF pulmonary exacerbation and pulmonary exacerbation severity score?

2. What is the optimal way for viral sampling?

3. What is the role of Virochip in routine viral identification?

4. How do respiratory viruses influence bacterial activities in chronically infected airways?

5. What influences the rate of respiratory viral clearance in CF respiratory tract?

6. What are the roles of anti-virals in CF?

7. What are the anti-viral properties of Azithromycin in CF?

Further understanding in the pathogenesis of viral infection in CF would be beneficial as this may provide insight to the above unresolved mysteries. At the moment, influenza vaccination and the use of neuraminidase inhibitors remain the only evidence based practice, albeit weak for the management of viral infections in $\mathrm{CF}$. 


\section{Author details}

Dennis Wat

Address all correspondence to: Dennis.Wat@lhch.nhs.uk

Liverpool Heart and Chest Hospital, Liverpool, United Kingdom

\section{References}

[1] Farrell PM. The prevalence of cystic fibrosis in the European Union. Journal of cystic fibrosis : official journal of the European Cystic Fibrosis Society. 2008;7(5):450-3.

[2] 2005 U.S. Cystic Fibrosis Foundation Annual Data Report to the Center Directors.. 2006.

[3] Riordan JR, Rommens JM, Kerem B, Alon N, Rozmahel R, Grzelczak Z, et al. Identification of the cystic fibrosis gene: cloning and characterization of complementary DNA. Science. 1989;245(4922):1066-73.

[4] Vawter GF, Shwachman H. Cystic fibrosis in adults: an autopsy study. Pathol Annu. 1979;14 Pt 2:357-82.

[5] Oppenheimer EH, Esterly JR. Pathology of cystic fibrosis review of the literature and comparison with 146 autopsied cases. Perspect Pediatr Pathol. 1975;2:241-78.

[6] Saiman L, Siegel J. Infection control in cystic fibrosis. Clin Microbiol Rev. 2004;17(1): 57-71.

[7] Rajan S, Saiman L. Pulmonary infections in patients with cystic fibrosis. Semin Respir Infect. 2002;17(1):47-56.

[8] Mahadeva R, Webb K, Westerbeek RC, Carroll NR, Dodd ME, Bilton D, et al. Clinical outcome in relation to care in centres specialising in cystic fibrosis: cross sectional study. Bmj. 1998;316(7147):1771-5.

[9] UK Cystic Fibrosis Registry Annual Data Report 2013: Summary 2014.

[10] Flume PA, Liou TG, Borowitz DS, Li H, Yen K, Ordonez CL, et al. Ivacaftor in subjects with cystic fibrosis who are homozygous for the F508del-CFTR mutation. Chest. 2012;142(3):718-24.

[11] Sawicki GS, Rasouliyan L, Pasta DJ, Regelmann WE, Wagener JS, Waltz DA, et al. The impact of incident methicillin resistant Staphylococcus aureus detection on pulmonary function in cystic fibrosis. Pediatr Pulmonol. 2008;43(11):1117-23. 
[12] Thomassen MJ, Demko CA, Klinger JD, Stern RC. Pseudomonas cepacia colonization among patients with cystic fibrosis. A new opportunist. Am Rev Respir Dis. 1985;131(5):791-6.

[13] Nixon GM, Armstrong DS, Carzino R, Carlin JB, Olinsky A, Robertson CF, et al. Clinical outcome after early Pseudomonas aeruginosa infection in cystic fibrosis. J Pediatr. 2001;138(5):699-704.

[14] Aaron SD, Ramotar K, Ferris W, Vandemheen K, Saginur R, Tullis E, et al. Adult cystic fibrosis exacerbations and new strains of Pseudomonas aeruginosa. Am J Respir Crit Care Med. 2004;169(7):811-5.

[15] Chattoraj SS, Ganesan S, Jones AM, Helm JM, Comstock AT, Bright-Thomas R, et al. Rhinovirus infection liberates planktonic bacteria from biofilm and increases chemokine responses in cystic fibrosis airway epithelial cells. Thorax. 2011;66(4):333-9.

[16] Johansen HK, Hoiby N. Seasonal onset of initial colonisation and chronic infection with Pseudomonas aeruginosa in patients with cystic fibrosis in Denmark. Thorax. 1992;47(2):109-11.

[17] de Almeida MB, Zerbinati RM, Tateno AF, Oliveira CM, Romao RM, Rodrigues JC, et al. Rhinovirus $C$ and respiratory exacerbations in children with cystic fibrosis. Emerging infectious diseases. 2010;16(6):996-9.

[18] Etherington C, Naseer R, Conway SP, Whitaker P, Denton M, Peckham DG. The role of respiratory viruses in adult patients with cystic fibrosis receiving intravenous antibiotics for a pulmonary exacerbation. Journal of cystic fibrosis : official journal of the European Cystic Fibrosis Society. 2014;13(1):49-55.

[19] Wat D, Doull I. Respiratory virus infections in cystic fibrosis. Paediatric respiratory reviews. 2003;4(3):172-7.

[20] Petersen NT, Hoiby N, Mordhorst CH, Lind K, Flensborg EW, Bruun B. Respiratory infections in cystic fibrosis patients caused by virus, chlamydia and mycoplasma-possible synergism with Pseudomonas aeruginosa. Acta paediatrica Scandinavica. 1981;70(5):623-8.

[21] Armstrong D, Grimwood K, Carlin JB, Carzino R, Hull J, Olinsky A, et al. Severe viral respiratory infections in infants with cystic fibrosis. Pediatric pulmonology. 1998;26(6):371-9.

[22] Hiatt PW, Grace SC, Kozinetz CA, Raboudi SH, Treece DG, Taber LH, et al. Effects of viral lower respiratory tract infection on lung function in infants with cystic fibrosis. Pediatrics. 1999;103(3):619-26.

[23] Pribble CG, Black PG, Bosso JA, Turner RB. Clinical manifestations of exacerbations of cystic fibrosis associated with nonbacterial infections. The Journal of pediatrics. 1990;117(2 Pt 1):200-4. 
[24] Ramsey BW, Gore EJ, Smith AL, Cooney MK, Redding GJ, Foy H. The effect of respiratory viral infections on patients with cystic fibrosis. American journal of diseases of children. 1989;143(6):662-8.

[25] Wang EE, Prober CG, Manson B, Corey M, Levison H. Association of respiratory viral infections with pulmonary deterioration in patients with cystic fibrosis. The New England journal of medicine. 1984;311(26):1653-8.

[26] Collinson J, Nicholson KG, Cancio E, Ashman J, Ireland DC, Hammersley V, et al. Effects of upper respiratory tract infections in patients with cystic fibrosis. Thorax. 1996;51(11):1115-22.

[27] Smyth AR, Smyth RL, Tong CY, Hart CA, Heaf DP. Effect of respiratory virus infections including rhinovirus on clinical status in cystic fibrosis. Archives of disease in childhood. 1995;73(2):117-20.

[28] Olesen HV, Nielsen LP, Schiotz PO. Viral and atypical bacterial infections in the outpatient pediatric cystic fibrosis clinic. Pediatric pulmonology. 2006;41(12):1197-204.

[29] Influenza virus vaccine live intranasal--MedImmune vaccines: CAIV-T, influenza vaccine live intranasal. Drugs in R\&D. 2003;4(5):312-9.

[30] Thompson WW, Shay DK, Weintraub E, Brammer L, Bridges CB, Cox NJ, et al. Influenza-associated hospitalizations in the United States. JAMA. 2004;292(11):1333-40.

[31] Ortiz JR, Neuzil KM, Victor JC, Wald A, Aitken ML, Goss CH. Influenza-associated cystic fibrosis pulmonary exacerbations. Chest. 2010;137(4):852-60.

[32] Chin M, De Zoysa M, Slinger R, Gaudet E, Vandemheen KL, Chan F, et al. Acute effects of viral respiratory tract infections on sputum bacterial density during CF pulmonary exacerbations. Journal of cystic fibrosis : official journal of the European Cystic Fibrosis Society. 2014.

[33] Punch G, Syrmis MW, Rose BR, Harbour C, Bye PT, Nissen MD, et al. Method for detection of respiratory viruses in the sputa of patients with cystic fibrosis. Eur J Clin Microbiol Infect Dis. 2005;24(1):54-7.

[34] Wat D, Gelder C, Hibbitts S, Bowler I, Pierrepoint M, Evans R, et al. Is there a role for influenza vaccination in cystic fibrosis? J Cyst Fibros. 2008;7(1):85-8.

[35] Flight WG, Bright-Thomas RJ, Tilston P, Mutton KJ, Guiver M, Morris J, et al. Incidence and clinical impact of respiratory viruses in adults with cystic fibrosis. Thorax. 2014;69(3):247-53.

[36] Wat D, Gelder C, Hibbitts S, Cafferty F, Bowler I, Pierrepoint M, et al. The role of respiratory viruses in cystic fibrosis. Journal of cystic fibrosis : official journal of the European Cystic Fibrosis Society. 2008;7(4):320-8. 
[37] Abman SH, Ogle JW, Harbeck RJ, Butler-Simon N, Hammond KB, Accurso FJ. Early bacteriologic, immunologic, and clinical courses of young infants with cystic fibrosis identified by neonatal screening. J Pediatr. 1991;119(2):211-7.

[38] Brasfield D, Hicks G, Soong S, Tiller RE. The chest roentgenogram in cystic fibrosis: a new scoring system. Pediatrics. 1979;63(1):24-9.

[39] Conway SP, Simmonds EJ, Littlewood JM. Acute severe deterioration in cystic fibrosis associated with influenza A virus infection. Thorax. 1992;47(2):112-4.

[40] Shwachman H, Kulczycki LL. Long-term study of one hundred five patients with cystic fibrosis; studies made over a five- to fourteen-year period. AMA J Dis Child. 1958;96(1):6-15.

[41] Chrispin AR, Norman AP. The systematic evaluation of the chest radiograph in cystic fibrosis. Pediatr Radiol. 1974;2(2):101-5.

[42] Punch G, Syrmis MW, Rose BR, Harbour C, Bye PT, Nissen MD, et al. Method for detection of respiratory viruses in the sputa of patients with cystic fibrosis. European journal of clinical microbiology \& infectious diseases : official publication of the European Society of Clinical Microbiology. 2005;24(1):54-7.

[43] Esposito S, Dacco V, Daleno C, Gambazza S, Montinaro V, Bisogno A, et al. Human rhinovirus infection in children with cystic fibrosis. Japanese journal of infectious diseases. 2014;67(5):399-401.

[44] Asner S, Waters V, Solomon M, Yau Y, Richardson SE, Grasemann H, et al. Role of respiratory viruses in pulmonary exacerbations in children with cystic fibrosis. Journal of cystic fibrosis : official journal of the European Cystic Fibrosis Society. 2012;11(5):433-9.

[45] Nokso-Koivisto J, Kinnari TJ, Lindahl P, Hovi T, Pitkaranta A. Human picornavirus and coronavirus RNA in nasopharynx of children without concurrent respiratory symptoms. Journal of medical virology. 2002;66(3):417-20.

[46] Nash EF, Whitmill R, Barker B, Rashid R, Whitehouse JL, Honeybourne D. Clinical outcomes of pandemic (H1N1) 2009 influenza (swine flu) in adults with cystic fibrosis. Thorax. 2011;66(3):259.

[47] Colombo C, Battezzati PM, Lucidi V, Magazzu G, Motta V, Alicandro G, et al. Influenza A/H1N1 in patients with cystic fibrosis in Italy: a multicentre cohort study. Thorax. 2011;66(3):260-1.

[48] Hoek RA, Paats MS, Pas SD, Bakker M, Hoogsteden HC, Boucher CA, et al. Incidence of viral respiratory pathogens causing exacerbations in adult cystic fibrosis patients. Scandinavian journal of infectious diseases. 2013;45(1):65-9. 
[49] Kurt-Jones EA, Popova L, Kwinn L, Haynes LM, Jones LP, Tripp RA, et al. Pattern recognition receptors TLR4 and CD14 mediate response to respiratory syncytial virus. Nat Immunol. 2000;1(5):398-401.

[50] Haynes LM, Moore DD, Kurt-Jones EA, Finberg RW, Anderson LJ, Tripp RA. Involvement of toll-like receptor 4 in innate immunity to respiratory syncytial virus. J Virol. 2001;75(22):10730-7.

[51] Qureshi ST, Medzhitov R. Toll-like receptors and their role in experimental models of microbial infection. Genes Immun. 2003;4(2):87-94.

[52] Seki M, Higashiyama Y, Tomono K, Yanagihara K, Ohno H, Kaneko Y, et al. Acute infection with influenza virus enhances suspectibility to fatal pneumonia following Streptococcus pneumoniae infection in mice with chronic pulmonary colonisation with Psudomonas aeruginosa. Clin Exp Immunol. 2004;137(1):35-40.

[53] Zheng S, De B, Choudhary S, Comhair S, Goggans T, Slee R, et al. Impaired innate host defense causes suspectibility to respiratory virus infections in cystic fibrosis. Immunity. 2003;18(5):619-30.

[54] Zheng S, Xu W, Bose S, Banerjee A, Haque S, Erzurum S. Impaired nitirc oxide synthase-2 signaling pathway in cystic fibrosis airway epithelium. Am J Physiol Lung Cell Mol Physiol. 2004;287(2):L374-81.

[55] Xu W, Zheng S, Goggans TM, Kiser P, Quinones-Mateu ME, Janocha AJ, et al. Cystic fibrosis and normal human airway epithelial cell response to influenza a viral infection. J Interferon Cytokine Res. 2006;26(9):609-27.

[56] Sutanto EN, Kicic A, Foo CJ, Stevens PT, Mullane D, Knight DA, et al. Innate inflammatory responses of pediatric cystic fibrosis airway epithelial cells: effects of nonviral and viral stimulation. Am J Respir Cell Mol Biol. 2011;44(6):761-7.

[57] Gielen V, Johnston SL, Edwards MR. Azithromycin induces anti-viral responses in bronchial epithelial cells. The European respiratory journal. 2010;36(3):646-54.

[58] Schogler A, Kopf BS, Edwards MR, Johnston SL, Casaulta C, Kieninger E, et al. Novel antiviral properties of azithromycin in cystic fibrosis airway epithelial cells. The European respiratory journal. 2015;45(2):428-39.

[59] Chiu CY, Rouskin S, Koshy A, Urisman A, Fischer K, Yagi S, et al. Microarray detection of human parainfluenzavirus 4 infection associated with respiratory failure in an immunocompetent adult. Clin Infect Dis. 2006;43(8):e71-6.

[60] Przyklenk B, Bauernfeind A, Bertele RM. Viral infections in the respiratory tract in patients with cystic fibrosis. Serodign Immunother Infect Dis. 1988(2):217-25.

[61] Walsh JJ, Dietlein LF, Low FN, Burch GE, Mogabgab WJ. Bronchotracheal response in human influenza. Type A, Asian strain, as studied by light and electron microscopic examination of bronchoscopic biopsies. Arch Intern Med. 1961;108:376-88. 
[62] Winther B, Gwaltney J, Hendley J. Respiratory virus infection of monolayer cultures of human nasal epithelial cells. Am Rev Respir Dis. 1990;141(4 Pt 1):839-45.

[63] Ohrui T, Yamaya M, Sekizawa K, Yamada N, Suzuki T, Terajima M, et al. Effects of rhinovirus infection on hydrogen peroxide- induced alterations of barrier function in the cultured human tracheal epithelium. Am J Respir Crit Care Med. 1998;158(1): 241-8.

[64] Igarashi Y, Skoner DP, Doyle WJ, White MV, Fireman P, Kaliner MA. Analysis of nasal secretions during experimental rhinovirus upper respiratory infections. J Allergy Clin Immunol. 1993;92(5):722-31.

[65] Sanford BA, Shelokov A, Ramsay MA. Bacterial adherence to virus-infected cells: a cell culture model of bacterial superinfection. J Infect Dis. 1978;137(2):176-81.

[66] Raza MW, Essery SD, Weir DM, Ogilvie MM, Elton RA, Blackwell CC. Infection with respiratory syncytial virus and water-soluble components of cigarette smoke alter production of tumour necrosis factor alpha and nitric oxide by human blood monocytes. FEMS Immunol Med Microbiol. 1999;24(4):387-94.

[67] Kim EY, Battaile JT, Patel AC, You Y, Agapov E, Grayson MH, et al. Persistent activation of an innate immune response translates respiratory viral infection into chronic lung disease. Nat Med. 2008;14(6):633-40.

[68] Murphy TF, Sethi S. Bacterial infection in chronic obstructive pulmonary disease. Am Rev Respir Dis. 1992;146(4):1067-83.

[69] Wilson R, Cole PJ. The effect of bacterial products on ciliary function. Am Rev Respir Dis. 1988;138(6 Pt 2):S49-53.

[70] Jiang Z, Nagata N, Molina E, Bakaletz LO, Hawkins H, Patel JA. Fimbria-mediated enhanced attachment of nontypeable Haemophilus influenzae to respiratory syncytial virus-infected respiratory epithelial cells. Infect Immun. 1999;67(1):187-92.

[71] White AJ, Gompertz S, Stockley RA. Chronic obstructive pulmonary disease. 6: The aetiology of exacerbations of chronic obstructive pulmonary disease. Thorax. 2003;58(1):73-80.

[72] Avadhanula V, Rodriguez CA, Devincenzo JP, Wang Y, Webby RJ, Ulett GC, et al. Respiratory viruses augment the adhesion of bacterial pathogens to respiratory epithelium in a viral species- and cell type-dependent manner. J Virol. 2006;80(4): 1629-36.

[73] Hament JM, Kimpen JL, Fleer A, Wolfs TF. Respiratory viral infection predisposing for bacterial disease: a concise review. FEMS Immunol Med Microbiol. 1999;26(3-4): 189-95. 
[74] Oliver BG, Lim S, Wark P, Laza-Stanca V, King N, Black JL, et al. Rhinovirus exposure impairs immune responses to bacterial products in human alveolar macrophages. Thorax. 2008;63(6):519-25.

[75] Thompson CI, Barclay WS, Zambon MC, Pickles RJ. Infection of human airway epithelium by human and avian strains of influenza a virus. J Virol. 2006;80(16):8060-8.

[76] Pittet LA, Hall-Stoodley L, Rutkowski MR, Harmsen AG. Influenza virus infection decreases tracheal mucociliary velocity and clearance of Streptococcus pneumoniae. Am J Respir Cell Mol Biol.42(4):450-60.

[77] de Vrankrijker AM, Wolfs TF, Ciofu O, Hoiby N, van der Ent CK, Poulsen SS, et al. Respiratory syncytial virus infection facilitates acute colonization of Pseudomonas aeruginosa in mice. J Med Virol. 2009;81(12):2096-103.

[78] Avadhanula V, Wang Y, Portner A, Adderson E. Nontypeable Haemophilus influenzae and Streptococcus pneumoniae bind respiratory syncytial virus glycoprotein. J Med Microbiol. 2007;56(Pt 9):1133-7.

[79] Stark JM, Stark MA, Colasurdo GN, LeVine AM. Decreased bacterial clearance from the lungs of mice following primary respiratory syncytial virus infection. J Med Virol. 2006;78(6):829-38.

[80] Kieninger E, Vareille M, Kopf BS, Blank F, Alves MP, Gisler FM, et al. Lack of an exaggerated inflammatory response on virus infection in cystic fibrosis. The European respiratory journal. 2012;39(2):297-304.

[81] Subrata LS, Bizzintino J, Mamessier E, Bosco A, McKenna KL, Wikstrom ME, et al. Interactions between innate antiviral and atopic immunoinflammatory pathways precipitate and sustain asthma exacerbations in children. Journal of immunology. 2009;183(4):2793-800.

[82] Perry AK, Chen G, Zheng D, Tang H, Cheng G. The host type I interferon response to viral and bacterial infections. Cell Res. 2005;15(6):407-22.

[83] Parker D, Cohen TS, Alhede M, Harfenist BS, Martin FJ, Prince A. Induction of type I interferon signaling by Pseudomonas aeruginosa is diminished in cystic fibrosis epithelial cells. Am J Respir Cell Mol Biol. 2012;46(1):6-13.

[84] Green HK, Andrews N, Fleming D, Zambon M, Pebody R. Mortality attributable to influenza in England and Wales prior to, during and after the 2009 pandemic. PloS one. 2013;8(12):e79360.

[85] Heinonen S, Silvennoinen H, Lehtinen P, Vainionpaa R, Ziegler T, Heikkinen T. Effectiveness of inactivated influenza vaccine in children aged 9 months to 3 years: an observational cohort study. Lancet Infect Dis. 2010;11(1):23-9.

[86] Dharmaraj P, Smyth RL. Vaccines for preventing influenza in people with cystic fibrosis. The Cochrane database of systematic reviews. 2014;3:Cd001753. 
[87] Harper SA, Bradley JS, Englund JA, File TM, Gravenstein S, Hayden FG, et al. Seasonal influenza in adults and children--diagnosis, treatment, chemoprophylaxis, and institutional outbreak management: clinical practice guidelines of the Infectious Diseases Society of America. Clin Infect Dis. 2009;48(8):1003-32.

[88] Katpally U, Fu TM, Freed DC, Casimiro DR, Smith TJ. Antibodies to the buried N terminus of rhinovirus VP4 exhibit cross-serotypic neutralization. J Virol. 2009;83(14): 7040-8.

[89] Kim HW, Canchola JG, Brandt CD, Pyles G, Chanock RM, Jensen K, et al. Respiratory syncytial virus disease in infants despite prior administration of antigenic inactivated vaccine. Am J Epidemiol. 1969;89(4):422-34.

[90] Giebels K, Marcotte JE, Podoba J, Rousseau C, Denis MH, Fauvel V, et al. Prophylaxis against respiratory syncytial virus in young children with cystic fibrosis. Pediatr Pulmonol. 2008;43(2):169-74.

[91] Robinson KA, Odelola OA, Saldanha IJ. Palivizumab for prophylaxis against respiratory syncytial virus infection in children with cystic fibrosis. The Cochrane database of systematic reviews. 2014;5:Cd007743.

[92] Schmidt AC, McAuliffe JM, Murphy BR, Collins PL. Recombinant bovine/human parainfluenza virus type 3 (B/HPIV3) expressing the respiratory syncytial virus (RSV) G and F proteins can be used to achieve simultaneous mucosal immunization against RSV and HPIV3. J Virol. 2001;75(10):4594-603.

[93] Shun-Shin M, Thompson M, Heneghan C, Perera R, Harnden A, Mant D. Neuraminidase inhibitors for treatment and prophylaxis of influenza in children: systematic review and meta-analysis of randomised controlled trials. BMJ. 2009;339:b3172.

[94] Yu H, Liao Q, Yuan Y, Zhou L, Xiang N, Huai Y, et al. Effectiveness of oseltamivir on disease progression and viral RNA shedding in patients with mild pandemic 2009 influenza A H1N1: opportunistic retrospective study of medical charts in China. BMJ. 2010;341:c4779.

[95] Bautista E, Chotpitayasunondh T, Gao Z, Harper SA, Shaw M, Uyeki TM, et al. Clinical aspects of pandemic 2009 influenza A (H1N1) virus infection. N Engl J Med. 2010;362(18):1708-19.

[96] Hayden FG, Treanor JJ, Betts RF, Lobo M, Esinhart JD, Hussey EK. Safety and efficacy of the neuraminidase inhibitor GG167 in experimental human influenza. Jama. 1996;275(4):295-9.

[97] Birnkrant D, Cox E. The Emergency Use Authorization of peramivir for treatment of 2009 H1N1 influenza. N Engl J Med. 2009;361(23):2204-7.

[98] Harter G, Zimmermann O, Maier L, Schubert A, Mertens T, Kern P, et al. Intravenous zanamivir for patients with pneumonitis due to pandemic (H1N1) 2009 influenza virus. Clin Infect Dis. 2010;50(9):1249-51. 
[99] Jagannath VA, Asokan GV, Fedorowicz Z, Lee TW. Neuraminidase inhibitors for the treatment of influenza infection in people with cystic fibrosis. The Cochrane database of systematic reviews. 2014;2:Cd008139.

[100] Yin M, Brust J, van Tieu H, Hammer S. Antiherpes, anti-hepatitis virus, and anti-respiratory virus agents. In: Richman D, Whiyley R, Hayden F, editors. Clinical virology, 3rd edition. Washington: ASM Press; 2009. p. 217-64.

[101] Rosner IK, Welliver RC, Edelson PJ, Geraci-Ciardullo K, Sun M. Effect of ribavirin therapy on respiratory syncytial virus-specific IgE and IgA responses after infection. J Infect Dis. 1987;155(5):1043-7.

[102] Hiatt PT, D Laber, L. Pulmonary function (PF) following treatment with ribavarin in infants hospitalised with RSV bronchiolitis. Am Rev Respir Dis. 1990;141:A624.

[103] Smith DW, Frankel LR, Mathers LH, Tang AT, Ariagno RL, Prober CG. A controlled trial of aerosolized ribavirin in infants receiving mechanical ventilation for severe respiratory syncytial virus infection. N Engl J Med. 1991;325(1):24-9.

[104] Hopkins P, McNeil K, Kermeen F, Musk M, McQueen E, Mackay I, et al. Human metapneumovirus in lung transplant recipients and comparison to respiratory syncytial virus. Am J Respir Crit Care Med. 2008;178(8):876-81.

[105] Bonney D, Razali H, Turner A, Will A. Successful treatment of human metapneumovirus pneumonia using combination therapy with intravenous ribavirin and immune globulin. Br J Haematol. 2009;145(5):667-9.

[106] Turner RB, Wecker MT, Pohl G, Witek TJ, McNally E, St George R, et al. Efficacy of tremacamra, a soluble intercellular adhesion molecule 1 , for experimental rhinovirus infection: a randomized clinical trial. Jama. 1999;281(19):1797-804.

[107] Suzuki T, Yamaya M, Sekizawa K, Hosoda M, Yamada N, Ishizuka S, et al. Erythromycin inhibits rhinovirus infection in cultured human tracheal epithelial cells. Am J Respir Crit Care Med. 2002;165(8):1113-8.

[108] Ledford RM, Patel NR, Demenczuk TM, Watanyar A, Herbertz T, Collett MS, et al. VP1 sequencing of all human rhinovirus serotypes: insights into genus phylogeny and susceptibility to antiviral capsid-binding compounds. J Virol. 2004;78(7):3663-74.

[109] Hayden FG, Turner RB, Gwaltney JM, Chi-Burris K, Gersten M, Hsyu P, et al. Phase II, randomized, double-blind, placebo-controlled studies of ruprintrivir nasal spray 2-percent suspension for prevention and treatment of experimentally induced rhinovirus colds in healthy volunteers. Antimicrobial agents and chemotherapy. 2003;47(12):3907-16.

[110] Turner RB. New considerations in the treatment and prevention of rhinovirus infections. Pediatr Ann. 2005;34(1):53-7. 
[111] Equi A, Balfour-Lynn IM, Bush A, Rosenthal M. Long term azithromycin in children with cystic fibrosis: a randomised, placebo-controlled crossover trial. Lancet. 2002;360(9338):978-84. 

Chapter 8

\title{
An Overview of Infections in Cystic Fibrosis Airways and the Role of Environmental Conditions on Pseudomonas aeruginosa Biofilm Formation and Viability
}

\author{
Cameron T. McDaniel, Warunya Panmanee and \\ Daniel J. Hassett
}

Additional information is available at the end of the chapter

http://dx.doi.org/10.5772/60897

\begin{abstract}
In this chapter, the authors review a major complication associated with cystic fibrosis (CF), problematic bacterial infections of the lungs. Infection by organisms such as Staphylococcus aureus, Burkholderia cepacia complex, and Pseudomonas aeruginosa (a major player in CF related infections) results in complications due to increased inflammation and production of virulence factors produced by the bacteria. In addition to these more canonical organisms associated with $\mathrm{CF}$ infection, emergingbacterial species have been found in the $\mathrm{CF}$, including anaerobes that have only within the past 5-10 years have been reported to exist in the lungs. P. aeruginosa has long been a cause of devastating infections, and is often seen as the "hallmark"organism associated with the disease. The authors describe the P. aeruginosa infection, including its conversion to a mucoid phenotype, as well as its ability to utilize the thicker airway surface layer associated with CF to grow in "mode two biofilms." Finally, the authors discuss treatments for bacterial infections, and some of the new advances that offerhope for treatment of CF symptoms and infections by multi-drug resistant organisms. Among these new treatments is the application of acidified nitrite, a nonantibiotic treatment that has been found to be effective at killing nonmucoid and mucoid variants of $P$. aeruginosa.
\end{abstract}

Keywords: Cystic Fibrosis, Infections, Bacteria, Pseudomonas aeruginosa, Biofilm, Acidified Nitrite 


\section{Introduction}

Cystic fibrosis (CF) is the second most common genetic disease in the United States, second only to sickle cell anemia. A mutation in the associated gene, cystic fibrosis transmembrane regulator (CFTR), results in the clinical symptoms seen for the disease. While the disease itself is devastating, it does not usually result in the immediate death of the patient. Rather, the bodily conditions that the CFTR mutation creates, especially in the lungs, results in the acquisition of problematic bacterial biofilm infections that remain in the thick, inspissated mucus layer for the remainder of the patient's life. This unique niche provides many complex nutrients that the bacteria utilize and offers an environment that is protected against the host's innate immune system. While only a few bacterial species have generally been thought of as dominating the $\mathrm{CF}$ lung, it has recently been revealed that there are many species inhabiting the lung, and that these species vary from normal flora to even obligate anaerobes [1]. While the exact role of all these bacterial species in the lung has not yet been determined, the clinical picture is becoming clearer.

When the genetic cause of CF was first identified in 1989 [2], the average lifespan of a CF patient was approximately 15 years of age. Since then, it has increased to roughly 40 [3, 4]. This is due to the tremendous efforts that have been made in determining new and improved means of treating patients suffering from $\mathrm{CF}$, including addressing physiological parameters and methods of treating bacterial infections.

This chapter will focus on the bacterial infections that develop during CF and the conditions of the lung that make it so favorable for bacterial infection. We will discuss some of the major bacterial species that contribute to the morbidity of the disease, and focus on perhaps the largest contributor to airway infection, Pseudomonas aeruginosa (PA). In addition, we will define some of the niches that the bacteria inhabit within the lung, and discuss treatment options for infected individuals.

\section{Overview of CF and the role of CFTR in the lung}

\subsection{CFTR: Role and function}

The function of CFTR in the lungs has been established previously, supporting the fact that its absence or modification (depending on the mutation) leads to the dangerous symptoms that are diagnostic hallmarks in CF patients. Although some canonically think of CFTR as only being influential in the development of the lung problems, CFTR is actually expressed in many areas of the body, including the liver, intestines, pancreas, skin, and reproductive organs (Figure 1). In all these cases, a defect in CFTR can cause many problems for the affected organ. The reason is due to the large role the transporter plays in the maintenance of osmotically balanced fluids in these tissues. CFTR is an anion channel found in the apical membrane of epithelial cells, primarily responsible for pumping chloride ions into the fluids surrounding the epithelial cells, and allowing for the passage of water from the epithelial cells into the fluid 
layers lining the cells (e.g, the pericilliary layer (PCL) of the airways) [5, 6, 7]. This allows the fluids to maintain their function, which, in the lung, is usually to facilitate clearance of opportunistic pathogens and cellular debris from the area or to transport the fluid to a different area (as is the case in the reproductive system). The transporter itself is ATP-driven, and is activated due to rising cAMP levels in the cells [7].

\section{Cystic Fibrosis}

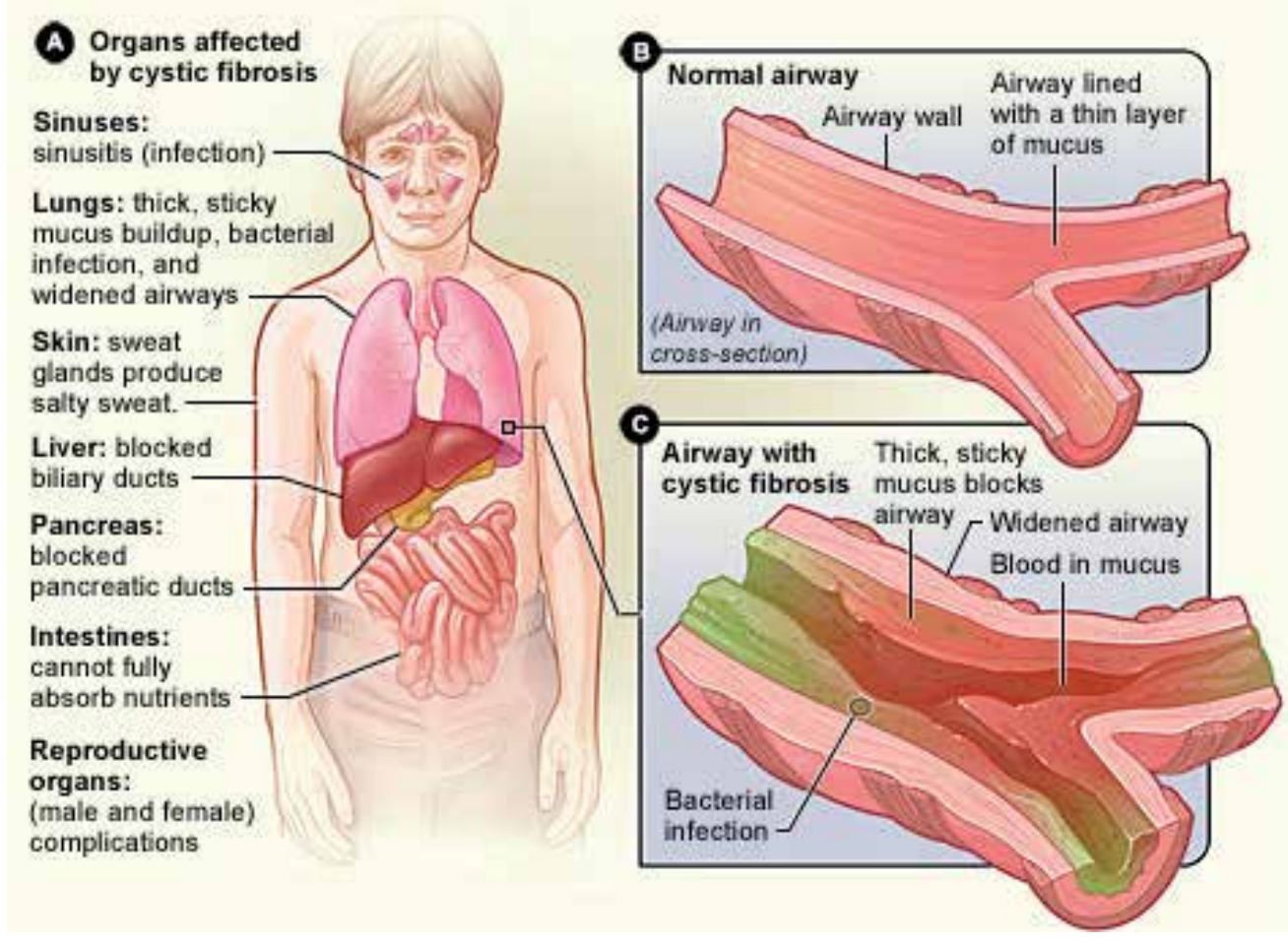

Figure 1. Organs affected by CF and its effect on airways.

A) An overview of the organs affected by $\mathrm{CF}$, with a brief description of the complications associated with it. B) A normal airway depicting open passages. C) A CF airway depicting the buildup of mucus, inflammation, and bacterial infection that will lead to further complications. (Source: National Heart, Lung, and Blood Institute; National Institutes of Health; U.S. Department of Health and Human Services. [8])

In cases where the CFTR is not active or only partially active, the consequences can be quite severe. This is due to a myriad of potential mutations in the CFTR gene. These mutations have been categorized into classes, as previously reviewed by Rowntree and Harris [9] and summated by the Cystic Fibrosis Foundation. As shown in Table 1, these classes focus on the means by which the CFTR is rendered dysfunctional, such as mutations affecting protein 
maturation (Class II) or those leading to dysregulation of Cl- conductance (Class IV) [9, 4]. The most common mutation is a deletion of a phenylalanine residue at position 508 of the protein, referred to as homozygous recessive $\Delta \mathrm{F} 508$ [7]. Although the exact reasoning of why this mutation was clinically deleterious was at first a mystery, it has come to be discovered that this leads to a misfolding of the channel, causing it to never reach the cell membrane and instead be destroyed in the Golgi apparatus. Although one could surmise that the loss of CFTR alone is not enough to cause harm and the body could compensate for this loss by redundant channels, it is also suspected that CFTR can help mediate the activation and use of other channels in the membrane. Thus, its loss may be far more reaching than simply its anion channeling properties. With this loss of function, the surrounding fluid begins to become osmotically imbalanced and often viscous and impervious to other ions [6]. With no new water coming into the fluids from the epithelial cells, the fluid layer begins to thicken, eventually forming mucus plugs in the respective organ. As such, the associated ducts are no longer able to perform their proper functions.

\begin{tabular}{|c|c|c|}
\hline Class & Impact on CFTR Protein & Examples of Mutations \\
\hline Class I & No functional CFTR protein created & $\begin{array}{l}\text { G542X, W1282X, R553X, 621+1G->T, } \\
1717-1 \mathrm{G}->\mathrm{A}\end{array}$ \\
\hline Class II & $\begin{array}{l}\text { CFTR protein is created, but misfolded, } \\
\text { keeping it from reaching the cell surface }\end{array}$ & $\begin{array}{l}\text { F508del, N1303K, 1507del, G85E, } \\
\text { R560T }\end{array}$ \\
\hline Class III & $\begin{array}{l}\text { CFTR protein is created and reaches cell surface, } \\
\text { but does not function properly }\end{array}$ & $\begin{array}{l}\text { G551D, S549N, V520F, L1077P, } \\
\text { G1244E }\end{array}$ \\
\hline Class IV & The opening in the CFTR protein ion channel is faulty & $\begin{array}{l}\text { R117H, D1152H, R347P, R334W, } \\
\text { L206W }\end{array}$ \\
\hline Class V & CFTR protein is created in insufficient quantities & $\begin{array}{l}3849+10 \mathrm{kbC}->\mathrm{T}, 2789+5 \mathrm{G}->\mathrm{A}, \\
\mathrm{A} 455 \mathrm{E}, 3272-26 \mathrm{~A}->\mathrm{G}, 3120 \mathrm{G}->\mathrm{A}\end{array}$ \\
\hline
\end{tabular}

Table 1. Classifications of CFTR mutations and their impact on the protein.

Although there are numerous mutations that have been associated with $\mathrm{CF}$, they can generally be broken down into five classes based on the way this mutation affects CFTR. This table briefly summarizes these classes and provides an example of each mutation [4].

\subsection{The CF lung}

Within the CF lung, the loss of functional CFTR is quite dramatic, and usually leads to the canonical respiratory symptoms associated with CF lung disease. A healthy functioning lung will have a thin, hydrated pericilliary mucus layer lining the airway. This mucus rests above 
the cilia of the epithelial cells in a biphasic layer [7]. The top layer is slightly more viscous than the bottom layer and serves to trap bacteria and particles that enter the lung. The bottom layer, referred to as the PCL, is much more fluid, and allows for the cilia to beat within it, pushing the entire mucus layer up the lung for expectoration [7, 10]. Through this mechanism, the lungs can clear bacteria and debris that has been inhaled or otherwise entered the airway passages. The PCL is kept hydrated by the action of the CFTR and other ion channels present in the epithelial cells lining the airway that also maintain the osmotic balance.

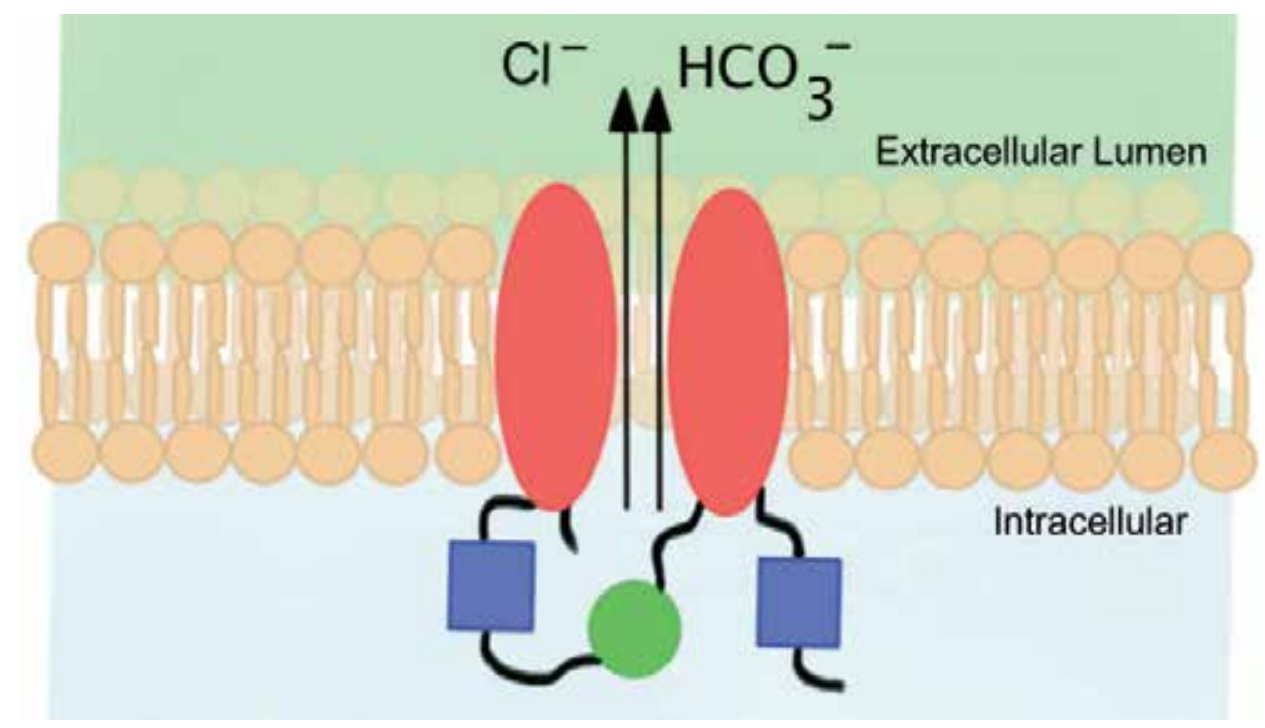

Figure 2. A model of the CFTR in the apical membrane of lung epithelia.

The CFTR, embedded in the apical membrane of epithelial cells, serves to transport anions, specifically chloride and bicarbonate, into the lumen of the associated organ. The CFTR is composed of several domains, including two transmembrane domains (red ovals), two nucleotide binding domains (blue, squares), and a regulatory domain that controls the opening of the transporter. The nucleotide binding domains use ATP to provide energy for the transport of the anions into the lumen bordering the cell.

However, in a case such as CF, where the CFTR is functionally absent and proper ion transport is lacking, the mucus layer begins to thicken. This is believed to be due to the primary transport of $\mathrm{Cl}^{-}[11,6,7]$ and the secondary transport of $\mathrm{HCO}_{3}^{-}$by CFTR (Figure 2). Recent studies have shown that the ability of CFTR to transport $\mathrm{HCO}_{3}{ }^{-}$is very important, as it seems to play a large role in the regulation of the mucin folding [7]. Mucin, the primary protein component of airway mucus, is a long chain-like, repetitive peptide that is heavily $\mathrm{O}$ - and $\mathrm{N}$-glycosylated. At the Cand N-terminal regions, the protein is rich in cysteine residues, which can lead to intermolecular disulfide bridges. These disulfide bridges will link the chains together, creating a larger oligomer. It is suspected that in a more acidic environment, the mucin molecules contract, causing the overall density of the mucus to increase. This causes impermeability issues [6] that 
can be devastating to the patient. In addition to the effects that decreased $\mathrm{HCO}_{3}^{-}$levels have on the density of the mucus, the general inability to transport anions across the apical membrane also affects the airway mucus layer. The PCL that lines the cilia of the epithelial cells is very sensitive to changes in water concentration. When the cells are not exporting significant amounts of ions, the PCL will then lose water as a sequela, resulting in it becoming denser and reducing the effectiveness of ciliary beating. This leads to an overall larger amount of material that cannot be cleared from the airway, causing a buildup or mucus plug.

In addition to the buildup of mucus as the disease progresses, the patient will experience several other symptoms as well. Commonly, the bronchi become inflamed, caused by an overreactive response from the immune system due to both mucus buildup (containing a plethora of bacterial components such as virulence factors, DNA, and cell debris from lysed bacteria or airway epithelial cells) and a potential infection. While the infections will be covered in more detail later in this chapter, bacteria such as Pseudomonas aeruginosa (PA), H. influen$z a e$, and $S$. aureus have been found to infect CF patients early in life (roughly 1 year of age) [1], and even if eradicated once, will often arise from a re-infection later in life. $P A$ is the organism most commonly associated with a decline in the clinical course of CF patients, as its ability to form biofilms and convert to a mucoid phenotype often provides a large level of resistance to antibiotics and other disease treatments.

Interestingly, the CF lung will also develop an oxygen gradient in its luminal mucus $[12,13]$. As mentioned earlier, the increased density of the mucus makes it more difficult for oxygen to diffuse across it freely and into the blood. While this has consequences for the overall health of the individual, it also has implications for growth of bacteria enmeshed within it. The oxygen gradient is severe enough that the basal layer of the mucus could be termed microaerobic, or in more severe cases, anaerobic. This leads to the growth and development of bacteria that would normally not be found in the lung, and eventually to the growth of the mucoid form of $P A$. This will be covered in more depth later in this chapter.

\subsection{Pathology of the CF lung}

As might be expected, the buildup of thick mucus in the CF lungs often has severe clinical implications The significant reduction or loss of mucus clearance often results in infection and leads to inflammation due to the dramatic $\sim 1,500$-fold increase in airway neutrophils [14]. Coupling the inflammation and buildup of mucus, it is not surprising that the overall lung capacity of $\mathrm{CF}$ patients decreases dramatically throughout life. This can be tested using a series of pulmonary function tests (PFTs) [15]. These often involve a spirometry test, which is a measure of the forced efflux volume in one second (FEV1), or how much air the patient can forcefully exhale in one second. This is a hallmark test for overall lung volume and strength. Clinically it has been shown that the FEV1 of a CF patient will be approximately $10 \%$ below the expected for a healthy individual of the same age [16].

In older patients, the prolonged effects of the disease often lead to chronic infections. These invading organisms can then be cultured and analyzed to determine the best treatment strategy. While we will be covering the type of infections further in this chapter, it is important to note that clinically, this also affects the patient in other ways, primarily leading to inflam- 
mation of the respiratory system and decreased airway capacity [17]. This inflammation is often brought on by increased neutrophil accumulation in the lungs, which not only serve to act as a preliminary means of immune defense, but also to recruit macrophages. These neutrophils and macrophages will phagocytose bacteria and dead immune cells, but also produce pro-inflammatory cytokines that exacerbate the inflammatory process. These cytokines not only attract other immune cells, but also serve as a trigger for the release of proteases and elastases by the immune cells. Normally, these help eradicate the bacteria that triggered this response, but theior over-production in the CF lung can actually damage epithelial cells, leading to the fibrotic nature of cells associated with this disease [18].

\section{Bacterial infections}

While CF airway disease is based on the genetic mutation of the CFTR gene, this is not usually what leads to the morbidity and mortality associated with the disease. Rather, an infectious agent that grows under the physiological conditions created by this mutation will lead to detrimental symptoms and eventual death. In the lungs, the buildup of thick mucus leads to decreased clearance of bacteria and provides a nutrient rich medium with which they can grow and even thrive. This mucus becomes colonized relatively easily with potentially several different species of bacteria at once $[19,16,20]$. Considering that infection is the major source of morbidity and mortality for CF patients, much research has focused on this aspect of the disease and different means by which to eradicate it. However, time has shown that this is not quite as easy as hoped, but the advancements of alternate treatments are helping this issue.

Bacterial colonization of the lungs of CF patients has been known for many decades. However, our understanding of what bacteria colonize the lungs has evolved dramatically. Early research identified that there were several species of bacteria that could colonize the lungs easily, and were often found associated with CF patients. By far, the most common (and most linked to severe progression of the disease) was $P A$. This gram-negative bacterium is often considered an opportunistic pathogen, existing naturally in soil, water, and in some cases, as part of the flora of human skin. However, in cases where the bacteria come into contact with immune-deficient or severely compromised tissues, they can grow quite prolifically. A common example is found with burn wounds, where $P A$ can colonize the exposed flesh and lead to the need for aggressive antibiotic treatment. This type of infection is often associated with a distinct "smell of grapes" and the potential for a green-blue color to develop, indicative of several of the toxins that $P A$ produces such as the blue phenazine antibiotic, pyocyanin. There are additional phenotypes that are seen with lung colonizing $P A$, the most dramatic of which was found in 1964 by Doggett et al. [21]. In that work, the authors noted that the multiple strains of $P A$ that they could isolate on agar from $C F$ patients all had a similar, mucoid phenotype. Although, at the time, the significance of this was not known, this mucoid phenotype is tightly linked to the overall progression of CF lung disease. It is indicative of the organism's ability to create a biofilm, a highly resistant matrix of exopolysaccharide and bacteria that allows survival in harsh environments. We will cover the details of biofilms 
further into this chapter, but it is important to note the significance they play in CF disease progression.

In addition to $P A$, three other major strains of bacteria were found associated with the CF lung. The first of these is Staphylococcus aureus, a gram-positive organism that is part of the normal skin flora. Often, S. aureus is found colonizing the nares of hosts, where it is hypothesized that it can travel to the lungs. It is often the first bacterium identified in CF patients, potentially due to a lack of proper immune defense within the newborns diagnosed with $C F[22,23]$. It often resides in the lungs for a prolonged period, although it can be eradicated with proper antibiotic treatment. Similar to $P A$ acquiring a mucoid variant, $S$. aureus can also acquire a different phenotype. Small colony variants have been seen associated with lung cultures, which have shown decreased expression of many of the virulence factors associated with S. aureus [24]. This could indicate that the bacteria are attempting to avoid the host immune system, allowing survival in the lungs for a longer duration. What potentially worsens the overall clinical course is the acquisition of MRSA in the lungs [23]. Although it is more rare than its antibiotic-sensitive counterpart, it is possible to acquire this strain nosocomially, or even develop the strain independently through the constant use of $\beta$-lactam antibiotics to treat existing,methicillinsensitive $S$. aureus (MSSA) infections. Interestingly, it has been found that in situations where a patient is infected with both $S$. aureus and $P A$, treatment of one can lead to the prominence of the other [25]. When patients were given anti-staphylococcal antibiotics, it was found that $P A$ infection could develop and fill the niche that the eradicated S. aureus no longer occupies. This is another unfortunate side effect of a $S$. aureus infection, that not only can its treatment lead to a potential development of MRSA but it can also cause an exacerbation of any existing $P A$ infection. This is of great clinical importance to note for younger patients who have not yet been colonized with $P A$.

The second major strain historically found in addition to $P A$ is Haemophilus influenzae. This bacterium is similar to $S$. aureus in that it is a normal commensal organism of the nasopharynx region, but can actually be found in normal lungs. As an individual progresses through life, the chances of being colonized with $H$. influenzae increases, rising from $20 \%$ in infants, to $50 \%$ of children, to more than $75 \%$ of adults [26]. This has led some to believe that $H$. influenzae is one of the earliest CF pathogens, and that it modifies the lung in a manner that allows for subsequent $P A$ infection [27]. The forms of $H$. influenzae that can colonize an individual vary, depending on the presence or absence of a capsule around the bacterium. When a capsule is absent, the bacterium is referred to as non-typeable H. influenzae (NTHi), and this form is most associated with respiratory infections [26]. Within the CF lungs, the bacteria attempt to attach to the epithelial cells underlying the PCL. Once attached, the cells will trigger innate immune responses, which can lead to initial inflammation processes [27]. After this, NTHi will try to evade the immune response, using a series of enzymes to destroy antibodies, and its ability to create microcolonies, and to invade the epithelial cells [26]. For this reason especially, NTHi is an important pathogen to be aware of for treatment strategies.

The last of the historically major three bacteria associated with CF, Burkholderia cepacia is often considered the most dangerous lung pathogen requiring rapid and rigorous medical attention. Although first identified as an individual bacterial species related to Pseudomonas, it has since 
been reclassified as a complex of genomovars (Burkholderia cepacia Complex, BCC), which have similar characteristics [28]. BCC is generally considered one of the main causes of pneumonia in immunocompromised individuals, which includes CF patients. BCC has been found to coinfect individuals with other $\mathrm{CF}$ associated bacteria, such as $P A$ [29]. The complex is also known to produce virulent factors, such as elastases and gelatinases, which can weaken cell-to-cell interactions [29]. In addition, BCC fills a unique niche in the CF lung by being able to invade macrophages that have made it to the lung. These tactics caused the bacterium to be of great concern when it was first identified in CF patients in 1984, but since then, epidemiological and clinical studies have greatly advanced treatment [28].

Several other genera of microbes, including Achromobacter, Pandorea, Alcaligenes, Stenotrophomonas, and Ralstonia, have also begun to be found in CF sputum in the past 20 years. These bacteria are quite often associated with more advanced stages of the disease, indicating that they may need the fertile ground of the CF lung to which they become accustomed before they can thrive $[19,16]$. Alcaligenes and Stenotrophomonas are increasingly found with a PA or BCC infection [19]. In addition, several fungal pathogens have been associated with $C F$ as well, such as Aspergillus fumigatus and Candida albicans. While these fungi have not been the primary focus of CF microbial treatment, recent evidence suggests that they can, in fact, become a problem for CF patients. They have been associated with an increase in inflammation, as well as a possibly more severe disease progression. Both host and fungal factors are equally important in determining if the fungus will become pathogenic [30].

Although each of these pathogens has been studied as a single organism in the CF pathology, limited efforts have been made into looking at the interaction between the bacteria. Considering that more often than not multiple genera of bacteria are found in the CF lung, this is an important aspect to consider. Research that has been studying this topic has focused generally on the interaction between $P A$ and another species of bacteria, as this is the most common pathogen associated with $C F$. For instance, work has been conducted to elucidate the relationship and interactions between $P A$ and BCC. In these cases, initial reports showed that BCC and $P A$ formed communal biofilms, where both microbes could be detected in a "biofilm-like" structure. They share similar quorum sensing molecules, and together could be promoting the growth of one another. However, further investigation began to see that these bacteria did not necessarily occupy the same biofilm structures [29]. In some cases, it could occur, and could be recapitulated in vitro. However, in other situations, the bacteria did not cooperate. It seemed that $P A$ was producing a secreted chemical that prevented the growth of BCC nearby, which was suspected to be pyocyanin [29]. This suggests that all of the relationships in CF may not be symbiotic or even mutually beneficial. Perhaps it depends on the timeline of when the infections are established. However, more work is needed to determine the actual relationship between these bacteria. The entire web of bacterial networks in the CF lung is not trivial, but deciphering how it works could potentially allow for more effective treatment of lung infections.

While this paradigm of "the big three" bacteria for CF persisted for several decades, along with the recent knowledge that several other species could infect the CF lung, a paradigm shift occurred around 2008, when it was discovered that there were obligate anaerobes present in 
the lungs of older CF patients [31]. Included in this group of bacteria were the genera Prevotella, Veillonella, Propionibacterium, and Actinomyces [31]. This indicated that at least part of the lung must become anaerobic during the disease, which added to the notion that the CF lung has a vast array of micro-environments within it, each of which can be colonized by different bacterial species. It also corroborated the evidence of anaerobic niches being produced in the mucus plugs, which to this point had only been associated with bacteria converting to anaerobic growth.

With the discovery of the aforementioned anaerobic bacteria, it became clear that there was most likely a temporal aspect of CF infections as well. Clinical evidence has shown that depending on the age of the patient, certain bacteria are more likely to be cultured with their sputum (Figure 3). Early in the patient's life, from birth until around ten years of age, it is common for patients to test positive for $S$. aureus; but later in life this infection seems to become less likely [25]. PA can also be acquired early in life, however, much effort is often put into trying to eliminate this infection. As a result, a patient may test positive for $P A$ several times throughout their life, even if the infections are not chronic. However, often by the patient's teenage years, $P A$ has established a persistent infection and may have already converted to its mucoid form [32], leading to $73 \%$ of adult CF patients harboring such an infection [33]. In contrast to the "major" bacteria of $\mathrm{CF}$, the newly discovered anaerobic bacteria generally do not infect a patient until later in life. This is associated with the development of more anaerobic niches in the lung, which is indicative of mucus buildup and thickening. This occurs later in life as the detrimental effects of a defective CFTR build and the inflammation worsens. Although treatment would still depend on cultures from the patients, establishing a general timeline is beneficial for physicians who need to create a treatment plan for their patients, and could allow for proactive treatment as the patient enters different phases of life.

\section{Prevalence of Respiratory Microorganisms by Age in 2013}

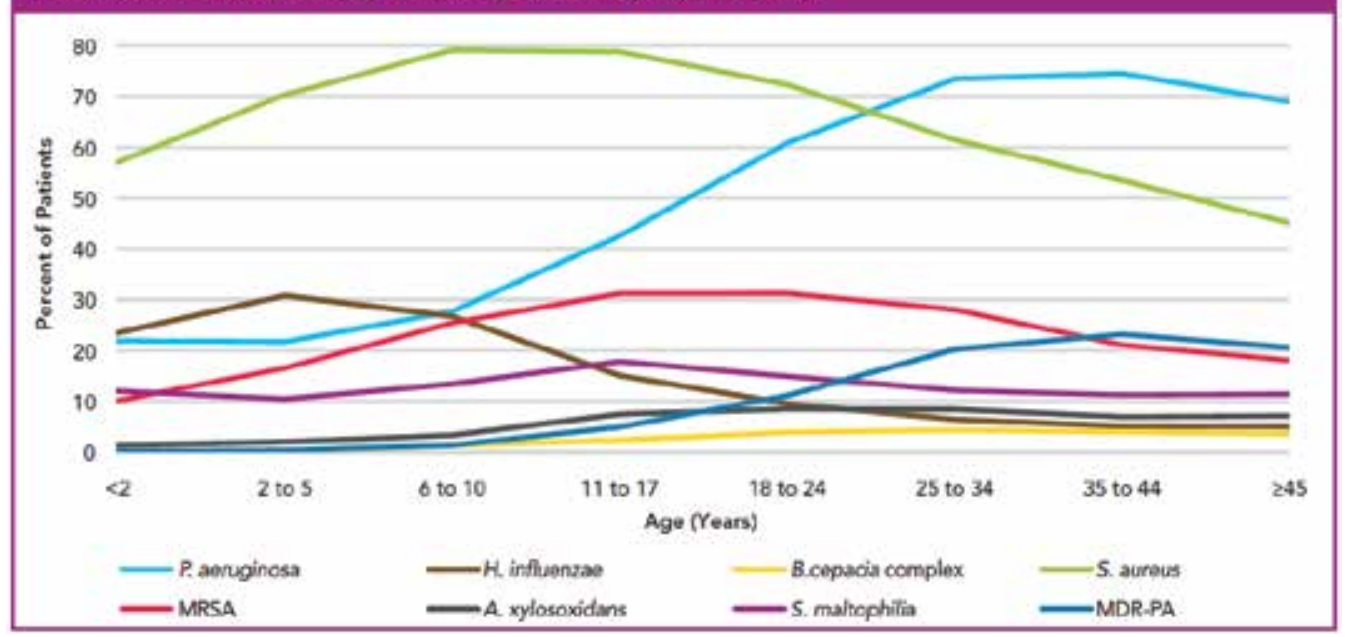

Figure 3. Prevalence of respiratory microorganisms by age in 2013. 
As a CF patient progresses through life, the likelihood of culturing positive for a particular microorganism changes. This is due mostly to the changing environment in the CF lungs. This graph depicts the percentage of patients registered in the Cystic Fibrosis Foundation patient registry who tested positive for a particular bacterium, separated by age [4].

Given the presence of anaerobic bacteria, this indicates that current antibiotic regimens for $\mathrm{CF}$ patients may have to be revisited. Normal antibiotic treatments include tobramycin, kanamycin, and several other antibiotics of the aminoglycoside class. Those in this class are generally ineffective against anaerobic bacteria due to their mechanism of entry. Aminoglycosides rely on the ability of an organism to respire, using either nitrate or oxygen as the terminal oxygen acceptor [34]. For fermenting bacteria, such as Actinomyces [35] and Prevotella [36], that means that this class of antibiotics is ineffective. In the case of $P A$, the organism exists as a facultative anaerobe that does use nitrate as a terminal oxygen acceptor. Although that should allow for these antibiotics to work, reports are mixed on the efficacy of the treatment alone, noting the ability for the bacteria to resist the action through a phenomenon termed "impermeability" [37]. It has been observed in multiple studies that CF-related $P A$ gains this ability, characterized by a lack of uptake [37]. This may be related to the innate ability of $P A$ to produce protective biofilms in its mucoid state, a mechanism already shown to be protective against antibiotic treatment. It may also be due to the action of certain genes involved in the biofilm formation, as one study found that biofilms upregulated tolA, whose gene product can alter LPS structure in such a way as to make it more resistant to aminoglycoside recognition, and bacteria which did not produce as much of this protein were far more susceptible to aminoglycosides [38].

\section{The major contributor: Pseudomonas aeruginosa}

Perhaps the most problematic and dangerous of the bacteria associated with CF is PA.PA is a gram-negative opportunistic pathogen that can sometimes be found as part of the normal human skin flora. However, in cases where the immune system is compromised in some way, the bacteria finds a way to infect a host, which can lead to severe complications. It is also perhaps the most deadly bacteria to be associated with $\mathrm{CF}$, as a patients life expectancy decreases by roughly 7 years if the patient cultures positive for $P A$ [37]. The reasoning for this is that $P A$ produces a large number of virulence factors that stimulate inflammation in the airway, and often converts to a mucoid phenotype that allows it to escape a number of host defenses and the use of many antibiotics.

\subsection{The role of quorum sensing in PA infections}

To control the expression of its virulence factors, $P A$ uses a bacterial "communication" system known as quorum sensing. This is not unique to $P A$, and has been shown to exist in a number of other bacteria, including E. coli, S. pyogenes, and S. aureus (another CF associated bacteria) [39]. However, much research has been conducted into the complex regulatory pathways associated with this system in $P A$, which controls not only the means by which the bacteria move in relation to each other, but also the expression of virulence factors that can affect the 
patient and the ability to convert into a biofilm [39]. Quorum sensing in $P A$ is accomplished by at least two regulatory pathways, the las and $r h l$ systems [40]. The las system is hierarchically first for sensing other molecules. The system is composed of two primary genes, las $R$ and lasI, respectively. The transcriptional activator gene las $R$, responds to environmental cues to produce the transcription factor LasR. LasR on its own, however, cannot properly interact with any genes. It needs a cofactor, specifically the quorum sensing autoinducer 3-oxo- $\mathrm{C}_{12}-\mathrm{HSL}$ (PAI-1) produced by the lasI gene product. This molecule can be taken up from other nearby bacteria that have released it into the supernatant and especially in biofilms [41]. When PAI-1 enters the bacteria, LasR forms a complex that is then able to act as a transcription factor. It has two major targets in the system, lasI and rhlR. Importantly, by activating lasI, the system begins an autoinduction loop that will maintain it in the cell and allow for the spread of signal to other bacteria. The gene encodes an enzyme, LasI, which is able to produce the 3 -oxo- $\mathrm{C}_{12}{ }^{-}$ HSL necessary to activate LasR. More active LasR results in the production of more LasI and PAI-1, amplifying the signaling effect.

However, the system looping and increasing would mean nothing if there was not an output somewhere. This output happens to be the second major system for quorum sensing, the $r h l$ system. This system behaves very similarly to the las system. After activation by the LasRPAI-1 complex, the RhlR transcription factor is produced. This requires a different signaling molecule, $\mathrm{C}_{4}$-HSL (PAI-2), with which to form a complex. Once the initial signal is received, the complex is formed and activates RhlI, which produces more PAI-2, and this, in turn, perpetuates this signaling process. At the end of this process, the bacteria result in having a buildup of RhlR-PAI-2 complex, which can help upregulate transcription of a number of virulence factors, including las $A$ and $\operatorname{las} B$ (elastase related enzymes necessary for invasion) [42, $40], x c p P$ and $x c p R$ (components of the type II secretion system), and even the stationary phase sigma factor gene rpoS, a gene linked to antibiotic resistance in $P A$ [40]. However, one of the most important secreted factors dependent on the $r h l$ system is the production of rhamnolipids, for which the system was named. These are important virulence factors that $P A$ produces that can lead to inflammation and associated immune responses.

Another important output of the las system is the Psedumonas quinolone signal (PQS) regulon. The PQS regulon is responsible for some of the phenotypic changes seen with $P A$ once it begins to grow in communities [39,40]. The regulon itself requires the induction of pqsR, the regulatory transcription factor for the rest of the genes. Once this is active, the transcription factor will homodimerize to activate the rest of the pqs genes, as well as the induction of phenazine genes (phnAB). Together, these will allow for the production of HHQ (2-heptyl-4quinolone, a precursor to PQS) and PQS signals that will not only allow for the autoinduction of the pathway, but also for the eventual production of pyocyanin and pyoverdin, important siderophores that also lead to the green color that is often seen with $P A$-laden CF sputum. In addition, this activates the production of Hydrogen Cyanide $(\mathrm{HCN})$, an important metabolite for $P A$ as well as a potential toxic agent for the patient and other bacteria.

\subsection{Alginate production and the conversion to mucoidy}

Although these quorum-sensing pathways are important for the virulence associated with $P A$, it is also important to note that this is not the only system involved in its infection of a CF 
patient. Perhaps more important for the chronic infection of patients are the genes and pathways involved in the conversion of $P A$ from its normal phenotype to its mucoid form. This mucoid phenotype has been known to be associated with CF infection, especially during chronic infection of patients $[43,31]$. It is characterized by the general loss of motility for the bacteria [44], and the overproduction of the viscous exopolysaccharide called alginate. Alginate surrounds the bacteria and creates a material that seems quite like mucus, hence the appropriate coining of the term "mucoid phenotype." Mucoid PA has also been associated with the production of biofilms and is involved in general resistance to several types of antibiotics and environmental stresses, such as dessication and nutrient depletion. However, this conversion is a complicated procedure that results in a signficantly altered regulatory network of several genes associated with alginate production.

The most important gene involved in the process of alginate production is algU (algT), a major component of the 12-gene operon that results in the production of alginate. AlgU is a extracytoplasmic sigma factor that is expressed at a low level in nonmucoid bacteria, and this basal level of protein is sequestered by the membrane spanning anti-sigma factor, MucA (Figure 4). This interaction is very important for the eventual conversion to the mucoid state. When MucA normally sequesters $\mathrm{Alg} \mathrm{U}$ in its nonmucoid state, the bacteria do not produce any significant amounts of alginate. However, under conditions of stress (oxidative, osmotic, heat shock), the bacteria will eliminate MucA through one of two means [45]. The first is the proteolytic destruction of MucA. In conditions where the bacteria are stressed due to nutrient depletion (iron, carbon, or phosphorus), dessication, or antibiotics, the bacteria can trigger the production of a protease that targets MucA. Upon the degradation of this protein, the bound $\mathrm{AlgU}$ is released into the cell to act as a sigma factor $\left(\sigma^{\mathrm{E}, 22}\right)$, in combination with several other proteins, to upregulate the operon responsible for alginate production, including most of the alg genes, such as $\operatorname{alg} D, \operatorname{alg} C$, and many others. Once this is accomplished, the cell will produce and secrete alginate, allowing for expression of the mucoid phenotype.

Under normal growth conditions in PA (Panel A), MucA (Blue) interacts with periplasmic MucB (Green) to allow for cytosolic sequestering of AlgU (Red). This prevents AlgU from acting as a transcription factor, effectively keeping mucoid genes inactivated. However, in times of stress, PA can either inactivate MucA through proteolysis (Panel B) or hypermutation (Panel C). In these cases, MucA is no longer able to interact with the periplasmic MucB, and the result is the loss of sequestered $\mathrm{AlgU}$, allowing it to now activate genes in the cell, including $\mathrm{AlgD}$ and related mucoidy genes. Adapted from Hassett et al., 2009 [46].

However, in cases where constitutive mucoidy is found, such as in CF patients chronically infected with $P A$, the phenotype is most often caused by a mutation within the mucA gene. This is suspected to still be an outcome of environmental stress (as the CF lung is often lacking water and may contain innate immune defenses). However, the result is not a temporary upregulation of alginate related genes, but rather, a hypermutable phenotype that will usually allow for mutations within the mucA gene to occur [45]. This hypermutability stems from the mutation of several genes associated with DNA repair, including the DNA mismatch repair system (MMR) and the deoxyguanine repair system (DO) [45], although errors from the DNA polymerase IV could also lead to these mutations. The most common mutation found associ- 


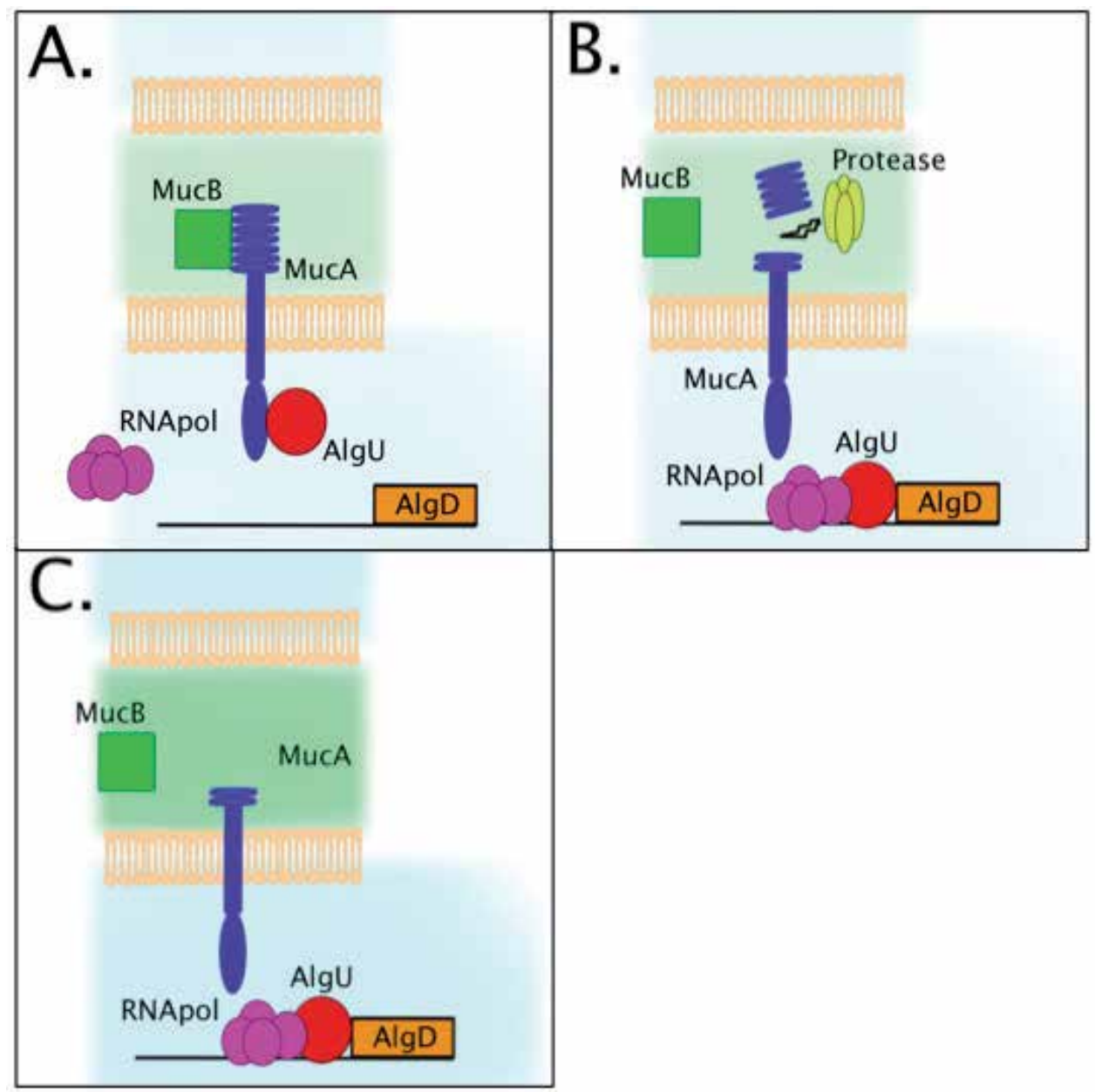

Figure 4. MucA interactions with AlgU.

ated with this is in $m u c A$. One such mutation, $m u c A 22$ (a deletion of $5 \mathrm{G}$ residues from bases 431-436), is commonly found, although several others have been associated with this phenotype. Because of these base deletions, the protein is truncated to $15.8 \mathrm{kDa}$, where it has lost its binding domain for AlgU. As a result, $\mathrm{AlgU}$ is constitutively active in the bacteria, resulting in a constant mucoid phenotype in vivo. A mucoid phenotype has also been associated with mutations in other $m u c$ genes, including $m u c B, m u c C$, and $m u c D$, however, these are not as frequent.

Once the patient acquires mucoid $P A$, their clinical course usually diminishes dramatically. This conversion is often a result of chronic $P A$ infection, which may not cause a drastic change in health immediately, but will cause chronic inflammation and lead to decreased lung function. Currently, much of the effort for treating CF infections is focused on dealing with biofilm associated bacteria, which includes the mucoid $P A$. 


\subsection{PA biofilm formation in CF patients}

Herein, we will focus on the biofilm formation of $P A$ on the biotic or abiotic surface, in vitro. There are five steps for biofilm development on this kind of surface (Figure 5). This process begins when (Step 1) the planktonic or free-living bacteria (Step 2) attach to the surface as show in Figure 5. Several genes and factors are required for this initial step of biofilm formation such as the expression of flagella, type IV pili, Cup fimbria, and the activation of sadB [47]. Next, (Step 3) cells begin to produce the extracellular matrix components that facilitate the bacteria joining together and forming microcolonies. The matrix contains polysaccharides, proteins and eDNA, which plays a role in the up to 1,000-fold increase in antibiotic resistance of the biofilms compared to planktonic bacteria cells [48, 49]. Then, (Step 4) the microcolonies will progress and mature into a thicker biofilm (maturation), thus resulting in the development of a gradient of oxygen in the biofilm. Here, the surface has a higher oxygen concentration than in the deeper part of the biofilm. In this case, the biofilm is composed of aerobic, micro-aerobic, and anaerobic environments. The factors that also play an important role in this maturation step are involved cell motility. For example, the creation of the mushroom cap-like structure (biofilm maturation) required pilA (a pilus component). This also involves parts of the quorum sensing system as it has been shown that a lasI mutant will form a flat and thin biofilm while rhlI or pqsA mutant form microcolonies lacking the mushroom caps [48]. Several genes have been reported to be involved in the biofilm development such as the response regulator of the GacA/GacS twocomponent regulatory system $(g a c A)$ that is involved with the extracellular matrix components, a sigma factor $(r p o S)$ that regulates a number of genes in the stationary phase that overlap with genes regulated by quorum sensing, the alginate biosysthesis regulator (algR) that is involved in type IV-mediated twitching motility, and a regulator of exopolysaccharide and Type III secretion (retS) [48, 50, 51, 52]. Finally, (Step 5) biofilm dispersion will occur where the biofilm components have been broken down or modified to release the surface proteins resulting in changes of the biofilm structure and also the forming of a new biofilm [47, 53]. Many genes have been studied in regard to biofilm dispersion. One such gene is $r h l A$, one of the genes required for rhamnolipid biosysthesis, which has been reported to be required for the dispersion of the bacteria from the center of the mushroom structure [54]. Another gene involved is the biofilm dispersion locus $(b d l A)$, which studies have shown may be a link between sensing environment cues, c-di-GMP levels and detachment of the biofilm [52].

In CF patients, $65 \%-80 \%$ of all microbial infections are biofilm related [48]. The CF lung provides a suitable environment for $P A$ to infect and form a biofilm. The normal airway epithelia cells are hydrated by a mucus that contains a mixture of electrolytes, glycoproteins such as mucins, protein, and lipids. The failure to elucidate free-living $P A$ by the inflammatory system combined with the bacterial defense mechanisms such as oxidative stress in a $\mathrm{CF}$ patient results in a vicious environment $[55,56]$. This environment can be oxygen depleted as well, causing it to be considered micro-aerobic or anaerobic [5]. Several studies have supported the ability of $P A$ to form a biofilm in the $C F$ lung, such as one that used a microscopic study of CF sputum, showing an increased resistance to antibiotics and phogocytosis because of the higher production of EPS in the biofilm [46]. This is a common reason to initiate biofilm development, as $P A$ biofilms resist phagocytosis by immune cells and are also more resistant 


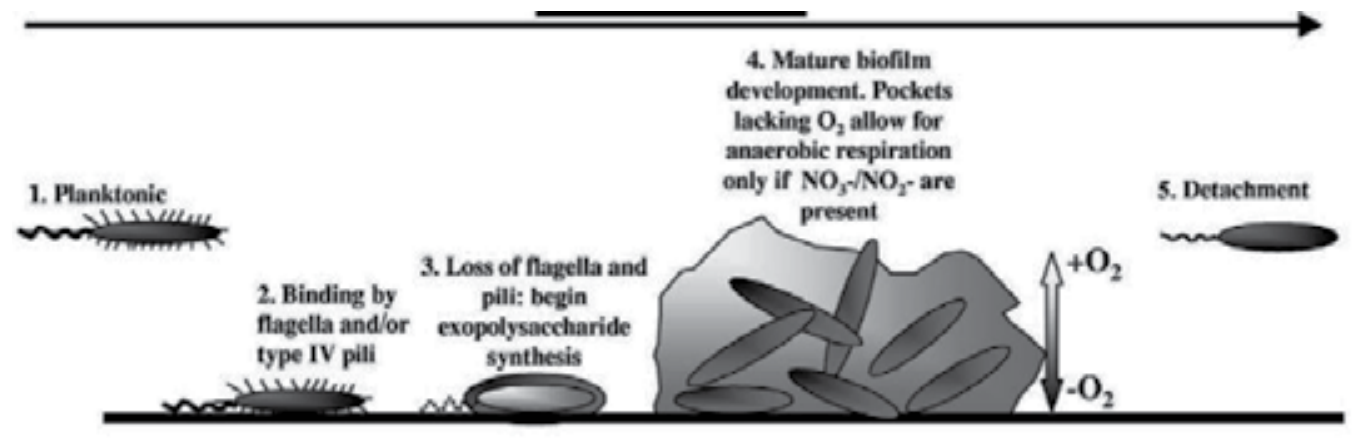

\section{Biotic or Abiotic Surface}

Figure 5. The five steps of Pseudomonas biofilm formation on a biotic or abiotic surface (in vitro).

to antibiotics than a free-living cell [55]. To form a biofilm in the CF lung, $P A$ must penetrate the mucus layer and enter into a hypoxic zone, where it can then form a biofilm with a quick transition from aerobic to anaerobic metabolism by using the alternative electron acceptors in the mucus such as nitrate $\left(\mathrm{NO}_{3}{ }^{-}\right)$, nitrite $\left(\mathrm{NO}_{2}{ }^{-}\right)$or nitrous oxide $\left(\mathrm{N}_{2} \mathrm{O}\right)$ through the respiratory process known as "denitrification" to support anaerobic growth. The ability of $P A$ to be able to grow in the anaerobic condition is an important factor for the formation of biofilms in the CF patient. $P A$ also can use alginate as an energy source for anaerobic growth [48]. Finally, these macrocolonies of $P A$ are formed during a chronic infection of CF [53]. The summary of the biofilm formation in CF patients is shown in Figure 6. Some of the important genes that are involved in the denitrification system are nitrate reductase (nar), nitrite reductase (nir), nitric oxide reductase (nor) and nitrous oxide reductase (nos). These genes are under tight regulation in the chromosome. One gene that has been found to regulate this process, oprF, is particularly important. It has been found that antibodies against this protein are elevated in CF patients, suggesting that it is upregulated in the bacteria. In addition, an oprF null mutant formed poor anaerobic biofilms due to no NIR activity [53]. Quorum sensing (QS) also regulates the genes in the denitrification system. One study showed that $r h l$ was required for an optimum balance of the denitrification pathway [13].

\subsection{Genetic alterations during $P A$ infection}

In addition to the changes that are phenotypically eminent such as during mucoid conversion, it is also important to note that additional genetic regulation is occurring in this environment. As mentioned earlier, the CF lung is not a hospitable environment for bacteria, due especially to the dessicated mucus layer, yet it is quite nutrient-rich. If the bacteria can benefit from this, then they will be able to survive and flourish in the lung. In order to do this, the bacteria must undergo several layers of genetic regulation in order to activate specific shunt pathways that will allow them to use the nutrients provided. In the case of $P A$, this has been studied extensively, but has required significant efforts to unravel, notably the creation of various synthetic media that represents CF lung ASL [57] as close as anything else to date. 


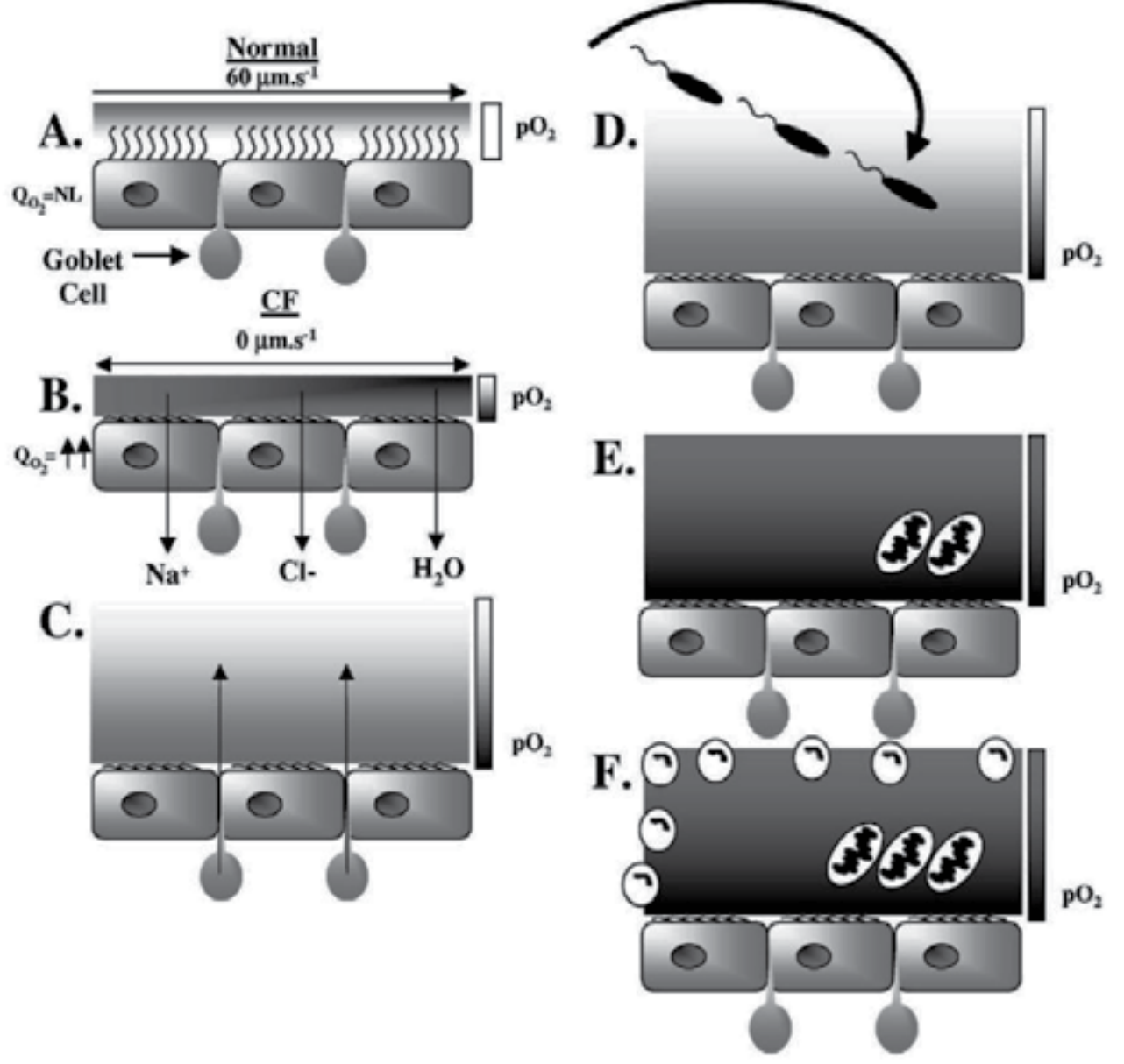

Figure 6. $P A$ biofilm formation in the CF lung. A shows the normal epithelia cells. B-F depict CF airway epithelia. B-C show the mucus formation as mentioned previously in the CF lung section of this chapter, resulting in the hypoxic zones in the CF lung. D-F illustrate the free-living cell bacteria moving into the hypoxic area (D). E shows the bacteria adjusting themselves from an aerobic to anaerobic condition and forming macrocolony, biofilm, in this environment. $\mathrm{F}$ shows the increasing amount of macrocolonies.

Early gene expression analyses showed several important changes in expression levels, but suffered from lack of application. Some of the first analyses were of general biofilm gene expression, which were important as a seminal work. For example, Whiteley et al. performed a transcriptional profiling analysis in 2001 that examined gene expression by $P A$ biofilms grown on granite pebbles [38]. They discovered that only a small subset of genes showed any change in expression (73 genes), roughly splitting 50:50 into up- and downregulated genes. This was quite interesting, as considering the relatively large change in phenotype between normal $P A$ and biofilm structure, it would seem that more changes would have had to occur. In addition, several genes promoting the resistance to certain antibiotics were upregulated, and when they treated the biofilms with antibiotics, a different gene expression profile was 
seen. This all suggested that biofilm bacteria might be able to respond better to antibiotics and environmental stresses than planktonic cells, as the biofilm is not only more inherently resistant, but the bacteria interior to the film experience a lower concentration of the stressor, allowing for genetic regulations and responses [38]. Following this paper, more attempts to analyze biofilm-related genes ensued. These studies involved mostly environmental biofilms, and not those associated with human infection. Eventually, as the prevalence of biofilms in disease became better known, it became important to discover if these changes in biofilm gene regulation were able to be transitioned over to this situation.

In this regard, several attempts to examine human-associated biofilm infections occurred, but it had to be verified that the gene expression difference was reliable, and not an artifact of the harvesting procedure. Some studies did well with this issue, collecting $P A$ from patients and analyzing both that sample and in vitro grown cells. These differences did seem quite significant, showing changes between the two samples that suggested a decrease in the regulatory machinery for biofilm cells rather than an upregulation of many of the biofilm associated genes (such as muc, alg, rhl, hcn, and plc) [58]. This was novel, as the biofilm conversion seemed much more complicated than simply via a dysregulatory pathway.

Other genes that were found to be changed in the CF lung associated samples seemed to actually be associated with the acquisition of nutrients in the surrounding area. For example, one such study found that there was an increase in genes associated with arginine metabolism and the glyoxylate shunt (a process found often with the $\beta$-oxidation of lipids to acetyl-CoA) [59]. This seems to indicate that the CF lung has a higher available amount of arginine and free lipids for degradation, potentially linked to the death of epithelial cells in the lung. This same study also found the cells generally change to a sessile state, where many of the genes associated with motility and chemotaxis are downregulated. This indicates that the cells initially colonizing the CF lung are able to prosper enough that the organisms can rapidly adapt to such conditions. This same down-regulation of motility genes is often seen with the conversion to a biofilm mode of growth. Once the bacteria have shifted to the mucoid state and are able to survive the thick, dessicated mucus, then they will be able to thrive in the lung. This can help explain why $P A$ is often such a major problem for the CF patients.

\section{Treatment strategies for $\mathrm{CF}$}

Considering the relative abundance of CF cases ( 70,000 world-wide), it is important that efforts are put forward into treating the symptoms that arise from the disease [60]. These symptoms can be very detrimental, especially in the case of serious bacterial infections and ensuing exacerbations. Initially, the treatments that were available were only focused on chest physical therapy for the buildup of airway mucus, but those efforts have evolved as current research has illuminated more on the pathophysiology of CF lung disease. An example of advanced treatment with continuing research was the initial attempts to provide gene therapy for CF. With the idea that complementing the mutated CFTR with wild-type CFTR would allow for functional ion transport, efforts were made to transfect cells with a functional copy 
of the gene using either a liposomal or viral vector. However, this eventually ended in failure, as the complementation strategy was unsuccessful and in some cases harmful. Specifically, adenovirus complementation caused a hyper-immune response, and DNA liposome transfection could not properly deliver the DNA to the nucleus [61]. Recent attempts are examining whether transfecting cells with mRNA coding for functional CFTR could be beneficial. However, this still requires much more research. With that in mind, treatment falls primarily into two main camps; the removal of the dessicated mucus, and the treatment of bacterial infections.

\subsection{Treatment of mucus buildup}

Even without the involvement of pathogens in the mucus plugs that develop in the CF lung, the plugs themselves can cause serious effects on the patients, specifically in their quality of life. As mentioned previously, these plugs develop from a lack of clearance of mucus associated with the pericilliary layer, resulting in a continually developing blockage and a decreased airflow that reaches the respiratory zone of the lungs. With reduced oxygen levels, the patients begin to suffer and can eventually become cyanotic. However, this is one of the more apparent symptoms of $\mathrm{CF}$, and has been a focus since the disease was first identified. Here, the primary mode of treatment has been physical percussion of the patient. This is termed as chest physical therapy (CPT) and has progressed much since it was initially developed. This treatment initially involved pounding on the patient's back in such a manner as to dislodge mucus that was clinging to the bronchi [62]. After dislodging the mucus, the patient would then cough up any that was loosened, but this process in general was quite painful and not desired by patients, even when it was successful. In an effort to make this a more effective procedure, technology began to be introduced into the process. Initially, a device similar to a back-pack, was created that performed the percussion automatically, rather than rely on the doctor or medical professional. Although this is effective in providing the same amount of force each time, the percussion itself was not eliminated from the process.

Following this, different methods were employed to physically break up the mucus. These have become more advanced in recent decades. Most are reliant on sound waves or vibrations to loosen the mucus that allows for its eventual clearance. These methods include masks and vests that patients don that create vibrations and devices that convert the exhaled air of a patient into vibrations [62]. In all of these, the pressure and pain of physical percussion is eliminated, but the positive effects remain. Even some chemical treatments are prescribed to help loosen the mucus, such as aerosolized sodium bicarbonate that can help return the mucus layer to its normal $\mathrm{pH}$ and allow for better clearance. Physicians also routinely prescribe bronchodilators and anti-inflammatory medications to patients to both increase the functional airway passage and to help clear some of the mucus from the airways. In some extreme cases, where this use of medication is ineffective or does not keep up with the overall progression of the disease, the patients may have to endure a lung transplant [62]. While this is occasionally effective, it usually results in the eventual buildup of mucus in the lungs once again, resulting in further treatment or potentially even another transplant. 


\subsection{Treatment of bacterial infections}

While the buildup of mucus within the lungs is quite problematic, it is often not what leads to the overall mortality or morbidity of CF patients. Rather, this is due to secondary infections that arise from this buildup. As stated earlier, this mucus is a rich, largely immobile niche of complex nutrients that enmesh the bacteria. In healthy lungs, the cilia are functioning properly with a thin PCL and clearing most pathogens out of the lungs. However, with the thick mucus, this is not the case, as the cilia do not have enough physical force to push it upwards. As a result, bacteria begin to infect the area. However, the precise location in the lungs where the infection resides is important for the choice of treatment methods. In general, the lungs are divided into two zones. The first is the conductive zone, which includes many of the preliminary branches of the bronchi and does not directly include the alveoli or any of the accompanying areas. The second is the respiratory zone, which includes many of the later branches of the airways and the alveoli. These differences are important due to the necessary changes for treatments. In the conductive zone, the tissue does not have quite as high a vascular exposure, but is much closer to the mouth. As such, infections in this area are often treated with aerosolized medicines. In contrast, the respiratory zone is much deeper into the lungs, but has much higher vascular exposure due to the presence of the alveoli in this zone. Treatments for this zone usually include oral or intravenous medications. While the mode of treatment is known, the actual ability to determine where the infection is occurring is dependent on a series of initial cultures from the patient, and the species present can change once treatment begins.

Once the type of treatment has been determined, the next step is determining what the effective antibiotic(s) is for the particular bacteria being targeted. This is dependent on the class of antibiotic and has to be administered in a patient-specific manner. In general, there are some that work more efficiently in certain patients than in others. For example, tobramycin, a potent aminoglycoside, has been approved by the FDA for clinical treatment of $P A$ in CF patients. However, various reports have shown an impenetrability of tobramycin in biofilms, an effect that may be due to the lowered levels of oxygen within the biofilms. The ability of an antibiotic to be active on biofilm bacteria is of the utmost importance when selecting a treatment strategy [33]. As mentioned previously, biofilms generally develop more resistance to certain antibiotics due to their differential metabolism, as well as penetration issues. Those at the surface of the biofilm are generally more metabolically active, while those in the middle of the biofilm projections and those at the base are generally far less active or even inactive. Ciofu et al. [63] showed that certain antibiotics will be more or less effective in these areas depending on their mode of action. Tobramycin is highly effective on the more oxygen-rich outer layer of metabolically active bacteria, but others such as colistin are better suited at dealing with the metabolically inactive bacteria further into the biofilm. In addition, both basic and clinical scientists are focusing better on the proper physiology of bacteria for treatment. This is something that is case-dependent, and cannot necessarily be generalized.

However, the efficiency of the antibiotics for long-term treatment of $P A$ infections has been worrisome, especially because of the development of multi-drug resistant PA (MRPA) [64]. Because of these issues, it is imperative to unravel new treatments that will work on bacteria that are growing under non-traditional conditions (e.g., anaerobic or microaerobic). That is 
why certain groups are evaluating the ability of other compounds for bactericidal action. One of the more notable treatments was discovered by our group in defining the use of acidified nitrite for the treatment of mucoid $P A$ infections. In 2006, we discovered that the application of acidified nitrite onto anaerobic $P A$, both planktonic and biofilm associated, virtually killed the organisms; however, the same effect was not quite seen aerobically [65]. The treatment also had to be administered at a slightly acidic $\mathrm{pH}$ (6.5) of the CF lung. This combination, however, was very effective. Further investigation showed that acidified nitrite was unstable and produced a toxic, bactericidal intermediate nitric oxide (NO). Although $P A$ normally proceeds through the route of denitrification, this process is overloaded by an excess of $\mathrm{NO}_{2}^{-}$, and subsequently NO. The enzymes involved in the detoxification process are not able to compensate for this overload, and the bacteria ultimately become poisoned. This occurs through the reaction of $\mathrm{NO}$ with sulfur from cysteines in the proteins in the cell, which produces Snitrosylated proteins (S-NO) that are targeted for degradation. In addition, NO also reacts with iron/heme containing proteins leading to the formation of dinitrosyl iron complexes. Considering the simplicity and remarkable efficacy of this treatment, this is an example of what to look for in the future.

\section{Future treatments}

While advancements in medicine are not written in stone, there are trends that are observable that can dictate what advancements should be expected. Most of these trends are collected on the website for the CF Foundation [66], which monitors all forms of data related to CF research. In this case, they maintain a log of what is in the pipeline for research, as well as commonly available treatments for patients. In general, the medications available fall into six categories: CFTR modulators, anti-inflammatory drugs, anti-infective drugs, nutrition, mucus alteration, and airway surface restoration. Much of the research on medications has been focused on antiinflammatory and anti-infective medications. While most of the anti-infective compounds have been modifications of available antibiotics into inhalable forms (such as aerosolized amikacin and vancomycin), the developments in anti-inflammatory medications are slightly more unique. One such example is the development of a drug that is targeting the Type III Secretion System of $P A$. This is a humanized monoclonal antibody directed against one of the protein components of the T3SS. The study states that its goal is to develop an anti-inflammatory molecule, which this antibody serves to do. By interacting with the T3SS, it will hopefully reduce any inflammation caused by sensing this molecule, and potentially reduce the amount of molecules secreted by this system. However, as they note, this is not a means to kill PA [66]. The antibody does not serve that purpose, so it is not able to function as an antibiotic in infected patients.

Another field that seems to be growing with potential treatments is the area of CFTR modulators. This field of treatment is focused on finding drugs that change the defective behavior of mutant CFTR protein. Since one form of mutant CFTR is produced yet does not make it to the apical membrane of the cells, some thought has gone into making it possible for the protein to make it to the surface, and hopefully in that process, fold properly to allow for a functioning 
anion channel. All of these are experimental compounds, but have made it to Phase 2 clinical trials at the time of this publication [66]. The first is a "potentiator", a compound that supposedly will be able to open a defective CFTR once it has reached the surface. While this is not meant for all forms of the disease, it is possible that this will have a great impact. Next is a "corrector" that is meant to move the folded protein to the correct location in the cell. This can help with proteins that have misfolded and are sent for degradation or are sent to a different membrane of the cell (e.g., basolateral). Finally, a synthetic signaling molecule has been developed that is meant to supplement decreased levels of S-nitrosoglutathione (an NO generator) in the cells of CF patients. This signaling molecule has been found to be decreased in these patients, and already evidence from the trial has shown that by supplementing this signal, there is increased and proper folding of the CFTR and function of the channel once it is in the membrane.

\section{Conclusions}

Bacterial infections of CF patients have been a cornerstone of treatment for decades, and that will not change going forward. Future efforts will be focused on finding alternative treatments that will be able to affect both planktonic and biofilm associated organisms in the lungs. This aspect of bacterial growth is most likely the key to effectively remove the organisms from the CF lung and improve the overall life expectancy for these patients. Already, great advances have been made in the last thirty years, as evidenced by not only the number of new and varying treatments, but also by the increased average life span for CF patients (40.7 years) [4]. While some research will still be focused on finding a way to directly treat the CFTR mutation, short-term research needs to be focused on discovering new and innovative treatment options.

\section{Acknowledgements}

The authors wish to thank previous support from the Daniel Tyler Health and Education Foundation (Fort Collins, CO); ARCH Biopartners (Toronto, CA); Cure Finders, Inc. (Sevierville, TN); and Cystic Fibrosis Research (Palo Alto, CA).

\section{Author details}

Cameron T. McDaniel, Warunya Panmanee and Daniel J. Hassett*

${ }^{*}$ Address all correspondence to: Daniel.Hassett@UC.Edu

University of Cincinnati, College of Medicine, Cincinnati, OH. Department of Molecular Genetics, Biochemistry, and Microbiology, USA 


\section{References}

[1] Starner TD, McCray PB Jr. Pathogenesis of early lung disease in cystic fibrosis: a window of opportunity to eradicate bacteria. Annals of Internal Medicine. 2005;145:816.

[2] Riordan JR1, Rommens JM, Kerem B, Alon N, Rozmahel R, Grzelczak Z, Zielenski J, Lok S, Plavsic N, Chou JL, et al. Identification of the cystic fibrosis gene: cloning and characterization of complementary DNA. Science. 1989 Sep 8;245(4922):1066-73.

[3] MacKenzie T, Gifford AH, Sabadosa KA, Quinton HB, Knapp EA, Goss CH, et al. Longevity of patients with cystic fibrosis in 2000 to 2010 and beyond: survival analysis of the cystic fibrosis foundation patient registry. Ann Intern Med. 2014;161:233-241. doi:10.7326/M13-0636

[4] Cystic Fibrosis Foundation. Cystic Fibrosis Foundation Patient Registry 2013 Annual Data Report to the Center Directors. Bethesda, Maryland.

[5] Coakley RD, Grubb BR, Paradiso AM, et al. Abnormal surface liquid pH regulation by cultured cystic fibrosis bronchial epithelium. PNAS. 2003;100:16083-16088.

[6] Gustafsson JK, Ermund A, Ambort D, et al. Bicarbonate and functional CFTR channel are required for proper mucin secretion and link cystic fibrosis with its mucus phenotype. The Journal of Experimental Medicine. 2012;209:1263-1272.

[7] Livraghi A, Randell SH. Cystic fibrosis and other respiratory diseases of impaired mucus clearance. Toxicologic Pathology. 2007;35:116-129.

[8] National Heart, Lung, and Blood Institute; National Institutes of Health; U.S. Department of Health and Human Services.

[9] Rowntree RK, Harris A. The phenotypic consequences of cftr mutations. Annals of Human Genetics. 2003;67(5):471-485.

[10] Tarran R, Button B, Boucher RC. Regulation of normal and cystic fibrosis airway surface liquid volume by phasic shear stress. Annu Rev Physiol. 2006;68:543-561.

[11] Boucher RC. An overview of the pathogenesis of cystic fibrosis lung disease. Adv Drug Deliv Rev. 2002;54:1359-1371.

[12] Worlitzsch D, Tarran R, Ulrich M, Schwab U, Cekici A, Meyer KC, Birrer P, Bellon G, Berger J, Weiss T, Botzenhart K, Yankaskas JR, Randell S, Boucher RC, Döring G. Effects of reduced mucus oxygen concentration in airway Pseudomonas infections of cystic fibrosis patients. J Clin Invest. 2002 Feb;109(3):317-25.

[13] Yoon SS, et al. Pseudomonas aeruginosa anaerobic respiration in biofilms: relationships to cystic fibrosis pathogenesis. Dev Cell. 2002;3(4):593-603. 
[14] Britigan BE, Hayek MB, Doebbeling BN, Fick RB Jr. Transferring and lactoferrin undergo proteolytic cleavage in the Pseudomonas aeruginosa-infected lungs of patients with cystic fibrosis. Infect Immun. 1993;61:5049-5055.

[15] NIH - National Heart, Blood, and Lung Institute. What are the signs and symptoms of Cystic Fibrosis? Available at: http://www.nhlbi.nih.gov/health/health-topics/ topics/cf/signs. Accessed January 4, 2015.

[16] Pulmonary exacerbations in cystic fibrosis with negative bacterial cultures. Pediatric pulmonology. 569.

[17] Elizur A, Cannon CL, Ferkol TW. Airway inflammation in cystic fibrosis. Chest. 2008;133:489.

[18] Armstrong D, Robinson L. Inflammation in the lungs of patients diagnosed with cystic fibrosis: association with Iron Deficiency. 2008;SURG 2(1).

[19] Microbiology of airway disease in a cohort of patients with Cystic Fibrosis. BMC Infectious Diseases. BioMed Central 4.

[20] Lyczak JB, Cannon CL, Pier GB. Lung infections associated with cystic fibrosis. Clinical Microbiology Reviews. 2002;15:194.

[21] Doggett RG, Harrison GM, Wallis ES. Comparison of some properties of Pseudomonas aeruginosa isolated from infections in persons with and without cystic fibrosis. J. Bacteriol. 1964;87:427-431.

[22] Localization of Staphylococcus aureus in infected airways of patients with cystic fibrosis and in a cell culture model of S. aureus adherence. Am J Respir Cell Mol Biol. American Thoracic Society - AJRCMB. 83.

[23] Christopher H, et. al. Review: Staphylococcus aureus and MRSA in cystic fibrosis. Journal of Cystic Fibrosis. 2011;10:298.

[24] Moisan H, Brouillette E, Jacob CL, Langlois-Bégin P, Michaud S, Malouin F. Transcription of virulence factors in Staphylococcus aureus small-colony variants isolated from cystic fibrosis patients is influenced by SigB. Journal of Bacteriology. 2006;188:64-76.

[25] Elborn JS. Treatment of Staphylococcus aureus in cystic fibrosis. Thorax. 1999;54:377-378.

[26] King P. Haemophilus influenzae and the lung (Haemophilus and the lung). Clinical and Translational Medicine. 2012;1:10.

[27] Starner TD, Zhang N, Kim GH, Apicella MA, McCray PB Jr. Haemophilus influenzae Forms Biofilms on Airway Epithelia. American Journal of Respiratory and Critical Care Medicine. 2006;174(2):213-220. 
[28] Mahenthiralingam E, Baldwin A, Dowson CG. 2007. Burkholderia cepacia complex bacteria: opportunistic pathogens with important natural biology. Journal of Applied Microbiology. 2007;104:1539-51.

[29] Schwab U, Abdullah LH, Perlmutt OS, et al. Localization of Burkholderia cepacia complex bacteria in cystic fibrosis lungs and interactions with Pseudomonas aeruginosa in hypoxic mucus. Infection and Immunity. 2014;82:4729-4745.

[30] Chotirmall SH, McElvaney NG. Fungi in the cystic fibrosis lung: bystanders or pathogens? International Journal of Biochemistry and Cell Biology. 2014;52: 161-173.

[31] Tunney MM, Field TR, Moriarty TF, et al. Detection of anaerobic bacteria in high numbers in sputum from patients with cystic fibrosis. Am J Respir Crit Care Med. 2008;177:995e1001.

[32] Frederiksen B, Koch C, Høiby N. Antibiotic treatment of initial colonization with Pseudomonas aeruginosa postpones chronic infection and prevents deterioration of pulmonary function in cystic fibrosis. Pediatr Pulmonol. 1997;23:330-335.

[33] Simon RH. Cystic fibrosis: Antibiotic therapy for lung disease. Available at: http:// www.uptodate.com/contents/cystic-fibrosis-antibiotic-therapy-for-lung-disease. Accessed January 6, 2015.

[34] Bryan LE, Kowand SK, Van Den Elzen HM. Biosynthesis; chemistry; mechanisms of action and resistance: mechanism of aminoglycoside antibiotic resistance in anaerobic bacteria: clostridium perfringens and bacteroides fragilis. Antimicrob. Agents Chemother. January 1979;15(1):7-13. doi:10.1128/AAC.15.1.7.

[35] Takahashi N, Kalfas S, Yamada T. Phosphorylating enzymes involved in glucose fermentation of Actinomyces naeslundii. J. Bacteriology. 1995;177(20): 5806-11.

[36] Downes J, Sutcliffe I, Tanner ACR, Wade WG. Prevotella marshii sp. Nov. and Prevotella baroniae sp. Nov., isolated from the human oral cavity. International Journal of Systematic and Evolutionary Microbiology. 2005;55(4):1551-55.

[37] Moffett KS. Pseudomonas aeruginosa in patients with cystic fibrosis. Infectious Disease and Antimicrobial Agents. http://www.antimicrobe.org/new/b260.asp

[38] Whiteley M, Bangera MG, Bumgarner RE, Parsek MR, Teitzel GM, Lory S, Greenberg EP. Gene expression in Pseudomonas aeruginosa biofilms. Nature. 25 October 2011;413;860-864.

[39] Smith RS, Iglewski BH. P. aeruginosa quorum-sensing systems and virulence. Curr Opin Microbiol. 2003;6:56-60.

[40] Nadal Jimenez P, Koch G, Thompson JA, Xavier KB, Cool RH, Quax WJ. The multiple signaling systems regulating virulence in Pseudomonas aeruginosa. Microbiology and Molecular Biology Reviews. 2012;76:46-65. 
[41] Singh, et al. Quorum-sensing signals indicate that cystic fibrosis lungs are infected with bacterial biofilms. Nature. 2000;407;762-4.

[42] Cowell BA, Twining SS, Hobden JA, Kwong MSF, Fleiszig SMJ. Mutation of lasA and lasB reduces Pseudomonas aeruginosa invasion of epithelial cells. Microbiology. 2003;149:2291-2299.

[43] Lam J, Chan R, Lam K, Costerton JW. Production of mucoid microcolonies by Pseudomonas aeruginosa within infected lungs in cystic fibrosis. Infection and Immunity. 1980;28:546-556.

[44] ES Garrett, D Perlegas, DJ Wozniak. Negative control of flagellum synthesis in Pseudomonas aeruginosa is modulated by the alternative sigma factor $\mathrm{AlgT}(\mathrm{AlgU})$. J Bacteriol. 1999;181:7401-7404.

[45] Okkotsu Y, Little AS, Schurr MJ. The Pseudomonas aeruginosa AlgZR two-component system coordinates multiple phenotypes. Cell. Infect. Microbiol. 2014.

[46] Hassett DJ, Sutton MD, Schurr MJ, Herr AB, Caldwell CC, Matu JO. Pseudomonas aeruginosa hypoxic or anaerobic biofilm infections within cystic fibrosis airways. Trends in Microbiology. March 2009;17(3):130-138.

[47] Tolker-Nielsen T. Pseudomonas aeruginosa biofilm infections: from molecular biofilm biology to new treatment possibilities. Acta Pathologica Microbiologica et Immunologica Scandinavica. December 2014;122(Supp 138):1-51.

[48] Wagner VE, Iglewski BH. P. aeruginosa biofilms in CF infection. Clinic Rev Allerg Immunol. 2008;35:124-134.

[49] Ishida H, Ishida Y, Kurosaka Y, Otani T, Sato K, Kobayashi H. In vitro and in vivo activities of levofloxacin against biofilm-producing Pseudomonas aeruginosa. Antimicrobial Agents and Chemotherapy. July 1998;1641-1645.

[50] Parkins MD, Ceri H, Storey DG. Pseudomonas aeruginosa GacA, a factor in multihost virulence, is also essential for biofilm formation. Molecular Microbiology. 2001;40(5): 1215-1226.

[51] Schuster M, et al. The Pseudomonas aeruginosa RpoS regulon and its relationship to quorum sensing. Mol Microbiol. 2004;51(4):973-985.

[52] Morgan R, Kohn S, Hwang SH, Hassett DJ, Sauer K. BdlA, a chemotaxis regulator essential for biofilm dispersion in Pseudomonas aeruginosa.

[53] Hassett DJ, Cuppoletti J, Trapnell B, Lymar SV, Rowe JJ, Yoon SS, Hilliard GM, Parvatiyar K, Kamani MC, Wozniak DJ, Hwang SH, McDermott TR. Urs A. Ochsner. Anaerobic metabolism and quorum sensing by Pseudomonas aeruginosa biofilms in chronically infected cystic fibrosis airways: rethinking antibiotic treatment strategies and drug targets. Advanced Drug Delivery Reviews. 2002;54:1425-1443. 
[54] Boles BR, Thoendel M, Singh PK. Rhamnolipids mediate detachment of Pseudomonas aeruginosa from biofilms. Mol Microbiol. 2005;57(5):1210-1223.

[55] Hansen C, Skov M. Evidence for the efficacy of aztreonam for inhalation solution in the management of Pseudomonas aeruginosa in patients with cystic fibrosis. Therapeutic Advances in Respiratory Disease. 2014;1-6.

[56] Hogardt M, Heesemann J. Microevolution of Pseudomonas aeruginosa to a chronic pathogen of the cystic fibrosis lung. Current Topics in Microbiology and Immunology. 2013;358:91-118.

[57] Fung C, Naughton S, Turnbull L, Tingpej P, Rose B, Arthur J, Hu H, Harmer C, Harbour C, Hassett DJ, Whitchurch CB, Manos J. Gene expression of Pseudomonas aeruginosa in a mucin-containing synthetic growth medium mimicking cystic fibrosis lung sputum. J Med Microbiol. Sep 2010;59(Pt 9):1089-100.

[58] Son MS, Matthews WJ Jr, Kang Y, Nguyen DT, Hoang TT. In vivo evidence of Pseudomonas aeruginosa nutrient acquisition and pathogenesis in the lungs of cystic fibrosis patients. Infect Immun. Nov 2007;75(11):5313-24.

[59] Hoboth C, Hoffmann R, Eichner A, Henke C, Schmoldt S, Imhof A, Heesemann J, Hogardt M. Dynamics of adaptive microevolution of hypermutable Pseudomonas aeruginosa during chronic pulmonary infection in patients with cystic fibrosis. J Infect Dis. 1 Jul 2009;200(1):118-30.

[60] Cystic Fibrosis Foundation. About CF. http://www.cff.org/AboutCF/. Accessed 2-1-2015.

[61] Conese M, Ascenzioni F, Boyd AC, et al. Gene and cell therapy for cystic fibrosis: From bench to bedside. Journal of Cystic Fibrosis. 2011;10(Supp 2):S114-S128.

[62] NIH - National Heart, Blood, and Lung Institute. How is Cystic Fibrosis treated? Available at: http://www.nhlbi.nih.gov/health/health-topics/topics/cf/treatment. Accessed January 4, 2015.

[63] Ciofu O, Tolker-Nielsen T, Jensen PØ, Wang H, Høiby N. Antimicrobial resistance, respiratory tract infections and role of biofilms in lung infections in cystic fibrosis patients. Adv Drug Deliv Rev.

[64] Luna RA, Millecker LA, Webb CR, et al. Molecular epidemiological surveillance of multidrug-resistant Pseudomonas aeruginosa isolates in a pediatric population of patients with cystic fibrosis and determination of risk factors for infection with the houston-1 strain. Journal of Clinical Microbiology. 2013;51:1237-1240.

[65] Yoon, SS, Coakley R, Lau GW, et al. Anaerobic killing of mucoid Pseudomonas aeruginosa by acidified nitrite derivatives under cystic fibrosis airway conditions. Journal of Clinical Investigation. 2006; 116: 436-446. 
[66] Cystic Fibrosis Foundation. Drug Development Pipeline. Available at: http:// www.cff.org/research/DrugDevelopmentPipeline/. Accessed January 5, 2015. 


\section{Section 4}

CFTR - Mutations and Modifiers 

Chapter 9

\title{
New Molecular Diagnosis Approaches - \\ From the Identification of Mutations \\ to their Characterization
}

\author{
Anne Bergougnoux, Magali Taulan-Cadars, \\ Mireille Claustres and Caroline Raynal \\ Additional information is available at the end of the chapter \\ http://dx.doi.org/10.5772/60679
}

\begin{abstract}
Molecular diagnosis of cystic fibrosis is based on the detection of mutation in the CFTR gene, identified in 1989. During the past 20 years, thanks to evolutions of diagnostic techniques, our knowledge of mutation spectrum and pathophysiological mechanisms involved in the disease has significantly improved. Sanger sequencing and quantitative methods greatly contributed to the identification of the 2,000 sequence variations reported worldwide in CFTR. We are now entering the new technological age with the generalisation of Next Generation Sequencing (NGS) technologies in diagnostics laboratories. These high throughput approaches allow scanning for the entire CFTR locus, including deep intronic regions, and in parallel other candidate genes that possibly influence the clinical evolution of patients. However, this powerful technology poses new challenge in test interpretation. In this chapter, we review the current and new technologies used in molecular diagnostics of cystic fibrosis, particularly NGS approaches. We also present current and new bioinformatics tools available for the interpretation of variants and in vitrolex vivo and in vivo techniques that can be used to improve the characterization of the functional impact of CFTR variations.
\end{abstract}

Keywords: Next generation sequencing, CFTR, sequence variations, interpretation, functional characterization 


\section{Introduction}

The autosomal recessive mode of inheritance of Cystic Fibrosis (CF) was suggested by Andersen and Hodges in 1945 when they described this disease as a pathological entity.

Since the discovery of the CFTR gene (and the predicted protein cystic fibrosis transmembrane conductance regulator) in 1989 [1], close to 2, 000 variations have been identified on this locus. CFTR gene studies represent one of the most frequent genetic analyses routinely performed worldwide, either to confirm the clinical diagnosis of CF or CFTR-related disorders (CFTRRDs), or to offer carrier testing, prenatal or pre-implantation genetic diagnosis.

The most common severe mutation, p.Phe $508 \mathrm{del}$, is found in approximately $70 \%$ of CF alleles of European descent (therefore it is present in $49 \%$ of homozygous and $42 \%$ of compound heterozygous CF patients), with significant variations depending on ethnicities [2]. There is a clear decreasing northwest to southwest gradient in p.Phe508del frequency across Europe. Only four other mutations represent more than 1\% of CF cases: p.Gly542*, p.Gly551Asp, p.Asn1303Lys, and p.Trp1282*. All other mutations are rare and many are private, only detected within a single family.

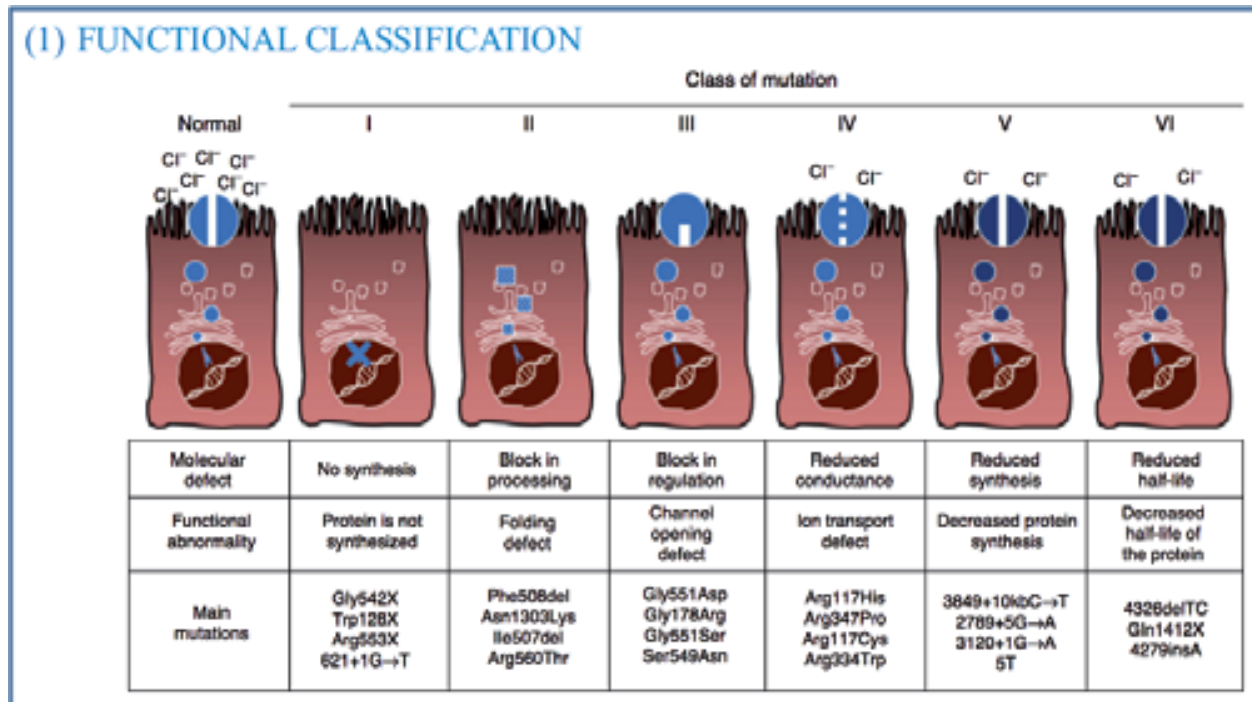

(2) CLINICAL CLASSIFICATION

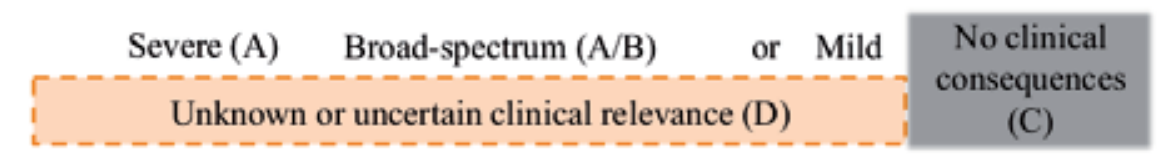

Figure 1. Two models for the classification of CFTR variants: (1) Functional: five classes of defective CFTR protein [3, 4, 5]; and (2) Clinical: four classes based on phenotypic expression in patients [6]. 
Therefore, a good knowledge of CFTR diseases and their molecular pathology is required when choosing tools and strategy and when interpreting results. Organization of regional or national networks of specialist clinical and molecular genetic laboratories is needed. Two models for the classification of sequence alterations have been proposed (Figure 1): (1) depending on their molecular impact on the CFTR chloride channel, which requires in vitro/in vivo functional assays, now less used due to the difficulty in accurately classifying the new reported variants [3,4] or (2) by their clinical consequences on patients based on detailed and prospective clinical evaluation of patients associated with molecular findings [5].

However, even if the most severe mutations were studied in vitro, mild mutations or variants of unknown (clinical) significance (VUCs or VUS) were barely analysed. Thus, only one third of CFTR variations are functionally classified. Finally, in CF, as in other genetic diseases, new molecular diagnosis techniques such as Next Generation Sequencing (NGS) allow the analysis of the whole CFTR locus (including deep intronic regions) but increase the number of VUCS reported on patients.

Therefore, geneticists and clinicians have to combine CFTR epidemiological databases, functional in vitro/in vivo analysis and exhaustive clinical data to perform pertinent genotype/ phenotype correlations in patients in order to provide appropriate genetic counselling to families.

\section{Technical aspects of molecular diagnosis}

The CFTR genetic testing strategy depends on the clinical and familial context and is classically performed step by step, as recommended [7]. As shown in Figure 2, tests are carried out in various situations, including confirmation of a clinical diagnosis, prenatal diagnosis (familial context or foetal suspicion of CF) and carrier screening.

A wide range of techniques is still used to identify CFTR gene sequence variations (presented in Table 1) and there is no gold standard or preferred method for routine testing. However, laboratories should be aware of the limitations of their chosen method (e.g. some mutations are not identified). Moreover, assay performance should always be verified before diagnostic use, even though commercial kits are CE-marked in vitro diagnostic devices (IVDD). In the perspective of accreditation (expected in France in 2020 for hospital diagnostic laboratories), all methods used in molecular biology should be validated before diagnostic use, notably by comparison with a reference method (Sanger sequencing for Single Nucleotide Variations and quantitative PCR for Copy Number Variations detection). This technical validation should be undertaken for NGS in the step 1 and 2 diagnosis.

Undetected CFTR mutations may lie within the introns or regulatory regions, which are not routinely explored but that will be soon analysed by NGS. Comparison between NGS and reference methods for the identification of CFTR variants on these unexplored regions will be a challenge. 


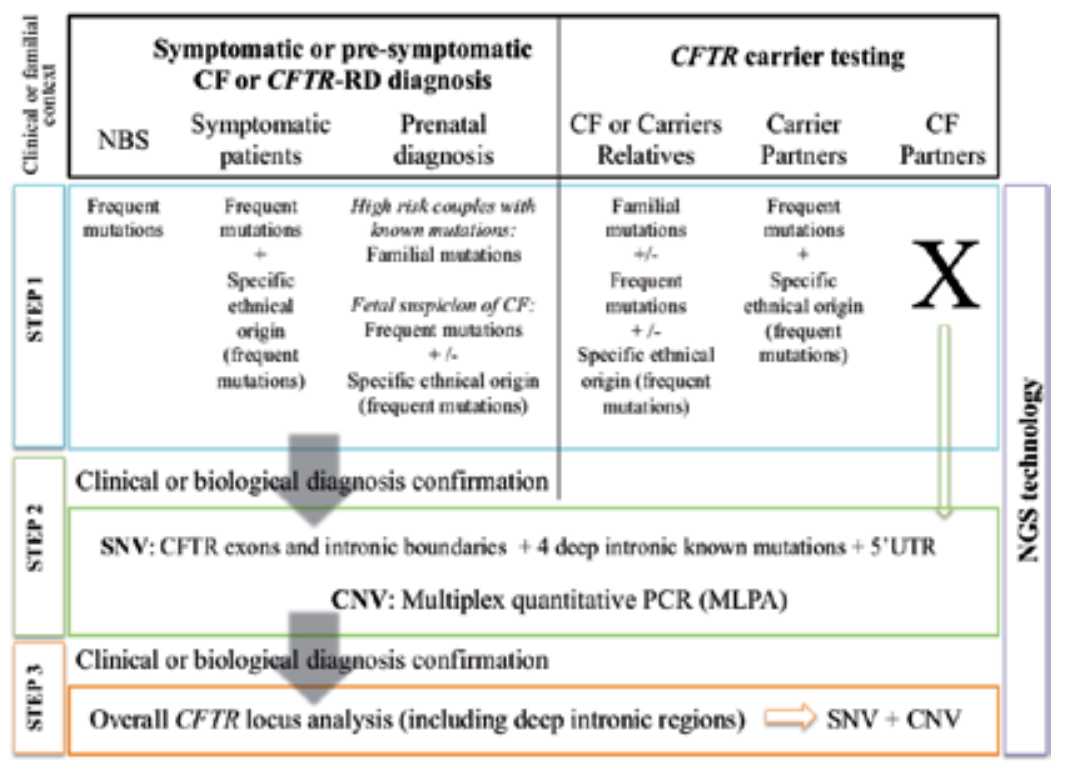

Figure 2. CF molecular diagnosis: a step-by-step strategy (modified from [7]). NBS: newborn screening; SNV: Single Nucleotide Variation; CNV: Copy Number Variation; MLPA: Multiplex Ligation-dependent Probe Amplification.

\subsection{Current techniques to detect $C F T R$ variants}

\subsubsection{Screening techniques (Step 1)}

These approaches consist of genotyping a panel of frequent CF mutations using commercial kits (Table 1) that classically cover more than $80 \%$ of CF known mutations in European populations. Additional search for mutations specific to certain regions or ethnicities (frequency higher than $1 \%$ of CF alleles in the targeted population) completes the analyses.

Data on disease and carrier frequencies or mutation frequencies in various populations are available in the WHO report [8] and should be accurately known and used by laboratories.

For many patients carrying CF-causing mutations included in commercial panels, CFTR molecular analysis generally stops at this step. There is no need for additional studies, except the confirmation of mutations by a second method, as recommended by international guidelines for genetics diagnosis.

\subsubsection{Scanning techniques (Step 2)}

The high heterogeneity of CFTR mutations in CF and CFTR-RD populations makes the complete molecular screening of the 27 exons and parts of the regulatory regions (5'UTR, 3'UTR and partial intronic regions) essential.

Therefore, the analysis of the CFTR locus can be performed as follows: 
- To detect SNV: scanning the 27 exons of the CFTR gene, intronic boundaries and four intronic regions with reported deep intronic mutations [9-12] and a part of the promoter. As an example, one can use Sanger sequencing with Single Condition Amplification Primers (SCAP) technique [13].

- To detect CNV (large CFTR rearrangements): scanning of exon by multiplex fluorescent quantitative PCR (Multiplex Ligation-dependent Probe Amplification or MLPA) or arrayCGH [14].

These robust methods allow the detection of more than $97 \%$ of CFTR mutations involved in CF.

\subsubsection{Advantages and limits}

Classical screening or scanning methods used to detect mutations (in step 1 and 2) have high specificity and sensitivity (Table 1). Older techniques such as RFLP (restriction fragment length polymorphism), DGGE (denaturing gradient gel electrophoresis) or DHPLC (denaturing high performance liquid chromatography) were widely used in the past 20 years but were difficult to set up and showed inconstant performance. They have been progressively replaced by easyto-use commercial kits, more appropriate for larger sample series. These kits allow PCR multiplexing and thus enable the detection of the most common CF mutations in one reaction. Sanger sequencing and quantitative PCR are the most effective to identify unknown mutations. However, all these classical methods are labour-intensive and time-consuming.

\begin{tabular}{|c|c|c|c|}
\hline Method name & Mutations detected & Advantages & Limits \\
\hline \multicolumn{4}{|c|}{ Screening (step 1) } \\
\hline Reverse dot blot hybridization & Up to 20 mutations per multiplex & \multirow{4}{*}{$\begin{array}{l}\text { Appropriate for large series } \\
\text { High specificity } \\
\text { Cost effective }\end{array}$} & $\begin{array}{l}\text { Partial hybridization if SNP on } \\
\text { probe }\end{array}$ \\
\hline $\begin{array}{l}\text { ARMS (amplification refractory } \\
\text { mutation system) }\end{array}$ & Up to 50 mutations & & $\begin{array}{c}\text { Primer Design difficult } \\
\text { Need of high agarose gel quality }\end{array}$ \\
\hline $\begin{array}{l}\text { OLA (Oligonucleotide ligation } \\
\text { assay) }\end{array}$ & 32 mutations (Abbott kit) & & $\begin{array}{c}\text { Partial hybridization if SNP on } \\
\text { probe }\end{array}$ \\
\hline NGS & 139 to 166 CFTR variants & & $\begin{array}{l}\text { Need of sufficient coverage } \\
\text { In development (Illumina) }\end{array}$ \\
\hline \multicolumn{4}{|c|}{ Scanning (Step 2) } \\
\hline $\begin{array}{l}\text { HRM (High Resolution Melting } \\
\text { curve analysis) }\end{array}$ & Heterozygous variants & $\begin{array}{l}\text { Appropriate for large series (e.g. } \\
\text { epidemiological studies) }\end{array}$ & $\begin{array}{c}\text { Exon by exon } \\
\text { Need of positive cases } \\
\text { Not adapted for homozygous } \\
\text { variants }\end{array}$ \\
\hline Sanger Sequencing & All sequenced bases & Close to $100 \%$ sensitivity & $\begin{array}{l}\text { Expensive method } \\
\text { Can not detect } \mathrm{CNV}\end{array}$ \\
\hline $\begin{array}{l}\text { Quantitative fluorescent multiplex } \\
\text { PCR }\end{array}$ & $\begin{array}{l}\text { Deletions, duplications, } \\
\text { insertions }\end{array}$ & Simple and rapid & $\begin{array}{c}\text { False positive if SNP on probe } \\
\text { Performance dependent on DNA } \\
\text { quality }\end{array}$ \\
\hline NGS (used for step 3) & All sequenced bases & $\begin{array}{l}\text { Appropriate for large series } \\
\text { Cost effective }\end{array}$ & $\begin{array}{l}\text { Need of sufficient coverage } \\
\text { In development (Illumina) }\end{array}$ \\
\hline
\end{tabular}

Table 1. Overview, advantages and limits of screening and scanning techniques used in molecular genetics diagnosis (adapted from [7]) 


\subsection{Next Generation Sequencing (NGS)}

\subsubsection{Principle of NGS technologies}

The development of Next Generation Sequencing (NGS) or Massively Parallel Sequencing (MPS) technologies with an immense capacity (up to 1 Terabase $(\mathrm{Tb})$ of data per run with the HiSeq2500 system, Illumina) is a major technical progress in the field of human genetics. Since 2004, three principal NGS platforms have been commercially available, including (i) 454 GS FLX \& GS junior from Roche (ii) Genome Analyzer, HiSeq \& MiSeq from Illumina and (iii) SOLiD \& Ion Torrent PGM from Life Technologies-Applied Biosystems (AB) [15]. These technologies differ in terms of sample library preparation workflow, enabling sequencing on any of the current NGS sequencers (e.g. Illumina, Life technologies, Roche). Since late 2004, three principal NGS firms developed sequencers commercially available (listed in Table 2).

454 pyrosequencing is a sequencing-by-synthesis method that measures the release of inorganic pyrophosphate upon incorporation of nucleotides, by converting it into luciferase chemiluminescent signals using a series of enzymatic reactions. Ion Torrent semiconductor technology is also based on a sequencing-by-synthesis approach but measures $\mathrm{pH}$ changes (instead of light) induced by the release of hydrogen ions as nucleotides are incorporated. SOLiD technology is a ligation-based sequencing system. DNA ligase is used to identify the nucleotide present at a given position in a DNA sequence; each base is read twice, which increases accuracy, even for homopolymeric regions. Base detection uses a mixture of labelled oligonucleotides, which queries the input strand with ligase. In Illumina system, clonal amplification is performed using a process termed 'bridge amplification' followed by two basic steps, initial priming and extending of the single-stranded, single-molecule template and bridge amplification of the immobilized template with immediately adjacent primers to form clusters. For sequencing, only dye-labelled terminators are added; then the sequence at that position is determined for all clusters; next, the dye is cleaved and another round of dyelabelled terminators is added.

\begin{tabular}{|c|c|c|c|c|}
\hline Platform & $\begin{array}{c}\text { GAII, HiSeq \& } \\
\text { MiSeq }\end{array}$ & Ion Torrent & SOLiD & GS FLX \& GS Junior \\
\hline Methodology & $\begin{array}{l}\text { Sequencing by synthesis } \\
\text { (Reversible termination) }\end{array}$ & Sequencing by synthesis & Sequencing by Ligation & $\begin{array}{l}\text { Sequencing by synthesis } \\
\text { (Pyrosequencing) }\end{array}$ \\
\hline Loading & $\begin{array}{l}\text { Adaptors on template } \\
\text { DNA bind high density } \\
\text { primers across surface of } \\
\text { slide }\end{array}$ & $\begin{array}{l}\text { Adaptors on template } \\
\text { DNA bind primers on } \\
\text { beads, one molecule per } \\
\text { bead }\end{array}$ & $\begin{array}{l}\text { Adaptors on template DNA } \\
\text { bind primers on beads, one } \\
\text { molecule per bead }\end{array}$ & $\begin{array}{c}\text { Adaptors on template DNA } \\
\text { bind primers on beads, one } \\
\text { molecule per bead }\end{array}$ \\
\hline Clonal amplification & $\begin{array}{c}\text { Bridge PCR: Surface } \\
\text { array on flow cell }\end{array}$ & $\begin{array}{c}\text { Emulsion PCR: clusters on } \\
\text { beads }\end{array}$ & $\begin{array}{l}\text { Emulsion PCR: clusters on } \\
\text { beads }\end{array}$ & $\begin{array}{c}\text { Emulsion PCR: clusters on } \\
\text { beads }\end{array}$ \\
\hline Parallelisation & $\begin{array}{l}\text { Random array on flow } \\
\text { cell }\end{array}$ & Beads loaded on a chip & $\begin{array}{l}\text { Beads bonded to high } \\
\text { density glass slide }\end{array}$ & $\begin{array}{l}\text { Beads loaded onto high } \\
\text { density plate }\end{array}$ \\
\hline Detection & Fluorescence & $\begin{array}{l}\mathrm{H}^{+} \text {ions (sensitive } \mathrm{pH} \\
\text { meter) }\end{array}$ & Fluorescence & Light (luciferase) \\
\hline
\end{tabular}

Table 2. Overview of the main NGS technologies 


\subsubsection{Target enrichment}

Targeted re-sequencing isolates genomic regions of interest in a sample library, allowing to focus efficiently and cost-effectively on a small subset of the genome, such as an exome, a particular chromosome, a set of genes or a region of interest such as a whole gene. Two main strategies can be envisioned: capture [16,17] or amplification relevant genomic DNA [18-20] as shown in Table 3.

\begin{tabular}{|c|c|c|c|c|c|c|}
\hline \multirow[b]{2}{*}{ Methods } & \multicolumn{3}{|c|}{ Capture/hybridisation-based method } & \multicolumn{3}{|c|}{ Amplicon-based method } \\
\hline & HaloPlex & $\begin{array}{c}\text { SureSelect } \\
\text { (Agilent)/SeqCa) } \\
\text { (NimbleGen) }\end{array}$ & Nextera (Illumina) & $\begin{array}{l}\text { Microdoplet } \\
\text { (RainDance } \\
\text { Technologies) }\end{array}$ & $\begin{array}{c}\text { Multiplex PCR } \\
\text { (AmpliSeq, Life } \\
\text { Technology \& } \\
\text { TrueSeq, } \\
\text { Illumina) }\end{array}$ & $\begin{array}{l}\text { Long-Range PCR } \\
\text { Qiagen \& "home" } \\
\text { design }\end{array}$ \\
\hline \multirow{3}{*}{ Main steps } & $\begin{array}{c}\text { Restriction enzyme } \\
\text { digestion }\end{array}$ & $\begin{array}{l}\text { Sonication/ } \\
\text { nebulisation }\end{array}$ & $\begin{array}{l}\text { Tagmentation with } \\
\text { transposon }\end{array}$ & Sonication & $\begin{array}{c}\text { PCR } \\
\text { amplification }\end{array}$ & PCR amplification \\
\hline & Probe hybridisation & $\begin{array}{c}\text { PCR amplificatio } \\
+ \text { probe } \\
\text { hybridisation }\end{array}$ & $\begin{array}{l}\text { PCR amplification } \\
\text { + probe } \\
\text { hybridisation }\end{array}$ & & & $\begin{array}{l}\text { Sonication/ } \\
\text { nebulisation }\end{array}$ \\
\hline & & & & & & $\begin{array}{l}\text { Ligation of } \\
\text { sequencing } \\
\text { adaptors }\end{array}$ \\
\hline
\end{tabular}

Table 3. Main strategies proposed for target enrichment

\subsubsection{Advantages and limits}

The main advantages of the technology are related to its capability to process a large number of samples in parallel. NGS technologies are time saving and lower the costs per patient, of step 1 and particularly step 2 molecular analyses. But, they also have significant limitations such as high error rates, enrichment of rare variants and large proportion of missing values, as well as the fact that most current analytical methods are designed for population-based association studies. With second generation sequencing, it is necessary to clonally amplify the isolated targets in order to generate sufficient signal for detection during the sequencing run generating clusters of many thousands of identical DNA targets. In addition, each NGS platform generates different read lengths that range from short (e.g. 35 bases) to long reads (over 500 bases). For a number of applications, including targeted re-sequencing, ChIP-Seq and RNA-Seq, short reads are highly informative and adequate. Conversely, longer reads are more suitable for de novo genome assembly, mapping of high homology regions (related gene family and pseudogenes) and sequencing of repetitive DNA regions, such as introns. This is an important consideration since short read length can make accurate assembly and alignment computationally challenging.

For some clinical applications, as NGS produces massive amounts of data, their analysis and interpretation are time-consuming, not trivial and a real challenge even if specific portions of a genome is analysed. 


\subsubsection{Bioinformatics software to analyse NGS data}

Then, generated data are analysed by bioinformatics tools. Quality criteria such as confidence or coverage are guarantees for optimal sensitivity and specificity of a sequencing run. Bioinformatics analyses are frequently performed by using software developed by sequencers' companies. The three steps include (1) base calling and quality score computation, (2) assembly and alignment and (3) variant calling and annotation. Laboratories developed their own 'inhouse' pipeline mainly to apply filters allowing an easy focus on causing-mutations. Others use free web resources to realign files including BWA (http://bio-bwa.sourceforge.net) or to perform a new variant calling such as Samtools and GATK [21-23]. Others serve as Viewer such as Integrative Genomics Viewer (http://www.broadinstitute.org/igv/). Galaxy website is an integrative platform offering the possibility to view and process files generated by NGS. Many databases, useful for variant annotation and sometimes inserted in others programs or pipelines, are publicly available including the 1000 Genomes Project [24] and the dbSNP database [25] (further described in part 3.1.1).

Many tracks are currently studied (i.e. improvement of bioinformatics tools, comparison of NGS approaches between CF laboratories, development of databases including newly detected NGS variants, publication of guidelines and definition of a diagnosis report model) to make CF molecular diagnosis by NGS suitable for different clinical and familial cases.

\subsubsection{Indications and choice of CFTR analysed regions}

In the case of Mendelian diseases caused by mutations in a single gene, like CFTR in Cystic Fibrosis, NGS sequencing of entire genome or exome is still useless and expensive. Collaborations between companies specialized in molecular diagnosis and academic laboratories recently led to the establishment of new molecular diagnosis tools based on NGS. Combination of (i) the enrichment of regions of interest by hybrid capture, circularization or Polymerase Chain Reaction (PCR) and (ii) high throughput sequencing now allows time-efficient and economical way to perform analyses. Use of NGS technology as a first intention is currently set up in laboratories, which will soon question the CF diagnosis strategy as a tree. As shown in Figure 2, in our actual CF molecular diagnosis strategy, NGS technology can be used instead of 'classical' techniques for the detection of a panel of common mutations (step 1) or to analyse the 'CFTR exome' (step 2). Technical manipulations are similar for both, but filters can be used to focus on regions that contain mutations. Multiplicom ${ }^{\circledR}$ and Illumina ${ }^{\circledR}$ propose a locus specific design to library preparation for molecular analysis of the 'CFTR exome'. CE-marked kits for in vitro diagnostic (CE-IVD) and efficient bioinformatics tools are commercialized. The latter could be performed by in-house pipelines or subcontract on commercial firms (Sophia Genetics ${ }^{\circledR}$ ). SNV and indels are correctly detected (high specificity and sensitivity) and CNV detection is now available for some amplicon-based methods.

Furthermore, in patients with definite CF clinical diagnosis (positive sweat test, CF clinical features) and who carry no or only one CF mutation, step 3 analysis could be ultimately proposed, in combination with the analysis of potential modifiers genes (see next section)

In literature, three studies reported NGS CFTR sequencing (Table 4) on CF patients, CF carriers or controls samples [18, 26, 27]. Abou Tayoun and colleagues [26] first proposed a proof-of- 
concept for a 'CFTR exome' analysis by NGS on 79 samples. Target enrichment was performed by PCR amplification (AmpliSeq Panel, Life technologies) and Sequencing on Ion Torrent Platform (PGM $®$ ). Their sequencing offered minimal coverage of 100X (depending on the Ion 314 or 318 chip used). Two others studies realized sequencing of the whole CFTR locus (close to $250 \mathrm{~kb}$ ) including deep intronic CFTR regions (Figure 2, Step 3). Trujillano et al. [27] reported the CFTR re-sequencing by hybridization capture on a custom NimbleGen SeqCap EZ Choise array using HiSeq2000 (Illumina ${ }^{\circledR}$ ) in a set of 92 samples. They highlighted the precise characterization of breakpoints of seven genomic rearrangements in CFTR.

We proposed a complete CFTR gene sequencing of DNA samples from patients with a confirmed CF clinical diagnosis but with an incomplete genotype [18]. Although large unexplored intronic regions might contain few mutations (about $1 \%-3 \%$ of $\mathrm{CF}$ mutations), we identified a new pathogenic mutation, which creates a pseudo-exon (Table 4). Moreover, we compared hybridization capture and Long-Range PCR to target enrichment and used a smallscale NGS platform for sequencing (GS Junior Sequencer, 454 Life Sciences ${ }^{\circledR}$ ). Some promising variants were then confirmed as deleterious by in vitro/ex vivo functional assays. However, for most detected intronic variants, classification will be a long and difficult way. This approach is currently under development for CF diagnosis.

\begin{tabular}{|c|c|c|c|c|}
\hline & Abou Tayoun et al., 2013 [25] & Trujillano et al., 2014 [26] & \multicolumn{2}{|l|}{ Bonini et al., 2015 [17] } \\
\hline Samples & $\begin{array}{l}57 \text { subjects already genotyped } \\
-22 \text { cell lines: } 24 \mathrm{CF}, 46 \mathrm{CF} \\
\text { carriers, } 9 \text { controls }\end{array}$ & $\begin{array}{l}92 \text { subjects already genotyped: } 45 \\
\text { CF, } 27 \text { CF carriers, } 20 \text { CFTR-RD }\end{array}$ & \multicolumn{2}{|c|}{$\begin{array}{l}18 \mathrm{CF} \text { subjects in whom only one mutation had } \\
\text { identified }\end{array}$} \\
\hline Study objectif & $\begin{array}{l}\text { Proof of concept of CFTR } \\
\text { assay by NGS }\end{array}$ & $\begin{array}{l}\text { Resequencing mutations } \\
\text { Validation technique }\end{array}$ & \multicolumn{2}{|c|}{$\begin{array}{l}\text { Identification of the second mutation } \\
\text { Comparison of two target enrichment } \\
\text { approaches }\end{array}$} \\
\hline \multicolumn{5}{|l|}{ Sequencing protocol } \\
\hline Regions analyzed & $\begin{array}{l}\text { All CFTR exons and 20bp of } \\
\text { all intron/splice sites }(10 \\
343 \mathrm{bp})\end{array}$ & $\begin{array}{l}\text { All CFTR locus }(208 \mathrm{~kb} \rightarrow 181 \mathrm{~kb} \\
\text { after masking repetitive DNA } \\
\text { elements) }\end{array}$ & $\begin{array}{l}\text { All CFTR locus }(250 \mathrm{~kb}) \\
2.1 \text { million probes } \\
(60-90 \mathrm{pb})\end{array}$ & $\begin{array}{l}36 \text { overlapping } \\
\text { fragments }(7.3-14.5 \mathrm{~kb})\end{array}$ \\
\hline Target enrichment & $\begin{array}{l}\text { Custom AmpliSeq panel (Life } \\
\text { technologies) }\end{array}$ & $\begin{array}{l}\text { Hybrid capture: Custom } \\
\text { NimbleGen SeqCap EZ }\end{array}$ & $\begin{array}{l}\text { Hybrid capture: Custom } \\
\text { NimbleGen SeqCap EZ }\end{array}$ & $\begin{array}{l}\text { Long-Range -PCR } \\
\text { (LR-PCR) enrichment }\end{array}$ \\
\hline $\begin{array}{l}\text { Initial DNA quantity } \\
\text { per sample }\end{array}$ & $20 \mathrm{ng}$ & & $500 \mathrm{ng}$ & 750ng \\
\hline Library preparation & $\begin{array}{l}2 \text { separate primer pools }(2 \times 36 \\
\text { amplicons), end-repair, } \\
\text { ligation to adaptators, } \\
\text { quantification of Library } \\
\text { pooled bar-coded libraries } \\
\text { Clonal PCR }\end{array}$ & $\begin{array}{l}\text { Sonication (Covaris } \\
\text { End-repair, A-tailing, ligation to } \\
\text { index adaptators (TrueSeq } \\
\text { protocol } \\
\text { CR), PCR amplification, } \\
\text { quantification of Library } \\
\text { (bioanalyzer), in-solution } \\
\text { hybridization }\end{array}$ & $\begin{array}{l}\text { Nebulization; End- } \\
\text { repair, ligation to } \\
\text { adaptators, } \\
\text { quantification of } \\
\text { Library, Clonal } \\
\text { emulsion PCR }\end{array}$ & $\begin{array}{l}\text { Nebulization; LR- } \\
\text { PCREnd-repair, } \\
\text { phosphorylation, } \\
\text { ligation to MID } \\
\text { adaptators, } \\
\text { quantification of } \\
\text { Library, } \\
\text { Clonal emulsion PCR }\end{array}$ \\
\hline $\begin{array}{l}\text { Sample Multiplexing } \\
\text { and cost }\end{array}$ & 5 to 35 samples & 8 to 24 samples & No multiplexing & 4 samples \\
\hline NGS technology & $\begin{array}{l}\text { 200bp single reads } \\
\text { Ion 314TM (10Mb) or Ion } 318 \\
(1 \mathrm{~Gb}) \\
\text { Ion torrent Personal Genome } \\
\text { Machine (PGM) }\end{array}$ & $\begin{array}{l}\text { 2x100bp paired-end reads } \\
\text { HiSeq } 2000 \text { instrument (Illumina) }\end{array}$ & $\begin{array}{l}\text { Single or Paired-end read } \\
\text { GS Junior Sequencer (454 }\end{array}$ & $\begin{array}{l}\text { ds, up to } 450 \text { bp } \\
4 \text { Life Sciences) }\end{array}$ \\
\hline
\end{tabular}




\begin{tabular}{|c|c|c|c|c|}
\hline & Abou Tayoun et al., 2013 [25] & Trujillano et al., 2014 [26] & Bonini et al., 2015 [17] & \\
\hline Mapping & Human genome & $\begin{array}{l}\text { Human genome (GATK pipeline } \\
\text { and picard-tools) }\end{array}$ & $\begin{array}{l}\text { Human Genome ( } 454 \\
\text { Roche GS Junior data } \\
\text { analysis pipeline) }\end{array}$ & $\begin{array}{l}\text { Chromosome } 7 \\
\text { (454 Roche GS Junior } \\
\text { data analysis } \\
\text { pipeline) }\end{array}$ \\
\hline Minimal coverage & & $\begin{array}{l}\text { Mean: } 231 X \\
99,7 \% \text { covered by minimum } 5 \\
\text { reads }\end{array}$ & $40 X$ & \\
\hline \multicolumn{5}{|c|}{ CFTR Sequencing statistics } \\
\hline Specificity & $97 \%$ ( 22 on 23 mutations) & $100 \%$ & ND & \\
\hline Sensitivity & $100 \%$ (23 on 23 mutations) & $100 \%$ (122 on 122 variants) & ND & \\
\hline Reproducibility & $\begin{array}{l}100 \% \text { ( } 56 \text { on } 56 \text { variants in } 3 \\
\text { independent runs) }\end{array}$ & $100 \%$ (8 on 8 variants) & ND & \\
\hline \multicolumn{5}{|l|}{ Bioinformatics tools } \\
\hline CNV & $\begin{array}{l}\text { Depth-Of-Coverage tool from } \\
\text { the Genome Analysis ToolKit } \\
\text { (GATK) } \\
\text {--> Log2 ratios <-0.7: deletion }\end{array}$ & $\begin{array}{l}\text { Pindel; Conifer; PeSV-Fisher } \\
5 \text { large deletions, } 1 \text { duplication and } \\
1 \text { genomic rearrangement }\end{array}$ & ND & \\
\hline SNV & $\begin{array}{l}7 \text { variants per sample on } \\
\text { average }\end{array}$ & $\begin{array}{l}115 \mathrm{SNV}+28 \text { InDels on average } \\
\text { per sample }\end{array}$ & \multicolumn{2}{|c|}{$\begin{array}{l}197 \text { variants on average per sample (118 in } \\
\text { introns) }\end{array}$} \\
\hline $\begin{array}{l}\text { Variant identification } \\
\text { and calling }\end{array}$ & Not notified & $\begin{array}{l}\text { Variant prediction tools: GATK } \\
\text { Unified Genotyper, samtools } \\
\text { mpileup, SHORE }\end{array}$ & \multicolumn{2}{|c|}{$\begin{array}{l}\text { Reference Mapper } \\
\text { Mutalyzer } \\
\text { SeqNext (JSI medical systems) }\end{array}$} \\
\hline Variant filter & $\begin{array}{l}\text { Min coverage: } 20 \text { reads } \\
\text { Threshold: } 25 \% \text { reads } \\
\text { Both strands }\end{array}$ & $\begin{array}{l}\text { GATK Variant filtration }(\mathrm{MQ}<30.0 ; \\
\text { QUAL }<25.0 ; \mathrm{QD}<4.0 ; \mathrm{DP}<5 ; \\
\mathrm{DP}<2000 ; \mathrm{GQ}<15) \\
\text { GATK Combine Variant }\end{array}$ & \multicolumn{2}{|c|}{$\begin{array}{l}\text { Threshold } 30 \% \text { of reads } \\
\text { Heterozygous: Read support: } 30-65 \% \\
\text { Homozygous }>70 \%\end{array}$} \\
\hline Databases & $\begin{array}{l}\text { Variant frequency }>1 \% \\
\text { dbSNP } \\
\text { Exome Variant Server } \\
\text { CFMDB } \\
\text { CFTR2 }\end{array}$ & $\begin{array}{l}1000 \text { Genomes project } \\
\text { dbSNP } \\
\text { Exome Variant Server } \\
\text { CFMDB } \\
\text { CFTR2 }\end{array}$ & \multicolumn{2}{|l|}{$\begin{array}{l}1000 \text { Genomes project } \\
\text { dbSNP }\end{array}$} \\
\hline In silico analysis & $\begin{array}{l}\text { SIFT } \\
\text { Mutation Taster }\end{array}$ & $\begin{array}{l}\text { Annovar } \\
\text { SIFT, PolyPhen2, PhyloP, Mutation } \\
\text { Taster, phastCons } \\
\text { UCSC Genome Browser }\end{array}$ & \multicolumn{2}{|c|}{$\begin{array}{l}\text { Annovar, HSF, MaxEnt, NNSplice, SIFT, } \\
\text { Polyphen2, PhyloP, UCSC Genome Browser, } \\
\text { Ensembl, MutationTaster }\end{array}$} \\
\hline \multicolumn{5}{|l|}{ Performance } \\
\hline Diagnostic rate & ND & $98.91 \%$ & \multicolumn{2}{|l|}{$88.9 \%(16 / 18)$} \\
\hline Particular cases & $\begin{array}{l}\text { False positive c.2052del } \\
\text { (2184delA) } \\
\text { Sanger sequencing to } \\
\text { determine the c. } \\
\text { 1210-34TG(11-13)T(5-9) } \\
\text { haplotype }\end{array}$ & $\begin{array}{l}\text { In-house script to determine the c. } \\
\text { 1210-34TG(11-13)T(5-9) haplotype }\end{array}$ & \multicolumn{2}{|c|}{$\begin{array}{l}\text { False positive c.2052del (2184delA) } \\
\text { Sanger sequencing to determine the c. } \\
\text { 1210-34TG(11-13);-12T(5-9) haplotype }\end{array}$} \\
\hline Advantages & $\begin{array}{l}\text { Robust, specific, limited } \\
\text { number of VUCs }\end{array}$ & $\begin{array}{l}\text { Breakpoints of large genomic } \\
\text { rearrangement accurately } \\
\text { determined }\end{array}$ & \multicolumn{2}{|c|}{$\begin{array}{l}\text { LR-PCR more adapted for complete gene } \\
\text { resequencing } \\
\text { New intronic mutations identified }\end{array}$} \\
\hline Limits & $\begin{array}{l}\text { SINE/LINE repeats } \\
\text { (amplification and mapping) } \\
\text { Homopolymer stretches } \\
\text { (Sequencing) }\end{array}$ & $\begin{array}{l}\text { GC rich genomic segments (target } \\
\text { enrichment) } \\
\text { Errors in index tags (sequencing) }\end{array}$ & \multicolumn{2}{|c|}{$\begin{array}{l}\text { SINE/LINE repeats (amplification and } \\
\text { mapping) } \\
\text { Homopolymer stretches (Sequencing) } \\
\text { Uncaptured region in intron } 3\end{array}$} \\
\hline
\end{tabular}

Table 4. Comparison of three NGS strategies for CFTR sequencing 


\subsection{Other loci}

With the perspectives of high throughput molecular diagnosis in genetics laboratories, CF, CFTR-RDs and CF-like diseases could be simultaneously explored in patients using NGS. Various gene panels could be investigated according to patients' phenotypes. Therefore, NGS approaches could contribute to (i) identify and confirm the implication of modifiers genes and (ii) improve molecular diagnosis of atypical Cystic Fibrosis, CFTR-RDs or CF-like diseases. Several sequence changes located in the so-called 'modifier genes' have been associated with progression of lung disease in CF patients. A decrease of pulmonary function measured by FEV1 (the forced expired volume after $1 \mathrm{~s}$ of blowing out) was associated with SNVs in EDNRA, ACER, IFRD1, IL8, MUC5AC and TGF- $\beta 1$ genes. Haplotype 8.1 and variants in MBL2 gene were related to Pseudomonas aeruginosa colonization [28, 29]. SNVs in SNAP23, PPP2R4, PPP2R1A and $K R T 19$ were recently associated with a decrease of lung function. Interactions between CFTR and these altered proteins may modify CFTR trafficking and membrane stability and therefore modify phenotype of CF patients [30]. Moreover, changes in other modifier genes were suspected to have an effect on intestinal obstruction (DCTN4, ADIPOR2 and MSRA genes), CFassociated diabetes (TCF7L2 gene) or liver disease (SERPIN1A1) [31].

CFTR-Related disorders comprise congenital bilateral absence of vas deferens (CBAVD), pancreatitis, diffuse bronchiectasis and nasal polyposis. Classically, two CFTR mutations (a severe and a mild mutation or two mild mutations) are found in well-characterized CBAVD patients [32]. In addition, Sharma et al. reported 2 SNVs on TGF- $\beta 1$ and ENDRA genes associated with urogenital anomalies [33]. Chronic pancreatitis is caused by CFTR variations in some cases, and mutations in CTRC, PRSS1, PRSS2 or SPINK1 are also involved and must be analysed.

The implication of CFTR in diffuse bronchiectasis or nasal polyposis is more controversial [34]; variants in other genes were previously reported as possibly causative. However, the identification of mutations in other genes with sufficient significance remains difficult and needs large patient cohorts. In fact, in airway tract diseases, the influence of environment (pollutants, drugs/ therapy and way of life) complicated the achievement of unbiased studies. Nevertheless, the hypothesis of oligogenism is supported by a study that reported mutations in $\mathrm{ENaC}$ channel genes (SCNN1A, SCNN1B, SCNN1G) or SERPIN1A1 in CF-like patients (borderline sweattest and suggestive CF clinical features without two CFTR mutations) [35].

Finally, a pleiotropic effect of $S L C 26 A 9$ on meconium ileus, pancreatic damage and lung disease has been identified [36], as well as SLC9A3 for meconium ileus and lung disease and SLC6A14 for meconium ileus and both lung disease and age at first $P$. aeruginosa infection [37]. Thus, the existence of pleiotropic effect of modifier genes on CF evolution may encourage the development of new therapeutic targets with multi-organ benefits.

\section{Functional characterization of CFTR sequence variations}

To provide appropriate diagnosis and prognosis to $\mathrm{CF}$ patients and also appropriate genetic counselling to families, the impact of variants identified by the techniques detailed above has to be functionally characterized. 
Some variants such as frequent mutations (found in more than $1 \%$ of CF mutated alleles), nonsense or frameshift mutations are readily classified as pathogenic mutations. However, the frequent identification of rare sequence alterations of unknown pathogenicity (VUCS, VUS) substantially complicates test interpretation. Moreover, their number will increase with the diffusion of NGS technologies. To facilitate classification of these variants, CF laboratories have to combine several tools like central mutation databases or CFTR locus specific databases, in silico prediction tools and ex vivo/in vivo functional analyses [38, 39].

\subsection{Epidemiological data and locus specific databases dedicated to CFTR}

\subsubsection{Core or central mutation databases}

Their goal is to collect all sequence variations detected in all genes and to describe each mutation briefly. These databases are used to assess the frequency of a variation (minor allele frequency (MAF) lower or higher than 1\%) in the general population (i.e. unaffected individuals). Since a MAF higher than $1 \%$ reflects a low probability for the variant to be pathogenic, such data may be highly informative for the interpretation of variants.

\subsubsection{The National Center for Biotechnology Information (NCBI) short genetic variations database} dbSNP

dbSNP [25] is the most comprehensive directory of single nucleotide variations. It catalogues short variations in nucleotide sequences from a wide range of organisms. Genetic Variations may be common, thus representing true polymorphisms, or they may be rare. Some of these rare human entries have additional information associated with them, including disease associations, genotype information and allele origin, as some variations are somatic rather than germ-line events. Genotypes and allele frequencies information for various populations from different studies, including data form the HapMap project, are also available.

\subsubsection{Databases specifically collecting data from NGS projects}

1000Genomes [24] aimed to find most genetic variants that have frequencies of at least $1 \%$ in samples from five populations: East Asian, South Asian, African, European and American ancestries. As in dbSNP, genotypes and allele frequencies information are available for a large number of variants [40].

Exome Variant Server [41] is a database that collects data of the NHLBI GO Exome Sequencing Project (ESP). This project aimed to discover novel genes and mechanisms contributing to various disorders by sequencing the protein coding regions of the human genome (i.e. exome) using NGS technology. As the CFTR gene is widely studied, this tool would not be of added value compared to dbSNP and 1000 Genomes.

\subsubsection{Locus Specific Databases (LSDBs) dedicated to CFTR}

LSDBs are now recognized as the best mode of collecting and curating lists of mutations related to human genetic diseases [42]. They compile in a single bioinformatics tool disease-causing 
and non-disease-causing sequence variations identified by genetics laboratories in families with a history of a given Mendelian disease. The most sophisticated ones integrate clinical and biological data, information on the geographic and/or ethnic origin, frequency of variations in the general population, mutation hot spots and all useful information for diagnosis, prognosis and the evaluation of genotype/phenotype relationships [43].

Here we choose to detail three LSDB dedicated to CFTR that provide complementary information for the interpretation and the characterization of variants identified in diagnostics practice.

\subsubsection{Cystic fibrosis mutation database}

The Cystic Fibrosis Mutation Database (CFMDB) [44] also called 'CFTR1' is an open access database dedicated to the collection of sequence variations in the CFTR gene for the international CF genetics research community. It was initiated by the Cystic Fibrosis Genetic Analysis Consortium (CFGAC) in 1989 and is maintained by the Cystic Fibrosis Centre at the Hospital for Sick Children in Toronto. CFMDB allows the direct submission of new variants by laboratories, by filling out an on- line standardized form with the possibility to detail phenotypic data, genotype (i.e. other variants identified in patient) or epidemiological data. The key point of this database is to collect the largest number of CFTR sequence variations identified in patients, relatives and partners. On the other side, because the submission procedure applies only to the initial report of each variant, CFMDB does not provide frequency data, available with the two other databases described below. Finally, contributors do not always follow HGVS recommendations and a same variant can be reported by several laboratories under different names, possibly leading to misinterpretation or misreporting in diagnosis reports.

\subsubsection{Clinical and functional translation of CFTR database CFTR2}

CFTR2 [45] is a website designed to provide information about specific CF mutations to patients, researchers and the general public. For each mutation included in the database, it provides information about whether a given mutation causes cystic fibrosis when combined with another CF-causing mutation and clinical and biological information (sweat chloride, lung function, pancreatic status and pseudomonas infection rates) in patients carrying the mutation. A specific section for health practitioners and scientists provides more in-depth and research-related information.

The goal of the CFTR2 project is to categorize all mutations seen in CF patients as diseasecausing (always resulting in CF when combined with another CF-causing mutation), neutral or mutation of varying clinical consequences (CF and CFTR-RD). Mutations that have not been fully analysed are considered of unknown clinical significance.

The major advantages of CFTR2 are (i) the collection of detailed clinical characteristics on large cohorts of individuals [46] that provide useful information related to a given genotype, and (ii) results of functional testing that are key arguments for their final interpretation [47].

However, this database only collects clinical and genetic data of CF patients (from national registers) that can lead to a bias of phenotypic spectrum assessment of several mutations 
considered as CF-causing mutations while they were also reported in CFTR-RD patients in trans of other CF-causing mutations.

\subsubsection{The French molecular database CFTR-France}

CFTR-France [48] has been developed since 2012 with the aim to collect, store and process any category of variants identified in the CFTR gene, thanks to the collaboration of nine French laboratories with high expertise in the molecular analyses of this gene. Its specificity is to compile and annotate any category of variations (disease-causing, non-disease-causing and variants of unknown clinical significance) that have been identified by collaborators in patients affected with CF or CFTR-RD, in foetuses with abnormal ultrasonography (e.g. echogenic bowel), newborns with pending or inconclusive diagnosis and asymptomatic individuals carrying at least one sequence variation on each CFTR gene (i.e. carrying two variations in trans). The database includes the main clinical data of these individuals, genetic information from familial segregation studies and various variant annotations (frequency in patients and controls populations, sequence homology, predicted or experimentally assessed functional impact, etc.), allowing the analysis of genotype/phenotype relationships.

Thus, CFTR-France, by collecting all phenotypes, reflects the phenotypic spectrum of a large number of mutations. It also reports mutations in complex alleles with association frequencies (related to all individuals recorded in the database), and gives the up-to-date HGVS nomenclature of mutations.

Data collected in CFTR-France are provided by level 2 (specialised) and reference laboratories, so that patients analysed only by level 1 laboratories (searching for the most common mutations) are not included in the database.

Note: Access to CFTR-France is currently restricted to collaborators. A public access program is in progress for the medical and scientific community and for patients and families.

\subsection{In silico prediction analyses}

\subsubsection{Variants located in exons and exon-intron boundaries}

\subsubsection{Prediction tools for the assessment of the impact on protein}

Prediction methods of amino acid substitutions use protein sequence, structure and/or annotation. Disease-causing mutations that affect protein function tend to occur at evolutionarily conserved sites and/or at key positions in protein structure. Multiple sequence alignment of orthologous sequences reveal what positions have been conserved through evolution, and these positions are supposed to be important for protein function. Annotation can enhance prediction for variants located in structurally and functionally important domains, but this information is often sparse.

The issue of the efficiency of prediction tools in assessing possible pathogenicity of missense variants in the CFTR gene is of major interest, since they constitute the vast majority of VUS 
identified in patients. Diagnostics laboratories frequently use those tools and particularly in problematic situations. Unfortunately their performance has not been clearly established and results (i.e. score of pathogenicity) may be discordant for a given variant.

Predictions of the impact of non-synonymous substitutions in CFTR are mainly based on multiple sequence alignment of orthologous sequences. Indeed, even if a partial 3D model of the CFTR protein has been established $[49,50]$, prediction tools do not take into account these elements in the final 'score of pathogenicity'. It is classically recommended to use several prediction tools to obtain concordant predictions that could be considered for variant interpretation.

Table 5 summarizes bioinformatics programs classically used by diagnostics laboratories [51-53] and the new software SuSPect [54].

\begin{tabular}{|c|c|c|}
\hline Method and Web site & Algorithm & Output \\
\hline PolyPhen 2 [54] & $\begin{array}{l}\text { Sequence conservation, structure to model position of } \\
\text { amino acid substitution, and SWISS-PROT annotation }\end{array}$ & $\begin{array}{c}\text { Score ranges from } 0 \text { to a positive } \\
\text { number, where } 0 \text { is neutral, and a high } \\
\text { positive number is damaging }\end{array}$ \\
\hline SIFT [55] & $\begin{array}{l}\text { Sequence homology: } \\
\text { scores are calculated using position-specific scoring } \\
\text { matrices with Dirichlet priors }\end{array}$ & $\begin{array}{l}\text { Score ranges from } 0 \text { to } 1 \text {, where } 0 \text { is } \\
\text { damaging and } 1 \text { is neutral }\end{array}$ \\
\hline Align GVGD [56] & $\begin{array}{l}\text { Multiple sequence alignments to characterise the } \\
\text { biochemical properties (composition, polarity and } \\
\text { volume) of the observed amino acids at each position }\end{array}$ & $\begin{array}{l}\text { The prediction classes form a spectrum } \\
\text { (C0, C15, C25, C35, C45, C55, C65) with } \\
\text { C65 most likely to interfere with } \\
\text { function and C0 least likely. }\end{array}$ \\
\hline SuSPect [57] & $\begin{array}{l}\text { Sequence-, structure*-and systems biology-based } \\
\text { features to predict the phenotypic effects of missense } \\
\text { mutations. } \\
\text { Algorithm trained using VariBench (contains } \\
\text { information for experimentally verified effects) } \\
\text { *Structure-based comparison with other ABC transporters }\end{array}$ & $\begin{array}{c}\text { Table of scores from } 0-100, \text { colour- } \\
\text { coded according to predicted } \\
\text { deleteriousness (blue=neutral, } \\
\text { red=disease-causing). } \\
\text { A score of } 50 \text { is recommended as a cut- } \\
\text { off between neutral and disease-causing } \\
\text { variants }\end{array}$ \\
\hline & Databases: RefSeq, dbSNP, Uniprot, InterPro, UCSC & \\
\hline $\begin{array}{c}\text { Alamut@ [58] } \\
\text { Commercial software (Interactive } \\
\text { Biosoftware) }\end{array}$ & $\begin{array}{l}\text { Genome Browser Database, PubMed, Ensembl. } \\
\text { And } \\
\text { Automated access to on-line prediction tools: } \\
\text { PolyPhen2, SIFT, Align GVGD } \\
\text { Access to splicing prediction tools (detailed in Section } \\
\text { III.2.1.2.) } \\
\text { "Alamut Batch" } \\
\text { High-throughput annotation software for NGS } \\
\text { analysis }\end{array}$ & $\begin{array}{l}\text { Summarized report of the results of } \\
\text { different prediction tools, according to } \\
\text { their output format } \\
\text { Designed for intensive variant } \\
\text { analysis workflows }\end{array}$ \\
\hline
\end{tabular}

Table 5. Bioinformatics tools for the prediction of amino acid changes: websites, characteristics and output format [55-59]

A recent work has emphasised the importance of sequence alignments on the performance of prediction tools [60]. The authors constructed custom multiple sequence alignments called phenotype-optimized sequence ensembles (POSEs) that was tested on a training set of CFTR mutations. 
A previous work already suggested that providing SIFT or PolyPhen-2 with custom alignments increased their performance relative to the default alignments employed by the algorithms [61]. This could explain that, if Alamut@ is a highly interesting tool with its ease of use, some predictions obtained by using each tool separately with a custom algorithm could differ from AlamutC results (obtained with default alignments).

\subsubsection{Prediction tools for the assessment of the impact on pre-mRNA splicing}

Splicing mechanisms comprise exon recognition within large pre-mRNA molecules and the precise removal of flanking introns. Three elements constitute the core splicing signals: the intronic branch point, the acceptor site (or 3'splice site), including an inconstant upstream polypyrimidine tract (PPT), and the donor site (or 5' splice site). These core human splice site motifs contain only a part of the information that defines exons, whereas the rest corresponds to less conserved splicing regulatory elements. The latter are located within the exon or flanking introns, promoting or inhibiting exon recognition through exonic/intronic splicing enhancers (ESE or ISE) or silencers (ESS or ISS), respectively (Figure 3).

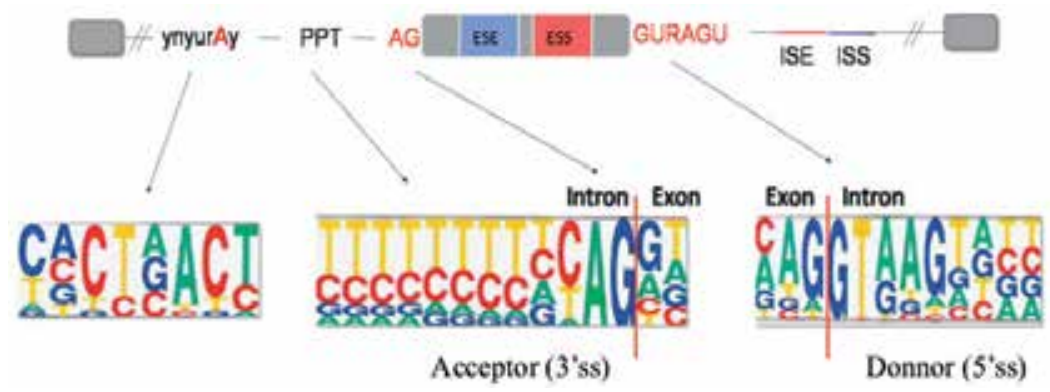

Figure 3. A schematic of key splicing motifs and regulatory elements. Adapted by Le Guédard-Méreuze S. from Wang and Burge, $2008[62,63]$.

Many bioinformatics tools have been developed to predict which splicing modification is the most probable for a given sequence variation - exon skipping, cryptic splice sites activation, use of de novo splice sites - or if the variant may be considered as neutral regarding its impact on splicing. Most algorithms were developed based on biostatistical and experimental analyses of information contained in the genomic sequence. They provide a score depending on the strength of the considered splice site. Indeed, the strength of splicing motifs is a key parameter to predict the impact of a sequence variation. Performance of these tools has been widely studied by comparing the results of predictions with experimental assays for various genes including CFTR [39, 63-66]. In 2012, Houdayer and collaborators performed a large-scale study of VUCS in BRCA genes in order to assess the performance of six prediction tools [67]. This work provided guidelines for the proper use of these tools and for the interpretation of prediction results.

Table 6 summarizes principle and main characteristics of the most 'popular' bioinformatics programs and ASSEDA, a recently developed program [68-73]. 


\begin{tabular}{|c|c|c|}
\hline Method and Web site & Algorithm & Output \\
\hline MaxEntScan [72] & $\begin{array}{l}\text { Based on a Maximum Entropy Model } \\
\text { (MEM): } \\
\text { Modelling the sequences of short sequence } \\
\text { motifs such as those involved in RNA } \\
\text { splicing which simultaneously accounts for } \\
\text { non-adjacent as well as adjacent } \\
\text { dependencies between positions. }\end{array}$ & \\
\hline \multirow{5}{*}{$\begin{array}{l}\text { Human Splicing Finder (HSF) [73] } \\
\text { Associates weight matrix model } \\
\text { (WMM) and } \\
\text { Maximum Entropy Model (MEM) }\end{array}$} & \multicolumn{2}{|c|}{ Consensus splice sites } \\
\hline & $\begin{array}{l}\text { HSF (WMM): Quantification of the relative } \\
\text { likelihood of candidate splice site sequence to } \\
\text { show coincidence with consensus sequence }\end{array}$ & $\begin{array}{c}\text { Numerical Score } \\
(0-100)\end{array}$ \\
\hline & $\begin{array}{l}\text { MaxEnt (MEM): Statistical approach } \\
\text { representing the least biased approximation fo } \\
\text { the distribution of sequence motifs, from } \\
\text { available data (real and decoy splice sites). } \\
\text { Incorporates local adjacent and non-adjacent } \\
\text { position dependencies }\end{array}$ & $\begin{array}{c}\text { Numerical Score } \\
(0-10)\end{array}$ \\
\hline & \multicolumn{2}{|c|}{ cis-regulatory elements } \\
\hline & $\begin{array}{c}\text { ESEfinder* } \\
\text { RESCUE-ESE* } \\
\text { Predicted PESE AND PESS Octamers from } \\
\text { Zhang \& Chasin } \\
\text { *See Prediction tools for cis-regulatory } \\
\text { elements }\end{array}$ & $\begin{array}{l}\text { See Prediction tools for cis-regulatory } \\
\text { elements }\end{array}$ \\
\hline NNSplice [74] & $\begin{array}{l}\text { Machine learning approach that recognizes } \\
\text { sequence patterns once it is trained with sets } \\
\text { of DNA sequences encompassing authentic } \\
\text { and decoy splice sites. } \\
\text { Based on a hidden Markov Model (HMM): } \\
\text { Incorporates local adjacent position } \\
\text { dependencies }\end{array}$ & Score between 0 and 1 \\
\hline $\begin{array}{l}\text { Automated Splice Site and Exon } \\
\text { Definition Analysis (ASSEDA) [75] } \\
\text { Restricted access: registration required }\end{array}$ & $\begin{array}{l}\text { Analyses splicing mutations according to } \\
\text { changes in total exon information: } \\
\text { information content of a spliced exon from } \\
\text { the cumulative contributions of sequences } \\
\text { recognized by the spliceosomal machinery } \\
\text { and the distribution distances separating } \\
\text { binding sites within the same exon. }\end{array}$ & $\begin{array}{l}\Delta R i_{\text {,total }} \text { values (differences in probability of } \\
\text { relative inclusion or exclusion of the wild } \\
\text { type and mutated exon in mature mRNA) }\end{array}$ \\
\hline \multicolumn{3}{|c|}{ Prediction tools for cis-regulatory elements } \\
\hline ESEfinder [76] & $\begin{array}{c}\text { Prediction of SR protein specific putative } \\
\text { ESE, based on an in vitro SELEX approach } \\
\text { dependent on addition of individual SR } \\
\text { proteins }\end{array}$ & ESE motif score \\
\hline $\begin{array}{c}\text { RESCUE-ESE [77] } \\
\text { (Relative enhancer and silencer } \\
\text { classification by unanimous } \\
\text { enrichment) }\end{array}$ & $\begin{array}{l}\text { Statistical approach based upon different } \\
\text { distribution of hexamers in exons and } \\
\text { introns with different properties (e.g. weak } \\
\text { and strong splice sites) }\end{array}$ & $\begin{array}{l}\text { Z-score } \\
\text { (Highlights extremely over- or under- } \\
\text { represented hexamers) }\end{array}$ \\
\hline
\end{tabular}

Table 6. Main characteristics of several Splicing prediction tools [74-79] 
It is important to note that consequences on splicing of exonic synonymous and non-synonymous CFTR variants must be assessed, as suggested by recent of experimental studies $[39,80]$.

\subsubsection{Deep intronic variants}

The examples of insertion of intronic sequences called pseudo-exons (or cryptic exons) in mature transcripts of various genes are becoming ever more numerous and their role in human diseases has been largely demonstrated. We saw in section 2.2 that NGS strategies currently allow scanning of CFTR deep intronic regions [18], resulting in a growing number of new identified deep intronic variants.

Bioinformatics tools described above, which assess the impact of variants on splicing, can also be used to evaluate deep intronic mutations. We tested these algorithms on mutations identified in CF patients after NGS sequencing of the entire CFTR locus and they showed satisfactory results [18]. Indeed, prediction tools allowed the selection of possible diseasecausing mutations (i.e. predicted impact on splicing by inclusion of pseudo-exons) and predictions were confirmed by in vitro functional studies using minigene constructs (see section 3.3.1.1) and by direct analysis of aberrant transcripts from nasal epithelial cells of patients (see 3.3.2.1).

\subsection{In vitro/ex vivo functional analyses}

\subsubsection{Cell lines transfection experiments}

The type of cells used for transfection depends on the tissue that is studied and the clinical context. Pulmonary (BEAS-2B, A549, Calu-3) or intestinal/colic (CACO-2, T84) immortalized cells (by SV40 or carcinoma) contain an appropriate concentration of transcriptional and splicing factors for CFTR protein synthesis. Cells stably transfected with mutated CFTR can also be used (CFBe41o-, CFPAC-1). Stable expression is usually obtained by lentivirus transduction and transient transfection by chemical agent (Polyfect, interferin). In this case, the endogenous CF molecular and cellular context (inflammation) should also be considered.

\subsubsection{Splicing assessment}

Minigenes are autonomic cyclic entity containing promoter and exons and are produced by clonal amplification in bacteria [81, 82]. They contain a genomic segment from the gene of interest (here CFTR) that includes exon and flanking intronic regions (length can range from ten to thousands of nucleotides, an average of $300 \mathrm{bp}$ ) or only intronic regions in the case of evaluation of potential creation of a pseudo-exon. To determine whether a mutation is responsible for altered splicing, minigenes can also include cis-regulatory elements if affected (ESE, ESS, ISE or/and ISS) [83]. These regions of interest are framed by two invariable exons, which are part of the system. Every assay of transfection in cell lines compares the wild-type and mutated (through directed mutagenesis) constructs [84]. All CFTR exons are needed to produce a mature and functional protein. Thus, a modification of transcript in the in vitro system suggests that the assessed CFTR change has a deleterious effect on exon splicing. An 
ever-increasing number of mini-gene studies have been performed to assess the pathogenicity of CFTR variants $[39,60,80,84]$. This strategy, despite its limitations, is of high interest in the overall strategy for the characterization of rare sequence variations.

\subsubsection{Expression vectors for the quantification of $m R N A$, protein or CFTR-specific chloride conductance}

Full-length CFTR cDNA is classically inserted in expression vector system (e.g. pcDNA3 or pTracer) upon a promoter that may be drug-activated (G418, tetracycline or doxycyclineactivation). To assess point variants or small indels, directed mutagenesis is carried out (usually QuikChange Mutagenesis kits®, Agilent Technologies). To assess molecular consequences of large rearrangements concerning one or more exons, a truncated CFTR cDNA can be inserted in the expression vector [85-87]. Transient or stable transfection can be performed in eukaryotic cells (describe in III.3.1. section). 3-HA tag (in the fourth loop of CFTR) can be introduced to easily visualize protein expression. Then, measurement of mRNA expression and evaluation of function and localization of the CFTR protein can be performed for each alternative transcript construct, compared to wild-type.

Automated real-time RT-PCR allows the relative straightforward quantification of mRNA transcripts with specific primers and appropriate reference genes for normalization. mRNA level informs about future protein quality and quantity.

Protein assessment consists in the implementation of complementary experiments for protein quantification, evaluation of its maturation or its cellular localization. Main techniques are detailed below. The effect of variants on CFTR expression and maturation is assessed based on the detection of immature (core-glycosylated, B band, 150-kD) and/or mature (additional glycosylation in the Golgi, C band $\sim 170-190 \mathrm{kDa}$ ) CFTR forms by immunoblotting. Long-term pulse-chase experiments can provide additional information on the lifetime of CFTR on cellular compartments [88]. Immunocytochemical assays (Immunofluorescence (IF) based) can highlight the cellular localization of the CFTR protein. However, most difficulties noted in IF experiments relate to non-specific antibody staining and the effect of sample processing on characteristics of cell development. Moreover, confusion between cell surface (where CFTR is active) and subsurface (where it would not) may occur. Therefore, more sensitive and specific antibodies as well as co-localization assays with other cell surface markers (such as $\beta$-tubulin or WGA) are needed. Finally, this remains a qualitative or semi-quantitative method.

CFTR function and activity, i.e. CFTR-specific chloride conductance, can be determined by patch-clamp electrophysiology, halide selective electrode technique, radioisotope efflux assays and by fluorescence-based halide efflux measurement. The use of a CFTR-activating appropriate drug (such as forskolin, isobutylmethylxanthine (IBMX), isoproterenol, terbutaline, genistein, adenosine, etc.) or ATP followed by specific CFTR inhibitor CFTRinh-172 permits CFTR-dependant or independent chloride transport, respectively. To date, the easiest approach developed consists in Iodide efflux based on fluorescence measurement. YFP fluorescence is dependent on YFP expression levels and iodide concentration. Compared with conventional plate-bound CFTR functional assays, the flow cytometric approach can be used to study CFTR function in cell suspension. It may be further adjusted to study CFTR function 
in heterologous cell populations using cell surface markers and selection of cells that display high CFTR function. Technical limitations include the need to perform this assay in specialized centres (using expensive imaging equipment).

All these methods offer the possibility to evaluate the functional consequences of molecular abnormalities on CFTR and finally improve the classification of variants.

\subsubsection{Ex vivo CF patients' cells assays}

\subsubsection{Analyses on primary airway cells and tissues}

Characterization of CFTR molecular defect can partially be performed directly after biopsies of tissues that show sufficient CFTR expression. Nasal or lung airway epitheliums are optimal. These tissues are accessible by minimally invasive interventions and display an endogenous expression of CFTR transcripts and protein. Moreover, nasal and bronchial epitheliums show the same cellular composition (ciliated, goblet, columnar and immune cells) although ratios differ slightly [89]. Since quantification and detection of aberrant splicing and quantification or localization of proteins are possible in human tissues, information that they bring is crucial to assess the effect of variants and to propose a functional classification. However, this approach has its limitations and requires other functional assays to perform large-scale genotype-phenotype correlation studies. In addition, especially for nasal tissue, the low quantity of cells collected (out of 500.000 cells per brushing) only allows 'one-shot' tests and hinders mechanistic assays. Moreover, highly variable CFTR expression in heterogeneous cell types, in healthy individuals and in p.Phe508del homozygous patients has been described, varying from 0 to $100 \%$ [90]. Other genetic (see below) or environmental parameters could also modify CFTR expression levels.

\subsubsection{Ex vivo culture of primary airway cells}

Culture of primary cells from CF patients can be performed with brushed nasal or bronchial cells after biopsies. Wild-type endogenous CFTR protein is expressed at the apical membrane of polarized cells. Therefore, in vitro monolayer culture seems no longer adapted. Obtaining polarized cells is promoted by air-liquid interface culture (ALI), proposed since the 2000s, by an ex vivo system of collagen-coated porous membrane on which cells are platted after a phase of monolayer amplification or directly after nasal brushing. Basal adherent cells differentiate in all airways epithelial cell types, which organize into a pseudostratified epithelium [91].

This model offers the opportunity to perform functional assays described above to determine CFTR dysfunction. Molecular defect induced by a specific mutation can be qualitatively determined if the cell donor is homozygous for this mutation.

Technical limitations such as bacterial or fungi contaminations or absence of adherence complicate culture of cells obtained from CF patients. Moreover, further studies are needed to determine if extrapolation is possible between observations in primary cells directly after 
brushing and after several weeks in culture media, particularly for quantitative level assessment.

\subsubsection{Intestinal current measurement (ICM)}

ICM was developed as a research tool to assess CFTR function in the 1990s and has been used as a diagnostic test since the early 2000s $[92,93]$. At least four superficial rectal biopsies per patient, obtained by suction, are needed and mounted on adequate tissue sliders. ICM consists in blocking epithelial sodium channels by amiloride and stimulates cAMP-mediated CFTRdepending chloride transport in a chloride-free solution with forskolin or IBMX. Cholinergic chloride transport and histaminic reaction were also evaluated and the sum of the response $\triangle \mathrm{Isc}_{\text {forskolin/IBMX+carbachol-histamine }}$ appears to be the best parameter to evaluate CFTR function by ICM, but reference values and ranges have not been established. This combination of ionic responses discriminates patients with CF from healthy subjects but not CF patients with pancreatic insufficiency (PI) or sufficiency (PS). ICM can detect a loss of CFTR function above $80 \%$, therefore CF carriers and CFTR-RD patients may not be identified by this method. Moreover, mild mutations could result in a false-negative ICM.

ICM is not altered by secondary damage on tissue and thus better reveals the primary CFTR dysfunction (compared with nasal potential difference, see below). A new functional CFTR assay using primary CF intestinal organoïds derived from patients and cultured in vitro may offer new tools to screen for therapy [94].

Finally, setup and maintenance of dedicated equipment by experienced and trained staff limits its use.

\subsubsection{In vivo biomarkers assays of CFTR function}

Sweat chloride Test (ST) and Nasal potential difference (NPD) measurement are used as diagnostic tests for CF. In atypical clinical context these in vivo tests can give additional arguments to further explore the CFTR locus (cf. Figure 2 that describes the molecular diagnostics step-by-step strategy). Furthermore, they provide complementary information for the interpretation of CFTR variations.

ST higher than $60 \mathrm{mmol} / \mathrm{L}$ is the 'gold standard' and discriminates between healthy and typical CF. Sweat electrolytes are higher in the most severely affected and are lower in those with mild mutation, who have partial rescue of channel function [95-97]. There are two major advantages for the use of sweat test in the evaluation of the CFTR mutation severity: (i) stability of the measure throughout life and (ii) non-invasive way of measurement which maintains skin integrity. Indeed, sweat electrolytes levels reflect the primary defect on the CFTR protein and do not highlight secondary consequences of its absence or dysfunction on affected organs. Furthermore, a new means for assessing the secretory function of CFTR has been recently developed based on $\beta$-adrenergic-mediated sweating. This method provides a unique evaluation of the purely secretory function of CFTR in vivo. It has been shown to be more sensitive in individuals carrying mutations that commonly exhibit normal or borderline sweat chloride rates, such as c.3718-2477C $>\mathrm{T}$ (legacy name: $3849+10 \mathrm{kbC}>\mathrm{T}$ ) [98]. 
The transepithelial NPD measurement estimates the net ion conductance across the nasal airway epithelium and indirectly measure CFTR activity. Sodium conductance is determined after amiloride perfusion (Na+). NPD parameters discriminate CF and CFTR-RD phenotypes and a correlation between NPD and disease severity has been shown [99], although there is an overlap in subjects with mild phenotype [97]. However, other sources of phenotypic variability (modifiers genes or environment) and technical limitations (i.e. maintenance of dedicated equipment and trained personnel) contribute to the inconsistencies of NPD across genotype-phenotype correlation studies. CFTR expression and NPD response can also be modified if nasal epithelium is affected by rhinosinusitis, polyposis or exposure to smoking [100]. However, despite the difficulty of setting up this technique, NPD assessment could be used as a complementary step to support CFTR dysfunction in inconclusive clinical cases and then to confirm the deleterious effect of CFTR variants identified in these patients.

\section{Conclusion}

New issues are emerging from the use of NGS technology in CF molecular diagnosis. On one hand, NGS approaches offer new possibilities by multiplexing samples and provide a wider coverage of the CFTR locus including deep intronic regions. NGS assay design can also include additional modifiers genes [31]. On the other hand, molecular diagnoses in emergency contexts challenge the possibility of sample multiplexing, and the increased number of VUCS will require complex functional analyses. However, as tools described above are constantly improving, the knowledge about CFTR variations is rapidly expanding, allowing geneticists and clinicians to provide patients with high quality information and adequate genetic counselling. Finally, the functional characterization of CFTR variations will provide rationale for a personalised medicine strategy driven by patients' genotype in the very near future.

\section{Acknowledgements}

We acknowledge all members of the technical team for CF molecular diagnosis: Jean-Pierre Altieri, Fanny Verneau and Lise Larrieu. We also thank the coordination team of the CFTRFrance genetic database: Corinne Bareil and Corinne Thèze and all the collaborating members of this project. We are also grateful to the French Network of laboratories specialized in molecular diagnosis of CF (GenMucoFrance). Finally, we thank the French Association against CF (Vaincre la Mucoviscidose) for its constant support.

\section{Author details}

Anne Bergougnoux ${ }^{1 *}$, Magali Taulan-Cadars ${ }^{2}$, Mireille Claustres $^{2}$ and Caroline Raynal ${ }^{1}$

*Address all correspondence to: anne.bergougnoux@inserm.fr 
1 Laboratory of Molecular Genetics, Montpellier Hospital, France

2 Montpellier University, France

\section{References}

[1] Riordan JR, Rommens JM, Kerem B, Alon N, Rozmahel R, Grzelczak Z, Zielenski J, Lok S, Plavsic N, Chou JL, et al. Identification of the cystic fibrosis gene: cloning and characterization of complementary DNA. Science. 1989 Sep 8;245(4922):1066-73. Erratum in: Science 1989 Sep 29;245(4925):1437.

[2] Casals T, Ramos MD, Giménez J, Larriba S, Nunes V, Estivill X. High heterogeneity for cystic fibrosis in Spanish families: 75 mutations account for $90 \%$ of chromosomes. Hum Genet. 1997 Dec;101(3):365-70.

[3] Tsui LC. The spectrum of cystic fibrosis mutations. Trends Genet. 1992 Nov;8(11): 392-8.

[4] Welsh MJ, Smith AE. Molecular mechanisms of CFTR chloride channel dysfunction in cystic fibrosis. Cell. 1993 Jul 2;73(7):1251-4. Review.

[5] Quintana-Gallego E, Delgado-Pecellín I, Calero Acuña C. CFTR protein repair therapy in cystic fibrosis. Arch Bronconeumol. 2014 Apr;50(4):146-50. doi: 10.1016/j.arbres. 2013.07.013. [Article in English, Spanish].

[6] Castellani C, Cuppens H, Macek M Jr, Cassiman JJ, Kerem E, Durie P, Tullis E, Assael BM, Bombieri C, Brown A, Casals T, Claustres M, Cutting GR, Dequeker E, Dodge J, Doull I, Farrell P, Ferec C, Girodon E, Johannesson M, Kerem B, Knowles M, Munck A, Pignatti PF, Radojkovic D, Rizzotti P, Schwarz M, Stuhrmann M, Tzetis M, Zielenski J, Elborn JS. Consensus on the use and interpretation of cystic fibrosis mutation analysis in clinical practice. J Cyst Fibros. 2008 May;7(3):179-96. doi: 10.1016/ j.jcf.2008.03.009. Review.

[7] Dequeker E, Stuhrmann M, Morris MA, Casals T, Castellani C, Claustres M, Cuppens H, des Georges M, Ferec C, Macek M, Pignatti PF, Scheffer H, Schwartz M, Witt M, Schwarz M, Girodon E. Best practice guidelines for molecular genetic diagnosis of cystic fibrosis and CFTR-related disorders--updated European recommendations. Eur J Hum Genet. 2009 Jan;17(1):51-65. doi: 10.1038/ejhg.2008.136.

[8] World Health Organisation Report "The molecular genetic epidemiology of cystic fibrosis". Available on: http://www.who.int/genomics/publications/en/ HGN_WB_04.02_report.pdf [Accessed: 2015-02-07]

[9] Abeliovich D, Lavon IP, Lerer I, Cohen T, Springer C, Avital A, Cutting GR. Screening for five mutations detects $97 \%$ of cystic fibrosis $(\mathrm{CF})$ chromosomes and predicts a 
carrier frequency of 1:29 in the Jewish Ashkenazi population. Am J Hum Genet. 1992 Nov;51(5):951-6.

[10] Chillón M, Dörk T, Casals T, Giménez J, Fonknechten N, Will K, Ramos D, Nunes V, Estivill X. A novel donor splice site in intron 11 of the CFTR gene, created by mutation $1811+1.6 \mathrm{kbA}-->\mathrm{G}$, produces a new exon: high frequency in Spanish cystic fibrosis chromosomes and association with severe phenotype. Am J Hum Genet. 1995 Mar; 56(3):623-9.

[11] Costantino L, Claut L, Paracchini V, Coviello DA, Colombo C, Porcaro L, Capasso P, Zanardelli M, Pizzamiglio G, Degiorgio D, Seia M. A novel donor splice site characterized by CFTR mRNA analysis induces a new pseudo-exon in CF patients. J Cyst Fibros. 2010 Dec;9(6):411-8. doi: 10.1016/j.jcf.2010.08.009.

[12] Faà V, Incani F, Meloni A, Corda D, Masala M, Baffico AM, Seia M, Cao A, Rosatelli MC. Characterization of a disease-associated mutation affecting a putative splicing regulatory element in intron $6 \mathrm{~b}$ of the cystic fibrosis transmembrane conductance regulator (CFTR) gene. J Biol Chem. 2009 Oct 30;284(44):30024-31. doi: 10.1074/ jbc.M109.032623. Epub 2009 Sep 15.

[13] Bareil C, Guittard C, Altieri JP, Templin C, Claustres M, des Georges M. Comprehensive and rapid genotyping of mutations and haplotypes in congenital bilateral absence of the vas deferens and other cystic fibrosis transmembrane conductance regulator-related disorders. J Mol Diagn. 2007 Nov;9(5):582-8.

[14] Costantino L, Rusconi D, Claut L, Colombo C, Novara F, Paracchini V, Porcaro L, Capasso $\mathrm{P}$, Zuffardi $\mathrm{O}$, Seia M. A wide methodological approach to identify a large duplication in CFTR gene in a CF patient uncharacterised by sequencing analysis. J Cyst Fibros. 2011 Dec;10(6):412-7. doi: 10.1016/j.jcf.2011.06.007.

[15] Metzker ML. Sequencing technologies - the next generation Nat Rev Genet. 2010 Jan; 11:31-46.

[16] Bodi K, Perera AG, Adams PS, Bintzler D, Dewar K, Grove DS, Kieleczawa J, Lyons RH, Neubert TA, Noll AC, Singh S, Steen R, Zianni M. Comparison of commercially available target enrichment methods for next-generation sequencing. J Biomol Tech. 2013 Jul;24(2):73-86. doi: 10.7171/jbt.13-2402-002.

[17] Chilamakuri CS, Lorenz S, Madoui MA, Vodák D, Sun J, Hovig E, Myklebost O, Meza-Zepeda LA. Performance comparison of four exome capture systems for deep sequencing. BMC Genomics. 2014 Jun 9;15:449. doi: 10.1186/1471-2164-15-449.

[18] Bonini J, Varilh J, Raynal C, Thèze C, Beyne E, Audrezet MP, Ferec C, Bienvenu T, Girodon E, Tuffery-Giraud S, Des Georges M, Claustres M, Taulan-Cadars M. Smallscale high-throughput sequencing-based identification of new therapeutic tools in cystic fibrosis. Genet Med. 2015 Jan 8. doi: 10.1038/gim.2014.194. 
[19] Tewhey R, et al. Microdroplet-based PCR enrichment for large-scale targeted sequencing. Nature Biotech. 2009;27:1025-31.

[20] Guo Y, Long J, He J, Li CI, Cai Q, Shu XO, Zheng W, Li C. Exome sequencing generates high quality data in non-target regions. BMC Genomics. 2012 May 20;13:194. doi: 10.1186/1471-2164-13-194.

[21] Li H, Handsaker B, Wysoker A, et al. The sequence alignment/map format and SAMtools. Bioinformatics. 2009;25:2078-9.

[22] McKenna A, Hanna M, Banks E, Sivachenko A, Cibulskis K, Kernytsky A, Garimella K, Altshuler D, Gabriel S, Daly M, DePristo MA. The genome analysis toolkit: a MapReduce framework for analyzing next-generation DNA sequencing data. Genome Res. 2010;20:1297-1303.

[23] Treangen TJ, Salzberg SL. Repetitive DNA and next-generation sequencing: computational challenges and solutions. Nat Rev Genet. 2011 Nov 29;13(1):36-46. doi: $10.1038 /$ nrg3117.

[24] 1000 Genomes Project Database. Available on: http://www.1000genomes.org/ [Accessed: 2015-02-07]

[25] NCBI Short Genetic Variations Database. Available on: http:// www.ncbi.nlm.nih.gov.gate2.inist.fr/projects/SNP/ [Accessed: 2015-02-07]

[26] Abou Tayoun AN, Tunkey CD, Pugh TJ, Ross T, Shah M, Lee CC, Harkins TT, Wells WA, Tafe LJ, Amos CI, Tsongalis GJ. A comprehensive assay for CFTR mutational analysis using next-generation sequencing. Clin Chem. 2013 Oct;59(10):1481-8. doi: 10.1373/clinchem.2013.206466.

[27] Trujillano D, Ramos MD, González J, Tornador C, Sotillo F, Escaramis G, Ossowski S, Armengol L, Casals T, Estivill X. Next generation diagnostics of cystic fibrosis and CFTR-related disorders by targeted multiplex high-coverage resequencing of CFTR. J Med Genet. 2013 Jul;50(7):455-62. doi: 10.1136/jmedgenet-2013-101602. Epub 2013 May 17.

[28] Guo X, Pace RG, Stonebraker JR, Commander CW, Dang AT, Drumm ML, Harris A, Zou F, Swallow DM, Wright FA, O'Neal WK, Knowles MR. Mucin variable number tandem repeat polymorphisms and severity of cystic fibrosis lung disease: significant association with MUC5AC. PLoS One. 2011;6(10):e25452. doi: 10.1371/journal.pone. 0025452. Epub 2011 Oct 6.

[29] Guillot L, Beucher J, Tabary O, Le Rouzic P, Clement A, Corvol H. Lung disease modifier genes in cystic fibrosis. Int J Biochem Cell Biol. 2014 Jul;52:83-93. doi: 10.1016/j.biocel.2014.02.011.

[30] Gisler FM, von Kanel T, Kraemer R, Schaller A, Gallati S. Identification of SNPs in the cystic fibrosis interactome influencing pulmonary progression in cystic fibrosis. Eur J Hum Genet. 2013 Apr;21(4):397-403. doi: 10.1038/ejhg.2012.181. 
[31] Cutting GR. Modifier genes in Mendelian disorders: the example of cystic fibrosis. Ann N Y Acad Sci. 2010 Dec;1214:57-69. doi: 10.1111/j.1749-6632.2010.05879.x. Review.

[32] Claustres M, Guittard C, Bozon D, Chevalier F, Verlingue C, Ferec C, Girodon E, Cazeneuve C, Bienvenu T, Lalau G, Dumur V, Feldmann D, Bieth E, Blayau M, Clavel C, Creveaux I, Malinge MC, Monnier N, Malzac P, Mittre H, Chomel JC, Bonnefont JP, Iron A, Chery M, Georges MD. Spectrum of CFTR mutations in cystic fibrosis and in congenital absence of the vas deferens in France. Hum Mutat. 2000;16(2):143-56.

[33] Sharma H, Mavuduru RS, Singh SK, Prasad R. Heterogeneous spectrum of mutations in CFTR gene from Indian patients with congenital absence of the vas deferens and their association with cystic fibrosis genetic modifiers. Mol Hum Reprod. 2014 Sep; 20(9):827-35. doi: 10.1093/molehr/gau047.

[34] Bergougnoux A, Viart V, Miro J, Bommart S, Molinari N, des Georges M, Claustres M, Chiron R, Taulan-Cadars M. Should diffuse bronchiectasis still be considered a CFTR-related disorder? J Cyst Fibros. 2015 pii: S1569-1993(15)00054-5.

[35] Ramos MD, Trujillano D, Olivar R, Sotillo F, Ossowski S, Manzanares J, Costa J, Gartner S, Oliva C, Quintana E, Gonzalez MI, Vazquez C, Estivill X, Casals T. Extensive sequence analysis of CFTR, SCNN1A, SCNN1B, SCNN1G and SERPINA1 suggests an oligogenic basis for cystic fibrosis-like phenotypes. Clin Genet. 2014 Jul;86(1):91-5. doi: $10.1111 /$ cge.12234.32

[36] Bakouh N, Bienvenu T, Thomas A, Ehrenfeld J, Liote H, Roussel D, Duquesnoy P, Farman N, Viel M, Cherif-Zahar B, Amselem S, Taam RA, Edelman A, Planelles G, Sermet-Gaudelus I. Characterization of SLC26A9 in patients with CF-like lung disease. Hum Mutat. 2013 Oct;34(10):1404-14. doi: 10.1002/humu.22382.

[37] Li W, Soave D, Miller MR, Keenan K, Lin F, Gong J, Chiang T, Stephenson AL, Durie $\mathrm{P}$, Rommens J, Sun L, Strug LJ. Unraveling the complex genetic model for cystic fibrosis: pleiotropic effects of modifier genes on early cystic fibrosis-related morbidities. Hum Genet. 2014 Feb;133(2):151-61. doi: 10.1007/s00439-013-1363-7.

[38] Practice guidelines for the Interpretation and Reporting of Unclassified Variants (UVs) in Clinical Molecular Genetics, 2007. Available on: http:// cmgsweb.shared.hosting.zen.co.uk/ [Accessed: 2015-02-07]

[39] Raynal C, Baux D, Theze C, Bareil C, Taulan M, Roux AF, Claustres M, Tuffery-Giraud S, des Georges M. A classification model relative to splicing for variants of unknown clinical significance: application to the CFTR gene. Hum Mutat. 2013;34:774-874.

[40] 1000 Genomes Project Consortium, Abecasis GR, Altshuler D, Auton A, Brooks LD, Durbin RM, Gibbs RA, Hurles ME, McVean GA. A map of human genome variation from population-scale sequencing. Nature. 2010 Oct 28;467(7319):1061-73. doi: 10.1038/nature09534. Erratum in: Nature 2011 May 26;473(7348):544. 
[41] NHLBI GO Exome Sequencing Project. Available on: http://evs.gs.washington.edu/ EVS/ [Accessed: 2015-02-07]

[42] Claustres M, Horaitis O, Vanevski M, Cotton RG. Time for a unified system of mutation description and reporting: a review of locus-specific mutation databases. Genome Res. 2002;12:680-8.

[43] Cotton RGH, Auerbach AD, Beckmann JS, Blumenfeld OO, Brookes AJ, Brown AE, et al. Recommendations for locus-specific databases and their curation. Hum Mutat. 2008;29(1):2-5.

[44] Cystic Fibrosis Mutation Database (CFMDB). Available on: www.genet.sickkids.on.ca/cftr/ [Accessed: 2015-02-07]

[45] Clinical and Functional Translation of CFTR (CFTR2), US CF Foundation, Johns Hopkins University, The Hospital for Sick Children, John Hopkins, USA. Available on: http://www.cftr2.org/ [Accessed: 2015-02-07]

[46] Castellani C, CFTR2 Team. CFTR2: how will it help care? Paediatr Respir Rev. 2013 May;14(Suppl 1):2-5. doi: 10.1016/j.prrv.2013.01.006.

[47] Sosnay PR, Siklosi KR, Van Goor F, Kaniecki K, Yu H, Sharma N, Ramalho AS, Amaral MD, Dorfman R, Zielenski J, Masica DL, Karchin R, et al. Defining the disease liability of variants in the cystic fibrosis transmembrane conductance regulator gene. Nat Genet. 2013;45:1160-7.

[48] CFTR-France. Available on: https://cftr.iurc.montp.inserm.fr/CFTR/ (Restricted access) [Accessed: 2015-02-07]

[49] He L1, Aleksandrov AA, Serohijos AW, Hegedus T, Aleksandrov LA, Cui L, Dokholyan NV, Riordan JR. Multiple membrane-cytoplasmic domain contacts in the cystic fibrosis transmembrane conductance regulator (CFTR) mediate regulation of channel gating. J Biol Chem. 2008 Sep 26;283(39):26383-90. doi: 10.1074/ jbc.M803894200.

[50] Mornon JP, Lehn P, Callebaut I. Atomic model of human cystic fibrosis transmembrane conductance regulator: membrane-spanning domains and coupling interfaces. Cell Mol Life Sci. 2008 Aug;65(16):2594-612. doi: 10.1007/s00018-008-8249-1.

[51] Adzhubei IA, Schmidt S, Peshkin L, Ramensky VE, Gerasimova A, Bork P, Kondrashov AS, Sunyaev SR. A method and server for predicting damaging missense mutations. Nat Methods. 2010 Apr;7(4):248-9. doi: 10.1038/nmeth0410-248.

[52] Ng PC, Henikoff S. SIFT: predicting amino acid changes that affect protein function. Nucleic Acids Res. 2003 Jul 1;31(13):3812-4.

[53] Tavtigian SV, Deffenbaugh AM, Yin L, Judkins T, Scholl T, Samollow PB, de Silva D, Zharkikh A, Thomas A. Comprehensive statistical study of 452 BRCA1 missense sub- 
stitutions with classification of eight recurrent substitutions as neutral. J Med Genet. 2006;43(4):295-305.

[54] Yates CM, Filippis I, Kelley LA, Sternberg MJ. SuSPect: enhanced prediction of single amino acid variant (SAV) phenotype using network features. J Mol Biol. 2014 Jul 15;426(14):2692-701. doi: 10.1016/j.jmb.2014.04.026.

[55] PolyPhen 2. Available on: http://genetics.bwh.harvard.edu/pph2/ [Accessed: 2015-02-08]

[56] SIFT. Available on: http://sift.jcvi.org/www/SIFT_BLink_submit.html [Accessed: 2015-02-08]

[57] Align GVGD. Available on: http://agvgd.iarc.fr/agvgd_input.php [Accessed: 2015-02-08]

[58] SuSPect. Available on: www.sbg.bio.ic.ac.uk/suspect [Accessed: 2015-02-08]

[59] Alamut@. http://www.interactive-biosoftware.com/ (Commercial software, not available online)

[60] Masica DL, Sosnay PR, Cutting GR, Karchin R. Phenotype-optimized sequence ensembles substantially improve prediction of disease-causing mutation in cystic fibrosis. Hum Mutat. 2012 Aug;33(8):1267-74. doi: 10.1002/humu.22110.

[61] Hicks S, Wheeler DA, Plon SE, Kimmel M. Prediction of missense mutation functionality depends on both the algorithm and sequence alignment employed. Hum Mutat. 2011;32:661-8.

[62] Le Guédard-Méreuze S. Mutations in splicing cis-regulatory elements: role in human genetic diseases [thesis in French]. Université Montpellier 1, 2009.

[63] Spurdle AB, Couch FJ, Hogervorst FB, Radice P, Sinilnikova OM, IARC Unclassified Genetic Variants Working Group. Prediction and assessment of splicing alterations: implications for clinical testing. Hum Mutat. 2008 Nov;29(11):1304-13. doi: 10.1002/ humu.20901.

[64] Vreeswijk MP, Kraan JN, van der Klift HM, Vink GR, Cornelisse CJ, Wijnen JT, Bakker E, van Asperen CJ, Devilee P. Intronic variants in BRCA1 and BRCA2 that affect RNA splicing can be reliably selected by splice-site prediction programs. Hum $\mathrm{Mu}-$ tat. 2009 Jan;30(1):107-14. doi: 10.1002/humu.20811.

[65] Sharma N, Sosnay PR, Ramalho AS, Douville C, Franca A, Gottschalk LB, Park J, Lee M, Vecchio-Pagan B, Raraigh KS, Amaral MD, Karchin R, Cutting GR. Experimental assessment of splicing variants using expression minigenes and comparison with in silico predictions. Hum Mutat. 2014 Oct;35(10):1249-59. doi: 10.1002/humu.22624.

[66] Houdayer C, Caux-Moncoutier V, Krieger S, Barrois M, Bonnet F, Bourdon V, Bronner M, Buisson M, Coulet F, Gaildrat P, Lefol C, Léone M, Mazoyer S, Muller D, Remenieras A, Révillion F, Rouleau E, Sokolowska J, Vert JP, Lidereau R, Soubrier F, 
Sobol H, Sevenet N, Bressac-de Paillerets B, Hardouin A, Tosi M, Sinilnikova OM, Stoppa-Lyonnet D. Guidelines for splicing analysis in molecular diagnosis derived from a set of 327 combined in silico/in vitro studies on BRCA1 and BRCA2 variants. Hum Mutat. 2012 Aug;33(8):1228-38.doi: 10.1002/humu.22101.

[67] Yeo G, Burge CB. Maximum entropy modeling of short sequence motifs with applications to RNA splicing signals. J Comput Biol. 2004;11(2-3):377-94.

[68] Desmet FO, Hamroun D, Lalande M, Collod-Béroud G, Claustres M, Béroud C. Human Splicing Finder: an online bioinformatics tool to predict splicing signals. Nucleic Acids Res. 2009 May;37(9):e67. doi: 10.1093/nar/gkp215.

[69] Shapiro MB, Senapathy P. RNA splice junctions of different classes of eukaryotes: sequence statistics and functional implications in gene expression. Nucleic Acids Res. 1987 Sep 11;15(17):7155-74.

[70] Reese MG, Eeckman FH, Kulp D, Haussler D. Improved splice site detection in Genie. J Comput Biol. 1997 Fall;4(3):311-23.

[71] Mucaki EJ, Shirley BC, Rogan PK. Prediction of mutant mRNA splice isoforms by information theory-based exon definition. Hum Mutat. 2013 Apr;34(4):557-65. doi: 10.1002/humu.22277.

[72] Cartegni L, Wang J, Zhu Z, Zhang MQ, Krainer AR. ESEfinder: a web resource to identify exonic splicing enhancers. Nucleic Acids Res. 2003;31:3568-71.

[73] Fairbrother WG, Yeh RF, Sharp PA, Burge CB. Predictive identification of exonic splicing enhancers in human genes. Science. 2002;297:1007-13.

[74] MaxEntScan. Available on: http://genes.mit.edu/burgelab/maxent/Xmaxentscan_scoreseq.html [Accessed: 2015-02-08]

[75] Human Splicing Finder, version 2.4.1. Available on: http://www.umd.be/HSF/ [Accessed: 2015-02-08]

[76] NNSplice. Available on: http://www.fruitfly.org/seq_tools/splice.html [Accessed: 2015-02-08]

[77] Automated Splice Site and Exon Definition Analyses (ASSEDA). Available on: http:// splice.uwo.ca/ (Restricted access: registration required) [Accessed: 2015-02-08]

[78] ESEfinder. Available on: http://rulai.cshl.edu/tools/ESE/ [Accessed: 2015-02-08]

[79] Relative enhancer and silencer classification by unanimous enrichment (RESCUE ESE). Available on: http://genes.mit.edu/burgelab/rescue-ese/ [Accessed: 2015-02-08]

[80] Aissat A, de Becdelievre A, Golmard L, Vasseur C, Costa C, Chaoui A, Martin N, Costes B, Goossens M, Girodon E, Fanen P, Hinzpeter, A. Combined computationalexperimental analyses of CFTR exon strength uncover predictability of exon- skipping level. Hum Mutat. 2013;34:873-81. 
[81] Burn TC, Connors TD, Klinger KW, Landes GM. Increased exon-trapping efficiency through modifications to the pSPL3 splicing vector. Gene. 1995;161(2):183-7.

[82] Cooper TA. Use of minigene systems to dissect alternative splicing elements. Methods. 2005 Dec;37(4):331-40.

[83] Singh G, Cooper TA. Minigene reporter for identification and analysis of cis elements and trans factors affecting pre-mRNA splicing. Biotechniques. 2006 Aug;41(2):177-81.

[84] Goina E, Fernandez-Alanis E, Pagani F. Approaches to study CFTR pre-mRNA splicing defects. Methods Mol Biol. 2011;741:155-69. doi: 10.1007/978-1-61779-117-8_11.

[85] Scott A, Petrykowska HM, Hefferon T, Gotea V, Elnitski L. Functional analysis of synonymous substitutions predicted to affect splicing of the CFTR gene. J Cyst Fibros. 2012 Dec;11(6):511-7. doi: 10.1016/j.jcf.2012.04.009.

[86] Taulan M, Guittard C, Theze C, Claustres M, Georges MD, A novel double 312 deletion underscores the importance of characterizing end points of the CFTR large 313 rearrangements. Eur J Hum Genet. 2009;17(12):1683-87.

[87] Taulan M, Viart V, Theze C, Guittard C, Altieri JP, Templin C, Mely L, Claustres M, des Georges M. Identification of a novel duplication CFTRdup2 and functional impact of large rearrangements identified in the CFTR gene. Gene. 2012 Jun 1;500(2): 194-8. doi: 10.1016/j.gene.2012.03.043.

[88] Aleksandrov AA, Kota P, Aleksandrov LA, He L, Jensen T, Cui L, Gentzsch M, Dokholyan NV, Riordan JR. Regulatory insertion removal restores maturation, stability and function of DeltaF508 CFTR. J Mol Biol. 2010 Aug 13;401(2):194-210. doi: 10.1016/ j.jmb.2010.06.019.

[89] Bergougnoux A, Claustres M, de Sario A. Nasal epithelial cells, a tool to study DNA methylation in airway diseases. Epigenomics. 2015 Feb;7(1):119-26. doi: 10.2217/epi. 14.65 .

[90] van Meegen MA, Terheggen-Lagro SW, Koymans KJ, van der Ent CK, Beekman JM. Apical CFTR expression in human nasal epithelium correlates with lung disease in cystic fibrosis. PLoS One. 2013;8(3):e57617. doi: 10.1371/journal.pone.0057617.

[91] Hajj R, Baranek T, Le Naour R, Lesimple P, Puchelle E, Coraux C. Basal cells of the human adult airway surface epithelium retain transit-amplifying cell properties. Stem Cells. 2007 Jan;25(1):139-48.

[92] Hirtz S, Gonska T, Seydewitz HH, Thomas J, Greiner P, Kuehr J, Brandis M, Eichler I, Rocha H, Lopes AI, Barreto C, Ramalho A, Amaral MD, Kunzelmann K, Mall M. CFTR Cl- channel function in native human colon correlates with the genotype and phenotype in cystic fibrosis. Gastroenterology. 2004 Oct;127(4):1085-95. 
[93] De Jonge HR, Ballmann M, Veeze H, Bronsveld I, Stanke F, Tümmler B, Sinaasappel M. Ex vivo CF diagnosis by intestinal current measurements (ICM) in small aperture, circulating Ussing chambers. J Cyst Fibros. 2004 Aug;3 Suppl 2:159-63.

[94] Dekkers JF, Wiegerinck CL, de Jonge HR, Bronsveld I, Janssens HM, de Winter-de Groot KM, Brandsma AM, de Jong NW, Bijvelds MJ, Scholte BJ, Nieuwenhuis EE, van den Brink S, Clevers H, van der Ent CK, Middendorp S, Beekman JM. A functional CFTR assay using primary cystic fibrosis intestinal organoids. Nat Med. 2013 Jul;19(7):939-45. doi: 10.1038/nm.3201.

[95] Farrell PM, Koscik RE. Sweat chloride concentrations in infants homozygous or heterozygous for F508 cystic fibrosis. Pediatrics. 1996 Apr;97(4):524-8.

[96] Davis PB, Schluchter MD, Konstan MW. Relation of sweat chloride concentration to severity of lung disease in cystic fibrosis. Pediatr Pulmonol. 2004 Sep;38(3):204-9.

[97] Wilschanski M, Dupuis A, Ellis L, Jarvi K, Zielenski J, Tullis E, Martin S, Corey M, Tsui LC, Durie P. Mutations in the cystic fibrosis transmembrane regulator gene and in vivo transepithelial potentials. Am J Respir Crit Care Med. 2006 Oct 1;174(7): 787-94. Epub 2006 Jul 13.

[98] Quinton P, Molyneux L, Ip W, Dupuis A, Avolio J, Tullis E, Conrad D, Shamsuddin AK, Durie P, Gonska T. $\beta$-adrenergic sweat secretion as a diagnostic test for cystic fibrosis. Am J Respir Crit Care Med. 2012 Oct 15;186(8):732-9. doi: 10.1164/rccm. 201205-0922OC.

[99] Ho LP, Samways JM, Porteous DJ, Dorin JR, Carothers A, Greening AP, Innes JA. Correlation between nasal potential difference measurements, genotype and clinical condition in patients with cystic fibrosis. Eur Respir J. 1997 Sep;10(9):2018-22.

[100] Bagheri-Hanson A, Nedwed S, Rueckes-Nilges C, Naehrlich L. Intestinal current measurement versus nasal potential difference measurements for diagnosis of cystic fibrosis: a case-control study. BMC Pulm Med. 2014 Oct 4;14:156. doi: 10.1186/1471-2466-14-156. 



\title{
Emerging Roles of microRNAs in Cystic Fibrosis - From Pathogenesis to Development of New Therapies
}

\author{
Sabrina Noel and Teresinha Leal
}

Additional information is available at the end of the chapter

\begin{abstract}
As essential components of the regulatory system of gene expression, microRNAs (miRNAs) have been shown to influence development, severity, prognosis, and/or progression of a variety of inherited diseases. Differential expression studies have evidenced an impact of miRNAs on lung disease development in chronic obstructive pulmonary disease (COPD), asthma, lung inflammation, consequences of smoke exposure and airway allergy in human and in animal models of the diseases. Recent clinical and cell-based studies have revealed specific alterations of miRNA expression in cystic fibrosis (CF). Here we critically review the major findings concerning altered miRNA expression in $\mathrm{CF}$ airway epithelium, in particular with respect to $\mathrm{CF}$ transmembrane conductance regulator (CFTR) expression, innate immunity, and epithelial differentiation. Finally, we explore strategies to exploit these changes with the aim of innovative therapeutic benefits.
\end{abstract}

Keywords: microRNA, cystic fibrosis, CFTR, lung pathophysiology, inflammation, epithelial differentiation, biomarkers

\section{Introduction}

Cystic Fibrosis (CF) is due to mutations in the CF transmembrane conductance regulator (CFTR) gene causing impairment of chloride ions exchanges through the apical membrane of epithelial cells. CF affects epithelia in a variety of organs, notably lung, intestine, pancreas and the reproductive system. The most common CFTR mutation, F508del, results in deletion of a 
phenylalanine at position 508 of the protein, and the mutated protein is retained in the endoplasmic reticulum and rapidly degraded via the endoplasmic reticulum-associated degradation pathway. Up to now, almost 2000 CFTR mutations have been identified (http:// genet.sickkids.on.ca). A good correlation can generally be observed between CFTR genotype and the gastrointestinal disease, the pancreatic status, and the reproductive tract abnormalities, but the lung disease outcome is difficult to predict based solely on CFTR genotype (http:// www.cftr2.org/). For example, it has been shown that siblings and monozygous twins, thus carrying the same CFTR genotype, even if living in the same environment and receiving the same medical care, may develop different spatial and temporal patterns in lung disease progression. The basis for variability in severity of CF lung disease is poorly understood and depends on concomitant expression of other genetic and environmental factors. Over the recent years, exploring the role of genetics/genomics (e.g. modifier genes, gene-environment interactions, epigenetics, etc.) has received growing attention in $\mathrm{CF}$, with the aim of unveiling basic mechanisms and bringing in better understanding of the pathophysiology of the disease, helping to predict its progression, and hopefully leading to specially designed novel therapeutic strategies.

Of the whole human genome, only a small fraction, the protein-coding part, has long attracted attention because of the pervasive role of genes in determining amino acid sequences of expressed proteins, leading to observable consequences of mutations. Later on, following the discovery of transduction factors regulating gene translation, the initial view of "junk DNA", corresponding to the majority of DNA, had to be revisited. Following the identification of nonmessenger RNA functions, the new concept of a network of non-coding transcriptome that regulates protein-coding expression emerged.

Non-coding RNAs are presently broadly categorized into three classes. The major class (well over $90 \%$ of total RNA) makes up the so-called housekeeping RNAs; they consist of small nuclear, small nucleolar, transfer, and ribosomal RNAs, the latter interacting with protein and transfer RNA to form the functional ribosome complex. The other classes of non-coding RNAs are long (>200) and short $(<200)$ ribonucleotides. The best characterized and most extensively studied family of non-coding RNAs is that of microRNAs (miRNAs), short (17--27 nucleotides in length) single-stranded RNA molecules, which negatively regulate the translation of messenger RNAs into proteins. Figure 1 summarizes the general mechanism of biogenesis of miRNAs. Highly phylogenetically conserved, they bind to the 3'UTR (untranslated region) of target mRNAs, thereby potently repressing the target mRNA translation into protein or favoring mRNA degradation. To date, more than 1800 mature miRNAs have been identified in the human genome (http://www.mirbase.org). Bioinformatics studies predict that miRNAs potentially regulate the expression of about $60 \%$ of human genes.

As essential components of the regulatory system of gene expression, miRNAs have been shown to influence the development, the severity, the prognosis and/or the progression of a number of inherited diseases [1]. Differential expression studies of miRNAs have evidenced an impact on lung disease development in chronic obstructive pulmonary disease (COPD), asthma, lung inflammation, consequences of smoke exposure and airway allergy in human and in animal models of the diseases [2]. Since marked inflammation is a major feature of $\mathrm{CF}$ 


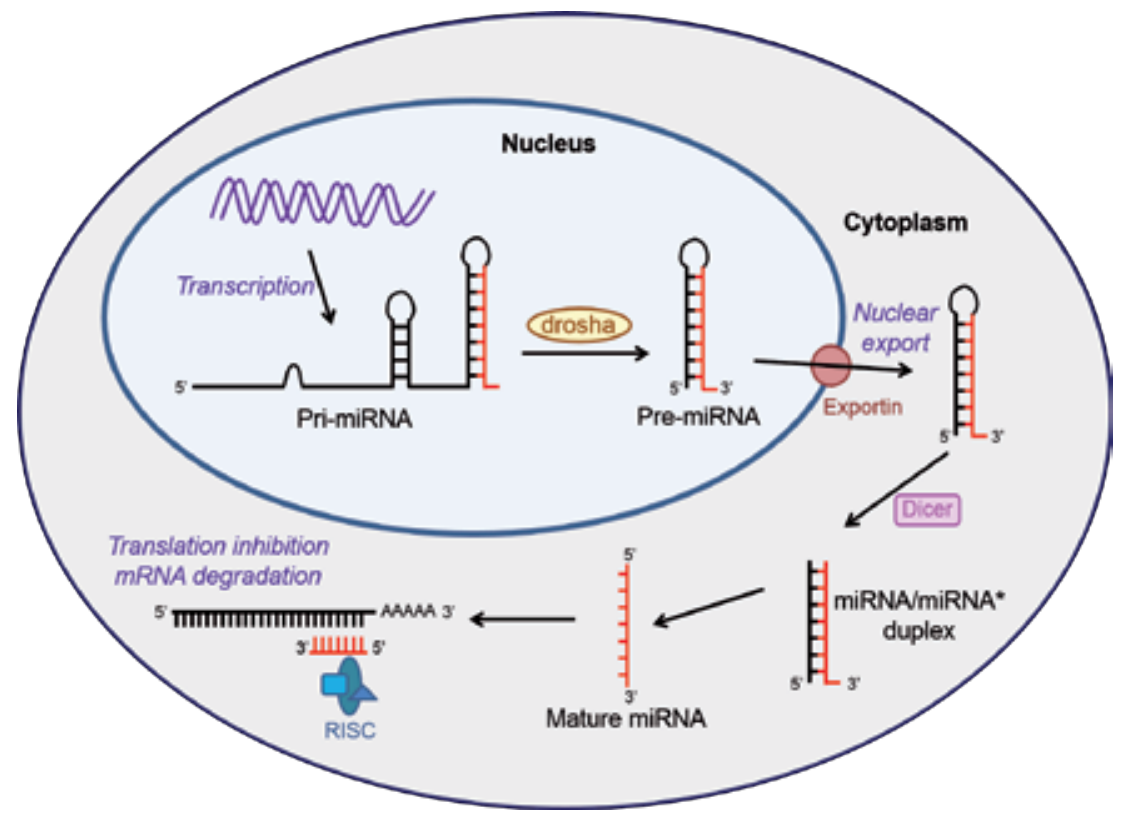

Figure 1. Biogenesis of microRNAs (miRNAs). miRNA genes are transcribed by RNA-polymerase 2 into primary miRNA (pri-miRNA) precursors. The pri-miRNAs are cleaved in the nucleus by a nuclear protein complex including the class 2 ribonuclease III Drosha to produce pre-miRNAs. Pre-miRNAs are exported to the cytoplasm via the nuclear export protein Exportin. In the cytoplasm, a pre-miRNA is cleaved by a protein complex including the helicase Dicer and form a duplex of miRNAs containing the mature miRNA bound to its complementary sequence (miRNA*). Duplexes are unwound and mature miRNAs bind to the 3'UTR of the target mRNA within the RNA-induced silencing complex to prevent translation by inhibition of ribosomes binding or mRNA degradation.

lung disease, miRNAs may be expected to play a role in its pathogenesis. Indeed, recent clinical and cell-based studies have revealed CF-specific alterations in miRNA expression. This point will be extensively discussed further below.

In this article, we review and highlight some of the most relevant published data focusing on miRNAs in CF. The first section deals with regulation of CFTR expression and modulation of CFTR trafficking. In the second section, key elements of inflammatory and innate immune responses in with $\mathrm{CF}$ airways are reviewed, focusing on the potential role of miRNAs in molecular pathways involved in lung inflammation. The third section discusses the potential role of miRNAs in lung development, differentiation and remodeling. The next section explores strategies to exploit miRNAs as biomarkers and potential therapies of CF disease. Finally, limitations in translating miRNAs from deeper knowledge of their role in pathogenesis to development of new therapies are highlighted.

\section{Regulation of CFTR expression}

The human CFTR gene is located on chromosome 7 and spans $189 \mathrm{~kb}$. Its mRNA transcript is $6.2 \mathrm{~kb}$ long and includes a $1.5 \mathrm{~kb} 3^{\prime} \mathrm{UTR}$ containing multiple potential binding sites for miRNAs. 
According to computational analyses using common bioinformatic programs such as TargetScan (http://www.targetscan.org), PicTar (http://pictar.mdc-berlin.de), and miRanda (http://www.microrna.org), CFTR 3'UTR contains almost 500 putative miRNA target sites, several of which have already been examined.

The main miRNAs involved in cystic fibrosis pathogenesis are summarized in figure 2.

Gillen et al. were the first to experimentally show that miRNAs could regulate CFTR expression [3]. Among the numerous miRNAs putatively repressing CFTR mRNA, twelve miRNAs (miR-145, miR-331-3p, miR-376a/b, miR-377, miR-384, miR-494, miR-600, miR-607, miR-939, miR-1246, miR-1290 and miR-1827) were able to decrease CFTR mRNA levels in the human Bronchial epithelial (HBE) 16HBE-14o-, colon carcinoma epithelial Caco-2 and the pancreatic adenocarcinoma PANC-1 cell lines. Direct targeting of the CFTR 3'UTR was evidenced for miR-145 and miR-494 in Caco-2 cells. The results [3] showed that the pattern of miRNAs effects on targeted CFTR 3'UTR varies with cell lines, suggesting that miRNA-driven gene expression could be tissue specific. Another study [4] demonstrated direct repression of CFTR expression by miR-101 and miR-494 in the HEK (human embryonic kidney) cell line expressing CFTR 3'UTR constructs. miR-101 and miR-494 seem to bind directly to the CFTR 3'UTR at positions 1508-1514 and 1140-1147, respectively. Downregulation of CFTR gene expression by miR-101 as well as by miR-144 and miR-145 was further confirmed in the 16HBE14o- cell line and in primary human airway cells, which are relevant models of CF lung disease [5, 6].

In a non-CF context, expression of miR-101 and miR-144 was previously shown to be upregulated in lungs upon exposure to air pollutants (such as cigarette smoke or cadmium) and correlated with loss of CFTR expression [4]. Hassan et al. [5] demonstrated upregulation of miR-101 in vivo in mice exposed to cigarette smoke, a condition associated with acquired loss of CFTR function [7]. miR-101 was also found highly expressed in lungs of COPD smoking patients in comparison with that of healthy non-smoking subjects. Moreover, miRNA expression profile analysis performed in the CF bronchial brushings in comparison to healthy controls showed high levels of miR-101, thus further reinforcing the potential role of miR-101 in CF [8]. Interestingly, synergistic effects between miR-101 and miR-494 were observed, although they are targeting different sites in CFTR 3'UTR [4]. Similar synergy was found for miR-509-3p and miR-494 [9]. Therefore, distinct miRNAs may act cooperatively to regulate CFTR expression and function in primary airway epithelial cells.

These miRNAs are likely able to modulate F508del-CFTR mRNA expression as well. Interestingly, increased expression of miR-145, miR-223 and miR-494 in vivo has been shown to correlate with decreased CFTR expression in bronchial epithelium of individuals bearing the F508del-CFTR mutation [3] as well as in CFBE41o- cells [10]. Experimental modulation of miRNA expression confirmed the hypothesis supporting the view that deregulation of miRNA may affect CFTR biogenesis in CF cells. Another study demonstrated that miRNA can indirectly influence CFTR biogenesis by modulating expression of other regulatory elements such as transcription factors [11]. It has been shown that modulating the transcription regulation factor SIN3A expression with miR-138 mimics increased biogenesis and cell surface expression of both wild-type and F508del-CFTR proteins. Interestingly, miR-138 indirectly 
prevented proteosomal degradation of F508del-CFTR mutant and favored its trafficking towards the apical membrane in HBEs from CF patients [11].

Altogether, these data are in favor of a role for several miRNAs in the post-transcriptional regulation of the CFTR channel synthesis and trafficking. Because several of them are deregulated in $\mathrm{CF}$, they could play a major role in CF lung pathology.

\section{Regulation of key elements of inflammation and innate immune system}

miRNAs are crucial regulators of innate and adaptive immune responses and their abnormal expression or function or both have been linked to multiple human inflammatory disorders [12]. The link between CFTR mutations and the mechanisms underlying disproportionate proinflammatory responses remains poorly understood. Aberrant release of proinflammatory mediators by epithelial and immune cells in CF includes hypersecretion of interleukin-8 (IL-8). The fact that this can be detected in lungs of children with CF [13] even during fetal life [14, 15] suggests that it is a constitutive process of CF. miRNA-based post-transcriptional regulations, known to be highly sensitive to a range of homeostasis signals, such as changes in hypoxia, $\mathrm{pH}$, ion concentration and osmolarity, have recently been considered as potentially regulating immune responses in CF, and more specifically IL-8 hyperproduction.

The first published contribution of miRNA involvement in CF [8] reported that miR-126 was found to be abundantly expressed in normal lungs and that it was reduced in lungs of patients with CF. This process cannot account for the pathophysiological mechanism of changes observed in patients with CF, based on the observation of high concentrations of IL-8 depending on constitutive activation of nuclear factor kappa B (NF- $\mathrm{B}$ ) [15-17]. Indeed, Oglesby et al. [8] found that in patients with $\mathrm{CF}$, miR-126 was strongly downregulated and expression of TOM1, which is itself a negative regulator of the NF- $\kappa B$ signaling pathway, was upregulated. On this basis, a decreased expression of IL- 8 would be expected, in contradiction to the wellestablished increased IL-8 production. These observations indicate that miR-126 alone does not play a role via the TOM1 cascade and that other factors may also be involved. Other miRNAs originated from host or part of this microbioma could also be involved. For example, Rao et al. [18] reported their finding of bacterial miR-146, which binds to a receptor of the TLR family, in sputum of CF patients infected with Pseudomonas aeruginosa.

As for other miRNAs, it has been suggested that expression of miR-155 might contribute to the activation of IL-8--dependent inflammation in patients with CF. Clinically, overexpression of miR-155 has been observed in lung epithelial cells and in neutrophils from patients with $\mathrm{CF}$ [18]. Interestingly, miR-155 expression was also elevated in primary CF bronchial epithelial cells and in IB-3 and CuFi-1 CF cell lines [16, 19]. Upregulating miR-155 expression in these cells increased production of IL-8 through activation of the PI3K/Akt signaling pathway [16]. Another study confirmed these results [17] by showing that high levels of miR-155 in sterile CF cells, reduced after exposure to the anti-inflammatory cytokine IL-10 or following inhibition 
of IL-1 $\beta$ signaling, was accompanied by a reduction of IL-8 production. These observations suggest a general role of miR-155 as an IL-8 expression regulator and consequently of the NF$\kappa B$ pathway $[16,17,19,20]$. The expression of miR-155 is also increased in patients with asthma, idiopathic pulmonary disease and acute lung injury [21]. It has been underlined that a tight control is required for the expression of a molecule such as miR-155 which is overexpressed in cancers of B-cell origin. It is noteworthy that a signaling molecule as small as miR-155 has such a dangerous potential when deregulated.

Although so far, few studies have focused on the role of miRNA as regulator of the immune response specifically in the CF context, it is of prime importance to consider other miRNAs, the expression of which is deregulated in inflammatory lungs, and which could be nonspecifically modulated in CF. CF cells display a new profile of miRNAs, including high expression of miR-215 which is a strong modulator of cell cycle through the p53-signaling pathway [17]. As an example of unspecific modulation, miR-509-3p and miR-494 (which directly target CFTR expression, as described above) are overexpressed in CF bronchial epithelial cells [9]. In addition, bacterial infection and tumor necrosis factor-alpha and IL-1 $\beta$ exposure increase miR-509-3p and miR-494 concentrations in part via the action of the NF-kB transcriptional activator complex. These findings, together with those showing that miR-494 is upregulated in CF cells $[9,10]$, support the idea of a role of miRNAs in inflammatory responses in $\mathrm{CF}$ respiratory epithelia, either directly by activation of transcription factors such as NF- $\kappa \mathrm{B}$, or indirectly by inhibition of CFTR expression.

Another crucial aspect of the immune response in lungs of patients with CF is the dysregulation of the protease-antiprotease balance which eventually leads to bronchiectasis [22]. Much attention has been given to serine proteases, in particular elastase secreted by neutrophils massively recruited during lung inflammation in patients with CF. However, other proteases secreted by epithelial cells themselves impact airway function. Recently, involvement of cysteine proteases cathepsin (CTS) B and S, overexpressed in lungs of patients with $\mathrm{CF}$, has been described [23]. CTSS is constitutively released at high levels by airways of patients with CF. Weldon et al. showed that the overproduction of CTSS in lungs of patients with CF was indirectly modulated by miR-31 in HBEs via repression of the transcription factor, interferon regulatory factor 1 (IRF1), which directly controls the CTSS gene [24]. It could be predicted that evidence will be brought up, showing that other proteases, such as metalloproteinases, often detected at high levels in CF patients sputum, are also regulated by miRNA-dependent mechanisms. Similarly, miRNAs could be involved in the expression of anti-proteases (e.g., $\alpha-1$ antitrypsin, secretory leucocyte protease inhibitor (SLPI), tissue inhibitors of metalloproteinase 1 (TIM-1)), opening new perspectives in development of novel therapies for CF and other lung diseases.

It is quite likely that a number of miRNAs could find several target points in the network of cellular and molecular players of the inflammation, imbalanced in CF. In turn, inflammatory responses themselves drive the expression of several miRNA species that either worsen the 
imbalance by increasing production of proinflammatory mediators or directly repress CFTR protein expression, forming a vicious circle.

\section{Regulation of lung cell development and differentiation}

Studies of miRNAs expression patterns have revealed that 27 miRNAs are differentially expressed during lung embryogenesis [25] following a characteristic pattern. miR-29a is highly expressed in late stages of lung development and in adult life [26]. In contrast, the miR-17-92 locus is highly expressed in undifferentiated lung epithelial cells and in lung cancer cells [27], and its expression progressively declines as differentiation progresses. Interestingly, miR-127 and miR-351 are transiently expressed during late phases of lung embryogenesis, first in the mesenchymal network, and then in epithelial cells. These observations suggest that miRNAs play distinct roles in the differentiation processes during the mesenchymal-to-epithelial transition.

The chronic inflammation in CF leads to irreversible airway tissue remodeling characterized by loss of multiciliated cells, gain in mesenchymal cells, goblet cells hyperplasia, and squamous metaplasia. Two miRNAs involved in multiciliogenesis have particularly attracted attention. miR-449a accumulates in bronchial epithelial cells during their transition to full differentiation [28]. Involvement of this particular miRNA in multiciliogenesis has been previously described [29]. Expression of miR-449a remains high in differentiated cells, indicating that it also plays a role in maintaining the multiciliated phenotype [29]. Conversely, expression of miR-455-3p, which negatively regulates the mucin 1 gene, is lost during the differentiation process of HBEs, reinforcing the control of epithelial (de)differentiation by miRNAs. Consequently, high expression of miR-449a and low expression of miR-455-3p can serve as biomarkers for the differentiation of bronchial epithelia. Further studies would be welcome to determine whether these miRNAs are deregulated in CF cells.

Airway epithelium dedifferentiation is part of tissue remodeling through induction of epithelial-mesenchymal transition (EMT). EMT is a process during which epithelial cells lose their phenotype, including loss of cell polarity and dissolution of cell-cell junctions, and acquire mesenchymal characteristics. Transforming growth factor (TGF) $\beta$ is a key mediator of EMT and epithelial remodeling. TGF- $\beta$ signaling was shown to be increased in CF lungs [30] and several miRNAs are regulated via the TGF- $\beta$ pathway [31]. Among them, miR-155 expression is reduced in human fibroblast cells upon exposure to TGF- $\beta$ [32]. Although it may contribute to reduce inflammation (through its effect of IL-8 secretion in HBEs described earlier), reduction of miR-155 expression at the airway level might contribute to loss of epithelial polarity. Moreover, another study utilizing epithelial cells indicated that miR-155 plays an important role in TGF- $\beta$-induced EMT as it facilitates tight junction dissolution [33]. Because CFTR expression is important to maintain epithelial differentiation and polarity [34], in particular in CF cells [35], it could be expected that at least some of the other miRNAs controlling CFTR expression might be involved in airway remodeling as well. Likewise, other 
miRNAs might non-specifically regulate epithelial differentiation in CF cells and could be involved in loss of epithelial polarity.

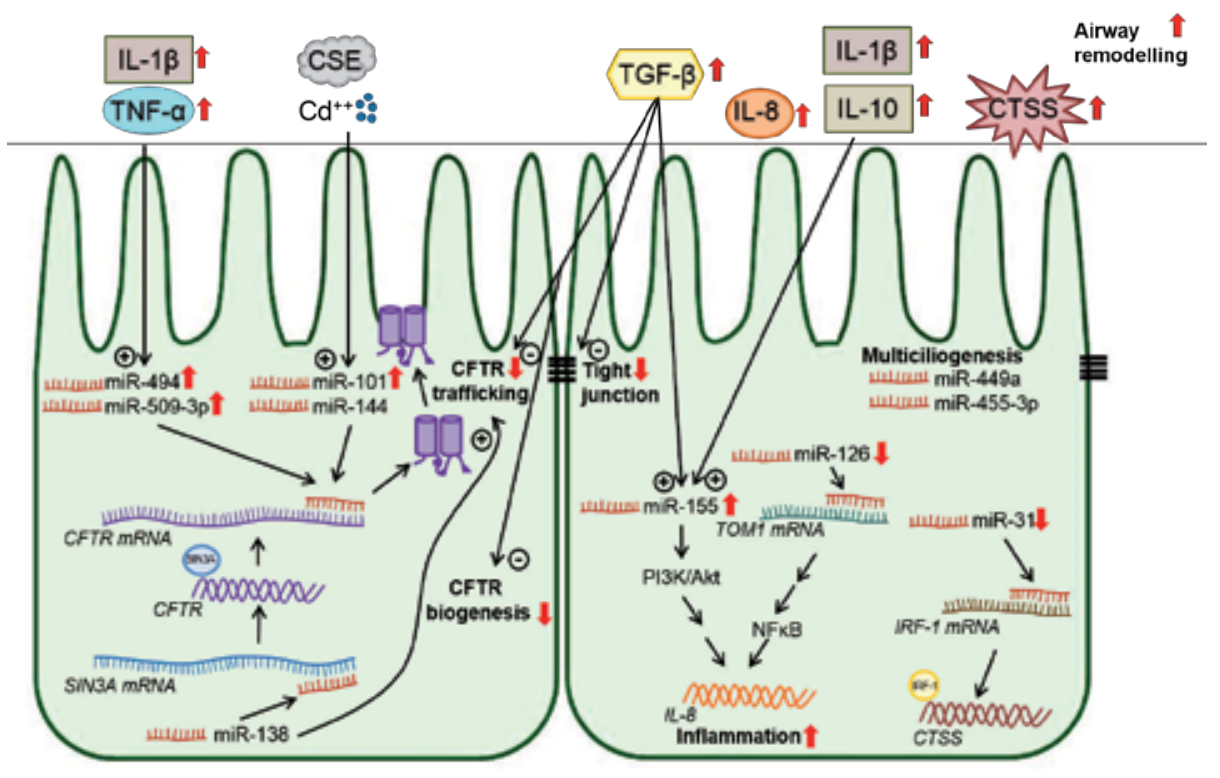

Figure 2. Main miRNAs involved in cystic fibrosis pathogenesis The left part depicts miRNA-dependent regulation of cystic fibrosis transmembrane conductance regulator (CFTR) biogenesis and trafficking. The right part zooms into the regulation of interleukin (IL)-8 production by miRNAs. miRNAs which may drive phenotypic changes on multiciliogenesis, airway remodeling and disorganization of tight junction are also represented. Red arrows depict changes reported in CF; up arrows indicate upregulated miRNAs and phenotype; and down arrows indicate miRNA and phenotype downregulation. CSE: cigarette smoke exposure; CTS: cathepsin.

MicroRNAs potentially altered and phenotypic consequences in CF are summarized in Table 1.

\begin{tabular}{|c|c|c|c|c|}
\hline Target & MicroRNA & Expression & Consequences & References \\
\hline CFTR & $\begin{array}{l}\operatorname{miR}-101,-144 \\
-494\end{array}$ & $\begin{array}{l}\text { Overexpressed in CF nasal and } \\
\text { bronchial biopsies }\end{array}$ & $\begin{array}{l}\text { Downregulation of CFTR } \\
\text { expression }\end{array}$ & $4,5,7,8,10$ \\
\hline CFTR & $\begin{array}{l}\operatorname{miR}-145,-223 \\
-494,509-3 p\end{array}$ & $\begin{array}{l}\text { Overexpressed in CF bronchial } \\
\text { brushings and CFBE41o- cell line }\end{array}$ & $\begin{array}{l}\text { Downregulation of CFTR } \\
\text { expression }\end{array}$ & $3,5,9,10$ \\
\hline SIN3A & miR-138 & & $\begin{array}{l}\text { Corrector of CFTR trafficking } \\
\text { Inhibition of proteosomal } \\
\text { degradation of F508del-CFTR, } \mathrm{Cl}^{-} \\
\text {permeability }\end{array}$ & 11 \\
\hline TOM1 & miR-126 & $\begin{array}{l}\text { Decreased expression in CF } \\
\text { bronchial brushing and CFBE41o- } \\
\text { cell line }\end{array}$ & $\begin{array}{l}\text { Unexplained downregulation of } \\
\text { NF- } \kappa B\end{array}$ & 8 \\
\hline
\end{tabular}




\begin{tabular}{|c|c|c|c|c|}
\hline Target & MicroRNA & Expression & Consequences & References \\
\hline $\begin{array}{l}\text { SHIP1 (Pi3K/ } \\
\text { Akt) }\end{array}$ & miR-155, -125 & $\begin{array}{l}\text { Overexpressed in CF neutrophils, in } \\
\text { primary CF bronchial epithelial cells } \\
\text { and in CF cell lines (IB-3 and CuFi-1) }\end{array}$ & $\begin{array}{l}\text { IL-8 hyperproduction } \\
\text { NF- } \kappa \text { B activation } \\
\text { Proapoptotic p53-signalling } \\
\text { pathway } \\
\text { EMT via TGF- } \beta \text { signalling }\end{array}$ & $\begin{array}{l}16,17,19,20 \\
32,33\end{array}$ \\
\hline \multirow[t]{5}{*}{ IRF-1 } & miR-31 & $\begin{array}{l}\text { Decreased expression in } \mathrm{CF} \\
\text { bronchial brushing }\end{array}$ & Cathepsin $S$ hyperproduction & 24 \\
\hline & $\operatorname{miR}-29 a$ & $\begin{array}{l}\text { Highly expressed in late stages of } \\
\text { lung development and in adult life }\end{array}$ & Lung embryogenesis & 26 \\
\hline & miR-127, -351 & $\begin{array}{l}\text { Transiently expressed during late } \\
\text { phases of lung embryogenesis }\end{array}$ & Modulation of lung development & 25 \\
\hline & miR-17-92 & $\begin{array}{l}\text { Highly expressed in undifferentiated } \\
\text { lung epithelial cells }\end{array}$ & dEMT & 27 \\
\hline & $\operatorname{miR}-449 a$ & $\begin{array}{l}\text { Accumulated in bronchial epithelial } \\
\text { cells }\end{array}$ & Multiciliogenesis, EMT & 28,29 \\
\hline MUC1 & $\operatorname{miR}-455-3 p$ & $\begin{array}{l}\text { Expressed in human bronchial } \\
\text { epithelial cells }\end{array}$ & $\begin{array}{l}\text { EMT } \\
\text { negative regulation of mucin } 1\end{array}$ & 29 \\
\hline
\end{tabular}

Table 1. microRNAs potentially altered and phenotypic consequences in cystic fibrosis (CF)

\section{5. miRNAs as biomarkers and potential therapies}

As in other diseases in which miRNAs have been involved, such as asthma, COPD, idiopathic pulmonary fibrosis, cancer and diabetes, further analyses of miRNA expression-function relationships will very likely reveal new genetic factors that could be targeted in therapy. There is also a great hope that miRNAs could be used as diagnostic markers and represent new prognostic factors that might influence the course of the CF disease.

The pathophysiology of CF is very variable from patient to patient and is only partly explained by the CFTR genotype. Two recent studies raised the hypothesis that profiling serum miRNome could identify miRNAs as potential prognostic biomarkers [16, 36]. First, elevated miR-155 serum levels have been detected in patients with $C F$, possibly reflecting its high expression in CF airway cells [16]. Second, a prototype study by Cook et al. has suggested that serum miRNAs could be used as diagnostic markers in CF liver disease [37]. Profiles of circulating miRNA levels in patients with $\mathrm{CF}$ liver disease were compared to those of $\mathrm{CF}$ patients without liver disease and of non-CF controls. For the first time, changes in circulating miRNA levels were identified in CF and they were correlated with disease status, suggesting that serum miRNA analysis may help predict early onset of hepatic fibrosis in CF [36]. It could 
be expected that new biomarkers of the course of the CF lung disease will be identified in the coming years.

It can also be foreseen that single nucleotide polymorphisms (SNPs) in the 3'UTR of miRNAtargeted genes, in particular CFTR, may explain phenotype variability. As an illustration, an SNP $\left(c^{*}{ }^{*} 1043 \mathrm{~A}>C\right.$ ) was identified in the $3^{\prime}$ UTR of CFTR in a patient with a CFTR-related disease [38]. CFTR-related diseases are clinical entities associated with CFTR dysfunction but that do not fulfil diagnostic criteria for classical CF (e.g., Congenital Bilateral Absence of Vas Deferens (CBAVD), chronic pancreatitis and disseminated bronchiectasis). This SNP was located within the binding site of two miRNAs including miR-509-3p (shown otherwise to directly target CFTR mRNA), and experimental data suggested that it might impair the regulation of gene expression. That could explain the mild phenotype, as this SNP would act as a mild mutation. Consequently, polymorphisms in the CFTR 3'UTR may play a role in the observed heterogeneous phenotype. Molecular analysis of the $3^{\prime}$ UTR of CFTR could therefore be performed as a differential diagnostic tool in patients presenting with suggestive clinical symptoms of $\mathrm{CF}$ but no mutation in the CFTR gene per se.

Modulating miRNA expression in vivo looks very appealing for developing new $\mathrm{CF}$ therapies [38]. Indeed miRNAs are short and need to be delivered only to the cytoplasm, as opposed to nucleus delivery required for DNA-based constructs. Moreover, miRNA-based therapies have several advantages over gene therapy strategies aiming at restoring CFTR expression. First, miRNA modulators are likely to target multiple genes in the context of a deregulated network. However, this might also be a major drawback as potential off-target effects may cause adverse phenotypes. Second, as in all cases of gene therapies, tissue-specific delivery remains a major issue in miRNA-based therapies. An interesting approach has been used by McKiernan et al. to successfully deliver miRNA replacement therapy in CFBE41o- cells [39]. They demonstrated that polyethyleneimine nanoparticles complexed with pre-miR-126 resulted in significant knock-down of TOM1, previously described as a direct target of miR-126 [8]. This result shows that polymeric nanoparticles may be used to effectively deliver miRNA replacement therapy with no adverse effects and may present a strong advantage in comparison to virus-based delivery strategies. As for any other disease for which miRNA-based therapy is considered as an attractive new option, factors controlling the stability of the miRNAs, the delivery systems and the off-target effects of miRNA-based therapies represent strong challenges for the future of development of such drugs.

\section{Limitations in translating miRNAs from deeper knowledge of their role in pathogenesis to development of new therapies}

Altogether, it is crucial to highlight important experimental considerations regarding miRNA investigations in CF. Expression profiles of miRNAs in human CF bronchial tissue often differ from study to study. The differences can be explained in part by the selection of tissue material. Indeed, the presence of inflammatory cytokines or non-resident, migratory cells (neutrophils, macrophages, etc.) or both infiltrating airway epithelium generate a non-negligible degree of heterogeneity in biopsy samples. Similar issues can be faced when analyzing miRNA expres- 
sion in human primary HBEs cultures. miRNA expression is strongly dependent on cell origin and differentiation state and on culture conditions. Accordingly, the air-liquid interface condition, which favors differentiation towards the epithelial phenotype, might influence miRNA expression. Moreover, although cultured, undifferentiated HBEs are quite homogenous, when switching to air-liquid interface condition, not only ciliated cells but also basal and goblet cells are found. In summary, any heterogeneity in cell population is likely to bring bias in miRNA expression profiles.

The large discrepancy among miRNA studies on CF disease may also indicate tissue- or organspecific expression patterns of miRNAs [40]. Consequently, miRNA gene targeting may be variable in different tissues and organs as well. This point is particularly relevant in $\mathrm{CF}$, a multisystemic disease. Similarly, studies aimed at profiling miRNAs in established CF mouse models may show differences that could be species specific. Substantial differences in technical approaches and statistical analyses are also important factors of variability and lack of reproducibility among studies.

Moving towards translation from bench to bedside, practical limitations including degradation and inactivation by nucleases, efficacy of intracellular delivery, short plasma elimination rates, renal and dose-limiting hemodynamic toxicities may hamper development of miRNA-based therapies. As miRNAs have pleiotropic intracellular effects on multiple signal transduction pathways, either single or combined therapies targeting CFTR expression, modulation of inflammation and cell differentiation might eventually be considered. This development could prove lengthy and full of traps and pitfalls before reaching a stage of translation to the clinical setting.

\section{Conclusion}

The study of miRNA in CF is still at an embryonic stage. To date, several studies have determined profiles of miRNAs in samples from target tissues such as lung and nasal biopsies, primary bronchial epithelial cell cultures or epithelial tissues from relevant mouse models. They have generated some indirect data suggesting a significant role of miRNA in controlling lung development, CFTR expression, as well as inflammation signaling pathways in CF.

The preliminary findings reviewed here form a solid basis for growing interest in miRNAs in $\mathrm{CF}$. The possibility that they may act as phenotype modifiers and that they could be used as diagnostic and prognostic biomarkers looks very attractive. With no doubt, further research on miRNA biogenesis and function in CF will expand widely in the coming years, shedding new light on disease variability and paving the way to innovative therapies.

\section{Acknowledgements}

SN is a research fellow with the Fonds Spéciaux de Recherche (FSR; Université catholique de Louvain, UCL) and Marie Curie actions of the European Commission. The authors are indebted to Professor Jean Lebacq for his critical review and editing. 


\section{Author details}

Sabrina Noel and Teresinha Leal ${ }^{*}$

*Address all correspondence to: teresinha.leal@uclouvain.be

Louvain Centre for Toxicology and Applied Pharmacology, Université Catholique de Louvain, Brussels, Belgium

The authors have no financial or personal conflict of interest.

\section{References}

[1] Mo YY. MicroRNA regulatory networks and human disease. Cell Mol Life Sci. 2012; 69(21): 3529-3531.

[2] Booton R, Lindsay MA. Emerging role of microRNAs and long noncoding RNAs in respiratory disease. Chest. 2014; 146(1): 193-204.

[3] Gillen AE, Gosalia N, Leir SH, Harris A. microRNA regulation of expression of the cystic fibrosis transmembrane conductance regulator gene. Biochem J. 2011; 438(1): 25-32.

[4] Megiorni F, Cialfi S, Dominici C, Quattrucci S, Pizzuti A. Synergistic post-transcriptional regulation of the cystic fibrosis transmembrane conductance regulator (CFTR) by miR-101 and miR-494 specific binding. PLoS ONE. 2011; 6(10): e26601.

[5] Hassan F, Nuovo GJ, Crawford M, Boyaka PN, Kirkby S, Nana-Sinkam SP, CormetBoyaka E. miR-101 and miR-144 regulate the expression of the CFTR chloride channel in the lung. PLoS ONE. 2012; 7(11): e50837.

[6] Viart V, Bergougnoux A, Bonini J, Varilh J, Chiron R, Tabary O, Molinari N, Claustres M, Taulan-Cadars M. Transcription factors and miRNAs that regulate fetal to adult CFTR expression change are new targets for cystic fibrosis. Eur Respir J. 2015; 45(1): 116-128.

[7] Cantin AM, Hanrahan JW, Bilodeau G, Ellis L, Dupuis A, Liao J, Zielenski J, Durie P. Cystic fibrosis transmembrane conductance regulator function is suppressed in cigarette smokers. Am J Respir Crit Care Med. 2006; 173(10): 1139-1144.

[8] Oglesby IK, Bray IM, Chotirmall SH, Stallings RL, O'Neill SJ, McElvaney NG, Greene CM. miR-126 is downregulated in cystic fibrosis airway epithelial cells and regulates TOM1 expression. J Immunol. 2010; 184(4): 1702-1709.

[9] Ramachandran S, Karp PH, Osterhaus SR, Jiang P, Wohlford-Lenane C, Lennox KA, Jacobi AM, Praekh K, Rose SD, Behlke MA, Xing Y, Welsh MJ, McCray PB Jr. Post- 
transcriptional regulation of cystic fibrosis transmembrane conductance regulator expression and function by microRNAs. Am J Respir Cell Mol Biol. 2013; 49(4): 544-551.

[10] Oglesby IK, Chotirmall SH, McElvaney NG, Greene CM. Regulation of cystic fibrosis transmembrane conductance regulator by microRNA-145, -223 , and -494 is altered in $\Delta$ F508 cystic fibrosis airway epithelium. J Immunol. 2013; 190(7): 3354-3362.

[11] Ramachandran S, Karp PH, Jiang P, Ostedgaard LS, Walz AE, Fisher JT, Keshavjee S, Lennox KA, Jacobi AM, Rose SD, Behlke MA, Welsh MJ, Xing Y, McCray PB Jr. A microRNA network regulates expression and biosynthesis of wild-type and $\triangle \mathrm{F} 508$ mutant cystic fibrosis transmembrane conductance regulator. Proc Natl Acad Sci U S A. 2012; 109(33): 13362-13367.

[12] Raisch J, Darfeuille-Michaud A, Nguyen HT. Role of microRNAs in the immune system, inflammation and cancer. World J Gastroenterol. 2013; 19(20): 2985-2996.

[13] Dean TP, Dai Y, Shute JK, Church MK, Warner JO. Interleukin-8 concentrations are elevated in bronchoalveolar lavage, sputum, and sera of children with cystic fibrosis. Pediatr Res. 1993; 34(2): 159-161.

[14] Tirouvanziam R, de Bentzmann S, Hubeau C, Hinnrasky J, Jacquot J, Péault B, Puchelle E. Inflammation and infection in naive human cystic fibrosis airway grafts. Am J Respir Cell Mol Biol. 2000; 23(2): 121-127.

[15] Khan TZ, Wagener JS, Bost T, Martinez J, Accurso FJ, Riches DW. Early pulmonary inflammation in infants with cystic fibrosis. Am J Respir Crit Care Med. 1995; 151(4): 1075-1082.

[16] Bhattacharyya S, Balakathiresan NS, Dalgard C, Gutti U, Armistead D, Jozwik C, Srivastava M, Pollard HB, Biswas R. Elevated miR-155 promotes inflammation in cystic fibrosis by driving hyperexpression of interleukin-8. J Biol Chem. 2011; 286(13): 11604-11615.

[17] Tsuchiya M, Kumar P, Bhattacharyya S, Chattoraj S, Srivastava M, Pollard HB, Biswas R. Differential regulation of inflammation by inflammatory mediators in cystic fibrosis lung epithelial cells. J Interferon Cytokine Res. 2013; 33(3): 121-129.

[18] Rao JR, Nelson D, Moore JE, Millar BC, Goldsmith CE, Rendall J, Elborn JS. Non-coding small (micro) RNAs of Pseudomonas aeruginosa isolated from clinical isolates from adult patients with cystic fibrosis. Br J Biomed Sci. 2010; 67(3): 126-132.

[19] Bhattacharyya S, Kumar P, Tsuchiya M, Bhattacharyya A, Biswas R. Regulation of miR-155 biogenesis in cystic fibrosis lung epithelial cells: Antagonistic role of two mRNA-destabilizing proteins, KSRP and TTP. Biochem Biophys Res Commun. 2013; 433(4): 484-488.

[20] Vigorito E, Kohlhaas S, Lu D, Leyland R. miR-155: An ancient regulator of the immune system. Immunol Rev. 2013; 253(1): 146-157. 
[21] Sessa R, Hata A. Role of microRNAs in lung development and pulmonary diseases. Pulm Circ. 2013; 3(2): 315-328.

[22] Voynow JA, Fischer BM, Zheng S. Proteases and cystic fibrosis. Int J Biochem Cell Biol. 2008; 40(6-7): 1238-1245.

[23] Martin SL, Moffitt KL, McDowell A, Greenan C, Bright-Thomas RJ, Jones AM, Webb AK, Elborn JS. Association of airway cathepsin B and S with inflammation in cystic fibrosis. Pediatr Pulmonol. 2010; 45(9): 860-868.

[24] Weldon S, Mc Nally P, McAuley DF, Oglesby IK, Wohlford-Lenana CL, Bartlett JA, Scott CJ, McElvaney NG, Greene CM, McCray PB Jr, Taggart CC. miR-31 dysregulation in cystic fibrosis airways contributes to increased pulmonary cathepsin $\mathrm{S}$ production. Am J Respir Crit Care Med. 2014; 190(2): 165-174.

[25] Bhaskaran M(1), Wang Y, Zhang H, Weng T, Baviskar P, Guo Y, Gou D, Liu L. MicroRNA-127 modulates fetal lung development. Physiol Genomics. 2009; 37(3): 268-278.

[26] Fabbri M, Garzon R, Cimmino A, Liu Z, Zanesi N, Callegari E, Liu S, Alder H, Costinean S, Fernandez-Cymering C, Volinia S, Guler G, Morrison CD, Chan KK, Marcucci G, Calin GA, Huebner K, Croce CM. MicroRNA-29 family reverts aberrant methylation in lung cancer by targeting DNA methyltransferases 3A and 3B. Proc Natl Acad Sci U S A. 2007; 104(40): 15805-15810.

[27] Hayashita Y, Osada H, Tatematsu Y, Yamada H, Yanagisawa K, Tomida S, Yatabe $Y$, Kawahara K, Sekido Y, Takahashi T. A polycistronic microRNA cluster, miR-17-92, is overexpressed in human lung cancers and enhances cell proliferation. Cancer Res. 2005; 65(21): 9628-9632.

[28] Martinez-Anton A, Sokolowska M, Hern S, Davis AS, Alsaaty S, Taubenberger JK, Sun J, Cai R, Danner RL, Eberlein M, Logun C, Shelhamer JH. Changes in microRNA and mRNA expression with differentiation of human bronchial epithelial cells. Am J Respir Cell Mol Biol. 2013; 49(3): 384-395.

[29] Marcet B, Chevalier B, Luxardi G, Coraux C, Zagarosi LE, Cibois M, Robbe-Sermesant K, Jolly T, Cardinaud B, Moreilhon C, Giovanni-Chami L, Nawrocki-Raby B, Birembaut $\mathrm{P}$, Waldmann $\mathrm{R}$, Kodjabachian L, Barbry L. Control of vertebrate multiciliogenesis by miR-449 through direct repression of the Delta/Notch pathway. Nat Cell Biol. 2011; 13(6): 693-699.

[30] Huaux F, Noel S, Dhooghe B, Panin N, Lo Re S, Lison D, Wallemacq P, Marbaix E, Scholte BJ, Lebecque P, Leal T. Dysregulated proinflammatory and fibrogenic phenotype of fibroblasts in cystic fibrosis. PLoS ONE. 2013; 8(5): e64341.

[31] Bowen T, Jenkins RH, Fraser DJ. MicroRNAs, transforming growth factor beta-1, and tissue fibrosis. J Pathol. 2013; 229(2): 274-285.

[32] Pottier N, Maurin T, Chevalier B, Puisséqur MP, Lebrigand K, Robbe-Sermesant K, Bertero T, Lino Cardenas CL, Courcot E, Rios G, Fourre S, Lo-Guidice JM, Marcet B, 
Cardinaud B, Barbry P, Mari B. Identification of keratinocyte growth factor as a target of microRNA-155 in lung fibroblasts: implication in epithelial-mesenchymal interactions. PLoS ONE. 2009; 4(8): e6718.

[33] Kong W, Yang H, He L, Zhao LL, Coppola D, Dalton WS, Cheng JQ. MicroRNA-155 is regulated by the transforming growth factor $\beta /$ Smad pathway and contributes to epithelial cell plasticity by targeting RhoA. Mol Cell Biol. 2008; 28(22): 6773-6784.

[34] Zhang JT, Jiang XH, Xie C, Cheng H, Da Dong J, Wang Y, Fok KL, Zhang XH, Sun TT, Tsang LL, Chen H, Sun XJ, Chung YW, Cai ZM, Jiang WG, Chan HC. Downregulation of CFTR promotes epithelial-to-mesenchymal transition and is associated with poor prognosis of breast cancer. Biochem Biophys Acta. 2013; 1833(12): 2961-2969.

[35] Snodgrass SM, Cihil KM, Cornuet PK, Myerburg MM, Swiatecka-Urban A. Tgf- $\beta 1$ inhibits $\mathrm{Cftr}$ biogenesis and prevents functional rescue of $\triangle \mathrm{F} 508-\mathrm{Cftr}$ in primary differentiated human bronchial epithelial cells. PLoS ONE. 2013; 8(5): e63167.

[36] Cook NL, Pereira TN, Lewindon PJ, Sheperd RW, Ramm GA. Circulating microRNAs as noninvasive diagnostic biomarkers of liver disease in children with cystic fibrosis. J Pediatr Gastroenterol Nutr. 2015; 60(2): 247-254.

[37] Amato F, Seia M, Giordano S, Elce A, Zarrilli F, Castaldo G, Tomaiuolo R. Gene mutation in microRNA target sites of CFTR gene: a novel pathogenetic mechanism in cystic fibrosis? PLoS ONE. 2013; 8(3): e60448.

[38] Hassan T, McKiernan PJ, McElvaney NG, Cryan SA, Greene CM. Therapeutic modulation of miRNA for the treatment of proinflammatory lung disease. Expert Rev Anti Infect Ther. 2012; 10(3): 359-368.

[39] McKiernan PJ, Cunningham O, Greene CM, Cryan SA. Targeting miRNA-based medicines to cystic fibrosis airway epithelial cells using nanotechnology. Int J Nanomedicine. 2013; 8: 3907-3915.

[40] Bazett M, Paun A, Haston CK. MicroRNA profiling of cystic fibrosis intestinal disease in mice. Mol Genet and Metab. 2011; 103(1): 38-43. 



\title{
New Research on the Importance of Cystic Fibrosis Transmembrane Conductance Regulator Function for Optimal Neutrophil Activity
}

\author{
Michelle M. White, Fatma Gargoum, Niall Browne, \\ Killian Hurley, Noel G. McElvaney and \\ Emer P. Reeves
}

Additional information is available at the end of the chapter

http://dx.doi.org/10.5772/60645

\begin{abstract}
Despite tremendous recent advances in our understanding of the molecular and cellular basis of cystic fibrosis (CF), there remains a paradox of why recruited neutrophils fail to eradicate bacterial infections in the airways of individuals with CF. The focus of this chapter is on new research authenticating the CF neutrophil as a key player in disease pathogenesis. Studies specifying intrinsic abnormalities due to a lack of cystic fibrosis transmembrane conductance regulator (CFTR) function, along with reports indicating reprogrammed cell activity secondary to chronic bacterial infection and inflammation, will be discussed.
\end{abstract}

Keywords: cystic fibrosis, cystic fibrosis transmembrane conductance regulator, neutrophil function

\section{Introduction}

Cystic fibrosis (CF) is a multi-system disease resulting from mutations in the gene encoding the cystic fibrosis transmembrane conductance regulator (CFTR). In excess of $1900 \mathrm{CF}$ mutations have been identified thus far [1], leading to misfolding of the CFTR protein and defective chloride $\left(\mathrm{Cl}^{-}\right)$transport across cell membranes. $\mathrm{CF}$ affects various organs including 
the pancreas, liver, and intestinal tract, but the most severe complications often occur in the lung. CF related symptoms, although variable among patients, are typically present early in life and increase in severity with age despite aggressive therapeutic intervention. CFTR absence or malfunction causes defective ion transport across the epithelium, reduction in pericillary liquid volume, and persistent mucus hypersecretion. As a consequence, mucus accumulates on the airway surface and leads to chronic bacterial infection, exacerbated airway inflammation, and lung injury. Pulmonary inflammation in the CF setting has been observed as early as infancy and there is evidence of structural lung disease present in children with $\mathrm{CF}$ as young as 10 weeks old, with $50 \%-70 \%$ of children presenting with bronchiectasis by $3-5$ years [2-6]. Additional studies have shown neutrophil-dominated airway inflammation in children with $\mathrm{CF}[7,8]$, and with increasing age elevated levels of proinflammatory mediators including interleukin (IL)-8 [9], tumour necrosis factor-alpha (TNF-alpha) [10], and leukotriene $\mathrm{B}_{4}\left(\mathrm{LTB}_{4}\right)[11]$ serve to escalate the chronic neutrophil presence in the pulmonary circulation. Indeed, key studies have demonstrated that neutrophil-released granule proteins, particularly neutrophil elastase (NE), play a crucial pathological role (Figure 1) [12, 13]. In the healthy lung, neutrophils represent approximately $1 \%$ of the inflammatory cells; however, they account for $60-70 \%$ of the total cell count in CF bronchial lavage fluid [14, 15] and $25 \%$ of CF children were positive for free NE activity in airway samples at 3 months old [16].

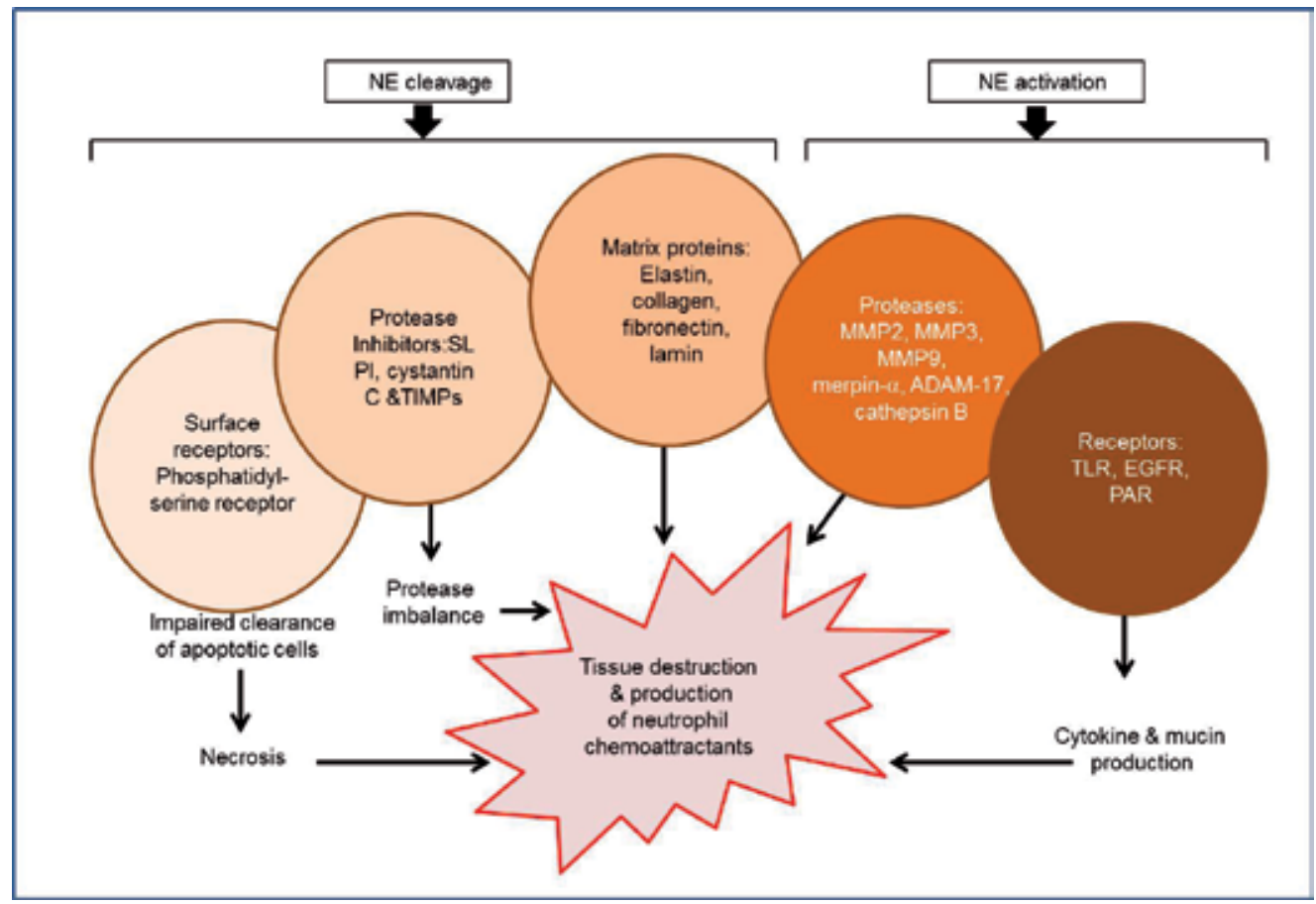

Figure 1. The potential effects of active NE in CF. Neutrophil elastase (NE) has the potential to cleave structural molecules and antiproteases causing an anti-protease imbalance and also activate enzymes and receptors that play a crucial pathological role in CF. Abbreviations used: NE, neutrophil elastase; SLPI, secretory leukocyte protease inhibitor; TIMPs, tissue inhibitors of metalloproteinases; MMP, metalloproteinase; TACE, tumour necrosis factor-alpha-converting enzyme; TLRs, toll-like receptors; EGFR, epidermal growth factor receptor; PAR, protease activated receptors. 
Despite advances in our understanding of the molecular and cellular basis of $\mathrm{CF}$, there persists an enigma as to why recruited neutrophils fail to eradicate bacterial infections in the lung. Support for the hypothesis that a genetic defect gives rise to dysregulated neutrophil responses in CF is shown by a study demonstrating upregulation of genes coding for both chemokines and proteins involved in signal transduction in CF [17]. A recent study has demonstrated that CXC chemokine receptors $1 / 2$ (CXCR1/CXCR2) haplotypes in CF modulate antibacterial neutrophil functions against $P$. aeruginosa [18]. In addition, CF circulating neutrophils display an altered toll-like receptor (TLR) expression when compared to blood neutrophils from healthy subjects, including reduced expression of TLR2 [19, 20]. A recently published study identified IFRD1 (interferon-related developmental regulator 1), a histone-deacetylasedependent transcriptional co-regulator expressed during terminal neutrophil differentiation, as a genetic modifier of CF disease severity. Neutrophils isolated from IFRD1 deficient mice exhibited impaired oxidative burst, bacterial clearance, and cytokine production leading to excessive bacterial burden and chronic infection of the lung [21]. Furthermore, levels of IFRD in bronchial epithelial cells with the $\Delta F 508$ mutation were significantly reduced but could be rescued by treatment with glutathione suggesting down-regulation of IFRD1 expression in response to oxidative stress [22].

The focus of this chapter is on new research authenticating the CF neutrophil as a key player in disease pathogenesis. Studies published in the previous 10 years specifying intrinsic abnormalities due to a lack of CFTR function, along with reports indicating reprogrammed cell activity secondary to chronic bacterial infection and inflammation will be discussed. Our review of the literature was carried out using the MEDLINE database (from 2005 to the year 2015), Google Scholar, and The Cochrane Library databases using several appropriate generic terms.

\section{The debate on CFTR expression by neutrophils}

The year 2015 marks the 26th anniversary of the identification and isolation of the CFTR gene from epithelial cells [23]. This initial discovery was vital in the development of CFTR-targeted pharmacotherapy and resulted in an explosion of research aimed at identifying the expression of CFTR mRNA transcripts and protein levels in epithelial cells and non-epithelial cells alike. Although CF is a pleiotropic disease that affects multiple organs, disease progression is most pronounced in the airways and therefore researchers initially studied the expression of CFTR in epithelial cells found within the lungs. In this regard, Yoshimura and colleagues (1991) demonstrated the expression of CFTR mRNA transcripts in human bronchial epithelial cells [24], and Trapnall et al. demonstrated equal expression of CFTR mRNA transcripts from isolated respiratory tract epithelial cells from healthy controls and homozygous and heterozygous $\triangle F 508$ patients with CF [25]. The level of research at this stage indicated that the expression of the CFTR was epithelial cell specific, yet the conclusions drawn from a further study by Yoshimura suggested that the CFTR-gene had characteristics of a house-keeping gene, suggesting that it may be present in other cell types [26]. This latter manuscript prompted researchers to examine CFTR mRNA transcripts in other cell types and in late 1991, Yoshimura 
demonstrated the expression of low levels of CFTR mRNA transcripts in non-epithelial cells, including T-lymphocytes, neutrophils, monocytes, and alveolar macrophages, all of human origin [26]. This was the first record of CFTR gene expression in a multitude of immune cell types, and the authors suggested an important role for CFTR $\mathrm{Cl}^{-}$transport. In agreement, it was later demonstrated that alveolar macrophages from CFTR deficient mice retained the ability to phagocytose and generate an oxidative burst, but exhibited defective killing of internalized bacteria due to impaired acidification of the phagosome. Of interest, in this later study CFTR protein was not detected in either murine or human neutrophils [27] and further studies have shown CFTR independent phagosomal acidification in macrophages [28, 29]. Nevertheless, the expression of CFTR mRNA transcripts in human macrophages was confirmed by Del Porto, who published that the bactericidal capabilities of macrophages was CFTR dependent, indicating an important functional role for the CFTR protein in these immune cells [30].

Up to this point, no connection between abnormal neutrophil function and the expression of CFTR protein in human neutrophils had been made, and whether or not neutrophils express functional CFTR was still the topic of great debate among leading scientists and clinicians [5, $8,13,14]$, with relevance for the development of CFTR-targeting pharmacotherapy. This is emanating from worldwide laboratories equally committed to the pursuit of knowledge on the cause for impaired neutrophil activity in CF and also the potential consequence of the loss of functional CFTR $\mathrm{Cl}^{-}$channels. Indeed, there is still great controversy as to the true nature of dysregulated neutrophil activity in CF and, for example, in 2010, McKeon and colleagues could only detect low levels of CFTR mRNA transcripts in human neutrophils by either reverse transcriptase polymerase chain reaction (RT-PCR) or real time PCR methods of amplification [31]. The authors could not detect CFTR protein expression in membrane or cytosolic fractions, or cell lysates from human neutrophils by Western blot analysis, suggesting that human neutrophils do not express CFTR protein and that the dysregulated neutrophil function in $\mathrm{CF}$ was due to the inflammatory status of the individual [31]. Similarly, a study by Morris et al., (2005) who investigated altered phagocytosis of neutrophils in CF due to cell priming, did not detect CFTR protein in human neutrophils by Western blot analysis [32].

Over the years, a number of reasons for not detecting CFTR protein in neutrophil cell fractions by Western blot analysis have been put forward and include the susceptibility of CFTR protein to degradation, a lack of reliable anti-CFTR antibodies, and also boiling of cell fractions prior to electrophoresis. As these issues were addressed researchers have detected CFTR protein in human neutrophils and have established functional roles for membrane associated CFTR. In line with this concept, in 2006, Painter and colleagues demonstrated the presence of CFTR protein in human neutrophil membrane phagolysosomes by confocal microscopy, and also verified the expression of CFTR at a mRNA level, and at a protein level by Western blot analysis [33]. Interestingly, in this same study results demonstrated the expression of CFTR protein by Western blot analysis in a human myeloid cell line (HL-60 cells) differentiated into neutrophillike cells [33]. This is in contrast to the study by Yoshimura et al., who could not detect CFTR mRNA transcripts in HL-60 cells [24]. Further studies have identified CFTR mRNA transcripts in differentiated HL-60 cells and demonstrated CFTR protein localisation to the phagocytic vacuole, strengthening the similarities between HL-60 cells and human neutrophils [34]. 
Furthermore, expression of CFTR protein was confirmed by Pohl et al. (2014) in healthy control neutrophils and levels were severely impaired in neutrophils isolated from people with $\mathrm{CF}$ carrying the $\triangle F 508$ or G551D mutation. By flow cytometry, this latter study also demonstrated that the percentage of CFTR positive cells significantly increased post phorbol ester stimulation [35], indicating upregulation of CFTR to the plasma membrane post activation. Interestingly, bacterial lipopolysaccharide (LPS)-stimulated murine neutrophils also demonstrated increased CFTR protein expression when compared to unstimulated cells, suggesting a role for CFTR in bacterial mediated neutrophil cell activation [36].

\section{The role of CFTR in neutrophils: oxygen dependent and independent mechanisms of microbial killing}

\subsection{Oxygen dependent killing}

The focus of this chapter will now turn to studies that have reported impaired neutrophil activity due to a lack of CFTR function. Of major relevance to bacterial killing in CF, reported defective killing of microbes due to conditions that prevail within the vacuole of phagocytosing neutrophils will be discussed. The process of neutrophil mediated bacterial clearance can be divided into two main processes: those that are oxygen independent and those that are oxygen dependent. With regards to oxidative mechanisms of microbial killing, the first indications that oxygen plays a role in the functionality of neutrophils was first discovered by Baldridge and Gerard [37]. By exposing canine neutrophils to bacteria they observed a significant increase in oxygen consumption [37], and later it was revealed that this increase in oxygen consumption was independent of mitochondrial respiration [38]. Following discovery of the respiratory burst, it was discovered that neutrophils from patients with chronic granulomatous disease (CGD) failed to mount a respiratory burst during phagocytosis and these individuals are characterised by an abnormality of neutrophil function and recurrent life-threatening infections $[39,40]$. CGD provides the most definitive evidence for the physiological and clinical importance of the respiratory burst, or alternatively referred to as the NADPH oxidase (nicotinamide adenine dinucleotide phosphate oxidase). Indeed, patients with CGD have played a vital role in understanding the structure and mechanism of the NADPH oxidase (Figure 2). The burst of oxygen consumption upon phagocytosis and the absence of this process in CGD leading to impaired bacterial killing was understood to indicate that the oxygen consumed was converted to antimicrobial oxidants. By exposing neutrophils to opsonised bacteria, Cohen and colleagues demonstrated that $99 \%$ of oxygen consumed was converted to superoxide $\left(\mathrm{O}_{2}^{-}\right)$[41] and the requirement of $\mathrm{O}_{2}^{-}$as a precursor to hydrogen peroxide $\left(\mathrm{H}_{2} \mathrm{O}_{2}\right)$ was confirmed [42]. It is not known what concentration of $\mathrm{H}_{2} \mathrm{O}_{2}$ is attained within the vacuole, with measurement from $0.01 \mu \mathrm{M}$ [42] to $100 \mathrm{mM}$ estimated, depending on the amount of phagocytosis and the intracellular $\mathrm{pH}$ [43]. These oxidants produced by neutrophils may also contribute to lung tissue damage and the shield against oxidant-modulated injury is the extracellular anti-oxidant glutathione; but this is significantly decreased in CF epithelial lining fluid [44-47]. Of major relevance is that CFTR has been linked to extracellular glutathione 
transport [48] and in paediatric reports of extremely high levels of protein oxidation have been detected in airway samples [49].
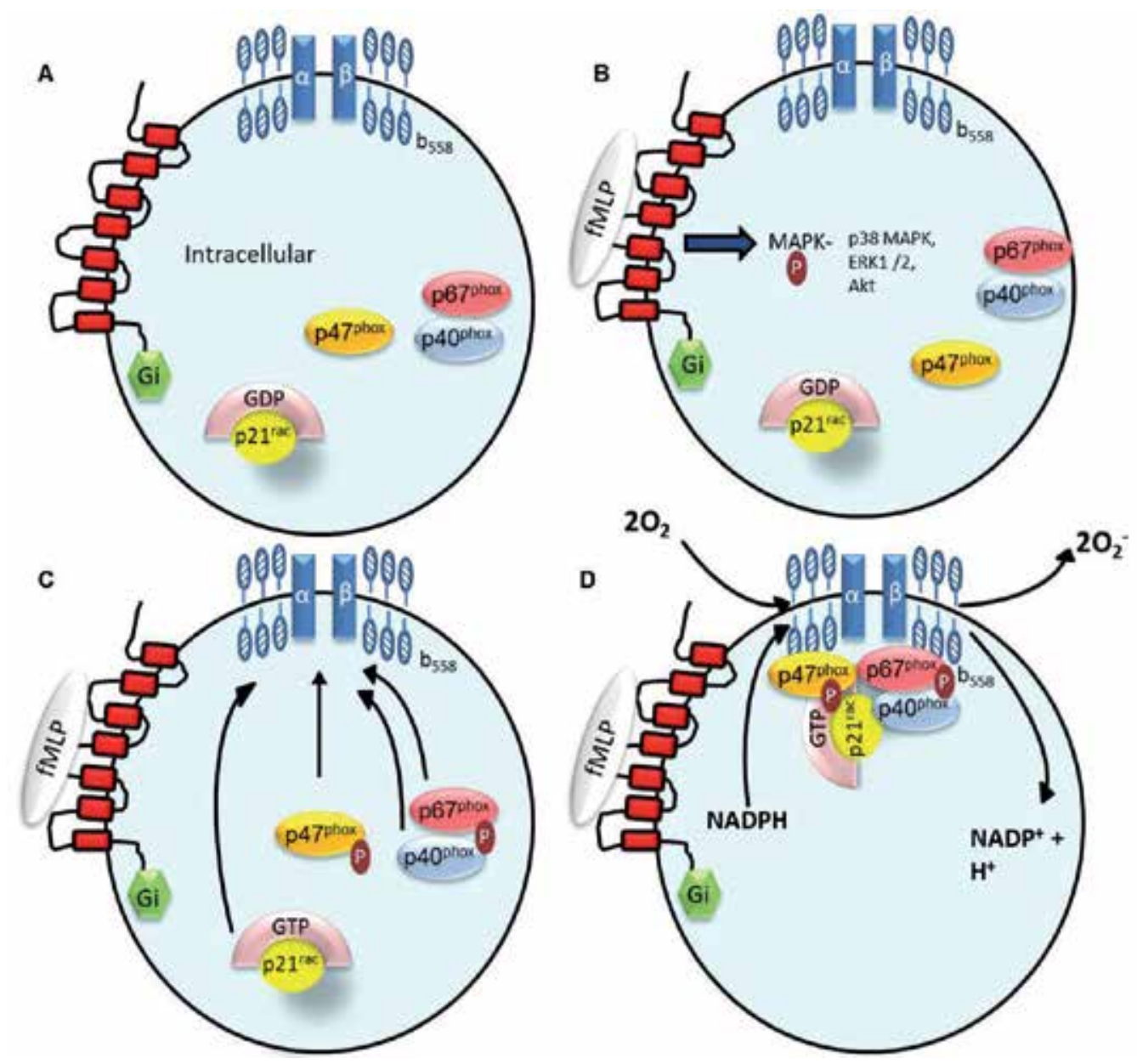

The neutrophil respiratory burst, or NADPH oxidase, generates superoxide $\left(\mathrm{O}_{2}{ }^{-}\right)$as the initial oxygen-derived reactive species in response to bacteria or a variety of soluble stimuli (fMLP). A) The enzyme complex is latent in quiescent unstimulated neutrophils and approximately $20 \%$ flavocytochrome $b_{558}$ is found in the plasma membrane pool, and $80 \%$ in the specific granules. The active site of this enzyme is located in an integral membrane cytochrome, $\mathrm{b}_{558}$, which consists of the two subunits gp91 ${ }^{\text {phox }}$ and $\mathrm{p} 21^{\text {phox }}(\alpha$ and $\beta$ subunits, respectively). B) Stimulation of the neutrophil by fMLP induces activation and phosphorylation (P) of a number of kinases including Akt. C) p21 $1^{\text {rac }}$ is converted into the active guanosine triphosphate GTP-bound form and the phosphorylation of the cytosolic components (p67phox, p47 phox and $\mathrm{p} 40^{\text {phox }}$ ) occurs. D) These subunits then translocate to the plasma membrane where they interact with cytochrome $b_{558}$ to initiate reactive oxygen species production.

Figure 2. Schematic illustration of the NADPH oxidase of resting and fMLP activated cells.

$\mathrm{H}_{2} \mathrm{O}_{2}$ generated during the oxidative burst has limited bactericidal properties and the bestdefined function of $\mathrm{H}_{2} \mathrm{O}_{2}$ in the antimicrobial activities of neutrophils comes from the function of $\mathrm{H}_{2} \mathrm{O}_{2}$ as a substrate for myeloperoxidase (MPO) in the presence of halides (chloride $\left(\mathrm{Cl}^{-}\right)$). Neutrophil MPO was initially called verdoperoxidase, and later its name subsequently 
changed to MPO. It is present in exceptionally high concentrations in neutrophils, with levels estimated to be no less than $5 \%$ of the dry weight of the cell. MPO is synthesized and packaged into azurophilic, also referred to as primary granules of neutrophils, during the promyelocyte stage of granulocyte development and is present in mature resting granulocytes.

Mature MPO is a $150 \mathrm{kDa}$ tetramer composed of two glycosylated 59-64 kDa heavy subunits and two unglycosylated $14 \mathrm{kDa}$ light subunits as a pair of protomers linked together by a disulphide bond. When the phagosome containing microorganisms fuses with cytoplasmic granules, MPO, along with the other components of the granules, is released into the vacuole. A role for MPO as a component of the antimicrobial armoury of neutrophils was proposed with the finding that MPO was strongly microbial when combined with $\mathrm{H}_{2} \mathrm{O}_{2}$ and a halide [50, 51]. $\mathrm{MPO}$ and $\mathrm{H}_{2} \mathrm{O}_{2}$ form an enzyme-substrate complex, which oxidises ions to the toxic agent hypohalous acid. Any of the halide ions $\left(\mathrm{I}^{-}, \mathrm{Br}^{-}, \mathrm{Cl}^{-}\right)$can be oxidised with iodide and bromide being more effective than $\mathrm{Cl}^{-}$on a molar basis [51]. It is more likely however that the neutrophil uses $\mathrm{Cl}^{-}$because it is present in high concentration in biological fluids, resulting in the formation of hypochlorous acid $(\mathrm{HOCl})$. There are three proposed means of $\mathrm{Cl}^{-}$transport to the phagosome: extracellular $\mathrm{Cl}^{-}$intake during phagocytosis of a pathogen, stored $\mathrm{Cl}^{-}$within granules released into the phagosome upon vesicle fusion, and passive or active transport of $\mathrm{Cl}^{-}$from the cytosol to the phagosome [52]. Only active or passive transport has been suggested to provide a constant supply of $\mathrm{Cl}^{-}$(Figure 3) [53] and two $\mathrm{Cl}^{-}$ion channels (ClCs), $\mathrm{ClC}-3$ and CFTR have been implicated in the transport of $\mathrm{Cl}^{-}$within the neutrophil and the phagosome $[33,54]$. The influx of protons to the phagosomal lumen by V-ATPase has been demonstrated to facilitate the transport of $\mathrm{Cl}^{-}$ions into the phagosome by $\mathrm{ClC}$ including the CFTR [55] (Figure 3).

It is generally believed that $\mathrm{HOCl}$ is the most bactericidal oxidant known to be produced by the neutrophil. Levels of $\mathrm{HOCl}$ produced are based on calculations made after phorbol ester stimulation, which results in the secretion of $\mathrm{O}_{2}^{-}$across the membrane to the surrounding supernatant and levels achieved are estimated at $80 \mu \mathrm{M}$.

Levels produced in the phagocytic vacuole have been estimated using chlorinated fluorescein as a specific marker for $\mathrm{HOCl}$ production and by use of this technique it was calculated that $11 \%$ of oxygen consumed was converted to $\mathrm{HOCl}$, resulting in $28 \mu \mathrm{M}$ within the phagosome [56]. $\mathrm{HOCl}$ is an extremely strong non-radical oxidant and bacterial targets include adenosine triphosphate (ATP)-generating systems [57], disruption of basement membranes or cell membranes, and fragmentation of proteins [58]. Chloramines are generated indirectly through the reaction of $\mathrm{HOCl}$ with amines, but are less reactive than $\mathrm{HOCl}$ but much more stable, and are therefore called "long lived oxidants." Because of the high intracellular concentration of the $\beta$-amino acid taurine, $N$-chlorotaurine is the major compound of low molecular weight long lived oxidants produced by neutrophils $[59,60]$. Of major relevance to bacterial killing in $\mathrm{CF}$ it was reported that $\mathrm{CF}$ neutrophils had impaired chlorination of internalised bacteria within the phagosome when compared to healthy control neutrophils [33]. The authors highlighted the role of CFTR in facilitating $\mathrm{Cl}^{-}$flux into the phagosome of neutrophils which was impaired in CF cells. This CFTR dysfunction resulted in limited availability of phagosomal $\mathrm{Cl}^{-}$required for the generation of $\mathrm{HOCl}$, resulting in reduced intravacuolar killing of Pseudo- 


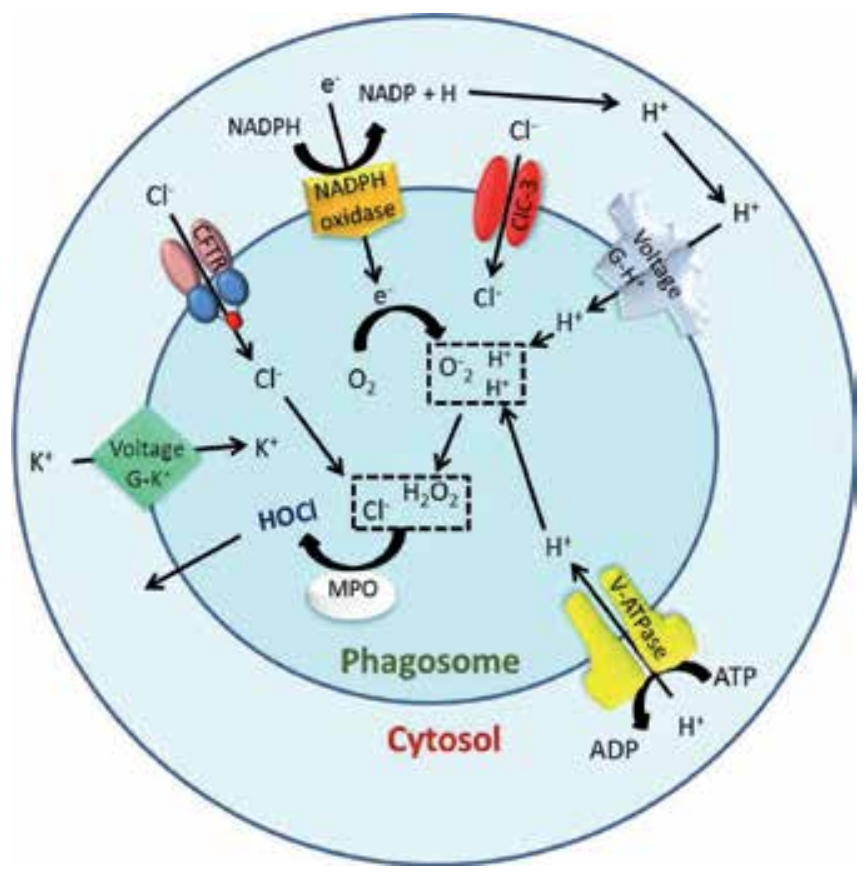

Upon activation of the NADPH oxidase, the cytochrome takes electrons from NADPH and passes them, via FAD and haem, to $\mathrm{O}_{2}$ in the following reaction: NADPH $+2 \mathrm{O}_{2} \rightarrow \mathrm{NADP}^{+}+\mathrm{H}^{+}+2 \mathrm{O}_{2}^{-}$. NADPH oxidase generates an electron current which is compensated through voltage gated ion influx of protons (Voltage G- $\mathrm{H}^{+}$) and potassium (Voltage G-K $\left.{ }^{+}\right)$. $\mathrm{Cl}^{-}$transport is facilitated by CFTR and also other channels including $\mathrm{ClC}-3$. The influx of $\mathrm{H}^{+}$to the phagosomal lumen by V-ATPase facilitates $\mathrm{Cl}^{-}$transport into the phagosome by $\mathrm{ClC}$ including the CFTR. $\mathrm{O}_{2}^{-}$is a mild oxidant and reductant with restricted biological activity. $\mathrm{H}_{2} \mathrm{O}_{2}$ is a slow acting oxidising agent and $\mathrm{HOCl}$ is a strong non-radical oxidant.

Figure 3. Suggested mechanism of $\mathrm{Cl}^{-}$and ion transport within the neutrophil phagosome.

monas aeruginosa, the archetype infecting organism in CF [33, 34, 53, 61]. Moreover, new data has shown that mutant CFTR fails to target to the neutrophil vacuole resulting in impaired intraphagosomal $\mathrm{HOCl}$ production and neutrophil microbial killing [62].

Having described the importance of MPO mediated halogenation for adequate microbial killing, there is also a need to mention that MPO deficiency occurs with a high prevalence and patients are not clinically afflicted by serious bacterial infections [63], with infections by Candida species being the main difficulty. Although more slowly than healthy cells, neutrophils deficient in MPO can kill bacteria in vitro [64], a result incompatible with the concept that $\mathrm{HOCl}$ is the major mediator of neutrophil bactericidal function in man. MPO deficient cells illustrate a prolonged respiratory burst resulting in increased levels of $\mathrm{H}_{2} \mathrm{O}_{2}$ and this coupled with nonoxidative methods of bacterial killing has been proposed to compensate for MPO deficiency. Furthermore, despite the fact that neutrophils of CGD patients do not produce $\mathrm{O}_{2}{ }^{-}$and $\mathrm{H}_{2} \mathrm{O}_{2}$, studies have shown that neutrophils from CGD patients are capable of killing significant numbers of phagocytosed bacteria and yeast $[65,66]$ and the presence of oxygen-independent microbial mechanisms in neutrophils is demonstrated by the ability of these cells to kill bacteria in the absence of oxygen [67]. 


\subsection{Oxygen independent microbial killing}

The presence of an oxygen independent microbial mechanism in neutrophils is demonstrated by the ability of these cells to kill bacteria under anaerobic conditions. Hirsch and colleagues reported that neutrophil lysates killed bacteria and that this effect was due to a substance they called phagocytin [68]. The component responsible for bacterial killing was localized to the cytoplasmic granules which are released into the phagocytic vacuole [69]. Neutrophil-derived microbial molecules are packaged within four distinct subgroups of granules (Figure 4) and are released either into the phagocytic vacuole or to the outside of the cell upon activation. Granule biogenesis follows the granulocyte differentiation pathway. The azurophilic (also referred to as primary) granules first emerge at the stage of the promyelocytes [70] and contain $\mathrm{MPO}$, the serine proteases neutrophil elastase (NE), cathepsin $\mathrm{G}$ and proteinase 3, defensins and bacterial permeability-increasing protein [71], and are considered as the true microbial compartment mobilized upon phagocytosis. Later in differentiation, at the metamyelocyte stage, specific granules containing lactoferrin [72], $18 \mathrm{kDa}$ human cathelicidin antimicrobial protein (hCAP-18], and lysozyme emerge [73], followed by a third population termed the gelatinase granules which predominantly contain gelatinase (matric metalloprotease (MMP)-9 and MMP-2), lysozyme, and leukolysin [74, 75]. A forth type of granule, called the secretory vesicles, appears at the stage of the mature neutrophil. Movement of the cytoplasmic granules following ingestion of bacteria was first observed by Robineaux and Frederic [76], and by use of chicken leukocytes with their large dense granules, Hirsch observed by phase contrast microscopy, degranulation and release of the granule contents directly into the phagocytic vacuole, by fusion of the granule membrane with the invaginated cell membrane [77]. As for any form of intracellular vesicle transport, degranulation is a tightly regulated process. Small GTPases of the Ras superfamily are known key regulators of cellular events including vesicle transport, cell division, control of cytoskeletal rearrangements, and nuclear assembly. Secondary and tertiary granules are tethered through a small GTPase Rab27a and its effector protein Munc13-4 [78] which interacts with the soluble N-ethylmaleimide association protein receptor (SNARE), a protein complex composed of vesicle-associated membrane proteins (VAMPs) on the vesicle surface and SNAP23 and STX4 on the plasma membrane [79-81]. Once the granule is docked the granule membrane fuses with the plasma membrane enabling release of granule contents. The small GTPase Rac2 has been shown necessary for the release of primary granules [82] but of interest, a sub-population of Rab27a positive primary granules was also found [83] with different Rab27a effectors Slp1 and Munc13-4 reported necessary for primary and tertiary granules release, respectively [84].

Although the neutrophil possesses an armoury of anti-microbial proteins and peptides, individual components have been shown to exert microbial effects. For example, NE has long been regarded as the major antibacterial protein and mice made homozygous for a disrupted NE gene have demonstrated impaired resistance to Klebsiella pneumonia and Escherichia coli sepsis [85]. A target for NE is the bacterial outer-membrane protein OmpA [86]. NE degradation of OmpA results in cell death as a result of loss of bacterial integrity by localized weakening of the cell wall followed by osmotic lysis [86]. The amino acid composition of cathepsin G shares $37 \%$ sequence homology with NE and plays a role in neutrophil responses against a 


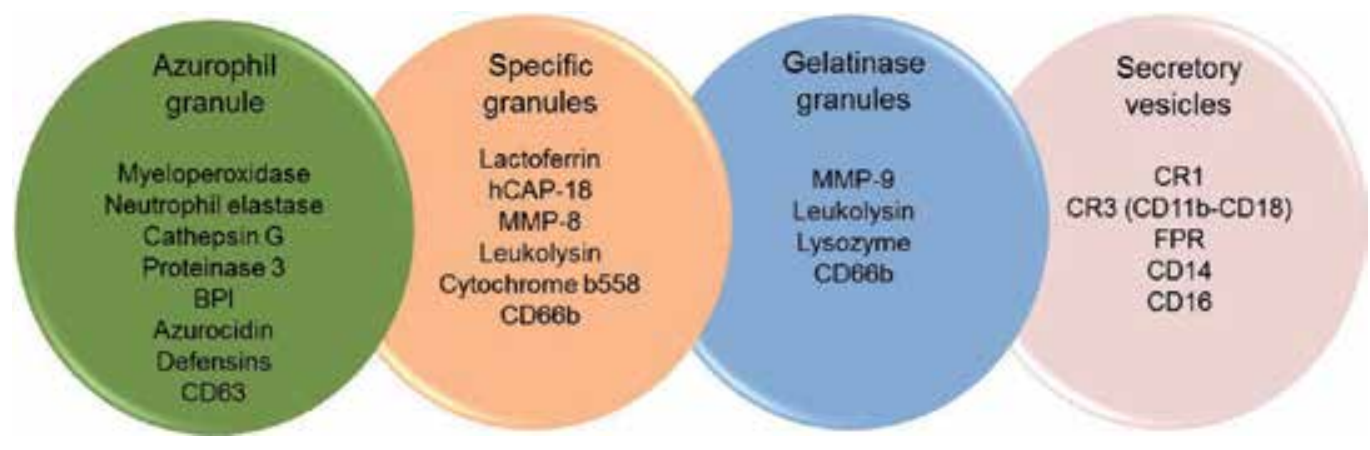

Figure 4. Granule and vesicle contents of the neutrophil. The most abundant organelles within the cytoplasm are the granules, which are membrane-bound organelles containing an array of antimicrobial proteins. Three major types have been identified, azurophilic, specific, and gelatinase-containing granules. A forth type of granule, called the secretory vesicles, are endocytic in nature and act as an internal reservoir of membrane/cytokine receptors.

variety of bacteria. Purified cathepsin G has been shown to inhibit the growth of several organisms including Staphylococcus aureus, E. coli, P. aeruginosa, and Neisseria gonorrhoea [87, 88]. Moreover, Tkalcevic and colleagues performed in vivo studies of mice deficient in cathepsin G, NE, or both and demonstrated the importance of cathepsin G in the successful clearance of Aspergillus fumigatus. Wild type mice almost completely cleared the fungal pathogen, while the single mutants showed an intermediate phenotype between the wild type and double mutants, thus establishing a critical role for both elastase and cathepsin $G$ in the control of fungal infection in vivo [89].

Examples of proteins stored in specific (secondary) and gelatinase (tertiary) granules include human lactoferrin and MMPs. Lactoferrin is a major component of the specific granules and is active against a variety of pathogens [90]. This protein binds to bacteria through its highly positively charged $\mathrm{N}$-terminus and displays antimicrobial properties against Gram-positive and Gram-negative bacteria by limiting the availability of environmental iron. However, since iron-saturated lactoferrin is also capable of killing certain bacteria, mechanisms other than iron depletion are involved. Further studies have indicated that peptides obtained after enzymatic hydrolysis of lactoferrin are much more effective in killing bacteria than is the intact protein. It is likely that the $N$-terminal cationic domain of human lactoferrin plays an essential role in the bactericidal activity and has been shown to be highly effective against infections with antibiotic-resistant $S$. aureus [91]. MMP-9 is stored in an inactive preform that requires activation by a serine protease such as NE. Its main function is the degradation of type $\mathrm{V}$ collagen in the extracellular matrix to aid migration to the site of infection [92]. The importance of MMP-9 in host defence was illustrated by a higher frequency of peritoneal sepsis in MMP-9 knockout mice due to impaired migration of neutrophils to the site of infection [93].

In spite of their original role in host defence, NE and proteinase 3 have been strongly implicated in the pulmonary pathology of CF. Indeed it has been shown that NE is the main mediator of proteolysis (Figure 1) but can also cause up-regulation of expression of other proteases including MMPs and cathepsins and as a result it has been proposed that neutralisation of NE activity is central to reducing the overall protease burden [94]. In line with this thought, NE 
has the ability to degrade structural proteins in the lung including elastin, collagen, and fibronectin and to promote IL-8 production by bronchial epithelial cells [95], to degrade antimicrobial peptides [96], and to degrade antiproteases including alpha-1 antitrypsin, SLPI [97], and elafin [98] leading to a protease/antiprotease imbalance [99]. Moreover, it has been shown that the process of primary granule release by $\mathrm{CF}$ neutrophils appears altered, as greater levels of NE [100] and MPO [101] were recorded in the extracellular fluids post stimulation with either CF airway samples, TNF-alpha and IL-8, or serum-opsonised particles. Of importance, altered cytosolic $\mathrm{pH}$ regulation in CF neutrophils has been demonstrated [102, 103], which could in turn influence the process of degranulation. In further support of increased primary granule release by CF neutrophils, MPO and NE levels have been reported to be present at significantly increased levels in airway samples from patients with CF compared to controls [49, 104]. Moreover, levels of NE degranulation were not significantly altered following intravenous antibiotic treatment of patients with $\mathrm{CF}$, indicating continued dysregulation of neutrophil activity even with clinical improvement [105]. While the mechanism for excessive primary granule release by CF neutrophils has not been fully investigated [104, 106], new research on the cause of reduced secondary and tertiary granule release has recently been revealed [35]. Contrary to increased release of secondary and tertiary granules by neutrophils of individuals with airway disease linked to alpha-1 antitrypsin deficiency (AATD) [107], evidence was presented indicating that abnormal CFTR function contributes to impaired neutrophil killing in CF due to inadequate Rab27a activation, which regulates the release of antimicrobial proteins from secondary and tertiary granules. In this study reduced degranulation of lactoferrin of secondary granules and MMP-9 of tertiary granules from patients with CF compared to healthy control cells was observed, an effect mirrored in healthy control cells post CFTR inhibition. Collectively results revealed that CFTR inhibition or dysfunction reduces cytosolic $\mathrm{Mg}^{2+}$ levels resulting in impaired Rab27a activity, ultimately reducing the CF neutrophils ability to kill bacterial pathogens [35].

\section{The involvement of CFTR in neutrophil adherence leading to migration}

Before describing the process leading to neutrophil adhesion, it is important to stress the complexity of studying neutrophil adhesion and migration. Blood neutrophils isolated from patients with CF are chronically exposed to pro-inflammatory cytokines, including $\mathrm{LTB}_{4}$ and IL-8, and pathogenic particles including fMLP (formyl-methionyl-leucyl-phenylalanine) and LPS, resulting in these cells being in a constant primed state. Therefore, independent of the expression of CFTR protein, neutrophils in CF may illustrate enhanced cell adherence and migration. To circumvent this dilemma and to eliminate the potential for bias towards inflammatory versus altered neutrophil adhesion due to a lack of CFTR function, an approach taken by researchers is to include neutrophils isolated from inflammatory control patients. For example, in 1998, Russell and colleagues demonstrated that L-selectin shedding is altered in patients with CF resulting in increased neutrophil adhesion in response to IL-8 and fMLP [108]. This paper reported that non-CF bronchiectasis patients did not possess alterations in Lselectin shedding, suggesting that the defect in L-selectin shedding is CF specific and could be 
a result of defective CFTR rather than the inflammatory status of the individual [108]. Indeed, CFTR expression in human neutrophils has provoked the idea that altered neutrophil migration and adhesion in CF could be caused by an intrinsic defect. Counteracting this concept however, Pohl et al. (2014) did not demonstrate impaired neutrophil migration in healthy control cells exposed to the CFTR inhibitor, CFTR(inh)-172, suggesting that altered neutrophil migration in $\mathrm{CF}$ is not inherent.

Circulating neutrophils generally adhere and migrate in response to pro-inflammatory mediators including TNF-alpha and pathogenic components including $\mathrm{N}$-formyl peptides produced by bacteria. Lipid mediators involved in neutrophil adhesion and chemotaxis include $\mathrm{LTB}_{4}[109,110]$, with significantly increased levels quantified in sputum of patients with CF [111]. The chemokine IL-8 is the main neutrophil chemo-attractant involved in CF lung neutrophil infiltration [112], and increased levels of IL-8 have been detected in bronchial lavage fluid and sputum of patients with CF [113]. IL-8 is produced by a number of cells, including fibroblasts [114], epithelial cells [115], and by neutrophils themselves [116]. Interestingly, neutrophils isolated from children with CF demonstrate increased migration to IL-8 [117], and also release significantly increased levels of the chemokine when compared to the blood neutrophils of the same donor, suggesting that the environment that the cell is found triggers disproportionate release of IL-8 [116].

Neutrophils migrate in a multistep process consisting of rolling, tight binding, diapedesis, and migration. Initially, E-selectin and P-selectin are upregulated on the epithelium cell surface, and reversibly bind to L-selectin found on the neutrophil cell surface. In turn, L-selectin is shed from the neutrophil membrane resulting in up-regulation of integrins LFA-1 (CD11a/CD18) and MAC-1 (CD11b/CD18) which can both bind to ICAM-1 on the epithelium. Research studies have demonstrated that activation of integrins is in part mediated by IL-8 [118], and this is particularly relevant in $\mathrm{CF}$ as studies involving infants and children have demonstrated increased expression of IL-8 and ICAM-1, possibly indicating intrinsic inflammatory changes at a very early stage in disease progression supporting cell adhesion $[119,120]$. This latter study is reinforced by data indicating that $\mathrm{CF}$ neutrophils show higher migratory responsiveness to IL-8 [117] supporting elevated numbers of neutrophils migrating to the airways. Moreover, neutrophil activation results in increased cytosolic $\mathrm{Ca}^{2+}$ levels triggering activation of calpain, a calcium dependent protease. Calpain has been demonstrated to liberate adhesion molecules $\mathrm{CD} 11 \mathrm{~b}$ and CD18, facilitating cell adhesion through the tight binding of integrins to epithelium cell surfaces [121]. Of major importance, studies have shown that CF neutrophils possess increased calpain activity, affecting cleavage of the cholesterol transporter caveolin-1, thereby modulating cholesterol trafficking to the plasma membrane [122]. Interestingly, and of major relevance to $\mathrm{CF}$, Solomkin et al. (2007) demonstrated that cholesterol depletion in human neutrophils results in increased cell adhesion [123].

\section{The involvement of CFTR in neutrophil apoptosis}

Timely and effective neutrophil programmed cell death is essential for the resolution of inflammation [124, 125] and abnormal neutrophil apoptosis is associated with decreased 
antimicrobial defences [126], incomplete microbial clearance, and sustained inflammation $[127,128]$. Three different pathways are involved in the regulation of apoptosis and particular attention will be given to signalling pathways that are relevant to the neutrophil as it has been recognised that neutrophils have unique mechanisms of cell death due to their short half-life and phagocytic activity (Figure 5). Essential to the regulation and execution of apoptosis are the caspases, a family of cytosolic proteases or cysteine-dependent aspartate-directed proteases, involved in all three pathways. The first pathway described is the mitochondrial or intrinsic pathway. This pathway responds to physical and chemical stress signals, including growth factor withdrawal, DNA damage, and endoplasmic stress, and is transduced by members of the Bcl-2 family, which ultimately trigger the mitochondrial outer membrane permeabilisation (MOMP). As a result, several pro-apoptotic mitochondrial proteins, the most important being cytochrome C, are released into the cytosol. Subsequently cytochrome C, apoptotic protease activating factor-1 (Apaf-1) and caspase-9 form the apoptosome [129-131], this results in the activation of caspase- 9 which thereafter activates caspase- 3 and initiates the execution of apoptosis. In addition, caspase- 8 can cleave Bid into truncated Bid (TBid), which ultimately triggers MOMP, thus providing a link from the intrinsic pathway to the extrinsic pathway.

The second pathway, the extrinsic or external death receptor pathway, is activated in response to extracellular signals such as FasL and TNF-alpha and is mediated by the binding of these members of the tumour necrosis factor family to death receptors on the cell surface (e.g., Fas, TNFR). The binding of the FasL and TNF-alpha to its cognate receptor results in the multimerisation of the death receptor and the formation of the death inducing signal complex (DISC), containing multiple adaptor molecules such as the Fas associated death domain (FADD) or TNF-R1-associated death domain (FADD) or TNF-R1-associtaed death domain (TRADD). The respective adaptor death domain then interacts with caspase-8 [132, 133], which in turn leads to the autolytic activation from pro-caspase- 8 to caspase- 8 . After activation and release of its active subunit (p18), caspase- 8 then activates caspase- 3 , which finally executes apoptosis by releasing caspase-activated DNase (CAD) from its inhibitor (ICAD) with DNA fragmentation as a consequence [134]. The third pathway is the ER stress pathway. This pathway is thought to involve the activation of caspases, increase in cytosolic calcium and proapoptotic transcription factors in response to stress signals such as hypoxia, accumulation of unfolded protein, and alteration in calcium homeostasis within the ER [135, 136].

In the neutrophil the enzymatic activity of active caspase-3, -7 , and -9 can be selectively inhibited by X-linked inhibitor of apoptosis (XIAP), a member of the conserved inhibitor of apoptosis (IAP) family of proteins [137]. Moreover, there are a number of key distinguishing features of neutrophil apoptosis. Firstly, the prominent role of the Bcl-2 homologue, Mcl-1, as a survival protein is central to the neutrophil's ability to undergo rapid apoptosis and may therefore limit the neutrophil's lifespan [138].

Mcl-1 is an unusual bcl-2 protein and can be rapidly turned over in the proteasome giving it a short half-life ( $2 \mathrm{~h}$ ) in the cell and is in contrast to the essential pro-apoptotic bcl-2 homologues, which are known to persist in the cell beyond $12 \mathrm{~h}$. A critical excess of pro- over anti-apoptotic homologues decides the fate of the neutrophil and this eventually leads to loss of mitochondrial membrane potential and the progression of apoptosis [139]. Secondly, the involvement of 


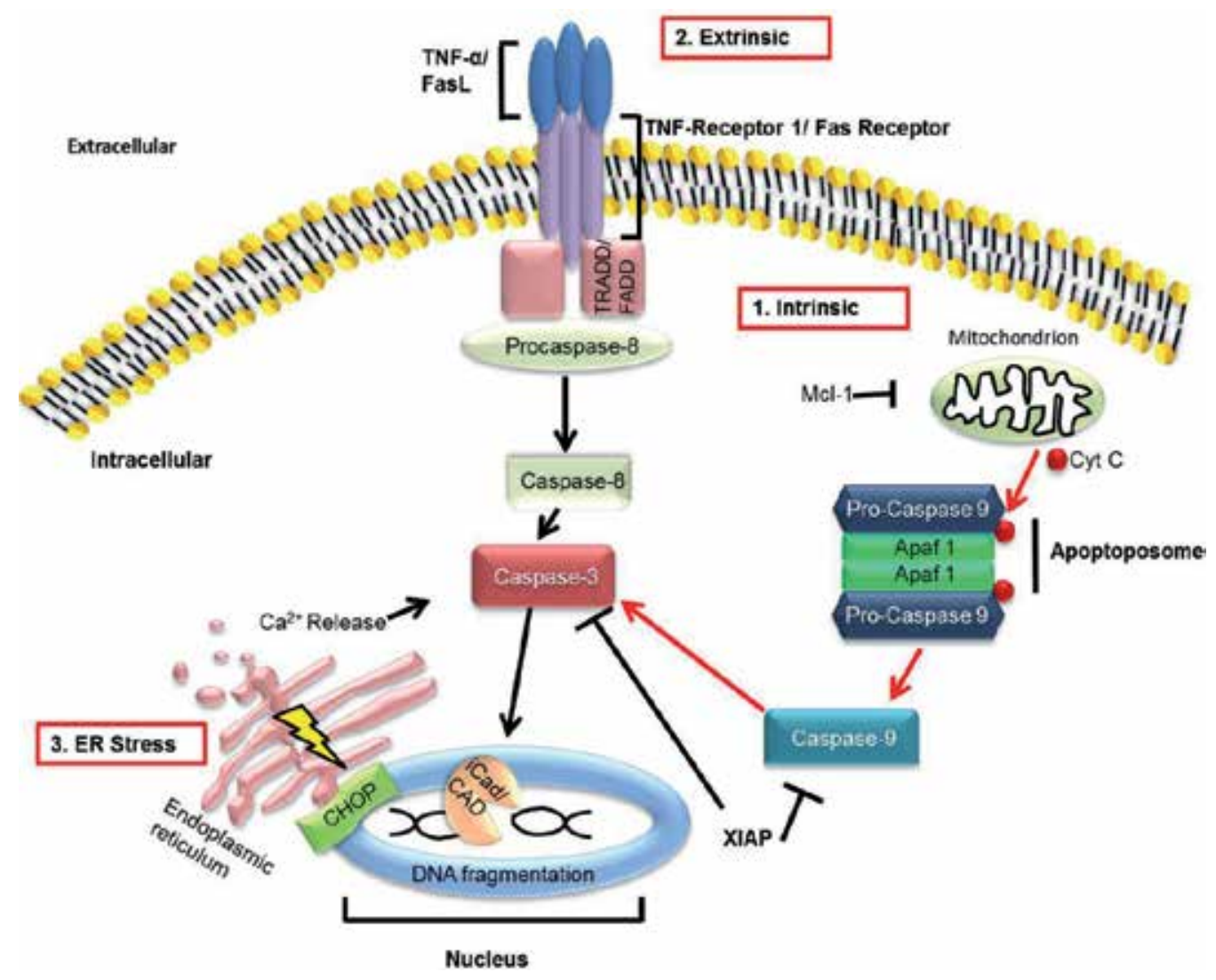

Figure 5. Summary schematic of apoptosis pathways in the neutrophil. [1] Intrinsic mitochondrial pathway (red): mitochondria release cytochrome $\mathrm{c}$ (Cyt C) in response to cellular stress. Together with apoptotic protease activating factor-1 (Apaf-1) and procaspase-9, Cyt $C$ will form the apoptosome complex. This results in the proteolytic activation of the procaspase. Mature caspase-9 can then proteolytically activate caspase-3. [2] Extrinsic/ligand death-receptor pathway (black): death factors such as tumour necrosis factor alpha (TNF-alpha) and Fas ligand (FasL) trigger apoptosis by binding on death receptors such as tumour necrosis factor receptor 1 (TNFR1) and Fas. The death receptors recruit procaspase- 8 by means of an adaptor protein, TNF-R1-associated death domain (TRADD), or Fas associated death domain protein (FADD). After cleavage the mature caspase-8 can then directly activate caspase-3. [3] Endoplasmic reticulum (ER) stress pathway (yellow bolt): the ER can also induce apoptosis as a reaction to ER stress by releasing intracytosolic calcium $\left(\mathrm{Ca}^{2+}\right)$ thereby activating effector caspases or through the expression of pro-apoptotic transcription factors such as CHOP. These different initiation pathways converge further downstream into activation of caspase- 3 . The effector caspase- 3 cleaves ICAD (inhibitor of $\mathrm{CAD}$ ) and releases it from CAD (caspase-activated DNase). CAD translocates from the cytoplasm to the nucleus and can now act as active endonuclease and fragment DNA. Inhibitors of apoptosis such as myeloid leukaemia cell differentiation protein (Mcl-1) and X-linked inhibitor of apoptosis (XIAP) act upstream against pro-apoptotic Bcl-2 family members at the mitochondria and downstream, directly inhibit caspases, respectively.

reactive oxygen species (ROS) is very specific to neutrophil apoptosis. The observation that neutrophils isolated from CGD patients which are known to be NADPH oxidase defective display a significant delayed spontaneous cell death relative to that of neutrophils from healthy donors is important [140]. This suggests that activation of the NADPH oxidase, with consequent production of ROS, is involved in spontaneous apoptosis and in regulating the programmed cell death of neutrophils during phagocytosis. 
In contrast to the reported accelerated apoptosis in neutrophils from patients with airways disease associated with AATD [141], prolonged neutrophil survival has been reported in people with CF independent of infectious state and mutation type [142]. In support of this concept, Moriceau et al. (2010) illustrated that neutrophils isolated from heterozygous asymptomatic parents of people with CF exhibited delayed apoptosis [143, 144]. Moreover, the expression of the apoptosis-inducing membrane receptor Fas and its ligand have been reported reduced on CF neutrophils [145] while in addition, NE can degrade the phosphatidylserine receptor on macrophages, further delaying the removal of apoptotic neutrophils in CF [146].

\section{The effect of CFTR mutation targeted therapy on neutrophil function}

There can be no doubt that our grasp and understanding of the complex physiology of CFTR protein function has progressed majorly and permitted the development of exciting new treatments designed to target the basic defects of CF. Along with this, continuing improvements in clinical care have allowed improved outcomes for patients with CF including reduced morbidity and preserved lung function. As CFTR has been shown to be an integral membrane channel on neutrophils, the influence of new CFTR therapeutics on neutrophil function is an area of intense interest.

Class I mutations are thought to affect $5 \%$ of the CF population in Western society and in this class of CFTR mutations, there is complete absence of stable CFTR protein (due to non-sense mutations from premature stop codons) and thus replacement of the defective CFTR gene or changes in how the protein is made is required. However, the focus relating to the research surrounding gene addition therapy is its potential use in all class mutations in $\mathrm{CF}$, with the ultimate aim of functional gene insertion and normalized CFTR expression. Studies on gene addition therapy have been developed to replace the mutant CFTR gene, and in this respect a phase II trial with non-viral lipid vector for DNA instillation has commenced. The study investigators have recommended monthly inhaled therapy for one-year duration (NCT 01621867). Further developments involve a lentiviral vector for gene therapy in this patient population [147] and the collective results of gene therapy on neutrophil function will be an exciting area of research in the future.

A second area of immense interest is the therapeutic use of aminoglycosides (e.g., gentamycin) and ataluren to cause read through of premature stop codons thereby allowing translation to continue to the end of transcription [148, 149]. Ataluren (PTC 124) is under investigation for its use and role in targeting premature stop codons. This compound has the potential to allow processing of premature stop codons, resulting in the production of normal length and functional CFTR, with insertion at the cell surface. Its beneficial effects have been proven with analysis of nasal chloride transport. The phase III trial in 238 patients with CF failed to achieve its primary end point (improvement in $\mathrm{FEV}_{1}$ ) at 48 weeks, except in a small subgroup of patients not on concomitant nebulised aminoglycoside treatment [150].

Class II (e.g., $\triangle F 508$ ) and III CFTR mutations (e.g., G551D) have seen the advent of CFTR corrector and potentiator therapies. Corrector agents facilitate appropriate protein folding and 
targeting to the cell membrane. The correctors lumacaftor (VX 809) or VX 661 are designed to promote increased quantities of $\triangle \mathrm{F} 508$ CFTR at the cell membrane surface. VX 809 has shown good results in vitro but it is likely that adequate correction of $\triangle$ F508 CFTR will need multicompound drug therapy $[147,151]$. The potentiator ivacaftor (VX 770) was originally developed to augment the activity and efficiency of the abnormal CFTR protein (Figure 6).

A

B

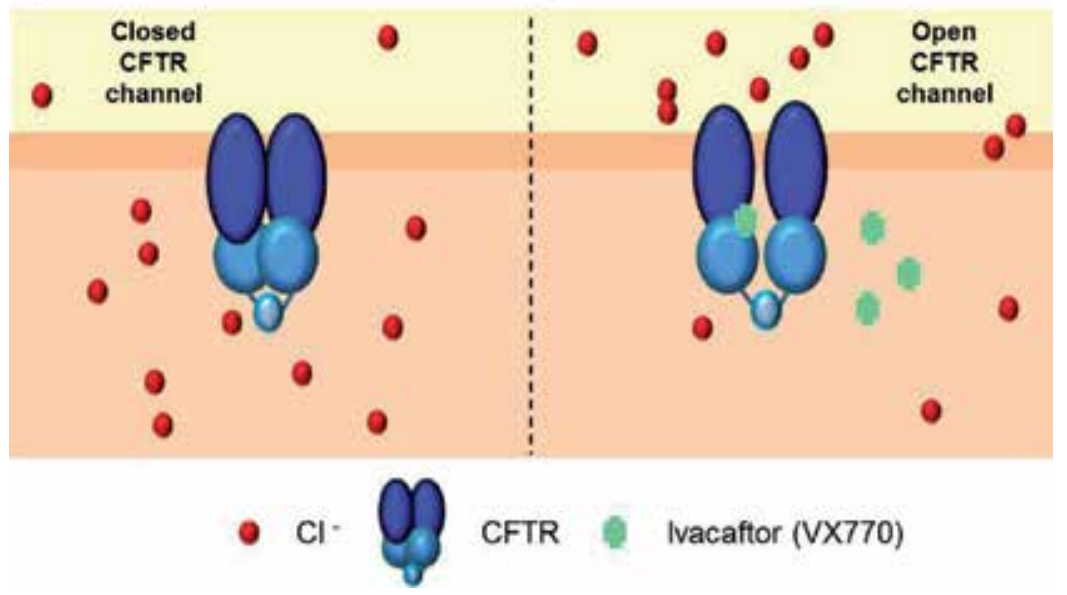

Figure 6. CFTR function in human epithelia. Panel (A) illustrates defective CFTR $\mathrm{Cl}^{-}$transport due to the G551D mutation. Image (B) illustrates corrected function post VX-770 (ivacaftor) treatment.

Use of this therapy involved a well-designed randomized, double blind, placebo-controlled trial. The study subjects had at least one G551D CFTR mutation and were randomly assigned to ivacaftor $150 \mathrm{mg}$ twice daily or placebo for 48 weeks. The treatment group showed a sustained improvement from baseline in FEV1 by $10 \%$ compared with the placebo group. They were also $55 \%$ less likely to suffer a pulmonary exacerbation compared with their placebo counter parts, had higher health scores, gained weight and normalization of their sweat chloride levels. These benefits were sustained for the duration of the trial and the frequency of adverse events in the two groups was equivocal [152]. A shortcoming of the use of ivacaftor is that only $2-3 \%$ of individuals with CF have the G551D mutation and research is currently underway to ascertain whether ivacaftor may be employed for other mutations. A multicentre, phase II trial on the use of the CFTR corrector lumacaftor together with the CFTR potentiator ivacaftor for the treatment of patients with CF with the $\triangle F 508$ CFTR mutation was performed. The primary outcome was change in sweat chloride concentration with combination therapy in the $\Delta F 508$ subjects, however a minimal effect on sweat chloride level was observed [153]. It is postulated that the reason behind this was that the potentiator rendered the CFTR protein less stable and increased its removal from the cell membrane. However, 
currently there is a very motivated area of research into double or triple combination treatment for synergistic $\triangle$ F508 CFTR correction [154].

Of relevance to the circulating neutrophil and as CFTR mRNA transcripts have been reported in neutrophils at an expression level similar to those found in monocytes and alveolar macrophages [24,33], the effect of CFTR corrector and potentiator therapies on neutrophil function is an anticipated area of research. In a study by Pohl et al. (2014), the mechanism leading to impaired degranulation by CF neutrophils was shown to involve altered ion homeostasis caused by defective CFTR function and significantly decreased levels of GTPbound Rab27a. Of major importance, treatment of G551D patients with ivacaftor, normalized neutrophil cytosolic ion levels, and activation of Rab27a thereby leading to increased degranulation and pseudomonal killing. These results confirm that intrinsic alterations of circulating neutrophils from patients with CF are corrected by ivacaftor thus illustrating additional clinical benefits for CFTR modulator therapy. In line with this concept, neutrophils of ivacaftor-treated subjects demonstrated decreased cell surface CD63, a marker of primary granule release [155].

\section{Conclusion}

A plethora of studies on neutrophil function in CF have been performed and demonstrate alterations in cellular activities including impaired microbial uptake [156, 157], defective intracellular kinase activation [116], cellular inactivation [158], and increased oxidant formation [106, 159]. Furthermore, an additional area of intense research has focused on persistent mammalian target of rapamycin (mTOR) and cyclic AMP response element binding protein (CREB) pathway activation in CF airway neutrophils [160], with more recent data suggesting that neutrophils express augmented cell surface nutrient transporter expression and glucose uptake, consistent with metabolic adaptation [161]. As the CF neutrophil may shape the inflammatory response and influence patient outcome, further research investigating the $\mathrm{CF}$ neutrophil is required. In addition, a promising development in the treatment of airway inflammation involves the correction of CFTR dysfunction. If CFTR dysfunction is corrected at a very early age, it is possible that neutrophil induced inflammation involving impaired trafficking, delayed apoptosis, impaired degranulation, and bacterial killing may be significantly curtailed.

\section{Acknowledgements}

We would like to thank the U.S. Cystic Fibrosis Foundation and Science Foundation Ireland under the Research Frontiers Programme (11/RFP/BMT/3094) and the Program for Research in Third Level Institutes administered by the Higher Education Authority in Ireland for support. 


\section{Author details}

Michelle M. White, Fatma Gargoum, Niall Browne, Killian Hurley, Noel G. McElvaney and Emer P. Reeves*

*Address all correspondence to: emerreeves@rcsi.ie

Respiratory Research Division, Department of Medicine, Royal College of Surgeons in Ireland, Education and Research Centre, Beaumont Hospital, Dublin, Ireland

\section{References}

[1] De Boeck K, Zolin A, Cuppens H, Olesen HV, Viviani L. The relative frequency of CFTR mutation classes in European patients with cystic fibrosis. J Cyst Fibros. 2014;13(4):403-9.

[2] Mott LS, Park J, Murray CP, Gangell CL, De Klerk NH, Robinson PJ, et al. Progression of early structural lung disease in young children with cystic fibrosis assessed using CT. Thorax. 2012;67(6):509-16.

[3] Sly PD, Brennan S, Gangell C, De Klerk N, Murray C, Mott L, et al. Lung disease at diagnosis in infants with cystic fibrosis detected by newborn screening. American Journal of Respiratory and Critical Care Medicine. 2009;180(2):146-52.

[4] Davis SD, Fordham LA, Brody AS, Noah TL, Retsch-Bogart GZ, Qaqish BF, et al. Computed tomography reflects lower airway inflammation and tracks changes in early cystic fibrosis. American Journal of Respiratory and Critical Care Medicine. 2007;175(9):943-50.

[5] Wainwright CE, Vidmar S, Armstrong DS, Byrnes CA, Carlin JB, Cheney J, et al. Effect of bronchoalveolar lavage-directed therapy on Pseudomonas aeruginosa infection and structural lung injury in children with cystic fibrosis: a randomized trial. JAMA : The Journal of the American Medical Association. 2011;306(2):163-71.

[6] Stick SM, Brennan S, Murray C, Douglas T, Von Ungern-Sternberg BS, Garratt LW, et al. Bronchiectasis in infants and preschool children diagnosed with cystic fibrosis after newborn screening. The Journal of Pediatrics. 2009;155(5):623-8 e1.

[7] Armstrong DS, Hook SM, Jamsen KM, Nixon GM, Carzino R, Carlin JB, et al. Lower airway inflammation in infants with cystic fibrosis detected by newborn screening. Pediatr Pulmonol. 2005;40(6):500-10.

[8] Rosenfeld M, Gibson RL, McNamara S, Emerson J, Burns JL, Castile R, et al. Early pulmonary infection, inflammation, and clinical outcomes in infants with cystic fibrosis. Pediatr Pulmonol. 2001;32(5):356-66. 
[9] Reeves EP, Williamson M, Byrne B, Bergin DA, Smith SG, Greally P, et al. IL-8 dictates glycosaminoglycan binding and stability of IL-18 in cystic fibrosis. J Immunol. 2010;184(3):1642-52.

[10] Mitola S, Sorbello V, Ponte E, Copreni E, Mascia C, Bardessono M, et al. Tumor necrosis factor-alpha in airway secretions from cystic fibrosis patients upregulate endothelial adhesion molecules and induce airway epithelial cell apoptosis: implications for cystic fibrosis lung disease. International Journal of Immunopathology and Pharmacology. 2008;21(4):851-65.

[11] Colombo C, Faelli N, Tirelli AS, Fortunato F, Biffi A, Claut L, et al. Analysis of inflammatory and immune response biomarkers in sputum and exhaled breath condensate by a multi-parametric biochip array in cystic fibrosis. International Journal of Immunopathology and Pharmacology. 2011;24(2):423-32.

[12] Birrer P, McElvaney NG, Rudeberg A, Sommer CW, Liechti-Gallati S, Kraemer R, et al. Protease-antiprotease imbalance in the lungs of children with cystic fibrosis. Am J Respir Crit Care Med. 1994;150(1):207-13.

[13] McElvaney NG, Nakamura H, Birrer P, Hebert CA, Wong WL, Alphonso M, et al. Modulation of airway inflammation in cystic fibrosis. In vivo suppression of interleukin-8 levels on the respiratory epithelial surface by aerosolization of recombinant secretory leukoprotease inhibitor. J Clin Invest. 1992;90(4):1296-301.

[14] Hartl D, Griese M, Kappler M, Zissel G, Reinhardt D, Rebhan C, et al. Pulmonary $\mathrm{T}(\mathrm{H}) 2$ response in Pseudomonas aeruginosa-infected patients with cystic fibrosis. J Allergy Clin Immunol. 2006;117(1):204-11.

[15] Reinhardt N, Chen CI, Loppow D, Schink T, Kleinau I, Jorres RA, et al. Cellular profiles of induced sputum in children with stable cystic fibrosis: comparison with BAL. Eur Respir J. 2003;22(3):497-502.

[16] Sly PD, Gangell CL, Chen L, Ware RS, Ranganathan S, Mott LS, et al. Risk factors for bronchiectasis in children with cystic fibrosis. N Engl J Med. 2013;368(21):1963-70.

[17] Adib-Conquy M, Pedron T, Petit-Bertron AF, Tabary O, Corvol H, Jacquot J, et al. Neutrophils in cystic fibrosis display a distinct gene expression pattern. Mol Med. 2008;14(1-2):36-44.

[18] Kormann MS, Hector A, Marcos V, Mays LE, Kappler M, Illig T, et al. CXCR1 and CXCR2 haplotypes synergistically modulate cystic fibrosis lung disease. Eur Respir J. 2012;39(6):1385-90.

[19] Jann NJ, Schmaler M, Ferracin F, Landmann R. TLR2 enhances NADPH oxidase activity and killing of Staphylococcus aureus by PMN. Immunol Lett. 2010;135(1-2): $17-23$. 
[20] Petit-Bertron AF, Tabary O, Corvol H, Jacquot J, Clement A, Cavaillon JM, et al. Circulating and airway neutrophils in cystic fibrosis display different TLR expression and responsiveness to interleukin-10. Cytokine. 2008;41(1):54-60.

[21] Gu Y, Harley IT, Henderson LB, Aronow BJ, Vietor I, Huber LA, et al. Identification of IFRD1 as a modifier gene for cystic fibrosis lung disease. Nature. 2009;458(7241): 1039-42.

[22] Blanchard E, Marie S, Riffault L, Bonora M, Tabary O, Clement A, et al. Reduced expression of Tis7/IFRD1 protein in murine and human cystic fibrosis airway epithelial cell models homozygous for the F508del-CFTR mutation. Biochem Biophys Res Commun. 2011;411(3):471-6.

[23] Riordan JR, Rommens JM, Kerem B, Alon N, Rozmahel R, Grzelczak Z, et al. Identification of the cystic fibrosis gene: cloning and characterization of complementary DNA. Science. 1989;245(4922):1066-73.

[24] Yoshimura K, Nakamura H, Trapnell BC, Chu CS, Dalemans W, Pavirani A, et al. Expression of the cystic fibrosis transmembrane conductance regulator gene in cells of non-epithelial origin. Nucleic Acids Res. 1991;19(19):5417-23.

[25] Trapnell BC, Chu CS, Paakko PK, Banks TC, Yoshimura K, Ferrans VJ, et al. Expression of the cystic fibrosis transmembrane conductance regulator gene in the respiratory tract of normal individuals and individuals with cystic fibrosis. Proc Natl Acad Sci USA. 1991;88(15):6565-9.

[26] Yoshimura K, Nakamura H, Trapnell BC, Dalemans W, Pavirani A, Lecocq JP, et al. The cystic fibrosis gene has a "housekeeping"-type promoter and is expressed at low levels in cells of epithelial origin. J Biol Chem. 1991;266(14):9140-4.

[27] Di A, Brown ME, Deriy LV, Li C, Szeto FL, Chen Y, et al. CFTR regulates phagosome acidification in macrophages and alters bactericidal activity. Nat Cell Biol. 2006;8(9): 933-44.

[28] Haggie PM, Verkman AS. Cystic fibrosis transmembrane conductance regulator-independent phagosomal acidification in macrophages. J Biol Chem. 2007;282(43): 31422-8.

[29] Steinberg BE, Huynh KK, Brodovitch A, Jabs S, Stauber T, Jentsch TJ, et al. A cation counterflux supports lysosomal acidification. J Cell Biol. 2010;189(7):1171-86.

[30] Del Porto P, Cifani N, Guarnieri S, Di Domenico EG, Mariggio MA, Spadaro F, et al. Dysfunctional CFTR alters the bactericidal activity of human macrophages against Pseudomonas aeruginosa. PLoS One. 2011;6(5):e19970.

[31] McKeon DJ, Cadwallader KA, Idris S, Cowburn AS, Pasteur MC, Barker H, et al. Cystic fibrosis neutrophils have normal intrinsic reactive oxygen species generation. Eur Respir J. 2010;35(6):1264-72. 
[32] Morris MR, Doull IJ, Dewitt S, Hallett MB. Reduced iC3b-mediated phagocytotic capacity of pulmonary neutrophils in cystic fibrosis. Clin Exp Immunol. 2005;142(1): 68-75.

[33] Painter RG, Valentine VG, Lanson NA, Jr., Leidal K, Zhang Q, Lombard G, et al. CFTR Expression in human neutrophils and the phagolysosomal chlorination defect in cystic fibrosis. Biochemistry. 2006;45(34):10260-9.

[34] Painter RG, Marrero L, Lombard GA, Valentine VG, Nauseef WM, Wang G. CFTRmediated halide transport in phagosomes of human neutrophils. J Leukoc Biol. 2010;87(5):933-42.

[35] Pohl K, Hayes E, Keenan J, Henry M, Meleady P, Molloy K, et al. A neutrophil intrinsic impairment affecting Rab27a and degranulation in cystic fibrosis is corrected by CFTR potentiator therapy. Blood. 2014;124(7):999-1009.

[36] Su X, Looney MR, Su HE, Lee JW, Song Y, Matthay MA. Role of CFTR expressed by neutrophils in modulating acute lung inflammation and injury in mice. Inflamm Res. 2011;60(7):619-32.

[37] Baldridge C, Gerard, RW. The extra respiratory burst of phagocytosis. Am J Phsiol. 1933;103:235-6.

[38] Sbarra AJ, Karnovsky ML. The biochemical basis of phagocytosis. I. Metabolic changes during the ingestion of particles by polymorphonuclear leukocytes. J Biol Chem. 1959;234(6):1355-62.

[39] Good RA, Quie PG, Windhorst DB, Page AR, Rodey GE, White J, et al. Fatal (chronic) granulomatous disease of childhood: a hereditary defect of leukocyte function. Semin Hematol. 1968;5(3):215-54.

[40] Holmes B, Page AR, Good RA. Studies of the metabolic activity of leukocytes from patients with a genetic abnormality of phagocytic function. J Clin Invest. 1967;46(9): 1422-32.

[41] Cohen MS, Metcalf JA, Root RK. Regulation of oxygen metabolism in human granulocytes: relationship between stimulus binding and oxidative response using plant lectins as probes. Blood. 1980;55(6):1003-10.

[42] Root RK, Metcalf JA. H2O2 release from human granulocytes during phagocytosis. Relationship to superoxide anion formation and cellular catabolism of $\mathrm{H} 2 \mathrm{O} 2$ : studies with normal and cytochalasin B-treated cells. J Clin Invest. 1977;60(6):1266-79.

[43] Iyer GYN IM, Quastel JH. Biochemical Aspects of Phagocytosis. Nature. 1961;192:535-41

[44] Galli F, Battistoni A, Gambari R, Pompella A, Bragonzi A, Pilolli F, et al. Oxidative stress and antioxidant therapy in cystic fibrosis. Biochim Biophys Acta. 2012;1822(5): 690-713. 
[45] Griese M, Ramakers J, Krasselt A, Starosta V, Van Koningsbruggen S, Fischer R, et al. Improvement of alveolar glutathione and lung function but not oxidative state in cystic fibrosis. Am J Respir Crit Care Med. 2004;169(7):822-8.

[46] Roum JH, Borok Z, McElvaney NG, Grimes GJ, Bokser AD, Buhl R, et al. Glutathione aerosol suppresses lung epithelial surface inflammatory cell-derived oxidants in cystic fibrosis. J Appl Physiol (1985). 1999;87(1):438-43.

[47] Roum JH, Buhl R, McElvaney NG, Borok Z, Crystal RG. Systemic deficiency of glutathione in cystic fibrosis. J Appl Physiol (1985). 1993;75(6):2419-24.

[48] Linsdell P, Hanrahan JW. Glutathione permeability of CFTR. Am J Physiol. 1998;275(1 Pt 1):C323-6.

[49] Kettle AJ, Chan T, Osberg I, Senthilmohan R, Chapman AL, Mocatta TJ, et al. Myeloperoxidase and protein oxidation in the airways of young children with cystic fibrosis. Am J Respir Crit Care Med. 2004;170(12):1317-23.

[50] Klebanoff SJ. Iodination of bacteria: a bactericidal mechanism. J Exp Med. 1967;126(6):1063-78.

[51] Klebanoff SJ. Myeloperoxidase-halide-hydrogen peroxide antibacterial system. J Bacteriol. 1968;95(6):2131-8.

[52] Winterbourn CC, Hampton MB, Livesey JH, Kettle AJ. Modeling the reactions of superoxide and myeloperoxidase in the neutrophil phagosome: implications for microbial killing. J Biol Chem. 2006;281(52):39860-9.

[53] Bonvillain RW, Painter RG, Adams DE, Viswanathan A, Lanson NA, Jr., Wang G. RNA interference against CFTR affects HL60-derived neutrophil microbicidal function. Free Radic Biol Med. 2010;49(12):1872-80.

[54] Moreland JG, Davis AP, Bailey G, Nauseef WM, Lamb FS. Anion channels, including $\mathrm{ClC}-3$, are required for normal neutrophil oxidative function, phagocytosis, and transendothelial migration. J Biol Chem. 2006;281(18):12277-88.

[55] Aiken ML, Painter RG, Zhou Y, Wang G. Chloride transport in functionally active phagosomes isolated from Human neutrophils. Free Radic Biol Med. 2012;53(12): 2308-17.

[56] Jiang Q, Griffin DA, Barofsky DF, Hurst JK. Intraphagosomal chlorination dynamics and yields determined using unique fluorescent bacterial mimics. Chemical Research in toxicology. 1997;10(10):1080-9.

[57] Barrette WC, Jr., Hannum DM, Wheeler WD, Hurst JK. General mechanism for the bacterial toxicity of hypochlorous acid: abolition of ATP production. Biochemistry. 1989;28(23):9172-8. 
[58] Albrich JM, Gilbaugh JH, 3rd, Callahan KB, Hurst JK. Effects of the putative neutrophil-generated toxin, hypochlorous acid, on membrane permeability and transport systems of Escherichia coli. J Clin Invest. 1986;78(1):177-84.

[59] Gottardi W, Nagl M. Chlorine covers on living bacteria: the initial step in antimicrobial action of active chlorine compounds. J Antimicrob Chemother. 2005;55(4):475-82.

[60] Gottardi W, Nagl M. N-chlorotaurine, a natural antiseptic with outstanding tolerability. J Antimicrob Chemother. 2010;65(3):399-409.

[61] Painter RG, Bonvillain RW, Valentine VG, Lombard GA, LaPlace SG, Nauseef WM, et al. The role of chloride anion and CFTR in killing of Pseudomonas aeruginosa by normal and CF neutrophils. J Leukoc Biol. 2008;83(6):1345-53.

[62] Ng HP, Zhou Y, Song K, Hodges CA, Drumm ML, Wang G. Neutrophil-mediated phagocytic host defense defect in myeloid Cftr-inactivated mice. PLoS One. 2014;9(9):e106813.

[63] Parry MF, Root RK, Metcalf JA, Delaney KK, Kaplow LS, Richar WJ. Myeloperoxidase deficiency: prevalence and clinical significance. Annals of Internal Medicine. 1981;95(3):293-301.

[64] Lehrer RI, Hanifin J, Cline MJ. Defective bactericidal activity in myeloperoxidase-deficient human neutrophils. Nature. 1969;223(5201):78-9.

[65] Reeves EP, Lu H, Jacobs HL, Messina CG, Bolsover S, Gabella G, et al. Killing activity of neutrophils is mediated through activation of proteases by $\mathrm{K}+$ flux. Nature. 2002;416(6878):291-7.

[66] Segal AW, Geisow M, Garcia R, Harper A, Miller R. The respiratory burst of phagocytic cells is associated with a rise in vacuolar pH. Nature. 1981;290(5805):406-9.

[67] Mandell GL. Bactericidal activity of aerobic and anaerobic polymorphonuclear neutrophils. Infect Immun. 1974;9(2):337-41.

[68] Hirsch JG. Phagocytin: a bactericidal substance from polymorphonuclear leucocytes. J Exp Med. 1956;103(5):589-611.

[69] Zucker-Franklin D, Hirsch JG. Electron microscope studies on the degranulation of rabbit peritoneal leukocytes during phagocytosis. J Exp Med. 1964;120:569-76.

[70] Fouret P, Du Bois RM, Bernaudin JF, Takahashi H, Ferrans VJ, Crystal RG. Expression of the neutrophil elastase gene during human bone marrow cell differentiation. J Exp Med. 1989;169(3):833-45.

[71] Lacy P. Mechanisms of degranulation in neutrophils. Allergy, asthma, and clinical immunology. Official Journal of the Canadian Society of Allergy and Clinical Immunology. 2006;2(3):98-108. 
[72] Esaguy N, Aguas AP, Silva MT. High-resolution localization of lactoferrin in human neutrophils: labeling of secondary granules and cell heterogeneity. J Leukoc Biol. 1989;46(1):51-62.

[73] Wang YB. Antibacterial mechanisms of lysozyme on Streptococcus mutans. Zhonghua ya yi xue hui za zhi Chinese Dental Journal / Dental Association, Republic of China. 1990;9(3):87-97.

[74] Gaggar A, Li Y, Weathington N, Winkler M, Kong M, Jackson P, et al. Matrix metalloprotease-9 dysregulation in lower airway secretions of cystic fibrosis patients. Am J Physiol Lung Cell Mol Physiol. 2007;293(1):L96-L104.

[75] Nie J, Pei D. Direct activation of pro-matrix metalloproteinase-2 by leukolysin/ membrane-type 6 matrix metalloproteinase/matrix metalloproteinase 25 at the asn(109)-Tyr bond. Cancer Res. 2003;63(20):6758-62.

[76] Robineaux J, Frederic J. [Contribution to the study of neutrophil granulations of the polynuclears by phase contrast microcinematography]. Comptes rendus des seances de la Societe de biologie et de ses filiales. 1955;149(5-6):486-9.

[77] Hirsch JG. Cinemicrophotographic observations on granule lysis in polymorphonuclear leucocytes during phagocytosis. J Exp Med. 1962;116:827-34.

[78] Neeft M, Wieffer M, De Jong AS, Negroiu G, Metz CH, Van Loon A, et al. Munc13-4 is an effector of rab27a and controls secretion of lysosomes in hematopoietic cells. Mol Biol Cell. 2005;16(2):731-41.

[79] Borregaard N, Sorensen OE, Theilgaard-Monch K. Neutrophil granules: a library of innate immunity proteins. Trends Immunol. 2007;28(8):340-5.

[80] Mollinedo F, Martin-Martin B, Calafat J, Nabokina SM, Lazo PA. Role of vesicle-associated membrane protein-2, through Q-soluble N-ethylmaleimide-sensitive factor attachment protein receptor/R-soluble N-ethylmaleimide-sensitive factor attachment protein receptor interaction, in the exocytosis of specific and tertiary granules of human neutrophils. J Immunol. 2003;170(2):1034-42.

[81] Stow JL, Manderson AP, Murray RZ. SNAREing immunity: the role of SNAREs in the immune system. Nat Rev Immunol. 2006;6(12):919-29.

[82] Abdel-Latif D, Steward M, Macdonald DL, Francis GA, Dinauer MC, Lacy P. Rac2 is critical for neutrophil primary granule exocytosis. Blood. 2004;104(3):832-9.

[83] Munafo DB, Johnson JL, Ellis BA, Rutschmann S, Beutler B, Catz SD. Rab27a is a key component of the secretory machinery of azurophilic granules in granulocytes. Biochem J. 2007;402(2):229-39.

[84] Brzezinska AA, Johnson JL, Munafo DB, Crozat K, Beutler B, Kiosses WB, et al. The Rab27a effectors JFC1/Slp1 and Munc13-4 regulate exocytosis of neutrophil granules. Traffic. 2008;9(12):2151-64. 
[85] Belaaouaj A, McCarthy R, Baumann M, Gao Z, Ley TJ, Abraham SN, et al. Mice lacking neutrophil elastase reveal impaired host defense against gram negative bacterial sepsis. Nat Med. 1998;4(5):615-8.

[86] Belaaouaj A, Kim KS, Shapiro SD. Degradation of outer membrane protein A in Escherichia coli killing by neutrophil elastase. Science. 2000;289(5482):1185-8.

[87] Odeberg H, Olsson I. Antibacterial activity of cationic proteins from human granulocytes. J Clin Invest. 1975;56(5):1118-24.

[88] Shafer WM, Onunka VC, Martin LE. Antigonococcal activity of human neutrophil cathepsin G. Infect Immun. 1986;54(1):184-8.

[89] Tkalcevic J, Novelli M, Phylactides M, Iredale JP, Segal AW, Roes J. Impaired immunity and enhanced resistance to endotoxin in the absence of neutrophil elastase and cathepsin G. Immunity. 2000;12(2):201-10.

[90] Arnold RR, Brewer M, Gauthier JJ. Bactericidal activity of human lactoferrin: sensitivity of a variety of microorganisms. Infect Immun. 1980;28(3):893-8.

[91] Nibbering PH, Ravensbergen E, Welling MM, Van Berkel LA, Van Berkel PH, Pauwels EK, et al. Human lactoferrin and peptides derived from its $\mathrm{N}$ terminus are highly effective against infections with antibiotic-resistant bacteria. Infect Immun. 2001;69(3):1469-76.

[92] Delclaux C, Delacourt C, D'Ortho MP, Boyer V, Lafuma C, Harf A. Role of gelatinase $\mathrm{B}$ and elastase in human polymorphonuclear neutrophil migration across basement membrane. Am J Respir Cell Mol Biol. 1996;14(3):288-95.

[93] Renckens R, Roelofs JJ, Florquin S, De Vos AF, Lijnen HR, Van't Veer C, et al. Matrix metalloproteinase-9 deficiency impairs host defense against abdominal sepsis. J Immunol. 2006;176(6):3735-41.

[94] Geraghty P, Greene CM, O'Mahony M, O'Neill SJ, Taggart CC, McElvaney NG. Secretory leucocyte protease inhibitor inhibits interferon-gamma-induced cathepsin $\mathrm{S}$ expression. J Biol Chem. 2007;282(46):33389-95.

[95] Walsh DE, Greene CM, Carroll TP, Taggart CC, Gallagher PM, O'Neill SJ, et al. Interleukin-8 up-regulation by neutrophil elastase is mediated by MyD88/IRAK/TRAF-6 in human bronchial epithelium. J Biol Chem. 2001;276(38):35494-9.

[96] Bergsson G, Reeves EP, McNally P, Chotirmall SH, Greene CM, Greally P, et al. LL-37 complexation with glycosaminoglycans in cystic fibrosis lungs inhibits antimicrobial activity, which can be restored by hypertonic saline. J Immunol. 2009;183(1): 543-51.

[97] Weldon S, McNally P, McElvaney NG, Elborn JS, McAuley DF, Wartelle J, et al. Decreased levels of secretory leucoprotease inhibitor in the Pseudomonas-infected cyst- 
ic fibrosis lung are due to neutrophil elastase degradation. J Immunol. 2009;183(12): 8148-56.

[98] Guyot N, Butler MW, McNally P, Weldon S, Greene CM, Levine RL, et al. Elafin, an elastase-specific inhibitor, is cleaved by its cognate enzyme neutrophil elastase in sputum from individuals with cystic fibrosis. J Biol Chem. 2008;283(47):32377-85.

[99] Birrer P, McElvaney NG, Rudeberg A, Sommer CW, Liechti-Gallati S, Kraemer R, et al. Protease-antiprotease imbalance in the lungs of children with cystic fibrosis. Am J Respir Crit Care Med. 1994;150(1):207-13.

[100] Taggart C, Coakley RJ, Greally P, Canny G, O'Neill SJ, McElvaney NG. Increased elastase release by $\mathrm{CF}$ neutrophils is mediated by tumor necrosis factor-alpha and interleukin-8. Am J Physiol Lung Cell Mol Physiol. 2000;278(1): L33-41.

[101] Koller DY, Urbanek R, Gotz M. Increased degranulation of eosinophil and neutrophil granulocytes in cystic fibrosis. Am J Respir Crit Care Med. 1995;152(2):629-33.

[102] Coakley RJ, Taggart C, Canny G, Greally P, O'Neill SJ, McElvaney NG. Altered intracellular $\mathrm{pH}$ regulation in neutrophils from patients with cystic fibrosis. Am J Physiol Lung Cell Mol Physiol. 2000;279(1):L66-74.

[103] Coakley RJ, Taggart C, McElvaney NG, O'Neill SJ. Cytosolic pH and the inflammatory microenvironment modulate cell death in human neutrophils after phagocytosis. Blood. 2002;100(9):3383-91.

[104] Taggart C, Coakley RJ, Greally P, Canny G, O'Neill SJ, McElvaney NG. Increased elastase release by CF neutrophils is mediated by tumor necrosis factor-alpha and interleukin-8. Am J Physiol Lung Cell Mol Physiol. 2000;278(1):L33-41.

[105] Brockbank S, Downey D, Elborn JS, Ennis M. Effect of cystic fibrosis exacerbations on neutrophil function. Int Immunopharmacol. 2005;5(3):601-8.

[106] Witko-Sarsat V, Allen RC, Paulais M, Nguyen AT, Bessou G, Lenoir G, et al. Disturbed myeloperoxidase-dependent activity of neutrophils in cystic fibrosis homozygotes and heterozygotes, and its correction by amiloride. J Immunol. 1996;157(6): 2728-35.

[107] Bergin DA, Reeves EP, Hurley K, Wolfe R, Jameel R, Fitzgerald S, et al. The circulating proteinase inhibitor alpha-1 antitrypsin regulates neutrophil degranulation and autoimmunity. Sci Transl Med. 2014;6(217):217ra1.

[108] Russell KJ, McRedmond J, Mukherji N, Costello C, Keatings V, Linnane S, et al. Neutrophil adhesion molecule surface expression and responsiveness in cystic fibrosis. Am J Respir Crit Care Med. 1998;157(3 Pt 1):756-61.

[109] Hoover RL, Karnovsky MJ, Austen KF, Corey EJ, Lewis RA. Leukotriene B4 action on endothelium mediates augmented neutrophil/endothelial adhesion. Proc Natl Acad Sci USA. 1984;81(7):2191-3. 
[110] Palmblad J, Malmsten CL, Uden AM, Radmark O, Engstedt L, Samuelsson B. Leukotriene B4 is a potent and stereospecific stimulator of neutrophil chemotaxis and adherence. Blood. 1981;58(3):658-61.

[111] O'Driscoll BR, Cromwell O, Kay AB. Sputum leukotrienes in obstructive airways diseases. Clin Exp Immunol. 1984;55(2):397-404.

[112] Dai Y, Dean TP, Church MK, Warner JO, Shute JK. Desensitisation of neutrophil responses by systemic interleukin 8 in cystic fibrosis. Thorax. 1994;49(9):867-71.

[113] Dean TP, Dai Y, Shute JK, Church MK, Warner JO. Interleukin-8 concentrations are elevated in bronchoalveolar lavage, sputum, and sera of children with cystic fibrosis. Pediatr Res. 1993;34(2):159-61.

[114] Smith RS, Fedyk ER, Springer TA, Mukaida N, Iglewski BH, Phipps RP. IL-8 production in human lung fibroblasts and epithelial cells activated by the Pseudomonas autoinducer N-3-oxododecanoyl homoserine lactone is transcriptionally regulated by NF-kappa B and activator protein-2. J Immunol. 2001;167(1):366-74.

[115] DiMango E, Zar HJ, Bryan R, Prince A. Diverse Pseudomonas aeruginosa gene products stimulate respiratory epithelial cells to produce interleukin-8. J Clin Invest. 1995;96(5):2204-10.

[116] Corvol H, Fitting C, Chadelat K, Jacquot J, Tabary O, Boule M, et al. Distinct cytokine production by lung and blood neutrophils from children with cystic fibrosis. Am J Physiol Lung Cell Mol Physiol. 2003;284(6):L997-1003.

[117] Brennan S, Cooper D, Sly PD. Directed neutrophil migration to IL-8 is increased in cystic fibrosis: a study of the effect of erythromycin. Thorax. 2001;56(1):62-4.

[118] Takami M, Terry V, Petruzzelli L. Signaling pathways involved in IL-8-dependent activation of adhesion through Mac-1. J Immunol. 2002;168(9):4559-66.

[119] Khan TZ, Wagener JS, Bost T, Martinez J, Accurso FJ, Riches DW. Early pulmonary inflammation in infants with cystic fibrosis. Am J Respir Crit Care Med. 1995;151(4): 1075-82.

[120] Verhaeghe C, Delbecque K, De Leval L, Oury C, Bours V. Early inflammation in the airways of a cystic fibrosis foetus. J Cyst Fibros. 2007;6(4):304-8.

[121] Dewitt S, Hallett MB. Cytosolic free $\mathrm{Ca}(2+)$ changes and calpain activation are required for beta integrin-accelerated phagocytosis by human neutrophils. J Cell Biol. 2002;159(1):181-9.

[122] White M AB, Cox S, McElvaney NG, Reeves EP. A neutrophil inflammatory impairment affects cell structure in cystic fibrosis and is indirectly corrected by CFTR potentiator therapy. The 28th Annual North American Cystic Fibrosis Conference; September 2014; Georgia World Congress Center, Atlanta, Georgia, USA Ped Pulmonol; 2014. p. ISSN 8755-6863. 
[123] Solomkin JS, Robinson CT, Cave CM, Ehmer B, Lentsch AB. Alterations in membrane cholesterol cause mobilization of lipid rafts from specific granules and prime human neutrophils for enhanced adherence-dependent oxidant production. Shock. 2007;28(3):334-8 .

[124] Witko-Sarsat V, Pederzoli-Ribeil M, Hirsch E, Sozzani S, Cassatella MA. Regulating neutrophil apoptosis: new players enter the game. Trends Immunol. 2011;32(3): 117-24.

[125] El Kebir D, Filep JG. Modulation of neutrophil apoptosis and the resolution of inflammation through beta2 Integrins. Front Immunol. 2013;4:60.

[126] Kennedy AD, DeLeo FR. Neutrophil apoptosis and the resolution of infection. Immunolog Res. 2009;43(1-3):25-61.

[127] Savill JS, Wyllie AH, Henson JE, Walport MJ, Henson PM, Haslett C. Macrophage phagocytosis of aging neutrophils in inflammation. Programmed cell death in the neutrophil leads to its recognition by macrophages. J Clin Invest. 1989;83(3):865-75.

[128] Nathan C. Neutrophils and immunity: challenges and opportunities. Nature Rev Immunology. 2006;6(3):173-82.

[129] Li P, Nijhawan D, Budihardjo I, Srinivasula SM, Ahmad M, Alnemri ES, et al. Cytochrome $\mathrm{c}$ and dATP-dependent formation of Apaf-1/caspase-9 complex initiates an apoptotic protease cascade. Cell. 1997;91(4):479-89.

[130] Liu X, Kim CN, Yang J, Jemmerson R, Wang X. Induction of apoptotic program in cell-free extracts: requirement for dATP and cytochrome c. Cell. 1996;86(1):147-57.

[131] Zou H, Henzel WJ, Liu X, Lutschg A, Wang X. Apaf-1, a human protein homologous to C. elegans CED-4, participates in cytochrome c-dependent activation of caspase-3. Cell. 1997;90(3):405-13.

[132] Kuwana T, Smith JJ, Muzio M, Dixit V, Newmeyer DD, Kornbluth S. Apoptosis induction by caspase- 8 is amplified through the mitochondrial release of cytochrome c. J Biol Chem. 1998;273(26):16589-94.

[133] Muzio M, Chinnaiyan AM, Kischkel FC, O'Rourke K, Shevchenko A, Ni J, et al. FLICE, a novel FADD-homologous ICE/CED-3-like protease, is recruited to the CD95 (Fas/APO-1) death--inducing signaling complex. Cell. 1996;85(6):817-27.

[134] Tang D, Kidd VJ. Cleavage of DFF-45/ICAD by multiple caspases is essential for its function during apoptosis. J Biol Chem. 1998;273(44):28549-52.

[135] Han J, Murthy R, Wood B, Song B, Wang S, Sun B, et al. ER stress signalling through eIF2alpha and CHOP, but not IRE1alpha, attenuates adipogenesis in mice. Diabetologia. 2013;56(4):911-24. 
[136] Rao RV, Hermel E, Castro-Obregon S, Del Rio G, Ellerby LM, Ellerby HM, et al. Coupling endoplasmic reticulum stress to the cell death program. Mechanism of caspase activation. J Biol Chem. 2001;276(36):33869-74.

[137] Altieri DC. Survivin and IAP proteins in cell-death mechanisms. Biochem J. 2010;430(2):199-205.

[138] Edwards SW, Derouet M, Howse M, Moots RJ. Regulation of neutrophil apoptosis by Mcl-1. Biochem Soc Trans. 2004;32(Pt3):489-92.

[139] Kroemer G, Galluzzi L, Brenner C. Mitochondrial membrane permeabilization in cell death. Physiol Rev. 2007;87(1):99-163.

[140] Kasahara Y, Iwai K, Yachie A, Ohta K, Konno A, Seki H, et al. Involvement of reactive oxygen intermediates in spontaneous and CD95 (Fas/APO-1)-mediated apoptosis of neutrophils. Blood. 1997;89(5):1748-53.

[141] Hurley K, Lacey N, O'Dwyer CA, Bergin DA, McElvaney OJ, O'Brien ME, et al. Alpha-1 antitrypsin augmentation therapy corrects accelerated neutrophil apoptosis in deficient individuals. J Immunol. 2014;193(8):3978-91.

[142] McKeon DJ, Condliffe AM, Cowburn AS, Cadwallader KC, Farahi N, Bilton D, et al. Prolonged survival of neutrophils from patients with Delta F508 CFTR mutations. Thorax. 2008;63(7):660-1.

[143] Moriceau S, Kantari C, Mocek J, Davezac N, Gabillet J, Guerrera IC, et al. Coronin-1 is associated with neutrophil survival and is cleaved during apoptosis: potential implication in neutrophils from cystic fibrosis patients. J Immunol. 2009;182(11): 7254-63.

[144] Moriceau S, Lenoir G, Witko-Sarsat V. In cystic fibrosis homozygotes and heterozygotes, neutrophil apoptosis is delayed and modulated by diamide or roscovitine: evidence for an innate neutrophil disturbance. J Innate Immun. 2010;2(3):260-6.

[145] Downey DG, Brockbank S, Martin SL, Ennis M, Elborn JS. The effect of treatment of cystic fibrosis pulmonary exacerbations on airways and systemic inflammation. Pediatr Pulmonol. 2007;42(8):729-35.

[146] Vandivier RW, Fadok VA, Hoffmann PR, Bratton DL, Penvari C, Brown KK, et al. Elastase-mediated phosphatidylserine receptor cleavage impairs apoptotic cell clearance in cystic fibrosis and bronchiectasis. J Clin Invest. 2002;109(5):661-70.

[147] Boyle MP, De Boeck K. A new era in the treatment of cystic fibrosis: correction of the underlying CFTR defect. Lancet Respir Med. 2013;1(2):158-63.

[148] Kerem E, Hirawat S, Armoni S, Yaakov Y, Shoseyov D, Cohen M, et al. Effectiveness of PTC124 treatment of cystic fibrosis caused by nonsense mutations: a prospective phase II trial. Lancet. 2008;372(9640):719-27.

[149] Wilschanski M, Famini C, Blau H, Rivlin J, Augarten A, Avital A, et al. A pilot study of the effect of gentamicin on nasal potential difference measurements in cystic fibro- 
sis patients carrying stop mutations. Am J Respir Crit Care Med. 2000;161(3 Pt 1): 860-5.

[150] Kerem E, Konstan MW, De Boeck K, Accurso FJ, Sermet-Gaudelus I, Wilschanski M, et al. Ataluren for the treatment of nonsense-mutation cystic fibrosis: a randomised, double-blind, placebo-controlled phase 3 trial. Lancet Respir Med. 2014;2(7):539-47.

[151] Amaral MD, Farinha CM. Rescuing mutant CFTR: a multi-task approach to a better outcome in treating cystic fibrosis. Curr Pharmaceut Design. 2013;19(19):3497-508.

[152] Ramsey BW, Davies J, McElvaney NG, Tullis E, Bell SC, Drevinek P, et al. A CFTR potentiator in patients with cystic fibrosis and the G551D mutation. N Engl J Med. 2011;365(18):1663-72.

[153] Boyle MP, Bell SC, Konstan MW, McColley SA, Rowe SM, Rietschel E, et al. A CFTR corrector (lumacaftor) and a CFTR potentiator (ivacaftor) for treatment of patients with cystic fibrosis who have a phe508del CFTR mutation: a phase 2 randomised controlled trial. Lancet Respir Med. 2014;2(7):527-38.

[154] Bell SC, De Boeck K, Amaral MD. New pharmacological approaches for cystic fibrosis: promises, progress, pitfalls. Pharmacol Therapeut. 2015;145:19-34.

[155] Bratcher PE, Rowe SM, Reeves G, Roberts T, Szul T, Harris WT, Tirouvanziam R, Gaggar A. Alterations in blood leukocytes of G551D-bearing cystic fibrosis patients undergoing treatment with ivacaftor. J Cyst Fibros. 2015 Mar 11. pii: S1569-1993(15)00050-8.

[156] Hartl D, Latzin P, Hordijk P, Marcos V, Rudolph C, Woischnik M, et al. Cleavage of CXCR1 on neutrophils disables bacterial killing in cystic fibrosis lung disease. Nat Med. 2007;13(12):1423-30.

[157] Alexis NE, Muhlebach MS, Peden DB, Noah TL. Attenuation of host defense function of lung phagocytes in young cystic fibrosis patients. J Cyst Fibros. 2006;5(1):17-25.

[158] Tirouvanziam R, Gernez Y, Conrad CK, Moss RB, Schrijver I, Dunn CE, et al. Profound functional and signaling changes in viable inflammatory neutrophils homing to cystic fibrosis airways. Proc Natl Acad Sci USA. 2008;105(11):4335-9.

[159] Witko-Sarsat V, Delacourt C, Rabier D, Bardet J, Nguyen AT, Descamps-Latscha B. Neutrophil-derived long-lived oxidants in cystic fibrosis sputum. Am J Respir Crit Care Med. 1995;152(6 Pt 1):1910-6.

[160] Makam M, Diaz D, Laval J, Gernez Y, Conrad CK, Dunn CE, et al. Activation of critical, host-induced, metabolic and stress pathways marks neutrophil entry into cystic fibrosis lungs. Proc Natl Acad Sci USA. 2009;106(14):5779-83.

[161] Laval J, Touhami J, Herzenberg LA, Conrad C, Taylor N, Battini JL, et al. Metabolic adaptation of neutrophils in cystic fibrosis airways involves distinct shifts in nutrient transporter expression. J Immunol. 2013;190(12):6043-50. 
Chapter 12

\title{
Role of Non-coding RNAs in Cystic Fibrosis
}

\author{
Jessica Varilh, Jennifer Bonini and \\ Magali Taulan-Cadars \\ Additional information is available at the end of the chapter \\ http://dx.doi.org/10.5772/60449
}

\begin{abstract}
Cystic Fibrosis (CF) is a common autosomal recessive disorder, caused by mutations in the Cystic Fibrosis Transmembrane conductance Regulator (CFTR) gene. CFTR gene expression is tightly controlled by transcriptional and post-transcriptional regulatory factors, resulting in complex spatial and temporal expression patterns. Here, we describe an overview of the findings about the contribution of ncRNAs, especially miRNAs, in physiological CFTR gene expression and in CF. Determination of mechanisms governing its expression is essential for developing new $\mathrm{CF}$ therapies. ncRNAs, including lncRNAs and miRNAs, could also contribute to CF progression and severity and their dysregulation in $\mathrm{CF}$ opens new perspectives for patient followup and treatment.
\end{abstract}

Keywords: CFTR gene expression, Cystic Fibrosis, non-coding RNA, lncRNA, miRNA

\section{Introduction}

Cystic Fibrosis (CF) is a common autosomal recessive disorder. Although in its classical form $\mathrm{CF}$ affects several organs, including the pancreas and the gastrointestinal and reproductive tracts, its morbidity is mainly due to pulmonary damages. This disorder is caused by mutations in the Cystic Fibrosis Transmembrane conductance Regulator (CFTR) gene. This gene displays great mutational heterogeneity, depending on the ethnic and phenotypic background, with almost 2,000 referenced CFTR alterations (genet.sickkids.on.ca/). The most common mutation 
is the p.Phe508del, whereas other mutations, located both in coding and non-coding regions, are rare or private. The p.Phe508del mutation induces aberrant protein folding, leading to endoplasmic reticulum (ER)-associated degradation, atypical intracellular trafficking and reduced stability of the CFTR protein at the apical membrane. Dysfunction or lack of the CFTR protein causes an obstructive lung disease characterized by impaired ion transport in the airway epithelium, accumulation of sticky mucus in the air space and chronic airway inflammation. Physiological CFTR expression is tightly controlled by transcriptional, post-transcriptional, translational and post-translational regulatory mechanisms, resulting in complex spatial and temporal expression patterns. Notwithstanding the importance of CFTR transcriptional regulation [1-3], CFTR expression can be modulated through other mechanisms. Indeed, epigenetic changes, such as DNA methylation or histone acetylation, also influence CFTR gene expression in different tissues [4-7]. Post-transcriptional controls also regulate its expression, for instance via the usage of upstream open reading frames (uORFs) encoded within the CFTR 5'UTR [8] and the 3'UTR that controls CFTR mRNA stability through ARE sequences (AU-rich elements) [9]. An emerging area of research is focusing on the role played by non-coding RNAs (ncRNAs), such as microRNAs (miRNAs), in CFTR gene expression. Starting from 2011, a few studies have shown the involvement of miRNAs in the physiological control of the complex spatio-temporal expression pattern of CFTR mRNA [10,11], including a recent work by our group [3]. Moreover, the implication of long non-coding RNAs (lncRNAs) and miRNAs in human diseases is well documented [12], including in inherited disorders [13] and lung diseases [14-17]. However, only few studies, described below, have hitherto been carried out on the role of ncRNAs in CF. This chapter is an overview of the findings about the role of ncRNAs in physiological CFTR gene expression and in CF.

\section{What are non-coding RNAs?}

Large expanses of the genome are transcribed into RNAs, but only a small portion of these RNAs encode proteins $[18,19]$. Many fundamental cellular processes rely on conserved ncRNAs, particularly on ribosomal RNAs (rRNAs), the ribosome RNA components that allow mRNA translation into proteins (Figure 1). Other roles are the transport of amino acids via transfer RNAs (tRNAs) and mRNA splicing through the implication of small nucleolar RNAs (snoRNAs). miRNAs and their crucial role as key modulators of post-transcriptional gene regulation were discovered more than 20 years ago [20]. In the last few years, lncRNAs have been identified as new modulators of key biological processes [21-23, 18]. Currently, ncRNAs are divided in two classes, based on their length; long ncRNAs (lncRNAs, $>200 \mathrm{nt}$ ) and short ncRNAs (<200 nt), such as miRNAs, small nucleolar RNAs (snoRNAs) and PIWI-interacting RNAs (piRNAs) [24].

Ribosomal RNA (rRNA) and transfer RNA (tRNA) are the most represented ncRNAs in humans. Long non-coding RNAs (lnc or long ncRNA) are longer than $200 \mathrm{nt}$ and are subdivided in five categories based on their genomic localization: pRNA (promoter-associated RNA), eRNA (enhancer-associated RNA), gsRNA (gene body-associated RNA), lincRNA (intergenic RNA) and NAT (Natural Antisense Transcript). Short non-coding RNAs (short 


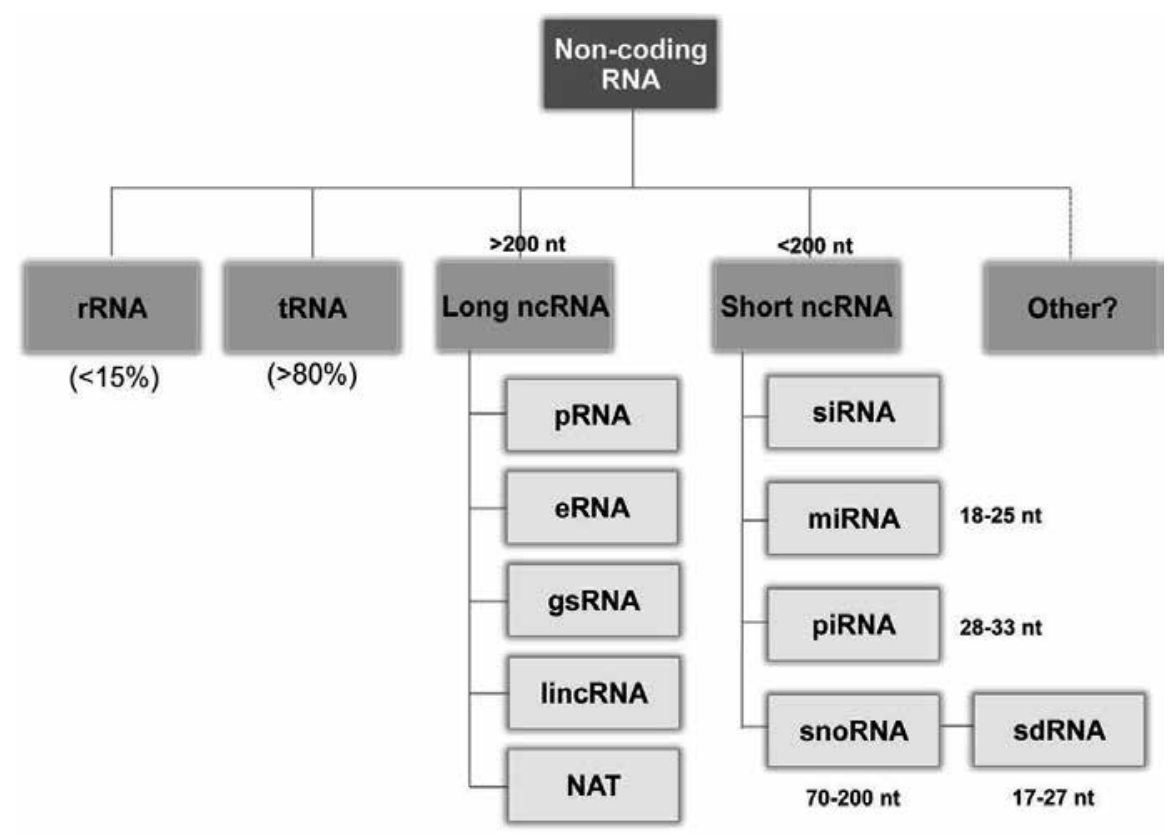

Figure 1. Non-coding RNAs.

ncRNAs) are smaller than $200 \mathrm{nt}$ and are subdivided in four classes based on their size and function: siRNA (small interfering RNA), miRNA (microRNA), piRNA (PIWI-interacting RNA), snoRNA (small nucleolar RNA) and derived snoRNA (sdRNA).

\subsection{Long non-coding RNAs}

lncRNAs include all ncRNAs longer than $200 \mathrm{nt}$ (except rRNA and tRNA). They constitute the bulk of the non-coding transcriptome [25].

\subsection{1. $\ln \mathrm{R} N A$ biogenesis}

It is thought that most lncRNAs originate within a 2-kb region surrounding the Transcription Start Site (TSS) of protein-coding genes (65\% of lncRNAs overlap with a promoter and are called pRNAs), or map to enhancer regions (19\%; named eRNAs), or derive from antisense transcripts that overlap with annotated gene bodies (5\%, called NATs), or are associated with the bodies of protein-coding genes (gsRNA, gene body-associated lncRNAs) [26, 27]. The remaining lncRNAs originate from more distal $(>2 \mathrm{~kb})$ unannotated regions $(11 \%)$ and are commonly referred to as long intervening or intergenic ncRNAs (lincRNAs) [28, 29] (Figure 2).

a-Promoter-associated RNAs (pRNA), b-Enhancer-associated RNAs (eRNA), c-Intronic and gene body-associated (sense) RNAs (gsRNA), d-Natural Antisense Transcripts (NAT), e-Long Intergenic RNAs (lincRNA). In the lower part of the figure are described the main lncRNA functions. 


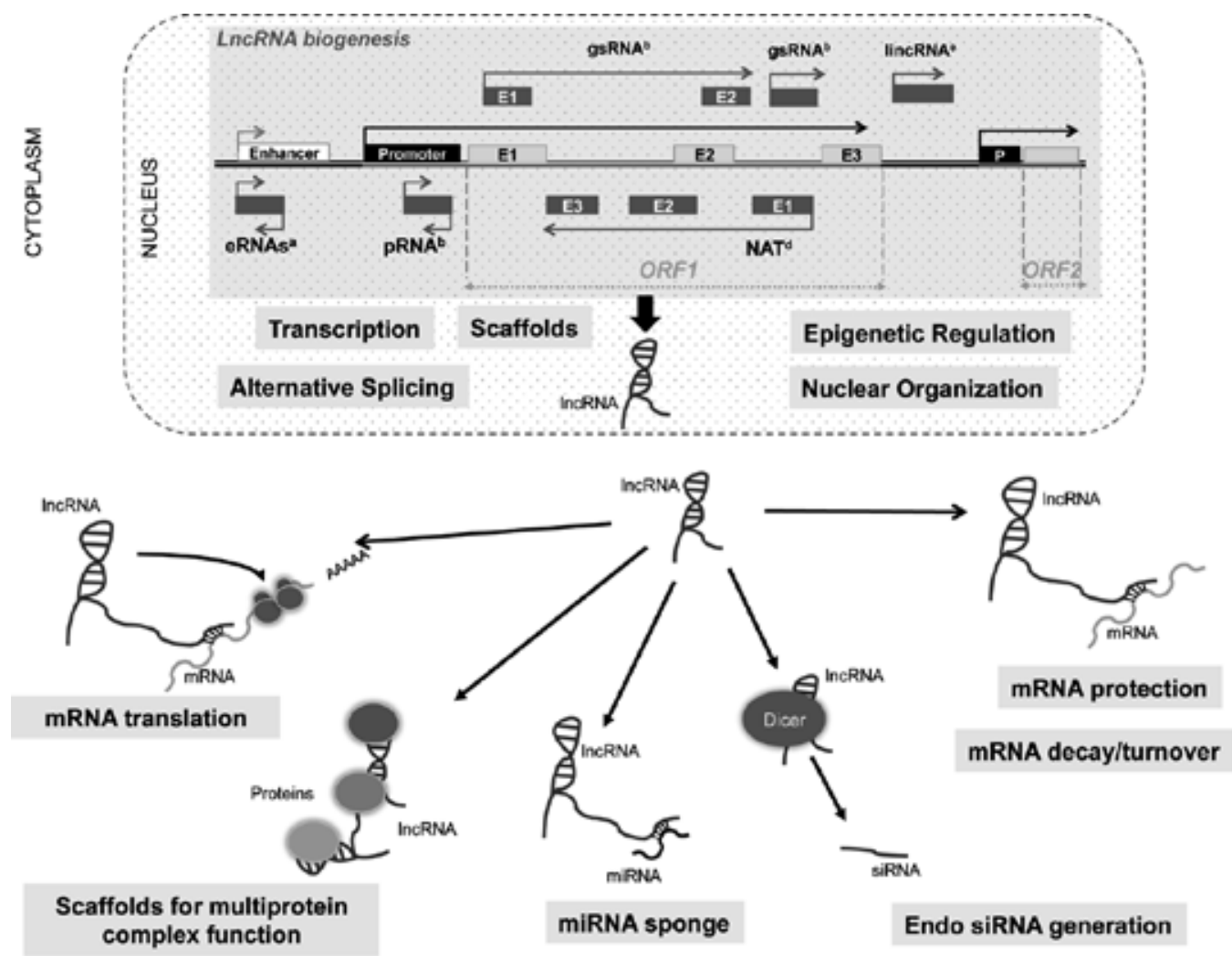

Figure 2. lncRNAs, from biogenesis to functions.

The finding that a large number of lncRNAs arise from loci close to protein-coding genes is consistent with previous genome-wide analyses of lncRNAs [30]. Although all studies agree that the $5^{\prime}$ end of IncRNAs, like for mRNAs, is capped by methylguanosine, their splicing status and their $3^{\prime}$-end processing have not been fully defined [26]. It is likely that splice site recognition occurs at low frequency at most lncRNA loci and that lncRNAs may be predominantly mono-exonic and non-polyadenylated [26]. Most lncRNAs are not translated [28] and their localization is predominantly nuclear [25].

\subsubsection{IncRNA functions}

IncRNAs have regulatory functions in different biological processes (Figure 2). Many of their functions are related to their capacity to bind to RNA, DNA and proteins. The founding member of the lncRNA family is Xist $(\sim 17 \mathrm{~kb})$. Xist originates from the silent $X$ chromosome in female cells and coats this chromosome during the early stages of development to establish epigenetic $X$ inactivation [31]. IncRNAs can be used as indicators of the transcriptional activity of a locus or a gene [19]. Their roles as scaffolds for nuclear processes, guides for ribonucleoprotein complexes or decoys have been described in the literature. Similarly to miRNAs, they can act as activators or repressors of protein expression. 
lncRNAs are considered to be more species, tissue and developmental stage-specific than mRNAs [32]. A growing number of studies show that lncRNA deregulation has a role in various diseases [33,34; for reviews: 35,36], including pulmonary disorders. Several reviews have discussed the role of lncRNAs and miRNAs in respiratory diseases $[16,17,37]$ and a recent work reported lncRNA involvement in CF (detailed in section 4.2.1).

\subsection{MicroRNAs}

\subsection{1. miRNA localization and biogenesis}

Animal miRNAs derive from the nuclear genome. In humans, the majority of canonical miRNAs are encoded by introns of non-coding or coding transcripts, but some miRNAs are encoded by exonic regions (Figure 3). Often, several miRNA loci are in close proximity, thus constituting a polycistronic transcription unit [38]. Most miRNAs use their host gene transcripts as carriers, but separate transcription from internal promoters remains possible. Generally, miRNAs in the same cluster are co-transcribed. Most miRNA genes located in introns of protein-coding genes share the promoter of the host gene [39]. miRNA genes often have multiple transcription start sites [40]. miRNA loci in intergenic regions apparently have their own transcriptional regulatory elements, thus constituting independent transcription units.

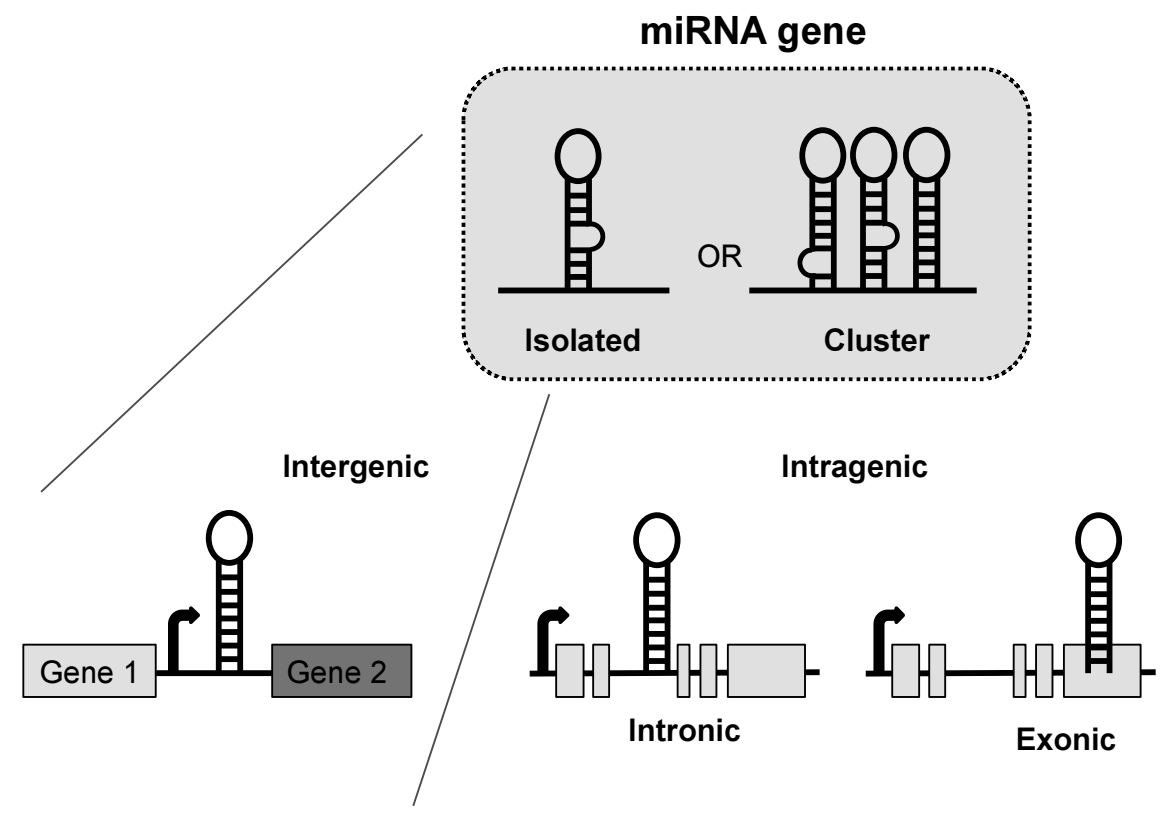

Figure 3. Genomic locations of miRNAs. miRNA genes, isolated or in clusters, are located in intergenic (ex: miR-494) or intragenic genome regions, including exons of non-coding (e.g. miR-155) or coding (e.g. miR-985) genes and introns of non-coding (e.g. the miR-15a $\sim 16-1$ cluster) or coding (e.g. miR-126) genes. 
miRNA biogenesis includes several steps. First, the gene coding for a given miRNA is transcribed by RNA polymerase II into a long primary transcript (pri-miRNA, ranging from $100 \mathrm{nt}$ to several kilobases). Some miRNA genes, especially those located in Alu elements, are transcribed by RNA polymerase III [41]. When several miRNAs are in a cluster, pri-miRNAs can contain multiple miRNAs. Transcription factors, such as p53, MYC, C/EBP, FOXA positively or negatively regulate miRNA transcription $[3,42,43]$. Epigenetic control, such as DNA methylation and histone modifications also contribute to miRNA gene regulation [44].

Then, several maturation steps are necessary for miRNA processing. Indeed, the long primiRNAs (typically over $1 \mathrm{~kb}$ ) contain stem-loop structures in which mature miRNA sequences are embedded. The nuclear RNase III Drosha acts by cropping the stem-loop to release small hairpin-shaped RNAs of $65 \mathrm{nt}$ in length (pre-miRNA) from the pri-miRNAs. To do this, Drosha, together with its cofactor DiGeorge Syndrome Critical Region 8 (DGCR8), forms the Microprocessor complex. As Drosha cleavage defines one end of the mature miRNA and thereby determines its specificity, it is important that the Microprocessor complex precisely recognizes and cleaves each pri-miRNA. Importantly, Drosha-mediated processing of intronic miRNAs does not affect splicing of the host pre-miRNA [45]. Multiple auxiliary factors could contribute to pri-miRNA maturation [46]. For example, three primary sequence determinants (the basal UG, CNNC and the apical GUG motifs) contribute to efficient processing of human primiRNAs. At least one of these three motifs is present in almost $80 \%$ of human miRNAs [46]. The splicing factor SRp20 (also called SRSF3) and the RNA helicase DDX17 bind to the CNNC motif and increase processing of human pri-miRNAs by Drosha. Moreover, the terminal loops of miRNA precursors are enriched in cis-elements that recruit regulatory proteins. For example, the splicing factors HNRPA1 and KSRP bind to the conserved terminal loops of some pri-miRNAs and facilitate Drosha-mediated processing [47-49].

Following Drosha processing, pre-miRNAs are exported in the cytoplasm where they are cleaved by Dicer near the terminal loop, liberating a small RNA duplex. Dicer, like Drosha, belongs to a family of RNase III-type endonucleases that act specifically on double-stranded RNA.

RNA duplexes include two mature miRNAs: one derived from the 5 ' strand and the other one from the 3' strand of the precursor (e.g. miR-27a-5p and miR-27a-3p). One is also called the 'guide' (miRNA) and is usually more biologically active than the other one (the 'passenger', often referred to as miRNA*). The passenger is normally degraded, but, in some cases, it can be functional [50]. The mature miRNA strand is subsequently incorporated in the RNAinduced silencing complex (RISC, or miRISC for miRNA-containing RISC, or miRNP for microribonucleoprotein), where it directly binds to a member of the Argonaute (AGO) protein family (four AGO members, AGO1 are the most frequently used).

To date, about 1,900 miRNAs (1,881 precursors and 2,588 mature miRNAs; GRCh38 human genome assembly) have been reported in the miRbase database (http://www.mirbase.org/). A substantial number of these miRNAs have dubious annotations and for nearly one-third of miRNA loci, there is no convincing evidence concerning the production of authentic miRNAs (miRbase). 


\subsection{2. miRNA roles}

miRNAs are small ncRNAs that can act in the nucleus and in the cytoplasm [51] through binding to RNA, DNA and proteins. They play an important role in the negative regulation of gene expression by base-pairing to partially complementary sites on the target mRNAs, usually in the 3' UTR part. Binding of an miRNA to its target mRNA, within the RISC complex, typically leads to translational repression and exonucleolytic mRNA decay. However, highly complementary targets can be cleaved endonucleolytically.

a.

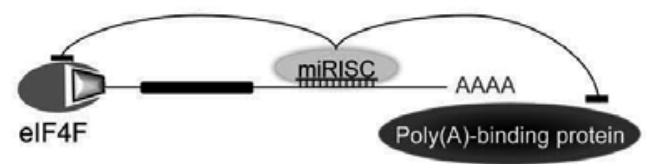

b.

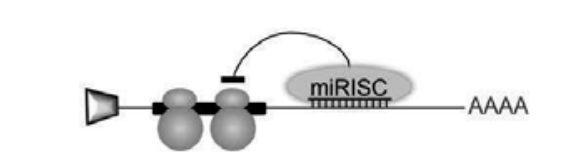

c.

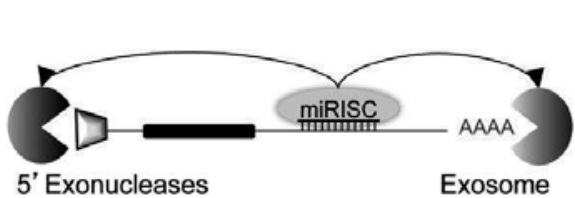

d.
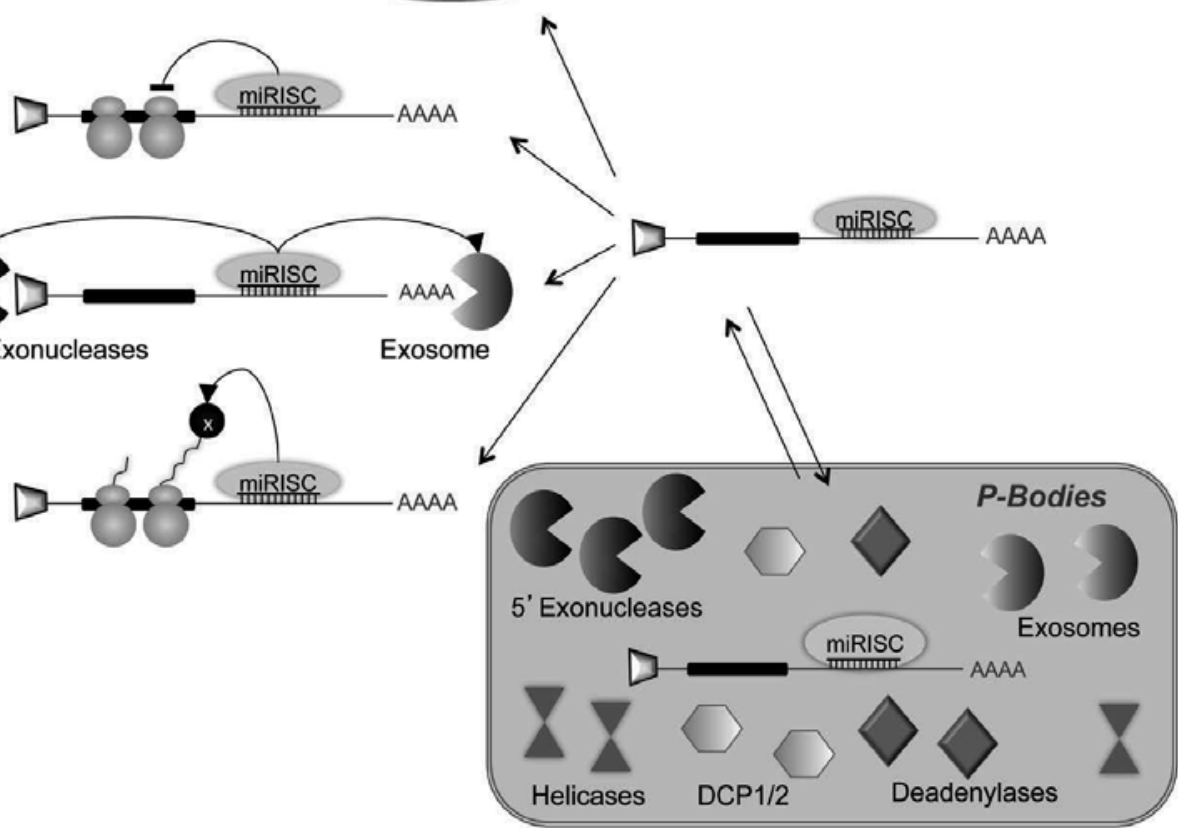

Figure 4. Main miRNA roles. a. Translation block by inhibiting cap and poly(A)-binding protein recognition. b. Elongation inhibition by slowing down elongation or ribosome 'drop-off'. c. Degradation by deadenylation and decapping. d. Proteolysis. Degradation of a nascent peptide. e. mRNA storage in P-bodies that contain exonucleases, RNA helicases, decapping enzymes, DCP1/2, exosomes, deadenylases.

Several miRNAs have a role in lung diseases, such as asthma, chronic obstructive pulmonary disease (COPD) and idiopathic pulmonary fibrosis (see Table 1). The studies reporting the involvement of miRNAs in $\mathrm{CF}$ are detailed in section 3.2.2. 


\begin{tabular}{|c|c|c|c|}
\hline Disease & MicroRNAs & Targets & References \\
\hline \multirow{5}{*}{ Asthma } & miR-106a & IL-10 & [81] \\
\hline & $\operatorname{miR}-21$ & IL-12 & [82] \\
\hline & miR-133a & RhoA & [83] \\
\hline & miR-26a & Glycogen synthase kinase $3 ß$ & [84] \\
\hline & let-7 & IL-13 & [85] \\
\hline \multirow{3}{*}{ COPD } & miR-181d & Interferon $\gamma$, collagen XVI $\alpha \mathrm{I}$ & [86] \\
\hline & miR-30c & Proto-cadherin & [86] \\
\hline & miR-146a & Prostaglandin E2 & [87] \\
\hline \multirow{3}{*}{ Idiopathic pulmonary fibrosis } & $\operatorname{miR}-21$ & SMAD7 & [88] \\
\hline & miR-155 & KGF & [89] \\
\hline & miR-let7d & HMGA2 & [90] \\
\hline
\end{tabular}

COPD, chronic obstructive pulmonary disease.

Table 1. Examples of pulmonary diseases in which miRNAs have a role

\section{Role of non-coding RNAs in the physiological regulation of CFTR gene expression}

As the function of lncRNAs has not been studied yet, we only present findings on the miRNA roles in the regulation of CFTR gene expression.

\section{1. miRNAs and CFTR gene expression}

CFTR gene expression is spatially and temporally regulated. Several studies have demonstrated the differential use of transcription start sites, depending on the tissue type or the developmental stage [52-55]. In the lung, CFTR transcripts can be detected early during embryo development (12th week of pregnancy) and their level progressively increases up to the 24th week of pregnancy. Thereafter, CFTR expression in the airways decreases and is repressed until after birth and remains very low during adult life $[56,60]$. The changes in CFTR protein expression in human foetuses are consistent with CFTR mRNA temporal pattern of expression [60]. In a recent study, we showed that miRNAs (miR-101, miR-145, miR-384) regulate the switch from strong foetal to very low CFTR expression after birth. Specifically, miR-101 and miR-145 negatively regulate the level of CFTR transcripts in adult lung cells, while they have no effect in foetal lung cells. miR-101 directly acts on its cognate site in the 3'UTR-CFTR in combination with an overlapping AU-rich element. Other studies showed that CFTR expression is also post-transcriptionally regulated by miRNAs, such as miR-145 and miR-494 [10,11]. Gillen et al. demonstrated that miR-145 is expressed in primary adult human airway epithelial cells, where CFTR expression is low, and directly acts on CFTR stability [10]. In addition to its 
specific role in mature lung cells, miR-101 decreases luciferase activity in an embryonic kidney cell line [11], whereas it does not affect CFTR mRNA stability in pancreatic cell lines [10], suggesting a potential role as a tissue-specific factor.

\subsection{Methods to investigate miRNA role in the regulation of CFTR gene expression}

Different approaches can be employed to investigate miRNA role in the regulation of CFTR gene expression, as depicted in Figure 5A.

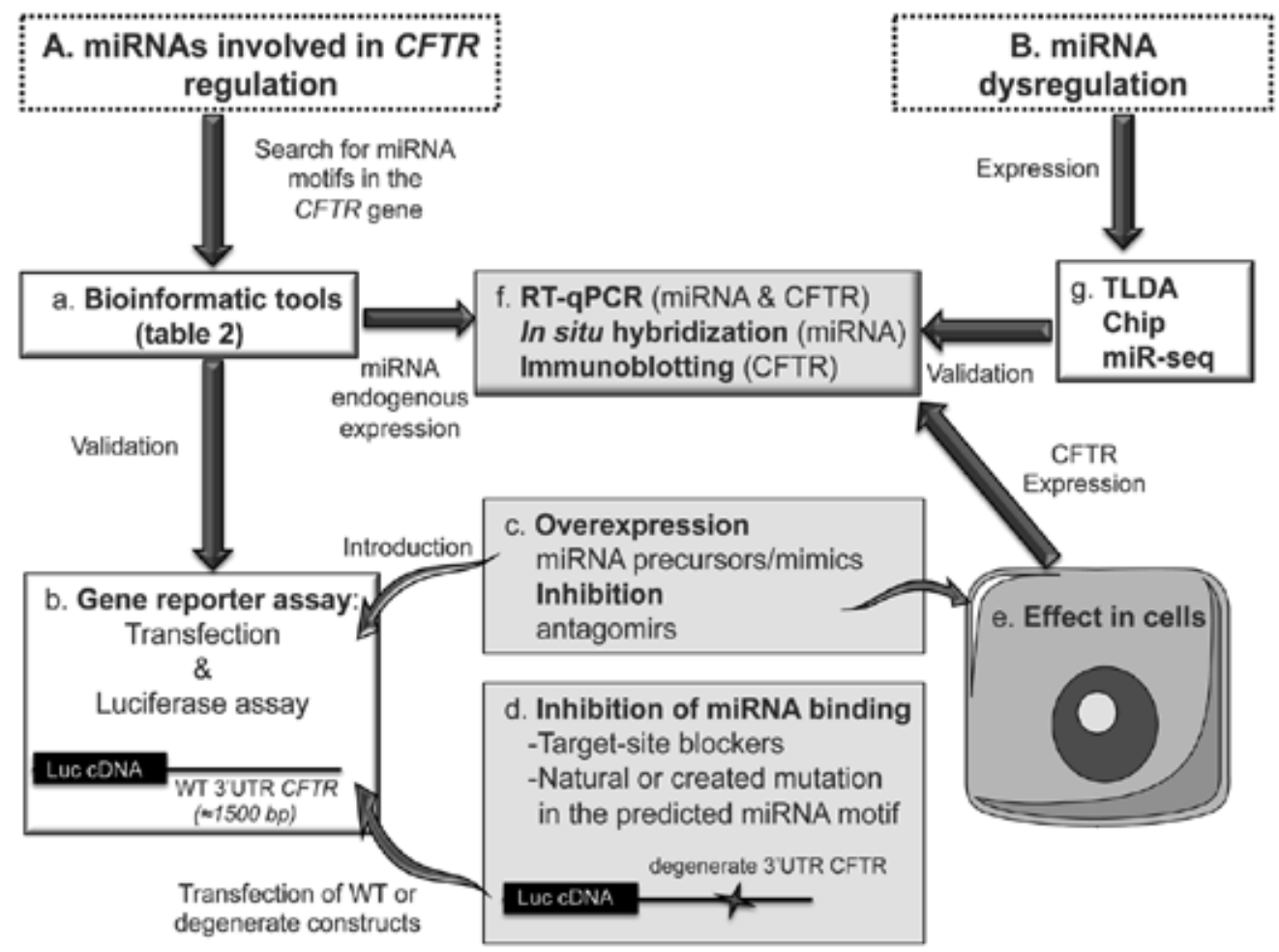

Figure 5. Approaches for miRNA study. A. Strategies used to study the involvement of miRNAs in the regulation of CFTR gene expression. B. Strategies to identify miRNAs that are deregulated in CF samples compared to non-CF samples. Chips for global miRNA profiling are commercialized by Agilent, Affimetrix and Exiqon. miR-seq (miRNA sequencing) is usually performed by Illumina platforms. Luc, luciferase; TLDA, TaqMan Array Micro Fluidic Cards (Applied Biosystems); WT, wild type.

\subsubsection{Predictive tools are freely available}

Predictive tools are necessary to assess the putative presence of miRNA binding motifs. A nonexhaustive list, including information about each program, is proposed in Table 2. Databases collecting all information on miRNAs are listed in Table 3. Although miRBase (http:// www.mirbase.org/) is the most used database and collects links for several predictive pro- 
grams, others exist. These tools have been developed to predict miRNA targets or the miRNAs that putatively bind to a selected gene. Some of them propose the possibility to input several miRNAs and/or several genes to identify integrated networks.

\begin{tabular}{|c|c|c|c|}
\hline Name & Website & Characteristics & References \\
\hline TargetScan & http://www.targetscan.org/ & $\begin{array}{l}\text { Target site prediction for mammals. Secondary } \\
\text { structure taken into account. }\end{array}$ & [91] \\
\hline miRanda & http://microrna.org/ & $\begin{array}{l}\text { Target site prediction for human, mouse, rat, fruitfly } \\
\text { and nematode. } \\
\text { Thermodynamic stability of RNA duplexes taken } \\
\text { into account. }\end{array}$ & [92] \\
\hline PicTar & http://www.pictar.org/ & $\begin{array}{l}\text { Algorithm for target-site prediction based on the } \\
\text { alignment of 3'UTR with predicted sites. Several } \\
\text { databases are used for vertebrates, flies, nematodes. }\end{array}$ & [93] \\
\hline RNAhybrid & $\begin{array}{l}\text { http://bibiserv.techfak.uni- } \\
\text { bielefeld.de/rnahybrid/ }\end{array}$ & $\begin{array}{l}\text { Tool for finding the minimum free energy } \\
\text { hybridization for long and mainly short RNAs, such } \\
\text { as microRNAs to one or more given targets. }\end{array}$ & [94] \\
\hline PITA & $\begin{array}{c}\text { http:// } \\
\text { genie.weizmann.ac.il/pubs/ } \\
\text { mir07/mir07_data.html }\end{array}$ & $\begin{array}{l}\text { Target-site prediction for human and other species } \\
\text { /that evaluates the microRNA targets accessibility as } \\
\text { a component analysis. }\end{array}$ & [95] \\
\hline miRDB & http://mirdb.org/miRDB/ & $\begin{array}{l}\text { Tool for miRNA target-site prediction and functional } \\
\text { annotation based on the mirTarget algorithm for all } \\
\text { known human, dog, rat, and chicken transcripts. }\end{array}$ & [96] \\
\hline DIANA-microT & $\begin{array}{l}\text { http:// } \\
\text { diana.cslab.ece.ntua.gr/ } \\
\text { microT/ }\end{array}$ & $\begin{array}{l}\text { Target-site prediction program taking intro account } \\
\text { both conserved and non-conserved miRNA } \\
\text { regulatory elements (MREs) and providing scores as } \\
\text { an indication of the expected fold change in protein } \\
\text { production. }\end{array}$ & {$[97]$} \\
\hline miRBase Target & http://www.mirbase.org/ & $\begin{array}{l}\text { Prediction of target sites based on alignment and } \\
\text { conservation. } \\
\text { Provides links to other mains programs for miRNA } \\
\text { target prediction (e.g., TargetScan, PicTar). }\end{array}$ & [98] \\
\hline miRTar & $\begin{array}{c}\text { http:// } \\
\text { mirtar.mbc.nctu.edu.tw/ } \\
\text { human/ }\end{array}$ & $\begin{array}{l}\text { Web-based system based on the analysis of } \\
\text { conserved sequences (seed or 3' miRNAs) using } \\
\text { external prediction tools (TargetScan, miRanda, } \\
\text { PITA, RNAhybrid). } \\
\text { Analyzes miRNA biological functions using the } \\
\text { KEGG pathway to draw a miRNA target interaction } \\
\text { network. }\end{array}$ & [99] \\
\hline miRNAMap & $\begin{array}{c}\text { http:// } \\
\text { mirnamap.mbc.nctu.edu.t } \\
\text { w/index.php }\end{array}$ & $\begin{array}{l}\text { Uses the computational tools, miRanda, RNAhybrid, } \\
\text { and TargetScan to identify miRNA targets in the } \\
\text { 3'UTR of target genes. }\end{array}$ & [100] \\
\hline
\end{tabular}




\begin{tabular}{|c|c|c|c|}
\hline Name & Website & Characteristics & References \\
\hline MiRonTop & $\begin{array}{l}\text { http://www.microarray.fr: } \\
\text { 8080/miRonTop/index }\end{array}$ & $\begin{array}{l}\text { Identification of miRNAs from DNA microarrays } \\
\text { and high-throughput sequencing data. } \\
\text { Uses several existing miRNA target prediction } \\
\text { approaches. }\end{array}$ & {$[101]$} \\
\hline MIR@nt@n & $\begin{array}{l}\text { http://maia.uni.lu/ } \\
\text { mironton.php }\end{array}$ & $\begin{array}{l}\text { Integrative approach for searching miRNAs target } \\
\text { sites, to build networks including motifs as } \\
\text { feedbacks and feedforward loops from lists of } \\
\text { molecular actors. }\end{array}$ & [102] \\
\hline ProMiR & http://bi.snu.ac.kr/ProMiR/ & $\begin{array}{l}\text { Prediction of potential conserved and non- } \\
\text { conserved microRNAs in a query sequence from } 60 \\
\text { to } 150 \text { nucleotides and clusters near known or } \\
\text { unknown miRNAs in various species. }\end{array}$ & [103] \\
\hline
\end{tabular}

Table 2. Free bioinformatics tools for miRNAs

\begin{tabular}{|c|c|c|c|}
\hline Name & Website & Characteristics & References \\
\hline miRBase & http://www.mirbase.org/ & $\begin{array}{l}\text { Collection of annotation, literature, genomic } \\
\text { coordinates for human miRNAs and from several } \\
\text { other species. } \\
\text { From deep sequencing datasets, uses the pattern of } \\
\text { mapped reads to assess the confidence in each } \\
\text { miRNA annotation. }\end{array}$ & [98] \\
\hline miRGen & $\begin{array}{c}\text { http:// } \\
\text { diana.cslab.ece.ntua.gr/ } \\
\text { mirgen/ }\end{array}$ & $\begin{array}{l}\text { Integrated database collecting relationships between } \\
\text { animal miRNAs, genomic annotation sets and } \\
\text { miRNA targets to a combination of used target } \\
\text { prediction programs. }\end{array}$ & [104] \\
\hline miRNAMap & $\begin{array}{l}\text { http:// } \\
\text { miRNAMap.mbc.nctu.edu. } \\
\text { tw/ }\end{array}$ & $\begin{array}{l}\text { Resource for collecting experimentally verified } \\
\text { microRNAs and verified miRNA target genes in } \\
\text { human and other metazoan genomes reducing the } \\
\text { false positive prediction rate of miRNA target sites. } \\
\text { Expression profiles by RT-qPCR of } 224 \text { human } \\
\text { miRNAs in } 18 \text { normal tissues. }\end{array}$ & [100] \\
\hline CoGemiR & http://cogemir.tigem.it/ & $\begin{array}{l}\text { Database offering an overview of the genomic } \\
\text { organization and conservation of microRNAs in } \\
\text { different metazoan species during evolution. }\end{array}$ & [105] \\
\hline miRanda & http://microrna.org/ & $\begin{array}{l}\text { Atlas of miRNA expression in human, mouse and } \\
\text { rat tissues based on small RNA library sequencing. }\end{array}$ & \\
\hline
\end{tabular}

Table 3. Free databases for miRNAs 


\subsubsection{Functional approaches}

To evaluate in vitro the impact of miRNAs on CFTR gene regulation, several approaches can be used (Figure $5 \mathrm{~A}$ ). a) First of all, in silico analysis by using bioinformatics tools can be performed (see 3.2.1). b) The role of specific miRNAs could be assessed in vitro by using airway cell lines and luciferase gene reporter assays, in which the luciferase coding sequence is under the control of the 3' UTR of CFTR, as previously described [11,3]. c) By transfecting mimics or precursors, miRNAs can be overexpressed and the relative level of luciferase expression reflects the effect of a given miRNA on the $3^{\prime} \mathrm{UTR}-C F T R$. Inhibitors may also be used to confirm the specific effect of the miRNA under study. d) To validate the direct effect of the identified miRNAs (for instance, miRNA-494 and miRNA-101), the cis element for miRNAs binding in the 3'UTR-CFTR can be mutated by site-directed mutagenesis (created mutation). Target-site blockers may also be used to inhibit binding to the tested motif [3]. e) To validate these effects, the endogenous level of CFTR mRNA following miRNA overexpression or down-regulation can be assessed using different techniques.

\section{Role of non-coding RNAs in CF}

\subsection{What is the impact of mutations in microRNA target sites on the CFTR gene?}

\subsubsection{Mutations in the 3'UTR of the CFTR gene}

Assessing the putative impact of single nucleotide polymorphisms (SNPs) in the $3^{\prime}$ UTR of CFTR is essential to define the pathological importance of these cis-regulatory motifs in noncoding regions, especially after the development of Next-Generation Sequencing (NGS) technologies. Recent work identified the SNP c. ${ }^{*} 1043 \mathrm{~A}>\mathrm{C}$ on the $3^{\prime} \mathrm{UTR}$ of the CFTR gene in one patient with a CFTR-related disorder and congenital bilateral absence of vas deferens [61]. This SNP is located in a region predicted to interact with miR-433 and miR-509-3p. Expression analysis demonstrated that the $c .{ }^{*} 1043 \mathrm{~A}>\mathrm{C}$ mutation increases the affinity for miR-509-3p and slightly decreases that for miR-433. In vitro, these two miRNAs reduces CFTR protein expression. The authors suggested that the very low expression of miR-509-3p in normal human bronchial epithelial (NHBE) cells could explain the mild phenotype caused by this mutation. Thus, the c. ${ }^{*} 1043 \mathrm{~A}>\mathrm{C}$ mutation, by acting as a mild CFTR mutation that enhances the affinity for an inhibitory miRNA, could represent a novel pathogenic mechanism in CF.

\subsubsection{Methods to investigate the impact of CFTR gene mutations}

Bioinformatics tools, such as miRNA binding site prediction programs (listed in Table 2), could be used to predict whether at a mutated position there is a cis-regulatory motif and whether it corresponds to an miRNA binding site. Moreover, tools like RNAhybrid could allow predicting the binding energy of the mutated motif. In vitro luciferase reporter gene assays together with mutagenesis approaches to create degenerate 3'UTR-CFTR sequences can be used to study the different variants (Figure 5Ad). 


\subsection{Deregulation of non-coding RNAs in CF}

Clinical manifestations of $\mathrm{CF}$ are various including chronic pulmonary inflammation and infection that strongly contribute to the morbidity and mortality of these patients [62]. Overinflammation precedes chronic infection that is then amplified by pathogens. Notably, the protease-antiprotease balance, which is responsible for lung remodelling, is disrupted in CF airways early in life and then this imbalance is chronically maintained [63]. Pulmonary tissues in patients with CF are usually infected by antibiotic-resistant Pseudomonas aeruginosa. Interleukin-8 (IL-8), IL-6 and Tumor Necrosis Factor alpha (TNF-alpha) are pro-inflammatory cytokines that are highly expressed in CF lung epithelial cells and allow the recruitment of neutrophils [62]. Lipopolysaccharide (LPS) and IL-1beta, which bind to Toll-like receptor 4 (TLR4) and IL-1R, respectively, are also involved. The identification of the molecular events involved in lung epithelium injury and repair is essential for understanding CF physiopathology. Expression levels of miRNAs physiologically vary greatly among tissues. Recent advances explored their effects on influencing signaling pathways in CF. Identification of deregulated miRNAs may offer possible future directions for clinical applications.

\subsubsection{Deregulated IncRNAs in $C F$}

To date, only one publication has reported aberrant expression of specific lncRNAs in CF bronchial epithelium in vivo [64]. To establish the lncRNA profiles of CF and non-CF bronchial epithelium, $10 \mathrm{CF}$ and 12 non-CF (controls) bronchial brushing samples were analyzed using a human lncRNA Array v3.0 (ArrayStar). In this way, more than 30586 lncRNAs and 26109 protein coding transcripts were evaluated. Overall, 1063 lncRNAs, most of which were intergenic, were differentially expressed in non-CF and CF bronchial brushing samples. RTqPCR analysis of the differential expression of well-known ncRNAs (XIST, MALAT1, HOTAIR, and TLR8 antisense) did not confirm the down-regulation of MALAT1 and HOTAIR in CF samples compared to controls. Interestingly, MALAT1 (lnc-SCYL1-1*) and HOTAIR (lncSMUG1-7*) have been described as oncogenic lncRNAs in lung cancer (*asterisks meaning the existence of several isoforms).

\subsubsection{Deregulated miRNAs in $C F$}

miRNA profiling studies identified various miRNAs with altered expression in CF (summarized in Figure 6). For instance, in vivo the expression of miR-145, miR-223 and miR-494 is increased in CF bronchial brushing samples (individuals with at least one p.Phe508del CFTR allele) compared to non-CF controls and correlates with decreased p.Phe508del CFTR expression. Moreover, these three miRNAs inhibit CFTR mRNA expression [65]. These authors also highlighted a relationship between their regulation and CFTR chloride channel activity. Specifically, they showed that treatment with inh-172, a specific inhibitor of the CFTR chloride channel, significantly increases the level of miR-145, miR-223 and miR-494 in 16 HBE14orespiratory epithelial cells. These data are in agreement with the hypothesis by Wenming $\mathrm{Xu}$ et al. [66] that CFTR chloride channel alteration affects the miRNA profile. Another study reported that miR-509-3p and miR-494 are increased in well-differentiated primary cultures of human CF but not of non-CF airway epithelia, [67]. Other miRNAs have been found to be 
deregulated in CF, such as miR-155, miR-126 and miR-31 [68]. miR-155 is overexpressed in IB3-1 CF cells compared to IB3-1 control cells and in ex vivo CF cells (bronchial brushing samples versus normal human bronchial epithelial cells and CF neutrophils CD66+ versus control cells). Moreover, in IB3 cells, miR-155 up-regulation is related to defective CFTR chloride channel activity (following exposure to inh-172). This leads to repression of SHIP1, a well-known effector in the regulation of inflammation, and consequently to activation of the PI3K/AKT pathway that stabilizes IL-8 mRNA through MAPK. Thus, miR-155 up-regulation contributes to the maintenance of a pro-inflammatory phenotype. Furthermore, miR-155antagomir leads to IL-8 down-regulation in IB3-1CF cells and could represent a candidate treatment for CF. The biogenesis of miR-155 has been in part elucidated and involves the inflammatory RNA binding proteins KSRP and TTP [69]. KSRP promotes miR-155 production, while TTP down-regulates, via miR-1, miR-155 mature expression in CF lung epithelial cells.

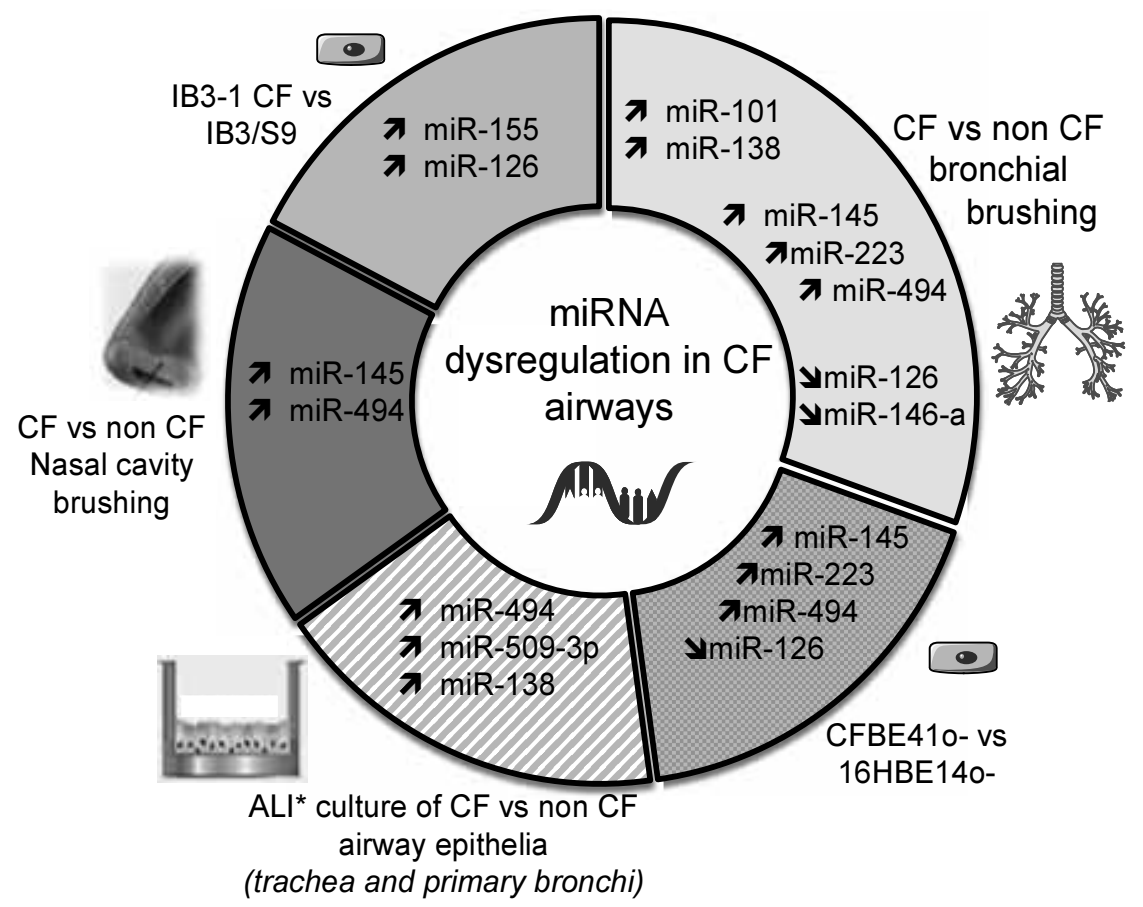

Figure 6. Deregulated miRNAs in different CF models. The schematic lists miRNAs that have been reported to be upregulated or down-regulated in CF (tissues, cell lines, primary cultures) versus (vs) non-CF samples. *ALI: Air-Liquid Interface.

Conversely, miR-126 down-regulation has an anti-inflammatory role to compensate the immunity response. Oglesby et al. [70] reported that miR-126 is consistently decreased in CF compared to non-CF airway epithelial cells and this reduction correlates with up-regulation of TOM1, a negative regulator of TLR2, TLR4, IL-1, IL-1 $\beta$ and TNF-alpha [71,72]. TOM1, which also negatively regulates NF- $\kappa \mathrm{B}$, may play an anti-inflammatory role in CF lung. The authors 
hypothesized that the observed reduction in miR-126 expression in CF cells may be due to ER stress induced by accumulation of misfolded p.Phe508del CFTR proteins. A complete analysis of the pathways triggered in CF cells seems essential to identify/develop novel treatments for CF.

Another study assessed the miRNA profile, by using Agilent microarray, of CF IB3-1 cells infected or not with Pseudomonas aeruginosa, a model mimicking the inflammatory response observed in pulmonary tissues of CF patients [73]. Two miRNAs (miR-93 and miR-494) were found to be strongly deregulated in infected cells. These two miRNAs are predicted to interact with the 3'UTR of IL-8 mRNA. Down-regulation of miR-93 was confirmed in two other bronchial epithelial cell lines (CF CuFi-1 and non-CF Nuli-1 cells). In non-infected CF cells, miR-93 is strongly expressed and is involved in IL-8 down-regulation combined with low NF$\kappa \mathrm{B}$ recruitment to the IL-8 promoter. In CF cells infected with Pseudomonas aeruginosa, the combined effects of high NF- $\kappa B$ recruitment to the $I L-8$ gene promoter and miR-93 downregulation lead to high IL-8 expression [73].

Finally, miR-31 is down-regulated in CF bronchial brushing cells compared to non-CF cells [74]. In CF epithelial cells, miR-31 negatively modulates the expression of IRF1, a transcription factor that regulates the level of cathepsin S (CTSS). CTSS is overexpressed in CF airways cell lines, such as bronchial (CFBE), tracheal (CFTE) and CF primary bronchial epithelial cells (CFPBECs) and has been detected in CF lung secretions. CTSS activates the epithelial sodium channel and cleaves and inactivates antimicrobial proteins such as surfactant $\mathrm{A}$, lactoferrin and members of the $\beta$-defensin family, thus contributing to lung inflammation in patients with CF [63-66]. Moreover, in a cohort of paediatric patients with CF, it was found that CTSS level correlates with the decline of lung function. Thus, miR-31 is a potential regulator of CTSS expression via IRF1 in CF epithelial cells [74].

\subsubsection{Methods to study deregulated non-coding RNA}

Methods to quantify miRNAs in CF and non-CF samples are depicted in Figure 5B. Approaches to identify dysregulated lncRNAs in CF samples are detailed in Figure 7 and databases for lncRNAs are listed in Table 4.

\begin{tabular}{|c|c|c|c|}
\hline Name & Website & Characteristics & References \\
\hline LNCipedia & http://www.lncipedia.org/ & $\begin{array}{l}\text { Integrated database of human lnc transcripts. } \\
\text { Specific annotation of lncRNAs. Algorithms to } \\
\text { / assess the protein-coding potential of transcripts. } \\
\text { LncRNA gene conservation between human, mouse } \\
\text { and zebrafish. }\end{array}$ & {$[106,107]$} \\
\hline lncRNA2Target & $\begin{array}{c}\text { http:// } \\
\text { www.lncrna2target.org/ }\end{array}$ & $\begin{array}{l}\text { Curated database with human lncRNA-to-target } \\
\text { gene. Target genes based on overexpression and } \\
\text { knockdown studies. }\end{array}$ & [108] \\
\hline
\end{tabular}




\begin{tabular}{|c|c|c|c|}
\hline Name & Website & Characteristics & References \\
\hline lncRNAWiki & $\begin{array}{l}\text { http://lncrna.big.ac.cn/ } \\
\text { index.php/Main_Page }\end{array}$ & $\begin{array}{l}\text { Component of ScienceWiki for community curation } \\
\text { of human lncRNAs. }\end{array}$ & [109] \\
\hline lncRNAdb & http://www.lncrnadb.org/ & $\begin{array}{l}\text { Database for functional eukaryotic lncRNAs. Allow } \\
\text { blast search of putative lncRNAs. } \\
\text { / Gene expression data, evolutionary conservation, } \\
\text { structural information, genomic context, subcellular } \\
\text { localization, functional evidence. }\end{array}$ & {$[110,111]$} \\
\hline NONCODE & http://www.noncode.org/ & $\begin{array}{l}\text { Integrated knowledge database dedicated to } \\
\text { ncRNAs. NONCODE specific ID for each ncRNA } \\
\text { with a conversion tool to RefSeq and Ensembl. } \\
\text { Includes all ncRNAs, except transfer RNAs and } \\
\text { ribosomal RNAs, ncRNA sequences and relative } \\
\text { information (expression, cellular location, } \\
\text { chromosomal information...). More than } 80 \% \text { of } \\
\text { entries are based on literature data. }\end{array}$ & {$[112,113]$} \\
\hline $\begin{array}{l}\text { NRED : ncRNA } \\
\text { expression } \\
\text { database }\end{array}$ & $\begin{array}{l}\text { http:// } \\
\text { nred.matticklab.com/cgi- } \\
\text { bin/ncrnadb.pl }\end{array}$ & $\begin{array}{l}\text { Gene expression repository for human and mouse } \\
\text { lncRNAs. } \\
\text { Includes both microarrays and in situ hybridization } \\
\text { data. } \\
\text { Includes evolutionary conservation, secondary } \\
\text { structure, genomic context. }\end{array}$ & [114] \\
\hline GENCODE & $\begin{array}{c}\text { http:// } \\
\text { www.gencodegenes.org/ }\end{array}$ & $\begin{array}{l}\text { Human lncRNAs catalog from manually annotated } \\
\text { genes } \\
\text { Data available via the UCSC Genome Browser }\end{array}$ & [119] \\
\hline $\begin{array}{l}\text { Human Body } \\
\text { Map lincRNAs }\end{array}$ & $\begin{array}{c}\text { http:// } \\
\text { www.broadinstitute.org/ } \\
\text { genome_bio/ } \\
\text { human_lincrnas/ }\end{array}$ & $\begin{array}{l}\text { Human reference catalog for lincRNAs } \\
\text { Expression data from RNAseq accross } 24 \text { tissues } \\
\text { and cell types. } \\
\text { lincRNA features (sequence, structure, } \\
\text { transcriptional and orthology features). }\end{array}$ & [115] \\
\hline fRNAdb & $\begin{array}{l}\text { http://www.ncrna.org/ } \\
\text { frnadb }\end{array}$ & $\begin{array}{l}\text { Database containing a large collection on non- } \\
\text { coding transcripts including annotated and non- } \\
\text { annotated sequences from the H-inv database, } \\
\text { NONCODE and RNAdb databases. }\end{array}$ & [116] \\
\hline NPInter & $\begin{array}{l}\text { http://www.bioinfo.org/ } \\
\text { NPInter/index.php }\end{array}$ & $\begin{array}{l}\text { Database integrating the diverse body of } \\
\text { experimental knowledge on functional interactions } \\
\text { between ncRNAs (except tRNAs and rRNAs) and } \\
\text { protein-related biomacromolecules such as proteins, } \\
\text { mRNA of genomic RNAs. } \\
\text { Functional interactions (both physical interactions } \\
\text { and other forms of interactions) eliciting a cellular } \\
\text { reaction. }\end{array}$ & [117] \\
\hline
\end{tabular}




\begin{tabular}{|c|c|c|c|}
\hline Name & Website & Characteristics & References \\
\hline $\ln C e \mathrm{DB}$ & $\begin{array}{c}\text { http://gyanxet-beta.com/ } \\
\text { lncedb/ }\end{array}$ & $\begin{array}{l}\text { Database of lncRNAs acting as competing } \\
\text { endogenous RNAs. Putative interactions between } \\
\text { lncRNAs and mRNA targets using algorithms and } \\
\text { in silico tools such as StarBase. }\end{array}$ & [118] \\
\hline lncRbase & $\begin{array}{l}\text { http:// } \\
\text { bicresources.jcbose.ac.in/ } \\
\text { zhumur/lncrbase/ }\end{array}$ & $\begin{array}{l}\text { Comprehensive database of human and mouse } \\
\text { lncRNAs. } \\
\text { Contains genomic location, overlapping small } \\
\text { ncRNAs, associated repeat elements and imprinted } \\
\text { genes and lncRNA promoter information. }\end{array}$ & [119] \\
\hline
\end{tabular}

Table 4. Free databases for lncRNAs

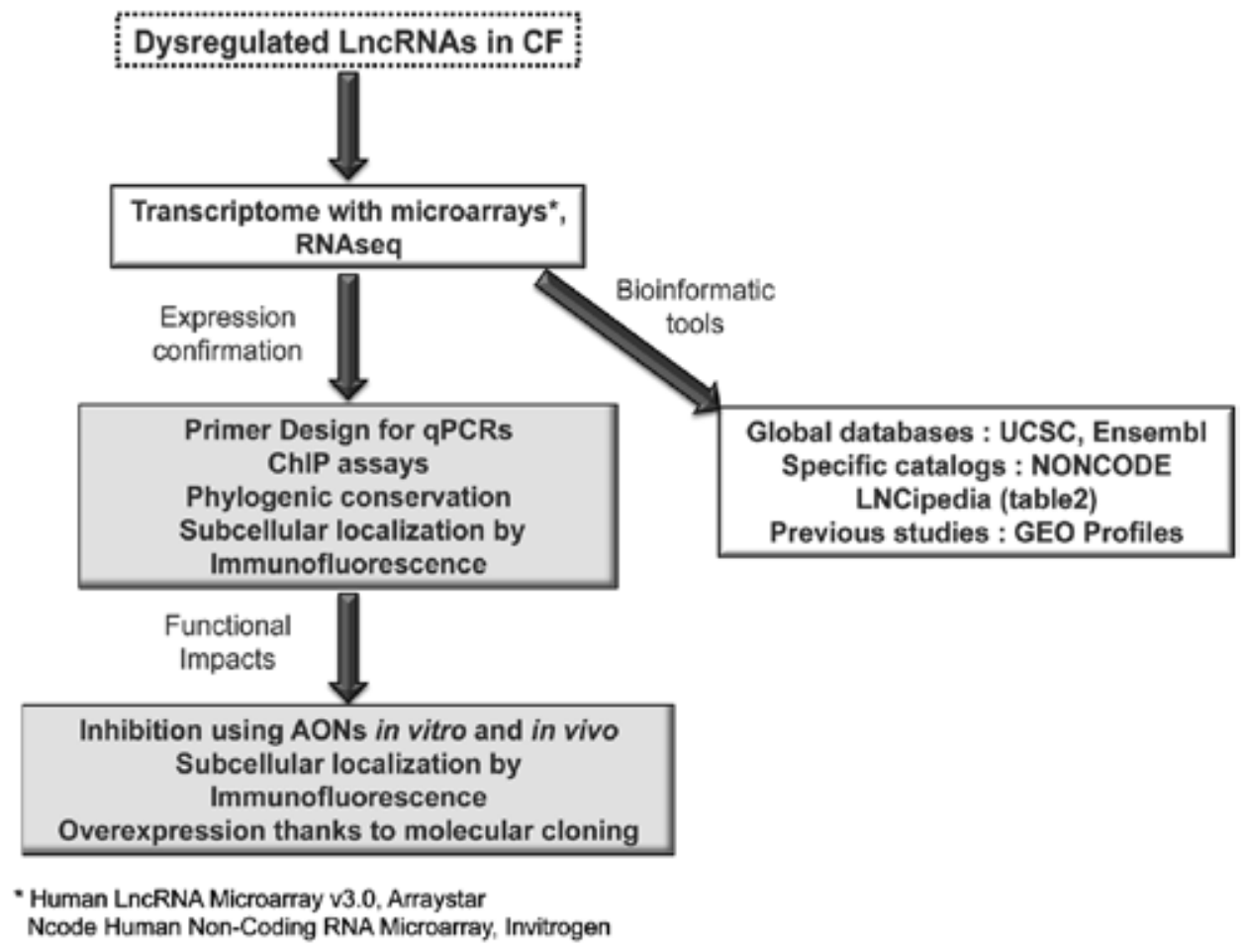

Figure 7. Strategies to study lncRNAs in CF and non-CF samples. Few techniques allow the quantitative analysis of lncRNAs in biological samples. RNAseq: global sequence screening is currently an easy option thanks to NGS and small RNA sequencing. This method allows detecting and quantitating in a biological sample all non-coding transcripts. However, as lncRNAs are very weakly expressed, this strategy might not be fully appropriate. To obtain a significant profile of ncRNAs, more than 180 million reads are required, whereas for protein-coding transcripts, less than 30 million are needed. Therefore, microarray analysis remains a powerful tool for global profiling of lncRNAs. In total, more than 30,586 lncRNAs have been analyzed based on the last lncRNA databases. AONs: antisense oligonucleotides. 


\subsection{Impact of mutations in lncRNA or miRNA genes}

To date, no ncRNA mutation has been described in CF. However, alterations in RNA sequence and/or structure can affect the synthesis, maturation and turnover of ncRNAs. Changes in RNA molecules can be introduced in different ways. For instance, SNPs may affect miRNA biogenesis. miRNA tailing can modify pre-miRNAs and mature miRNAs. RNA editing can modify nucleotide sequences of RNA transcripts. NGS technologies, including exome sequencing and complete re-sequencing of the CFTR gene, could reveal mutations in lncRNA sequences that may affect CF severity and outcome.

\section{Targeting miRNA as new putative therapeutic tool}

\subsection{Could miRNAs help in improving CF treatment?}

A recent work demonstrated that miR-138 mimics might restore CFTR-Phe508del expression and functional chloride transport. However, the authors stressed that miR-138 mimics may also have undesired effects, because miR-138 targets SIN3A, a highly conserved transcriptional repressor that regulates many genes [67]. Another anti-miRNA agent has been exploited as inhibitor of miR-509-3p, which is involved in the regulation of the CFTR gene. Recently, we reported that miRNA function can be blocked by targeting the CFTR gene with blockers. We designed blockers to prevent the binding of several miRNAs specifically to the 3'UTR-CFTR and tested them in well-differentiated primary human nasal epithelial cells from healthy individuals and patients with CF carrying the p.Phe508del CFTR mutation. These molecules rescued CFTR chloride channel activity by increasing CFTR mRNA and protein levels. This is in agreement with previous studies showing that complementation of just $6-10 \%$ of CFTR transcripts leads to the production of enough CFTR to maintain normal chloride transport in epithelia [75]. These data are supported by findings that the presence of a naturally occurring sequence variation in the CFTR promoter, in cis of a severe mutation, increases transcription. This allows the production of enough CFTR protein to reach the apical membrane cells and partially restore CFTR channel function, thus leading to a moderate CF phenotype despite the presence of a severe disease-causing mutation [76]. Similarly, stabilization of p.Phe508del CFTR protein has been associated with increased p.Phe508del CFTR channel activity [77].

\subsection{Assays and molecules}

As depicted in Figure 5Ad, inhibitors or target-site blocker oligonucleotides have been previously used to restore CFTR expression. Tests have been performed by incorporating inhibitors that induce degradation of the targeted endogenous miRNA or with oligonucleotides that block miRNA binding to the $3^{\prime}$ UTR of CFTR in cell lines, primary cultures and reconstituted epithelium 


\section{Conclusion and remarks}

The identification of cis- and trans-regulators and pathways involved in CFTR gene expression is essential for developing new CF targeted therapies. Over the past four decades, therapies for CF have focused entirely on symptoms to improve patients' quality of life. The first treatment (VX-770) targeted the basic defect in p.Gly551Asp-CFTR (1.6\% of patients with CF worldwide) [78]. The new molecule VX-809 has been evaluated in patients carrying the p.Phe508del CFTR mutation; however, on its own it does not have clear effects [79] and clinical trials testing the combination of different molecules are in progress. The mechanisms responsible for the phenotype severity are not well understood yet. Mutational heterogeneity and complex alleles influence CF severity. Moreover, the role of few modifier genes has been established [80]. ncRNAs could also contribute to CF progression and severity and their dysregulation in $\mathrm{CF}$ opens new perspectives for patient follow-up and treatment.

\section{Author details}

Jessica Varilh ${ }^{1}$, Jennifer Bonini ${ }^{2}$ and Magali Taulan-Cadars ${ }^{2 *}$

*Address all correspondence to: magali.taulan@inserm.fr

1 Laboratoire de Génétique Moléculaire, CHU Montpellier, France

2 Laboratoire de Génétique de Maladies Rares, Université Montpellier, UFR de Médecine, Montpellier, France

\section{References}

[1] McCarthy VA, Harris A. The CFTR gene and regulation of its expression. Pediatr Pulmonol. 2005;40(1): 1-8.

[2] Viart V, Varilh J, Lopez E, René C, Claustres M, et al. Phosphorylated C/EBP $\beta$ influences a complex network involving YY1 and USF2 in lung epithelial cells. PLoS One. 2013;8(4): e60211.

[3] Viart V, Bergougnoux A, Bonini J, Varilh J, Chiron R, et al. Transcription factors and miRNAs that regulate fetal to adult CFTR expression change are new targets for cystic fibrosis. Eur Respir J. 2015;45(1): 116-28.

[4] Bartoszewski, R, Rab A, Twitty G, Stevenson L, Fortenberry J, et al. The mechanism of cystic fibrosis transmembrane conductance regulator transcriptional repression during the unfolded protein response. J Biol Chem. 2008;283: 12154-65. 
[5] Li, S, Aufiero B, Schiltz RL, Walsh MJ. Regulation of the homeodomain CCAAT displacement/cut protein function by histone acetyltransferases p300/CREB-binding protein (CBP)-associated factor and CBP. Proc Natl Acad Sci USA. 2000;97: 7166-71.

[6] Paul T, Li S, Khurana S, Leleiko NS, Walsh MJ. The epigenetic signature of CFTR expression is co-ordinated via chromatin acetylation through a complex intronic element. Biochem J. 2007;408: 317-26.

[7] Bergougnoux A, Rivals I, Liquori A, Raynal C, Varilh J, et al. A balance between activating and repressive histone modifications regulates cystic fibrosis transmembrane conductance regulator (CFTR) expression in vivo. Epigenetics. 2014;9(7): 1007-17.

[8] Davies Wl, Vandenberg Ji, Sayeed Ra, Trezise Ae. Cardiac expression of the cystic fibrosis transmembrane conductance regulator involves novel exon 1 usage to produce a unique amino-terminal protein. J Biol Chem. 2004;279(16): 15877-87.

[9] Baudouin-Legros M, Hinzpeter A, Jaulmes A, Brouillard F, Costes B, Fanen P, Edelman A.Cell-specific posttranscriptional regulation of CFTR gene expression via influence of MAPK cascades on 3'UTR part of transcripts. Am J Physiol Cell Physiol. 2005 Nov;289(5): C1240-50. Epub 2005 Jun 8.PMID:15944206

[10] Gillen AE, Gosalia N, Leir SH, Harris A. MicroRNA regulation of expression of the cystic fibrosis transmembrane conductance regulator gene. Biochem J. 2011;438: 25-32.

[11] Megiorni F, Cialfi S, Dominici C, Quattrucci S, Pizzuti A. Synergistic Post-Transcriptional Regulation of the Cystic Fibrosis Transmembrane conductance Regulator (CFTR) by miR-101 and miR-494 Specific Binding. PLoS One. 2011;6: e26601.

[12] Bartels CL, Tsongalis GJ. MicroRNAs: novel biomarkers for human cancer. Clin Chem. 2009;55(4): 623-31.

[13] Twayana S, Legnini I, Cesana M, Cacchiarelli D, Morlando M, et al. Biogenesis and function of non-coding RNAs in muscle differentiation and in Duchenne muscular dystrophy. Biochem Soc Trans. 2013;41(4): 844-9.

[14] Moffatt MF. Genes in asthma: new genes and new ways.Curr Opin Allergy Clin Immunol. 2008;8(5): 411-17.

[15] Nana-Sinkam SP, Karsies T, Riscili B, Ezzie M, Piper M. Lung microRNA: from development to disease. Expert Rev Respir Med. 2009;3(4): 373-85.

[16] Mestdagh P, Vandesompele J, Brusselle G, Vermaelen K. Non-coding RNAs and respiratory disease. Thorax. 2014. pii: thoraxjnl-2014-206404.

[17] Booton R, Lindsay MA. Emerging role of MicroRNAs and long noncoding RNAs in respiratory disease. Chest. 2014;146(1): 193-204.

[18] Morris KV, Mattick JS. The rise of regulatory RNA. Nat Rev Genet. 2014;15(6): 423-37. 
[19] Derrien T, Guigo R, Johnson R. The long non-coding RNAs : a new player in the « dark matter ». Front Genet. 2012;2:107204.

[20] Lee RC, Feinbaum RL, Ambros V. The C. elegans heterochronic gene lin-4 encodes small RNAs with antisense complementary to lin-14. Cell. 1993;75(5): 843-54.

[21] $\mathrm{Hu}$ W, Alvarez-Dominguez JR, Lodish HF. Regulation of mammalian cell differenciation by long non-coding RNAs. EMBO Rep. 2012;13(11): 971-83.

[22] Fatica A, Bozzoni I. Long non-coding RNAs: new players in cell differenciation and development. Nat Rev Genet. 2014;15(1): 7-21.

[23] Michalik KM, You X, Manavski Y, Doddaballapur A, Zörnig M, et al. Long noncoding RNA MALAT1 regulates endothelial cell function and vessel growth. Circ Res. 2014;114(9): 1389-97.

[24] Santosh B, Varshney A, Yadava PK. Non-coding RNAs: biological functions and applications. Cell Biochem Funct. 2015;33(1): 14-22.

[25] Derrien T, Johnson R, Bussotti G, Tanzer A, Djebali S, et al. The GENCODE v7 catalog of human long noncoding RNAs: analysis of their gene structure, evolution and expression. Genome Res. 2012;22(9): 1775-89.

[26] Bonasio R, Shiekhattar R. Regulation of transcription by long noncoding RNAs. Annu Rev Genet. 2014;48: 433-55.

[27] Sigova AA, Mullen AC, Molinie B, Gupta S, Orlando DA, et al. Divergent transcription of long noncoding RNA/mRNA gene pairs in embryonic stem cells. Proc Natl Acad Sci USA. 2013;110: 2876-81.

[28] Guttman M, Amit I, Garber M, French C, Lin MF, et al. Chromatin signature reveals over a thousand highly conserved large non-coding RNAs in mammals. Nature. 2009;458: 223-27.

[29] Ulitsky I, Shkumatava A, Jan CH, Sive H, Bartel DP. Conserved function of lincRNAs in vertebrate embryonic development despite rapid sequence evolution. Cell. 2011;147: 1537-50.

[30] Van Bakel H, Nislow C, Blencowe BJ, Hughes TR. Most "dark matter" transcripts are associated with known genes. PLOS Biol. 2010; 8:e1000371.

[31] Clemson CM, McNeil JA, Willard HF, Lawrence JB. XIST RNA paints the inactive X chromosome at interphase: evidence for a novel RNA involved in nuclear/chromosome structure. J Cell Biol. 1996;132: 259-75.

[32] Djebali S, Davis CA, Merkel A, Dobin A, Lassmann T, et al. Landscape of transcription in human cells. Nature. 2012; 89: 101-8. 
[33] Cho SF, Chang YC, Chang CS, Lin SF, Liu YC, et al. MALAT1 long non-coding RNA is overexpressed in multiple myeloma and may serve as a marker to predict disease progression. BMC Cancer. 2014;14: 809.

[34] Sun T, Ye H, Wu CL, Lee GS, Kantoff PW. Emerging players in prostate cancer: long non-coding RNAs. Am J Clin Exp Urol. 2014;2(4): 294-9.

[35] Li J, Xuan Z, Liu C. Long non-coding RNAs and complex human diseases. Int J Mol Sci. 2013;14(9): 18790-808.

[36] Maass PG, Luft FC, Bähring S. Long non-coding RNA in health and disease. J Mol Med (Berl). 2014;92(4): 337-46.

[37] Vencken SF, Greene CM, McKiernan PJ. Non-coding RNA as lung disease biomarkers. Thorax. 2014. pii: thoraxjnl-2014-206193.

[38] Lee Y, Jeon K, Lee JT, Kim S, Kim VN. MicroRNA maturation: stepwise processing and subcellular localization. EMBO J. 2002;21(17): 4663-70.

[39] Monteys AM, Spengler RM, Wan J, Tecedor L, Lennox KA, et al. Structure and activity of putative intronic miRNA promoters. RNA 16, 495-505 (2010)

[40] Ozsolak F, Poling LL, Wang Z, Liu H, Liu XS, et al. Chromatin structure analyses identify miRNA promoters. Genes Dev. 22, 3172-83 (2008)

[41] Borchert GM, Lanier W, Davidson BL. RNA polymerase III transcribes human microRNAs. Nat Struct Mol Biol. 2006;13(12): 1097-101.

[42] Kim, VN, Han J and Siomi MC. Biogenesis of small RNAs in animals. Nature Rev Mol. Cell Biol. 2009;10: 126-39.

[43] Krol J, Loedige I, Filipowicz W. The widespread regulation of microRNA biogenesis, function and decay. Nature Rev Genet. 2010;11: 597-610.

[44] Davis-Dusenbery BN, Hata A. Mechanisms of control of microRNA biogenesis. J Biochem. 2010;148: 381-92.

[45] Kim YK, Kim VN. Processing of intronic microRNAs. EMBO J. 2007;26(3):775-83.

[46] Auyeung VC, Ulitsky I, McGeary SE, Bartel DP. Beyond secondary structure: primary-sequence determinants license pri-miRNA hairpins for processing. Cell. 2013;152: 844-58.

[47] Guil S, Caceres JF. The multifunctional RNA-binding protein hnRNP A1 is required for processing of miR-18a. Nature Struct Mol Biol. 2007;14: 591-96.

[48] Trabucchi M, Briata P, Garcia-Mayoral M, Haase AD, Filipowicz W, et al. The RNAbinding protein KSRP promotes the biogenesis of a subset of microRNAs. Nature. 2009;459: 1010-14. 
[49] Michlewski G, Guil S, Semple CA, Caceres JF. Posttranscriptional regulation of miRNAs harboring conserved terminal loops. Mol Cell. 2008;32: 383-93.

[50] Bartel DP. MicroRNAs: target recognition and regulatory functions. Cell. 2009;136(2): 215-33.

[51] Salmanidis M, Pillman K, Goodall G, Bracken C. Direct transcriptional regulation by nuclear microRNAs. Int J Biochem Cell Biol. 2014;54: 304-11.

[52] Chou JL, Rozmahel R, Tsui LC, et al. Characterization of the promoter region of the cystic fibrosis transmembrane conductance regulator gene. J Biol Chem. 1991;266: 24471-76.

[53] Yoshimura K, Nakamura H, Trapnell BC, Dalemans W, Pavirani A, et al. The cystic fibrosis gene has a "housekeeping"-type promoter and is expressed at low levels in cells of epithelial origin. J Biol Chem. 1991; 266: 9140-44.

[54] Koh J, Sferra TJ, Collins FS. Characterization of the cystic fibrosis transmembrane conductance regulator promoter region. Chromatin context and tissue-specificity. J Biol Chem. 1993; 268: 15912-21.

[55] White NL, Higgins CF, Trezise AE. Tissue-specific in vivo transcription start sites of the human and murine cystic fibrosis genes. Hum Mol Genet. 1998;7: 363-9.

[56] Harris A, Chalkley G, Goodman S, Coleman L. Expression of the cystic fibrosis gene in human development. Development. 1991;113: 305-10.

[57] McCray PB Jr, Reenstra WW, Louie E, et al. Expression of CFTR and presence of cAMP-mediated fluid secretion in human fetal lung. Am J Physiol. 1992;262: L472L481.

[58] Trezise AE, Chambers JA, Wardle CJ, Gould S, Harris A. Expression of the cystic fibrosis gene in human foetal tissues. Hum Mol Genet. 1993;2: 213-18.

[59] Tizzano EF, O'Brodovich H, Chitayat D, Bènichou JC, Buchwald M. Regional expression of CFTR in developing human respiratory tissues. Am J Respir Cell Mol Biol. 1994;10: 355-62.

[60] Marcorelles P, Montier T, Gillet D, Lagarde N, Ferec C. Evolution of CFTR protein distribution in lung tissue from normal and $\mathrm{CF}$ human fetuses. Pediatr Pulmonol. 2007; 42: 1032-40.

[61] Amato F, Seia M, Giordano S, Elce A, Zarrilli F, et al. Gene mutation in microRNA target sites of CFTR gene: a novel pathogenetic mechanism in cystic fibrosis? PLoS One. 2013;8(3): e60448.

[62] Bonfield TL, Panuska JR, Konstan MW, Hilliard KA, Hilliard JB, et al. Inflammatory cytokines in cystic fibrosis lungs. Am J Respir Crit Care Med. 1995;152(6 Pt 1): 111-18. 
[63] Birrer P, McElvaney NG, Rüdeberg A, Sommer CW, Liechti-Gallati S, et al. Proteaseantiprotease imbalance in the lungs of children with cystic fibrosis. Am J Respir Crit Care Med. 1994;150(1): 207-13.

[64] McKiernan PJ, Molloy K, Cryan SA, McElvaney NG, Greene CM. Long noncoding RNA are aberrantly expressed in vivo in the cystic fibrosis bronchial epithelium. Int J Biochem Cell Biol. 2014;52: 184-91.

[65] Oglesby IK, Chotirmall SH, McElvaney NG, Greene CM. Regulation of cystic fibrosis transmembrane conductance regulator by microRNA-145, -223 , and -494 is altered in $\Delta$ F508 cystic fibrosis airway epithelium. J Immunol. 2013;190(7): 3354-62.

[66] Xu W, Hui C, Yu SS, Jing C, Chan HC. MicroRNAs and cystic fibrosis-an epigenetic perspective. Cell Biol Int. 2011;35(5): 463-6.

[67] Ramachandran S, Karp PH, Osterhaus SR, Jiang P, Wohlford-Lenane C, et al. Posttranscriptional regulation of cystic fibrosis transmembrane conductance regulator expression and function by microRNAs. Am J Respir Cell Mol Biol. 2013; 49(4): 544-51.

[68] Bhattacharyya S, Balakathiresan NS, Dalgard C, Gutti U, Armistead D, et al. Elevated miR-155 promotes inflammation in cystic fibrosis by driving hyperexpression of interleukin-8. J Biol Chem. 2011;286(13): 11604-15.

[69] Bhattacharyya S, Kumar P, Tsuchiya M, Bhattacharyya A, Biswas R. Regulation of miR-155 biogenesis in cystic fibrosis lung epithelial cells: antagonistic role of two mRNA-destabilizing proteins, KSRP and TTP. Biochem Biophys Res Commun. 2013;433(4): 484-8.

[70] Oglesby IK, Bray IM, Chotirmall SH, Stallings RL, O'Neill SJ, et al. miR-126 is downregulated in cystic fibrosis airway epithelial cells and regulates TOM1 expression. J Immunol. 2010;184(4): 1702-9.

[71] Katoh Y, Shiba Y, Mitsuhashi H, Yanagida Y, Takatsu H, et al. Tollip and Tom1 form a complex and recruit ubiquitin-conjugated proteins onto early endosomes. J Biol Chem. 2004;279(23): 24435-43.

[72] Yamakami M, Yokosawa H. Tom1 (target of Myb 1) is a novel negative regulator of interleukin-1- and tumor necrosis factor-induced signaling pathways. Biol Pharm Bull. 2004;27(4): 564-6.

[73] Fabbri E, Borgatti M, Montagner G, Bianchi N, Finotti A, et al. Expression of microRNA-93 and Interleukin-8 during Pseudomonas aeruginosa-mediated induction of proinflammatory responses. Am J Respir Cell Mol Biol. 2014;50(6): 1144-55.

[74] Weldon S, McNally P, McAuley DF, Oglesby IK, Wohlford-Lenane CL, et al. Taggart CC.miR-31 dysregulation in cystic fibrosis airways contributes to increased pulmonary cathepsin S production. Am J Respir Crit Care Med. 2014;190(2): 165-74. 
[75] Broackes-Carter FC, Mouchel N, Gill D, Hyde S, Bassett J, et al. Temporal regulation of CFTR expression during ovine lung development: implications for CF gene therapy. Hum Mol Genet. 2002;11(2): 125-31.

[76] Romey MC, Pallares-Ruiz N, Mange A, Mettling C, Peytavi R, et al. A naturally occurring sequence variation that creates a $\mathrm{YY} 1$ element is associated with increased cystic fibrosis transmembrane conductance regulator gene expression. J Biol Chem. 2000;275(5): 3561-7.

[77] Hutt DM, Herman D, Rodrigues AP, Noel S, Pilewski JM, et al. Reduced histone deacetylase 7 activity restores function to misfolded CFTR in cystic fibrosis. Nat Chem Biol. 2010;6(1): 25-33.

[78] Ramsey BW, Davies J, McElvaney NG, Tullis E, Bell SC, et al. VX08-770-102 Study Group. A CFTR potentiator in patients with cystic fibrosis and the G551D mutation. N Engl J Med. 2011;365(18): 1663-72.

[79] Clancy JP, Jain M. Personalized medicine in cystic fibrosis: dawning of a new era. Am J Respir Crit Care Med. 2012;186(7): 593-7.

[80] Knowles MR, Drumm M. The influence of genetics on cystic fibrosis phenotypes. Cold Spring Harb Perspect Med. 2012;2(12).

[81] Sharma A, Kumar M, Aich J, Hariharan M, Brahmachari SK, et al. Posttranscriptional regulation of interleukin-10 expression by hsa-miR-106a. Proc Natl Acad Sci USA. 2009;106(14):5761-6.

[82] Lu TX, Munitz A, Rothenberg ME. MicroRNA-21 is up-regulated in allergic airway inflammation and regulates IL-12p35 expression. J Immunol. 2009;182(8): 4994-5002.

[83] Chiba Y, Tanabe M, Goto K, Sakai H, Misawa M. Down-regulation of miR-133a contributes to up-regulation of Rhoa in bronchial smooth muscle cells. Am J Respir Crit Care Med. 2009;180(8): 713-9.

[84] Mohamed JS, Lopez MA, Boriek AM. Mechanical stretch up-regulates microRNA-26a and induces human airway smooth muscle hypertrophy by suppressing glycogen synthase kinase-3ß. J Biol Chem. 2010;285(38): 29336-47.

[85] Polikepahad S, Knight JM, Naghavi AO, Oplt T, Creighton CJ, et al. Proinflammatory role for let-7 microRNAS in experimental asthma. J Biol Chem. 2010;285(39): 30139-49.

[86] Christenson, S., J. Campbell, J. Zeskind, J. Mcdonough, P. Sanchez, et al. MicroRNA as regulators of gene expression changes that occur with the progression of emphysema. Am J Respir Crit Care Med. 2010;181: pp. A2024.

[87] Sato T, Liu X, Nelson A, Nakanishi M, Kanaji N, et al. Reduced miR-146a increases prostaglandin $\mathrm{E}$ in chronic obstructive pulmonary disease fibroblasts. Am J Respir Crit Care Med. 2010;182(8): 1020-9. 
[88] Liu G, Friggeri A, Yang Y, Milosevic J, Ding Q, et al. miR-21 mediates fibrogenic activation of pulmonary fibroblasts and lung fibrosis. J Exp Med. 2010;207(8): 1589-97.

[89] Pottier N, Maurin T, Chevalier B, Puisségur MP, Lebrigand K, et al. Identification of keratinocyte growth factor as a target of microRNA-155 in lung fibroblasts: implication in epithelial-mesenchymal interactions. PLoS One. 2009;4(8): e6718.

[90] Pandit KV, Corcoran D, Yousef H, Yarlagadda M, Tzouvelekis A, et al. Inhibition and role of let-7d in idiopathic pulmonary fibrosis. Am J Respir Crit Care Med. 2010;182(2): 220-9.

[91] Friedman, RC, Farh KK, Burge CB, Bartel DP. Most mammalian mRNAs are conserved targets of microRNAs. Genome Res. 2009;19: 92-105.

[92] John B, Enright AJ, Aravin A, Tuschl T, Sander C, et al. Human MicroRNA targets. PLoS Biol. 2004;2: e363.

[93] Krek A, Grun D, Poy MN, Wolf R, Rosenberg L, et al. Combinatorial microRNA target predictions. Nat Genet. 2005;7: 495-500.

[94] Rehmsmeier M, Steffen P, Hochsmann M, Giegerich R. Fast and effective prediction of microRNA/target duplexes. RNA. 2004;10: 1507-17.

[95] Kertesz M, Iovino N, Unnerstall U, Gaul U, Segal E. The role of site accessibility in microRNA target recognition. Nat Genet. 2007;39: 1278-84.

[96] Wong N, Wang X. miRDB: an online resource for microRNA target prediction and functional annotations. Nucleic Acids Res. 2015 Jan 28;43 (Database issue):D146-52.

[97] Maragkakis M, Alexiou P, Papadopoulos GL, Reczko M, Dalamagas T, et al. Accurate microRNA target prediction correlates with protein repression levels. BMC Bioinformatics. 2009;10: 295.

[98] Kozomara A, Griffiths-Jones S. miRBase: annotating high confidence microRNAs using deep sequencing data. Nucleic Acids Res. 2014;42 (Database issue):D68-73.

[99] Hsu JB, Chiu CM, Hsu SD, Huang WY, Chien CH, et al. miRTar: an integrated system for identifying miRNA-target interactions in human. BMC Bioinformatics. 2011;12: 300 .

[100] Hsu SD, Chu CH, Tsou AP, Chen SJ, Chen HC et al. miRNAMap 2.0: genomic maps of microRNAs in metazoan genomes. Nucleic Acids Res. 2008;36: D165-169.

[101] Le Brigand K, Robbe-Sermesant K., Mari B, Barbry P. MiRonTop: mining microRNAs targets across large scale gene expression studies. Bioinformatics. 2010;26: 3131-32.

[102] Le Bechec A, Portales-Casamar E, Vetter G, Moes M, Zindy PJ et al. MIR@NT@N: a framework integrating transcription factors, microRNAs and their targets to identify sub-network motifs in a meta-regulation network model. BMC Bioinformatics. 2011;12: 67 . 
[103] Nam JW, Kim J, Kim SK, Zhang BT. ProMiR II: a web server for the probabilistic prediction of clustered, nonclustered, conserved and nonconserved microRNAs. Nucleic Acids Res. 2006;34: W455-458.

[104] Alexiou P, Vergoulis T, Gleditzsch M, Prekas G, Dalamagas T et al. miRGen 2.0: a database of microRNA genomic information and regulation. Nucleic Acids Res. 2010;38 (Database issue): D137-41.

[105] Maselli V, Di Bernardo D, Banfi S. CoGemiR: a comparative genomics microRNA database. BMC Genomics. 2008;9: 457.

[106] Volders PJ, Helsens K, Wang X, Menten B, Martens L et al. LNCipedia: a database for annotated human lncRNA transcript sequences and structures. Nucleic Acids Res. 2013;41(Database issue): D246-51.

[107] Volders PJ, Verheggen K, Menschaert G, Vandepoele K, Martens L2 et al. An update on LNCipedia: a database for annotated human lncRNA sequences. Nucleic Acids Res. 2015;43(Database issue): D174-80.

[108] Jiang Q, Wang J, Wu X, Ma R, Zhang T et al. LncRNA2Target: a database for differentially expressed genes after lncRNA knockdown or overexpression. Nucleic Acids Res. 2015;43 (Database issue): D193-6.

[109] Ma L, Li A, Zou D, Xu X, Xia L et al. LncRNAWiki: harnessing community knowledge in collaborative curation of human long non-coding RNAs. Nucleic Acids Res. 2015;43 (Database issue): D187-92.

[110] Amaral PP, Clark MB, Gascoigne DK, Dinger ME, Mattick JS. IncRNAdb: a reference database for long noncoding RNAs. Nucleic Acids Res. 2011;39 (Database issue): D146-51.

[111] Quek XC, Thomson DW, Maag JL, Bartonicek N, Signal B et al. lncRNAdb v2.0: expanding the reference database for functional long noncoding RNAs. Nucleic Acids Res. 2015;43 (Database issue):D168-73.

[112] Xie C, Yuan J, Li H, Li M, Zhao G et al. NONCODEv4: exploring the world of long non-coding RNA genes. Nucleic Acids Res. 2014;42(Database issue): D98-103.

[113] Liu C, Bai B, Skogerbø G, Cai L, Deng W et al. NONCODE: an integrated knowledge database of non-coding RNAs. Nucleic Acids Res. 2005;33 (Database issue): D112-5.

[114] Dinger ME, Pang KC, Mercer TR, Crowe ML, Grimmond SM et al. NRED: a database of long noncoding RNA expression. Nucleic Acids Res. 2009;37(Database issue): D122-6.

[115] Cabili MN, Trapnell C, Goff L, Koziol M, Tazon-Vega B et al. Integrative annotation of human large intergenic noncoding RNAs reveals global properties and specific subclasses. Genes Dev. 2011;25(18):1915-27. 
[116] Kin T, Yamada K, Terai G, Okida H, Yoshinari Y et al. fRNAdb: a platform for mining/annotating functional RNA candidates from non-coding RNA sequences. Nucleic Acids Res. 2007;35 (Database issue):D145-8.

[117] Yuan J, Wu W, Xie C, Zhao G, Zhao Y et al. NPInter v2.0: an updated database of ncRNA interactions. Nucleic Acids Res. 2014;42(Database issue): D104-8.

[118] Das S, Ghosal S, Sen R, Chakrabarti J. lnCeDB: database of human long noncoding RNA acting as competing endogenous RNA. PLoS One. 2014;9(6): e98965.

[119] Chakraborty S, Deb A, Maji RK, Saha S, Ghosh Z. LncRBase: an enriched resource for lncRNA information. PLoS One. 2014;9(9): e108010. 


\section{Section 5}

Cystic Fibrosis: Genetic Correction 

Chapter 13

\title{
Therapy for Cystic Fibrosis Caused by Nonsense Mutations
}

\author{
Roberto Gambari, Giulia Breveglieri, \\ Francesca Salvatori, Alessia Finotti and \\ Monica Borgatti
}

Additional information is available at the end of the chapter

http://dx.doi.org/10.5772/61053

\begin{abstract}
Nonsense mutations cover about $10 \%$ of cystic fibrosis (CF) patients and generate premature termination codons (PTCs) leading to premature translational termination and causing the synthesis of truncated non-functional or partially functional CFTR (cystic fibrosis transmembrane conductance regulator) protein. The read-through approach is the suppression of translation terminations at PTCs and it has been developed as a therapeutic strategy to restore full-length protein using aminoglycoside antibiotics or PTC124. Phenotypic consequences of PTCs can be exacerbated by the nonsense-mediated mRNA decay (NMD) pathway, which detects and degrades mRNA containing PTC. Therefore, modulation of NMD is also of interest as a potential target for suppression therapy. Not all PTCs are susceptible to the read-through treatment alone, especially where the nonsense mutations are combined with other CFTR mutations. For example, many CF patients present the highly frequent F508del CF mutation, causing an alteration of the cell membrane positioning of the CFTR channel. Pharmacological correctors that rescue the trafficking of F508del CFTR may overcome this defect. A combined administration of correctors/potentiators, readthrough molecules, and/or NMD inhibitors, depending on the genotype of the CF patients, could be the basis for the design of a personalized therapeutic approach.
\end{abstract}

Keywords: Cystic fibrosis, PTCs, read-through, NMD inhibitors, correctors, potentiators 


\section{Introduction}

\subsection{Classes of mutation in cystic fibrosis}

The CFTR protein is a cAMP-regulated chloride channel that resides in the apical membrane of epithelial cells of different organs such as the intestines, lung, pancreas, and sweat glands. However, the main pathologic symptom of cystic fibrosis (CF) is respiratory disease with recurring infections, inflammation, and obstructions that induce progressive lung damage and possible respiratory difficulties [1].

More than 1,900 different CFTR mutations have been reported so far and can be generally categorized into five classes (Table 1). Approximately $40 \%$ of CFTR mutations belong to class I. Nonsense mutations cover $10 \%$ of CF patients, but in some populations specific nonsense mutations can occur in up to $50 \%$ of CF subjects. For example, G542X mutation, the most common CFTR nonsense mutation, has been found in $2 \%$ of Caucasian CF patients. The most frequent mutation of class II is the F508del mutation (a deletion of the phenylalanine residue at position 508) found in 75\% of CF patients. While for class III, the G551D mutation has been found in approximately $4 \%$ of CF patients. Class I-III mutations determine an important defect in CFTR function and cause a severe CF phenotype, while mutations belonging to Classes IV$\mathrm{V}$ permit a residual CFTR protein function and induce a milder CF phenotype. There is general consensus on the fact that in order to ameliorate the CF phenotype, the restoration of at least 5-35\% of normal CFTR function is necessary [1-4].

\begin{tabular}{|c|c|c|c|c|c|}
\hline Class & I & II & III & IV & $\mathbf{V}$ \\
\hline $\begin{array}{l}\text { Types of } \\
\text { mutations }\end{array}$ & $\begin{array}{l}\text { Nonsense; Frame } \\
\text { shift }\end{array}$ & Missense & Missense & Missense & Missense \\
\hline Defect on CFTR & No expression & $\begin{array}{l}\text { No correct } \\
\text { localization to cell } \\
\text { membrane }\end{array}$ & $\begin{array}{l}\text { No chloride } \\
\text { channel function }\end{array}$ & $\begin{array}{l}\text { Reduced chloride } \\
\text { channel } \\
\text { conductance }\end{array}$ & $\begin{array}{l}\text { Reduced } \\
\text { expression }\end{array}$ \\
\hline Examples & G542, W1282X & F508del & G551D & R117H; R334W & A445E \\
\hline $\begin{array}{l}\text { Possible } \\
\text { pharmacological } \\
\text { therapy }\end{array}$ & Read-through & Correctors & Potentiators & Potentiators & Potentiators \\
\hline
\end{tabular}

Table 1. Principal classes of CF mutations and therapeutic intervention.

\subsection{PTCs and NMD mechanism}

Premature termination codons (PTCs) lead to premature translational termination resulting in absent functional proteins. Different inherited and acquired diseases can be attributable to PTCs, such as cystic fibrosis, Duchenne muscular dystrophy (DMD), mucopolysaccharidosis, spinal muscular atrophy (SMA) type I and type II, X-linked nephrogenic diabetes insipidus, rhinitis pigmentosa $X$-linked, nephropathic cystinosis, and $\beta$-thalassemia [5]. 
PTCs are responsible for about $10 \%$ of CF cases worldwide, but are particularly common in Ashkenazi Jews (40\% of Israeli CF patients) [6]. The translation of PCT-containing mRNAs leads to the formation of truncated CFTR, in most cases lacking in its function [7].

During molecular evolution, a mechanism to eliminate these aberrant mRNAs containing PTCs has been developed, known as nonsense-mediated mRNA decay (NMD). This process of quality control is observed in all eukaryotic organisms and has an important role in the regulation of many cellular functions. Its physiological substrates act in a wide range of processes such as transcription, DNA repair, cell growth, intracellular transport, and NMD itself. Through this process, the mRNA levels containing PTCs are reduced but not completely eliminated, inducing a reduced level of proteins, which are synthesized in a truncated form [5].

In mammals the NMD process operates by the recognition of a premature stop codon. It is observed that the ability of PTCs in inducing NMD depends on its location with respect to the sequence downstream and associated proteins [8]. The NMD process often occurs in mammalian cells after the splicing of pre-mRNA and, in most cases, is controlled by the exon junction complex (EJC). This complex consists of at least 10 proteins binding to regions consisting of 20-24 nucleotides upstream of the exon-exon junction and including UPF2 and $\mathrm{UPF} 3$ proteins.

According to the currently proposed models of the NMD mechanism, for the majority of mRNAs, the stop codons that are localized more than 50-55 nucleotides upstream of an exonexon junction are recognized as premature, since the EJC is found downstream to the stop codon. The EJC is recognized by UPF2 and UPF3 and ribosomes start to translate the mRNA until reaching the PTC. The termination of the translation is triggered by the recognition of PTC s by eRF1 and eRF3, release factors recruited by ribosomes. These proteins then recruit the NMD key factor UPF1, which in turn binds to the SMG1 kinase. These four proteins (eRF1, eRF3, UPF1, SMG1) constitute the SURF complex, responsible for ribosome blocking. When the PTC is upstream of this EJC, UPF1 at the termination site may interact with UPF2 (associated with EJC) causing the phosphorylation of UPF1 by SMG1 and the dissociation of release factors (eRF1 and eRF3). The phosphorylated UPF1 recruits additional factors (SMG5, SMG6, and SMG7) triggering NMD. Once the NMD is stimulated, target transcripts are degraded by $5^{\prime}$ and $3^{\prime}$ exonucleases [5].

\section{PTCs and NMD mechanism as targets for CF therapy}

\subsection{The read-through approach in CF}

With the aim of overcoming the effects of the presence of PTCs in CF, several drugs were studied, such as aminoglycoside antibiotics and PTC124 (PTC Therapeutics), and found to be able to incorporate a random amino acid at the PTC position of mRNAs. This induces a ribosomal read-through of the premature, but not of the natural termination codons, restoring CFTR function in CF patients [9-10]. These treatments may provide a means of restoring clinically relevant levels of protein function in patients carrying PTCs in the mutated gene, 
and introduce new hope for the development of a pharmacologic approach for the cure of several important genetic diseases, including CF [9-10].

\subsubsection{Aminoglycosides}

Aminoglycoside antibiotics selectively bind to ribosomes (at decoding aminoacyl site, A-site) and cause the insertion of a near cognate amino-acyl tRNA into this ribosomal A site, allowing ribosome read-through at PTC and production of a full-length protein [11].

Previous studies have shown that a variety of aminoglycosides are able to suppress CFTR nonsense mutations and restore functional CFTR protein levels not only in mouse models, but also in human clinical trials [1,12-16].

Gentamicin and amikacin are able to suppress the common G542X nonsense mutation in a transgenic CF mouse model, restoring approximately 20-30\% of full-length CFTR protein, suggesting these compounds as read-through molecules to treat CF patients carrying PTCs [1]. In particular, the CFTR nonsense transgenic mouse model carrying a human CFTR cDNA with G542X nonsense mutation was used to screen the read-through activity of different aminoglycosides [1,12]. Five to thirty-four $\mathrm{mg} / \mathrm{kg}$ of gentamicin was administered by daily subcutaneous injections for 14 days and found to induce a $22 \%$ increase of the wild-type CFTR function. Similar results were observed with amikacin, used at $15-170 \mathrm{mg} / \mathrm{kg}$, while tobramycin was much less efficient than these two aminoglycosides in rescuing CFTR function $[1,12,13]$.

In humans, Wilschanski et al. [1,14] reported a study where 0.9 mgs of gentamicin were administered daily via nasal drops for 14 days in nine CF patients. The data obtained demonstrated a significant improvement in chloride conductance by nasal potential difference (PD) measurements. Full-length CFTR protein was also detected in the nasal epithelia of treated CF patients. On the contrary, patients homozygous for the F508del mutation, used as a control group, did not show improvements in the CFTR function or restoration of CFTR protein [14].

Clancy et al. [15] reported a study where $2.5 \mathrm{mg} / \mathrm{kg}$ of gentamicin was administered intravenously every 8 hours for one week to $10 \mathrm{CF}$ patients. They reported a three-fold increased incidence of nasal PD readings in the direction of chloride secretion in treated PTC subjects relative to CF controls. Airway chloride secretion was also stronger, with a high level of chloride secretion not detected in the control subjects, while sweat chloride measurements were not increased. In recent studies [1, 16], two different groups (11 CF patients with nonsense mutations and $18 \mathrm{CF}$ patients without nonsense mutations) were treated with $3.6 \mathrm{mg}$ of gentamicin or tobramycin daily by a nasal spray device for 28 days. The results indicated no improvement in chloride ion transport or CFTR localization using these two aminoglycosides. The authors underlined that nasally administered aminoglycosides did not produce detectable improvement in CFTR function because the CF subjects were heterozygous for different CFTR mutations [16]. This suggests that not all premature stop mutations are susceptible to the readthrough treatment alone in order to ameliorate the phenotype of CF patients carrying nonsense mutations. When the read-through approach is combined with the use of potentiators/ correctors of CFTR, however, the clinical outcome may be much more positive and this strategy could delineate a personalized therapeutic approach based on the CF genotype of patients [16]. 
A recent study using tobramycin was performed in yeast models with modulated NMD by UPF1 deletion [17]. Yeast strains were transformed with the Renilla/Firefly dual-luciferase reporter plasmids carrying either nonsense (UAG, UGA, UAA) or sense (CAG, CGA, CAA) codon between the luciferase genes. Tobramycin, a aminoglycoside antibiotic normally used to treat Pseudomonas aeruginosa pulmonary infection in CF patients, exhibited read-through ability on PTCs preferentially in the absence of NMD mechanism. These findings could be an explanation of the lower activity of tobramycin in restoring CFTR synthesis observed in mouse models and human clinical trials, while the data indicate possible combined treatment of tobramycin in the presence of NMD inhibitors.

Most of the novel aminoglycoside molecules have been synthetized with the major aim of optimizing their antibacterial activity, and not with the objective of obtaining the highest possible read-through action on PTCs. Current clinical studies investigating this class of molecules were performed using only commercially available aminoglycosides, such as gentamicin, amikacin, and tobramycin [14-18]. The general conclusion of these studies is that tobramycin presented a low efficiency as PTC suppressors, while gentamicin was efficient but toxic [14-18]. Alternative options to gentamicin could be amikacin [13] or paromomycin [19], two aminoglycosides with lower cellular toxicity, but not yet studied in human clinical trials as read-through molecules.

A future goal should be the design and synthesis of new aminoglycosides with high readthrough activity and low toxicity. Baasov et al. [18] reported the synthesis of a series of new derivatives of paromomycin proposed as read-through molecules in vitro. In particular NB30, a pseudo-trisaccharide derivative, had higher read-through activity compared to paromomycin and gentamicin and reduced toxicity [20,21]. Another derivate, NB124, presented a more efficient read-through activity than gentamycin. It restored full-length CFTR expression and chloride transport in Fischer rat thyroid cells stably transduced with a CFTR-G542XcDNA transgene and $7 \%$ of wild-type CFTR function in primary human bronchial epithelial CF cells with G542X/delF508 genotype [22].

These studies suggest that the reduction of the efficiency of translation termination, such as in the case of induction of ribosomal read-through, is a potential strategy for developing treatments of genetic diseases caused by PTCs. It is known that aminoglycosides cause toxic side effects that do not seem directly associated with their ability to suppress PTC. Monitoring the read-through efficiency of aminoglycosides with low toxic side effects is required and the synthesis of novel aminoglycoside derivatives exhibiting better parameters of administration to the patient, as well as availability and lower toxicity in vivo, is of interest to the research community.

\subsubsection{PTC124}

PTC124 (3-(5-(2-fluorophenyl)-1,2,4-oxadiazol-3-yl)-benzoic acid), also known as Ataluren, is a small non-aminoglycoside molecule that has been suggested to allow PTC read-through even though its target has yet to be identified [23]. It is an orally available and non-toxic small molecule, identified by HTS and is the first drug in its class. PTC124 appears to allow cellular machinery to read-through premature stop codons in mRNA, enabling the translation process 
to produce full-length, functional proteins [24]. In a transgenic mouse model carrying CFTR with nonsense mutations, $0.9 \mathrm{mg} / \mathrm{ml}$ of PTC124 was administered orally for 14-21 days. The results reported a partial CFTR expression at the apical surface of intestinal tissues and a restoration of normal CFTR function up to $24 \%$ with dose given in a liquid diet or $29 \%$ with at dose of $15-60 \mathrm{mg} / \mathrm{kg}$ daily by subcutaneous injections $[1,25]$.

PTC124 was studied in two different Phase II human clinical trials with modest results in terms of efficacy [26-27]. In one study [26], PTC124 was orally administered to 23 CF patients carrying nonsense mutations. They were treated with $16 \mathrm{mg} / \mathrm{kg}$ daily of PTC124 for 14 days, then after 14 days without treatment, with a dose of $40 \mathrm{mg} / \mathrm{kg}$ daily for further 14 days. An improvement of chloride conductance was observed by nasal potential difference measurements after the two treatments, where approximately half of the patients showed measurements within the normal range $[1,26]$. Another Phase II trial was conducted with a similar treatment schedule for 12 weeks in 19 CF patients carrying nonsense mutations [27]. An increased nasal chloride transport in approximately $60 \%$ of treated patients was reported. CFTR function was restored more efficiently with increased time of treatment [1,27]. PTC124 has also been examined in 30 pediatric CF patients with nonsense mutations [1, 28]. PTC124 was administered in two 14day cycles as described earlier. Approximately $50 \%$ of the treated patients partially restored CFTR-mediated nasal epithelial chloride transport and full-length CFTR protein at the apical membrane of nasal epithelial cells, with only mild or no adverse effects.

Despite these successes in Phase II trials $[11,27,28]$, PTC124 did not significantly improve the primary end-point in large Phase III clinical trials. A study [29], enrolling patients from 36 sites in 11 countries in North America and Europe, showed no improvement in the primary endpoint (forced expiratory volume in the first second, $\mathrm{FEV}_{1} \%$ ) as predicted. It did however demonstrate conservation of lung function compared to placebo in a predefined subset of subjects who were not treated with the inhaled antibiotic tobramycin, known to influence the efficacy of PTC124 [29,30].

The development of novel small molecules having efficient read-through activity on PTCs and restoring CFTR function with low toxicity should be considered as a major issue in CF research. The CF read-through therapy clinical trials indicate that both gentamicin and PTC124 are able to restore CFTR protein in a fraction of CF patients with PTCs. Longer-term studies will be required to determine whether the therapeutic effects found in patients upon short-term treatment can be realized, and if sufficient CFTR can be rescued to reduce pathological respiratory aspects of CF [1]. The NMD inhibitors studied may lead to the discovery of biomolecules to be employed in combination with read-through correctors, in order to achieve clinically relevant results.

\subsection{NMD inhibitors in $\mathrm{CF}$}

PTCs located $>50-55$ nucleotides upstream of an exon-exon junction can be minimized by NMD in order to detect and degrade mRNA containing PTC and minimize the negative consequences of nonsense mutations [5]. This should be carefully considered in the context of the development of read-through approach for restoring CFTR function. NMD is a cellular mechanism, aimed to detect and degrade PTC containing mRNA [31-34] and as part of an 
mRNA surveillance system, it has a crucial role in preventing accumulation of aberrant transcripts and their translation [32,34]. Down-modulation of NMD is an important therapeutic goal, in order to (a) maintain mRNA levels suitable for read-through correction and (b) restore sufficient full-length protein expression levels. The major molecular consequences of nonsense mutations are the promotion of premature translational termination and NMD. These two features are strictly associated.

The efficiency of restoration of correct translation using read-through molecules, with respect to modulation of NMD is still an unresolved problem. Differences in NMD efficiency of CFTR transcripts carrying the W1282X mutation among different epithelial cell lines have been reported [35], and in some CF cells the NMD of all transcripts was efficient and in others NMD was less efficient. Down regulation of NMD in cells carrying the W1282X mutation was reported to increase the level of CFTR nonsense transcripts and enhanced the CFTR chloride channel activity in response to gentamicin [9].

Data published to date indicate that the efficiency of PTC read-through is low [10], probably due to degradation of PTC-mRNA by NMD mechanism, depleting substrates available for read-through correction. Modulation of NMD, aimed to increase CFTR mRNA available to PTC read-through, could be another important therapeutic goal [9]. A possible strategy to modulate NMD mechanism may be based on novel molecules acting as NMD inhibitors. These molecules should not affect physiological substrates of NMD mechanism.

Recently Wang et al. [36] have demonstrated that a modest depletion (80\% of the activity of untreated cells) of UPF1 or UPF2 through shRNA could suppress NMD activity without affecting the proliferation or survival of cells. This suggests that pharmacologic NMD inhibition could be proposed as a therapeutic approach with limited toxicity or side effects. In particular, the same group identified NMD inhibitors (called NMDIs) acting against targeting SMG7-UPF1 complex obtained from a virtual library screening, with various chemical backbones and acting at nanomolar concentration [37]. It was demonstrated that the combination of a read-through drug and NMDIs increased protein expression and relative biological functions of a PTC-mutated p53. These novel data are promising, as previous pharmacologic inhibitors, useful in investigating the NMD mechanism, have been found to be toxic, did not synergize with read-through molecules and had an unknown mechanism of action $[38,39]$.

Amlexanox, a drug used for over 30 years orally or topically to treat asthma and aphthous ulcers [40,41], has also been identified [42] as a potent NMD inhibitor. Amlexanox is not citotoxic, does not inhibit general translation, and does not affect natural NMD substrate expression. This drug presents a dual activity of interest in CF research, that is, induction of an increased level of PTC mRNA, and induction of efficient expression of full-length and functional CFTR protein. The novelty of amlexanox is the ability to both inhibit NMD and facilitate PTC read-through, in contrast to other read-through molecules that present only readthrough activity strongly influenced by the identity of the PTC and its nucleotide environment [43]. Amlexanox in combination with PTC124 induces an increase of CFTR channel activity in cells with heterozygous genotype for nonsense mutations [43]. 
The clinical use of these molecules may be possible after further studies relative to the shortand long-term effects of potent NMD inhibition as the combination of a read-through approach with pharmacologic NMD inhibition is an appealing therapeutic strategy for CF patients carrying nonsense mutations.

\subsection{Treatment of CF heterozygous patients for nonsense mutations}

Clancy's group [16] nasally administered aminoglycosides did not produce detectable changes in CFTR function in subjects heterozygous for a variety of stop codon mutations within CFTR, suggesting the need to perform a combined therapy based on the most potent suppressors of nonsense mutations co-delivered with CFTR potentiators and/or correctors [16]. Many CF patients heterozygous for premature stop mutations carry a genotype with F508del. Initial results of a Phase II trial of VX-809, a lead F508del CFTR corrector developed by Vertex Pharmaceuticals (Cambridge, Massachusetts, USA), established that systemic administration of the compound for 4 weeks modestly improved sweat chloride at the highest dose tested compared with placebo [44]. VX-809 is reported to normalize the gating of corrected F508delCFTR but has no direct potentiating action suggesting that one straightforward approach could be the co-administration with a potentiator of CFTR channel gating [11,44,45]. If CF patients have nonsense mutations together with F508del, a cocktail of potentiators of CFTR channel gating, CFTR correctors, and read-through molecules could be proposed as a therapeutic strategy.

Xue $X$ et al. [22] reported a novel synthetic aminoglycoside based on NB124 that efficiently restored CFTR function in primary human bronchial epithelial (HBE) CF cells carrying PTC (G542X/delF508). The efficacy of NB124 was further enhanced by addition of the oral CFTR potentiator Ivacaftor (VX-770) to airway cells expressing CFTR PTCs [22]. Ivacaftor exhibited excellent activity and pharmacokinetic properties, providing the opportunity to treat the underlying cause of CF in combination with CFTR correctors and read-through molecules, depending on the CFTR mutations [46].

Many new compounds are expected to be proposed in the future for the potentiation and correction of CFTR. One example is 4,6, $4^{\prime}$-trimethylangelicin (TMA), a psoralen-related compound, which obtained the orphan drug designation from the EMA and is already in clinical use for psoriasis. Tamanini et al. [47] demonstrated that TMA, at nanomolar concentrations, inhibited the expression of the IL- 8 gene in bronchial epithelial cells in which the inflammatory response has been challenged with $P$. aeruginosa. The acute addition ( 15 minutes treatment) of $250 \mathrm{nM}$ TMA potentiated FSK-stimulated chloride secretion in airway cell monolayers expressing wild type CFTR or in CF cells in which F508del CFTR was already rescued to the apical membrane by overexpressing the interacting protein NHERF1. More recently, the same group [48] found that long pre-incubation with nanomolar concentrations of TMA was able to effectively restore both F508del CFTR-dependent chloride secretion and F508del CFTR cell surface expression in both primary or secondary airway cell monolayers homozygous for F508del mutation. These results indicate that TMA, besides its anti-inflammatory and potentiator activities, also displays corrector properties, suggesting that this 
compound (or more effective analogues) could be a potential candidate for combination therapies with read-through molecules.

Other CFTR potentiator and/or corrector molecules with different mechanisms of action have been recently reported in literature [11]. These include the cyanoquinoline CoPo-22 [49], the inhibitor Hsp90 co-chaperone Aha1 [50], phosphodiesterase type V (PDE5) inhibitors [51-53], the isoflavones genistein, and curcumin [54]. Additional studies concerning their mechanism of action and toxicity are required before clinical testing, and combination with read-through molecules can be considered.

\section{Conclusions}

More than 1,900 different CFTR mutations have been reported in CF patients, $10 \%$ being nonsense mutations and $75 \%$ represented by the most frequent CF F508del mutation [1,11]. In order to ameliorate the CF phenotype, restoration of at least $5-35 \%$ of normal CFTR function is required [2-4]. Several CFTR modulator therapies have been investigated in vitro and in late phase clinical trials, including CFTR potentiators, correctors, and PTC read-through molecules [1,2-5,9-16,21-30,37-39,42,45]. To achieve substantial clinical benefit in CF, a combined administration of two correctors with synergistic action $[55,56]$ or a corrector with a potentiator that counteracts distinct conformational defects in trafficking and activation of F508del CFTR [57, 58] are required.

Current open clinical trials, excluding studies with unknown status, are being conducted on Ivacaftor and PTC124 (Table 2).

Ivacaftor alone is not sufficient to efficiently alter CFTR activity in patients with CF homozygous for F508del [59]. The difficulty in achieving higher levels of correction is due to the fact that the F508del mutation induces multiple conformational defects in the mutant protein.

In current clinical trials (Table 2) Ivacaftor is being tested in combination with CFTR correctors such as Lumacaftor (VX-809, Vertex Pharmaceuticals, Cambridge, Massachusetts, USA) and VX-661 (Vertex Pharmaceuticals) in order to correct the protein misfolding and increase the CFTR localization to the cell surface.

VX-809 is selective for F508del CFTR and enhances chloride secretion to $15 \%$ of that found in non-CF human bronchial cells $[45,48,55]$. Preclinical research $[45,60]$ and known effects of the F508 deletion on gating in addition to cellular processing [56-58] support the combination strategy of VX-809 (or VX-661, an alternate CFTR corrector to VX-809) with Ivacaftor in current human clinical trials (Table 2).

Alternatively, dual-acting molecules (such as TMA [47,48], aminoarylthiazole [61], and cyanoquinoline $[49,62]$ derivatives) that both correct F508del CFTR and potentiate the F508del CFTR-dependent chloride permeability has been proposed, avoiding multiple combined administration. 


\begin{tabular}{|c|c|c|}
\hline Molecule & Title of Study & ClinicalTrials.gov Identifier \\
\hline \multirow{9}{*}{ Ivacaftor(VX770; Kalydeco) } & $\begin{array}{l}\text { Ivacaftor (Kalydeco) and Insulin in Cystic } \\
\text { Fibrosis (CF) }\end{array}$ & NCT02039986 \\
\hline & $\begin{array}{l}\text { Short Term Effects of Ivacaftor in Non-G551D } \\
\text { Cystic Fibrosis Patients }\end{array}$ & NCT01784419 \\
\hline & $\begin{array}{l}\text { Ivacaftor in French Patients with Cystic } \\
\text { Fibrosis and a G551D Mutation } \\
\text { (IVACAFTOR1) }\end{array}$ & NCT02194881 \\
\hline & $\begin{array}{l}\text { A Phase } 3 \text { Study to Evaluate the Efficacy and } \\
\text { Safety of Ivacaftor and VX-661 in } \\
\text { Combination with Ivacaftor in Subjects Aged } \\
12 \text { Years and Older with Cystic Fibrosis, } \\
\text { Heterozygous for the F508del-CFTR Mutation }\end{array}$ & NCT02392234 \\
\hline & $\begin{array}{l}\text { A Phase } 3 \text { Study of VX-661 in Combination } \\
\text { with Ivacaftor in Subjects Aged } 12 \text { Years and } \\
\text { Older with Cystic Fibrosis, Who Have One } \\
\text { F508del-CFTR Mutation and a Second } \\
\text { Mutation that Has Been Demonstrated to Be } \\
\text { Clinically Responsive to Ivacaftor }\end{array}$ & NCT02412111 \\
\hline & $\begin{array}{l}\text { Study to Evaluate Lumacaftor and Ivacaftor } \\
\text { Combination Therapy in Subjects } 12 \text { Years } \\
\text { and Older with Advanced Lung Disease } \\
\text { (Phase III) }\end{array}$ & NCT02390219 \\
\hline & $\begin{array}{l}\text { Airway Infection, Inflammatory Markers and } \\
\text { Exercise Capacity in Patients with Cystic } \\
\text { Fibrosis and at Least One G551D Mutation } \\
\text { Taking VX-770 (Ivacaftor) (Phase IV) }\end{array}$ & NCT01937325 \\
\hline & $\begin{array}{l}\text { A Randomized, Double-Blind, Placebo- } \\
\text { Controlled, Parallel-Group Study to Evaluate } \\
\text { the Efficacy and Safety of VX-661 in } \\
\text { Combination with Ivacaftor (Phase III) }\end{array}$ & NCT02347657 \\
\hline & $\begin{array}{l}\text { (Study: Vertex IIS) Does Ivacaftor Alter Wild } \\
\text { Type CFTR-Open Probability in the Sweat } \\
\text { Gland Secretory Coil? }\end{array}$ & NCT02310789 \\
\hline Ataluren(PTC124) & $\begin{array}{l}\text { Study of Ataluren in Nonsense Mutation } \\
\text { Cystic Fibrosis (ACT CF), Phase III }\end{array}$ & NCT02139306 \\
\hline N91115 & $\begin{array}{l}\text { Study of N91115 in Patients with Cystic } \\
\text { Fibrosis Homozygous F508del-CFTR } \\
\text { Mutation (SNO4) (Phase I) }\end{array}$ & NCT02275936 \\
\hline Glycerol Phenylbutyrate & $\begin{array}{l}\text { Glycerol Phenylbutyrate Corrector Therapy } \\
\text { For CF (GPBA) }\end{array}$ & NCT02323100 \\
\hline FDL169 & $\begin{array}{l}\text { FTIH - Single and Repeat Oral Doses of } \\
\text { FDL169 in Healthy Volunteers (Phase I) }\end{array}$ & NCT02359357 \\
\hline
\end{tabular}

Table 2. Current open clinical trials on CFTR modulators (source ClinicalTrials.gov). 
Other ongoing human clinical trials are investigating novel CFTR modulators on F508del such as N91115, glycerol phenylbutyrate, FDL169 (Table 2). N91115 is currently in Phase I. In next Phase II this molecule will be studied in addition to Lumacaftor/Ivacaftor.

The combination of CFTR potentiators and correctors could result in effective therapies in a large proportion of patients with CF (Table 2).

Other CFTR modulator classes include molecules with read-through activity, such as PTC124 currently in Phase III clinical study. Based on efficacy in vitro and in animal models $[10,25,63]$, PTC124 was studied in a series of conflicting Phase II trials [26-28,63]. A long-term study has demonstrated no improvement in FEV1 \% predicted, the primary endpoint. Conversely a small effect on lung function was demonstrated in a predefined subset of individuals untreated with inhaled antibiotics that can modify the efficiency of PTC124 [29,63]. So further clinical studies for this compound should be necessary to demonstrate its activity in $\mathrm{CF}$ as read-through therapeutic approach also in combination with NMD inhibitors or potentiators/correctors as reported in studies in vitro [22,43].

Results to date suggest, in the case of nonsense mutations, efficient and therapeutic restoration of a functional CFTR channel may be possible drugs administered in combination.

In conclusion, it is essential to stratify CF patients with the aim of creating a personalized therapy based on the use of a combination of drugs targeted at specific classes of mutations. This approach represents an appealing therapeutic strategy and should be the subject of further investigation.

\section{Acknowledgements}

This work was supported by contributions from Fondazione Cariparo (Cassa di Risparmio di Padova e Rovigo) to RG, Italian Cystic Fibrosis Research Foundation (FFC\#2/2010 and FFC\#1/2012) to M.B and (FFC\#17/2010 and FFC\#8/2014) to RG.

Our thanks to Dr. Amanda J Neville MSB for her comments and corrections of the English text.

\section{Author details}

Roberto Gambari ${ }^{1,2}$, Giulia Breveglieri ${ }^{1,2}$, Francesca Salvatori ${ }^{1,2}$, Alessia Finotti ${ }^{1}$ and Monica Borgatti ${ }^{1 *}$

*Address all correspondence to: monica.borgatti@unife.it

1 Department of Life Sciences and Biotechnology, Biochemistry and Molecular Biology Section, University of Ferrara, Ferrara, Italy

2 Biotechnology Center, University of Ferrara, Ferrara, Italy 


\section{References}

[1] Keeling KM, Wang D, Conard SE, Bedwell DM. Suppression of premature termination codons as a therapeutic approach. Critical Reviews in Biochemistry and Molecular Biology. 2012;47:444-463. DOI: 10.3109/10409238.2012.694846.

[2] Kerem E. Pharmacologic therapy for stop mutations: how much CFTR activity is enough? Current Opinion in Pulmonary Medicine. 2004;10:547-552.

[3] Zhang L, Button B, Gabriel SE, Burkett S, Yan Y, Skiadopoulos MH, Dang Yl, Vogel LN, Mckay T, Mengos A, Boucher RC, Collins PL, Pickles RJ. CFTR delivery to 25\% of surface epithelial cells restores normal rates of mucus transport to human cystic fibrosis airway epithelium. PLoS Biology. 2009;7:e1000155. DOI: 10.1371/journal.pbio. 1000155.

[4] Amaral MD. Processing of CFTR: traversing the cellular maze--how much CFTR needs to go through to avoid cystic fibrosis? Pediatric Pulmonology. 2005;39:479-491.

[5] Linde L, Kerem B. Introducing sense into nonsense in treatments of human genetic diseases. Trends Genetics. 2008;24:552-563. DOI: 10.1016/j.tig.2008.08.010.

[6] Bobadilla JL, Macek M Jr, Fine JP, Farrell PM. Cystic fibrosis: a worldwide analysis of CFTR mutations--correlation with incidence data and application to screening. $\mathrm{Hu}-$ man Mutation. 2002;19:575-606.

[7] Nicholson P, Yepiskoposyan H, Metze S, Zamudio Orozco R, Kleinschmidt N, Mühlemann O. Nonsense-mediated mRNA decay in human cell: mechanistic insights, functions beyond quality control and double-life of NMD factors. Cellular and Molecular Life Sciences 2010;67:677-700. DOI: 10.1007/s00018-009-0177-1.

[8] Behm-Ansmant I, Kashima I, Rehwinkel J, Saulière J, Wittkop N, Izaurralde E. mRNA quality control: an ancient machinery recognizes and degrades mRNAs with nonsense codons. FEBS Letters. 2007;58:2845-2853.

[9] Linde L, Boelz S, Nissim-Rafinia M, Oren YS, Wilschanski M, Yaacov Y, Virgilis D, Neu-Yilik G, Kulozik AE, Kerem E, Kerem B. Nonsense-mediated mRNA decay affects nonsense transcript levels and governs response of cystic fibrosis patients to gentamicin. The Journal of Clinical Investigation. 2007;1173:683-692.

[10] Welch EM, Barton ER, Zhuo J, Tomizawa Y, Friesen WJ, Trifillis P, Paushkin S, Patel M, Trotta CR, Hwang S, Wilde RG, Karp G, Takasugi J, Chen G, Jones S, Ren H, Moon YC, Corson D, Turpoff AA, Campbell JA, Conn MM, Khan A, Almstead NG, Hedrick J, Mollin A, Risher N, Weetall M, Yeh S, Branstrom AA, Colacino JM, Babiak J, Ju WD, Hirawat S, Northcutt VJ, Miller LL, Spatrick P, He F, Kawana M, Feng H, Jacobson A, Peltz SW, Sweeney HL. PTC124 targets genetic disorders caused by nonsense mutations. Nature. 2007;447:87-91. 
[11] Ikpa PT, Bijvelds MJ, de Jonge HR. Cystic fibrosis: toward personalized therapies. The International Journal of Biochemistry \& Cell Biology. 2014;52:192-200. DOI: 10.1016/j.biocel.2014.02.008.

[12] Du M, Jones JR, Lanier J, Keeling KM, Lindsey JR, Tousson A, Bebok Z, Whitsett JA, Dey CR, Colledge WH, Evans MJ, Sorscher EJ, Bedwell DM. Aminoglycoside suppression of a premature stop mutation in a Cftr-/- mouse carrying a human CFTRG542X transgene. Journal of Molecular Medicine. 2002;80:595-604.

[13] Du M, Keeling KM, Fan L, Liu X, Kovacs T, Sorscher E, Bedwell DM. Clinical doses of amikacin provide more effective suppression of the human CFTR-G542X stop mutation than gentamicin in a transgenic CF mouse model. Journal of Molecular Medicine. 2006;84:573-582.

[14] Wilschanski M, Yahav Y, Yaacov Y, Blau H, Bentur L, Rivlin J, Aviram M, BdolahAbram T, Bebok Z, Shushi L, Kerem B, Kerem E. Gentamicin-induced correction of CFTR function in patients with cystic fibrosis and CFTR stop mutations. The New England Journal Medicine. 2003;349:1433-1441.

[15] Clancy JP, Bebok Z, Ruiz F, King C, Jones J, Walker L, Greer H, Hong J, Wing L, Macaluso M, Lyrene R, Sorscher EJ, Bedwell DM. Evidence that systemic gentamicin suppresses premature stop mutations in patients with cystic fibrosis. American Journal Respiratory and Critical Care Medicine. 2001;163:1683-1692.

[16] Clancy JP, Rowe SM, Bebok Z, Aitken ML, Gibson R, Zeitlin P, Berclaz P, Moss R, Knowles MR, Oster RA, Mayer-Hamblett N, Ramsey B. No detectable improvements in cystic fibrosis transmembrane conductance regulator by nasal aminoglycosides in patients with cystic fibrosis with stop mutations. American Journal of Respiratory Cell and Molecular Biology. 2007;37:57-66.

[17] Altamura N, Castaldo R, Finotti A, Breveglieri G, Salvatori F, Zuccato C, Gambari R, Panin GC, Borgatti M. Tobramycin is a suppressor of premature termination codons. Journal of Cystic Fibrosis. 2013;12:806-811. DOI: 10.1016/j.jcf.2013.02.007.

[18] Hainrichson M, Nudelman I, Baasov T. Designer aminoglycosides: the race to develop improved antibiotics and compounds for the treatment of human genetic diseases. Organic \& Biomolecular Chemistry. 2008;6:227-239. DOI: 10.1039/b712690p.

[19] Howard MT, Anderson CB, Fass U, Khatri S, Gesteland RF, Atkins JF, Flanigan KM. Readthrough of dystrophin stop codon mutations induced by aminoglycosides. Annals of Neurology. 2004;55:422-426.

[20] Nudelman I, Rebibo-Sabbah A, Shallom-Shezifi D, Hainrichson M, Stahl I, Ben-Yosef $\mathrm{T}$, Baasov T. Redesign of aminoglycosides for treatment of human genetic diseases caused by premature stop mutations. Bioorganic \& Medicinal Chemistry Letters. 2006;16:6310-6315. 
[21] Rebibo-Sabbah A, Nudelman I, Ahmed ZM, Baasov T, Ben-Yosef T. In vitro and ex vivo suppression by aminoglycosides of PCDH15 nonsense mutations underlying type 1 Usher syndrome. Human Genetics. 2007;122:373-381.

[22] Xue X, Mutyam V, Tang L, Biswas S, Du M, Jackson LA, Dai Y, Belakhov V, Shalev M, Chen F, Schacht J, J Bridges R, Baasov T, Hong J, Bedwell DM, Rowe SM. Synthetic aminoglycosides efficiently suppress cystic fibrosis transmembrane conductance regulator nonsense mutations and are enhanced by ivacaftor. American Journal of Respiratory Cell and Molecular Biology. 2014;50:805-816. DOI: 10.1165/rcmb. 2013-0282OC.

[23] Lentini L, Melfi R, Di Leonardo A, Spinello A, Barone G, Pace A, Palumbo Piccionello A, Pibiri I. Toward a rationale for the PTC124 (Ataluren) promoted readthrough of premature stop codons: a computational approach and GFP-reporter cell-based assay. Molecular Pharmaceutics. 2014;11:653-664. DOI: 10.1021/mp400230s.

[24] Ryan NJ. Ataluren: first global approval. Drugs. 2014;74:1709-1714. DOI: 10.1007/ s40265-014-0287-4.

[25] Du M, Liu X, Welch EM, Hirawat S, Peltz SW, Bedwell DM. PTC124 is an orally bioavailable compound that promotes suppression of the human CFTR-G542X nonsense allele in a CF mouse model. Proceedings of the National Academy of Sciences of the United States of America. 2008;105:2064-2069. DOI: 10.1073/pnas.0711795105.

[26] Kerem E, Hirawat S, Armoni S, Yaakov Y, Shoseyov D, Cohen M, Nissimrafinia M, Blau H, Rivlin J, Aviram M, Elfring GL, Northcutt VJ, Miller LL, Kerem B, Wilschanski M. Effectiveness of PTC124 treatment of cystic fibrosis caused by nonsense mutations: a prospective phase II trial. Lancet. 2008;372:719-727. DOI: 10.1016/ S0140-6736(08)61168-X.

[27] Wilschanski M, Miller LL, Shoseyov D, Blau H, Rivlin J, Aviram M, Cohen M, Armoni S, Yaakov Y, Pugatch T, Cohen-Cymberknoh M, Miller NL, Reha A, Northcutt VJ, Hirawat S, Donnelly K, Elfring GL, Ajayi T, Kerem E. Chronic ataluren (PTC124) treatment of nonsense mutation cystic fibrosis. The European Respiratory Journal. 2011;38:59-69. DOI: 10.1183/09031936.00120910.

[28] Sermet-Gaudelus I, Boeck KD, Casimir GJ, Vermeulen F, Leal T, Mogenet A, Roussel D, Fritsch J, Hanssens L, Hirawat S, Miller NL, Constantine S, Reha A, Ajayi T, Elfring GL, Miller LL. Ataluren (PTC124) induces cystic fibrosis transmembrane conductance regulator protein expression and activity in children with nonsense mutation cystic fibrosis. American Journal Respiratory and Critical Care Medicine 2010;182:1262-1272. DOI: 10.1164/Rccm.201001-0137oc.

[29] Kerem E, Konstan MW, De Boeck K, Accurso FJ, Sermet-Gaudelus I, Wilschanski M, Elborn JS, Melotti P, Bronsveld I, Fajac I, Malfroot A, Rosenbluth DB, Walker PA, McColley SA, Knoop C, Quattrucci S, Rietschel E, Zeitlin PL, Barth J, Elfring GL, Welch EM, Branstrom A, Spiegel RJ, Peltz SW, Ajayi T, Rowe SM. Cystic Fibrosis Ataluren Study Group. Ataluren for the treatment of nonsense-mutation cystic fibro- 
sis: a randomised, double-blind, placebo-controlled phase 3 trial. The Lancet Respiratory Medicine. 2014; 2:539-547. DOI: 10.1016/S2213-2600(14)70100-6.

[30] Bell SC, De Boeck K, Amaral MD. New pharmacological approaches for cystic fibrosis: promises, progress, pitfalls. Pharmacology \& Therapeutics. 2015;145:19-34. DOI: 10.1016/j.pharmthera.2014.06.005.

[31] Bhuvanagiri M, Schlitter AM, Hentze MW et al. NMD: RNA biology meets human genetic medicine. The Biochemical Journal. 2010;430:365-377. DOI: 10.1042/ BJ20100699.

[32] Khajavi M, Inoue K, Lupski JR. Nonsense-mediated mRNA decay modulates clinical outcome of genetic disease. European Journal of Human Genetics 2006;14:1074-1081.

[33] Nicholson P, Yepiskoposyan H, Metze S, Zamudio Orozco R, Kleinschmidt N, Mühlemann O. Nonsense-mediated mRNA decay in human cells: mechanistic insights, functions beyond quality control and the double-life of NMD factors. Cellular and Molecular Life Sciences. 2010;67:677-700. DOI: 10.1007/s00018-009-0177-1.

[34] Stalder L, Muhlemann O. The meaning of nonsense. Trends in Cell Biology 2008;18:315-321. DOI: 10.1016/j.tcb.2008.04.005.

[35] Linde L, Boelz S, Neu-Yilik G, Kulozik AE, Kerem B. The efficiency of nonsensemediated mRNA decay is an inherent character and varies among different cells. European Journal of Human Genetics. 2007;15:1156-1162.

[36] Wang D, Zavadil J, Martin L, Parisi F, Friedman E, Levy D, Harding H, Ron D, Gardner LB. Inhibition of nonsense-mediated RNA decay by the tumor microenvironment promotes tumorigenesis. Molecular and Cellular Biology 2011;31:3670-3680. DOI: 10.1128/MCB.05704-11.

[37] Martin L, Grigoryan A, Wang D, Wang J, Breda L(1), Rivella S, Cardozo T, Gardner LB. Identification and characterization of small molecules that inhibit nonsensemediated RNA decay and suppress nonsense p53 mutations. Cancer Research. 2014;74:3104-3113. DOI: 10.1158/0008-5472.CAN-13-2235.

[38] Keeling KM, Wang D, Dai Y, Murugesan S, Chenna B, Clark J, Belakhov V, Kandasamy J, Velu SE, Baasov T, Bedwell DM. Attenuation of nonsense-mediated mRNA decay enhances in vivo nonsense suppression. PLoS ONE 2013;8:e60478. DOI: 10.1371/ journal.pone.0060478.

[39] Durand S, Cougot N, Mahuteau-Betzer F, Nguyen CH, Grierson DS, Bertrand E, Tazi $\mathrm{J}$, Lejeune F. Inhibition of nonsense-mediated mRNA decay (NMD) by a new chemical molecule reveals the dynamic of NMD factors in P-bodies. The Journal of Cell Biology 2007;178:1145-1160.

[40] Saijo T, Kuriki H, Ashida Y, Makino H, Maki Y. Mechanism of the action of amoxanox (AA-673), an orally active antiallergic agent. International Archives of Allergy and Applied Immunology 1985;78:43-50. 
[41] Meng W, Dong Y, Liu J, Wang Z, Zhong X, Chen R, Zhou H, Lin M, Jiang L, Gao F, $\mathrm{Xu} \mathrm{T}$, Chen $\mathrm{Q}$, Zeng X. A clinical evaluation of amlexanox oral adhesive pellicles in the treatment of recurrent aphthous stomatitis and comparison with amlexanox oral tablets: a randomized, placebo controlled, blinded, multicenter clinical trial. Trials 2009;10:30. DOI: 10.1186/1745-6215-10-30.

[42] Gonzalez-Hilarion S, Beghyn T, Jia J, Debreuck N, Berte G, Mamchaoui K, Mouly V, Gruenert DC, Déprez B, Lejeune F. Rescue of nonsense mutations by amlexanox in human cells. Orphanet Journal of Rare Diseases 2012;7:58. DOI: 10.1186/1750-1172-7-58.

[43] Bidou L, Hatin I, Perez N, Allamand V, Panthier JJ, Rousset JP. Premature stop codons involved in muscular dystrophies show a broad spectrum of readthrough efficiencies in response to gentamicin treatment. Gene Therapy. 2004;11:619-627.

[44] Sloane PA, Rowe SM. Cystic fibrosis transmembrane conductance regulator protein repair as a therapeutic strategy in cystic fibrosis. Current Opinion in Pulmonary Medicine. 2010;16:591-597. DOI: 10.1097/MCP.0b013e32833f1d00.

[45] Van Goor F, Hadida S, Grootenhuis PD, Burton B, Stack JH, Straley KS, Decker CJ, Miller M, McCartney J, Olson ER, Wine JJ, Frizzell RA, Ashlock M, Negulescu PA. Correction of the F508del-CFTR protein processing defect in vitro by the investigationaldrug VX-809. Proceedings of the National Academy of Sciences of the United States of America. 2011;108:18843-18848. DOI: 10.1073/pnas.1105787108.

[46] Hadida S, Van Goor F, Zhou J, Arumugam V, McCartney J, Hazlewood A, Decker C, Negulescu P, Grootenhuis PD. Discovery of N-(2,4-di-tert-butyl-5-hydroxyphenyl)-4oxo-1,4-dihydroquinoline-3-carboxamide (VX-770, ivacaftor), a potent and orally bioavailable CFTR potentiator. Journal of Medicinal Chemistry. 2014;57:9776-9795. DOI: 10.1021/jm5012808.

[47] Tamanini A, Borgatti M, Finotti A, Piccagli L, Bezzerri V, Favia M, Guerra L, Lampronti I, Bianchi N, Dall'Acqua F, Vedaldi D, Salvador A, Fabbri E, Mancini I, Nicolis E, Casavola V, Cabrini G, Gambari R. Trimethylangelicin reduces IL-8 transcription and potentiates CFTR function. American Journal of Physiology. Lung Cellular and Molecular Physiology. 2011;300:L380-90. DOI: 10.1152/ajplung.00129.2010.

[48] Favia M, Mancini MT, Bezzerri V, Guerra L, Laselva O, Abbattiscianni AC, Debellis L, Reshkin SJ, Gambari R, Cabrini G, Casavola V. Trimethylangelicin promotes the functional rescue of mutant F508del CFTR protein in cystic fibrosis airway cells. American Journal of Physiology. Lung Cellular and Molecular Physiology. 2014;307:L48-61. DOI: 10.1152/ajplung.00305.2013.

[49] Phuan PW, Yang B, Knapp JM, Wood AB, Lukacs GL, Kurth MJ, Verkman AS. Cyanoquinolines with independent corrector and potentiator activities restore DeltaPhe508-cystic fibrosis transmembrane conductance regulator chloride channel 
function in cystic fibrosis. Molecular Pharmacology. 2011;80:683-693. DOI: 10.1124/ mol.111.073056.

[50] Koulov AV, LaPointe P, Lu B, Razvi A, Coppinger J, Dong MQ, Matteson J, Laister R, Arrowsmith C, Yates JR 3rd, Balch WE. Biological and structural basis for Aha1 regulation of Hsp90 ATPase activity in maintaining proteostasis in the human disease cystic fibrosis. Molecular Biology of the Cell. 2010;21:871-884. DOI: 10.1091/ mbc.E09-12-1017.

[51] Dhooghe B, Noel S, Bouzin C, Behets-Wydemans G, Leal T. Correction of chloride transport and mislocalization of CFTR protein by vardenafil in the gastrointestinal tract of cystic fibrosis mice. PLoS One. 2013;8:e77314. DOI: 10.1371/journal.pone. 0077314.

[52] Leier G, Bangel-Ruland N, Sobczak K, Knieper Y, Weber WM. Sildenafil acts as potentiator and corrector of CFTR but might be not suitable for the treatment of CF lung disease. Cellular Physiology and Biochemistry: International Journal of Experimental Cellular Physiology, Biochemistry, and Pharmacology. 2012;29:775-790. DOI: $10.1159 / 000265129$.

[53] Noel S, Dhooghe B, Leal T. PDE5 inhibitors as potential tools in the treatment of cystic fibrosis. Frontiers in Pharmacology 2012;3:167.

[54] Sohma Y, Yu YC, Hwang TC. Curcumin and genistein: the combined effects on disease-associated CFTR mutants and their clinical implications. Current Pharmaceutical Design. 2013;19:3521-3528.

[55] Ren HY, Grove DE, De La Rosa O, Houck SA, Sopha P, Van Goor F, Hoffman BJ, Cyr DM. VX-809 corrects folding defects in cystic fibrosis transmembrane conductance regulator protein through action on membrane-spanning domain 1. Molecular Biology of the Cell. 2013;24:3016-3024. DOI: 10.1091/mbc.E13-05-0240.

[56] Holleran JP, Glover ML, Peters KW, Bertrand CA, Watkins SC, Jarvik JW, Frizzell RA. Pharmacological rescue of the mutant cystic fibrosis transmembrane conductance regulator (CFTR) detected by use of a novel fluorescence platform. Molecular Medicine. 2012;18: 685-696. DOI: 10.2119/molmed.2012.00001.

[57] Okiyoneda T, Veit G, Dekkers JF, Bagdany M, Soya N, Xu H, Roldan A, Verkman AS, Kurth M, Simon A, Hegedus T, Beekman JM, Lukacs GL. Mechanism-based corrector combination restores DeltaF508- CFTR folding and function. Nature Chemical Biology. 2013;9:444-454. DOI: 10.1038/nchembio.1253.

[58] Verkman AS, Galietta LJ. Chloride channels as drug targets. Nature Reviews. Drug Discovery. 2009;8:153-171. DOI: 10.1038/nrd2780.

[59] Flume PA, Liou TG, Borowitz DS, Li H, Yen K, Ordoñez CL, Geller DE. VX 08-770-104 Study Group. Ivacaftor in subjects with cystic fibrosis who are homozygous for the F508del-CFTR mutation. Chest. 2012;142:718-724. 
[60] Van Goor F, Hadida S, Grootenhuis PD, Burton B, Cao D, Neuberger T, Turnbull A, Singh A, Joubran J, Hazlewood A, Zhou J, McCartney J, Arumugam V, Decker C, Yang J, Young C, Olson ER, Wine JJ, Frizzell RA, Ashlock M, Negulescu P. Rescue of CF airway epithelial cell function in vitro by a CFTR potentiator, VX-770. Proceedings of the National Academy of Sciences of the United States of America. 2009;106:18825-18830. DOI: 10.1073/pnas.0904709106.

[61] Pedemonte N, Tomati V, Sondo E, Caci E, Millo E, Armirotti A, Damonte G, ZegarraMoran O, Galietta LJ. Dual activity of aminoarylthiazoles on the trafficking and gating defects of the cystic fibrosis transmembrane conductance regulator chloride channel caused by cystic fibrosis mutations. The Journal of Biological Chemistry. 2011;286:15215-15226. DOI: 10.1074/jbc.M110.184267.

[62] Knapp JM, Wood AB, Phuan PW, Lodewyk MW, Tantillo DJ, Verkman AS, Kurth MJ. Structure-activity relationships of cyanoquinolines with corrector-potentiator activity in $\triangle \mathrm{F} 508$ cystic fibrosis transmembrane conductance regulator protein. Journal of Medicinal Chemistry. 2012;55:1242-1251. DOI: 10.1021/jm201372q.

[63] Rowe SM, Borowitz DS, Burns JL, Clancy JP, Donaldson SH, Retsch-Bogart G, Sagel SD, Ramsey BW. Progress in cystic fibrosis and the CF Therapeutics Development Network. Thorax. 2012;67:882-890. DOI: 10.1136/thoraxjnl-2012-202550. 
Chapter 14

\title{
Integrating Viral and Nonviral Vectors for Cystic Fibrosis Gene Therapy in the Airways
}

\author{
Ashley L Cooney, Paul B McCray, Jr and \\ Patrick L. Sinn \\ Additional information is available at the end of the chapter \\ http://dx.doi.org/10.5772/60977
}

\begin{abstract}
An important goal for cystic fibrosis (CF) gene therapy is to achieve long-term functional correction. While many vector options have been evaluated, integrating vectors have the greatest potential to maintain stable expression over time without a requirement for repeated administration. In this chapter, we discuss the importance of correcting the appropriate cell types, options for integrating vectors, animal models for $\mathrm{CF}$ gene therapy, and clinically relevant endpoint measurements. Lentiviral vectors are a promising option for CF gene therapy, as they integrate into the host genome and persistently express a transgene of interest. Airway cell tropism can be conferred by pseudotyping. Nonviral vectors such as DNA transposons can also integrate into the genome. Recent advances in hybrid viral/transposon vector technology improve the ability to deliver transposons to the airways in vivo. Integrating vector technology and new animal models have allowed considerable progress toward the goal of using gene therapy to correct life-long genetic diseases such as CF.
\end{abstract}

Keywords: lentivirus, transposons, animal models, progenitor cells, genotoxicity

\section{Introduction}

Cystic fibrosis (CF) is caused by mutations in the cystic fibrosis transmembrane conductance regulator (CFTR) gene, a cAMP-regulated anion channel. While our knowledge of CFTR function has advanced greatly since the discovery of the gene in 1989, CF remains fatal [1, 2]. 
While CF is a multi-organ system disease, most people with CF die of progressive lung disease that begins early in childhood and is characterized by chronic bacterial infection and inflammation [2]. Nearly $90 \%$ of CF patients have at least one copy of the $\Delta$ F508 mutation, but there are $>2,000$ disease causing mutations that result in a range of disease severities [2]. These mutations can be divided into six classes based on the type and consequence of the mutation (Table 1): class I, no synthesis; class II, defective processing; class III, defective regulation; class IV, altered conductance; class V, reduced synthesis; and class VI, accelerated turnover [3]. However, new mutations continue to be identified and one mutation may fit into more than one category by disrupting CFTR transcription, protein trafficking, or protein regulation in more than one way. Pharmacologic approaches aimed at activating alternative ion transport pathways [4-7], reducing inflammation [8,9], and inhibiting or eliminating bacterial infection $[10,11]$ are active areas of therapeutic development. There is also intense interest in identifying interventions that might restore function to the mutant protein $[5,6,12,13]$. The promise of restoring function to mutant protein was recently validated in a clinical trial for the CFTR conductance mutation G551D present in $2 \%-3 \%$ of CF patients [6]. However, unlike small molecule potentiators or correctors, a CFTR gene replacement approach would be efficacious regardless of the disease causing mutation and is potentially a single dose, life-long curative therapeutic strategy for a devastating disease.

\begin{tabular}{lllll}
\hline Class & $\sim$ Frequency & Mutation Type & Common Representative & CFTR Protein Outcome \\
\hline I & $10 \%$ & Nonsense, splice & G542X & No CFTR \\
\hline II & $70 \%$ & Missense & $\Delta$ F508 & Defective Processing \\
\hline III & $2 \%-3 \%$ & Missense & G511D & Defective Regulation \\
\hline IV & $<2 \%$ & Missense & R117H & Altered Conductance \\
\hline V & $<1 \%$ & Missense, splice & $3349+10 \mathrm{~KB}$ & Reduced Synthesis \\
\hline VI & $<1 \%$ & Missense & N287Y & Accelerated Turnover \\
\hline
\end{tabular}

Table 1. Six classes of mutations in CFTR that result in CF disease [3, 14, 15].

There are a great number of vector options for CFTR gene delivery. Non-integrating viral vectors (i.e., adenovirus or adeno-associated virus) and non-integrating nonviral vectors (i.e., plasmid DNA or in vitro transcribed RNA) each have important attributes and have resulted in significant advances in the CF gene therapy field (reviewed in [16-18]). However, potential limitations to these episomal expression systems may include gradual decreases in transgene expression over time and limiting host immune responses following vector readministration. These pitfalls could be avoided if a therapeutic transgene is stably integrated into the genome of a progenitor cell population. Thus, in this chapter, we focus on the use of integrating vectors for gene delivery, although some of the topics covered will be relevant to multiple vector systems.

This chapter discusses the common and emerging options for integrating vectors and efforts to deliver integrating vectors to CF animal models. Defining "curing CF with gene therapy" 
is not nearly as simple as it appears at first glance. As such, we explore a variety of relevant outcome measures. In addition, there are challenges that need to be taken into consideration for pre-clinical and clinical in vivo studies, such as: delivery, efficiency, persistence, the potential for insertional mutagenesis, and cell types to transduce.

\section{Correcting the appropriate cells}

Because pulmonary disease is generally the most life-limiting complication of $\mathrm{CF}$, gene therapy strategies focus on lung delivery of CFTR. Regardless of the gene delivery tool, an important consideration for CF gene therapy is the target cell. In the proximal airways, CFTR is normally most abundant in surface epithelial cells including ciliated cells, surface columnar cells, and submucosal gland epithelia (SMGs) [19]; in distal airways only superficial epithelia express CFTR. With this information in mind, we face two important questions. 1) What cell types need to be transduced to attain lasting expression? 2) What percentage of cells needs to be transduced to correct CF lung disease? A goal of gene transfer to the pulmonary epithelium with integrating vectors is to correct the CFTR defect in a population of cells that could pass the corrected gene to their progeny, thus eliminating the need for vector readministration. There appear to be several epithelial cell types in the lung that provide these functions, which has led to controversy regarding which cells to target for CF gene therapy. Arguments can be made in support of the necessity to correct basal cells [20,21] and non-ciliated columnar cells of the airways [22-24], SMGs [25-27], club cells [28, 29], and alveolar type II cells [30, 31] in the distal lung.

Compelling evidence from both in vitro and in vivo studies indicate that basal cells are multipotent proximal airway progenitor cells that repopulate pulmonary epithelia under normal conditions and during regeneration (reviewed in [32-34]). Cell-labeling experiments with transgenic mice show that basal cells give rise to labeled basal, ciliated, and club cells, thus fulfilling the definition of progenitor cells $[35,36]$. Several studies suggest that basal cells from human trachea or bronchi will repopulate denuded tracheal xenografts or differentiated epithelial cells in vitro [37-40]. Hematopoietic stem cells are an example showing that a single stem cell type can reconstitute a whole organ; however, there is no convincing evidence that a multipotent airway stem cell is capable of replenishing all regions of the intrapulmonary epithelium. The current literature supports that tracheal, bronchiolar, and alveolar epithelia are maintained by regionally distinct progenitor cell lineages.

What percentage of cells needs to be transduced to functionally correct the CF phenotype in vivo? This is one of the most important questions in the field of CF gene therapy, but remains unanswered. At least five studies examined the relationship between percentage of cells expressing CFTR and transepithelial $\mathrm{Cl}^{-}$secretion [41-45]. With relatively good agreement, they suggest that expressing CFTR in $5 \%-15 \%$ of cells restores $\mathrm{Cl}^{-}$secretion to near wild-type levels. As such, the benchmark of correcting $10 \%$ of the cells is often cited. However, there are many caveats to this number. Indeed one limitation is that many of these studies were performed using in vitro models. In addition, as discussed below, other studies suggest that defective 
$\mathrm{HCO}_{3}^{-}$transport through CFTR might be more relevant to early disease pathogenesis than $\mathrm{Cl}^{-}$secretion [46-49]. The relationships between $\mathrm{HCO}_{3}^{-}$secretion, airway surface liquid $\mathrm{pH}$, bacterial killing, mucociliary clearance, and mucus viscosity may be as important as $\mathrm{Cl}^{-}$ secretion as metrics for disease correction. The short answer to the question posed above is "we do not know"; however, given current animal models and improved vector technologies, the experiments necessary to address the question are feasible. Indeed, as we discuss below, existing vector technologies are being optimized for lung gene transfer and novel integrating vectors are being engineered.

\section{Options for integrating gene delivery}

\subsection{Lentivirus}

Lentiviruses comprise a genus of the virus family Retroviridae. All retroviruses are defined by the ability to reverse transcribe their RNA genome and integrate proviral DNA into the genome of the host cell [50]. Several features of lentiviral vectors (LVs) make them attractive vehicles for delivering therapeutic genes, including their large coding capacity, efficient gene transfer, persistent expression, directed tropism via pseudotyping, and lack of virus-encoded proteins that could elicit undesirable immune responses [51-53]. Unlike gamma-retroviral vectors such as murine Moloney leukemia virus (MMLV)-based vectors, the pre-integration complex of lentiviruses can transverse the nuclear envelope and integrate its cargo into the genomes of non-dividing cells [54]. The first recombinant lentiviral vectors were based on human immunodeficiency virus type-1 (HIV-1) and remain the most widely used lentiviral vector for gene transfer applications [55]. The earliest recombinant HIV-1 viruses were created in the late 1980s and were used to study HIV-1 biology [56].

Beginning in the 1990s and continuing to this day, there have been significant strides in improving the safety and utility of HIV-based gene transfer vectors (reviewed in [50]). To reduce pathogenicity and render the vector replication incompetent, most modern HIV production systems are divided into four expression plasmids (Figure 1A): 1) a plasmid containing the transgene of interest flanked by the HIV long terminal repeats (LTRs); 2) a packaging plasmid expressing the necessary structural and enzymatic proteins; 3) a separate plasmid expressing HIV rev; and 4) an envelope glycoprotein expression plasmid. Multiple viral proteins, such as nef, vif, vpu, env, and vpr, have been deleted as well as much of the U3 region of the $3^{\prime} \mathrm{LTR}$. This latter deletion removes promoter and enhancer activity from the LTR and has been termed a self-inactivating (SIN) modified vector [57]. The woodchuck hepatitis virus posttranscriptional regulatory element (wPRE) is a common addition to LV vectors and functions to increase RNA stability, resulting in higher levels of transgene expression [58, 59]. 

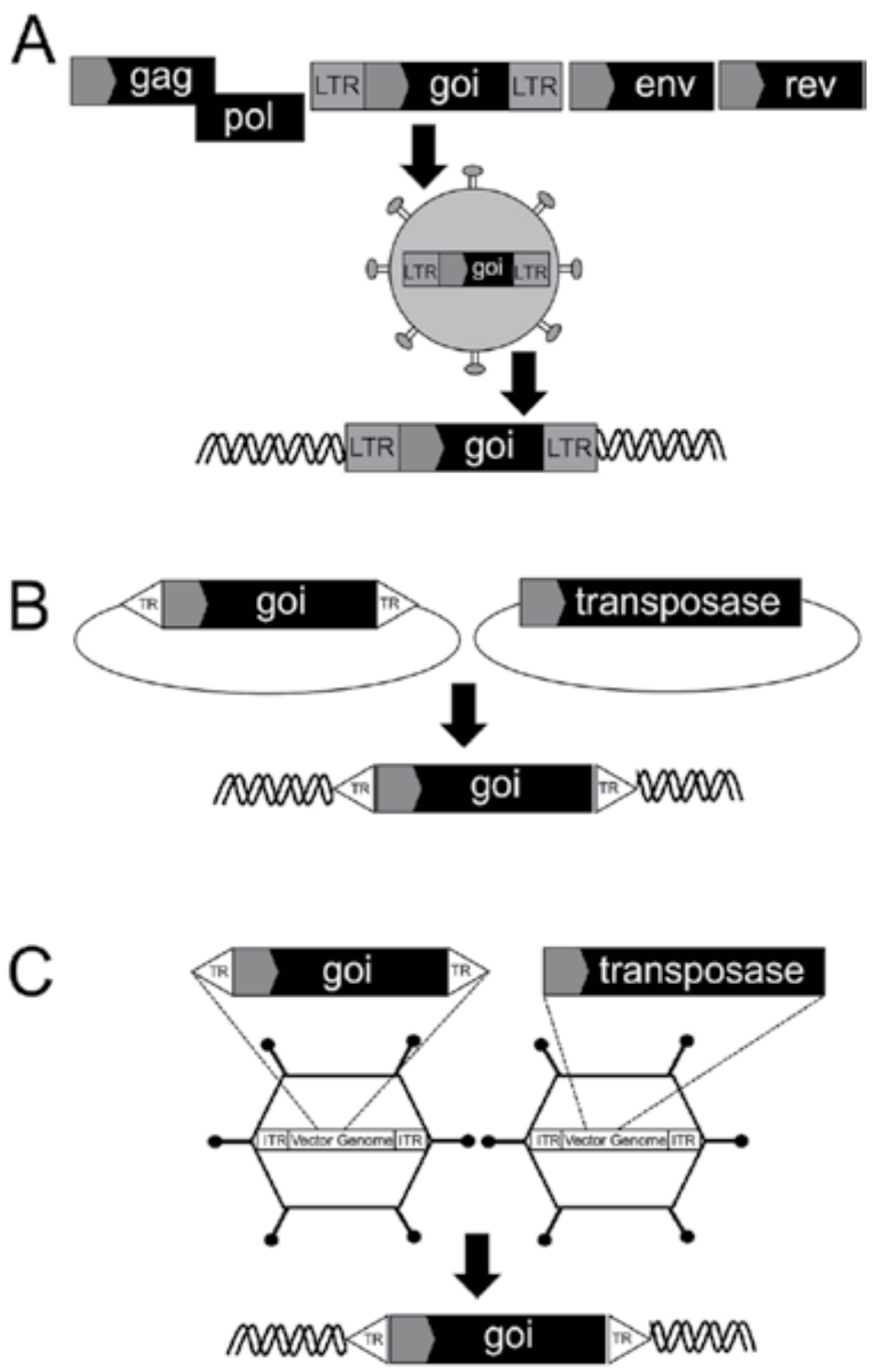

Figure 1. Schematics of integrating transgene delivery systems. A) HIV-based lentiviral vectors are produced by four plasmid transfection. The gag/pol plasmid supplies structural and enzymatic proteins; the env plasmid supplies the envelope glycoprotein (typically VSV-G); rev is delivered in trans via its own plasmid. The "gene of interest" (goi) is flanked by the long terminal repeats (LTRs) and driven by a heterologous promoter. Only the genetic material flanked by the LTRs is packaged and integrated into the host genome. B) Recombinant DNA transposons are typically delivered as a two-part plasmid system. Transposase catalyzes the transposition of the genetic material flanked by the appropriate terminal repeats (TRs) from the plasmid and into the host genome. C) DNA transposons can also be delivered by viral vectors to improve delivery efficiency. One viral vector carries the DNA transposon and the other carries the transposase. The goi is flanked by the transposon TRs, which in turn are flanked by the viral vector inverted terminal repeats (ITRs). Once inside the cell, transposition functions as described in B. 
HIV-based LV delivery of CFTR is a promising option for CF gene therapy. In proof of principle experiments using reporter genes, HIV conferred gene transfer to both ciliated and basal cells of the mouse, sheep, marmoset, and ferret airways [60]. Xenografts transduced with HIV expressing CFTR achieved functional correction as assessed by the measurement of transepithelial potential difference [61]. In a CF mouse model, delivery of CFTR by HIV resulted in sustainable transgene expression for 18 months in ciliated, non-ciliated, and basal cells [53] and partially recovered the anion channel defect [62]. These findings support HIV as a vector candidate for CF gene therapy. Further studies in a large animal CF model to restore anion channel defect to rescue $\mathrm{pH}$ and bacterial killing are important next steps in validating this vector for CF gene therapy.

For LVs, the native envelope glycoprotein is deleted and a heterologous envelope is supplied. This strategy, termed pseudotyping, modifies vector tropism. Glycoproteins from a wide variety of enveloped viruses can be used to package LVs and, as will be discussed, multiple groups have identified envelopes that confer lung gene transfer. The envelope glycoprotein from vesicular stomatitis virus (VSV-G) efficiently pseudotypes LVs, confers wide tropism, and is the most commonly used envelope glycoprotein. However, in well-differentiated airway epithelial cells, VSV-G pseudotyped LV (VSVG-LV) preferentially transduces the basolateral surface $[63,64]$. By using pretreatments or vehicles that transiently disrupt epithelial tight junctions, VSVG-LV accesses the basolateral surface of airway cells following luminal delivery [65]. This strategy has also been shown to greatly improve VSVG-LV in vivo gene transfer efficiency in the lungs of mice [62].

Feline immunodeficiency virus (FIV) is a non-primate LV with a less complex genome than HIV. Unlike HIV, wild-type FIV naturally lacks tat, vpr, vpu, and nef. In addition, FIV-based vector has vif deleted. Additional modifications to the FIV vector, such as deleting the major splice donor, lead to a higher transduction efficiency and transgene expression [58]. Reporter gene studies demonstrate that FIV transduces cells in the conducting airways, bronchioles, and alveoli [66]. FIV-mediated delivery of CFTR corrects the anion channel defect in airway epithelia [65]. FIV pseudotyped with the baculovirus envelope glycoprotein (GP64) preferentially transduces polarized airway epithelia at the apical surface [63], results in persistent gene expression in mice [63], and supports gene transfer to airways of pigs [66]. Additionally, FIV vectors can be readministered to mouse airways without blocking immune responses [67]. To prolong virus exposure to the airways immediately following delivery, formulating vector with a viscoelastic gel increases transduction efficiency in vivo [68, 69].

Simian immunodeficiency virus (SIV)-based LV also successfully transduces airway epithelia. SIV carries little pathogenicity for its own host; therefore, modified strains of SIV could potentially be a safer alternative to HIV-based LVs [70]. In studies using Sendai virus envelope proteins (F and HN) pseudotyped SIV (F/HN-SIV), a single dose persisted for the lifetime in the nasal epithelia of a mouse and achieved a dose-dependent increase in reporter gene expression upon vector readministration [71]. In vitro, F/HN-SIV carrying CFTR can generate functional chloride channels [72]. Additionally, SIV transduction results in persistent transgene expression in both differentiated human airway cells and freshly excised human lung tissue [71]. 
Equine Infectious Anemia Virus (EIAV) is another non-primate lentivirus that has been investigated as a gene transfer vector. It has been studied extensively for neurological disease applications, such as Parkinson's disease [73]. EIAV pseudotyped with the influenza HA envelope can transduce neonatal mouse airways, most notably, the nasal and lung epithelium. Readministration of HA-EIAV resulted in decreased gene transfer efficiency [74].

\subsection{DNA transposons}

Recombinant DNA transposons are integrating nonviral vectors that confer efficient and stable transgene expression in a variety of cell types. The DNA transposon Sleeping Beauty (SB) was resurrected from defective copies of a Tc1-like fish element. SB transposes its genetic cargo into host genomic loci using its catalytic transposase activity [75]. Recombinant DNA transposons used for gene transfer applications are comprised of a two-part system; one encodes terminal repeats (TRs) flanking a transgene of interest (transposon), and the other a catalytic protein responsible for transposition (transposase) (Figure 1B). Since the discovery of SB, other transposons such as piggyBac and Tol2 have also been used in gene transfer applications (reviewed in [76, 77]). DNA transposons are attractive tools for gene therapy because they have a large carrying capacity and integrate into the genome.

Generally, transposon-based vectors are delivered as plasmids. This poses limitations for delivery to some somatic cell types. To improve delivery, several formulations have been investigated. Belur and colleagues described a protocol for complexing SB with polyethyleneimine (PEI) for delivery to the airways of mice [78]. The efficiency of gene transfer was approximated to be $1 \%-3 \% 2$ months post delivery. The use of liposomes has also been investigated as an alternative transposon delivery strategy. Somatic delivery of liposomeprotamine formulated SB resulted in a higher transduction efficiency than Lipofectamine 2000 while maintaining low toxicity and biocompatibility [79]. This non-viral vector approach overcomes biological barriers and allows for chromosomal integration; however, to date, this strategy has not been applied to delivering CFTR to the in vivo airways.

\subsection{Hybrid DNA transposon/viral vectors}

Delivery of plasmid-based transposon vectors to somatic cells in vivo is inefficient. To overcome this limitation, multiple groups have used viral vectors to deliver transposon components (Figure 1C). Recombinant DNA transposons delivered by non-integrating viral vectors, termed hybrid vectors, are intended to combine the advantage of efficient transduction of the viral vector with persistent expression of the transposon, creating an integrating vector. Adenovirus (Ad) [80, 81], adeno-associated virus (AAV) [82], and integrase-deficient lentivirus (ID-LV) [83, 84] have all been investigated as delivery tools for DNA transposons. An integration analysis of SB delivered by ID-HIV suggests a similar, near random, pattern as is observed for plasmid-delivered SB [83].

Yant et al. showed that SB delivered by a helper-dependent Ad vector can integrate into the host chromosome in a transposase-dependent mechanism [75, 80]. Interestingly, in this system, the initial transposition of SB out of the Ad genome required the use of Flp-recombinase to 
successfully deliver the transposon transgene [80]. More recently, the generation of a hybrid piggy Bac/Ad and piggyBac/AAV facilitated delivery of a nonviral transposon. However, unlike $\mathrm{SB}$, neither piggyBac/Ad nor piggyBac/AAV required an extra recombinase step for transposition to occur [81]. In contrast to the hybrid SB, piggyBac has not exhibited overexpression inhibition or limitations on size of the genetic cargo [85]. PiggyBac/Ad hybrid vector successfully delivered a CFTR expression cassette to primary airway epithelial cultures in vitro that corrected the anion transport defect up to 4 months in culture. In reporter gene studies, transgene expression persisted for the 1-year duration of the experiment in mice [81].

\section{Animal models of cystic fibrosis}

Animal models serve an important testing ground for somatic cell gene transfer applications. Mice with null mutations [86-89], specific disease associated CFTR mutations [90-92], and conditional CFTR null alleles [93] have contributed to the understanding of molecular mechanisms of CF. However, mice do not recapitulate several aspects of CF lung disease pathogenesis. As discussed above, many studies evaluating integrating gene transfer vector delivery to the lungs of mice have been conducted. For these reasons, we now discuss efforts to deliver integrating vector to new animal models $C F$.

\subsection{CF rat}

Tuggle and colleagues used zinc finger nucleases to disrupt CFTR exon 3 in rats [94]. CFTR ${ }^{-/-}$ rats recapitulate many aspects of human disease including intestinal obstruction, obstruction of the vas deferens, and abnormalities in nasal mucus production. It is currently not clear if CFTR null rats develop lung disease. To date, no gene correction studies have been reported in this novel model.

\subsection{CF ferret}

CFTR null ferrets were developed using AAV-mediated gene targeting in somatic cells, nuclear transfer, and cloning [95]. Unlike CF mice, CF ferrets develop early and reproducible lung infections that make it a promising platform for testing lung-directed $\mathrm{CF}$ therapies [95]. There are several potential reasons for these species differences. First, $\mathrm{Ca}^{++}$-activated $\mathrm{Cl}^{-}$channels in the mouse airway may compensate for cAMP-mediated CFTR $\mathrm{Cl}^{-}$transport [96, 97], a pathway that appears to be less active in humans or ferrets [95, 98-100]. Second, in humans and ferrets, goblet cells are the predominant secretory cell type of the cartilaginous airways [101-104], whereas in mice the analogous secretory cell type is the club cell $[105,106]$. Third, SMGs are virtually absent in murine cartilaginous airways, with only a handful in the most proximal regions of the trachea [106, 107]. SMGs are important for airway innate immunity in the ferret [108] and humans [109, 110], and a potentially valuable site for CFTR expression [111-114].

Lentiviral gene transfer to the wild-type neonatal ferrets using EIAV- and FIV-based vectors expressing fluorescent reporter genes was recently reported [115]. The EIAV was pseudotyped with hemagglutinin (HA) from avian influenza A virus [74] and the FIV vector was pseudo- 
typed with GP64 [63]. A liquid bolus of the vector was delivered to newborn ferrets via a tracheal incision. Significant transgene infection was noted in respiratory epithelia of all lobes in both the conducting and small airways with both vectors. Cmielewski and colleagues delivered VSVG-HIV expressing the LacZ reporter to the lungs of 7-8-week-old ferrets [116]. Considerably less gene transfer was observed as compared to the HA or GP64 pseudotyped LV gene transfer in the neonatal ferrets. Currently, it is unclear if these differences are due to the vector pseudotype, age of the ferrets, or delivery protocol; however, these data suggest that ferrets may be useful pre-clinical models for lentiviral vector development.

\subsection{CF pig}

Pigs are an important model for many studies of human cardiovascular diseases, injury and repair, surfactants, inflammation, and pulmonary diseases (reviewed in [101]). Compared to rodents, the pig lung is anatomically and physiologically more similar to humans $[117,118]$ and has been studied extensively in xenotransplantation. The prenatal maturation of the pig lung is similar to humans and includes extensive alveolarization [119]. Pig airway branching and cell composition is much more akin to human airways than to those of mice. The cell types comprising the conducting airway epithelium in pigs and humans are similar, and notably lack the high percentage of club cells typical of mice. The pig bronchial epithelium is pseudostratified and contains ciliated, basal, and goblet cells, and abundant SMGs (reviewed in [101]). Importantly, the distribution of SMGs in the conducting airways and the CFTRdependent and -independent secretion of liquid and macromolecules is similar to humans [112, 120-122].

Pigs with CFTR null and $\triangle$ F508 knock-in alleles were generated by AAV-mediated homologous recombination and somatic cell nuclear transfer [99]. Breeding heterozygous male and females generated homozygous $C_{F T R^{--}}$pigs, and their striking neonatal phenotype was described [99, 123]. Newborn CF pigs exhibit severe disease similar to humans including pancreatic insufficiency, meconium ileus with intestinal obstruction, absence of the vas deferens, and evidence of liver and gall bladder disease [123]. Importantly, CFTR null and $\triangle F 508$ pigs spontaneously develop lung disease with many features similar to humans with CF including bacterial infection, inflammation, abnormal mucociliary clearance, bronchiectasis, and remodeling.

In a recent study, we compared HIV- and FIV-based lentiviral vectors in well-differentiated human and pig airway epithelia [66]. FIV transduced pig airway epithelia with greater efficacy than HIV, but both FIV and HIV transduced human airway epithelia with equal efficacy [66]. We further screened a number of envelope glycoproteins and identified GP64 as one of the most efficient pseudotypes for transduction and persistent expression in both pig and human epithelial cells [66]. A mCherry marker virus was delivered to wild-type pigs 4 weeks of age. A bolus dose of GP64-FIV vector was delivered to the ethmoid sinuses or to the tracheal lobe through a catheter threaded through the suction channel of a pediatric bronchoscope. We estimated the range of transduction efficiencies in the pig airways to be from $<1$ to $7 \%$. In future studies, we will deliver CFTR expressing vector to CF pigs to 
determine the preferred gene transfer targets and the level of CFTR correction required to prevent or slow disease progression

\section{Outcome measures}

In pre-clinical studies of CF gene therapy, it is vital to define metrics of correction before the studies are initiated. It would be naïve to simply deliver vector to the airways and "look and see" if the airway disease is cured. CF is a complex disease with many phenotypic features and clearly defining the disease progression in an untreated CF animal model is vital. As experience is gained with new animal models, additional assays for correction will be established and refined. Importantly, these metrics may apply to multiple gene correction but may not be feasible in all animal models.

\subsection{Quantitative real-time PCR and CFTR protein expression}

Quantitative real-time RT-PCR is a sensitive assay for measuring vector expressed CFTR mRNA [123]. At progressive time-points post vector delivery, whole tissue or brushings of nasal or tracheobronchial epithelia can be obtained. Silent mutations can be engineered into the vector expressed CFTR cDNA so that transgene expression can be differentiated from endogenous CFTR. Using a similar strategy, vector genome copy number can be estimated. Genomic DNA from a portion of the same tissue or epithelial brushings can be purified and copy number estimated by normalizing to endogenous DNA and a standard curve. Appropriate controls would include wild type and untreated affected littermates. To identify cells expressing the CFTR protein, immunohistochemistry and immunofluorescence protocols have been reported [123]. Using these approaches, the percentage of cells expressing CFTR and the cell types expressing CFTR can be determined [124].

\subsection{Functional correction}

Nasal potential difference (NPD) is an established assay for demonstrating in vivo correction of CFTR-dependent $\mathrm{Cl}^{-}$transport [125-127]. For many of these studies, vector was delivered nasally and the NPD was used as evidence of CFTR complementation. Ideally, for integrating vectors, predelivery, early post-delivery, and late post-delivery timepoints in the intrapulmonary airways should be measured. As early as 1 week after gene transfer, the nasal voltage and its response to amiloride, low $\mathrm{Cl}^{-}$, and cAMP agonists can be measured [90, 123, 128]. Animals could be followed with serial monthly nasal voltage measurements over a 12-month or longer period to document persistence of expression. Importantly, CFTR is an anion channel that conducts both $\mathrm{Cl}^{-}$and $\mathrm{HCO}_{3}{ }^{-}$. As mentioned above, correcting $~ 10 \%$ of cells is often cited as a benchmark for restoring $\mathrm{Cl}^{-}$transport and correcting the clinical phenotype. However, other studies also suggest that defective $\mathrm{HCO}_{3}{ }^{-}$transport might be relevant to disease. Multiple studies of CF mouse cervical mucus [46, 47], CF mouse small intestinal mucus [48], and human CF nasal SMGs [49] support the importance of CFTR-dependent $\mathrm{HCO}_{3}^{-}$transport in $\mathrm{CF}$ pathogenesis. Loss of CFTR-dependent $\mathrm{HCO}_{3}{ }^{-}$transport acidifies liquid produced by surface 
epithelia [129, 130] and secretions from SMGs [49]. Thus, measurements of $\mathrm{HCO}_{3}^{-}$transport may also be an important metric of functional correction.

As a result of abnormal CFTR-dependent $\mathrm{HCO}_{3}^{-}$secretion, airway surface liquid $\mathrm{pH}$ is acidified. The ASL of primary cultures of CF pig airway epithelia [130], newborn CF pig airways [130], and the nasal $\mathrm{pH}$ of newborn babies with $\mathrm{CF}$ [131] is acidic. In CF pigs, the acidity has been shown to impair bacterial killing [130]. In addition, there are new techniques to measure mucus viscosity, mucociliary clearance (MCT), and lung function in large animal models [101]. Importantly, defects in airway MCT and SMG mucus detachment recently were identified in CF [132]. The bacterial killing defect is a quantifiable characteristic of CF airways. Bacterial killing is impaired as a result of reduced bicarbonate anion secretion and increasing ASL $\mathrm{pH}$ rescues bacterial killing [130].

\subsection{Reduced infection and inflammation}

A goal of gene therapy for $\mathrm{CF}$ is to prevent the onset or reduce the progression of lung disease. Signs of reduced infection and inflammation in treated animals can be visually inspected in the airways. Bronchoscopy can be used to detect signs of inflammation, such as mucosal inflammation and excessive purulent secretions. Total cell counts, cell differentials, and cytokine levels are obtained from bronchoalveolar lavage (BAL) as standard assay for infection and inflammation. In addition, standard quantitative microbiologic techniques are used to identify and quantify BAL bacteria [133]. Biopsies can also be obtained from larger animal models such as the CF pig. These samples can also be used for sequencing-based analyses. As we learn more about the disease progression in new animal models of CF, improved metrics of functional correction are being developed. High-resolution computerized tomography (HRCT) facilitates detailed structural analysis of the airways [101]. HRCT scans can discern anatomic changes in the airways over time in control and treated animals [134].

\section{Challenges to pulmonary gene transfer with integrating vectors}

\subsection{Delivery}

The lung is an attractive target for gene therapy because, unlike most other tissues, the vector can be topically delivered. Vector delivery to the airways of mice, rats, and newborn ferrets is most easily accomplished by nasal or intratracheal bolus delivery of vector resuspended in a liquid vehicle such as buffered saline [135], LPC [60, 62], or a viscoelastic gel [68, 69]. In small animals, bolus delivery using a relatively small volume of vector $(25-50 \mu \mathrm{l})$ can achieve widespread gene expression throughout the airways. However, in large animal models such as pigs or sheep (and ultimately humans), aerosolization will likely be required to achieve a widespread pulmonary distribution. In general, devices for generating airborne vector fall into three categories: aerosolizing catheters, nebulizers, and atomizers. All of these devices convert liquids into particles small enough to be respired. 
Aerosolizing catheters convert liquids into particles at the point of expulsion. Typically, an aerosolizing catheter is first passed into the trachea and then the vector is instilled. The Microsprayer ${ }^{\circledR}$ (PennCentury) and the AeroProbe ${ }^{\circledR}$ catheter (Trudell Medical International) are examples of delivery systems for this application. According to the manufacturer, it is possible to generate particles with aerodynamic diameters of $4-8 \mu \mathrm{m}$. The Trudell AeroProbe was previously used to aerosolize helper-dependent adenovirus vectors to rabbit airways [136, 137] and to deliver Sendai virus vectors to sheep [138]. Since Sendai virus and lentiviruses are both enveloped, it is likely that this approach is feasible with lentiviral vectors. In addition, aerosolized VSVG-LVs have been successfully delivered to the airways of mice [139].

Successful vector aerosolization has been reported in mice [140], rabbits,[136, 137], pig [141] and sheep airways [142,143]. By $2-3$ weeks of age, wild type pigs are large enough to be sedated and have a pediatric bronchoscope passed into the trachea. The AeroProbe ${ }^{\circledR}$ catheter can be passed to the carina via the bronchoscope suction channel with the animal breathing spontaneously. In this way, an integrating vector can be aerosolized and targeted to specific bronchial segments.

In the case of nebulizers, the liquid is first converted into mist and then passively inhaled. Using this strategy, a plasmid-based vector was delivered to the airways of CF patients in a phase IIB gene therapy trial [144]. This approach could potentially be used to deliver DNA transposon or hybrid vectors. However, this strategy may not be feasible with enveloped viral vectors because this class of vectors may not be stable enough to withstand nebulization. In addition, nebulization requires a large volume of concentrated material; therefore, would be the least economic delivery strategy for LVs.

Atomizers are a subclass of aerosolizing catheters that deliver larger sized particles. An atomizer, such as the MADgic ${ }^{\mathrm{TM}}$ (LMA) atomizer, delivers large droplets $(\sim 30-90 \mu \mathrm{m}$ in diameter), which may vary in size depending on the force applied to the syringe plunger. This type of device is often used to topically deliver medications to the airways [145]. Our group has observed that this type of atomizer is an effective delivery device for multiple viral vectors including FIV, particularly when formulated with a viscoelastic material such as methylcellulose.

\subsection{Insertional mutagenesis}

Since persistent gene expression from lentiviral vectors requires genomic integration, they show promise for treating life-long genetic diseases; however, there is inherent risk when introducing a transgene with integrating vectors. Insertional mutagenesis may disrupt normal cell functions by inactivating an essential host gene or inappropriately causing expression of an undesirable gene. The risk will vary depending on the vector used, the transgene cassette, and the cell type targeted. In many cases, enhancer effects pose the greatest danger. So far, malignant cell transformation after vector-mediated insertional mutagenesis has only been observed in three clinical entities (X-linked severe combined immunodeficiency (SCID-X1), chronic granulomatous disease (CGD), and Wiskott-Aldrich syndrome (WAS)), all of which occurred in conjunction with the use of first-generation gamma-retroviral vectors harboring LTRs with strong enhancer/promoter sequences [146-150]. These studies were conducted in 
immunocompromised patients where gene transfer conferred a selective advantage to corrected cells. The vector and disease settings likely influenced the risks for insertional mutagenesis and subsequent clonal expansion. Modern LVs are engineered to lack enhancer/ promoter sequences within the LTRs and delivering CFTR to somatic cells has no known selective advantage. Considerable effort has been put toward mapping integration patterns and determining the functional consequences of LVs and retroviral vectors (reviewed in [151, 152]). LV integration analyses conducted on adrenoleukodystrophy clinical trial patients demonstrate that the genomic distribution maintain a polyclonal pattern [153]. Montini and colleagues demonstrated that LV integrations, even at high vector titer loads, did not accelerate tumorigenesis in tumor prone mice. In contrast, gamma-retroviral vector transduction triggered a dose-dependent acceleration of tumor onset [154].

The burden of proof has fallen on LV researchers to demonstrate that LVs do not cause cancer via insertional mutagenesis. It is unlikely that this can ever be demonstrated with absolute certainty; however, the evidence to date suggests that current LVs are considerably safer than the gamma-retroviral vectors that were first brought to clinical trials. In fact, results from human clinical trials using LVs are encouraging and the feasibility of gene therapy for monogenetic diseases is now firmly established [155]. Recent promising examples include Wiskott-Aldrich Syndrome [156, 157], metachromatic leukodystrophy [158, 159], acute lymphoid leukemia [160], lymphoma [161, 162], and multiple primary immuno-deficiencies [163].

\section{Conclusions}

Within a year of the discovery of CFTR, investigators validated the concept that gene replacement could reverse the ion transport defect in vitro, suggesting that gene therapy may be possible $[164,165]$. We and others have demonstrated that CFTR delivery by integrating vectors can correct the $\mathrm{CF}$ anion defect in vitro and in vivo, and although further pre-clinical trials are warranted, there is great potential for translating this strategy to the clinic. As discussed, estimates of the percent of CF epithelia requiring correction vary and there is debate about which cell types must be corrected to achieve phenotypic correction; however, interest in $\mathrm{CF}$ gene therapy remains strong as barriers to gene transfer are identified, outcome measures are established, CF animal models with lung disease are developed, and better delivery systems are engineered. Demonstration of corrective gene transfer to pristine newborn lungs in $\mathrm{CF}$ animal models is a vital first step before looking ahead to correcting more diseased lungs. Newborn screening for CF is now established in all 50 states, allowing early disease detection. This offers an opportunity to introduce an integrating therapeutic gene transfer vector to the airway epithelium prior to the onset of chronic infection and inflammation. This strategy is a potentially life-long curative therapy regardless of the disease-causing mutation. 


\section{Acknowledgements}

We acknowledge the support of the University of Iowa DNA Sequencing Core, In Vitro Models and Cell Culture Core, Viral Vector Core, and Cell Morphology Core. This work was supported by the National Institutes of Health R01 HL-105821 (PLS), P01 HL-51670 (PBM), and the Cystic Fibrosis Foundation SINN14G0 (PLS). Core facilities were partially supported by the National Institutes of Health: P01 HL-51670, P01 HL-091842 and the Center for Gene Therapy for Cystic Fibrosis P30 DK-54759.

\section{Author details}

Ashley L Cooney ${ }^{1}$, Paul B McCray, $\mathrm{Jr}^{1,2}$ and Patrick L. Sinn ${ }^{2}$

*Address all correspondence to: patrick-sinn@uiowa.edu

1 Department of Microbiology, Carver College of Medicine, The University of Iowa, Iowa City, IA, USA

2 Department of Pediatrics, Carver College of Medicine, The University of Iowa, Iowa City, IA, USA

\section{References}

[1] Rowe SM, Miller S, Sorscher EJ. Cystic fibrosis. The New England journal of medicine. 2005 May 12;352(19):1992-2001. PubMed PMID: 15888700.

[2] Davis PB. Cystic fibrosis since 1938. American journal of respiratory and critical care medicine. 2006 Mar 1;173(5):475-82. PubMed PMID: 16126935.

[3] Welsh MJ, Smith AE. Molecular mechanisms of CFTR chloride channel dysfunction in cystic fibrosis. Cell. 1993 Jul 2;73(7):1251-4. PubMed PMID: 7686820.

[4] Clunes MT, Boucher RC. Front-runners for pharmacotherapeutic correction of the airway ion transport defect in cystic fibrosis. Current opinion in pharmacology. 2008 Jun;8(3):292-9. PubMed PMID: 18468487. PubMed Central PMCID: 2517236.

[5] Van Goor F, Hadida S, Grootenhuis PD, Burton B, Stack JH, Straley KS, et al. Correction of the F508del-CFTR protein processing defect in vitro by the investigational drug VX-809. Proceedings of the National Academy of Sciences of the United States of America. 2011 Nov 15;108(46):18843-8. PubMed PMID: 21976485. PubMed Central PMCID: 3219147.

[6] Accurso FJ, Rowe SM, Clancy JP, Boyle MP, Dunitz JM, Durie PR, et al. Effect of VX-770 in persons with cystic fibrosis and the G551D-CFTR mutation. The New Eng- 
land journal of medicine. 2010 Nov 18;363(21):1991-2003. PubMed PMID: 21083385. PubMed Central PMCID: 3148255.

[7] Clancy JP, Rowe SM, Accurso FJ, Aitken ML, Amin RS, Ashlock MA, et al. Results of a phase IIa study of VX-809, an investigational CFTR corrector compound, in subjects with cystic fibrosis homozygous for the F508del-CFTR mutation. Thorax. 2012 Jan; 67(1):12-8. PubMed PMID: 21825083. PubMed Central PMCID: 3746507.

[8] Konstan MW, Byard PJ, Hoppel CL, Davis PB. Effect of high-dose ibuprofen in patients with cystic fibrosis. The New England journal of medicine. $1995 \mathrm{Mar}$ 30;332(13):848-54. PubMed PMID: 7503838.

[9] Saiman L, Marshall BC, Mayer-Hamblett N, Burns JL, Quittner AL, Cibene DA, et al. Azithromycin in patients with cystic fibrosis chronically infected with Pseudomonas aeruginosa: A randomized controlled trial. JAMA: The journal of the American Medical Association. 2003 Oct 1;290(13):1749-56. PubMed PMID: 14519709.

[10] Gibson RL, Emerson J, McNamara S, Burns JL, Rosenfeld M, Yunker A, et al. Significant microbiological effect of inhaled tobramycin in young children with cystic fibrosis. American journal of respiratory and critical care medicine. 2003 Mar 15;167(6): 841-9. PubMed PMID: 12480612.

[11] Ramsey BW, Banks-Schlegel S, Accurso FJ, Boucher RC, Cutting GR, Engelhardt JF, et al. Future directions in early cystic fibrosis lung disease research: An NHLBI workshop report. American journal of respiratory and critical care medicine. 2012 Apr 15;185(8):887-92. PubMed PMID: 22312017. PubMed Central PMCID: 3360572.

[12] Pedemonte N, Lukacs GL, Du K, Caci E, Zegarra-Moran O, Galietta LJ, et al. Smallmolecule correctors of defective DeltaF508-CFTR cellular processing identified by high-throughput screening. The journal of clinical investigation. 2005 Sep;115(9): 2564-71. PubMed PMID: 16127463. PubMed Central PMCID: 1190372.

[13] Sondo E, Tomati V, Caci E, Esposito AI, Pfeffer U, Pedemonte N, et al. Rescue of the mutant CFTR chloride channel by pharmacological correctors and low temperature analyzed by gene expression profiling. American journal of physiology Cell physiology. 2011 Oct;301(4):C872-85. PubMed PMID: 21753184. PubMed Central PMCID: 3512166 .

[14] MacDonald KD, McKenzie KR, Zeitlin PL. Cystic fibrosis transmembrane regulator protein mutations: 'class' opportunity for novel drug innovation. Paediatric drugs. 2007;9(1):1-10. PubMed PMID: 17291132.

[15] Rogan MP, Stoltz DA, Hornick DB. Cystic fibrosis transmembrane conductance regulator intracellular processing, trafficking, and opportunities for mutation-specific treatment. Chest. 2011 Jun;139(6):1480-90. PubMed PMID: 21652558. 
[16] Pringle IA, Hyde SC, Gill DR. Non-viral vectors in cystic fibrosis gene therapy: Recent developments and future prospects. Expert opinion on biological therapy. 2009 Aug;9(8):991-1003. PubMed PMID: 19545217.

[17] Sinn PL, Anthony RM, McCray PB, Jr. Genetic therapies for cystic fibrosis lung disease. Human molecular genetics. 2011 Apr 15;20(R1):R79-86. PubMed PMID: 21422098. PubMed Central PMCID: 3095059.

[18] Griesenbach U, Alton EW. Moving forward: Cystic fibrosis gene therapy. Human molecular genetics. 2013 Oct 15;22(R1):R52-8. PubMed PMID: 23918661.

[19] Kreda SM, Mall M, Mengos A, Rochelle L, Yankaskas J, Riordan JR, et al. Characterization of wild-type and deltaF508 cystic fibrosis transmembrane regulator in human respiratory epithelia. Molecular biology of the cell. 2005 May;16(5):2154-67. PubMed PMID: 15716351. PubMed Central PMCID: 1087225.

[20] Curradi G, Walters MS, Ding BS, Rafii S, Hackett NR, Crystal RG. Airway basal cell vascular endothelial growth factor-mediated cross-talk regulates endothelial cell-dependent growth support of human airway basal cells. Cellular and molecular life sciences: CMLS. 2012 Jul;69(13):2217-31. PubMed PMID: 22382924. PubMed Central PMCID: 3633460.

[21] Staudt MR, Buro-Auriemma LJ, Walters MS, Salit J, Vincent T, Shaykhiev R, et al. Airway Basal stem/progenitor cells have diminished capacity to regenerate airway epithelium in chronic obstructive pulmonary disease. American journal of respiratory and critical care medicine. 2014 Oct 15;190(8):955-8. PubMed PMID: 25317467. PubMed Central PMCID: 4299582.

[22] Randell SH. Progenitor-progeny relationships in airway epithelium. Chest. 1992;101:11S-6S.

[23] Ford JR, Terzaghi-Howe M. Basal cells are the progenitors of primary tracheal epithelial cell cultures. Experimental cell research. 1992;198:69-77.

[24] Hong KU, Reynolds SD, Watkins S, Fuchs E, Stripp BR. Basal cells are a multipotent progenitor capable of renewing the bronchial epithelium. The American journal of pathology. 2004 Feb;164(2):577-88. PubMed PMID: 14742263.

[25] Engelhardt JF, Schlossberg H, Yankaskas JR, Dudus L. Progenitor cells of the adult human airway involved in submucosal gland development. Development. 1995;121:2031-46.

[26] Borthwick DW, Shahbazian M, Krantz QT, Dorin JR, Randell SH. Evidence for stemcell niches in the tracheal epithelium. American journal of respiratory cell \& molecular biology. 2001;24(6):662-70.

[27] Liu X, Engelhardt JF. The glandular stem/progenitor cell niche in airway development and repair. Proceedings of the American Thoracic Society. 2008 Aug 15;5(6): 682-8. PubMed PMID: 18684717. PubMed Central PMCID: 2645260. 
[28] Evans MJ, Johnson LV, Stephens RJ, Freeman G. Renewal of the terminal bronchiolar epithelium in the rat following exposure to $\mathrm{NO}_{2}$ or $\mathrm{O}_{3}$. Laboratory investigation. 1976;35:246-57.

[29] Hong KU, Reynolds SD, Giangreco A, Hurley CM, Stripp BR. Clara cell secretory protein-expressing cells of the airway neuroepithelial body microenvironment include a label-retaining subset and are critical for epithelial renewal after progenitor cell depletion. American journal of respiratory cell \& molecular biology. 2001;24(6): 671-81.

[30] Evans MJ, Cabral LJ, Stephens RJ, Freeman G. Transformation of alveolar type 2 cells to type 1 cells following exposure to $\mathrm{NO}_{2}$. Experimental \& molecular pathology. 1975;22:142-50.

[31] Adamson IY, Bowden DH. The type 2 cell as progenitor of alveolar epithelial regeneration. A cytodynamic study in mice after exposure to oxygen. Laboratory investigation. 1974;30:35-42.

[32] Rawlins EL, Hogan BL. Epithelial stem cells of the lung: Privileged few or opportunities for many? Development. 2006 Jul;133(13):2455-65. PubMed PMID: 16735479.

[33] Rock JR, Randell SH, Hogan BL. Airway basal stem cells: A perspective on their roles in epithelial homeostasis and remodeling. Disease models \& mechanisms. 2010 SepOct;3(9-10):545-56. PubMed PMID: 20699479. PubMed Central PMCID: 2931533.

[34] Randell SH. Airway epithelial stem cells and the pathophysiology of chronic obstructive pulmonary disease. Proceedings of the American Thoracic Society. 2006 Nov; 3(8):718-25. PubMed PMID: 17065380. PubMed Central PMCID: 2647659.

[35] Rock JR, Onaitis MW, Rawlins EL, Lu Y, Clark CP, Xue Y, et al. Basal cells as stem cells of the mouse trachea and human airway epithelium. Proceedings of the National Academy of Sciences of the United States of America. 2009 Aug 4;106(31):12771-5. PubMed PMID: 19625615. PubMed Central PMCID: 2714281.

[36] Hong KU, Reynolds SD, Watkins S, Fuchs E, Stripp BR. In vivo differentiation potential of tracheal basal cells: Evidence for multipotent and unipotent subpopulations. American journal of physiology lung cellular and molecular physiology. 2004 Apr; 286(4):L643-9. PubMed PMID: 12871857.

[37] Delplanque A, Coraux C, Tirouvanziam R, Khazaal I, Puchelle E, Ambros P, et al. Epithelial stem cell-mediated development of the human respiratory mucosa in SCID mice. Journal of cell science. 2000 Mar;113 (Pt 5):767-78. PubMed PMID: 10671367.

[38] Hackett TL, Shaheen F, Johnson A, Wadsworth S, Pechkovsky DV, Jacoby DB, et al. Characterization of side population cells from human airway epithelium. Stem cells. 2008 Oct;26(10):2576-85. PubMed PMID: 18653771. PubMed Central PMCID: 2849005. 
[39] Hajj R, Baranek T, Le Naour R, Lesimple P, Puchelle E, Coraux C. Basal cells of the human adult airway surface epithelium retain transit-amplifying cell properties. Stem cells. 2007 Jan;25(1):139-48. PubMed PMID: 17008423.

[40] Li X, Rossen N, Sinn PL, Hornick AL, Steines BR, Karp PH, et al. Integrin alpha6beta4 identifies human distal lung epithelial progenitor cells with potential as a cellbased therapy for cystic fibrosis lung disease. PloS one. 2013;8(12):e83624. PubMed PMID: 24349537. PubMed Central PMCID: 3861522.

[41] Johnson LG, Olsen JC, Sarkadi B, Moore KL, Swanstrom R, Boucher RC. Efficiency of gene transfer for restoration of normal airway epithelia function in cystic fibrosis. Nature genetics. 1992;2:21-5.

[42] Goldman MJ, Yang Y, Wilson JM. Gene therapy in a xenograft model of cystic fibrosis lung corrects chloride transport more effectively than the sodium defect. Nature genetics. 1995 Feb;9(2):126-31. PubMed PMID: 7719338.

[43] Farmen SL, Karp PH, Ng P, Palmer DJ, Koehler DR, Hu J, et al. Gene transfer of CFTR to airway epithelia: Low levels of expression are sufficient to correct Cl-transport and overexpression can generate basolateral CFTR. American journal of physiology Lung cellular and molecular physiology. 2005 Dec;289(6):L1123-30. PubMed PMID: 16085675.

[44] Zhang L, Button B, Gabriel SE, Burkett S, Yan Y, Skiadopoulos MH, et al. CFTR delivery to $25 \%$ of surface epithelial cells restores normal rates of mucus transport to human cystic fibrosis airway epithelium. PLoS biology. 2009 Jul;7(7):e1000155. PubMed PMID: 19621064. PubMed Central PMCID: 2705187.

[45] Dannhoffer L, Blouquit-Laye S, Regnier A, Chinet T. Functional properties of mixed cystic fibrosis and normal bronchial epithelial cell cultures. American journal of respiratory cell and molecular biology. 2009 Jun;40(6):717-23. PubMed PMID: 19011164.

[46] Garcia MA, Yang N, Quinton PM. Normal mouse intestinal mucus release requires cystic fibrosis transmembrane regulator-dependent bicarbonate secretion. The journal of clinical investigation. 2009 Sep;119(9):2613-22. PubMed PMID: 19726884. PubMed Central PMCID: 2735925.

[47] Muchekehu RW, Quinton PM. A new role for bicarbonate secretion in cervico-uterine mucus release. The journal of physiology. 2010 Jul 1;588(Pt 13):2329-42. PubMed PMID: 20478977. PubMed Central PMCID: 2915510.

[48] Gustafsson JK, Ermund A, Ambort D, Johansson ME, Nilsson HE, Thorell K, et al. Bicarbonate and functional CFTR channel are required for proper mucin secretion and link cystic fibrosis with its mucus phenotype. The journal of experimental medicine. 2012 Jul 2;209(7):1263-72. PubMed PMID: 22711878. PubMed Central PMCID: 3405509 . 
[49] Song Y, Salinas D, Nielson DW, Verkman AS. Hyperacidity of secreted fluid from submucosal glands in early cystic fibrosis. American journal of physiology Cell physiology. 2006 Mar;290(3):C741-9. PubMed PMID: 16207791.

[50] Sakuma T, Barry MA, Ikeda Y. Lentiviral vectors: Basic to translational. The biochemical journal. 2012 May 1;443(3):603-18. PubMed PMID: 22507128.

[51] McCormack JE, Martineau D, DePolo N, Maifert S, Akabarian L, Townsend K, et al. Anti-vector immunoglobulin induced by retroviral vectors. Human gene therapy. 1997;8:1263-73.

[52] Sinn PL, Sauter SL, McCray PB, Jr. Gene therapy progress and prospects: Development of improved lentiviral and retroviral vectors - design, biosafety, and production. Gene therapy. 2005 Jul;12(14):1089-98. PubMed PMID: 16003340.

[53] Stocker AG, Kremer KL, Koldej R, Miller DS, Anson DS, Parsons DW. Single-dose lentiviral gene transfer for lifetime airway gene expression. The journal of gene medicine. 2009 Oct;11(10):861-7. PubMed PMID: 19634193.

[54] Brady T, Bushman FD. Nondividing cells: A safer bet for integrating vectors? Molecular therapy: The journal of the American Society of gene therapy. 2011 Apr;19(4): 640-1. PubMed PMID: 21455211. PubMed Central PMCID: 3070107.

[55] Franz K, Singh A, Weinberger LS. Lentiviral vectors to study stochastic noise in gene expression. Methods in enzymology. 2011;497:603-22. PubMed PMID: 21601105.

[56] Terwilliger EF, Godin B, Sodroski JG, Haseltine WA. Construction and use of a replication-competent human immunodeficiency virus (HIV-1) that expresses the chloramphenicol acetyltransferase enzyme. Proceedings of the National Academy of Sciences of the United States of America. 1989 May;86(10):3857-61. PubMed PMID: 2726755. PubMed Central PMCID: 287240.

[57] Zufferey R, Dull T, Mandel RJ, Bukovsky A, Quiroz D, Naldini L, et al. Self-inactivating lentivirus vector for safe and efficient in vivo gene delivery. Journal of virology. 1998;72(12):9873-80.

[58] Sinn PL, Goreham-Voss JD, Arias AC, Hickey MA, Maury W, Chikkanna-Gowda CP, et al. Enhanced gene expression conferred by stepwise modification of a non-primate lentiviral vector. Human Gene Therapy. 2007;18:1244-52.

[59] Zufferey R, Donello JE, Trono D, Hope TJ. Woodchuck hepatitis virus posttranscriptional regulatory element enhances expression of transgenes delivered by retroviral vectors. Journal of virology. 1999 Apr;73(4):2886-92. PubMed PMID: 10074136. PubMed Central PMCID: 104046.

[60] Cmielewski P, Donnelley M, Parsons DW. Long-term therapeutic and reporter gene expression in lentiviral vector treated cystic fibrosis mice. The journal of gene medicine. 2014 Sep-Oct;16(9-10):291-9. PubMed PMID: 25130650. 
[61] Goldman MJ, Lee PS, Yang JS, Wilson JM. Lentiviral vectors for gene therapy of cystic fibrosis. Human gene therapy. 1997;8(18):2261-8.

[62] Limberis M, Anson DS, Fuller M, Parsons DW. Recovery of airway cystic fibrosis transmembrane conductance regulator function in mice with cystic fibrosis after single-dose lentivirus-mediated gene transfer. Human gene therapy. 2002 Nov 1;13(16): 1961-70. PubMed PMID: 12427306.

[63] Sinn PL, Burnight ER, Hickey MA, Blissard GW, McCray PB, Jr. Persistent gene expression in mouse nasal epithelia following feline immunodeficiency virus-based vector gene transfer. Journal of virology. 2005 Oct;79(20):12818-27. PubMed PMID: 16188984. PubMed Central PMCID: 1235842.

[64] Sinn PL, Hickey MA, Staber PD, Dylla DE, Jeffers SA, Davidson BL, et al. Lentivirus vectors pseudotyped with filoviral envelope glycoproteins transduce airway epithelia from the apical surface independently of folate receptor alpha. Journal of virology. 2003 May;77(10):5902-10. PubMed PMID: 12719583. PubMed Central PMCID: 154009 .

[65] Wang G, Slepushkin V, Zabner J, Keshavjee S, Johnston JC, Sauter SL, et al. Feline immunodeficiency virus vectors persistently transduce nondividing airway epithelia and correct the cystic fibrosis defect. The journal of clinical investigation. 1999 Dec; 104(11):R55-62. PubMed PMID: 10587528. PubMed Central PMCID: 483477.

[66] Sinn PL, Cooney AL, Oakland M, Dylla DE, Wallen TJ, Pezzulo AA, et al. Lentiviral vector gene transfer to porcine airways. Molecular therapy Nucleic acids. 2012;1:e56. PubMed PMID: 23187455. PubMed Central PMCID: 3511674.

[67] Sinn PL, Arias AC, Brogden KA, McCray PB, Jr. Lentivirus vector can be readministered to nasal epithelia without blocking immune responses. Journal of virology. 2008 Nov;82(21):10684-92. PubMed PMID: 18768988. PubMed Central PMCID: 2573216.

[68] Sinn PL, Shah A, Donovan M, McCray PB, Jr. Viscoelastic gel formulations enhance airway epithelial gene transfer with viral vectors. American journal of respiratory cell and molecular biology. 2005;32:404-10.

[69] Griesenbach U, Meng C, Farley R, Wasowicz MY, Munkonge FM, Chan M, et al. The use of carboxymethylcellulose gel to increase non-viral gene transfer in mouse airways. Biomaterials. 2010 Mar;31(9):2665-72. PubMed PMID: 20022367. PubMed Central PMCID: 4148698.

[70] Nakajima T, Nakamaru K, Ido E, Terao K, Hayami M, Hasegawa M. Development of novel simian immunodeficiency virus vectors carrying a dual gene expression system. Human gene therapy. 2000 Sep 1;11(13):1863-74. PubMed PMID: 10986559.

[71] Griesenbach U, Inoue M, Meng C, Farley R, Chan M, Newman NK, et al. Assessment of $\mathrm{F} / \mathrm{HN}$-pseudotyped lentivirus as a clinically relevant vector for lung gene therapy. 
American journal of respiratory and critical care medicine. 2012 Nov 1;186(9):846-56. PubMed PMID: 22955314. PubMed Central PMCID: 3530223.

[72] Mitomo K, Griesenbach U, Inoue M, Somerton L, Meng C, Akiba E, et al. Toward gene therapy for cystic fibrosis using a lentivirus pseudotyped with Sendai virus envelopes. Molecular therapy: The journal of the American Society of Gene Therapy. 2010 Jun;18(6):1173-82. PubMed PMID: 20332767. PubMed Central PMCID: 2889732.

[73] Valori CF, Ning K, Wyles M, Azzouz M. Development and applications of non-HIVbased lentiviral vectors in neurological disorders. Current gene therapy. 2008 Dec; 8(6):406-18. PubMed PMID: 19075624.

[74] Patel M, Giddings AM, Sechelski J, Olsen JC. High efficiency gene transfer to airways of mice using influenza hemagglutinin pseudotyped lentiviral vectors. The journal of gene medicine. 2013 Jan;15(1):51-62. PubMed PMID: 23319179.

[75] Yant SR, Meuse L, Chiu W, Ivics Z, Izsvak Z, Kay MA. Somatic integration and longterm transgene expression in normal and haemophilic mice using a DNA transposon system. Nature genetics. 2000 May;25(1):35-41. PubMed PMID: 10802653.

[76] Meir YJ, Wu SC. Transposon-based vector systems for gene therapy clinical trials: Challenges and considerations. Chang Gung medical journal. 2011 Nov-Dec;34(6): 565-79. PubMed PMID: 22196059.

[77] Huang X, Guo H, Tammana S, Jung YC, Mellgren E, Bassi P, et al. Gene transfer efficiency and genome-wide integration profiling of Sleeping Beauty, Tol2, and piggyBac transposons in human primary T cells. Molecular therapy: The journal of the American Society of Gene Therapy. 2010 Oct;18(10):1803-13. PubMed PMID: 20606646. PubMed Central PMCID: 2951558.

[78] Belur LR, Podetz-Pedersen K, Frandsen J, McIvor RS. Lung-directed gene therapy in mice using the nonviral sleeping beauty transposon system. Nature protocols. 2007;2(12):3146-52. PubMed PMID: 18079714.

[79] Ma K, Wang DD, Lin Y, Wang J, Petrenko V, Mao C. Synergetic targeted delivery of sleeping-beauty transposon system to mesenchymal stem cells using LPD nanoparticles modified with a phage-displayed targeting peptide. Advanced functional materials. 2013 Mar 6;23(9):1172-81. PubMed PMID: 23885226. PubMed Central PMCID: 3718568 .

[80] Yant SR, Ehrhardt A, Mikkelsen JG, Meuse L, Pham T, Kay MA. Transposition from a gutless adeno-transposon vector stabilizes transgene expression in vivo. Nature biotechnology. 2002 Oct;20(10):999-1005. PubMed PMID: 12244327.

[81] Cooney AL, Singh BK, Sinn PL. Hybrid nonviral/viral vector systems for improved piggyBac DNA transposon in vivo delivery. Molecular therapy: The journal of the American Society of Gene Therapy. 2015 Apr;23(4):667-74. PubMed PMID: 25557623.

[82] Zhang W, Solanki M, Muther N, Ebel M, Wang J, Sun C, et al. Hybrid adeno-associated viral vectors utilizing transposase-mediated somatic integration for stable trans- 
gene expression in human cells. PloS one. 2013;8(10):e76771. PubMed PMID: 24116154. PubMed Central PMCID: 3792901.

[83] Vink CA, Gaspar HB, Gabriel R, Schmidt M, McIvor RS, Thrasher AJ, et al. Sleeping beauty transposition from nonintegrating lentivirus. Molecular therapy: The journal of the American Society of Gene Therapy. 2009 Jul;17(7):1197-204. PubMed PMID: 19417741. PubMed Central PMCID: 2835211.

[84] Staunstrup NH, Moldt B, Mates L, Villesen P, Jakobsen M, Ivics Z, et al. Hybrid lentivirus-transposon vectors with a random integration profile in human cells. Molecular therapy: The journal of the American Society of Gene Therapy. 2009 Jul;17(7): 1205-14. PubMed PMID: 19240688. PubMed Central PMCID: 2835219.

[85] Hackett CS, Geurts AM, Hackett PB. Predicting preferential DNA vector insertion sites: Implications for functional genomics and gene therapy. Genome biology. 2007;8 Suppl 1:S12. PubMed PMID: 18047689. PubMed Central PMCID: 2106846.

[86] Clarke LL, Grubb BR, Gabriel SE, Smithies O, Koller BH, Boucher RC. Defective epithelial chloride transport in a gene-targeted mouse model of cystic fibrosis. Science. 1992 Aug 21;257(5073):1125-8. PubMed PMID: 1380724.

[87] O'Neal WK, Hasty P, McCray PB, Jr., Casey B, Rivera-Perez J, Welsh MJ, et al. A severe phenotype in mice with a duplication of exon 3 in the cystic fibrosis locus. Human molecular genetics. 1993 Oct;2(10):1561-9. PubMed PMID: 7505691.

[88] Dorin JR, Dickinson P, Alton EW, Smith SN, Geddes DM, Stevenson BJ, et al. Cystic fibrosis in the mouse by targeted insertional mutagenesis. Nature. 1992 Sep 17;359(6392):211-5. PubMed PMID: 1382232.

[89] Ratcliff R, Evans MJ, Cuthbert AW, MacVinish LJ, Foster D, Anderson JR, et al. Production of a severe cystic fibrosis mutation in mice by gene targeting. Nature genetics. 1993 May;4(1):35-41. PubMed PMID: 7685652.

[90] Zeiher BG, Eichwald E, Zabner J, Smith JJ, Puga AP, McCray PB, Jr., et al. A mouse model for the delta F508 allele of cystic fibrosis. The journal of clinical investigation. 1995 Oct;96(4):2051-64. PubMed PMID: 7560099. PubMed Central PMCID: 185844.

[91] Delaney SJ, Alton EW, Smith SN, Lunn DP, Farley R, Lovelock PK, et al. Cystic fibrosis mice carrying the missense mutation G551D replicate human genotype-phenotype correlations. The EMBO journal. 1996 Mar 1;15(5):955-63. PubMed PMID: 8605891. PubMed Central PMCID: 449990.

[92] Colledge WH, Abella BS, Southern KW, Ratcliff R, Jiang C, Cheng SH, et al. Generation and characterization of a delta F508 cystic fibrosis mouse model. Nature genetics. 1995 Aug;10(4):445-52. PubMed PMID: 7545494.

[93] Hodges CA, Cotton CU, Palmert MR, Drumm ML. Generation of a conditional null allele for Cftr in mice. Genesis. 2008 Oct;46(10):546-52. PubMed PMID: 18802965. PubMed Central PMCID: 2711445. 
[94] Tuggle KL, Birket SE, Cui X, Hong J, Warren J, Reid L, et al. Characterization of defects in ion transport and tissue development in cystic fibrosis transmembrane conductance regulator (CFTR)-knockout rats. PloS one. 2014;9(3):e91253. PubMed PMID: 24608905. PubMed Central PMCID: 3946746.

[95] Sun X, Sui H, Fisher JT, Yan Z, Liu X, Cho HJ, et al. Disease phenotype of a ferret CFTR-knockout model of cystic fibrosis. The journal of clinical investigation. 2010 Sep;120(9):3149-60. PubMed PMID: 20739752. PubMed Central PMCID: 2929732.

[96] Grubb BR, Vick RN, Boucher RC. Hyperabsorption of $\mathrm{Na}+$ and raised $\mathrm{Ca}(2+)$-mediated $\mathrm{Cl}$-secretion in nasal epithelia of CF mice. The American journal of physiology. 1994 May;266(5 Pt 1):C1478-83. PubMed PMID: 7515571.

[97] Liu X, Yan Z, Luo M, Engelhardt JF. Species-specific differences in mouse and human airway epithelial biology of recombinant adeno-associated virus transduction. American journal of respiratory cell and molecular biology. 2006 Jan;34(1):56-64. PubMed PMID: 16195538. PubMed Central PMCID: 1752084.

[98] Liu X, Luo M, Zhang L, Ding W, Yan Z, Engelhardt JF. Bioelectric properties of chloride channels in human, pig, ferret, and mouse airway epithelia. American journal of respiratory cell and molecular biology. 2007 Mar;36(3):313-23. PubMed PMID: 17008635. PubMed Central PMCID: 1894945.

[99] Rogers CS, Hao Y, Rokhlina T, Samuel M, Stoltz DA, Li Y, et al. Production of CFTRnull and CFTR-DeltaF508 heterozygous pigs by adeno-associated virus-mediated gene targeting and somatic cell nuclear transfer. The journal of clinical investigation. 2008 Apr;118(4):1571-7. PubMed PMID: 18324337. PubMed Central PMCID: 2265103.

[100] Fisher JT, Tyler SR, Zhang Y, Lee BJ, Liu X, Sun X, et al. Bioelectric characterization of epithelia from neonatal CFTR knockout ferrets. American journal of respiratory cell and molecular biology. 2013 Nov;49(5):837-44. PubMed PMID: 23782101. PubMed Central PMCID: 3931095.

[101] Rogers CS, Abraham WM, Brogden KA, Engelhardt JF, Fisher JT, McCray PB, Jr., et al. The porcine lung as a potential model for cystic fibrosis. American journal of physiology lung cellular and molecular physiology. 2008 Aug;295(2):L240-63. PubMed PMID: 18487356. PubMed Central PMCID: 2519845.

[102] Mercer RR, Russell ML, Roggli VL, Crapo JD. Cell number and distribution in human and rat airways. American journal of respiratory cell and molecular biology. 1994 Jun;10(6):613-24. PubMed PMID: 8003339.

[103] Wang X, Zhang Y, Amberson A, Engelhardt JF. New models of the tracheal airway define the glandular contribution to airway surface fluid and electrolyte composition. American journal of respiratory cell and molecular biology. 2001 Feb;24(2):195202. PubMed PMID: 11159054. 
[104] Jacob S, Poddar S. Mucous cells of the tracheobronchial tree in the ferret. Histochemistry. 1982;73(4):599-605. PubMed PMID: 6175610.

[105] Plopper CG, Mariassy AT, Wilson DW, Alley JL, Nishio SJ, Nettesheim P. Comparison of nonciliated tracheal epithelial cells in six mammalian species: Ultrastructure and population densities. Experimental lung research. 1983 Dec;5(4):281-94. PubMed PMID: 6662075.

[106] Pack RJ, Al-Ugaily LH, Morris G, Widdicombe JG. The distribution and structure of cells in the tracheal epithelium of the mouse. Cell and tissue research. 1980;208(1):6584. PubMed PMID: 6248229.

[107] Widdicombe JH, Chen LL, Sporer H, Choi HK, Pecson IS, Bastacky SJ. Distribution of tracheal and laryngeal mucous glands in some rodents and the rabbit. Journal of anatomy. 2001 Feb;198(Pt 2):207-21. PubMed PMID: 11273045. PubMed Central PMCID: 1468211.

[108] Dajani R, Zhang Y, Taft PJ, Travis SM, Starner TD, Olsen A, et al. Lysozyme secretion by submucosal glands protects the airway from bacterial infection. American journal of respiratory cell and molecular biology. 2005 Jun;32(6):548-52. PubMed PMID: 15746432. PubMed Central PMCID: 2715323.

[109] Wine JJ, Joo NS. Submucosal glands and airway defense. Proceedings of the American Thoracic Society. 2004;1(1):47-53. PubMed PMID: 16113412.

[110] Verkman AS, Song Y, Thiagarajah JR. Role of airway surface liquid and submucosal glands in cystic fibrosis lung disease. American journal of physiology Cell physiology. 2003 Jan;284(1):C2-15. PubMed PMID: 12475759.

[111] Engelhardt JF, Yankaskas JR, Ernst SA, Yang Y, Marino CR, Boucher RC, et al. Submucosal glands are the predominant site of CFTR expression in the human bronchus. Nature genetics. 1992;2(3):240-8.

[112] Choi HK, Finkbeiner WE, Widdicombe JH. A comparative study of mammalian tracheal mucous glands. Journal of anatomy. 2000 Oct;197 Pt 3:361-72. PubMed PMID: 11117623. PubMed Central PMCID: 1468138.

[113] Ballard ST, Trout L, Bebok Z, Sorscher EJ, Crews A. CFTR involvement in chloride, bicarbonate, and liquid secretion by airway submucosal glands. The American journal of physiology. 1999 Oct;277(4 Pt 1):L694-9. PubMed PMID: 10516209.

[114] Sehgal A, Presente A, Engelhardt JF. Developmental expression patterns of CFTR in ferret tracheal surface airway and submucosal gland epithelia. American journal of respiratory cell and molecular biology. 1996 Jul;15(1):122-31. PubMed PMID: 8679216.

[115] Yan Z, Stewart ZA, Sinn PL, Olsen JC, Hu J, McCray PB, Jr., et al. Ferret and pig models of cystic fibrosis: Prospects and promise for gene therapy. Human gene therapy clinical development. 2015 Feb 12. PubMed PMID: 25675143. 
[116] Cmielewski P, Farrow N, Donnelley M, McIntyre C, Penny-Dimri J, Kuchel T, et al. Transduction of ferret airway epithelia using a pre-treatment and lentiviral gene vector. BMC pulmonary medicine. 2014;14:183. PubMed PMID: 25413892. PubMed Central PMCID: 4258949.

[117] Pabst R. The respiratory immune system of pigs. Veterinary immunology and immunopathology. 1996 Nov;54(1-4):191-5. PubMed PMID: 8988865.

[118] Maina JN, van Gils P. Morphometric characterization of the airway and vascular systems of the lung of the domestic pig, Sus scrofa: Comparison of the airway, arterial and venous systems. Comparative biochemistry and physiology Part A, Molecular \& integrative physiology. 2001 Nov;130(4):781-98. PubMed PMID: 11691614.

[119] Baskerville A. Histological and ultrastructural observations on the development of the lung of the fetal pig. Acta anatomica. 1976;95(2):218-33. PubMed PMID: 961354.

[120] Inglis SK, Corboz MR, Taylor AE, Ballard ST. In situ visualization of bronchial submucosal glands and their secretory response to acetylcholine. The American journal of physiology. 1997 Feb;272(2 Pt 1):L203-10. PubMed PMID: 9124370.

[121] Inglis SK, Corboz MR, Ballard ST. Effect of anion secretion inhibitors on mucin content of airway submucosal gland ducts. The American journal of physiology. 1998 May;274(5 Pt 1):L762-6. PubMed PMID: 9612291.

[122] Joo NS, Irokawa T, Wu JV, Robbins RC, Whyte RI, Wine JJ. Absent secretion to vasoactive intestinal peptide in cystic fibrosis airway glands. The journal of biological chemistry. 2002 Dec 27;277(52):50710-5. PubMed PMID: 12368280.

[123] Rogers CS, Stoltz DA, Meyerholz DK, Ostedgaard LS, Rokhlina T, Taft PJ, et al. Disruption of the CFTR gene produces a model of cystic fibrosis in newborn pigs. Science. 2008 Sep 26;321(5897):1837-41. PubMed PMID: 18818360. PubMed Central PMCID: 2570747.

[124] Gibson UE, Heid CA, Williams PM. A novel method for real time quantitative RTPCR. Genome research. 1996 Oct;6(10):995-1001. PubMed PMID: 8908519.

[125] Saussereau EL, Roussel D, Diallo S, Debarbieux L, Edelman A, Sermet-Gaudelus I. Characterization of nasal potential difference in cftr knockout and F508del-CFTR mice. PloS one. 2013;8(3):e57317. PubMed PMID: 23505426. PubMed Central PMCID: 3591431.

[126] Griesenbach U, Smith SN, Farley R, Singh C, Alton EW. Validation of nasal potential difference measurements in gut-corrected CF knockout mice. American journal of respiratory cell and molecular biology. 2008 Oct;39(4):490-6. PubMed PMID: 18458238.

[127] Salinas DB, Pedemonte N, Muanprasat C, Finkbeiner WF, Nielson DW, Verkman AS. CFTR involvement in nasal potential differences in mice and pigs studied using a 
thiazolidinone CFTR inhibitor. American journal of physiology Lung cellular and molecular physiology. 2004 Nov;287(5):L936-43. PubMed PMID: 15246976.

[128] Fasbender A, Marshall J, Moninger TO, Grunst T, Cheng S, Welsh MJ. Effect of colipids in enhancing cationic lipid-mediated gene transfer in vitro and in vivo. Gene therapy. 1997 Jul;4(7):716-25. PubMed PMID: 9282173.

[129] Coakley RD, Grubb BR, Paradiso AM, Gatzy JT, Johnson LG, Kreda SM, et al. Abnormal surface liquid $\mathrm{pH}$ regulation by cultured cystic fibrosis bronchial epithelium. Proceedings of the National Academy of Sciences of the United States of America. 2003 Dec 23;100(26):16083-8. PubMed PMID: 14668433. PubMed Central PMCID: 307696.

[130] Pezzulo AA, Tang XX, Hoegger MJ, Alaiwa MH, Ramachandran S, Moninger TO, et al. Reduced airway surface $\mathrm{pH}$ impairs bacterial killing in the porcine cystic fibrosis lung. Nature. 2012 Jul 5;487(7405):109-13. PubMed PMID: 22763554. PubMed Central PMCID: 3390761.

[131] Abou Alaiwa MH, Beer AM, Pezzulo AA, Launspach JL, Horan RA, Stoltz DA, et al. Neonates with cystic fibrosis have a reduced nasal liquid $\mathrm{pH}$; a small pilot study. Journal of cystic fibrosis: Official journal of the European Cystic Fibrosis Society. 2014 Jul;13(4):373-7. PubMed PMID: 24418186. PubMed Central PMCID: 4060428.

[132] Hoegger MJ, Fischer AJ, McMenimen JD, Ostedgaard LS, Tucker AJ, Awadalla MA, et al. Cystic fibrosis. Impaired mucus detachment disrupts mucociliary transport in a piglet model of cystic fibrosis. Science. 2014 Aug 15;345(6198):818-22. PubMed PMID: 25124441.

[133] Lyczak JB, Cannon CL, Pier GB. Lung infections associated with cystic fibrosis. Clinical microbiology reviews. 2002 Apr;15(2):194-222. PubMed PMID: 11932230. PubMed Central PMCID: 118069.

[134] Dodd JD, Lavelle LP, Fabre A, Brady D. Imaging in cystic fibrosis and non-cystic fibrosis bronchiectasis. Seminars in respiratory and critical care medicine. 2015 Apr; 36(2):194-206. PubMed PMID: 25826587.

[135] Kobinger GP, Weiner DJ, Yu QC, Wilson JM. Filovirus-pseudotyped lentiviral vector can efficiently and stably transduce airway epithelia in vivo. Nature biotechnology. 2001 Mar;19(3):225-30. PubMed PMID: 11231554.

[136] Koehler DR, Martin B, Corey M, Palmer D, Ng P, Tanswell AK, et al. Readministration of helper-dependent adenovirus to mouse lung. Gene therapy. 2006 May;13(9): 773-80. PubMed PMID: 16437131.

[137] Koehler DR, Frndova H, Leung K, Louca E, Palmer D, Ng P, et al. Aerosol delivery of an enhanced helper-dependent adenovirus formulation to rabbit lung using an intratracheal catheter. The journal of gene medicine. 2005 Nov;7(11):1409-20. PubMed PMID: 15999396. 
[138] Griesenbach U, McLachlan G, Owaki T, Somerton L, Shu T, Baker A, et al. Validation of recombinant Sendai virus in a non-natural host model. Gene therapy. 2011 Feb; 18(2):182-8. PubMed PMID: 20962870.

[139] Hwang SK, Kwon JT, Park SJ, Chang SH, Lee ES, Chung YS, et al. Lentivirus-mediated carboxyl-terminal modulator protein gene transfection via aerosol in lungs of Kras null mice. Gene therapy. 2007 Dec;14(24):1721-30. PubMed PMID: 17960162.

[140] Koehler DR, Sajjan U, Chow YH, Martin B, Kent G, Tanswell AK, et al. Protection of Cftr knockout mice from acute lung infection by a helper-dependent adenoviral vector expressing Cftr in airway epithelia. Proceedings of the National Academy of Sciences of the United States of America. 2003;100(26):15364-9. Epub 2003 Dec 12.

[141] Cao H, Machuca TN, Yeung JC, Wu J, Du K, Duan C, et al. Efficient gene delivery to pig airway epithelia and submucosal glands using helper-dependent adenoviral vectors. Molecular therapy Nucleic acids. 2013;2:e127. PubMed PMID: 24104599. PubMed Central PMCID: 3890457.

[142] McLachlan G, Baker A, Tennant P, Gordon C, Vrettou C, Renwick L, et al. Optimizing aerosol gene delivery and expression in the ovine lung. Molecular therapy: The journal of the American Society of Gene Therapy. 2007 Feb;15(2):348-54. PubMed PMID: 17235313.

[143] Davies LA, McLachlan G, Sumner-Jones SG, Ferguson D, Baker A, Tennant P, et al. Enhanced lung gene expression after aerosol delivery of concentrated pDNA/PEI complexes. Molecular therapy: The journal of the American Society of Gene Therapy. 2008 Jul;16(7):1283-90. PubMed PMID: 18500249.

[144] Alton EW, Boyd AC, Cheng SH, Cunningham S, Davies JC, Gill DR, et al. A randomised, double-blind, placebo-controlled phase IIB clinical trial of repeated application of gene therapy in patients with cystic fibrosis. Thorax. 2013 Nov;68(11):1075-7. PubMed PMID: 23525080.

[145] Xue FS, Yang QY, Liao X, Liu JH, Tong SY. Topical anesthesia of the airway using fibreoptic bronchoscope and the MADgic atomizer in patients with predicted difficult intubation. Canadian journal of anaesthesia = Journal canadien d'anesthesie. 2007 Nov;54(11):951-2. PubMed PMID: 17975244.

[146] Hacein-Bey-Abina S, Von Kalle C, Schmidt M, McCormack MP, Wulffraat N, Leboulch $\mathrm{P}$, et al. LMO2-associated clonal $\mathrm{T}$ cell proliferation in two patients after gene therapy for SCID-X1. Science. 2003;302(5644):415-9.

[147] Howe SJ, Mansour MR, Schwarzwaelder K, Bartholomae C, Hubank M, Kempski H, et al. Insertional mutagenesis combined with acquired somatic mutations causes leukemogenesis following gene therapy of SCID-X1 patients. The journal of clinical investigation. 2008 Sep;118(9):3143-50. PubMed PMID: 18688286. PubMed Central PMCID: 2496964. 
[148] Boztug K, Schmidt M, Schwarzer A, Banerjee PP, Diez IA, Dewey RA, et al. Stem-cell gene therapy for the Wiskott-Aldrich syndrome. The New England journal of medicine. 2010 Nov 11;363(20):1918-27. PubMed PMID: 21067383. PubMed Central PMCID: 3064520.

[149] Stein S, Ott MG, Schultze-Strasser S, Jauch A, Burwinkel B, Kinner A, et al. Genomic instability and myelodysplasia with monosomy 7 consequent to EVI1 activation after gene therapy for chronic granulomatous disease. Nature medicine. 2010 Feb;16(2): 198-204. PubMed PMID: 20098431.

[150] Persons DA, Baum C. Solving the problem of gamma-retroviral vectors containing long terminal repeats. Molecular therapy: The journal of the American Society of Gene Therapy. 2011 Feb;19(2):229-31. PubMed PMID: 21289636. PubMed Central PMCID: 3034864.

[151] Rothe M, Modlich U, Schambach A. Biosafety challenges for use of lentiviral vectors in gene therapy. Current gene therapy. 2013 Dec;13(6):453-68. PubMed PMID: 24195603.

[152] Biasco L, Baricordi C, Aiuti A. Retroviral integrations in gene therapy trials. Molecular therapy: The journal of the American Society of Gene Therapy. 2012 Apr;20(4): 709-16. PubMed PMID: 22252453. PubMed Central PMCID: 3321603.

[153] Biffi A, Bartolomae CC, Cesana D, Cartier N, Aubourg P, Ranzani M, et al. Lentiviral vector common integration sites in preclinical models and a clinical trial reflect a benign integration bias and not oncogenic selection. Blood. 2011 May 19;117(20):5332-9. PubMed PMID: 21403130.

[154] Montini E, Cesana D, Schmidt M, Sanvito F, Ponzoni M, Bartholomae C, et al. Hematopoietic stem cell gene transfer in a tumor-prone mouse model uncovers low genotoxicity of lentiviral vector integration. Nature biotechnology. 2006 Jun;24(6):687-96. PubMed PMID: 16732270.

[155] McGarrity GJ, Hoyah G, Winemiller A, Andre K, Stein D, Blick G, et al. Patient monitoring and follow-up in lentiviral clinical trials. The journal of gene medicine. 2013 Feb;15(2):78-82. PubMed PMID: 23322669.

[156] Aiuti A, Bacchetta R, Seger R, Villa A, Cavazzana-Calvo M. Gene therapy for primary immunodeficiencies: Part 2. Current opinion in immunology. 2012 Oct;24(5): 585-91. PubMed PMID: 22909900.

[157] Aiuti A, Biasco L, Scaramuzza S, Ferrua F, Cicalese MP, Baricordi C, et al. Lentiviral hematopoietic stem cell gene therapy in patients with Wiskott-Aldrich syndrome. Science. 2013 Aug 23;341(6148):1233151. PubMed PMID: 23845947.

[158] Cartier N, Hacein-Bey-Abina S, Bartholomae CC, Veres G, Schmidt M, Kutschera I, et al. Hematopoietic stem cell gene therapy with a lentiviral vector in X-linked adrenoleukodystrophy. Science. 2009 Nov 6;326(5954):818-23. PubMed PMID: 19892975. 
[159] Biffi A, Montini E, Lorioli L, Cesani M, Fumagalli F, Plati T, et al. Lentiviral hematopoietic stem cell gene therapy benefits metachromatic leukodystrophy. Science. 2013 Aug 23;341(6148):1233158. PubMed PMID: 23845948.

[160] Grupp SA, Kalos M, Barrett D, Aplenc R, Porter DL, Rheingold SR, et al. Chimeric antigen receptor-modified $\mathrm{T}$ cells for acute lymphoid leukemia. The New England journal of medicine. 2013 Apr 18;368(16):1509-18. PubMed PMID: 23527958.

[161] Budde LE, Zhang MM, Shustov AR, Pagel JM, Gooley TA, Oliveira GR, et al. A phase I study of pulse high-dose vorinostat (V) plus rituximab (R), ifosphamide, carboplatin, and etoposide (ICE) in patients with relapsed lymphoma. British journal of haematology. 2013 Apr;161(2):183-91. PubMed PMID: 23356514. PubMed Central PMCID: 3618618.

[162] Budde LE, Berger C, Lin Y, Wang J, Lin X, Frayo SE, et al. Combining a CD20 chimeric antigen receptor and an inducible caspase 9 suicide switch to improve the efficacy and safety of $\mathrm{T}$ cell adoptive immunotherapy for lymphoma. PloS one. 2013;8(12):e82742. PubMed PMID: 24358223. PubMed Central PMCID: 3866194.

[163] Farinelli G, Capo V, Scaramuzza S, Aiuti A. Lentiviral vectors for the treatment of primary immunodeficiencies. Journal of inherited metabolic disease. 2014 Mar 12. PubMed PMID: 24619149.

[164] Drumm ML, Pope HA, Cliff WH, Rommens JM, Marvin SA, Tsui LC, et al. Correction of the cystic fibrosis defect in vitro by retrovirus-mediated gene transfer. Cell. 1990 Sep 21;62(6):1227-33. PubMed PMID: 1698126.

[165] Rich DP, Anderson MP, Gregory RJ, Cheng SH, Paul S, Jefferson DM, et al. Expression of cystic fibrosis transmembrane conductance regulator corrects defective chloride channel regulation in cystic fibrosis airway epithelial cells. Nature. 1990 Sep 27;347(6291):358-63. PubMed PMID: 1699126. 

Chapter 15

\title{
In vivo Gene Correction of Cystic Fibrosis
}

\author{
Michael Sebastian Daniel Kormann \\ Additional information is available at the end of the chapter
}

\begin{abstract}
The cystic fibrosis transmembrane conductance regulator (CFTR) gene was cloned over two decades ago and a vast number of pre-clinical and clinical studies have been performed since that time. Despite this progress, a true "cure" for the disease has not been achieved, partly because the lung is a major barrier for intruders, making it exceedingly difficult for new pharmaceutical formulations to penetrate target cells. Safety-engineered viral vectors, such as adeno-associated viral vectors (AAVs) or integrase-defective lentiviruses, have been used with moderate success in temporarily supplementing the expression of critical proteins. However, stability and safety concerns often dampen the effects of these approaches. With emerging technologies, such as modified messenger (mRNA) and new genome editing strategies, scientists are now exploring the possibility of not only supplementing defective proteins, but instead, correcting the genetic defects at their source. This chapter will highlight the theoretical possibilities and primary data in pre-clinical models supporting the efforts toward in vivo gene correction of cystic fibrosis (CF).
\end{abstract}

Keywords: Cystic fibrosis (CF), gene correction, messenger RNA (mRNA), zinc finger nuclease (ZFN), transcription activator-like effector nuclease (TALEN), clustered regularly interspaced short palindromic repeats (CRISPR), CRISPR/Cas9, dimeric CRISPR RNA-guided FokI

\section{Introduction}

Cystic fibrosis (CF) is the most prevalent life-shortening autosomal recessive disorder in Caucasian populations [1]. Occurring in 1 out of every 3,500 newborns in the United States, 
and 1 out of every 2,000-3,000 in the European Union, CF affects more than 70,000 individuals worldwide [2]. Chronic lung disease is the major factor contributing to morbidity and mortality among CF patients, as abnormal airway secretions and chronic endobronchial infection lead to progressive airway obstruction. In addition to the respiratory tract, the disease may also affect the pancreas, liver, kidneys, intestine, and reproductive system [3].

Disease severity varies greatly among those with $\mathrm{CF}$, depending largely upon the degree to which the lungs are affected. However, eventual deterioration of the lungs leading to airway obstruction and death is inevitable, and for many years the average CF patient was not expected to reach adulthood [2]. Over the course of the past three decades, advancements in modern medicine have allowed physicians to postpone debilitating changes to the lungs, slowing the progression of disease and allowing many individuals with CF to live well into their 50 s or 60 s. Despite these advances in current therapy, the median age of survival remains only 33.4 years [2], emphasizing the need for novel therapeutic approaches to further improve patient outcomes in CF.

\section{The pathophysiology of cystic fibrosis}

Cystic fibrosis is the direct result of a mutation in both alleles of CFTR. This gene is responsible for encoding the CFTR protein, a chloride ion channel anchored in the plasma membrane of lung cells, pancreatic cells, sweat and other exocrine glands. Functionally, CFTR is important for the production and movement of sweat, digestive fluids, and mucus across the membrane, where mutations in the encoding gene may result in impaired anion secretion and hyperabsorption of sodium across epithelia [4-6].

Over 1500 different mutations have been described in the CFTR gene, each leading to different defects in the CFTR protein itself [7]. In the most common mutation, the deletion of phenylalanine $(\mathrm{F})$ from position $508(\Delta \mathrm{F} 508)$, improper protein folding results in the degradation of CFTR by the cell, which limits the amount of CFTR that reaches the epithelial cell surface. $\triangle$ F508-CFTR accounts for approximately $70 \%$ of CF cases worldwide and $90 \%$ of those occurring in the United States [7].

Alternative mutations in CFTR may result in truncation of the protein via premature stop codons, prevention of proper processing, folding, or trafficking to the plasma membrane, or interference with the chloride channel's ion transport ability, leading to poor gating or conductance [8]. A patient's specific CFTR gene mutation often dictates the severity of his or her disease, as well as the availability of drugs designed to target their particular protein defect.

In addition to mutations within CFTR itself, polymorphisms in other genes may also modify disease severity in patients with CF $[9,10]$. For instance, genetic variation in the gene encoding transforming growth factor $\beta 1$ (TGF $\beta 1$ ) has been associated with more severe pulmonary phenotypes predictive of poorer long-term outcomes [9]. Polymorphisms in the histone-deacetylase-dependent transcriptional co-regulator, IFRD1, have also been shown to modulate the pathogenesis of CF lung disease through the regulation of neutrophil effector function $[10,11]$. 
Traditional management strategies for CF typically involve the use of antibiotics to treat infection as well as agents or mechanical devices to improve mucus clearance and prevent damage to the lungs. Non-CFTR ion channel agents, for instance, are small molecules designed to normalize the transport of sodium and chloride by targeting non-CFTR ion channels expressed by epithelial cells. Osmotic agents, or inhaled hypertonic solutions, have also been employed to restore airway surface liquid by drawing liquid out of the airway epithelium and into the mucus [12].

In more recent years, the characterization of CFTR mutations and genetic modifiers have provided numerous targets for the development of novel therapies aimed at treating the underlying cause, rather than symptoms, of the disease. These agents are designed to directly compensate for CFTR mutations in one of three main ways:

- Overcoming the specific functional defect in the patient's CFTR protein (Figure 1),

- Supplementing the cell with a functional copy of the CFTR protein (Figure 2), or

- Repairing the CFTR gene mutation at its source (Figure 3).

\section{Targeting functional defects in CFTR Protein: Small molecule CFTR modulators}

A number of small molecules have been designed to overcome the functional defects in CFTR protein caused by upstream gene mutations (Figure 1). These CFTR modulators can be classified into three families according to the specific functional defect that they target: premature stop codon suppressors, correctors, and potentiators [13].

Premature stop codon suppressors, otherwise known as production correctors or readthrough agents, encourage the cell to overlook any premature stop codons transcribed within the CFTR mRNA (Figure 1A). They instruct cellular ribosomes to read-through these premature termination codons as the mRNA is being translated into protein. This encourages the production of full-length CFTR. Several CFTR mutations interfere with proper protein processing, resulting in misfolded CFTR that is degraded by the cell. Correctors focus on improving the processing and transport of CFTR protein to the cell surface (Figure 1B). By ensuring that CFTR is processed and folded correctly, the protein can be trafficked to the plasma membrane where it functions. Additional CFTR mutations allow this chloride ion channel to arrive at the plasma membrane, but cause defects in its gating or conductance ability. Potentiators work on these defects, to enhance opening of the channel or increasing the flow of chloride ions (Figure 1C) [13].

Lumacaftor (VX-809, Vertex Pharmaceuticals), for instance, is a CFTR corrector that increases trafficking of $\triangle F 508-C F T R$ to the epithelial cell surface [14]. Lumacaftor has also been used in combination with ivacaftor (VX-770, Vertex Pharmaceuticals, trade name Kalydeco), a CFTR potentiator that improves the transport of chloride through CFTR channels rendered dysfunctional by G551D or R560T missense mutations. By binding to the channels and inducing 
A

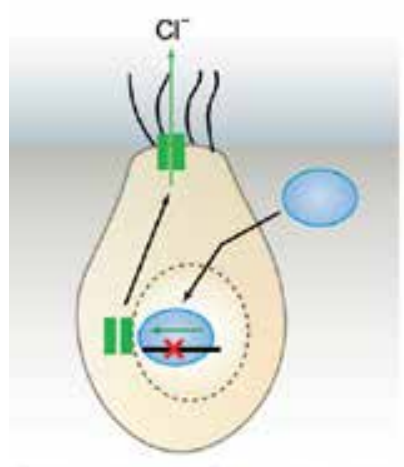

Premature stop codon suppressors help read-through of premature stop-codons that would normally lead to truncated and degraded CFTR protein
B

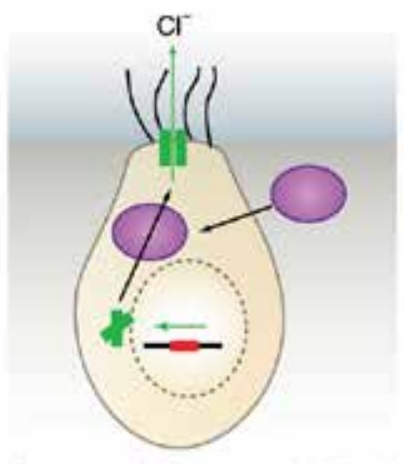

Correctors facilitate proper folding of CFIR and its location to the cell surface; the CFTR would otherwise be degraded.
C

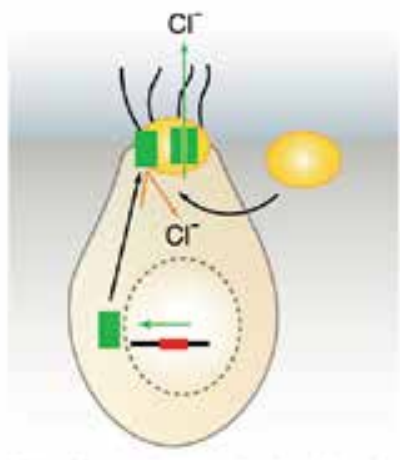

Potentiators improve the function of CFIR. That is possible when defective CFIR reaches the cell surtace but does not function properly.

Figure 1. Small molecule CFTR modulators target functional defects within the CFTR protein. Three types of CFTR modulators have been developed: premature stop codon suppressors (A), correctors (B), and potentiators (C). These small molecules act by targeting the transcription, translation, protein processing, membrane trafficking, and ion transport functionality of the CFTR protein, respectively.

a non-conventional mode of gating, ivacaftor increases the probability that the chloride channel is open [14]. Another small molecule CFTR modulator, ataluren (PTC124, PTC Therapeutics, trade name Translarna in the EU), is a production corrector that makes ribosomes less sensitive to G542X or W1282X nonsense mutations [15]. Overcoming these premature stop codons allows the synthesis of full-length, functional CFTR. Together, these small molecule modulators focus on addressing the functional defects in a patient's own CFTR protein.

\section{Gene therapy: Gene supplementation and transcript supplementation therapy}

Instead of addressing the functional deficit in a patient's endogenous CFTR, another approach involves supplementing the cell with an exogenous, functional copy of the protein (Figure 2). This can occur in one of several ways: through gene, transcript, or protein replacement therapy. By delivering a functional copy of the CFTR, subsequent mRNA transcript, or protein itself, the cell may regain enough CFTR function to halt the progression of disease. Protein replacement strategies have met limited success, as the therapeutic protein is often metabolized before it can enter the target tissue. Gene and transcript therapy approaches, however, continue to 
be investigated. Unlike CFTR modulators, these approaches have the potential to be used for all CF patients, regardless of the type of mutation they carry.

A

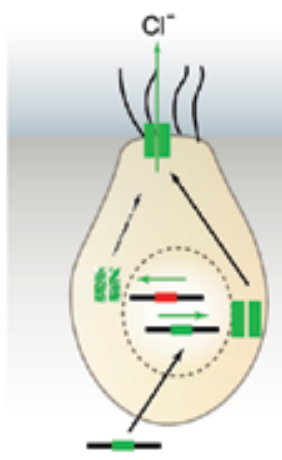

Gene Supplementation Therapy using a correct version of CFTR transfected or transduced into the respective target cells. Note that the DNA has to enter the nucleus to be transcribed, which is a major barrier in gene therapy.
B

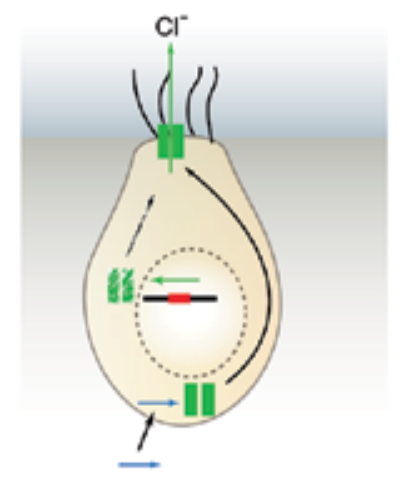

Transcript Supplementation Therapy using a correct version of CFTRmRNA transfected into the respective target cells. Note the mRNA is actively producing CFIR alreacly in the cytoplasm, thereby circumventing the nuclear membrane.
C

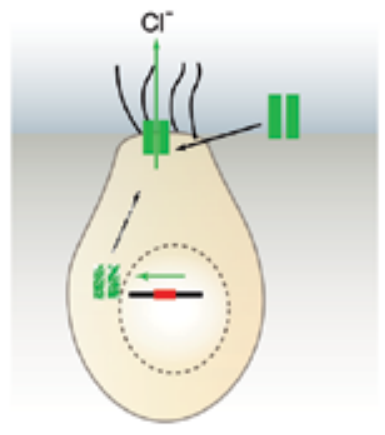

Protein supplementation therapy using a correct version of CFTR transfected or transduced into the respective target cells. Note that protein delivery is often ineflective and it is difficult to include all natural post-protein-modifications.

\section{$\longrightarrow$ DNA $\longrightarrow$ modified mRNA $\longrightarrow$ endogenous mRNA \\ correct(ed) sequence \\ defective sequence}

Figure 2. Gene, transcript, and protein replacement therapy supplement cells with a functional copy of the CFTR protein. Supplementing the cell with functional CFTR cDNA (A), mRNA transcripts (B), or CFTR protein (C) is another method of overcoming the genetic defects underlying Cystic Fibrosis.

\subsection{Gene supplementation therapy}

The two main forms of gene supplementation therapy are defined by the vehicle used to deliver functional cDNA to the cell: this consists of either viral or non-viral vectors (Figure 2A).

Non-viral vectors are typically comprised of plasmid DNA (pDNA) complexed with carrier molecules, such as cationic lipids or polymers. By binding to the negatively charged pDNA, these molecules either condense or encapsulate the DNA, forming lipoplexes or polyplexes that are then thought to be endocytosed by the cell [16]. In the absence of non-human, viral protein components, it is believed that non-viral vectors may incite minimal immune activation and increase the opportunity for repeat administration. However, even pDNA expression is often limited by $\mathrm{CpG}$ motifs that induce strong immune responses through innate immune receptors, such as Toll-like receptor 9 (TLR9) [17]. In addition, non-viral vectors are typically much less efficient than viral vectors at transfecting slowly dividing mammalian cells. This is due to the fact that viruses have evolved efficient strategies for improving cell entry, endosomal escape, cytoplasmic trafficking, and nuclear uptake, all of which make them naturally skilled vehicles for delivering therapeutic cDNA to the cell nucleus [16]. 
Viral vectors have been designed to harness these evolutionary advantages, while removing components of the viral genome that may cause harm. The ideal viral vector should be replication defective, non-immunogenic, and avoid integrating into actively transcribed genes. Random integration events into an oncogene or tumor suppressor may cause insertional mutagenesis leading to cell death or cancer.

Adenoviral vectors (Ad) engineered to be devoid of the viral genome were the first to be utilized for CF gene supplementation therapy. These vectors have the advantage of being nonintegrating, with a natural tropism for the lung. In clinical trials using Ad-CFTR, low levels of gene transfer and partial correction of chloride transport in nasal epithelium were observed in some patients [16]. However, issues such as dose-dependent lung inflammation and humoral and cellular immune responses preventing repeat administration remained limiting factors.

Adeno-associated viral vectors (AAV) also remain largely episomal inside the nucleus, minimizing the threat of insertional mutagenesis. Over 130 serotypes of AAV have been identified, with each viral capsid demonstrating its own unique transduction profile [18]. Capsids from AAV1, 5, 6, 8, and 9 may be the most efficient for transducing cells of the airway epithelium [19]. In addition, the creation of hybrid AAV capsids, such as AAV6.2, may allow the customization of vectors optimized for transducing the desired target cell. Early phase I trials with AAV2.CFTR showed limited efficacy, due in part to the use of a non-lung-tropic AAV2 serotype, limited packaging space for an optimal promoter (CFTR cDNA uses $4.7 \mathrm{~kb}$ of the vector's $\sim 5 \mathrm{~kb}$ packaging capacity), as well as AAV capsid-specific immune responses limiting repeat administration [16]. Strategies aimed at minimizing adaptive immunity to AAV vectors or reducing the need for repeat administration continue within the field. Removing CPG motifs from AAV vectors or designing hybrid AAV capsids has been shown to reduce innate and adaptive immune responses following intramuscular delivery [20,21]. Targeting AAV delivery to progenitor cells in mouse lung also shows promise as a means of avoiding lung cell turnover and circumventing the need for redelivery [22].

Lentiviral vectors based on recombinant human (HIV), simian (SIV), feline (FIV), and equine (EIV) immunodeficiency viruses have also been investigated for gene replacement therapy [16]. Lentiviral vectors are pseudotyped with the envelope proteins from various viruses to increase tissue tropism. The vesicular stomatitis virus G (VSV-G) envelope glycoprotein has most commonly been incorporated, although the $\mathrm{F}$ and $\mathrm{HN}$ proteins from murine parainfluenza virus type 1 , or Sendai virus $(\mathrm{SeV})$, may improve airway transduction. Studies with $\mathrm{SeV}$-pseudotyped lentiviral vectors have accommodated repeat administration to murine airways in pre-clinical studies [23]. Should repeat administration also be feasible in human subjects, the two major remaining limitations to lentiviral use include safety concerns over genomic integration and scale-up of vector production. The concern over vector integration came to the forefront in 2003, when the integration of a retroviral vector used to treat X-linked severe combined immunodeficiency (X-SCID) triggered unexpected activation of a protooncogene leading to leukemia in nearly half of the trial's participants [24,25]. 


\subsection{Transcript supplementation therapy}

In recent years, transcript supplementation therapy has been introduced as an alternative to gene replacement therapy (Figure 2B). As mRNA transcripts are not capable of integrating into the chromosome, the threat of insertional mutagenesis is completely void. Following uptake via receptor-mediated endocytosis and lysosomal trafficking, mRNA also completely avoids the rate-limiting step of nuclear entry, being translated rapidly and efficiently directly in the cytoplasm [26]. With its naturally short half-life, mRNA transcripts are particularly useful for applications where short bursts of protein expression are desired. However, the addition of chemical modifications mimicking endogenous mRNA modification schemes has increased expression and stability, while decreasing immune responses. One major benefit to the use of chemically modified mRNA is the ability to readminister the vector as necessary.

The use of mRNA itself has long been appealing as an alternative to gene-based delivery vehicles. Unfortunately, for many years researchers were unable to use in vitro transcribed mRNAs to upregulate protein expression in vivo, as these transcripts were immediately recognized and destroyed by the immune system following injection [27]. Recent work has shown that by completely substituting uridine with pseudouridine during mRNA synthesis, the binding affinity of mRNA to innate immune receptors can be reduced, making systemic in vivo application possible $[28,29]$. More recent work has shown that partial substitution of combinations of various nucleotide modifications, more closely mimicking those observed in endogenous transcripts, can yield mRNA transcripts with further increased stability, specifically in murine lung [30,31]. This emphasizes that the design of mRNA may have substantially different effects in specific organs in vivo compared with in vitro use.

In one recent study, transcript therapy with chemically modified surfactant protein $B(S P-B)$ mRNA exhibited success in achieving therapeutic levels of protein expression in a murine model of SP-B deficiency [30]. Repeated intratracheal administration of modified Foxp3 mRNA to murine lung was also shown to alleviate asthma symptoms in two different models of experimental asthma [31]. Both of these models demonstrate the efficacy of nucleotide modified mRNA in achieving therapeutic levels of protein expression in the lung following repeated, in vivo delivery. As a vehicle for delivery, modified mRNA may present a safer alternative to viral and non-viral DNA-based approaches, as immune activation can be efficiently prevented and the possibility of genomic integration is eliminated. Importantly, however, due to the short half-life of mRNA, the benefits of modified mRNA transcripts may be better utilized outside of direct transcript supplementation.

\section{Repairing the CFTR mutation: Gene correction with genome-editing nucleases}

With residual limitations in optimizing gene and transcript supplementation therapies for $\mathrm{CF}$ lung disease, a new field has begun to emerge: aiming to correct, rather than supplement, the defective gene. Compared to gene or transcript replacement approaches, "gene correction" 
aims to replace the defective portion of the CFTR gene with a normal allele at its natural chromosomal location (Figure 3). The repair of a mutant gene directly at its original locus has two major advantages. Most importantly, the corrected gene remains under control of its endogenous promoter, hence assuring life-long expression and native regulation in the cell. Moreover, depending on the delivery vehicle(s) used, gene correction has the potential to avoid the involvement of foreign DNA, thus reducing the risk of insertional mutagenesis.

A

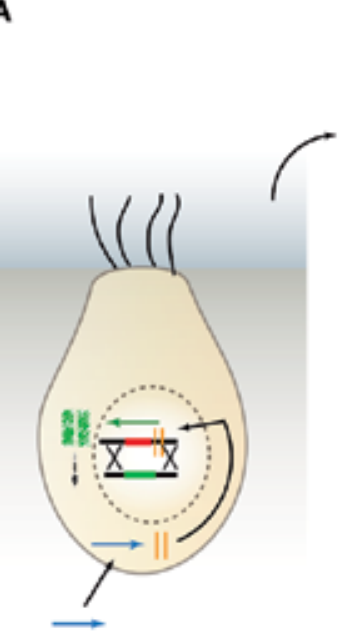

Gene Correction using nuclease encoding, chemically modified mRNA (nec-mRNA) and a donor template comprising homologous arms.

The SSEs create double strand breaks (DSBs), which stimulates homologous recombination, making it possible to exchange the defect locus with a correct locus (included in the donor vector)

Site-specific nucleases (SSEs): |

- ZFNs

- TALENs

- CRISPRICAS9

- NEXTGEN CRISPRUFokI

encoded as plasmid, viral vector or modified mRNA

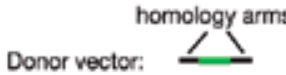

- plasmid DNA / ssDNA

- viral vector such as AAVs or lentivinuses

- containing homology arms

\section{B}

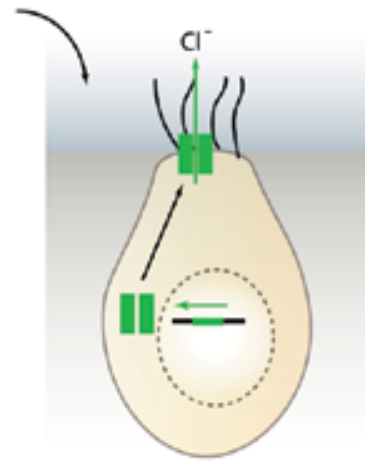

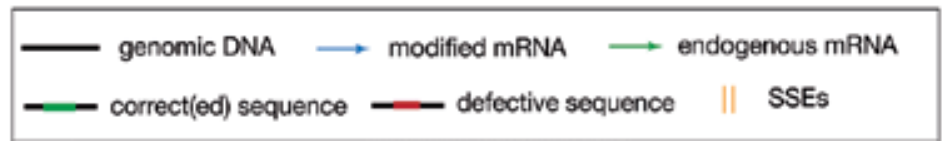

Figure 3. Gene correction approaches aim to repair the CFTR gene mutation directly at the endogenous chromosomal locus. The use of mRNA-encoded site-specific nucleases (SSE) can introduce double-strand breaks (DSB) near the genetic defect(s). If a donor vector with corrected sequence is also delivered to the cell, it can be utilized as a template for homologous repair (HR) as the cell works to repair the DSB (A). Successful homology-directed repair (HDR) will result in a corrected chromosomal locus, even after the SSE and donor template delivery vehicles are no longer expressed (B).

Several lines of investigation into viable gene correction approaches have been pursued. These involve the use of genome-editing nucleases, such as ZFNs, TALENs, or CRISPR-based systems, to take advantage of the cell's natural damage repair pathways. In this strategy, delivery of a site-specific-endonuclease (SSE) or SSE pair elicits a double-strand break (DSB) in the defective gene near the site of an unwanted mutation or sequence, initiating cellular repair mechanisms including homologous recombination (HR) and non-homologous endjoining (NHEJ).

NHEJ, an error-prone process, can be utilized to initiate mutations that essentially disrupt or knock out an undesirable gene. As NHEJ repairs the DSB by ligating the broken strands together, this process commonly results in small insertions or deletions of base pairs, known as indels. The generation of indels at the repair site can cause frame-shift mutations that prevent the protein from being properly transcribed and translated. This concept was dem- 
onstrated in 2008, when investigators utilized ZFNs and NHEJ to disrupt the HIV co-receptor, CCR5, rendering human $\mathrm{CD} 4^{+}$T cells more difficult for the HIV virus to transfect [32]. Through this mechanism, multiple nuclease pairs can also be utilized to create two DSBs, where NHEJ may completely cut out large segments of unwanted genomic sequence [33,34].

Alternatively, a donor template can be delivered to the cell in addition to the nuclease(s), and used as a guide for directing HR, in a process referred to as homology-directed repair (HDR). In HDR, an extra-chromosomal donor fragment or "repair template" contains regions of significant homology up- and downstream of the DSB site. In between the homology arms, the repair template houses the desired, corrected sequence. Once the nuclease has cleaved, the regions of homology will be used as a template for rebuilding the site. As a result, the mutationfree sequence housed between homology arms is incorporated into the chromosome (Figure 3).

In cases where the patient is homozygous for the target allele, the uncleaved copy of the allele may be favored as a template for HR, decreasing efficiency. As such, it is important to provide the repair template in excess, to ensure that the target cell favors the repair template over the sister chromatid. It is also important to note that even when a repair template is provided in excess for HR, SSE binding and cleavage can also occur at off-target sites, which may initiate NHEJ. In the case of an off-target cutting event, NHEJ can cause unexpected mutations that may beharmful to the cell. As a result, potential off-target binding sites of theSSE should be predicted in silico and subsequently sequenced to monitor for deleterious off-target effects. Overall, HDR can be utilized to correct individual point mutations, as well as to insert larger fragments, such as complete copies of functional cDNA, into desired sites. To date, gene correction has been achieved in vitro, ex vivo, as well as directly in murine liver and lung [35-37].

Each of the available nuclease technologies utilizes a different method for recognizing specific sites and initiating DSB cleavage. In the following section, we will discuss the various mechanisms of action, as well as the pros and cons of each technology. It is important to note that in order to be safe for use in a clinical setting, nuclease technology must meet the following criteria:

- Minimal off-target activity:

- High binding specificity

- Transient nuclease expression

- High cleavage efficiency

- Delivery to the cell using vectors that minimize insertional mutagenesis risk

\subsection{Zinc finger nucleases}

Zinc fingers are a common DNA-binding protein that can be found in nearly half of all transcription factors in the human genome [38]. These naturally abundant proteins can be reengineered to recognize and bind specific target sequences. ZFN technology takes advantage of this by attaching a DNA-cleaving nuclease to the zinc finger-binding domain. The result is a site-specific binding protein that can cleave a strand of DNA at a precise location. 
ZFNs utilize the non-specific DNA cleavage domain from the FokI restriction endonuclease to confer their cleavage activity [39]. Their design traditionally incorporates the wild-type FokI cleavage domain; however, more recent studies have utilized variants with improved cleavage activity or specificity [40-42]. Functioning as a dimer, the FokI domain requires two ZFN constructs, working together as a pair: one ZFN binds to a sequence immediately upstream of the intended cut site, while the other targets sequence immediately downstream of the cut site on the complementary strand (Figure 4A). This alignment places the C-terminal nucleases at a desired distance apart across the cut site, where they dimerize and create a DSB. Proper spacing of binding and cleavage domains is critical for optimal DSB induction.

A
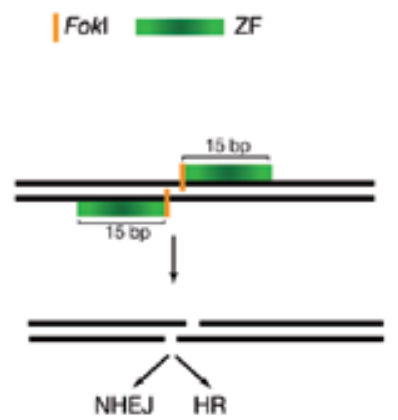

D |Fok|

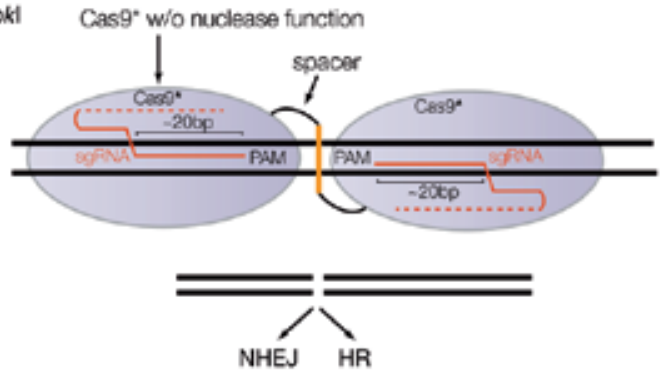

B

|Fok

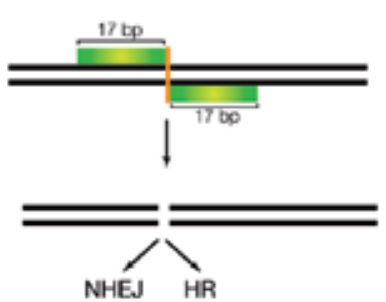

C | RuvC | HNH

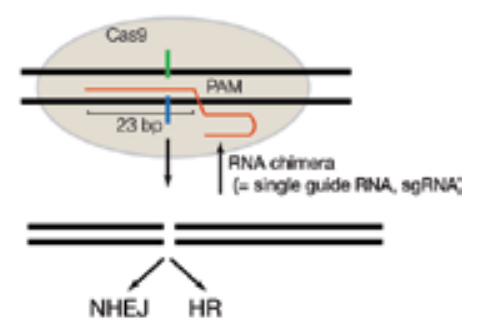

E

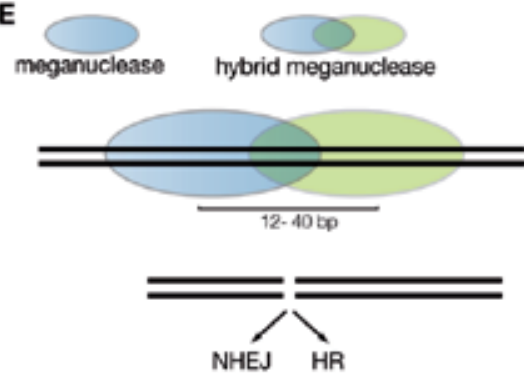

Figure 4. Genome-editing nucleases: Mechanisms of action. An overview of the mechanisms of DNA binding and cleavage used by ZFNs (A), TALENs (B), CRISPR/Cas9 (C), the dimeric CRISPR RNA-guided FokI system (D), and meganucleases (E). PAM, protospacer adjacent motif; sgRNA, single guide RNA; RuvC, nuclease domain in Cas9 that cleaves the non-target strand of DNA; HNH, nuclease domain in Cas9 that cleaves the target strand of DNA.

Numerous evidence has supported the use of ZFNs for targeted gene editing in multiple species, including mice, rats, rabbits, pigs, plants, and zebrafish [38]. The use of this platform has also extended to the manipulation of stem cell populations ex vivo. In one seminal study, ZFNs facilitated targeted disruption of CCR5, a co-receptor involved in HIV entry [32]. By introducing the CCR5- $\triangle 32$ mutation into ex vivo expanded $C D 4^{+} \mathrm{T}$ cells, followed by engraftment into HIV-1 infected mice, these target cells no longer expressed functional CCR5 entry receptors, making them more resistant to infection. Further studies utilized a dual strategy to target both of the HIV entry co-receptors, CCR5 and CXCR4 [43]. The use of ZFNs was also 
successfully demonstrated in vivo in murine liver, to correct a model of hemophilia B, or factor IX (FIX) deficiency [36].

Despite this, ZFNs are not without their limitations. First, they are relatively difficult to engineer and expensive to purchase commercially, leaving them inaccessible to the majority of investigators. Secondly, not all sequences can be targeted by ZFNs, restricting their use in certain applications. And lastly, the specificity of ZFN pairs is not $100 \%$, resulting in the potential for off-target cleavage events and related damage to occur. In the event of low specificity, off-target DSB induction may overwhelm cellular repair machinery leading to chromosomal rearrangements and/or cell death. These instances may also support random integration of donor DNA into undesirable locations, which has the potential to interfere with tumor suppressors, proto-oncogenes, or other actively transcribed genes [24,25].

\subsection{Transcription activator-like effector nucleases}

Transcription activator-like effector (TAL effector, or TALE) proteins are secreted by Xanthomonas bacteria upon infecting various species of plant. They function by binding to promoter sequences in the host to upregulate plant genes that are beneficial to bacterial infection. Similar to the concept of ZFNs, TALENs are built by fusing the DNA binding domain of a TAL effector to a DNA cleavage domain with nuclease activity [44-47]. The DNA binding domain consists of multiple repeats of a 33-34 amino acid sequence, where all but the 12th and 13th amino acids are highly conserved. By selecting a combination of repeat segments with the appropriate variable regions (Repeat Variable Diresidues, or RVDs), specific DNA binding domains can be engineered.

TALENs utilize the same non-specific DNA cleavage domain from the FokI endonuclease to confer cleavage activity. As a result, this strategy also requires two TALENs to work together as a pair, binding non-palindromic sequences on complementary strands of DNA. Proper positioning of the DNA binding and cleavage domains around the cute site allows the FokI endonuclease domains to dimerize and produce a site-specific DSB (Figure 4B) [48,49].

Compared with ZFNs, TALENs can cleave a broader, more comprehensive range of DNA sequences. In addition, they tend to be more accurate, reducing the potential for off-target cleavage events. Furthermore, as a result of the fast ligation-based automatable solid-phase high-throughput (FLASH) system reported in 2012, large-scale assembly of TALENs has also become a more efficient and cost-effective alternative [50].

\subsection{CRISPR/Cas9}

In 2012, the use of a novel genome-editing tool was described in human cell culture [51]. In bacteria and archaea, clustered regularly interspaced short palindromic repeats (CRISPR) work together with Cas genes to form a prokaryotic adaptive immune system that protects against foreign genetic elements such as plasmids or phages. Upon detecting viral DNA, for instance, this system converts segments of the foreign DNA into CRISPR RNAs (crRNA); the crRNA then combines with a trans-activating crRNA (tracrRNA). The crRNA-tracrRNA 
combination then guides a Cas9 DNA nuclease to a specific location within the viral DNA, called the protospacer, where a DSB is induced.

Investigators discovered that by designing a new crRNA and combining it with the tracrRNA, a "single-guide RNA" (sgRNA) could be produced that would direct the Cas9 nuclease activity to any desired location. Studies have shown that delivery of two components, the Cas 9 nuclease and a corresponding sgRNA (containing both the crRNA and tracrRNA), were sufficient to elicit cleavage in a desired gene [52-55]. Hence, by retargeting the crRNA portion of the sgRNA, a site-specific genome-editing tool could be developed (Figure 4C).

Unlike ZFN and TALEN strategies, the nuclease cleavage domain in the CRISPR/Cas9 system is not fused to the DNA binding domain: instead, these are delivered to the cell in two separate components (Figure 4C). As a result of this design, only a single DNA binding domain has to be created. As this single protein-binding domain is significantly shorter than those required for TALEN or ZFN designs, CRISPR/Cas9 components are significantly easier and more cost effective to synthesize, making this technology more widely available to the research community at large. Despite lower costs and greater accessibility, the functional activity of CRISPR/ Cas systems appears to be equal to or greater than their ZFN and TALEN counterparts.

As the CRISPR/Cas9 system is a relatively new genome engineering technology, it will be important for the field to thoroughly study any potential shortcomings. For instance, since a relatively short DNA binding domain and cleavage site are utilized, the risk of low specificity and potential off-target recognition may be greater [56].

\subsection{Dimeric CRISPR RNA-guided FokI nucleases}

In an effort to reduce the risk of unwanted off-target mutations associated with monomeric CRISPR/Cas9 nucleases, a modified dimeric version has now been developed [57]. Where the monomeric Cas9 nuclease is recruited by one sgRNA of only 100 nucleotides in length (with 17-20 nucleotides of complementarity to the target), dimerization offers an attractive strategy for improving the binding specificity of the Cas9 system (Figure 4D).

In this approach, a wild-type FokI nuclease domain is fused to a catalytically inactive Cas9 (dCas9) protein. Two such FokI-dCas9 fusions are recruited by two corresponding guide RNAs, where both are required to bind their respective target sites in order for FokI dimerization and DSB induction to occur (Figure 4D). An appropriately designed spacer and protospacer adjacent motif (PAM) are also critical for driving efficient cleavage. Overall, this RNA-guided FokI nuclease (RFN) strategy has been shown to elicit robust genome editing efficiencies while reducing known off-target mutations to undetectable levels [57].

\subsection{Meganucleases}

Meganucleases are another form of endonuclease utilized for genome editing approaches. They are unique in that their DNA recognition and cleavage functions are naturally combined in a single domain. There are five classes available, where I-SceI, I-CreI, and I-DmoI are perhaps the most widely used. Consisting of a large recognition site of 12 to 40 base pairs, meganu- 
cleases also offer high specificity and precision; however, historically, they were only capable of tolerating minor variations in their recognition site sequence, decreasing the probability of an available meganuclease for each desired application. In recent years, investigators have begun customizing meganucleases to expand their targeting repertoire. Two main approaches have been taken: modifying the specificity of existing meganucleases, and/or developing chimeric meganucleases with new recognition sites. In the latter approach, by fusing the DNAbinding domains of two different meganucleases, functional heterodimers can be designed for optimal efficacy and specificity (Figure 4E).

\section{The best vehicle for the job}

In genome editing, the identification of components capable of eliciting HDR is only half the battle. Efficiently delivering those components to the target cell can be an equally important hurdle to overcome. Unlike gene and transcript replacement therapies, where the goal is to achieve stable, long-term expression of a supplemental protein, gene editing has the advantage of requiring only short-term expression of the foreign components in the cell. Once these components have been expressed, induced a DSB, and triggered HDR, their presence is no longer necessary. In fact, in the attempt to minimize unwanted off-target cleavage activity and prolonged DSB induction, the ideal nuclease-delivery vehicle should only be transiently expressed.

Additionally, without the need for integration to promote stable expression, non-integrating delivery vehicles are also preferable, to minimize the risk of insertional mutagenesis. Delivery vehicles must also be capable of transducing target cells efficiently, to facilitate gene correction in enough cells to overcome the initial defect. To summarize, since transient expression of the nuclease is sufficient for stable modification of the genome, the ideal nuclease delivery vehicle should be:

- Short-lived,

- Non-integrating, and

- Able to enter target cells efficiently.

A variety of vectors have been utilized to deliver genome-editing reagents to the cell. Initial in vivo studies have included AAV viral vectors as well as integrase-defective lentiviruses. Using AAV-encoded ZFNs, for instance, Li and colleagues demonstrated direct in vivo gene correction using HDR in a murine model of FIX deficiency [36]. Despite these successes, early in vivo strategies have not fulfilled two of the critical components for nuclease delivery vehicles: transience and lack of potential integration. In addition, with respect to translation of these approaches to CF airway disease, none of these strategies reported targeting cells in the lung.

Due to its short half-life and inability to integrate into the genome, modified mRNA is gaining interest as an ideal vector for site-specific nuclease delivery: one that would address two of the main outstanding issues previously discussed. Transient expression of mRNA-encoded 
nucleases would also minimize the long-term threat of off-target events associated with the use of stably expressing, and possibly integrating viral vectors.

\section{Gene correction in the lung}

In a recent study, nuclease-encoding chemically modified mRNA (nec-mRNA) was described as a novel vehicle for delivering genome-editing components directly to the lung [37]. Using a murine model of SP-B deficiency, nec-mRNA-encoded ZFNs were able to demonstrate the first report of life-prolonging gene correction specifically within lung tissue.

In the transgenic mouse model of SP-B deficiency, SP-B cDNA is under the control of a Tetracycline-inducible promoter [58]. Administration of doxycycline allows SP-B to be expressed at wild-type levels. If doxycycline is removed, SP-B expression drops and mice begin to exhibit phenotypic changes similar to those seen in humans with the disease: thickened alveolar walls, heavy cellular infiltration, increased macrophages and neutrophils, interstitial edema, congestion, augmented cytokines in the lavage, a significant drop in lung function, and acute onset of respiratory distress leading to death within days $[59,60]$.

In order to demonstrate the value of nec-mRNA for lung-based genome-editing applications, this report utilized HDR to insert a constitutive CAG promoter immediately upstream of the SP-B cDNA. The resulting doxycycline-independent expression was able to significantly prolong the life of treated mice [37].

While this study was not performed in a humanized mouse model, the approach was able to demonstrate that extra-chromosomal nec-mRNA is capable of transducing airway epithelial cells, expressing genome-editing reagents, and achieving HDR rates sufficient for therapeutic levels of protein expression. Main findings from the study include:

i. mRNA modification schemes can be customized to optimize expression and minimize immunity.

ii. Intratracheal delivery of nec-mRNA is able to target airway epithelial cells.

iii. Complexing nec-mRNA to chitosan-coated nanoparticles can increase transduction efficiency in the lung.

iv. nec-mRNA expression is transient in comparison to AAV-encoded ZFNs.

v. nec-mRNA-mediated ZFN delivery can facilitate HDR rates comparable to AAVencoded ZFNs.

\section{Hurdles to success: Limitations to gene correction for cystic fibrosis}

The lung has evolved with natural defense mechanisms against foreign pathogens. As such, a number of intracellular and extracellular barriers must be overcome in order to target new 
technologies to the lung [61]. In addition to this, the lungs of CF patients are even more difficult to target, owing to the increased airway mucus (sputum) lining the lungs. Especially in more progressed CF patients, thickened mucus linings have proven prohibitive in several gene replacement therapy approaches. Hida and colleagues reported that expectorated sputum from CF patients effectively traps and slows the diffusion of both Ad and AAV viral vectors [62]. For instance, where the sputum penetration of muco-inert nanoparticles is reduced by only 40-fold compared with pure water, that of Ad and AAV particles is slowed by 3,000-fold and 12,000-fold, respectively. Poor penetration of mucus layers may be a major component preventing effective viral gene replacement therapy or the use of viral vectors to deliver genome-editing components for $\mathrm{CF}$.

Limits to stable expression of functional CFTR also play an important role. For gene replacement therapy approaches, this may include immune responses against the vector capsid, the inability to re-administer, as well as turnover of CFTR-expressing lung cells. For gene correction approaches, transient expression of nucleases does not destabilize the downstream effects of HDR. However, lung cell turnover continues to be an issue, making the possibility of readministration important.

The levels of CFTR expression required to halt the progression of CF lung disease remain largely unknown, as well as the cell types most suited as targets. While airway epithelial cells are generally considered to be the ideal target, airway histology and entry receptor expression patterns may impact the ability of this cell type to be transduced. The absence of adenoviral entry receptors on the apical surface of airway epithelium, for instance, is one of the major reasons that adenoviral gene therapy vectors are no longer pursued. Furthermore, turnover of these terminally differentiated cells will eventually require therapeutics to be redelivered to new target cells.

In addition to the most relevant cell type, another question remains: is it preferable to obtain low levels of CFTR in a high percentage of cells, or high levels of CFTR in only 10\% of cells? Gene replacement and correction approaches are more likely to attain the latter, although it remains unknown whether this will be sufficient to show therapeutic effect. One recent study has shown that restoration of normal mucus transport rates in cultured CF human airway epithelial cells required at least $25 \%$ of surface epithelial cells to be targeted by CFTR gene replacement therapy [63]. Whether this figure will translate to clinical benefit in an in vivo setting remains to be seen.

\section{Supplemental strategies and future directions}

Due to the limitations of targeting the lung, it remains unclear whether novel replacement and correction approaches will find success for Cystic Fibrosis lung disease. Efforts to overcome these barriers remain the subject of further investigation.

As one recent study demonstrated, combining nec-mRNA with chitosan-coated poly(lactideco-glycolide) nanoparticles may be one viable method for overcoming the CF sputum barrier to lung cell targeting $[37,64]$. Recent progress in the development of mucus penetrating 
nanoparticles (MPP) may provide an opportunity to further overcome this barrier [65]. Drugloaded MPPs with non-adhesive coatings have been shown to penetrate mucus layers at rates nearly as fast as pure water. These developments may allow the penetration of delivery vehicles to airway epithelium, without reducing the protective function of the mucus itself. Furthermore, adjuvant regimens of N-acetylcysteine (NAC) with or without recombinant human DNase (rhDNase) were used to increase diffusivity of nanocomplexed, non-viral gene delivery vectors through sputum layers [66]. This strategy was able to increase gene expression by $~ 12$-fold, making it another potential avenue for improving targeting in the lungs of $\mathrm{CF}$ patients. Complexes of pDNA or mRNA with GL67:DOPE:DMPE-PEG5000 (GL67) liposomes have also been described as a potential avenue for augmenting non-viral respiratory gene transfer [67]. Overall, developments in nanoparticle technology combined with advancements in aerosol-delivery devices may hold promise for the field.

Route of administration may be an important consideration as well, especially given the tendency for inhaled therapeutics to be entrapped in the mucus layer. Intratracheal highpressure spraying approaches have been effective in targeting airway epithelial cells in preclinical models [30,31,37], although efficiency is likely to decrease in the face of CF sputum. Preliminary evidence supports the claim that intravenous routes of administration may also target airway cells efficiently, while avoiding the barriers to a direct airway approach.

The continued development of humanized animal models of $\mathrm{CF}$, including mouse, pig, and ferret models, will further our ability to investigate novel therapeutic strategies [68,69]. An early mouse model, CFTR ${ }^{\text {tmluNC }}$, knocked out murine CFTR through a stop codon in exon 10; however, these mice showed a drastic drop in survival rates due to severe intestinal obstruction $[70,71]$. To overcome lethal intestinal defects, the mice were then 'gut-corrected' with a human CFTR construct driven by an intestinal-specific FABP promoter [72]. Studies in the FABPhCFTR/Cftr ${ }^{\text {tmiluc }}$ gut-corrected model have demonstrated that the human CFTR protein is indeed functional in mice. Using this or other models as a foundation, it may be possible to introduce a transgenic construct containing a mutated human CFTR driven by a lung-specific promoter. Creating humanized mice expressing the CFTR- $\triangle F 508$ mutation, for instance, may offer an excellent tool for studying gene correction using nucleases and repair templates designed for direct translation to the clinic.

In addition to the development of novel animal models, the identification of human lung stem cell populations has offered new hope for overcoming the issue of lung cell turnover [73]. If genome-editing vehicles could be efficiently targeted to lung stem cell populations, such as bronchioalveolar stem cells (BASCs) [74], Clara cells [75], or alveolar type II (ATII) progenitors [76], HDR in these self-renewing populations could support indefinite CFTR production.

Engineering strategies to minimize the risk of off-target cleavage and donor integration will also continue to be an important area of development. Along these lines, it will be critical to more thoroughly define standardized parameters for measuring off-target effects. State-of-theart techniques that can be used for measuring outcome parameters will also aid in assessing overall efficacy. Combining efforts to overcome these barriers to lung targeting, cell turnover, proper animal models, and off-target effects will enable the field to make continued progress toward a novel gene correction strategy for the treatment of Cystic Fibrosis. 


\section{Conclusion}

Over two decades since the cloning of the CFTR gene, numerous strategies have been investigated to identify clinically relevant genetic variants, target cells of the airway, and overcome deleterious mutations. Rather than masking symptoms of the disease, novel therapies strive to address the underlying genetic cause of the Cystic Fibrosis phenotype. Agents have approached this goal with varying strategies, including attempts to overcome the patient's functional CFTR defects, supplement their cells with a functional copy of the protein, or directly repair genomic mutations at their source. Innovations in viral and non-viral delivery vehicles and methods for overcoming barriers to lung targeting have allowed for promising progress in recent years. Coupled with novel genome-editing reagents, such as ZFNs, TALENs, and the CRISPR/Cas9 system, the promise of a novel therapeutic approach is becoming an increasingly attainable goal within the field. Further advancement in minimizing off-target activity, increasing the efficiency of site-specific cleavage, and optimizing robust, transient, non-integrating nuclease delivery vehicles will bring us closer to achieving stable modification of the genome in the race toward in vivo gene correction of Cystic Fibrosis.

\section{Author details}

Michael Sebastian Daniel Kormann

Address all correspondence to: kormann.michael@gmail.com

University Children's Clinic Department of Pediatrics I, Pediatric Infectiology \& Immunology, Translational Genomics and Gene Therapy in Pediatrics, University of Tübingen, Tübingen, Germany

\section{References}

[1] Tobias ES, Connor M, Ferguson-Smith M. Essential Medical Genetics. John Wiley \& Sons; 2011.

[2] WHO I Genes and human disease. WHO. n.d. http://www.who.int/genomics/public/ geneticdiseases/en/index2.html (accessed February 16, 2015).

[3] O'Sullivan BP, Freedman SD. Cystic fibrosis. Lancet. 2009;373:1891-904. DOI:10.1016/ S0140-6736(09)60327-5.

[4] Stutts MJ, Canessa CM, Olsen JC, Hamrick M, Cohn JA, Rossier BC, et al. CFTR as a cAMP-dependent regulator of sodium channels. Science. 1995;269:847-50. 
[5] Mall M, Hipper A, Greger R, Kunzelmann K. Wild type but not deltaF508 CFTR inhibits $\mathrm{Na}+$ conductance when coexpressed in Xenopus oocytes. FEBS Letters. 1996;381:47-52.

[6] Matsui H, Grubb BR, Tarran R, Randell SH, Gatzy JT, Davis CW, et al. Evidence for periciliary liquid layer depletion, not abnormal ion composition, in the pathogenesis of cystic fibrosis airways disease. Cell. 1998;95:1005-15.

[7] Bobadilla JL, Macek M, Fine JP, Farrell PM. Cystic fibrosis: a worldwide analysis of CFTR mutations--correlation with incidence data and application to screening. $\mathrm{Hu}^{-}$ man Mutation. 2002;19:575-606. DOI:10.1002/humu.10041.

[8] Rowe SM, Miller S, Sorscher EJ. Cystic fibrosis. The New England Journal of Medicine. 2005;352:1992-2001. DOI:10.1056/NEJMra043184.

[9] Drumm ML, Konstan MW, Schluchter MD, Handler A, Pace R, Zou F, et al. Genetic modifiers of lung disease in cystic fibrosis. The New England Journal of Medicine. 2005;353:1443-53. DOI:10.1056/NEJMoa051469.

[10] Gu Y, Harley ITW, Henderson LB, Aronow BJ, Vietor I, Huber LA, et al. Identification of IFRD1 as a modifier gene for cystic fibrosis lung disease. Nature. 2009;458:1039-42. DOI:10.1038/nature07811.

[11] Hector A, Kormann M, Kammermeier J, Burdi S, Marcos V, Rieber N, et al. Expression and regulation of interferon-related development regulator-1 in cystic fibrosis neutrophils. American Journal of Respiratory Cell and Molecular Biology. 2013;48:71-7. DOI:10.1165/rcmb.2012-0061OC.

[12] Anderson P. Emerging therapies in cystic fibrosis. Therapeutic Advances in Respiratory Disease. 2010;4:177-85. DOI:10.1177/1753465810371107.

[13] Rogan MP, Stoltz DA, Hornick DB. Cystic fibrosis transmembrane conductance regulator intracellular processing, trafficking, and opportunities for mutation-specific treatment. Chest. 2011;139:1480-90. DOI:10.1378/chest.10-2077.

[14] Boyle MP, Bell SC, Konstan MW, McColley SA, Rowe SM, Rietschel E, et al. A CFTR corrector (lumacaftor) and a CFTR potentiator (ivacaftor) for treatment of patients with cystic fibrosis who have a phe508del CFTR mutation: a phase 2 randomised controlled trial. The Lancet Respiratory Medicine. 2014;2:527-38. DOI:10.1016/ S2213-2600(14)70132-8.

[15] Kerem E, Konstan MW, De Boeck K, Accurso FJ, Sermet-Gaudelus I, Wilschanski M, et al. Ataluren for the treatment of nonsense-mutation cystic fibrosis: a randomised, double-blind, placebo-controlled phase 3 trial. The Lancet Respiratory Medicine. 2014;2:539-47. DOI:10.1016/S2213-2600(14)70100-6.

[16] Griesenbach U, Alton EWFW. Moving forward: cystic fibrosis gene therapy. Human Molecular Genetics. 2013;22:R52-8. DOI:10.1093/hmg/ddt372. 
[17] Zhou R, Norton JE, Zhang N, Dean DA. Electroporation-mediated transfer of plasmids to the lung results in reduced TLR9 signaling and inflammation. Gene Therapy. 2007;14:775-80. DOI:10.1038/sj.gt.3302936.

[18] Gao G, Vandenberghe LH, Wilson JM. New recombinant serotypes of AAV vectors. Current Gene Therapy. 2005;5:285-97.

[19] Limberis MP, Vandenberghe LH, Zhang L, Pickles RJ, Wilson JM. Transduction efficiencies of novel AAV vectors in mouse airway epithelium in vivo and human ciliated airway epithelium in vitro. Molecular Therapy: The Journal of the American Society of Gene Therapy. 2009;17:294-301. DOI:10.1038/mt.2008.261.

[20] Faust SM, Bell P, Cutler BJ, Ashley SN, Zhu Y, Rabinowitz JE, et al. CpG-depleted adeno-associated virus vectors evade immune detection. The Journal of Clinical Investigation. 2013;123:2994-3001. DOI:10.1172/JCI68205.

[21] Mays LE, Wang L, Tenney R, Bell P, Nam H-J, Lin J, et al. Mapping the structural determinants responsible for enhanced $\mathrm{T}$ cell activation to the immunogenic adenoassociated virus capsid from isolate rhesus 32.33. Journal of Virology. 2013;87:947385. DOI:10.1128/JVI.00596-13.

[22] Liu X, Luo M, Guo C, Yan Z, Wang Y, Lei-Butters DCM, et al. Analysis of adeno-associated virus progenitor cell transduction in mouse lung. Molecular Therapy: The Journal of the American Society of Gene Therapy. 2009;17:285-93. DOI:10.1038/mt. 2008.248.

[23] Mitomo K, Griesenbach U, Inoue M, Somerton L, Meng C, Akiba E, et al. Toward gene therapy for cystic fibrosis using a lentivirus pseudotyped with Sendai virus envelopes. Molecular Therapy: The Journal of the American Society of Gene Therapy. 2010;18:1173-82. DOI:10.1038/mt.2010.13.

[24] Hacein-Bey-Abina S, von Kalle C, Schmidt M, Le Deist F, Wulffraat N, McIntyre E, et al. A serious adverse event after successful gene therapy for X-linked severe combined immunodeficiency. The New England Journal of Medicine. 2003;348:255-6. DOI:10.1056/NEJM200301163480314.

[25] Hacein-Bey-Abina S, Von Kalle C, Schmidt M, McCormack MP, Wulffraat N, Leboulch $\mathrm{P}$, et al. LMO2-associated clonal $\mathrm{T}$ cell proliferation in two patients after gene therapy for SCID-X1. Science. 2003;302:415-9. DOI:10.1126/science.1088547.

[26] Lorenz C, Fotin-Mleczek M, Roth G, Becker C, Dam TC, Verdurmen WPR, et al. Protein expression from exogenous mRNA: uptake by receptor-mediated endocytosis and trafficking via the lysosomal pathway. RNA Biology. 2011;8:627-36. DOI: 10.4161/rna.8.4.15394.

[27] Kariko K, Muramatsu H, Ludwig J, Weissman D. Generating the optimal mRNA for therapy: HPLC purification eliminates immune activation and improves translation 
of nucleoside-modified, protein-encoding mRNA. Nucleic Acids Research. 2011;39:e142. DOI:10.1093/nar/gkr695.

[28] Kariko K, Muramatsu H, Welsh FA, Ludwig J, Kato H, Akira S, et al. Incorporation of pseudouridine into mRNA yields superior nonimmunogenic vector with increased translational capacity and biological stability. Molecular Therapy. 2008;16:1833-40. DOI:10.1038/mt.2008.200.

[29] Kariko K, Muramatsu H, Keller JM, Weissman D. Increased erythropoiesis in mice injected with submicrogram quantities of pseudouridine-containing mRNA encoding erythropoietin. Molecular Therapy. 2012;20:948-53. DOI:10.1038/mt.2012.7.

[30] Kormann MS, Hasenpusch G, Aneja MK, Nica G, Flemmer AW, Herber-Jonat S, et al. Expression of therapeutic proteins after delivery of chemically modified mRNA in mice. Nature Biotechnology. 2011;29:154-7. DOI:10.1038/nbt.1733.

[31] Mays LE, Ammon-Treiber S, Mothes B, Alkhaled M, Rottenberger J, Muller-Hermelink ES, et al. Modified Foxp3 mRNA protects against asthma through an IL-10-dependent mechanism. The Journal of Clinical Investigation. 2013;123:1216-28. DOI: 10.1172/JCI65351.

[32] Perez EE, Wang J, Miller JC, Jouvenot Y, Kim KA, Liu O, et al. Establishment of HIV-1 resistance in CD4+ T cells by genome editing using zinc-finger nucleases. Nature Biotechnology. 2008;26:808-16. DOI:10.1038/nbt1410.

[33] Mali P, Yang L, Esvelt KM, Aach J, Guell M, DiCarlo JE, et al. RNA-guided human genome engineering via Cas9. Science. 2013;339:823-6. DOI:10.1126/science.1232033.

[34] Cong L, Ran FA, Cox D, Lin S, Barretto R, Habib N, et al. Multiplex genome engineering using CRISPR/Cas systems. Science. 2013;339:819-23. DOI:10.1126/science. 1231143.

[35] Cox DBT, Platt RJ, Zhang F. Therapeutic genome editing: prospects and challenges. Nature Medicine. 2015;21:121-31. DOI:10.1038/nm.3793.

[36] Li H, Haurigot V, Doyon Y, Li T, Wong SY, Bhagwat AS, et al. In vivo genome editing restores haemostasis in a mouse model of haemophilia. Nature. 2011;475:217-21. DOI:10.1038/nature10177.

[37] Dewerth A, Mays L, Mahiny A, et al. In vivo genome editing using nuclease-encoding mRNA corrects SP-B deficiency. Nature Biotechnology. 2015. Manuscript accepted.

[38] Segal DJ. Bacteria herald a new era of gene editing. eLife. 2013;2:e00563. DOI:10.7554/ eLife.00563.

[39] Urnov FD, Rebar EJ, Holmes MC, Zhang HS, Gregory PD. Genome editing with engineered zinc finger nucleases. Nature Reviews Genetics. 2010;11:636-46. DOI: $10.1038 /$ nrg2842. 
[40] Doyon Y, Vo TD, Mendel MC, Greenberg SG, Wang J, Xia DF, et al. Enhancing zincfinger-nuclease activity with improved obligate heterodimeric architectures. Nature Methods. 2011;8:74-9. DOI:10.1038/nmeth.1539.

[41] Szczepek M, Brondani V, Büchel J, Serrano L, Segal DJ, Cathomen T. Structure-based redesign of the dimerization interface reduces the toxicity of zinc-finger nucleases. Nature Biotechnology. 2007;25:786-93. DOI:10.1038/nbt1317.

[42] Guo J, Gaj T, Barbas CF. Directed evolution of an enhanced and highly efficient FokI cleavage domain for zinc finger nucleases. Journal of Molecular Biology. 2010;400:96107. DOI:10.1016/j.jmb.2010.04.060.

[43] Didigu CA, Wilen CB, Wang J, Duong J, Secreto AJ, Danet-Desnoyers GA, et al. Simultaneous zinc-finger nuclease editing of the HIV coreceptors ccr5 and cxcr4 protects CD4+ T cells from HIV-1 infection. Blood. 2014;123:61-9. DOI:10.1182/ blood-2013-08-521229.

[44] Rusk N. TALEs for the masses. Nature Methods. 2011;8:197.

[45] Miller JC, Tan S, Qiao G, Barlow KA, Wang J, Xia DF, et al. A TALE nuclease architecture for efficient genome editing. Nature Biotechnology. 2011;29:143-8. DOI: 10.1038/nbt.1755.

[46] Boch J. TALEs of genome targeting. Nature Biotechnology. 2011;29:135-6. DOI: 10.1038/nbt.1767.

[47] Zhang F, Cong L, Lodato S, Kosuri S, Church GM, Arlotta P. Efficient construction of sequence-specific TAL effectors for modulating mammalian transcription. Nature Biotechnology. 2011;29:149-53. DOI:10.1038/nbt.1775.

[48] Mussolino C, Cathomen T. TALE nucleases: tailored genome engineering made easy. Current Opinion in Biotechnology. 2012;23:644-50. DOI:10.1016/j.copbio.2012.01.013.

[49] Bedell VM, Wang Y, Campbell JM, Poshusta TL, Starker CG, Krug RG, et al. In vivo genome editing using a high-efficiency TALEN system. Nature. 2012;491:114-8. DOI: 10.1038/nature11537.

[50] Reyon D, Tsai SQ, Khayter C, Foden JA, Sander JD, Joung JK. FLASH assembly of TALENs for high-throughput genome editing. Nature Biotechnology. 2012;30:460-5. DOI:10.1038/nbt.2170.

[51] Jinek M, Chylinski K, Fonfara I, Hauer M, Doudna JA, Charpentier E. A programmable dual-RNA-guided DNA endonuclease in adaptive bacterial immunity. Science (New York, NY). 2012;337:816-21. DOI:10.1126/science.1225829.

[52] Jinek M, East A, Cheng A, Lin S, Ma E, Doudna J. RNA-programmed genome editing in human cells. eLife. 2013;2:e00471. DOI:10.7554/eLife.00471. 
[53] Cho SW, Kim S, Kim JM, Kim J-S. Targeted genome engineering in human cells with the Cas9 RNA-guided endonuclease. Nature Biotechnology. 2013;31:230-2. DOI: 10.1038/nbt.2507.

[54] Hwang WY, Fu Y, Reyon D, Maeder ML, Tsai SQ, Sander JD, et al. Efficient genome editing in zebrafish using a CRISPR-Cas system. Nature Biotechnology. 2013;31:2279. DOI:10.1038/nbt.2501.

[55] Barrangou R. RNA-mediated programmable DNA cleavage. Nature Biotechnology. 2012;30:836-8. DOI:10.1038/nbt.2357.

[56] Lin Y, Cradick TJ, Brown MT, Deshmukh H, Ranjan P, Sarode N, et al. CRISPR/Cas9 systems have off-target activity with insertions or deletions between target DNA and guide RNA sequences. Nucleic Acids Research. 2014;42:7473-85. DOI:10.1093/nar/ gku402.

[57] Tsai SQ, Wyvekens N, Khayter C, Foden JA, Thapar V, Reyon D, et al. Dimeric CRISPR RNA-guided FokI nucleases for highly specific genome editing. Nature Biotechnology. 2014;32:569-76. DOI:10.1038/nbt.2908.

[58] Schultze N, Burki Y, Lang Y, Certa U, Bluethmann H. Efficient control of gene expression by single step integration of the tetracycline system in transgenic mice. Nature Biotechnology. 1996;14:499-503. DOI:10.1038/nbt0496-499.

[59] Nogee LM. Alterations in SP-B and SP-C expression in neonatal lung disease. The Annual Review of Physiology. 2004;66:601-23. DOI:10.1146/annurev.physiol. 66.032102.134711.

[60] Clark JC, Wert SE, Bachurski CJ, Stahlman MT, Stripp BR, Weaver TE, et al. Targeted disruption of the surfactant protein B gene disrupts surfactant homeostasis, causing respiratory failure in newborn mice. Proceedings of the National Academy of Sciences of the United States of America. 1995;92:7794-8.

[61] Ruge CA, Kirch J, Lehr C-M. Pulmonary drug delivery: from generating aerosols to overcoming biological barriers-therapeutic possibilities and technological challenges. The Lancet Respiratory Medicine. 2013;1:402-13. DOI:10.1016/S2213-2600(13)70072-9.

[62] Hida K, Lai SK, Suk JS, Won SY, Boyle MP, Hanes J. Common gene therapy viral vectors do not efficiently penetrate sputum from cystic fibrosis patients. PloS One. 2011;6:e19919. DOI:10.1371/journal.pone.0019919.

[63] Zhang L, Button B, Gabriel SE, Burkett S, Yan Y, Skiadopoulos MH, et al. CFTR delivery to $25 \%$ of surface epithelial cells restores normal rates of mucus transport to human cystic fibrosis airway epithelium. PLoS Biology. 2009;7:e1000155. DOI: 10.1371/journal.pbio.1000155.

[64] Nafee N, Taetz S, Schneider M, Schaefer UF, Lehr CM. Chitosan-coated PLGA nanoparticles for DNA/RNA delivery: effect of the formulation parameters on complexa- 
tion and transfection of antisense oligonucleotides. Nanomedicine: Nanotechnology, Biology, and Medicine. 2007;3:173-83. DOI:10.1016/j.nano.2007.03.006.

[65] Ensign LM, Schneider C, Suk JS, Cone R, Hanes J. Mucus penetrating nanoparticles: biophysical tool and method of drug and gene delivery. Advanced Materials (Deerfield Beach, FL). 2012;24:3887-94.

[66] Suk JS, Boylan NJ, Trehan K, Tang BC, Schneider CS, Lin J-MG, et al. N-acetylcysteine enhances cystic fibrosis sputum penetration and airway gene transfer by highly compacted DNA nanoparticles. Molecular Therapy: The Journal of the American Society of Gene Therapy. 2011;19:1981-9. DOI:10.1038/mt.2011.160.

[67] Andries O, De Filette M, Rejman J, De Smedt SC, Demeester J, Van Poucke M, et al. Comparison of the gene transfer efficiency of mRNA/GL67 and pDNA/GL67 complexes in respiratory cells. Molecular Pharmaceutics. 2012;9:2136-45. DOI:10.1021/ mp200604h.

[68] Yan Z, Stewart ZA, Sinn PL, Olsen JC, Hu J, McCray PB, et al. Ferret and pig models of cystic fibrosis: prospects and promise for gene therapy. Human Gene Therapy Clinical Development. 2015. DOI:10.1089/humc.2014.154.

[69] Olivier AK, Gibson-Corley KN, Meyerholz DK. Animal models of cystic fibrosis: gastrointestinal, pancreatic and hepatobiliary disease and pathophysiology. American Journal of Physiology Gastrointestinal and Liver Physiology. 2015:ajpgi.00146.2014. DOI:10.1152/ajpgi.00146.2014.

[70] Snouwaert JN, Brigman KK, Latour AM, Malouf NN, Boucher RC, Smithies O, et al. An animal model for cystic fibrosis made by gene targeting. Science (New York, NY). 1992;257:1083-8.

[71] Clarke LL, Grubb BR, Gabriel SE, Smithies O, Koller BH, Boucher RC. Defective epithelial chloride transport in a gene-targeted mouse model of cystic fibrosis. Science (New York, NY). 1992;257:1125-8.

[72] Zhou L, Dey CR, Wert SE, DuVall MD, Frizzell RA, Whitsett JA. Correction of lethal intestinal defect in a mouse model of cystic fibrosis by human CFTR. Science (New York, NY). 1994;266:1705-8.

[73] Kajstura J, Rota M, Hall SR, Hosoda T, D'Amario D, Sanada F, et al. Evidence for human lung stem cells. The New England Journal of Medicine. 2011;364:1795-806. DOI: 10.1056/NEJMoa1101324.

[74] Kim CFB, Jackson EL, Woolfenden AE, Lawrence S, Babar I, Vogel S, et al. Identification of bronchioalveolar stem cells in normal lung and lung cancer. Cell. 2005;121:823-35. DOI:10.1016/j.cell.2005.03.032.

[75] Hong KU, Reynolds SD, Giangreco A, Hurley CM, Stripp BR. Clara cell secretory protein-expressing cells of the airway neuroepithelial body microenvironment include a label-retaining subset and are critical for epithelial renewal after progenitor 
cell depletion. American Journal of Respiratory Cell and Molecular Biology. 2001;24:671-81. DOI:10.1165/ajrcmb.24.6.4498.

[76] Fujino N, Kubo H, Suzuki T, Ota C, Hegab AE, He M, et al. Isolation of alveolar epithelial type II progenitor cells from adult human lungs. Laboratory Investigation; a Journal of Technical Methods and Pathology. 2011;91:363-78. DOI:10.1038/labinvest. 2010.187. 



\section{Edited by Dennis Wat}

Cystic Fibrosis in the Light of New Research provides the latest research and clinical evidence that will be useful for clinicians, scientists and researchers to further their knowledge around this fascinating condition. The authors have brought along their expertise and wealth of knowledge to produce this book, including the basic science that underlies the disease, the burden of bacterial and viral infections, immunologic aspects of CF, a variety of clinical measurements to predict prognosis and novel therapies including gene therapy.

This book will be invaluable and entertaining for anyone who is involved in the care of patients with cystic fibrosis. 